\author{
Mon ograph \\ urn:1sid:zoobank.org:pub:7394D45E-46E1-453C-BF7E-1FE1B2CEBB0A
}

\title{
Revisions of Holocnemus and Crossopriza: the spotted-leg clade of Smeringopinae (Araneae, Pholcidae)
}

\author{
Bernhard A. HUBER ${ }^{\circledR}$ \\ Zoological Research Museum Alexander Koenig, Bonn, Germany. \\ Email: b.huber@leibniz-zfmk.de \\ urn:lsid:zoobank.org:author:33607F65-19BF-4DC9-94FD-4BB88CED455F
}

\begin{abstract}
The genera Holocnemus Simon, 1873 and Crossopriza Simon, 1893 are revised. Together with Stygopholcus Kratochvíl, 1932 (revised recently) and the newly described genus Maghreba gen. nov., they constitute the spotted-leg clade within the northern clade of Smeringopinae. Males and females in this group are characterized by dark marks on the leg femora and tibiae. The native area of the spotted-leg clade ranges from northern Africa and the Mediterranean to Central Asia and NW India. A morphological cladistic analysis suggests that Holocnemus is paraphyletic while Crossopriza is monophyletic, but morphology seems only partly adequate to resolve phylogenetic relationships convincingly. The genus Holocnemus includes four species, all of which are redescribed: H. pluchei (Scopoli, 1763); H. reini (C. Koch, 1873) comb. nov. (transferred from Pholcus); H. caudatus (Dufour, 1820); and H. hispanicus Wiehle, 1933. The genus Maghreba gen. nov. includes eight species from NW Africa: M. aurouxi (Barrientos, 2019) gen. et comb. nov. (transferred from Holocnemus; redescribed, female newly described) and seven newly described species. The genus Crossopriza includes six previously described species (of which five are redescribed), and 18 newly described species. The Madagascan C. nigrescens Millot, 1946 is synonymized with $C$. lyoni (Blackwall, 1867). All new species are described on the basis of both sexes.
\end{abstract}

Keywords. Taxonomy, sexual dimorphism, synanthropic, stridulation, gynander.

Huber B.A. 2022. Revisions of Holocnemus and Crossopriza: the spotted-leg clade of Smeringopinae (Araneae, Pholcidae). European Journal of Taxonomy 795: 1-241. https://doi.org/10.5852/ejt.2022.795.1663

\section{Table of contents}

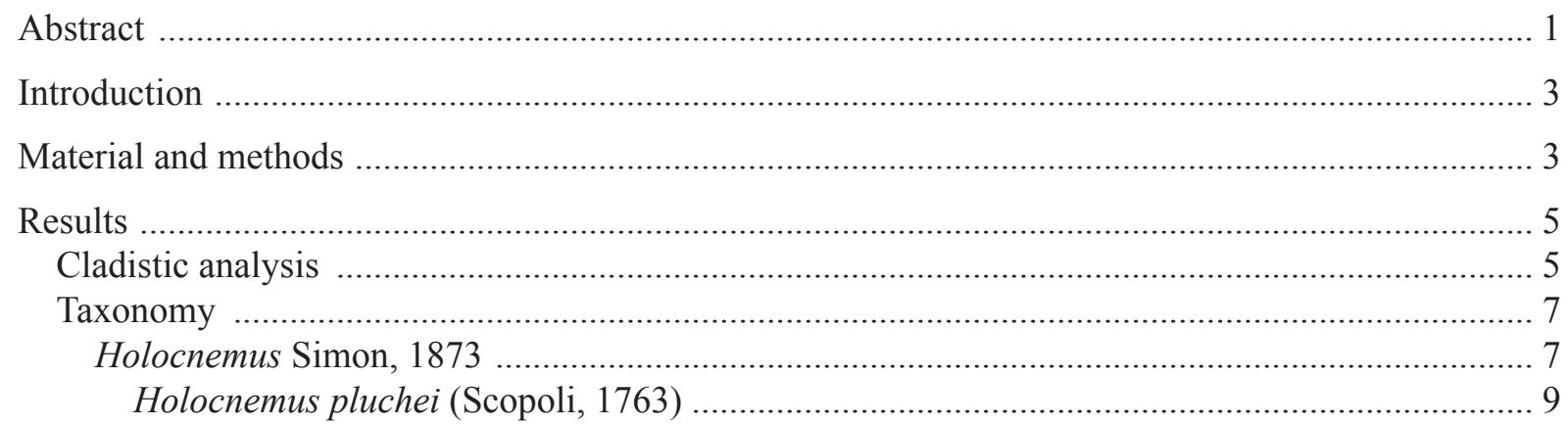




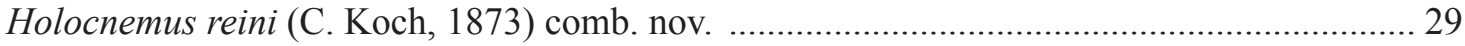

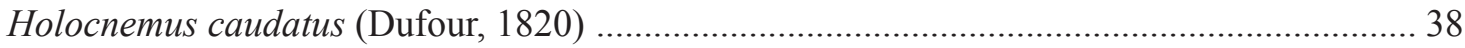

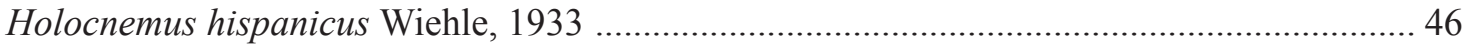

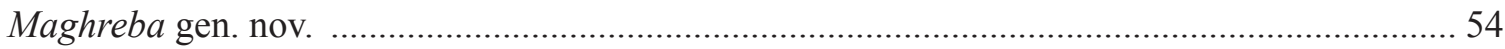

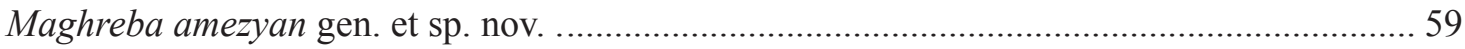

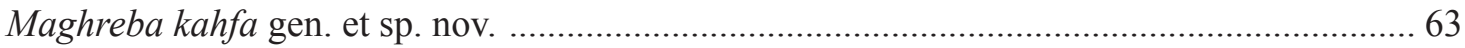

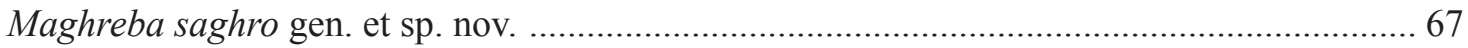

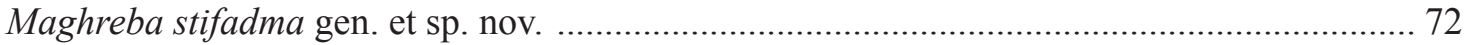

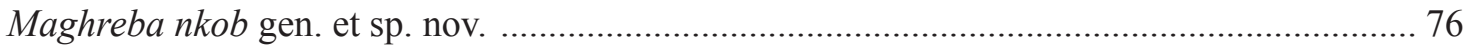

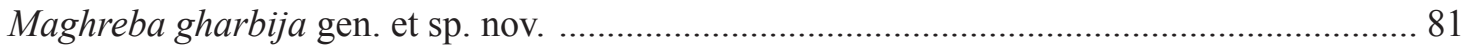

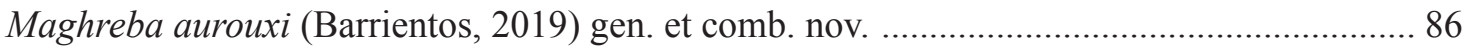

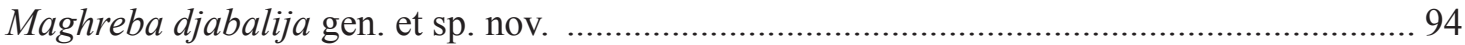

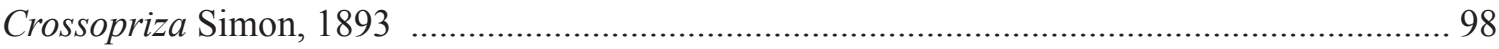

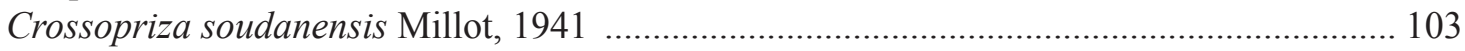

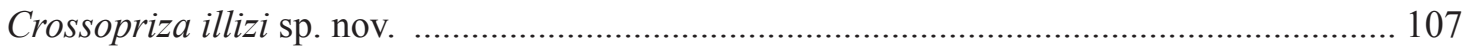

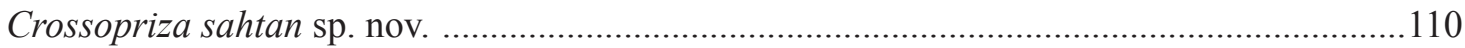

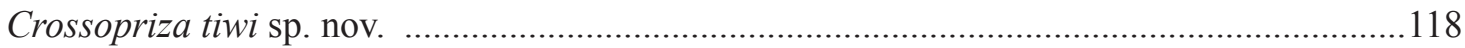

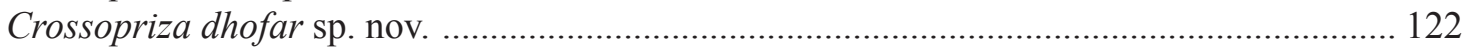

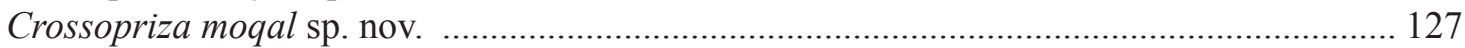

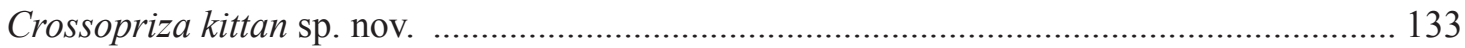

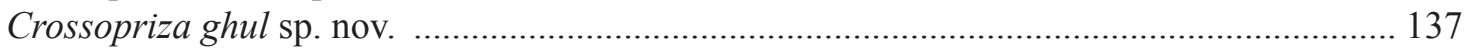

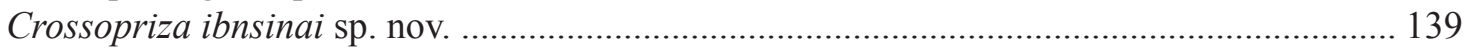

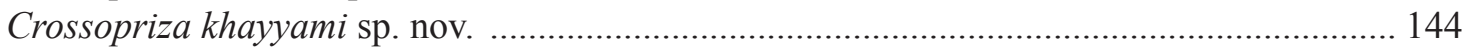

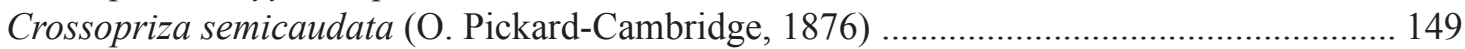

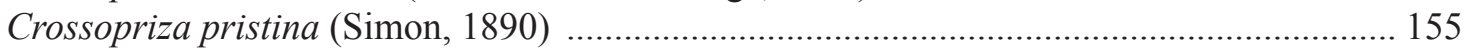

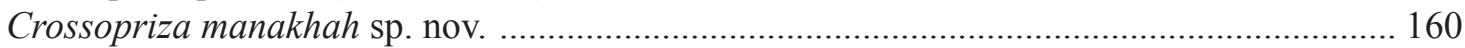

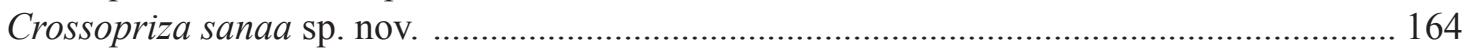

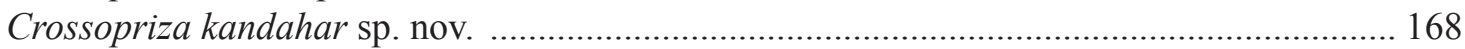

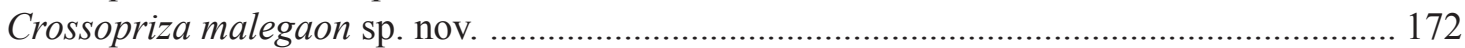

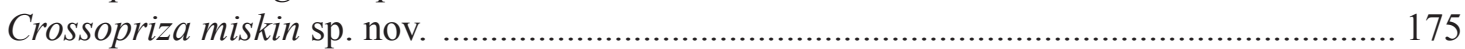

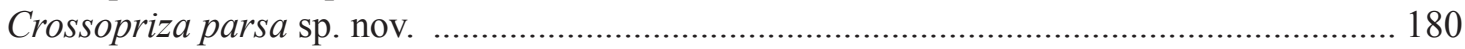

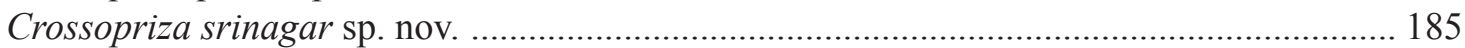

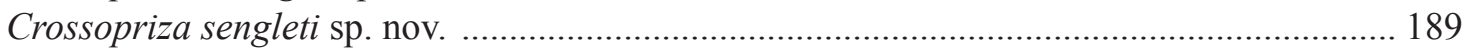

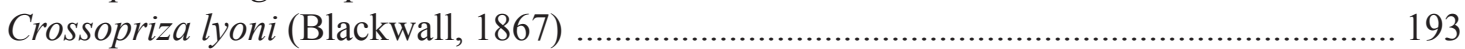

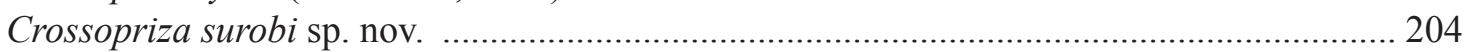

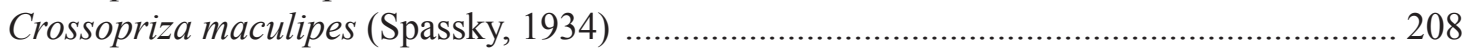

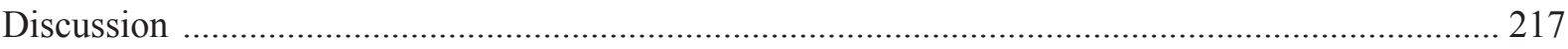

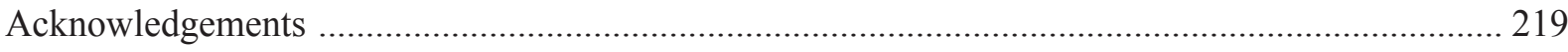

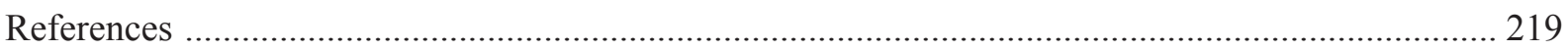

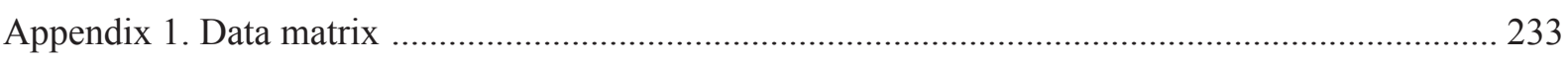

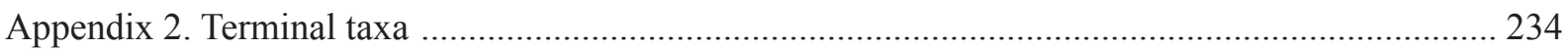

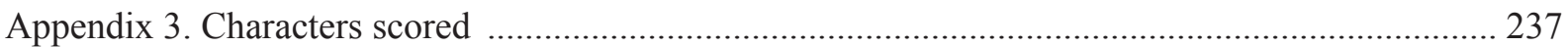




\section{Introduction}

The subfamily Smeringopinae Simon, 1893 was originally restricted to Africa, the Mediterranean, and the Middle East up to Central Asia and India. A few species have followed humans around the globe and are widely known due to their close association with houses and other constructions. Among these, Smeringopus pallidus (Blackwall, 1858) and Crossopriza lyoni (Blackwall, 1867) remain largely restricted to tropical and subtropical regions, while Holocnemus pluchei (Scopoli, 1763) has been spreading to more temperate regions (Huber 2012; herein).

Most representatives of Smeringopinae are medium-sized to large species (mean male carapace width $1.8 \mathrm{~mm}$; mean male tibia 1 length $12.4 \mathrm{~mm}$ ). Of approximately 650 Pholcidae C.L. Koch, 1850 species with a male carapace width of less than $1 \mathrm{~mm}$, only one is a representative of Smeringopinae (Huber 2021b). The relatively large size, together with the relatively exposed life-style of many species explains that the group includes some of the oldest names in Pholcidae. In fact, Holocnemus pluchei was the very first pholcid spider to be formally described. Nevertheless, the systematics of the subfamily has long been very poorly known. Ten years ago, only 56 nominal species were 'known to science' (Huber 2011b), many of them with names yet unidentifiable using the existing literature.

Starting in 2012, I have tried to alleviate this situation with a series of taxonomic revisions and cladistic analyses. The first two monographs dealt with the southern clade of Smeringopinae, the Sub-Saharan sister-genera Smeringopus Simon, 1890 and Smeringopina Kraus, 1957 (Huber 2012, 2013). The third and fourth monographs focused on the West Asian and SE European genera Hoplopholcus Kulczyński, 1908 and Stygopholcus Kratochvíl, 1932 (Huber 2020; Huber et al. 2021). The remaining genera (except for the small Seychellois endemic genus Cenemus Saaristo, 2001) appeared like the most problematic. Wiehle's (1933) meticulous struggle (and failure) to find a convincing morphological distinction between Crossopriza Simon, 1893 and Holocnemus Simon, 1873 testifies both to the long history of the problem and to its intricacy. Making things even worse, new material collected over the last decades in northwestern Africa (mainly Morocco) has complicated the situation further. These Moroccan and Algerian species (one of which was recently described as Holocnemus aurouxi Barrientos, 2019) seemed intermediate between Holocnemus and Crossopriza yet distinct from both. Molecular data have not so far resolved the phylogeny of this group, mainly because of insufficient taxon-sampling (Dimitrov et al. 2013; Eberle et al. 2018).

The present paper was originally intended to terminate this series on Smeringopinae and to finally clarify the systematics of Holocnemus, Crossopriza, and the mysterious northwest African taxa. However, the available data allowed only a first step, i.e., a detailed redescription of all previously described species and the description of new species currently available in collections. A second step clearly has to follow: a step, in which phylogenetic relationships can finally be resolved convincingly. The present paper presents a first comprehensive morphological cladistic analysis of Holocnemus and Crossopriza, but this is included primarily in order to point out characters that appear phylogenetically informative. Parts of the cladogram are unstable and thus neither convincing nor final. A more convincing solution will probably require the use of genomic data.

With now 158 species, Smeringopinae is still a relatively small subfamily, but one in which (1) almost all species are diagnosable, (2) most species have been included in phylogenetic analyses, and (3) distribution and diversity patterns can be described with reasonable accuracy.

\section{Material and methods}

This study is based on the examination of over 3500 adult specimens deposited in the 41 collections listed below. Taxonomic descriptions follow the style of recent publications on Smeringopinae (e.g., 
Huber 2020; Huber et al. 2021; based on Huber 2000). Measurements were done on a dissecting microscope with an ocular grid and are in mm unless otherwise noted; eye measurements are $\pm 5 \mu \mathrm{m}$; diameters of femora were measured at half length. Photos were made with a Nikon Coolpix 995 digital camera $(2048 \times 1536$ pixels $)$ mounted on a Nikon SMZ 18 stereo microscope or a Leitz Dialux 20 compound microscope. CombineZP (https://combinezp.software.informer.com/) was used for stacking photos. Drawings are partly based on photos that were traced on a light table and later improved under a dissecting microscope, or they were directly drawn with a Leitz Dialux 20 compound microscope using a drawing tube. Cleared epigyna were stained with chlorazol black. The number of decimals in coordinates is supposed to give a rough indication about the accuracy of the locality data: four decimals means that the collecting site (or cave entrance) is within about $10 \mathrm{~m}$ of the indicated spot; three decimals: within $\sim 100 \mathrm{~m}$; two decimals: within $\sim 1 \mathrm{~km}$; one decimal: within $\sim 10 \mathrm{~km}$. Distribution maps were generated with ArcMap ver. 10.0. For SEM photos, specimens were dried in hexamethyldisilazane (HMDS) (Brown 1993), and photographed with a Zeiss Sigma 300 VP scanning electron microscope. SEM data are presented within the descriptions but are usually not based on the specific specimen described. The same is also often true for other illustrations, implying that no differences were seen between the described and the illustrated specimen. The exact origins of the illustrated specimens are given in the figure legends. Larger series of specimens were often split into two vials (with two registry numbers); unless otherwise noted, the first vial contains the males, the second vial contains the females and juveniles.

The numerical cladistic analyses were done using NONA ver. 2 (Goloboff 1993), Pee-Wee ver. 2.8 (Goloboff 1997), and TNT ver. 1.1 (Goloboff et al. 2004, 2008). The matrix is shown in Appendix 1; it can be downloaded at http://www.pholcidae.de/matrices.html; terminal taxa and characters scored are given in Appendices 2 and 3. Of the 40 characters, 38 are binary; the two non-binary characters $(37,40)$ are treated as non-additive. Cladogram analysis was done with Winclada ver. 1.00.08 (Nixon 2002). See Cladistic analysis section below for details of the analyses.

\section{Institutional abbreviations}

$\begin{array}{ll}\text { AMNH } & =\text { American Museum of Natural History, New York, USA } \\ \text { AMS } & =\text { Australian Museum, Sydney, Australia } \\ \text { BMNH } & =\text { Natural History Museum, London, UK } \\ \text { BPBM } & =\text { Bishop Museum, Honolulu, Hawaii, USA } \\ \text { CAS } & =\text { California Academy of Sciences, San Francisco, USA } \\ \text { CBSU } & =\text { Collection of Biology of Shiraz University, Iran } \\ \text { CEM } & =\text { Collection Eduardo Morano, Toledo, Spain } \\ \text { CJL } & =\text { Collection Jørgen Lissner, Aarhus, Denmark } \\ \text { CJVK } & =\text { Collection Johan Van Keer, Kapelle-op-den-Bos, Belgium } \\ \text { CMK } & =\text { Collection Marjan Komnenov, Skopje, Macedonia } \\ \text { CPO } & =\text { Collection Pierre Oger, Waret l'Evêque, Belgium } \\ \text { CRB } & =\text { Collection Robert Bosmans, Gent, Belgium } \\ \text { CRBA } & =\text { Biodiversity Resource Center of the University of Barcelona, Spain } \\ \text { HECO } & =\text { Hope Entomological Collection, Oxford, Great Britain } \\ \text { HISR } & =\text { Hellenic Institute of Speleological Research, Iraklion, Greece } \\ \text { MACN } & =\text { Museo Argentino de Ciencias Naturales, Buenos Aires, Argentina } \\ \text { MCN } & =\text { Museu de Ciências Naturais, Porto Alegre, Brazil } \\ \text { MCP } & =\text { Pontificia Universidade Católica do Rio Grande do Sul, Porto Alegre, Brazil } \\ \text { MCZ } & =\text { Museum of Comparative Zoology, Cambridge, USA } \\ \text { MELN } & =\text { Museo Entomologico, S.E.A., León, Nicaragua } \\ \text { MGAB } & =\text { Muzeul Național de Istorie Naturală Grigore Antipa, Bucharest, Romania } \\ \text { MHNG } & =\text { Muséum d'histoire naturelle, Genève, Switzerland }\end{array}$




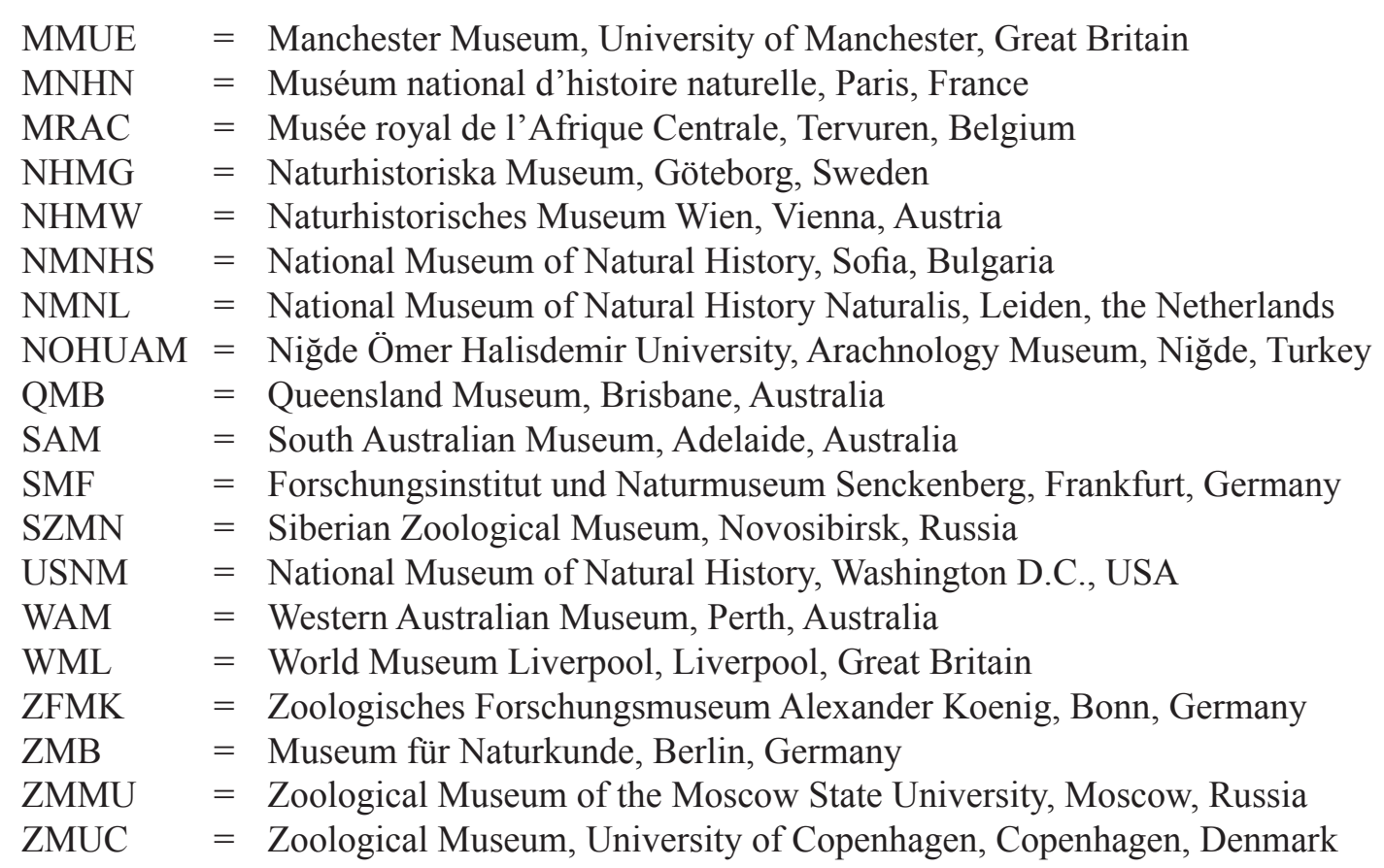

\section{Other abbreviations used in the text}

$\begin{array}{ll}\mathrm{ALE} & =\text { anterior lateral eye }(\mathrm{s}) \\ \mathrm{ALS} & =\text { anterior lateral spinneret(s) } \\ \mathrm{AME} & =\text { anterior median eye }(\mathrm{s}) \\ \text { a.s.l./b.s.l. } & =\text { above sea level/below sea level } \\ \mathrm{L} / \mathrm{d} & =\text { length/diameter } \\ \mathrm{PME} & =\text { posterior median eye(s) } \\ \mathrm{PMS} & =\text { posterior median spinneret(s) }\end{array}$

Abbreviations used in figures only are explained in the figure legends.

\section{Results}

\section{Cladistic analysis}

Using NONA with hold/100, mult*200 (or hold/10; mult*10.000), and amb- for the matrix in Appendix 1 and equal character weights resulted in a single most parsimonious cladogram with a length of 60 $(\mathrm{Ci}=70 ; \mathrm{Ri}=90)$ (Fig. 1). The implicit enumeration algorithm in TNT (using a matrix from which the 18 species with identical coding to other taxa had been deleted) resulted in a single tree with the identical topology. Successive weighting in NONA (with the consistency index as weighting function) also resulted in the same single most parsimonious cladogram.

Implied weighting in Pee-Wee (which resolves character conflict in favor of the characters that have less homoplasy) was used with various settings of the constant of concavity (conc $=1-6$ ) to explore the stability of different clades under different weighting regimes. Differences to the tree shown in Fig. 1 occurred only with conc $=1$ and conc $=2$. Both settings resulted in the same two most parsimonious cladograms that shared a noteworthy feature: Hoplopholcus was placed inside the spotted-leg clade, as sister to Stygopholcus. As a result, Maghreba gen. nov. was no longer sister of Stygopholcus but either sister of Holocnemus hispanicus or in a trichotomy with H. hispanicus and Crossopriza. Other than that, the main results were similar to those using equal character weights and successive character weighting: non-monophyly of Holocnemus; monophyly of Hoplopholcus, 


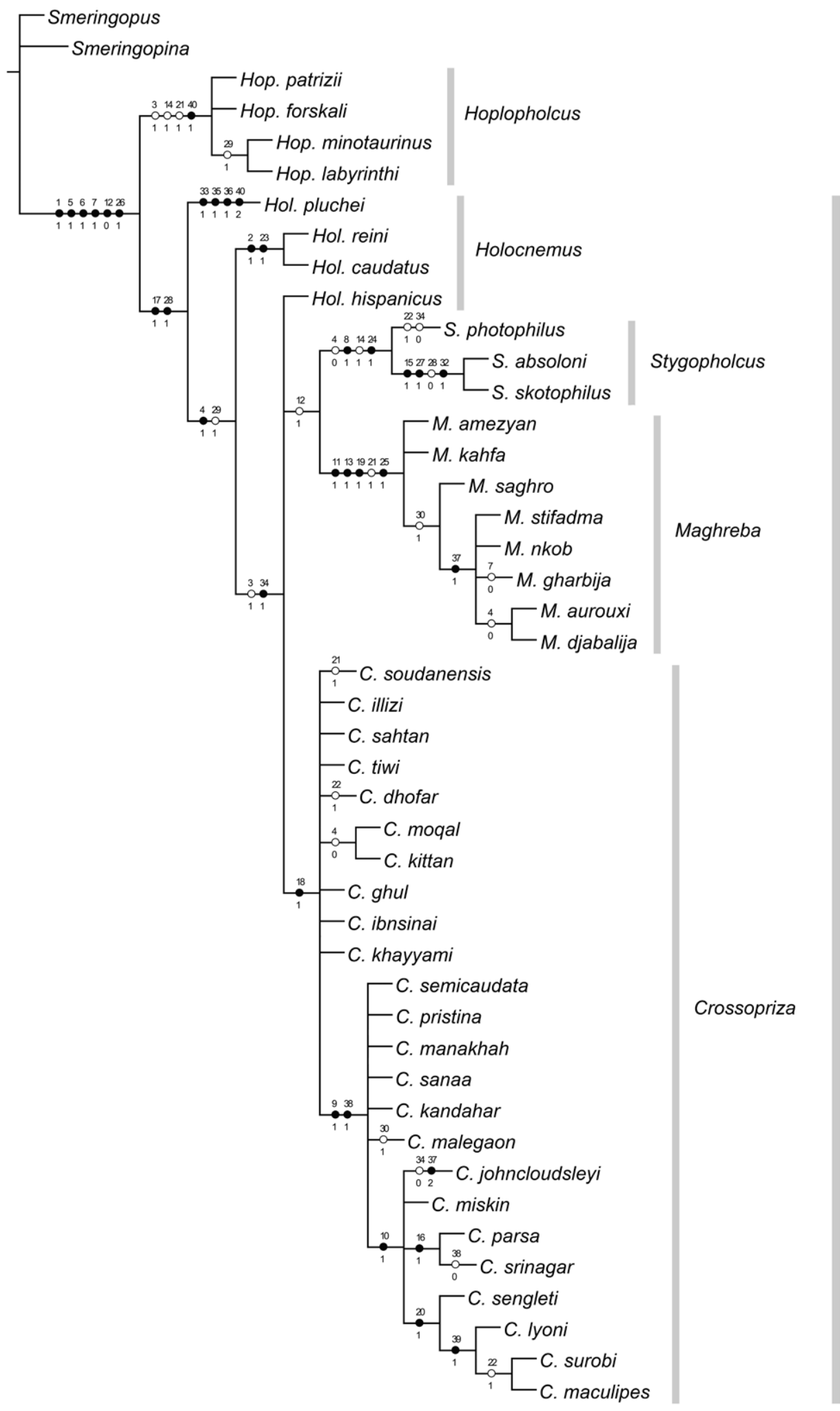

Fig. 1. Single most parsimonious cladogram of the spotted-leg clade resulting from analyses of the matrix in Appendix 1 using equal character weights and successive character weighting. Only unambigous character changes are shown. See Appendix 3 for differences in analyses using implied character weighting with conc $=1$ and 2 . 
Stygopholcus, Maghreba gen. nov., and Crossopriza. The cladogram shown in Fig. 1 is preferred because it does not require a regain of the retrolateral trichobothrium on tibia 1 (which the Pee-Wee cladograms do). All differences of the Pee-Wee cladograms are detailed in Appendix 3 (see characters $3,4,14,17,28,29,31,34)$.

\section{Taxonomy}

Class Arachnida Lamarck, 1801

Order Araneae Clerck, 1757

Family Pholcidae C.L. Koch, 1850

Holocnemus Simon, 1873

Holocnemus Simon, 1873: 48 (type species: Aranea pluchei Scopoli, 1763).

Holocnemus - Simon 1893: 471. — Wiehle 1933: 241.

\section{Remark}

Holocnemus is an artificial non-monophyletic group (see Relationships below) that is here maintained for the lack of a convincing better solution. The four included species can be weakly diagnosed by sharing the synapomorphies of the spotted-leg clade (i.e., spotted legs and prolateral hump on the procursus) while maintaining the plesiomorphic long abdomen. A comprehensive description of a nonmonophyletic genus appears of little value. The type species $H$. pluchei is unique in many ways and may eventually end up in its own monotypic genus. The species description below will thus probably serve the entire genus. For the other three species, I also refer to the redescriptions below.

\section{Relationships}

There is strong evidence that the four species currently included in Holocnemus are part of the spotted-leg clade of Smeringopinae, together with the genera Crossopriza, Maghreba gen. nov., and Stygopholcus. Beyond that, however, relationships continue to be obscure. All available evidence suggests that Holocnemus as currently construed is not monophyletic. The cladistic analysis places the type species $H$. pluchei as sister to all other representatives of the spotted-leg clade, and the other species

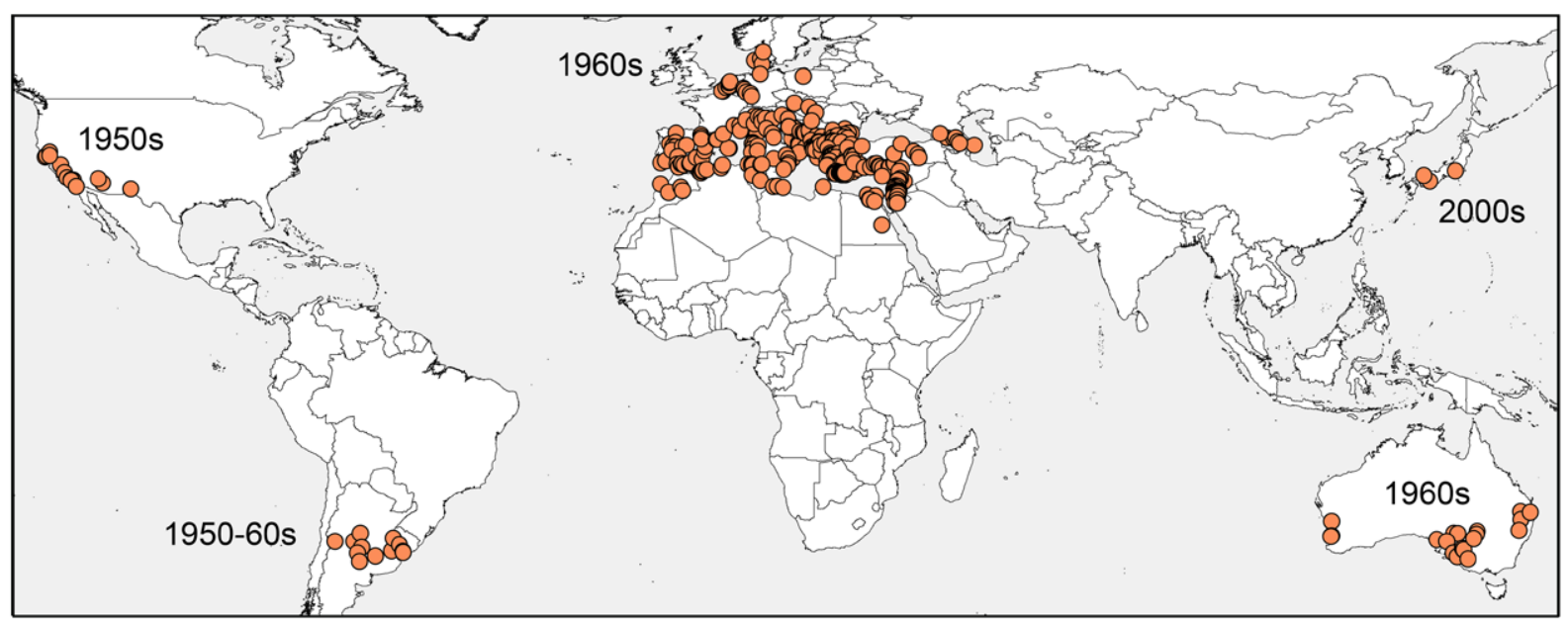

Fig. 2. Known distribution of Holocnemus pluchei (Scopoli, 1763), with approximate decades in which the species appeared in North America, South America, Central Europe, Australia, and Japan; see Distribution section for details. 


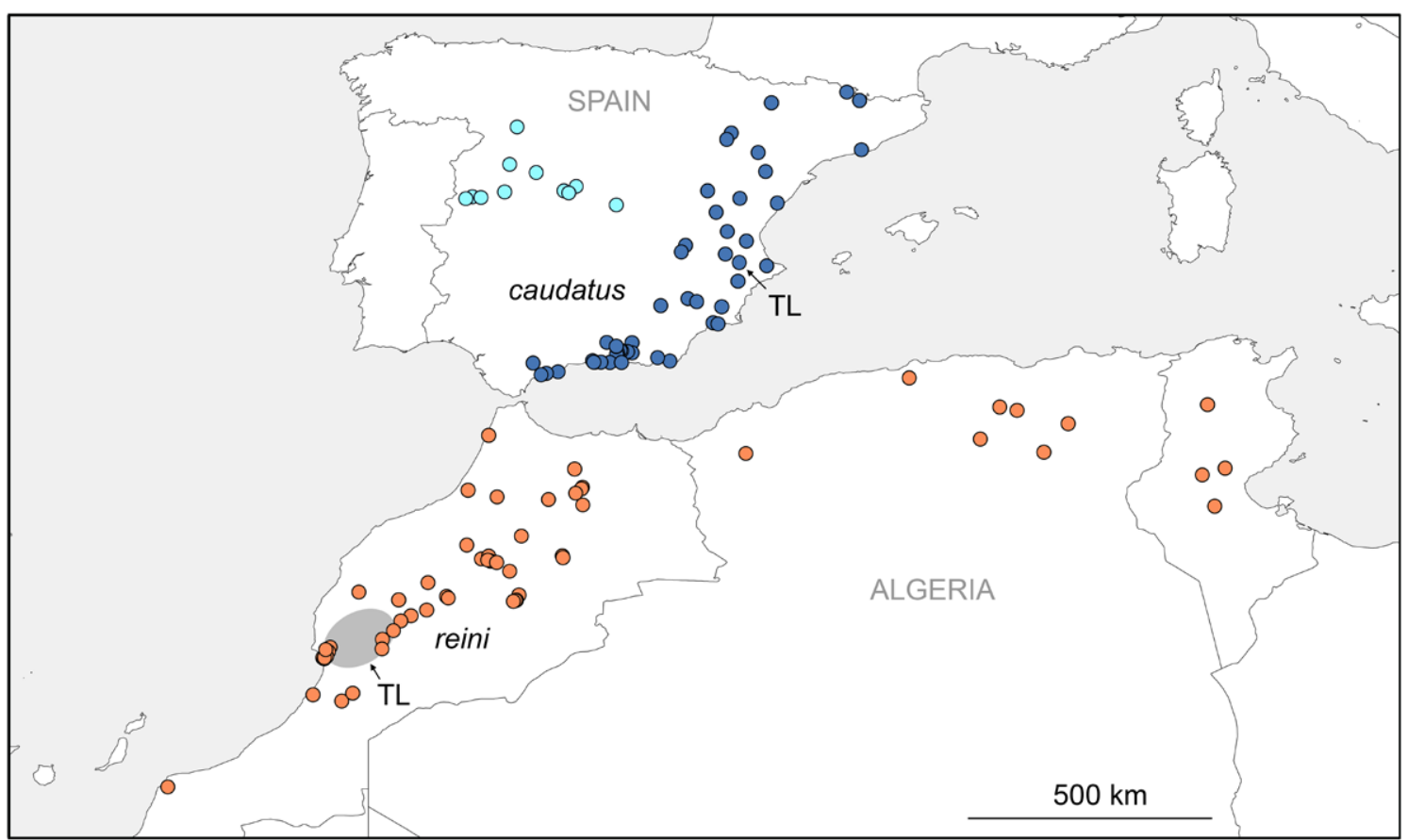

Fig. 3. Known distributions of Holocnemus reini (C. Koch, 1873) comb. nov. and H. caudatus (Dufour, 1820). Light blue: dubious records; see Distribution section for details. Abbreviation: $\mathrm{TL}=$ type localities (the type locality of $H$. reini is not exactly known; see text for details).

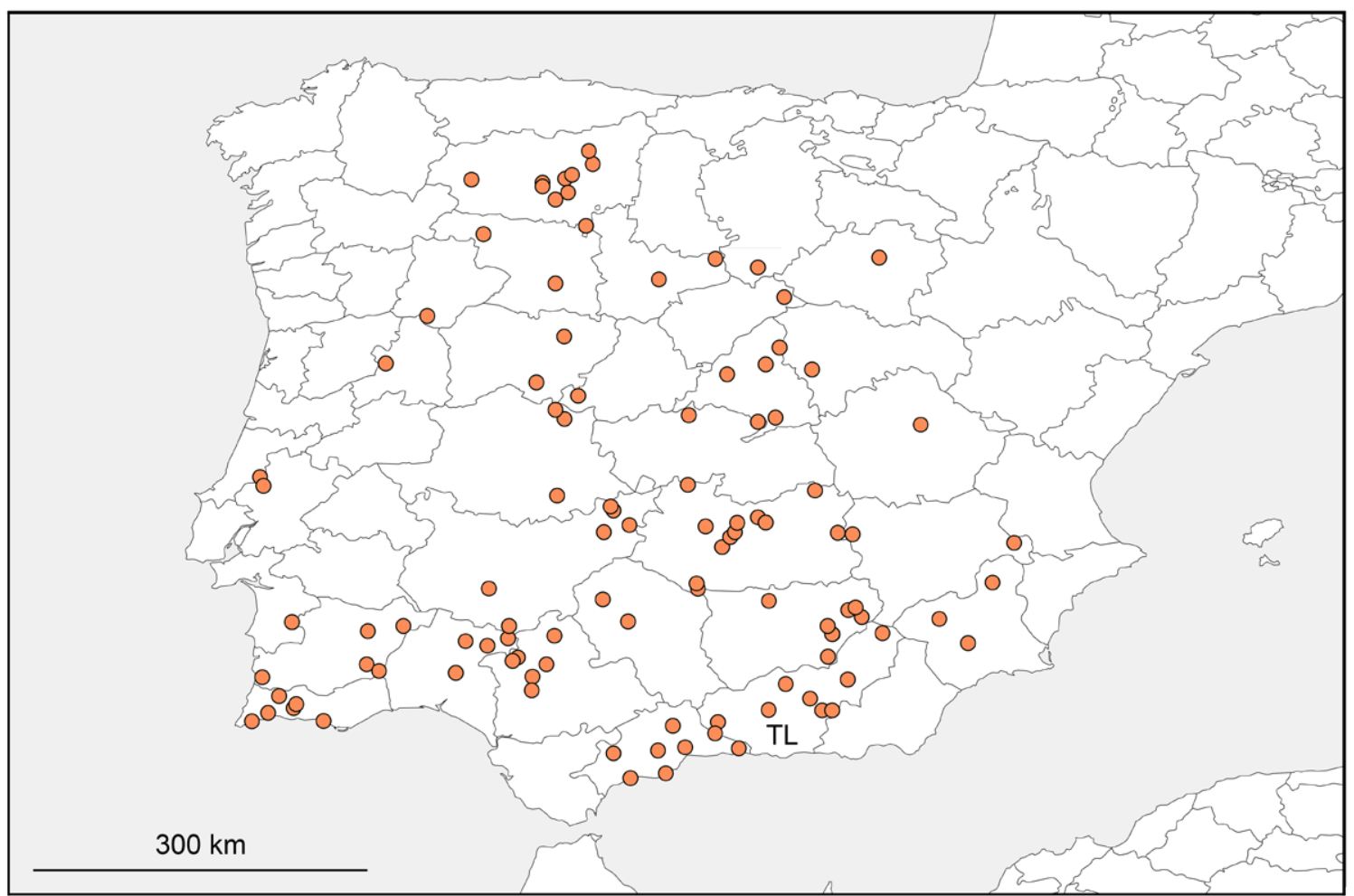

Fig 4. Known distribution of Holocnemus hispanicus Wiehle, 1933 on the Iberian Peninsula. Abbreviation: $\mathrm{TL}=$ type locality. 
in a paraphyletic comb (Fig. 1). Support for several of these nodes is weak, however, suggesting that a genomic analysis (in preparation) may well propose a different view of relationships. For that reason, no formal taxonomic changes are implemented here.

\section{Distribution}

The type species $H$. pluchei is widely distributed around the Mediterranean, and has relatively recently expanded towards the north and east and to other continents (Fig. 2). The other three species are restricted to the western Mediterranean: H. reini to northwestern Africa (Fig. 3), H. caudatus and H. hispanicus to the Iberian Peninsula (Figs 3-4).

\section{Natural history}

See individual sections on natural history below.

\section{Composition}

Currently four nominal species. The species number of this 'group' is not likely to change substantially (or not at all) in the future, considering the size of the spiders, their relatively overt life-style (compared to most other Smeringopinae), and their geographic distribution.

\section{Identification key}

1. Female with enlarged palps (Figs 29-30), with median process on sternum (Figs 31, 58); male with pair of distinctive processes on distal bulbal sclerite (Figs 24, 26) H. pluchei (Scopoli, 1763)

- Female with regular thin palps, without median process on sternum; males with very different distal bulbal sclerites (Figs 66-68, 139-141)

2. Sternum in male and female unmodified (i.e., without marginal indentations); male cheliceral apophyses with one modified hair each (Figs 142, 150); procursus ventral sclerite not covered retrolaterally by membrane (Fig. 138); distal bulbal sclerite trapezoidal in prolateral view (Figs 139, 154); epigynum with two long, anteriorly converging pockets (furrows) (Figs 133-134, 162)

H. hispanicus Wiehle, 1933

- Sternum in male and female with distinct marginal indentations (Figs 81, 112); male cheliceral apophyses with 2-3 modified hairs each (Figs 64, 82-83, 115); procursus with distinctive retrolateral membrane partly covering ventral sclerite (Figs 63, 117); distal bulbal sclerite triangular in prolateral view (Figs 66, 92); epigynum with pair of pockets in different arrangement (Figs 69, 96, 99, 128) ...

3. Wide distances between male cheliceral apophyses (70-80\% of cheliceral maximum width) and between female epigynal pockets $(>0.5 \mathrm{~mm})$ H. reini comb. nov. (C. Koch, 1873)

- Small distances between male cheliceral apophyses (50-60\% of cheliceral maximum width) and between female epigynal pockets $(<0.4 \mathrm{~mm})$ H. caudatus (Dufour, 1820)

\section{Holocnemus pluchei (Scopoli, 1763)}

Figs 2, 5-6, 13-58

Aranea pluchii Scopoli, 1763: 404.

Aranea rivulata Forsskål, 1775: 86. Synonymized with Pholcus pluchii by Simon 1866: 122 (but: Simon 1873).

Pholcus impressus C.L. Koch, 1837: 99, pl. 137 fig. 313. Synonymized with H. rivulatus by Simon 1873: 49.

Pholcus barbarus Lucas, 1846: 237, pl. 15 fig. 1, 1a-f. Synonymized with H. rivulatus by Simon 1873: 49.

Pholcus ruralis Blackwall, 1858: 432. Synonymized by Simon 1873: 49. 
Aranea rivulata - Forsskål 1776: 7, pl. 24 fig. f.

Aranea pluchii? (question mark in original publication) - Rossi 1790: 134.

Pholcus rivulatus - Savigny \& Audouin 1826: 140, pl. 3 fig. 12. — Walckenaer 1837: 653. — PickardCambridge 1872: 277; 1876: 566. — L. Koch 1875: 25 (specimens from Cairo).

Pholcus impressus - C.L. Koch 1850: 31. — Simon 1866: 122.

Pholcus pluchii-Simon 1866: 122, pl. 2 fig. 11.

Pholcus barbarus - Simon 1866: 123. — C. Koch 1873: 113.

Holocnemus rivulatus - Simon 1873: 49; 1874: 256; 1875: pl. 4 fig. 16; 1885: 28; 1893: 471; 1908: 427. —Damin 1900: 21. — Strand 1908: 93. — Lessert 1910: 89. — Franganillo 1925: 35; 1926c: 70.

Holocnemus pluchii - Roewer 1928: 120. — Kratochvíl 1932: 2, figs 1, 3-4. — Drensky 1936: 55; 1939: 248. — Kolosváry 1938: 65. — Senglet 1971: 354. — Timm 1976: 70, figs 1-2. — Nicolić \& Polenec 1981: 20. — Kritscher 1996: 123.

Holocnemus pluchei - Simon 1914: 237. - Dalmas 1920: 59. — Wiehle 1933: 242, 247, figs 2a, 7c. — Kratochvíl 1940: 7, fig. 1b, d. — Caporiacco 1948: 38. — Roewer 1959: 10. — Brignoli 1971a: 82, figs 1-6; 1971b: 130; 1971c: 257; 1976: 559; 1977: 31; 1978: 486; 1979: 189; 1984: 289. — Constantini 1975: 90. — Wunderlich 1980: 227, figs 31-32. — Barrientos \& Ferrández 1982: 82. — Roth 1985: B-33-1; 1994: 145. — Jakob \& Dingle 1990: 95. — Porter \& Jakob 1990: 313. — Jakob 1991: 711. — Heimer \& Nentwig 1991: 40, fig. 80.1-3. — Melic 1994: 15. — Huber 1995: 291, figs 1a, 2, 4, 6-8, 10a; 2000: figs 14, 120, 176; 2001: 136; 2014: 140. — Jäger 1995: 20; 2000: 51. - Johnson \& Jakob 1999: 957. — Van Keer \& Van Keer 2001: 82; 2004: 79. — Deltshev et al. 2003: 12; 2011: 130; 2013: 7. — Gasparo 2003: 62. — Hajer \& Řeháková 2003: 345. — Topçu et al. 2005: 289. — Ferrández et al. 2006: 77. — Kovács et al. 2006: 9, figs 1-3. — Van Keer 2007: 48. — Laborda \& Simó 2008: 262. — Kunt et al. 2010: 32. —Calbacho-Rosa et al. 2010: 1267; 2013: 407; 2019a: 1, fig. 1; 2019b: 1, figs 1-3. - Van Helsdingen 2010: 27. — Drakšić \& Katušić 2011: 168. - Dutto et al. 2011: 1040. - Vetter et al. 2011: 601. — Bosmans et al. 2013: 24. — Türkeș \& Karabulut 2013: 619. - Benhadi-Marín et al. 2013: 75, fig. 2 (C1-3). — Reiser \& Neumann 2014: 24. — Gajić \& Grbić 2016: 54. — Naumova et al. 2016: 434; 2019: 76. — Rozwałka et al. 2016: 73. — Barrientos \& Bosco Febrer 2017: 22. —Cargnelutti et al. 2018: 616; 2020: 536. — Lecigne 2018: 50, pl. 1 figs h-i, photo 3. - Ponomarev et al. 2019: 233, figs 2-4. — Kumada 2021: 17-19, figs 1-9.

\section{Misidentifications}

Pholcus rivulatus - L. Koch 1875: 25 (specimens from Massaua: Crossopriza sp. juveniles; see Distribution below).

Pholcus rivulatus - Leardi in Airaghi 1902: 348 (India; identity unclear; see Distribution below).

Holocnemus pluchei - Roewer 1960: 40 (Afghanistan; juvenile from Rig-Revan: Pholcus sp.; for other specimens, see Crossopriza ibnsinai sp. nov.).

\section{Diagnosis}

Easily distinguished from all other known Smeringopinae by enlarged female palps (Figs 29-30), by median process on female sternum (Figs 31,58), and by pair of distinctive processes on male distal bulbal sclerite (Figs 24, 26); also by shape of procursus (Figs 20-22; distinct prolateral proximal process, short distal ventral sclerite), by female external and internal genitalia (Figs 27-28, 36-42; epigynum anteriorly with pair of sculptured areas; internal ventral arc with pair of strong pockets), and by heavily sclerotized female pedicel opposing pair of small sclerites on abdomen (Fig. 32).

\section{Type material}

\section{Syntypes of Aranea pluchii}

SLOVENIA • Unknown number; "Carniola" [part of present-day Slovenia], possibly Idria [Scopoli's place of residence at the time]; $46.00^{\circ} \mathrm{N}, 14.03^{\circ} \mathrm{E} ; 1759-1762$; G.A. Scopoli leg.; probably lost. 


\section{Syntypes of Aranea rivulata}

EGYPT • Unknown number; Cairo; $\sim 30.0^{\circ} \mathrm{N}, 31.2^{\circ} \mathrm{E}$; probably 1762; P. Forsskål leg.; probably lost.

\section{Syntypes of Pholcus impressus}

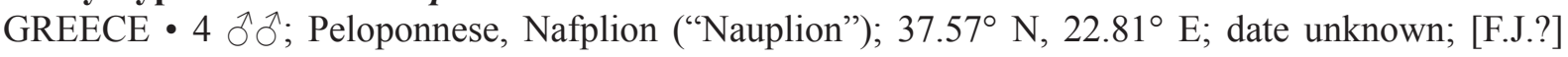
Schuch leg.; probably lost.

Syntypes of Pholcus barbarus (examined)

ALGERIA $-4 \hat{\jmath} \widehat{\partial}, 4$ q 9 , + juvs (possible syntypes); near Algiers; $36.75^{\circ} \mathrm{N}, 3.05^{\circ} \mathrm{E} ; 1839-1842$; P.H. Lucas leg.; with two labels: "Algerie, Lucas, Holocnemus rivulatus Forsk. sub barbarus Type" and "Holocnemus rivulatus Forsk (sub barbarus) type, Algerie, Lucas”; MNHN.

\section{Syntypes of Pholcus ruralis}

ALGERIA - Unknown number (possibly only 1 đ); locality not specified; possibly 1856; Gray and Clarck leg.; probably lost.

\section{Material examined}

Arranged from West to East and (within longitudes) from North to South.

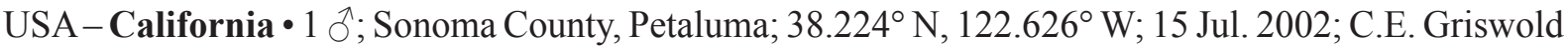
leg.; on house; CAS 9027116 part 1 क; Napa County, Lake Hennessey, below Lonn Dam; $38.48^{\circ} \mathrm{N}$, $122.38^{\circ}$ W; 11 May 1985; L. Vincent leg.; CAS 9027422 • 1 \%; Los Angeles County, Diamond Bar; $34.03^{\circ} \mathrm{N}, 117.80^{\circ} \mathrm{W} ; 23$ Sep. 1999; C. Yang leg.; CAS $9027418 \bullet 1$ \%; Orange County, Yorba Linda; $33.889^{\circ}$ N, $117.812^{\circ}$ W; 2 May 2000; S. Clayton leg.; CAS 9027393 • 1 + ; San Diego County, northern end of Harbison Canyon; $32.811^{\circ} \mathrm{N}, 116.844^{\circ} \mathrm{W} ; 250 \mathrm{~m}$ a.s.1.; 7 Jul. 2012; under stones and wood panels in scrubland; A. Schönhofer leg.; SMF.

ARGENTINA - Mendoza • 1 क; Mendoza; 32.89 ${ }^{\circ}$ S, $68.84^{\circ}$ W; 900 m a.s.1.; 30-31 Mar. 1965; H. Levi

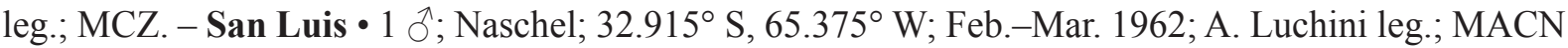

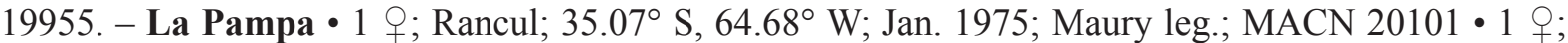
Santa Rosa [de Toay]; $36.62^{\circ} \mathrm{S}, 64.29^{\circ} \mathrm{W}$; date and collector unknown; MACN 20039. - Buenos Aires - 1 đ’; Carlos Casares; $35.62^{\circ}$ S, 61.36 ${ }^{\circ}$ W; 27 Feb.-2 Mar. 1980; collector unknown; MACN 20087 • 1 `, 2 우, 2 juvs; Buenos Aires, Cerrito; $34.60^{\circ}$ S, $58.38^{\circ}$ W; 19 Sep. 1972; C. Cesari leg.; in basement; MACN 20080.

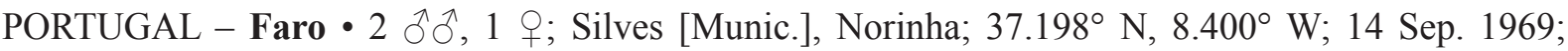
A. Senglet leg.; MHNG.

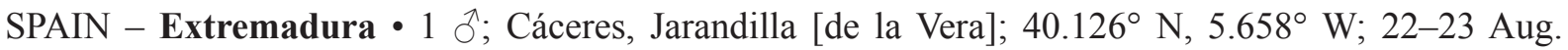

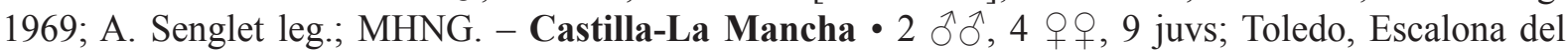

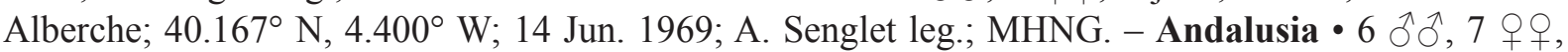

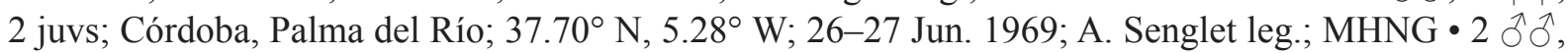
2 우; Córdoba, Peñarroya; $38.30^{\circ}$ N, 5.27 W 30 Jun. 1969; A. Senglet leg.; MHNG • 2 우; Cádiz,

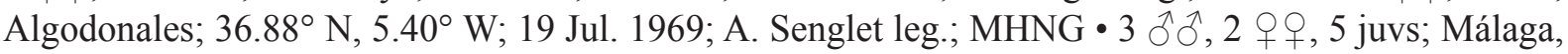
Estepona; $36.43^{\circ} \mathrm{N}, 5.15^{\circ} \mathrm{W}$; 24-26 Jul. 1969; A. Senglet leg.; MHNG • 2 + + ; Málaga, Valle de Abdalajís; $36.93^{\circ} \mathrm{N}, 4.68^{\circ} \mathrm{W} ; 28$ Jul. 1969; A. Senglet leg.; MHNG • 4 qo ; Málaga, Antequera; $37.02^{\circ} \mathrm{N}, 4.56^{\circ} \mathrm{W}$; $1-2$ Aug. 1969; A. Senglet leg.; MHNG • 3 đô, 5 우; Málaga, Torre del Mar; $36.75^{\circ}$ N, $4.10^{\circ}$ W; 27 Jul. 1969; A. Senglet leg.; MHNG • 2 q 9 ; Granada, Ugíjar; $36.96^{\circ}$ N, $3.05^{\circ}$ W; 15 Jul. 1971; A. Senglet leg.; MHNG • 3 q 9 ; Granada, Huéneja; $37.176^{\circ}$ N, 2.948 ${ }^{\circ}$ W; 16 Jul. 1971; A. Senglet leg.; MHNG • 1 o ; Sevilla; $37.39^{\circ}$ N, $5.98^{\circ}$ W; 16 Jun. 1953; A. Comellini leg.; MHNG. - Region of Murcia • 1 क ; Caravaca [de la Cruz]; $38.104^{\circ}$ N, $1.860^{\circ}$ W; 5 Jul. 1971; A. Senglet leg.; 
MHNG • 1 क; San Pedro del Pinatar; $37.83^{\circ}$ N, $0.78^{\circ}$ W; Sep. 1984; G. Hormiga leg.; USNM • 1 ; Fortuna, Cueva Las Magras; $38.290^{\circ} \mathrm{N}, 1.143^{\circ} \mathrm{W}$; 30 Jan. 2006; "ACP” leg.; CRBA 1146. - Valencian Community • $4 \overbrace{}^{\Uparrow} \partial^{\lambda}, 3$ 우 ; Valencia, La Albufera; $39.30^{\circ} \mathrm{N}, 0.33^{\circ} \mathrm{W}$; 16 Jun. 1971; A. Senglet leg.;

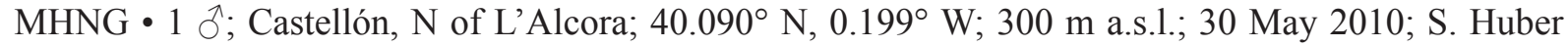
and A. Schönhofer leg.; at stone walls; SMF. - Catalonia - 1 đ; Barcelona, Gelida; $41.44^{\circ} \mathrm{N}, 1.86^{\circ} \mathrm{E}$; 14 Jun. 1971; A. Senglet leg.; MHNG -2 $\widehat{\jmath}, 1$ q, 3 juvs; near Barcelona, Cerdanyola [del Vallès]; $41.49^{\circ} \mathrm{N}, 2.14^{\circ}$ E; 7 Dec. 2004; D. Dimitrov leg.; SMF 56746.

FRANCE - Pyrénées-Orientales • 1 đิ, 2 q $\odot$; Banyuls; $42.48^{\circ} \mathrm{N}, 3.13^{\circ} \mathrm{E}$; date and collector unknown;

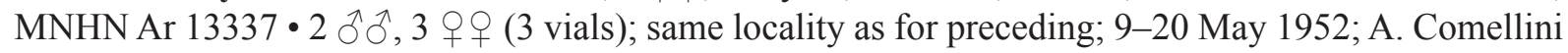
leg.; MHNG • 1 क; same locality as for preceding; no further data; MHNG. 11 \% ; Cerbère; $42.44^{\circ} \mathrm{N}$,

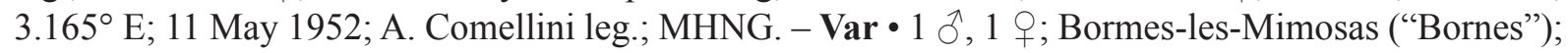

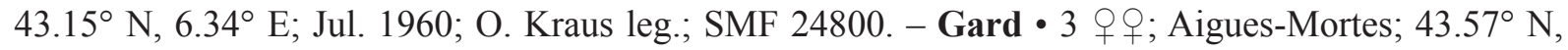
4.19 $\mathrm{E}$; 8 May 1952; A Comellini leg.; MHNG. - Corsica • 1 ô, 1 q; Propriano; $41.67^{\circ} \mathrm{N}, 8.91^{\circ} \mathrm{E}$; 29 May 1971; A. Senglet leg.; MHNG • 1 đ, 7 우, 1 juv.; Ponte Leccia; $42.463^{\circ}$ N, $9.207^{\circ}$ E; 19 Sep.

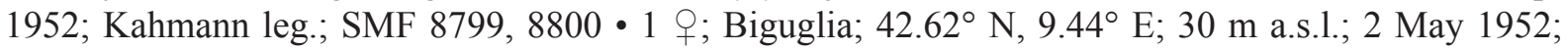
Kahmann leg.; SMF 8801.

BELGIUM - East Flanders • 1 क; near Gent; $51.10^{\circ}$ N, 3.70 E; Jul. 2004; B.A. Huber leg.; ZFMK Ar 12608.

GERMANY - Nordrhein-Westfalen • 1 ๙ , 4 $\uparrow \uparrow, 6$ juvs; Bonn, Botanical Garden (?) ("Treibhaus"); $50.724^{\circ}$ N, $7.091^{\circ}$ E; Nov. 1967; E. Kullmann leg.; ZFMK Ar 5219. - Rheinland-Pfalz • 1 क ; Hatzenport; $50.2265^{\circ} \mathrm{N}, 7.4108^{\circ}$ E; 80 m a.s.1.; 26 Sep. 2014; B.A. Huber leg.; among wooden boards outside of building; ZFMK Ar 12304 • 1 क; Mainz, Draisberghof; $48.987^{\circ}$ N, $8.203^{\circ}$ E; 1 Feb. 1998; P. Jäger leg.;

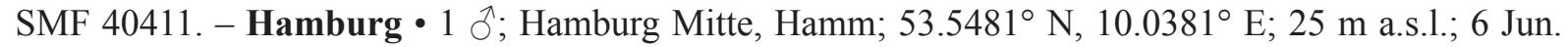
2019; B.A. Huber leg.; in building; ZFMK Ar 22292.

AUSTRIA - Vienna $11 \hat{\jmath}, 3$ 우 (in pure ethanol); Brigittenau; $48.2283^{\circ} \mathrm{N}, 16.3710^{\circ} \mathrm{E}$; Aug. 2001; B.A. Huber leg.; at windows in attic (fifth floor); ZFMK G64 to G66.

ITALY - Sardinia • $1 \partial^{7}$; S. Pietro; $40.717^{\circ} \mathrm{N}, 8.550^{\circ}$ E; 15 Aug. 1960; E. Kullmann leg.; ZFMK Ar 10705 - 1 q, 1 juv.; Castelsardo; 40.91 N, 8.71 E; 11 Jun. 1958; E. Kullmann leg.; ZFMK Ar $10746 \bullet 1$ o; Lago Bunnari; $40.716^{\circ} \mathrm{N}, 8.634^{\circ}$ E; 11 Apr. 1952; collector unknown; SMF $9273 \bullet 1$ đo, 2 juvs; Ozieri; $40.58^{\circ}$ N, 9.00 E; 23 Sep. 1968; A. Senglet leg.; MHNG • 2 q 9,1 juv.; Terralba; $39.72^{\circ} \mathrm{N}, 8.63^{\circ} \mathrm{E}$; 4 Aug. 1960; E. Kullmann leg.; ZFMK Ar $10696 \bullet 1$ ô, 1 q, 1 juv.; Muravera, Nuraghe; $39.318^{\circ}$ N,

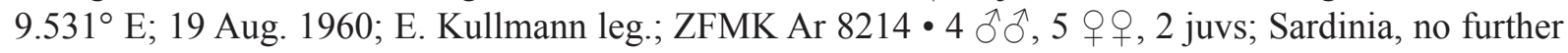

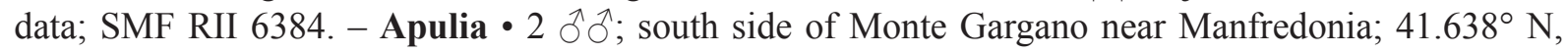
$15.890^{\circ}$ E; 60 m a.s.1.; 7 Apr. 2006; A. Schönhofer and J. Hillen leg.; under stones in cultivated field, Opuntia field, native rock; SMF. - Sicily $1{ }^{\top}$; Syracuse, Viale Ermocrate; $37.07^{\circ} \mathrm{N}, 15.27^{\circ} \mathrm{E} ; 18 \mathrm{Aug}$. 1993; B.A. Huber leg.; ZFMK Ar 5217 • 1 ô, 1 q; Syracuse, Theatre; 37.074 N, $15.279^{\circ}$ E; 18 Aug. 1993; B.A. Huber leg.; ZFMK Ar 5218.

CROATIA • 1 P; Karlovac, Ogulin; $45.266^{\circ}$ N, $15.233^{\circ}$ E; 320 m a.s.1.; 9 May 2010; J. Altmann and

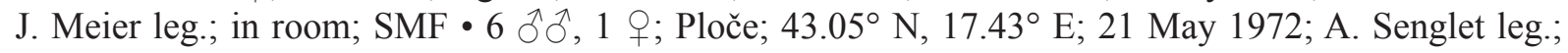
MHNG • 1 क ; Dubrovnik, Mt Srđ slopes; $42.65^{\circ}$ N, $18.10^{\circ}$ E; 0-400 m a.s.1.; 21 Apr. 1971; J. Murphy leg.; MMUE Murphy \#5477.

BOSNIA AND HERZEGOVINA • 1 ○े, 2 우; Popovo-Polje, Zavala; $42.848^{\circ} \mathrm{N}, 17.979^{\circ}$ E; 19 Sep. 1970; A. Senglet leg.; MHNG • 2 숫, 2 우우; Popovo-Polje, Sedlari; $42.826^{\circ}$ N, $18.056^{\circ}$ E; 18 Sep.

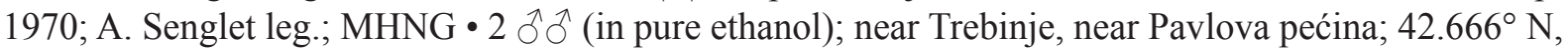


$18.308^{\circ}$ E; 400 m a.s.1.; 26 May 2014; B.A. Huber and M. Komnenov leg.; under rocks; ZFMK Bal10, Bal19.

MONTENEGRO • 1 §, 4 ㅇ; Podgorica, Velje Brdo, Megara Cave; $42.463^{\circ} \mathrm{N}, 19.199^{\circ} \mathrm{E} ; 17$ Feb.

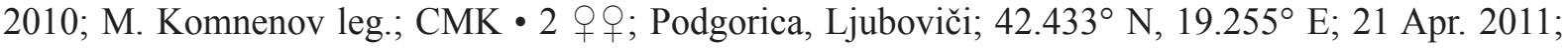
M. Komnenov leg.; artificial tunnel; CMK.

ALBANIA • 1 q; Kulla ë Lumës ("Kula Lums"); $42.098^{\circ}$ N, $20.418^{\circ}$ E; 1918 ; collector unknown; SMF RII $3726 \bullet 1$ q, 1 juv.; Durrës; $41.33^{\circ}$ N, $19.45^{\circ}$ E; 26 May 1993; P. Stoev and C. Deltshev leg.; NMNHS - 1 O; Përmet, near Petrani; $40.208^{\circ}$ N, $20.419^{\circ}$ E; 12 May 1995; B. Petrov and P. Stoev leg.; artificial gallery; NMNHS.

NORTH MACEDONIA • 1 क; Gevgelija; 41.14 N, 22.50 E; 10 Jul. 1956; Lamel leg.; HECO.

BULGARIA - Blagoevgrad • 1 ô, 1 क , 1 juv.; Blagoevgrad; $42.014^{\circ} \mathrm{N}, 23.097^{\circ} \mathrm{E} ; 380 \mathrm{~m}$ a.s.1.; 2 Aug. 2005; P. Jäger leg.; SMF 40643 • 6 우; N foothills of Krupnishna Planina Mt Range, $\sim 0.2 \mathrm{~km}$ NNE of Krupnik; $41.850^{\circ} \mathrm{N}, 23.125^{\circ} \mathrm{E}$; $375 \mathrm{~m}$ a.s.1.; 12 Aug. 2005; A.V. Gromov leg.; ZMMU • 1 ○े, 2 q ; Sandanski; $41.566^{\circ}$ N, $23.280^{\circ}$ E; 28 Jul. 1972; A. Senglet leg.; MHNG • 1 ð, 3 우; $\sim 0.5 \mathrm{~km} \mathrm{~S}$ of Banya; $41.873^{\circ}$ N, $23.528^{\circ}$ E; 825 m a.s.1.; 11 Aug. 2005; A.V. Gromov leg.; ZMMU. - Pazardzhik •

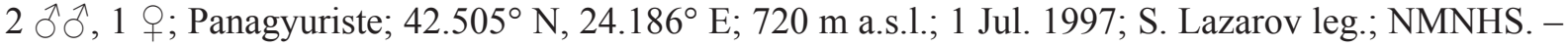
Ruse・ 1 今, 2 우; Byala; $43.46^{\circ} \mathrm{N}, 25.74^{\circ} \mathrm{E}$; 2013; collector unknown; MGAB.

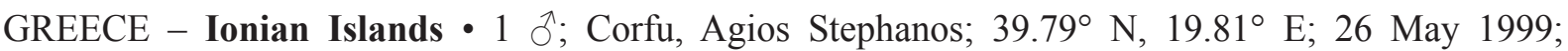
A. Russell-Smith leg.; ZFMK Ar 22293 • 1 ô, 1 o ; Corfu, Loutses; $39.786^{\circ} \mathrm{N}, 19.880^{\circ} \mathrm{E}$; 20 Sep. 1972; A. Senglet leg.; MHNG • 3 đิ ô, 1 क; Corfu, Agios Matheos; $39.495^{\circ}$ N, $19.872^{\circ}$ E; 18 Sep. 1972; A. Senglet leg.; MHNG • 1 ô, 1 q; Pantocrator, Spilaio Katsampa near Strinilas; $39.744^{\circ}$ N, $19.839^{\circ}$ E; 650 m a.s.1.; 12 May 1974; B. Hauser leg.; MHNG • 1 क ; Pantocrator, Spilaio Katsouri; $39.743^{\circ} \mathrm{N}, 19.925^{\circ} \mathrm{E} ; 130 \mathrm{~m}$ a.s.l.; 29 Apr. 1975; D. Tzanoudakis leg.; MHNG • 1 ð, 1 o; Lefkada,

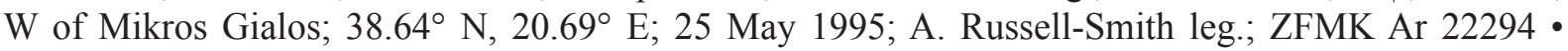

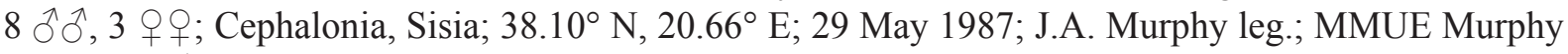
\#A231 • 1 đ, 2 O 9 ; same collection data as for preceding; reared from eggs; MMUE Murphy \#15938 • 1 q; Cephalonia, "Patra"; date unknown; Malicky leg.; SMF • 1 q; Cephalonia, "Castle Hill”"; 19 May 1987; J.A. Murphy leg.; MMUE Murphy \#15013 • 1 §, 1 क; Cephalonia, no further locality data; 21 May 1987; J.A Murphy leg.; MMUE Murphy \#16161. - Epirus • 1 \&, 1 juv.; Igoumenitsa; $39.50^{\circ} \mathrm{N}$, $20.27^{\circ}$ E; data unknown; F. Sauer leg.; SMF. - Central Macedonia • 1 đ; Yerakini ("Gerakina"); $40.27^{\circ} \mathrm{N}, 23.45^{\circ} \mathrm{E} ; 14$ Apr. 1978; J.A. Murphy leg.; stony area; MMUE Murphy \#6830 • 2 우; "Halkidiki"; 6 Apr. 1978; J.A. Murphy leg.; MMUE Murphy \#6709. - Eastern Macedonia and Thrace - 1 ; Limni Vistonis ("Limni Bouroú"); $41.05^{\circ}$ N, 25.10 E; Sep. 1984; collector unknown; SMF. Thessaly • 2 え $\partial^{\lambda}, 2$ q $\circ$; Larissa, "Omôlion (Tembe)"; $39.88^{\circ} \mathrm{N}, 22.58^{\circ} \mathrm{E}$ (coordinates between Omolio and Tempi); 17 Jun. 1970; A. Senglet leg.; MHNG. - Central Greece • 1 ô; Euboea, Lichada/Istiaia; $38.9^{\circ} \mathrm{N}, 23.0^{\circ} \mathrm{E}$ (coordinates between Lichada and Istiaia); 30 Aug. 1972; A. Senglet leg.; MHNG •

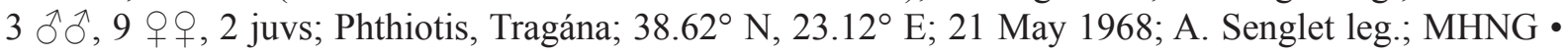
$2{ }^{\Uparrow}{ }^{\jmath}, 2$ 우 $ᄋ$, 1 juv.; Phthiotis, Theologus/Atalanti (Glyphada); $38.62^{\circ} \mathrm{N}, 23.10^{\circ} \mathrm{E}$ (coordinates between Theologos and Atalanti); 20 Jun. 1970; A. Senglet leg.; MHNG • 1 ภ, 1 q; Phthiotis, near Malesina;

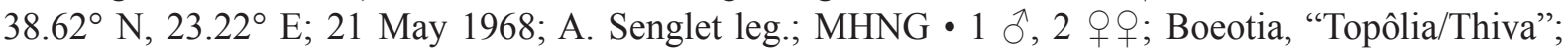
$38.32^{\circ}$ N, $23.32^{\circ}$ E (coordinates of Thiva); 25 Aug. 1970; A. Senglet leg.; MHNG • 1 ð’; Euboea, Kato Steni; $38.571^{\circ}$ N, 23.825 E; 2 Sep. 1972; A. Senglet leg.; MHNG • 1 Oे, 1 juv.; Northern Sporades,

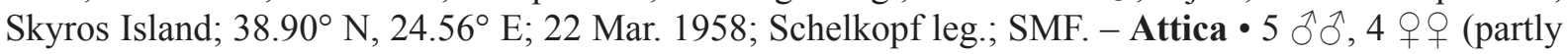

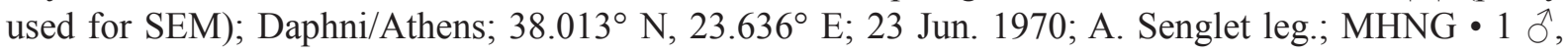
1 ; ; same locality as for preceding; 19 Jun. 1968; A. Senglet leg.; MHNG $\bullet 2$ +, , 1 juv.; Athens, 
Akropolis; $37.971^{\circ} \mathrm{N}, 23.727^{\circ} \mathrm{E}$; date and collector unknown; SMF RII $3724 \cdot 4$ juvs; same locality as for preceding; May 1926; F. Roewer leg.; SMF • 3 수, 2 우우 ; Athens, near Koropi; $37.900^{\circ} \mathrm{N}$, $23.867^{\circ}$ E; 19 Mar. 2007; A. Schönhofer leg.; under stones in olive grove and phrygana; SMF • 1 ; ; Vari, Spilaio Nympholyptou; 37.858 N, 23.802 E; 25 Feb. 2018; N. Fytrou and E. Zenzefyli leg.; HISR 3042 • 3 우, 1 juv.; near Var, "Havara"; 27 Jan. 1954; P. Strinati leg.; MHNG. - Peloponnese •

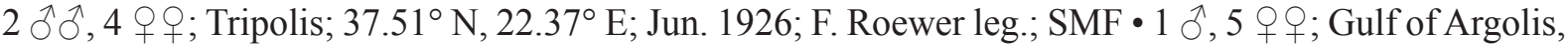

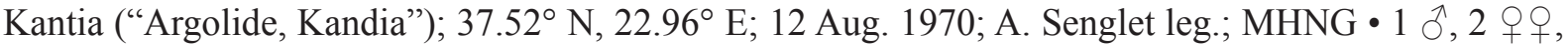
1 juv.; NE of Gytheio ("Yithion"); 1977; Kinzelbach leg.; SMF 37616 • 1 q; Laconia, Pyrgos Dirou; 36.625 N, 22.380 E; 18 Aug. 1970; A. Senglet leg.; MHNG. - North Aegean • 2 đ̊ ô; Chios, Emboria

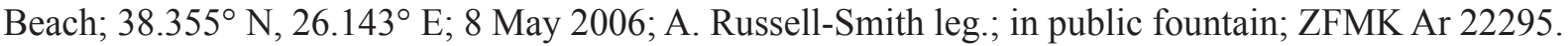
- South Aegean - 4 우; Paros, Naousa; 37.12 N, 25.24 E; 21 Jun. 1968; A. Senglet leg.; MHNG •

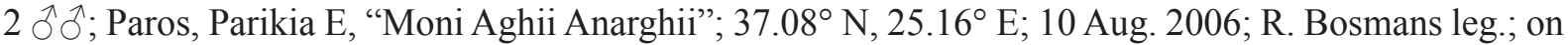

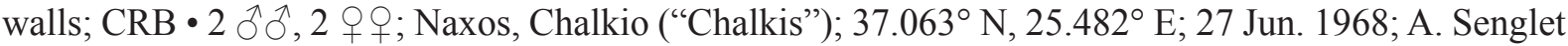

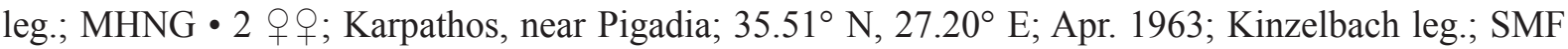

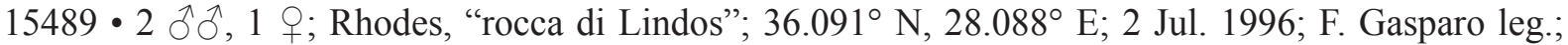
ZFMK Ar 22296. - Crete・ 1 ㄱ, 2 우; Chania, Azogires Palaeochoras; $35.272^{\circ}$ N, $23.719^{\circ}$ E; 4-6 Aug.

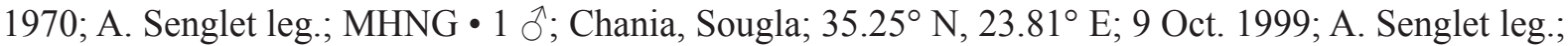
MHNG • 3 q 9 ; Chania, Halepa ("Chalepa"); $35.52^{\circ} \mathrm{N}, 24.05^{\circ} \mathrm{E}$; date and collector unknown; SMF RII

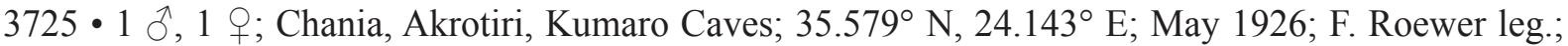

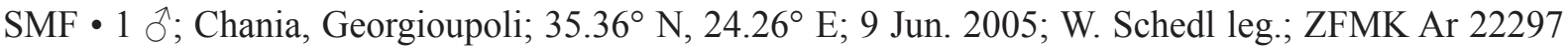

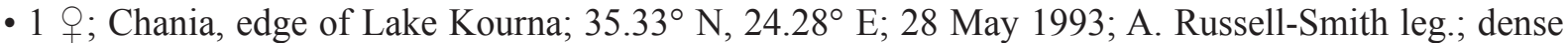

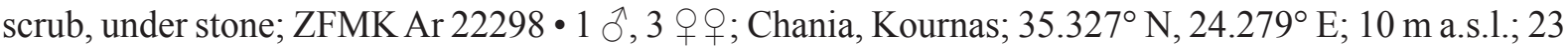
Mar. 2007; A. Schönhofer leg.; under stones, mostly open terrain; SMF • 1 ภ, 1 q; Chania, Grambousa, Spilaio Agiou Ioanni Prodromou; 5 Mar. 1995; K. Paragamian leg.; HISR 1773 • 1 ô, 1 ㅇ, 2 juvs; Chania, Hora Sfakion ("Sfakia"); $35.20^{\circ}$ N, 24.14 E E; 17 Jul. 2009; H. Eikamp and U. Kluge leg.; SMF - 1 đ, 1 क; Rethymno, Choumeri/Perama; $35.35^{\circ}$ N, 24.72 E; 1 Jul. 1970; A. Senglet leg.; MHNG • 1 क; Rethymno, Anogia; $35.24^{\circ}$ N, $24.87^{\circ}$ E; 11 May 2006; H. Eikamp and U. Kluge leg.; SMF 56588

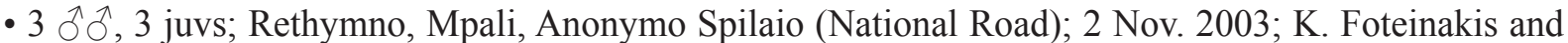
K. Paragamian leg.; HISR 2428 • 1 ð, 4 juvs; Heraklion, Gournes; $35.32^{\circ}$ N, 25.28 ${ }^{\circ}$ E; 3 May 2008; H. Eikamp and U. Kluge leg.; SMF • 1 o; same locality as for preceding; 15 May 2010; K. Eckl leg.;

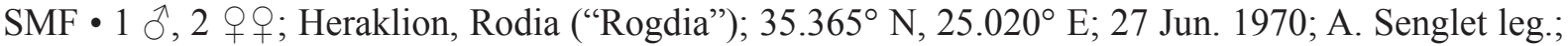

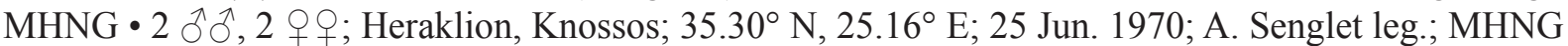

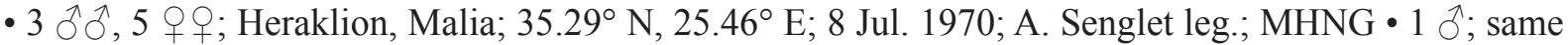
locality as for preceding; 11 May 2008; H. Eikamp and U. Kluge leg.; SMF $\bullet 2 \hat{\jmath} \hat{\delta}, 1$ q; Heraklion,

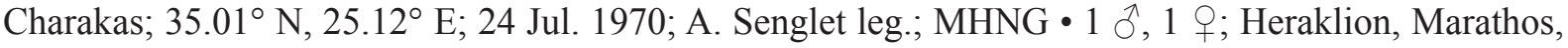

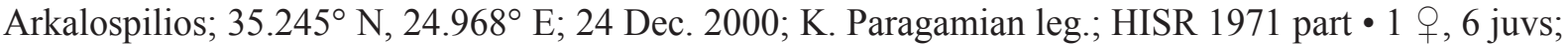
Heraklion, Kastri; $35.00^{\circ}$ N, $25.37^{\circ}$ E; 7 May 2008; H. Eikamp and U. Kluge leg.; SMF・ 1 \&; Heraklion, Kastri, Keratokambos; $35.000^{\circ}$ N, 25.375 ${ }^{\circ}$ E; 14 May 2006; H. Eikamp and U. Kluge leg.; SMF $56589 \bullet$

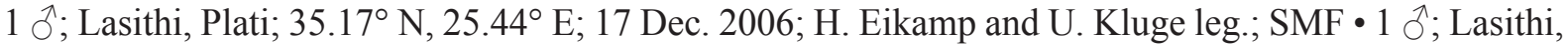

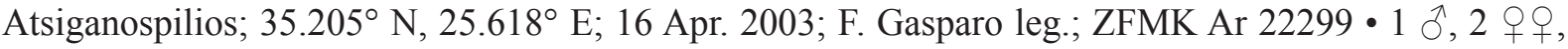
1 juv.; Lasithi, Agia Fotia; $35.02^{\circ}$ N, 25.87 E; 6 Jul. 2007; H. Eikamp and U. Kluge leg.; SMF $57161 \bullet$ 2 우; Lasithi, Bembonas, Tripti Mts; $35.1^{\circ}$ N, 25.9 E; 10 May 2009; K. Eckl and H. Eikamp leg.; SMF

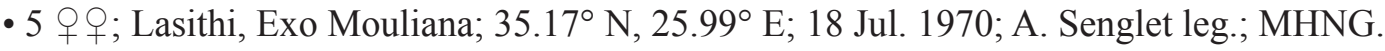

TURKEY - Çanakkale • 1 , , 1 juv.; Kavak River, near bridge of road Keşan-Gallipoli (“Gelibolu”); $40.60^{\circ} \mathrm{N}, 26.87^{\circ} \mathrm{E}$; date and collector unknown; SMF 11 o; Truva, "84/15"; $39.95^{\circ} \mathrm{N}, 26.24^{\circ} \mathrm{E}$; date and collector unknown; SMF. - Izmir • 1 \%; Vişneli, "Fetrek-2 Cave"; $38.346^{\circ} \mathrm{N}, 27.421^{\circ} \mathrm{E}$; 310 m a.s.1.; 5 Jun. 2009; Y.M. Marusik leg.; ZFMK Ar 5469 • 1 ô, 1 o ; Çiğli (airport); $38.516^{\circ} \mathrm{N}$, 27.013ํE; 15 Apr. 1973; A Vigna leg.; MHNG • 1 क; "Agamemnon”; 10 May 1975; C. Bisuchet and

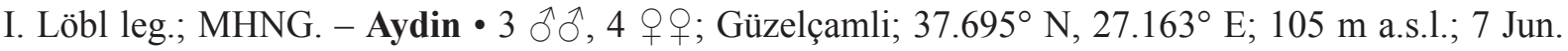


2009; Y.M. Marusik leg.; ZFMK Ar 5215. - Bursa • 2 qq, 1 juv.; Görükle, Uludağ Univ. Campus; 40.226 ${ }^{\circ}$ N, 28.869 ${ }^{\circ}$ E; 420 m a.s.1.; 2-3 Jun. 2009; Y.M. Marusik leg.; ZFMK Ar 5468. - Muğla • 1 ð, 1 O; Dalyan to Kaunos ("Cavnos") path; $36.82^{\circ}$ N, 28.61 E; 18 May 1997; A. Russell-Smith leg.;

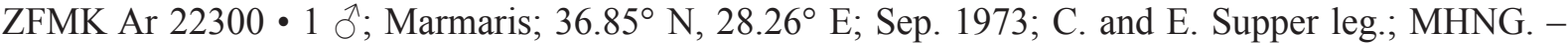
Antalya $\bullet 1 \delta^{\circ}$; Xanthos ruins; $36.356^{\circ}$ N, $29.318^{\circ}$ E; 7 Mar. 1977; Kinzelbach leg.; SMF $37622 \bullet 1 \overbrace{}^{\wedge}$ (in pure ethanol); Kemer District, Çıralı; $36.399^{\circ}$ N, 30.475 E; 10 m a.s.1.; 3 Aug. 2016; H. Öztürk leg.; in

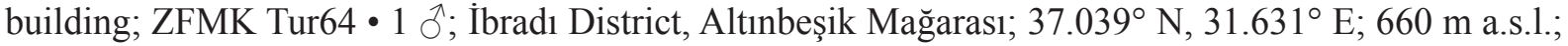

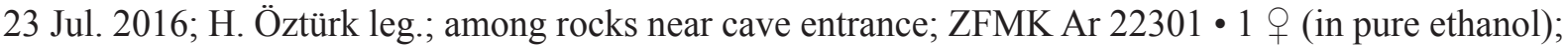
same collection data as for preceding; ZFMK Tur46 • 1 o, 3 우; Alanya Kalesi, above Kleopatra beach; $36.539^{\circ}$ N, $31.992^{\circ}$ E; 2 Nov. 2013; S. Huber leg.; ZFMK Ar 22302 • 1 đ, 1 क; Alanya District, Cüceler Mağarası; 36.489 ${ }^{\circ} \mathrm{N}, 32.276^{\circ} \mathrm{E}$; $270 \mathrm{~m}$ a.s.1.; 31 Jul. 2016; H. Öztürk leg.; among rocks near cave entrance; ZFMK Ar 22303. - Mersin • 1 đ̃; Anamur District, Köşekbükü Astım Mağarası; $36.127^{\circ} \mathrm{N}$, $32.760^{\circ} \mathrm{E}$; $130 \mathrm{~m}$ a.s.1.; $29 \mathrm{Jul}$. 2016; H. Öztürk leg.; in cave near entrance; ZFMK Ar $22304 \bullet 1$ O; NE of Silifke, above Narlıkuyu; $36.45^{\circ} \mathrm{N}, 34.10^{\circ} \mathrm{E}$; 24 Apr. 2012; J. Altmann and J. Meier leg.; maquis; SMF

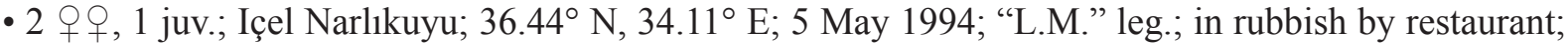

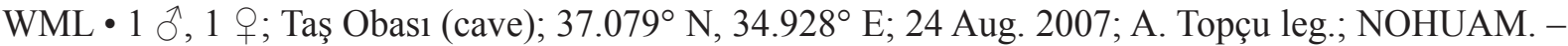

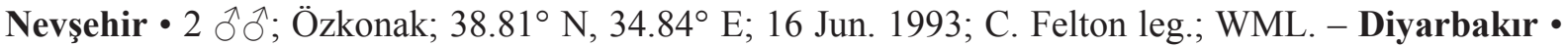

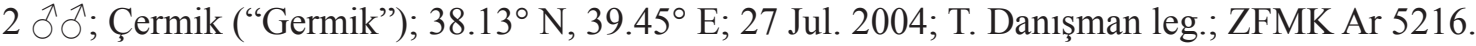

CYPRUS - Limassol • 1 ô, 1 q, 1 juv.; Pissouri; $34.67^{\circ}$ N, 32.70 E; 27 Apr. 1982; J. Murphy leg.; beach litter; MMUE Murphy \#12617. - Famagusta • 2 우, 3 juvs; Fig Tree Bay, "Cave I"; $35.01^{\circ} \mathrm{N}$, 34.06 E; 23 Oct. 1996; P. Strinati leg.; MHNG.

GEORGIA - Tbilisi • 1 o ; Tbilisi, Dighom I; $41.778^{\circ}$ N, 44.701 E; 17 Jul. 2019; Karalashvili, Seropian

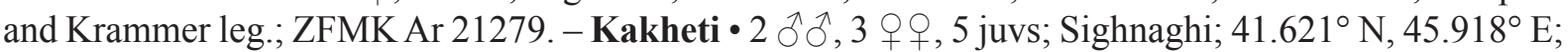
9 Jul. 2019; Karalashvili and Krammer leg.; on houses; ZFMK Ar 21372, Ar 21373 • 1 ô; Lagodekhi National Park; $41.847^{\circ}$ N, 46.284 ${ }^{\circ}$ E; 9 Jul. 2019; Karalashvili and Krammer leg.; forest; ZFMK $\operatorname{Ar} 21261 \cdot 1$ o, 1 क , 2 juvs; Vashlovani National Park, bungalows near border to Azerbaijan; $41.111^{\circ} \mathrm{N}$, 46.647 E; 26-27 Jul. 2019; Karalashvili and Krammer leg.; steppe; ZFMK Ar 21569, Ar 21570.

SYRIA 1 \& , 1 juv.; Latakia, ruins of Ugarit, Ra's as-Samra, creek $\mathrm{N}$ of Tell Ugarit; $35.602^{\circ} \mathrm{N}, 35.783^{\circ} \mathrm{E}$; 4 Mar. 1979; R. Kinzelbach leg.; SMF • 4 우, 3 juvs; Jayrud ("Djeroud"); $33.807^{\circ}$ N, $36.742^{\circ}$ E; 1911; H. Gadeau de Kerville leg.; in house; MNHN • 1 § ; between Hamaa and Aleppo, "Kaha al Suban" (locality not identified); 500 m a.s.1.; 9 Apr. 1982; collector unknown; SMF.

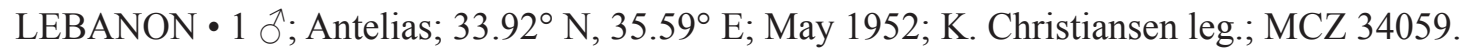

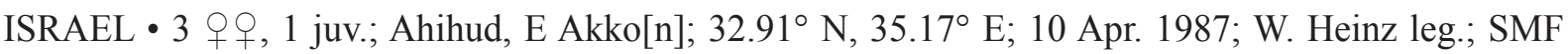
- 1 क; Haifa Distr., Karmiya Ridge, Mt Karmel; $32.7185^{\circ}$ N, 35.0065 ${ }^{\circ}$ E; 280 m a.s.1.; 17 Sep. 2013; B.A. Huber, S. Aharon and E. Gavish-Regev leg.; among rocks; ZFMK Ar 22305 • 1 \& (in pure ethanol); Haifa Distr., Mt Karmel, Oren Cave; 32.7145 N, 34.975 E; 70 m a.s.1.; 17 Sep. 2013; B.A. Huber, S. Aharon, and E. Gavish-Regev leg.; in cave; ZFMK Isr57 • 2 ô, 1 q ; $\mathrm{N}$ of Teverya ("Tiberias"); $32.80^{\circ} \mathrm{N}, 35.52^{\circ} \mathrm{E}$; stony meadow; 4 Mar. 1975; H. Levi, G. Levy, and G. Tsabor leg.; MCZ 34062 - 1 J, 1 q; Northern Distr., Mt Berenice, S of Teverya; $32.777^{\circ}$ N, 35.541 ${ }^{\circ}$ E; -120 m b.s.1.; 16 Sep. 2013; B.A. Huber, S. Aharon, and E. Gavish-Regev leg.; among rocks; ZFMK Ar $22306 \bullet 1$ q (in pure ethanol); same collection data as for preceding; ZFMK Isr39 1 1 , 1 क ; Northern Distr., near Afula, HaGilbo'a Reserve; $32.52^{\circ}$ N, 35.38 ${ }^{\circ}$ E; 300-500 m a.s.l.; 7 Apr. 1987; W. Heinz leg.; SMF • 1 \%;

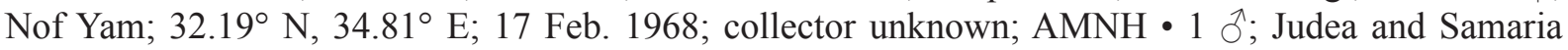
Distr., Memorial Ha-Biq'a, NE Peza'el [Fatsa'el]; 32.0524 N, 35.4589 E; -210 m b.s.1.; 15 Sep. 2013; B.A. Huber, S. Aharon, and E. Gavish-Regev leg.; in cave; ZFMK Ar 22307 • 1 \&; near Ramla, Kibbuz 
Gezer; $31.88^{\circ}$ N, $34.92^{\circ}$ E; 11 Aug. 1984; H. Feldmann leg.; indoors on ceiling; AMNH • 1 q; Central Distr., Modi'in; $31.8951^{\circ} \mathrm{N}, 34.9618^{\circ}$ E; $160 \mathrm{~m}$ a.s.1.; 8 Sep. 2013; B.A. Huber, S. Aharon, and E. Gavish-Regev leg.; among rocks; ZFMK Ar 22308 • 1 + , 2 juvs; near Ramallah, Bayt Ghur al-Tahta;

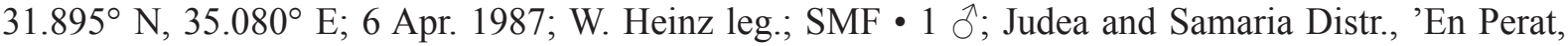

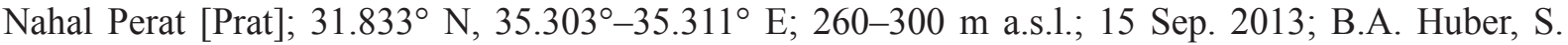
Aharon, and E. Gavish-Regev leg.; among rocks; ZFMK Ar 22309 • 1 क ; Jerusalem; $31.77^{\circ} \mathrm{N}, 35.21^{\circ} \mathrm{E}$; Apr. 1952; Khuri leg.; MCZ 34064 2 ठึ̄, 2 우, 2 juvs; 21 km E of Jerusalem, Jericho Road, wadi in hills; 23 Feb. 1975; H.W. Levi and G. Levy leg.; MCZ 34067 • 1 o, 3 juvs; Hebron, Beit Kahil; $31.57^{\circ}$ N, $35.07^{\circ}$ E; $\sim 800$ m a.s.1.; 30 Apr. 1987; W. Heinz leg.; SMF • 6 ô $^{\lambda}, 2$ q $q$,, 3 juvs; Dead Sea, Ein Feshkha; $31.716^{\circ}$ N, 35.452 ${ }^{\circ}$ E; 27 Feb. 1975; H. Levi, P. Amitai, and G. Levy leg.; MCZ $34061 \bullet$ 1 ô, 1 O ; Southern Distr., Nahal Dawid (David), 'En Gedi; $31.47^{\circ}$ N, $35.39^{\circ}$ E; -250 to -300 m b.s.l.; 10 Sep. 2013; B.A. Huber, S. Aharon, and E. Gavish-Regev leg.; among rocks; ZFMK Ar $22310 \bullet 1$ o (in pure ethanol); same date as for preceding; ZFMK Isr59・1 $\partial^{2}, 2$ 우영 Southern Distr., Nahal Boqeq, W of 'En Boqeq; $31.1992^{\circ}$ N, 35.3571 ${ }^{\circ}$ E; -350 m b.s.l.; 8 Sep. 2013; B.A. Huber, S. Aharon, and E. Gavish-Regev leg.; among rocks; ZFMK Ar 22311 • 1 ô; Southern Distr., NW of 'En Tamar, Nahal Zin; $30.991^{\circ} \mathrm{N}$, $35.347^{\circ}$ E; $-340 \mathrm{~m}$ a.s.1.; 9 Sep. 2013; B.A. Huber, S. Aharon, and E. Gavish-Regev leg.; among rocks; ZFMK Ar 22312 • 1 ô, 1 q; Negev, Mash'abei Sadeh, Golda Park; $31.0185^{\circ}$ N, $34.7645^{\circ}$ E; 340 m a.s.1.; 6 Sep. 2011; P. Jäger leg.; stony desert, under stones; SMF • 1 juv.; Negev, Mash'abei Sadeh, 1.5 air km S of Golda Park; $31.0026^{\circ}$ N, $34.7576^{\circ}$ E; 340 m a.s.l.; 7 Sep. 2011; P.

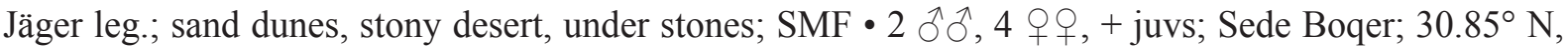

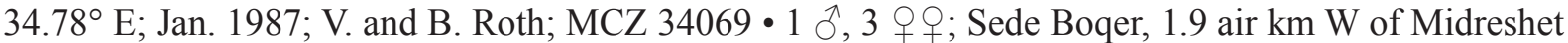
Ben Gurion; $30.8554^{\circ}$ N, $34.7642^{\circ}$ E; 530 m a.s.1.; 8 Sep. 2011; P. Jäger leg.; stony desert, bushes, under stones; SMF 1 1 (in pure ethanol); same collection data as for preceding; SMF $\bullet 2$ q $O$; same collection

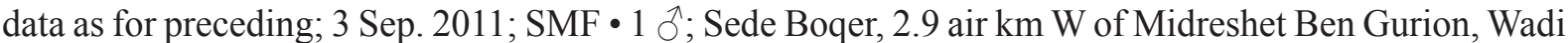

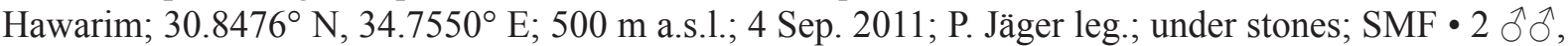
1 q, 2 juvs; Sede Boqer, Ein Avedat, 3.3 air km W of Midreshet Ben Gurion; $30.8486^{\circ} \mathrm{N}, 34.7492^{\circ} \mathrm{E}$; 400-480 m a.s.1.; 4 Sep. 2011; P. Jäger leg.; shrubs, under stones, in small caves; SMF • 6 juvs; same locality as for preceding; 6 Sep. 2011; P. Jäger leg.; stream with water, reed, shrubs, under stones; SMF

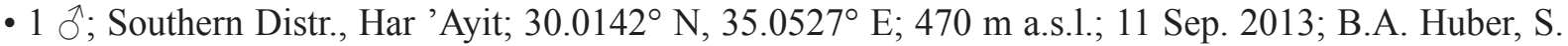
Aharon, and E. Gavish-Regev leg.; in deep crevice; ZFMK Ar 22313 • 1 o ; Southern Distr., Samar; $29.8342^{\circ}$ N, $35.0215^{\circ}$ E; 100 m a.s.l.; 11 Sep. 2013; B.A. Huber, S. Aharon, and E. Gavish-Regev leg.; in building; ZFMK Ar 22314.

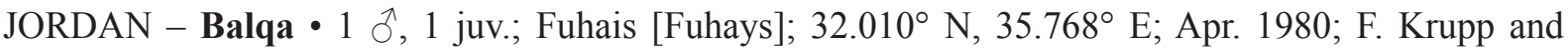
W. Schneider leg.; SMF. - Madaba • 2 우, 2 juvs; Mt Nebo; $31.77^{\circ}$ N, 35.73 E; Jul. 1989; E. Hyazin leg.; MRAC $169974 \cdot 2$ 우, 1 juv.; Hammamat az-Zarqa' Ma'in, hot springs, $150 \mathrm{~m}$ to Dead Sea;

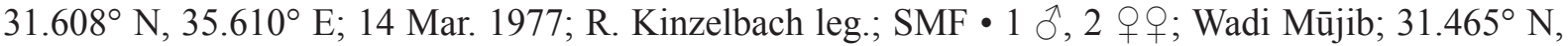
35.578 E; -380 m b.s.l.; among rocks; 14 Sep. 2013; B.A. Huber leg.; ZFMK Ar 22315. - Kerak •

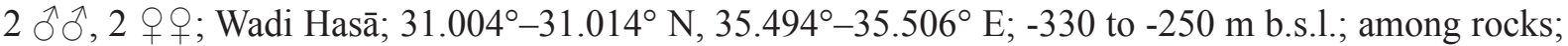
14 Sep. 2013; B.A. Huber leg.; ZFMK Ar 22316 • 2 $\widehat{\jmath}, 2$ 우 (in pure ethanol); same collection data

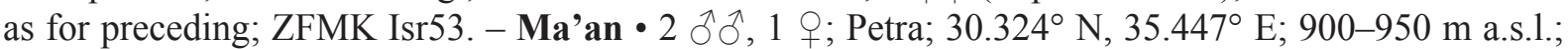
13 Sep. 2013; B.A. Huber leg.; among rocks; ZFMK Ar 22317 • 1 ㅇ, 1 juv. (in pure ethanol); same collection data as for preceding; ZFMK Isr37. - Aqaba • 1 đ̊; Wadi Rām; $29.7405^{\circ} \mathrm{N}, 35.4574^{\circ} \mathrm{E}$; 830 m a.s.1.; 12 Sep. 2013; B.A. Huber leg.; among rocks; ZFMK Ar $22318 \bullet 1$ o, 1 juv. (in pure ethanol); same collection data as for preceding; ZFMK Isr52 • 1 ô; Wadi Rām; $29.683^{\circ} \mathrm{N}, 35.450^{\circ} \mathrm{N}$; 9 Apr. 2004; J. Altmann leg.; SMF.

MOROCCO - Béni Mellal-Khénifra • 1 ô, 1 क (in pure ethanol); Imi n'Ifri; $31.724^{\circ} \mathrm{N}, 6.972^{\circ} \mathrm{W}$;

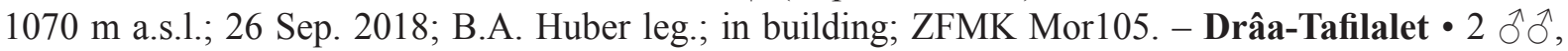
3 우, 1 juv.; Midelt; $32.68^{\circ}$ N, 4.73 W; 19 Dec. 1986; V. and B. Roth leg.; CAS • 1 \%; 22 km N of 

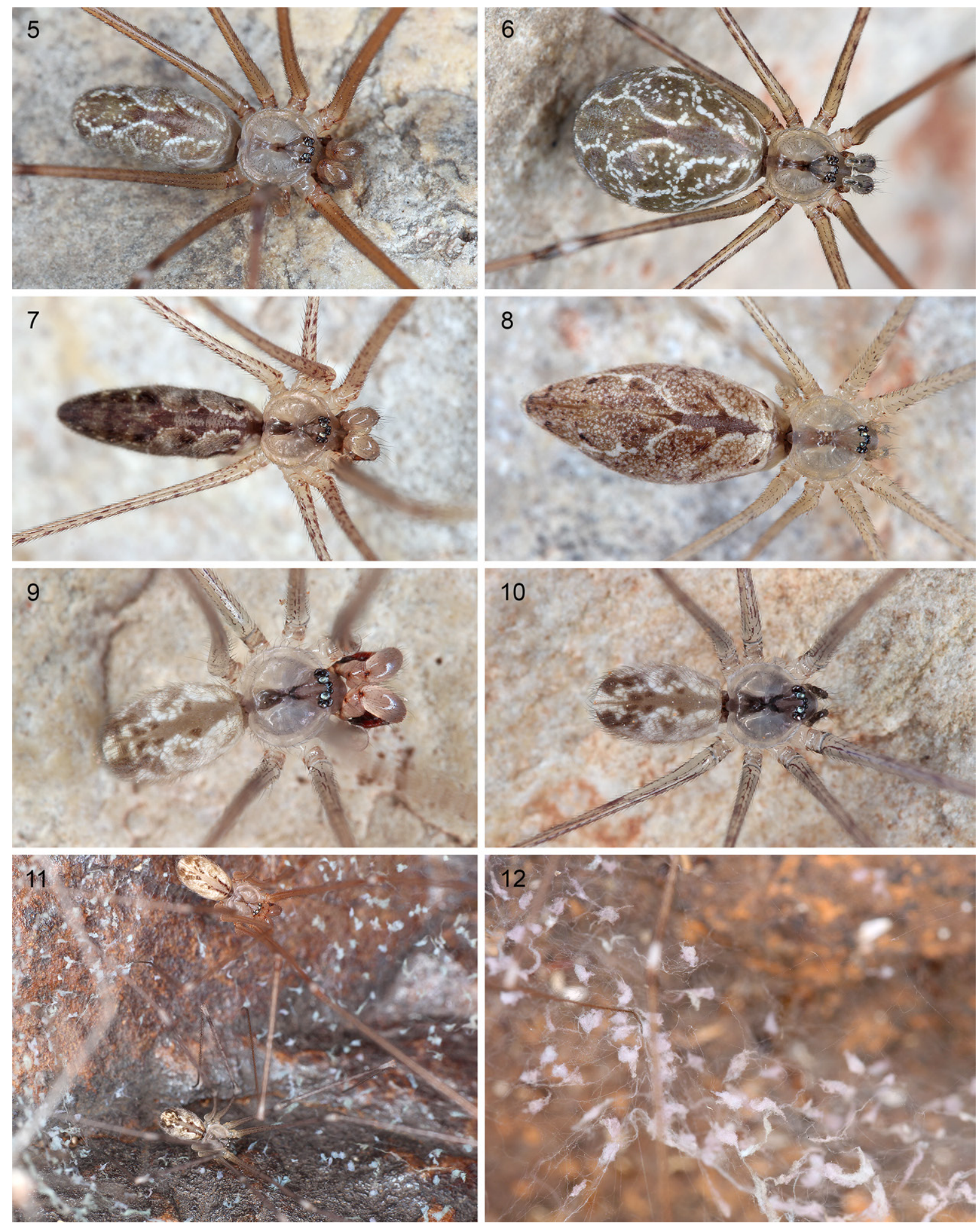

Figs 5-12. Live specimens and webs. 5-6. Holocnemus pluchei (Scopoli, 1763); male and female from Morocco, Imi n'Ifri. 7-8. Holocnemus reini (C. Koch, 1873) comb. nov.; male from Morocco, near Tilamizene; female from Morocco, W of Tamtetoucht. 9-10. Maghreba amezyan gen. et sp. nov., male and female from Morocco, between Lakhssas and Bouizakarne. 11-12. Maghreba amezyan gen. et sp. nov., pair in spotted web, and detail of web with silk puffs, SW of Ida Ougnidif. 
Errachida, between Sefrou and Tanout ou Filal; $32.04^{\circ}$ N, $4.42^{\circ}$ W; 24 Dec. 1986; V. and B. Roth leg.; CAS 9027138.

ALGERIA - Médéa • 2 đô, 1 q ; Médéa; $36.26^{\circ}$ N, $2.75^{\circ}$ E; date unknown; P.-H. Lucas leg.; MNHN.

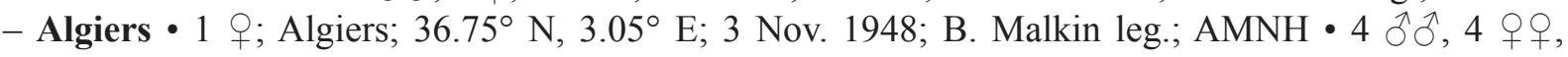
+ juvs (possible syntypes of Pholcus barbarus Lucas); near Algiers; $36.75^{\circ} \mathrm{N}, 3.05^{\circ} \mathrm{E}$; date unknown (between 1839 and 1842); P.-H. Lucas leg.; MNHN. - Tozeur • 2 우; Tozeur; 33.92 ${ }^{\circ}$ N, 8.12 ${ }^{\circ}$ E; Jul. 1972; E. and C. Supper leg.; MHNG.

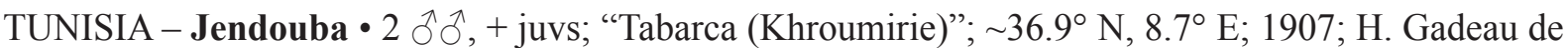
Kerville leg.; MNHN • 1 ^̊, 3 o 9,1 juv. (2 vials); Ayn Darahim (“Ain-Draham”); $36.78^{\circ} \mathrm{N}, 8.69^{\circ} \mathrm{E}$; 1899; G. Seurat leg.; MNHN. - Tunis • 1 §, 2 q 9 , 1 juv.; Carthago; $36.86^{\circ}$ N, $10.33^{\circ}$ E; $11-12$ Oct. 1948; B. Malkin leg.; AMNH • 1 क; La Marsa; $36.89^{\circ}$ N, $10.32^{\circ}$ E; Jul. 1972; E. and C. Supper leg.;

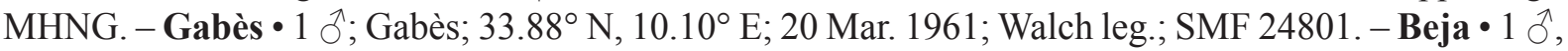
1 juv.; Beja; $36.73^{\circ} \mathrm{N}, 9.19^{\circ}$ E; 23 Mar. 2006; A. López and C. Ribera leg.; CRBA 1271. - Kairouan •

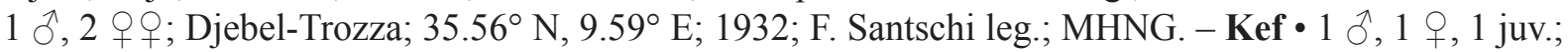
El Kef; $36.17^{\circ}$ N, $8.70^{\circ}$ E; Jul. 1972; E. and C. Supper leg.; MHNG.

LIBYA - Al Marquab • 1 क; Leptis Magna; 32.63 N, 14.29 E; 18-26 Aug. 1948; B. Malkin leg.;

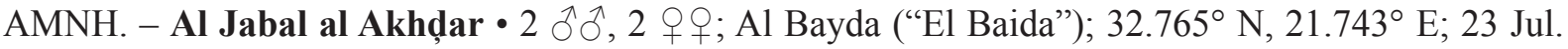
1979; C. Goodnight and N. Barbash leg.; AMNH.

EGYPT - Dakahlia • 6 えへ, 6 우, 6 juvs; Mansoura ("Mansurah"); $31.04^{\circ} \mathrm{N}, 31.38^{\circ} \mathrm{E}$; date unknown; I. Sörensen leg.; ZMUC. - Beheira - 3 ô $^{\lambda}, 8$ 우, + juvs; Bir Hooker; $30.38^{\circ}$ N, $30.35^{\circ}$ E; 1901; J. Dewitz leg.; MNHN. - Cairo • 1 o ; Maadi; $29.96^{\circ} \mathrm{N}, 31.26^{\circ} \mathrm{E}$; further collection data on label unclear; MHNG • 1 क; Cairo [city]; $30.0^{\circ} \mathrm{N}, 31.2^{\circ} \mathrm{E}$; date and collector unknown; SMF Roewer \#4783.

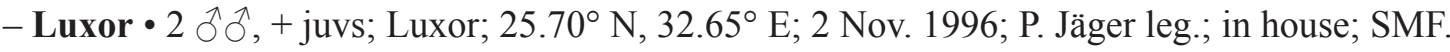

AUSTRALIA - Western Australia • 1 क, 1 juv.; Morawa Motel; 29.217 S, 116.017 E; 23 May 1996; M.S. Harvey leg.; in building; WAM • 1 ${ }^{\lambda}, 1$ juv.; West Swan, behind Caversham Wildlife Park; $31.850^{\circ} \mathrm{S}, 115.983^{\circ} \mathrm{E} ; 17$ May 1992; J.M. Waldock leg.; on building; WAM 99/1790 • 1 § ; Maylands; $31.93^{\circ} \mathrm{S}, 115.90^{\circ} \mathrm{E} ; 7$ Jan. 1992; J.M. Waldock leg.; WAM 99/1579 • 1 क ; same collection data as for preceding; 27 Dec. 1991; WAM 99/1578 • $1{ }^{\text {J}}$; East Victoria Park; $31.983^{\circ} \mathrm{S}, 115.900^{\circ} \mathrm{E} ; 15$ Jan. 1997; J.M. Waldock leg.; WAM 99/2097 • 1 \%; same collection data as for preceding; 13 Sep. 1992; in house; WAM 99/1776 • 1 \%; same collection data as for preceding; 19 Nov. 1994; WAM 99/1752. - South Australia - 1 O , 1 o; Pondanna Outstation; $32.56^{\circ} \mathrm{S}, 135.55^{\circ} \mathrm{E}$; 10 Dec. 1989; D. Hirst leg.; SAM 99/680 • 1 \&; Flinders Range National Park, Oraparina; $31.37^{\circ}$ S, $138.73^{\circ}$ E; Dec. 1984; B. Guerin leg.; SAM 99/679 • 1 q, 1 juv.; Erudine Station via Yunta; $31.43^{\circ} \mathrm{S}, 139.43^{\circ}$ E; 22 Apr. 1980; J. McEntee

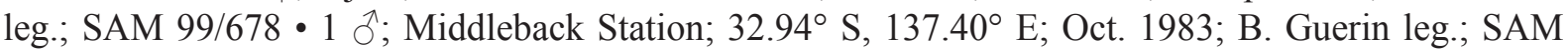
99/682 • 1 O; Mt Lofty Ranges, Hawthorndene; $35.02^{\circ}$ S, $138.63^{\circ}$ E; 18 Jan. 1992; L.N. Nicolson leg.; in garden; SAM 99/683 • 2 우, 2 juvs; Stoneleigh Park near Meningie; $35.70^{\circ} \mathrm{S}, 139.35^{\circ}$ E; Mar. 1969; A.W. Forbes leg.; SAM 99/684 • $1{ }^{\AA}$, 2 juvs; Murray Range near Kingston; $34.2^{\circ} \mathrm{S}, 140.3^{\circ} \mathrm{E} ; 17$ Dec.

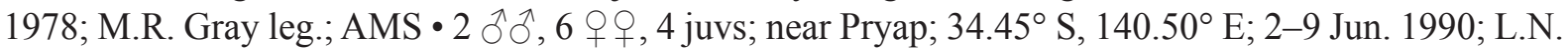
Nicolson leg.; SAM 99/670 1 ð̊, 6 우, 2 juvs; $7 \mathrm{~km} \mathrm{~W}$ of Loxton; $34.45^{\circ} \mathrm{S}, 140.50^{\circ} \mathrm{E} ; 20$ May 1990; L.N. Nicolson leg.; shed and house verandah; SAM 99/662 • 1 万ै; Loxton; $34.45^{\circ} \mathrm{S}, 140.57^{\circ} \mathrm{E}$; 11 Jun. 1990; L.N. Nicolson leg.; in house; SAM 99/669. - Queensland • 1 q; south side of Lake Broadwater; $27.357^{\circ}$ S, $151.098^{\circ}$ E; $1-15$ Nov. 1984; V. Wood leg.; buildings; QMB S49733 • 2 우; SW of Dalby, Lake Broadwater; $27.35^{\circ} \mathrm{S}, 151.10^{\circ} \mathrm{E}$; 9 Dec. 1987; J. Gallon leg.; cottage; QMB S14509 • 1 ○ं; Texas; $28.85^{\circ} \mathrm{S}, 151.17^{\circ} \mathrm{E}$; date unknown; Harvey and Katzman leg.; in building; WAM. - New South Wales - 1 Ô, 1 q; Duntroon Station; 31.03 S, $143.04^{\circ}$ E; Oct.-Nov. 1980; D. Hirst leg.; SAM 99/655 • 1 q, 

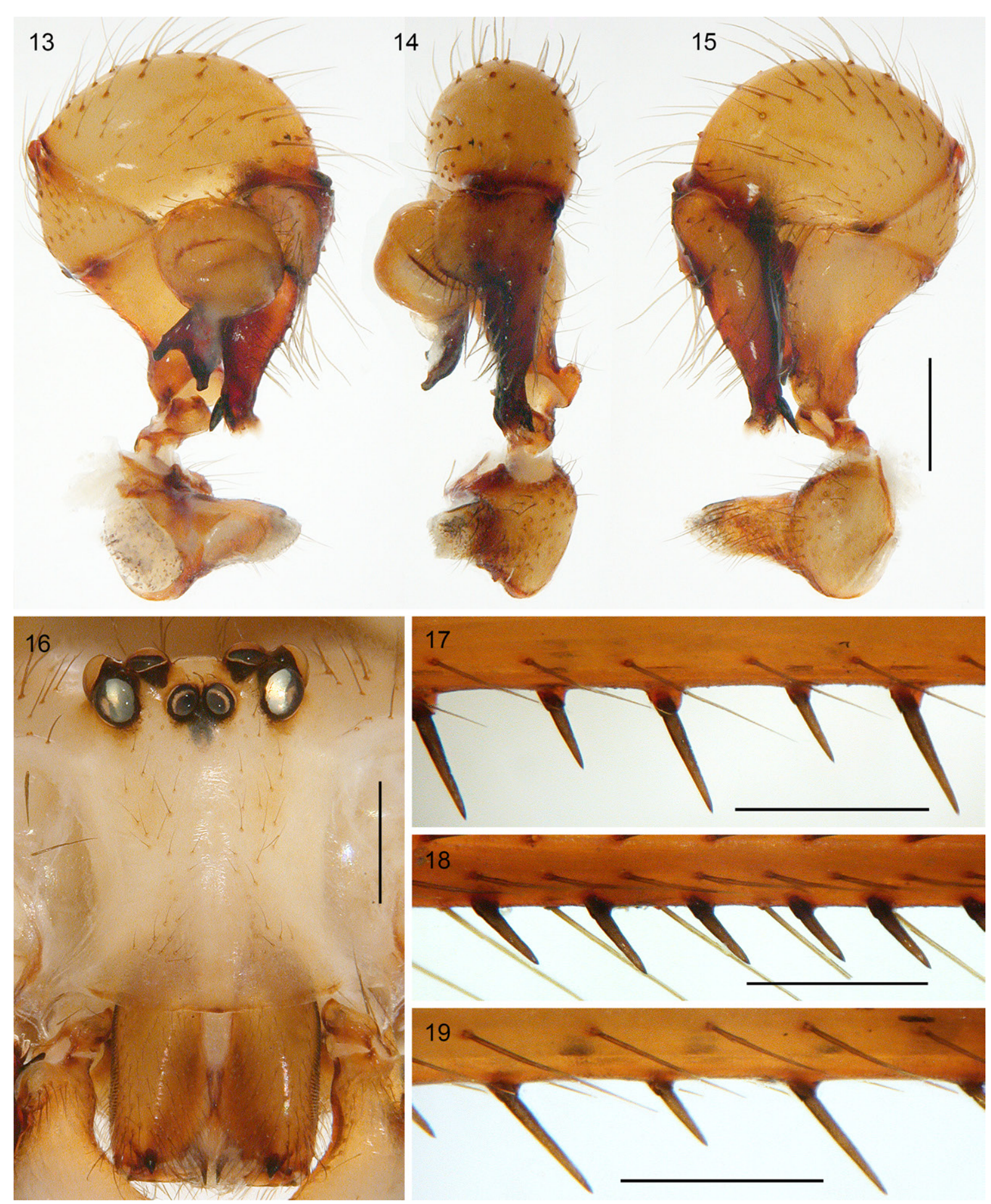

Figs 13-19. Holocnemus pluchei (Scopoli, 1763). 13-15. Male from Israel, S of Teverya (ZFMK Ar 22306); left palp, prolateral, dorsal, and retrolateral views. 16. Male from Israel, Samar (ZFMK Ar 22314); ocular area, clypeus, and chelicerae, frontal view. 17-19. Male from Israel, S of Teverya (ZFMK Ar 22306); spines on femur 1, tibia 1, and femur 2 (right legs, retrolateral views). Scale bars = $0.5 \mathrm{~mm}$. 
2 juvs; Weinteriga; $31.60^{\circ}$ S, $142.95^{\circ}$ E; 15 Apr. 1981; D. Hirst leg.; SAM 99/659 • 2 えðぇ; Menindee

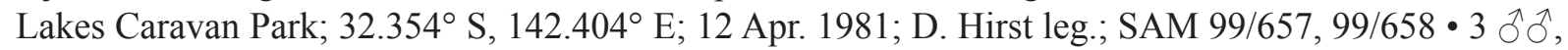
1 juv.; Sulcor; 30.85 S, $150.81^{\circ}$ E; 27 May 1995; S. Eberhard leg.; cave, twilight zone; AMS KS 49263.

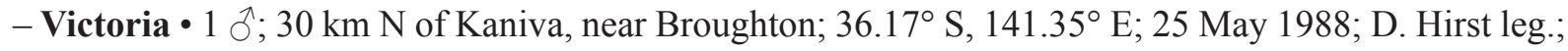
SAM 99/661.

\section{Redescription}

Male (Israel, ZFMK Ar 22306)

Measurements. Total length 6.0, carapace width 2.3. Distance PME-PME $220 \mu \mathrm{m}$; diameter PME $140 \times 150 \mu \mathrm{m}$; distance PME-ALE $70 \mu \mathrm{m}$; diameter AME $125 \mu \mathrm{m}$; distance AME-AME $25 \mu \mathrm{m}$. Leg 1: $45.4(12.9+1.1+12.7+16.4+2.3)$, tibia $2: 8.1$, tibia $3: 6.0$, tibia $4: 7.2$; tibia $1 \mathrm{~L} / \mathrm{d}: 42$; femora $1-4$ diameters: $0.46,0.39,0.37,0.38$.

COLOR (in ethanol). Carapace ochre-yellow, posterior part of ocular area brown; clypeus not darkened; sternum dark brown to black; legs ochre-yellow, with darker rings on femora (subdistally) and tibiae (proximally and subdistally), with oval to elongate black marks on femora and tibiae; abdomen ochregray, with dark and whitish marks dorsally and laterally; ventrally with distinct black median band, partly disrupted, with three parallel longitudinal marks behind gonopore.

Body. Habitus as in Fig. 5. Ocular area slightly raised; each secondary eye with small accompanying elevation (Fig. 43). Deep thoracic pit and pair of shallow furrows diverging from pit toward posterior margin. Clypeus unmodified (contra Calbacho-Rosa et al. 2019b; see Discussion), only rim more sclerotized than in female. Sternum wider than long (1.5/1.1), unmodified (i.e., without indentations as in $H$. caudatus and H. reini). Abdomen cylindrical, dorso-posteriorly weakly angular. Gonopore with four epiandrous spigots (Fig. 55). ALS with one widened spigot and one pointed spigot; PMS with two pointed spigots (Fig. 56).

Chelicerae. As in Fig. 16; see also Huber (1995: figs 1a, 4a; 2000: fig. 14); with pair of frontal lateral apophyses, each with one large modified cone-shaped hair (Fig. 54); distance between tips of modified hairs: $390 \mu \mathrm{m}$; without proximal frontal protrusion; lateral stridulatory ridges distinct (Fig. 47), distances between ridges $\sim 12 \mu \mathrm{m}$.

PALPS. As is Figs 13-15; coxa with rounded retrolateral hump (not a distinct apophysis); trochanter barely modified (slightly protruding ventrally); femur curved towards dorsal, distally widened but without ventral protrusion, proximally with prolateral stridulatory pick, without retrolateral transversal line, with prominent retrolateral proximal process; femur-patella joints only slightly shifted toward prolateral side; tibia large compared to femur, tibia-tarsus joints shifted toward retrolateral side; tarsal organ capsulate (Fig. 51); tarsus without macrotrichia; procursus (Figs 20-22) straight, dorsal hairs not or only slightly curved upwards; proximally on prolateral-ventral side with prominent process free of hairs (arrow in Fig. 20), procursus tip with strong but short ventral sclerite, membranous elements on dorsal and prolateral side, and 4-5 hair-like transparent processes on retrolateral side (Fig. 49); genital bulb (Figs 23-26) with simple basal sclerite connected to distal (main) sclerite; distal sclerite with two distinctive processes; sperm duct opening on membranous area on prolateral side at basis of distal bulbal sclerite (arrow in Fig. 50).

LEGs. With single ventral rows of spines on femur $1(\sim 31)$, tibia $1(\sim 38)$ and femur $2(\sim 14)$ (Figs 1719); without curved hairs; few vertical hairs; retrolateral trichobothrium of tibia 1 at $4 \%$; prolateral trichobothrium present on all tibiae; tarsal pseudosegments indistinct and irregular (cf. Fig. 35) except 2-3 distally; all tarsal organs capsulate with simple round rim (Fig. 53). 

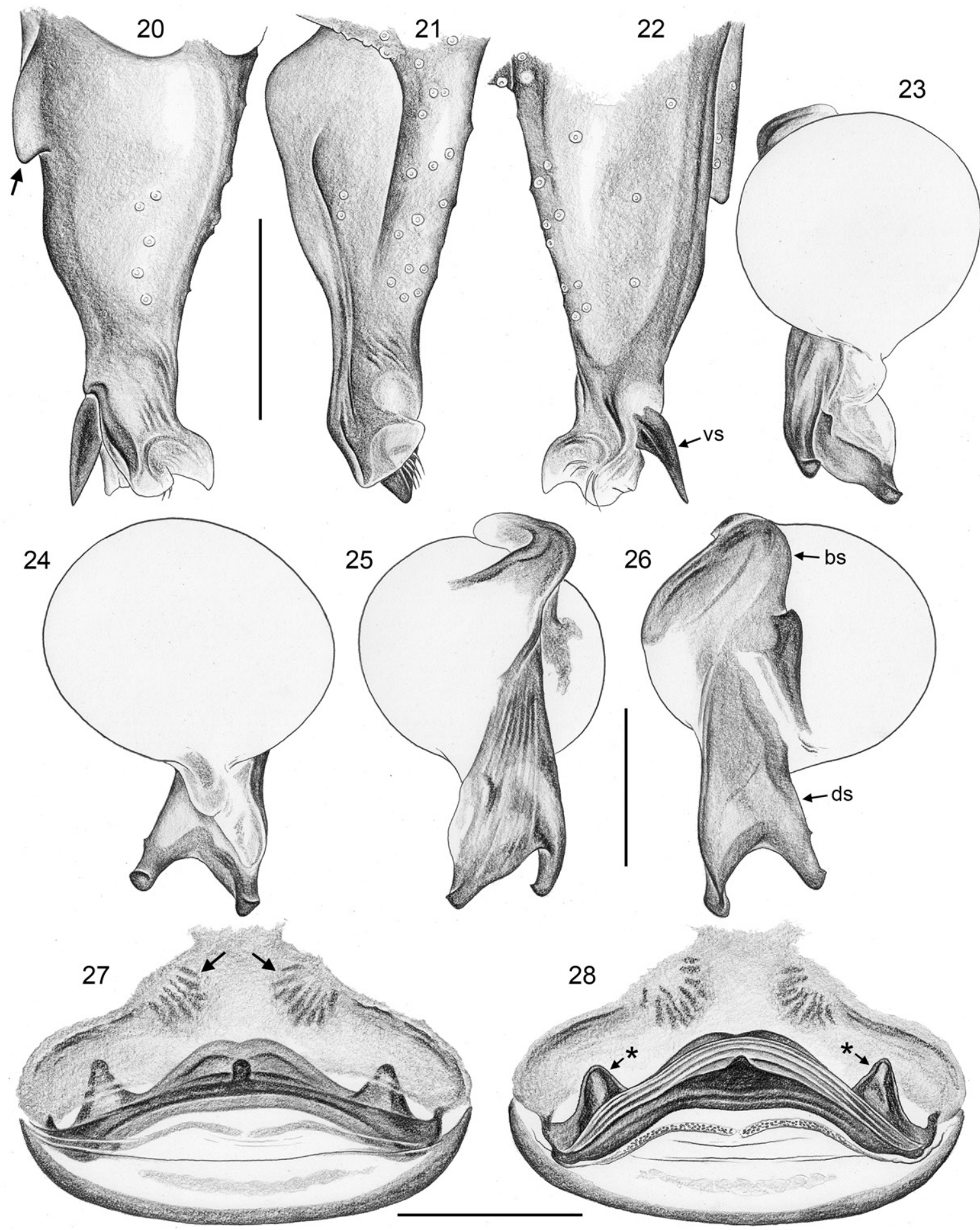

Figs 20-28. Holocnemus pluchei (Scopoli, 1763); male from Israel, S of Teverya (ZFMK Ar 22306), female from Georgia, Sighnaghi (ZFMK Ar 21373). 20-22. Left procursus, prolateral, dorsal, and retrolateral views; arrow: prolateral-ventral process. 23-26. Left genital bulb, ventral, prolateral, dorsal, and retrolateral views. 27-28. Cleared female genitalia, ventral and dorsal views; arrow: pair of sculptured areas; asterisks: pair of internal pockets. Abbreviations: bs = basal sclerite; $d s=$ distal sclerite; vs $=$ ventral sclerite. Scale bars: $20-26=0.3 \mathrm{~mm} ; 27-28=0.5 \mathrm{~mm}$. 
Male (variation)

Tibia 1 in 95 males: 7.9-15.5 (mean 11.6) (mean tibia 1 length in 204 males in Jakob \& Dingle 1990: 10.67); body length 3.5-7.5. Most specimens with more or less distinct dark median band on carapace; sternum sometimes with distinct black radial marks; abdomen sometimes without dark marks, but always with white marks in distinctive pattern. Smaller males with fewer spines, often without spines on tibia 1 and femur 2. Distance between tips of modified hairs on cheliceral apophyses: $280-390 \mu \mathrm{m}$. Gonopore with $4-5$ epiandrous spigots.

\section{Female}

In general similar to male (Fig. 6) but palp with strongly widened distal segments (tibia and tarsus; Figs 29-30), without joints between tibia and tarsus; with strong median process posteriorly on sternum (Figs 31,58 ) possibly acting against ventral process anteriorly on abdomen (Figs 38, 57; but see Discussion); with fewer but stronger stridulatory ridges (Fig. 48; see also Huber 1995; Huber 2021a: fig. 12), distances between stridulatory ridges $\sim 13.5 \mu \mathrm{m}$; without spines on legs; dark marks on femora and tibiae (Fig. 34) often more distinct than in males; with strong anterior sclerite dorsally on pedicel possibly acting against pair of sclerotized areas anteriorly on abdomen (Fig. 32). Without stridulatory apparatus between carapace and abdomen. Tibia 1 in 140 females: 7.2-14.1 (mean 10.5) (mean tibia 1 length in 172 females in Jakob \& Dingle 1990: 10.05); body length: $4.0-8.0 \mathrm{~mm}$. Epigynum as in Figs 36-39 and 57; main epigynal plate wide, weakly protruding, posteriorly whitish, anteriorly with pair of depressions $\sim 280-300 \mu \mathrm{m}$ apart, with sculptured cuticle (cf. Huber 1995: fig. 6c), median internal round element and pair of lateral internal triangular elements usually visible in uncleared specimens; posterior epigynal plate large but simple; large anterior plate in front of epigynum with pair of low humps and distinct anterior elevated rim (arrow in Fig. 38). Internal genitalia (Figs 27-28, 40-42) with elongate pore plates in transversal position narrowing medially, dorsal arc relatively simple, ventral arc with distinctive pair of lateral sclerotized pockets, medially widened, with ventral median process (pocket?). Spigots as in male.

\section{Natural history}

Together with Pholcus phalangioides (Fuesslin, 1775), H. pluchei is probably the best studied pholcid spider with respect to its biology. Much of what we know about $H$. pluchei has been studied in the USA and Argentina, i.e., using non-native populations. Whether they differ in any significant way from Mediterranean populations has never been studied. Data on egg numbers (see below) suggest that such differences may exist.

Around the Mediterranean, H. pluchei is ubiquitous in almost any habitat suitable for its web, including places fully exposed to the sun. Occasionally, H. pluchei lives in peripheral parts of webs of the araneid Cyrtophora citricola (Forsskål, 1775), together with the cobweb spider Argyrodes argyrodes (Walckenaer, 1841) (Blanke 1972; Hajer 1995; Leborgne et al. 1998; Hajer \& Řeháková 2003). In Central Europe, where $H$. pluchei has been massively spreading over the last decades, it may be displacing P. phalangioides, at least in warm/dry/light parts of buildings, close to windows (Jäger 2000; Van Keer 2007). In the southwestern USA, $H$. pluchei is often the dominant spider on outside surfaces in urban areas around homes and other structures (Vetter et al. 2011). It sometimes occurs is high densities: up to 600 spiders were estimated to inhabit a $3 \times 15 \mathrm{~m}$ juniper bush in California (Blanchong et al. 1995).

The usual web of $H$. pluchei is dome-shaped, as in most space-dwelling pholcids. A skeleton made of $1.2 \mu \mathrm{m}$ fibrils is filled with $0.8-0.9 \mu \mathrm{m}$ fibrils (Hajer \& Řeháková 2003). Occasionally, webs are densely covered with silk puffs, a common behavior in Smeringopinae that interferes with a clear view of the spider. The fact that silk puffs are produced 3-4 days before molting and before egg-laying supports the view that puffs may protect the spider from visual predators when it is most vulnerable (Hajer \& Řeháková 2003). Unusual spherical webs are built by females before egg-laying. Females remain in 
these small spheres (diameter $\sim 5 \mathrm{~cm}$ ) until the spiderlings hatch, and females do not prey during this time (Sedey \& Jakob 1998; Jäger 2000; Hajer \& Řeháková 2003).

Holocnemus pluchei is facultatively group-living, i.e., several conspecifics of all sizes may share a web (Jakob 1991). Groups are constantly forming and disintegrating as the spiders move frequently among webs, depending on size, feeding status, and presence or absence of conspecifics (Jakob 2004). Surprisingly, small spiders in a group seem to pay a high prize for group living: they feed less than
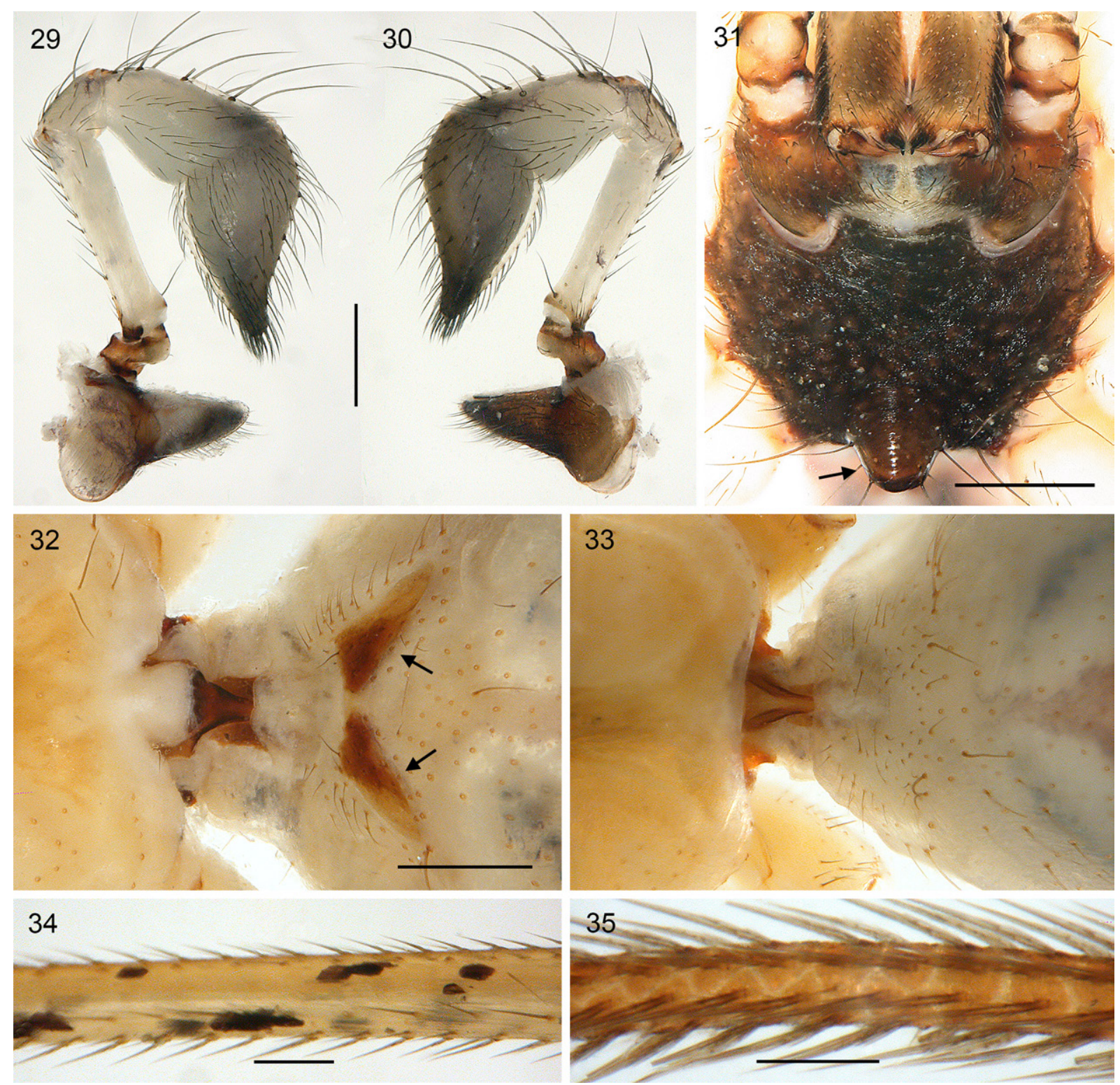

Figs 29-35. Holocnemus pluchei (Scopoli, 1763). 29-30. Female from Georgia, Sighnaghi (ZFMK Ar 21373); left palp, prolateral and retrolateral views. 31. Female from Georgia, Sighnaghi (ZFMK Ar 21373); sternum, ventral (slightly frontal) view; arrow: posterior process. 32-33. Female and male from Bulgaria, Blagoevgrad (SMF), showing modified female pedicel and pair of plates frontally on female abdomen (arrows). 34. Female from Israel, Mount Karmel (ZFMK Ar 22305); left femur 3 ventral view. 35. Female from Israel, $S$ of Teverya (ZFMK Ar 22306); female tarsus, showing irregular pseudosegmentation. Scale bars: $29-33=0.5 \mathrm{~mm} ; 34=0.2 \mathrm{~mm} ; 35=0.1 \mathrm{~mm}$. 

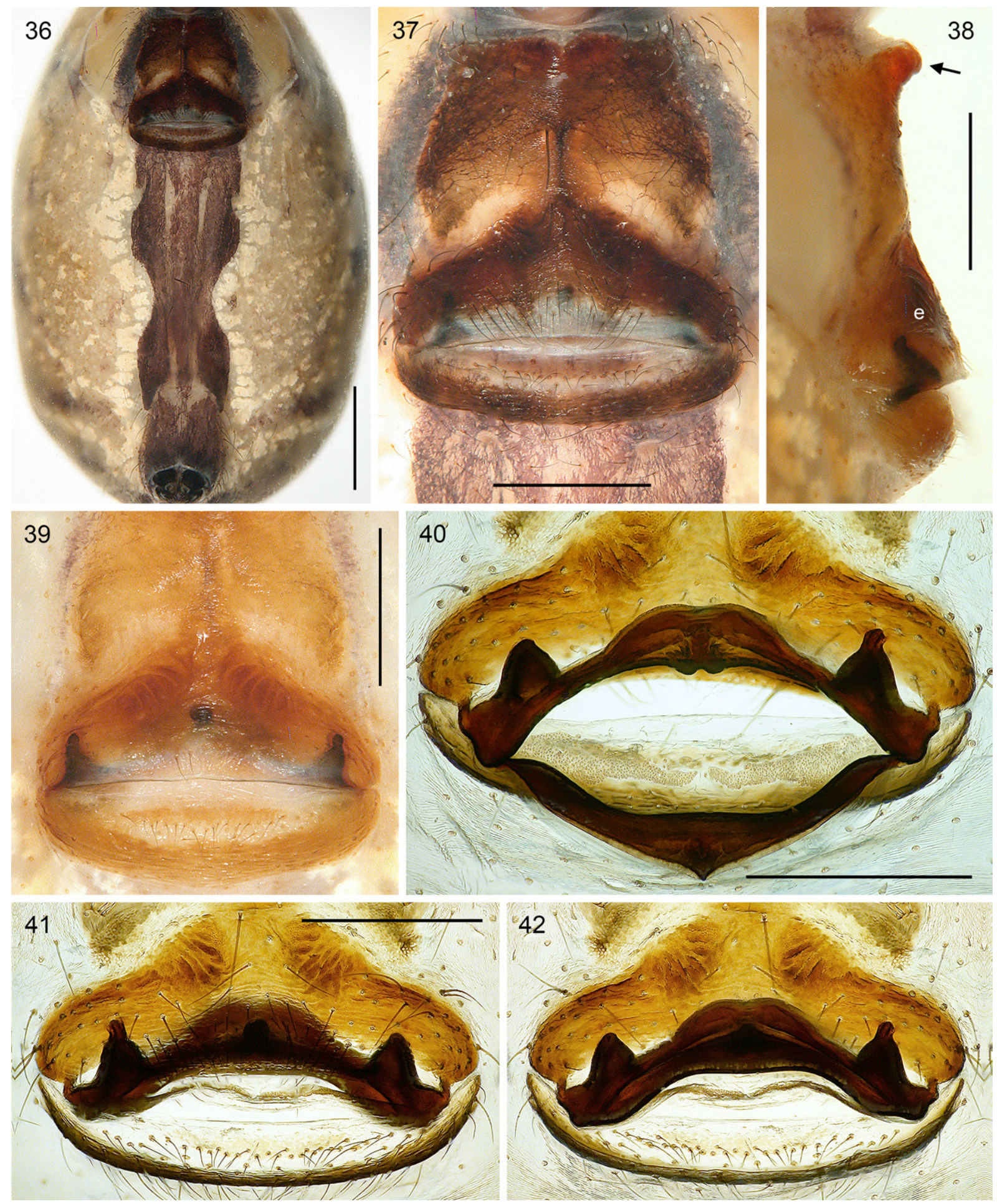

Figs 36-42. Holocnemus pluchei (Scopoli, 1763). 36-37. Female from Georgia, Sighnaghi (ZFMK Ar 21373), abdomen and epigynum, ventral views. 38-39. Female from Jordan, Petra (ZFMK Ar 22317), epigynum lateral and ventral views; arrow: anterior process. 40-42. Female from Georgia, Sighnaghi (ZFMK Ar 21373), cleared genitalia, dorsal view with dorsal arc tilted backwards, ventral view, and regular dorsal view. Abbreviation: $\mathrm{e}=$ epigynum. Scale bars: $36=1.0 \mathrm{~mm} ; 37-42=0.5 \mathrm{~mm}$. 

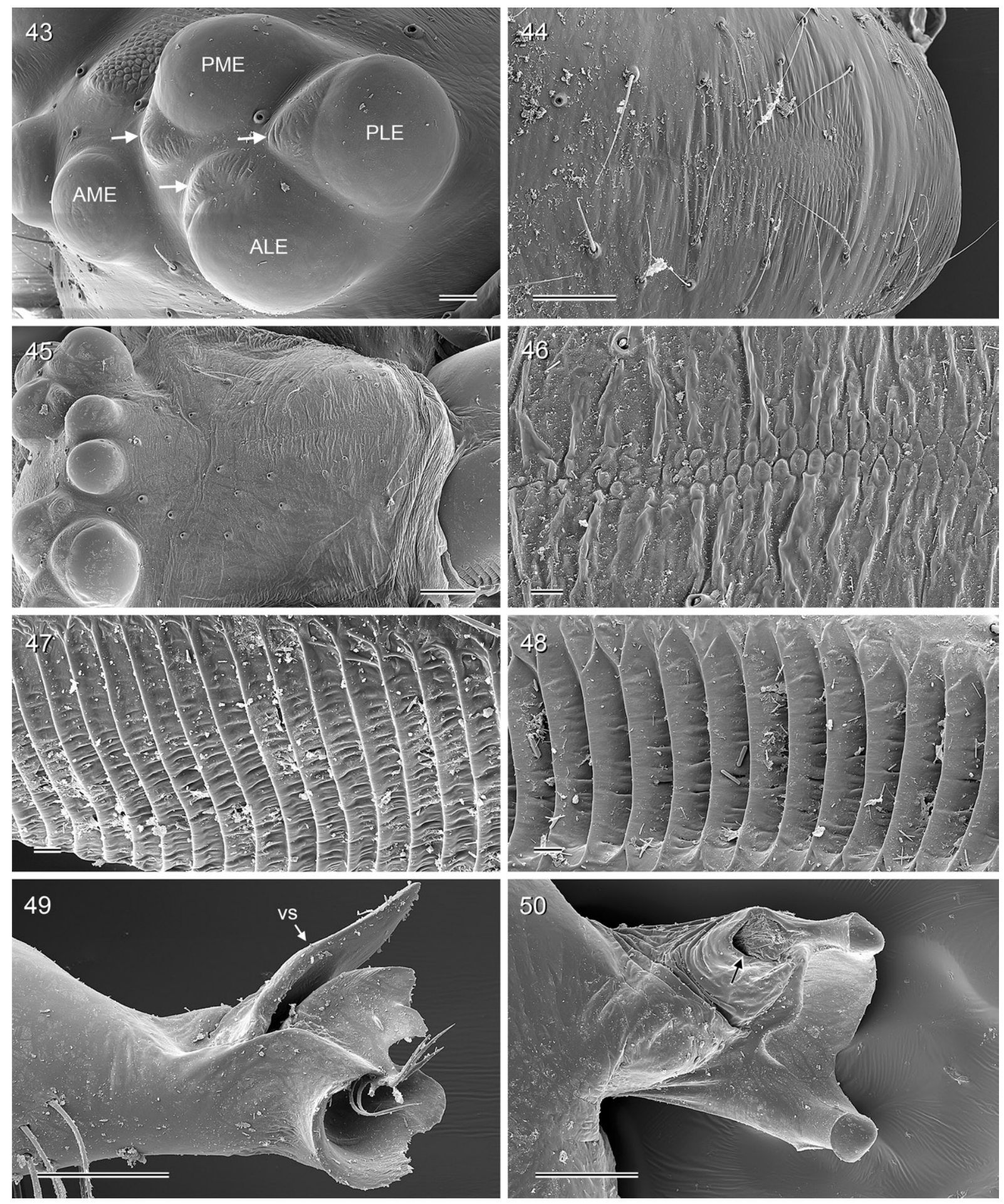

Figs 43-50. Holocnemus pluchei (Scopoli, 1763); male and female from Greece, Daphni/Athens (MHNG). 43. Left male ocular area, oblique view; arrows: 'accessory lenses'. 44. Male clypeus, frontal-dorsal view. 45. Female ocular area and clypeus, oblique frontal view. 46. Detail of preceding figure. 47-48. Stridulatory files on male and female chelicerae. 49. Left procursus, retrolateral view. 50. Distal (main) sclerite of left genital bulb, prolateral (slightly distal) view; arrow: sperm duct opening. Abbreviation: vs $=$ ventral sclerite. Scale bars: $43=30 \mu \mathrm{m} ; 44-45,49-50=100 \mu \mathrm{m} ; 46-48=10 \mu \mathrm{m}$. 
solitary siblings and larger companions, they tend to lose contests, and they are sometimes cannibalized (Gerhardt 1927; Jakob 1991; Jakob et al. 2000). The reason they join conspecifics may be the cost of building an own web: it takes a spiderling at least 5 days to feed enough to build a web (Jakob 1991), and webs are not recycled (Jakob et al. 2000).

Sperm uptake was described by Gerhardt (1927). First, a line of silk between the tips of legs 3 is moved against the gonopore until the sperm droplet emerges. Then the line with the drop is brought to the chelicerae from where it is taken up by the genital bulbs. In alternating movements, each bulb contacts the drop three times for approximately $30 \mathrm{~s}$ each. Within the bulb, sperm remains viable at least for several weeks (99.6\% viable after two weeks; Cargnelutti et al. 2020).

Basic aspects of courtship and copulation have been described by Gerhardt (1927), Huber (1995), and Dutto et al. (2011). Both males and females (and juveniles) use their chelicerae to stridulate, but in different contexts. While male stridulation seems to have a luring function, female stridulation conveys a negative message, mainly to males (signaling non-receptivity), but also to other females and maybe even to other species (e.g., the web-invading Pholcus phalangioides) (Huber 1995; Dutto et al. 2011). The stridulation of juveniles has never been studied. Female stridulatory behavior has been found to be consistent, i.e., individual females differ consistently in the frequency of stridulating during consecutive inter-sexual encounters (Calbacho-Rosa et al. 2019a).

In copulations with virgin females, sperm is transferred during a first phase that is characterized by rhythmic simultaneous movements of the male palps (Cargnelutti et al. 2018). This is followed by a second phase where the palps remain inserted but seemingly immobile. The significance of this second phase is only partly understood. It might be a form of mate-guarding, and the duration of the immobile phase positively affected sperm viability in females (which decreased more rapidly in females than in males) (Cargnelutti et al. 2020).

Copulations with non-virgin females are common; they take longer ( 40 vs 30 min; Kaster \& Jakob 1997), and they follow a slightly different pattern. They start with non-rhythmic alternating movements of only partially inserted palps (only procursus inserted) during which sperm of previous males is partially removed (Calbacho-Rosa et al. 2013). Second males fertilize an average of $\sim 65-83 \%$ of the eggs, but the actual values range from 0 to $100 \%$ (Kaster \& Jakob 1997). Second-male sperm precedence depends on timing: it is strongest in the few hours after copulation (Calbacho-Rosa et al. 2010) (explaining the pattern of mate guarding; see below).

The projection on the female sternum has been interpreted as a structure that controls the intensity and range of male palpal movements during copulation (Calbacho-Rosa et al. 2019b). These authors support their conclusion mainly by the fact that the projection contacts the male clypeus at the moment of maximum palpal contraction. They exclude a stidulatory function (with the "pre-epigynum") because the structures do not contact each other during intersexual interactions (but see Discussion).

The biological significance of the dorsal female modification (pedicel, abdomen) has never been studied (it has apparently not even been described before). It may function during abdominal twitching, a component of low-level aggressive interactions (Jakob 1991, 1994). Abdomen twitching was the only antagonistic behavioral component that differed between sexes, but it was males (that lack pedicelabdomen modifications) that performed more twitches (Blanchong et al. 1995). Escalating fights involve the legs, and Johnson \& Jakob (1999) found that 7-8\% of spiders in natural populations were missing at least one leg, usually (in $86 \%$ of the cases) one of the anterior two pairs. However, leg loss does not significantly affect the male's ability to compete over webs and prey (Johnson \& Jakob 1999). 

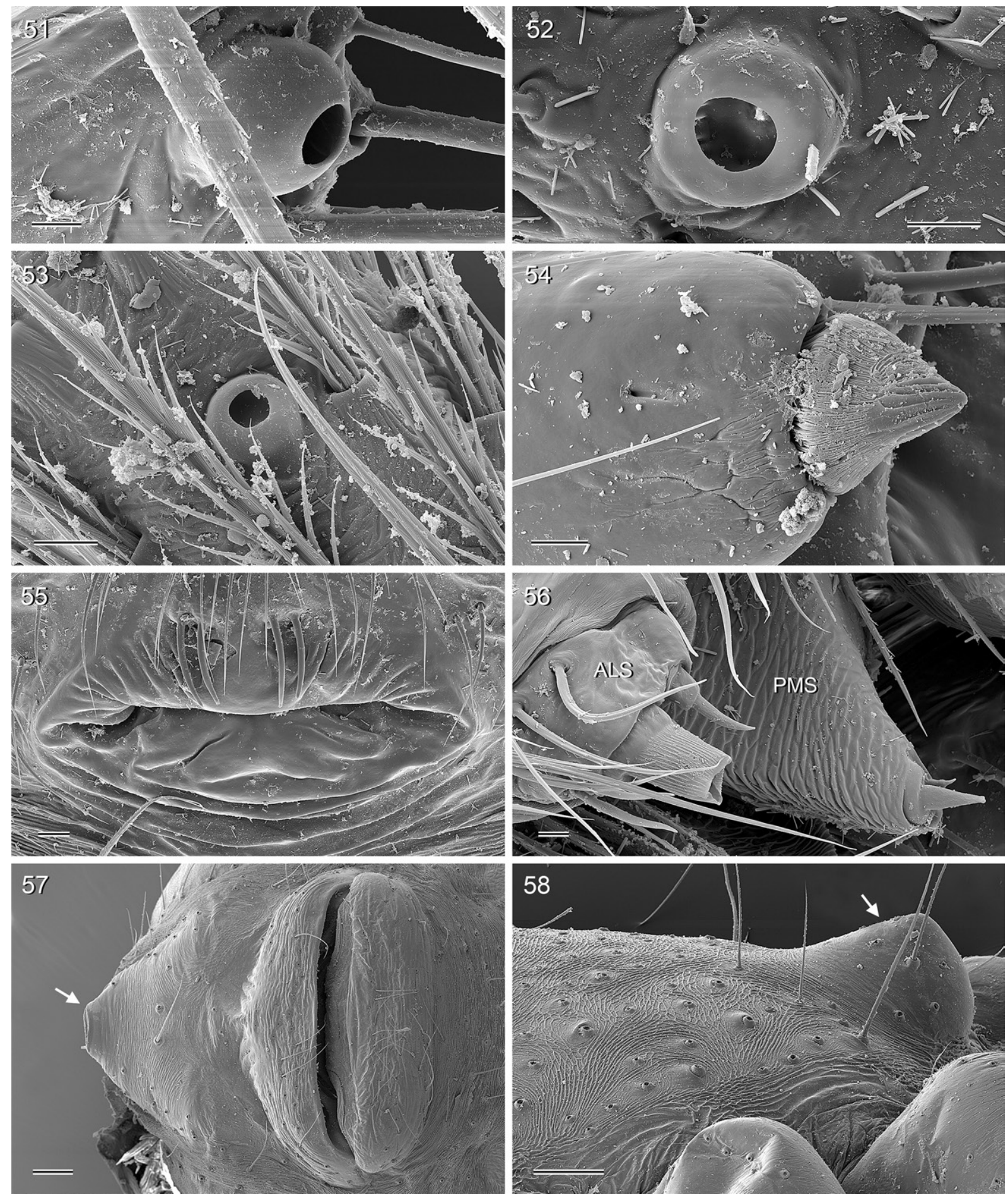

Figs 51-58. Holocnemus pluchei (Scopoli, 1763); male and female from Greece, Daphni/Athens (MHNG). 51-52. Male and female palpal tarsal organs. 53. Tarsal organ on male tarsus 4. 54. Tip of male cheliceral apophysis. 55. Male gonopore. 56. Male spinnerets. 57. Epigynum and anterior abdominal process (arrow). 58. Female sternum, showing posterior process (arrow). Scale bars: 51-54, $56=10 \mu \mathrm{m}$; $55=20 \mu \mathrm{m} ; 57-58=100 \mu \mathrm{m}$. 
Reports on mate guarding are somewhat contradictory. For Californian populations, Kaster \& Jakob (1997) report the absence of guarding; Sedey \& Jakob (1998) found that most females with eggs were initially accompanied by at least one male. In Argentinean populations, Calbacho-Rosa et al. (2010) observed post-copulatory mate guarding: males stayed close to females for approximately 12-24 hrs after copulation, and actively defended females (or rather their own investment) from intruding males. This does not seem to imply chivalrous behavior: males do not cede prey to females, and they win interactions as often as females do (Blanchong et al. 1995).

Females produce several egg-sacs per season, and Hajer \& Řeháková (2003) reported a maximum of eight clutches in the lab. As usual in pholcids (Huber \& Eberle 2021), larger females lay more eggs (and thus produce heavier clutches), but egg weight is not affected by female body size (Skow \& Jakob 2003). Kaster \& Jakob (1997), working on Californian populations, reported a mean clutch size of 33 (9-70), while Ahmed (2021), working on Egyptian spiders, reported a mean of 89. While Ahmed's (2021) publication is flawed in many respects, this number is not unfeasible. A single egg-sac of a female from Croatia reported in Huber \& Eberle (2021) was estimated at having 77 eggs.

While caring for an egg-sac, females are reluctant to remate, but occasionally they do, especially at a late stage of embryo development (Calbacho-Rosa et al. 2017). In order to mate, they have to temporarily suspend the egg-sac in the web, which supposedly carries some risk for the eggs, especially from (conspecific) predators (Calbacho-Rosa et al. 2017). Egg-sac carrying has also been shown to protect the eggs from fungi (Calbacho-Rosa et al. 2017), but this is not likely to have a significant effect during the relatively short time of mating.

Developmental time depends on food level, and well-fed spiders often reach maturity after five molts, while poorly-fed spiders usually need six molts (Jakob \& Dingle 1990). However, spiders maturing after six molts are on average larger, irrespective of food level (Jakob \& Dingle 1990). When legs were removed at the third instar, they were not regenerated (Johnson \& Jakob 1999).

When disturbed, H. pluchei starts to move vigorously like many long-legged pholcids. However, the movement has been described as 'bouncing', different from the 'whirling' of P. phalangioides (Jackson et al. 1993). When bouncing, H. pluchei lifts and lowers the body by flexing and extending the femurpatella and tibia-metatarsus joints at an amplitude of 2-20 $\mathrm{cm}$ and at a rate of 5-10/s (Jackson et al. 1993). This is thought to protect the spider from visual predators, and it may also interfere with a predator's movement in the web (Jackson 1992; Jackson et al. 1993). An alternative strategy is to leap out of the web and 'play dead' (Jackson et al. 1993).

\section{Distribution}

The original distribution of $H$. pluchei is the Mediterranean or part of it. Compared to some other synanthropic and anthropophilic pholcids, it has established permanent colonies in other regions relatively recently (Fig. 2). Surprisingly, this seems to have happened within a relatively short period of time around the world. For the USA, Vetter et al. (2011) did a survey among regional arachnologists and concluded that H. pluchei had been introduced in the San Francisco Bay area in the 1950s. The oldest South American records date back to the early 1960s (Argentina; see Material examined). By that time, the species was present in at least two provinces, suggesting that it may well have been introduced in the 1950s as well. The species is now very common in central Argentina as well as in Uruguay (Laborda \& Simó 2008).

The oldest confirmed Central European and Australian records are also from the 1960s (Germany: 1962; South Australia: 1969; see Material examined section). Older records from the Netherlands ( $19^{\text {th }}$ century) are dubious (van Helsdingen 2010). In Central Europe the species has been spreading massively since the 1990s (Van Keer \& Van Keer 2001; Reiser \& Neumann 2014) and has relatively recently reached 
countries like Denmark (oldest record 2006; https://www.danmarks-edderkopper.dk/), Great Britain (oldest record 2004; https://www.britishspiders.org.uk/), and the Caucasus (Ponomarev et al. 2019). The oldest known record for Japan is from 2008 (Kumada 2021), suggesting that the introduction to Japan happened relatively recently.

L. Koch's (1875) record for Massaua (Eritrea) is based on misidentified juvenile specimens of Crossopriza, presumably C. pristina (photos kindly provided by M. Tavano, 30 Jan. 2014). Leardi in Airaghi's (1902) record for Mahé (India) could not be checked but it here also considered to be based on misidentified specimens.

Holocnemus reini (C. Koch, 1873) comb. nov.

Figs 3, 7-8, 59-96

Pholcus reini C. Koch, 1873: 113.

Holocnemus caudatus (misidentification) - Barrientos et al. 2019: 12.

Crossopriza sp. - Hajer \& Řeháková 2003: 345-354.

\section{Remarks}

While Roewer (1955) considered Pholcus reini a nomen dubium ("nicht zu deuten"), Bonnet (1958) treated it as an available name. Platnick (2000), in his first online catalogue, adopted Roewer's view, and Pholcus reini has been listed as a nomen dubium ever since (World Spider Catalog 2021). The single male type specimen is apparently lost. It is not in SMF, in contrast to the types of at least one other species collected during the same expedition and published in the same paper (Ocypete fritschi C. Koch, 1873, now Eusparassus fritschi). However, even though C. Koch's (1873) original description does not provide any figure, it gives some details (size, ventral color pattern) that fit only two pholcid species known to be present in Morocco: Holocnemus pluchei and the species here interpreted to represent Holocnemus reini. Since C. Koch (1873) distinguished between Pholcus barbarus (now H. pluchei) and $P$. reini, it seems reasonable to assume that C. Koch's name does not refer to H. pluchei but to the species treated here.

I have not seen the specimens of Barrientos et al. (2019) but since all newly examined Moroccan specimens were $H$. reini rather than the similar $H$. caudatus, the specimens of Barrientos et al. (2019) are regarded as misidentified. Vocher specimens of Hajer \& Řeháková's (2003) “Crossopriza sp." were examined (see below).

\section{Diagnosis}

Easily distinguished from most known Smeringopinae (except $H$. caudatus) by deep indentations on sternum (Fig. 81; in males and females; deepest pair between coxae 4), by shape of procursus (Fig. 63; distinctive retrolateral membrane), by shape of genital bulb (Figs 66, 92; distal bulbal sclerite triangular in prolateral view, with one large and two small rounded processes); from known congeners and representatives of Crossopriza also by 2-3 modified hairs on each male cheliceral apophysis (Figs 64, 82-83; rather than one); from $H$. caudatus only by slightly wider distances between male cheliceral apophyses (70-80\% of cheliceral maximum width) and between female epigynal pockets ( $>$ $0.5 \mathrm{~mm})$.

\section{Type material}

Holotype

MOROCCO • ${ }^{1}$; Mtuga plateau (roughly in the area between Essaouira, Marrakesh, and Agadir); 1872; K. von Fritsch and J. Rein leg.; apparently lost. 


\section{Material examined}

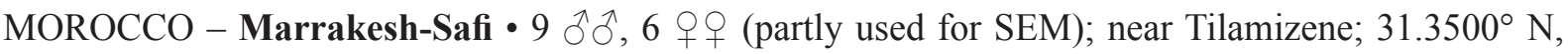
7.7645 ${ }^{\circ}$ W; 950 m a.s.1.; 11 Sep. 2018; B.A. Huber leg.; near ground; ZFMK Ar $22319 \bullet 2$ $q$, 1 juv. (in pure ethanol); same collection data as for preceding; ZFMK Mor72 1 §, 1 O; High Atlas, near Asni;

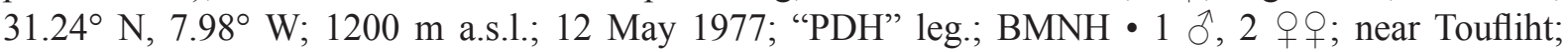
$31.4715^{\circ} \mathrm{N}, 7.4332^{\circ} \mathrm{W} ; 1465 \mathrm{~m}$ a.s.1.; 27 Sep. 2018; B.A. Huber leg.; among rocks near ground above ravine; ZFMK Ar 22320 • 1 q (in pure ethanol); same collection data as for preceding; ZFMK Mor108 • 1 q; near Rakte; $31.033^{\circ} \mathrm{N}, 8.137^{\circ} \mathrm{W} ; 1060 \mathrm{~m}$ a.s.1.; 12 Sep. 2018; B.A. Huber leg.; among rocks in dry

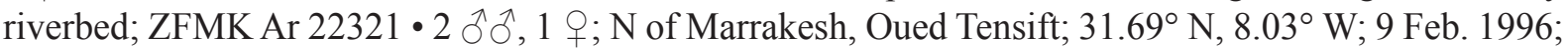
J. Van Keer leg.; under stones; CJVK • 1 क; same locality as for preceding; in and along riverbed; 9 Feb.

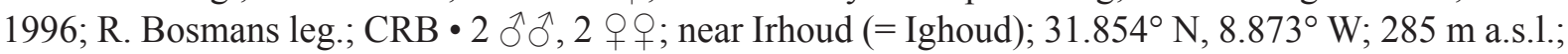
29 Sep. 2018; B.A. Huber leg.; along river and among cacti; ZFMK Ar $22323 \cdot 1$ o ; NE of Tamelelt [Tamallalt], El Kelaa des Sraghna; $32.055^{\circ}$ N, $7.400^{\circ}$ W; 590 m a.s.l.; 16 Apr. 2012; J. Van Keer leg.; stones in wasteland; CJVK. - Souss-Massa - 1 Oे, 1 क; S of Tizi n'Test at R203; 30.850 ${ }^{\circ} \mathrm{N}, 8.375^{\circ} \mathrm{W}$; 1580 m a.s.1.; 12 Sep. 2018; B.A. Huber leg.; among rocks near ground; ZFMK Ar $22322 \bullet 1$ juv. (in pure ethanol); same collection data as for preceding; ZFMK Mor75 1 त, 1 juv.; $80 \mathrm{~km}$ E of Taroudant,

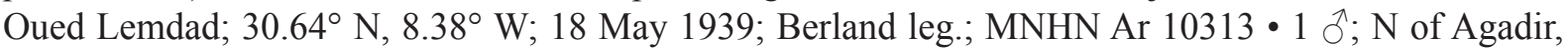
Imouzzer; $30.678^{\circ} \mathrm{N}$, 9.483 $\mathrm{W}$; $960 \mathrm{~m}$ a.s.1.; 8 Sep. 2014; S. Huber leg.; small cave near waterfall; ZFMK Ar 22326 • 1 q; $\mathrm{N}$ of Agadir, Imouzzer; $30.678^{\circ} \mathrm{N}, 9.482^{\circ} \mathrm{W} ; 960 \mathrm{~m}$ a.s.1.; 27 Nov. 2016; S. Huber leg.; olive tree plantation; ZFMK Ar $22327 \bullet 2$ ○े, 1 o ; Paradise Valley; $30.588^{\circ} \mathrm{N}, 9.528^{\circ} \mathrm{W}$; 305 m a.s.l.; 13 Sep. 2018; B.A. Huber leg.; among rocks near ground; ZFMK Ar $22328 \bullet 2$ 우 (in pure ethanol); same collection data as for preceding; ZFMK Mor77•1 $\$$; $7 \mathrm{~km} \mathrm{~N}$ of Agadir, Anza; $30.45^{\circ} \mathrm{N}$,

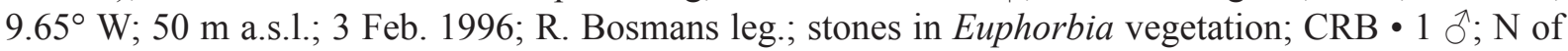
Agadir, road Agadir-Alma; 30.486 ${ }^{\circ}$ N, 9.565 ${ }^{\circ}$ W; 440 m a.s.l.; 27 Nov. 2016; S. Huber leg.; ZFMK Ar 22329 4 우, 1 juv.; Agadir, path to Kasbah Hill; $30.430^{\circ}$ N, 9.619 ${ }^{\circ}$ W; 110 m a.s.l.; 7 Sep. 2014; S. Huber leg.; ZFMK Ar 22330 • 1 §ं; same locality as for preceding; 60 m a.s.1.; 28 Nov. 2016; S. Huber

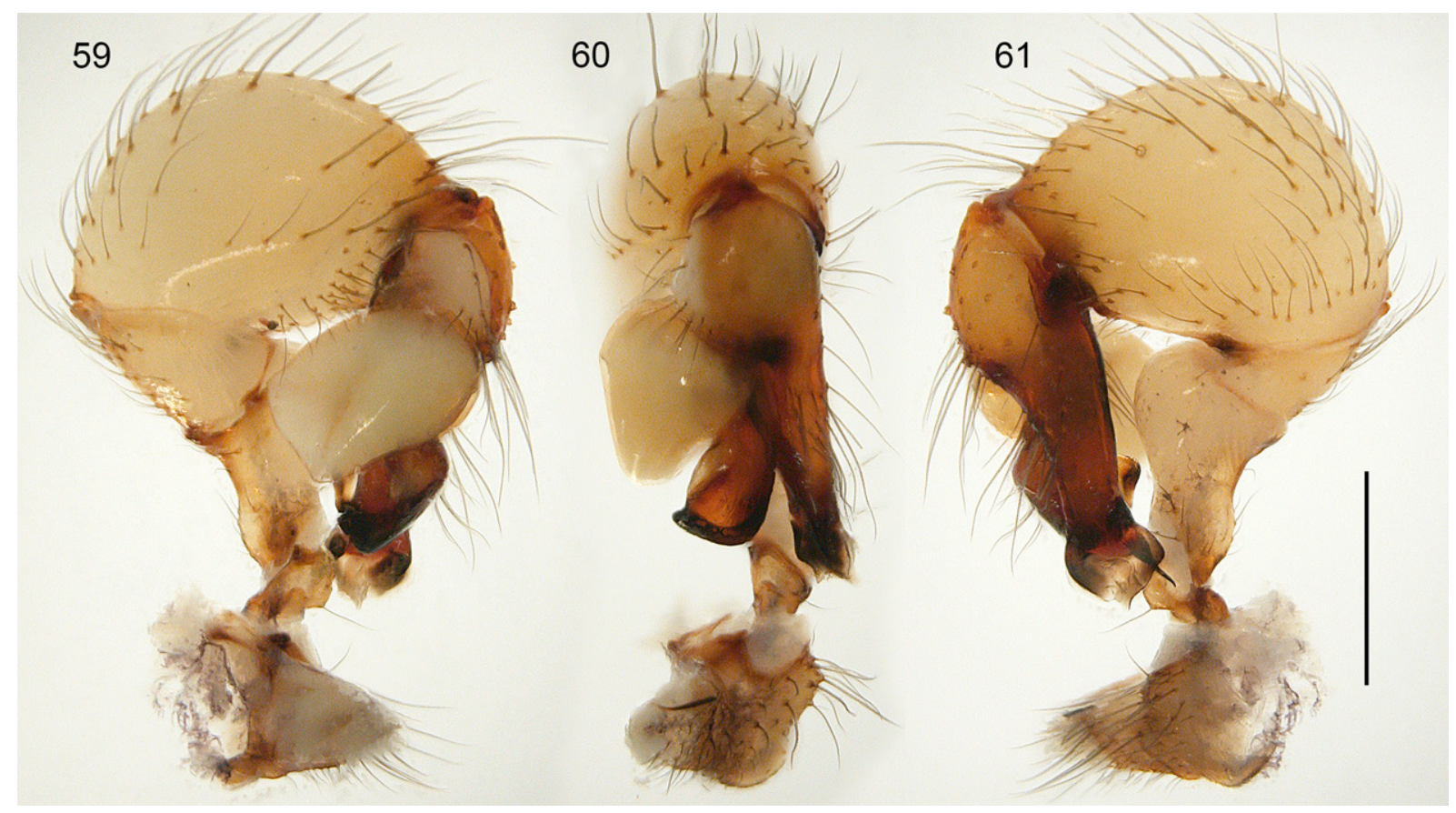

Figs 59-61. Holocnemus reini (C. Koch, 1873) comb. nov.; male from Morocco, near Tilamizene (ZFMK Ar 22319); left palp, prolateral, dorsal, and retrolateral views. Scale bar $=0.5 \mathrm{~mm}$. 

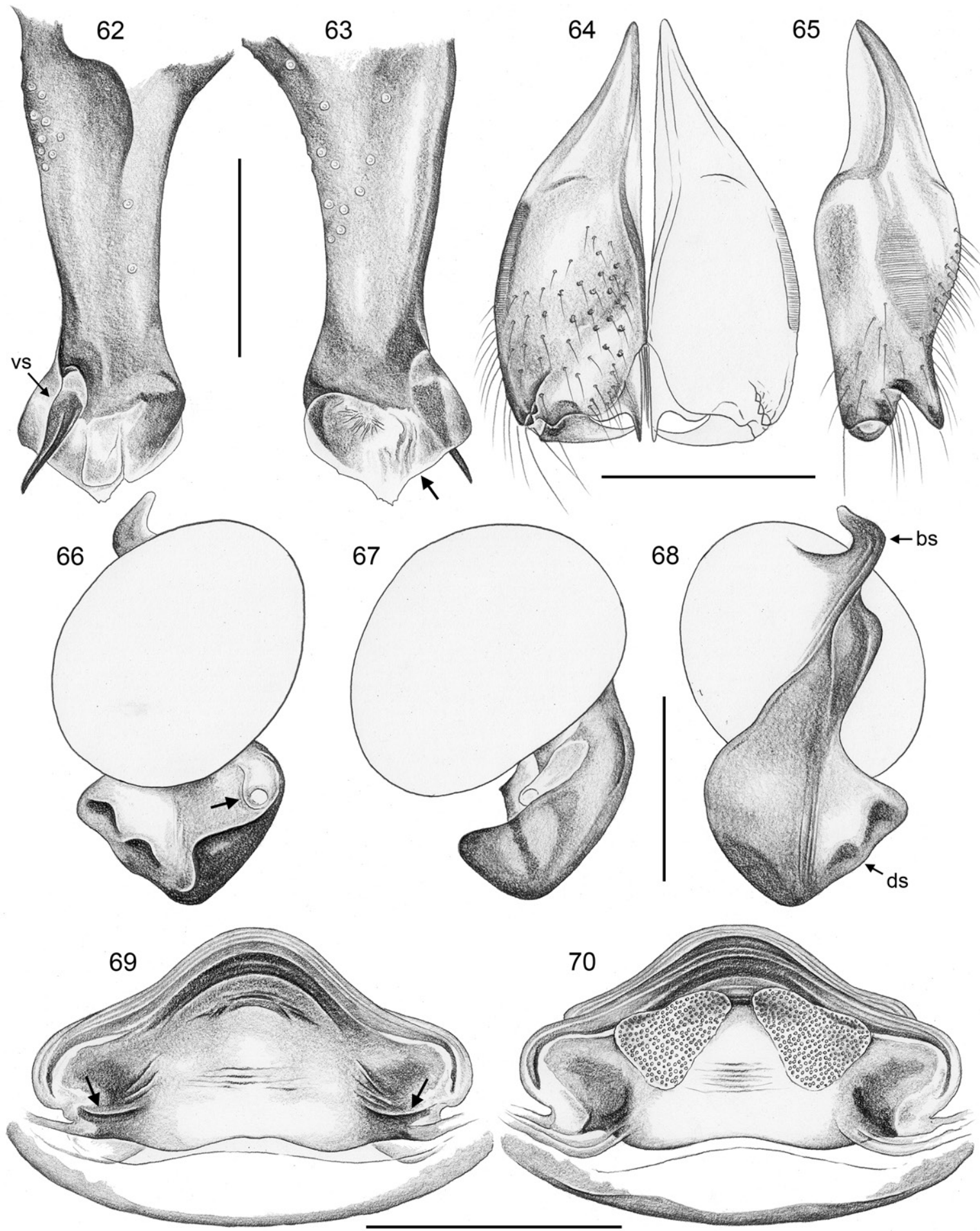

Figs 62-70. Holocnemus reini (C. Koch, 1873) comb. nov.; male from Morocco, near Tilamizene (ZFMK Ar 22319), female from Morocco, Al Kamoun (CAS 9027140). 62-63. Left procursus, prolateral and retrolateral views; arrow: retrolateral membrane. 64-65. Male chelicerae, frontal and lateral views. 66-68. Left genital bulb, prolateral, dorsal, and retrolateral views; arrow: sperm duct opening. 69-70. Cleared female genitalia, ventral and dorsal views; arrows: pockets. Abbreviations: bs $=$ basal sclerite; $d s=$ distal sclerite; $v s=$ ventral sclerite. Scale bars: $62-63,66-68=0.3 \mathrm{~mm} ; 64-65$, $69-70=0.5 \mathrm{~mm}$. 
leg.; ZFMK Ar 22331 • 1 क; wadi $\sim 5 \mathrm{~km} \mathrm{NE}$ of Agadir; $30.456^{\circ}$ N, $9.610^{\circ} \mathrm{W} ; 150 \mathrm{~m}$ a.s.1.; 14 Sep. 2014;

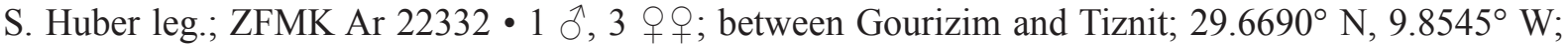

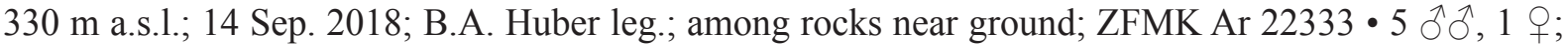
near Jamaa Idaoussemlal; 29.5306 N, 9.2375 W; 1220 m a.s.l.; 15 Sep. 2018; B.A. Huber leg.; along small brook; ZFMK Ar 22334 • 1 o, 1 \& (in pure ethanol); same collection data as for preceding; ZFMK Mor84 - 1 गे; SW of Tafraoute; $29.696^{\circ}$ N, 9.010 W; 1025 m a.s.1.; 15 Sep. 2018; B.A. Huber leg.;

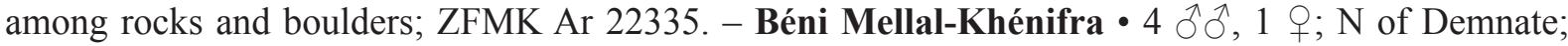
$31.761^{\circ} \mathrm{N}, 7.006^{\circ} \mathrm{W} ; 895 \mathrm{~m}$ a.s.1.; 26 Sep. 2018; B.A. Huber leg.; among rocks near small cave; ZFMK

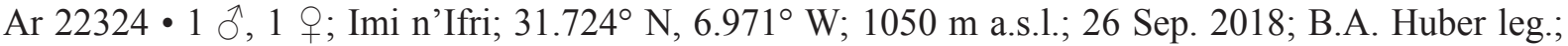

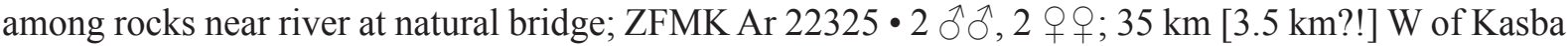
Tadla, Al Kamoun ("Kamoune"); 32.56 N, 6.26 W; 21 Dec. 1986; V. and B. Roth leg.; CAS 9027140

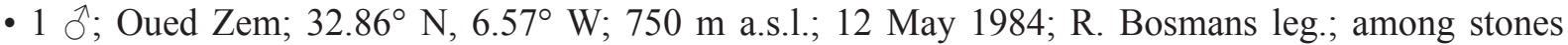
in Pinus halepensis plantation; CRB - 1 juv.; E of Kasba Tadla, SW Ait-Roadi; $32.62^{\circ} \mathrm{N}, 6.11^{\circ} \mathrm{W}$;
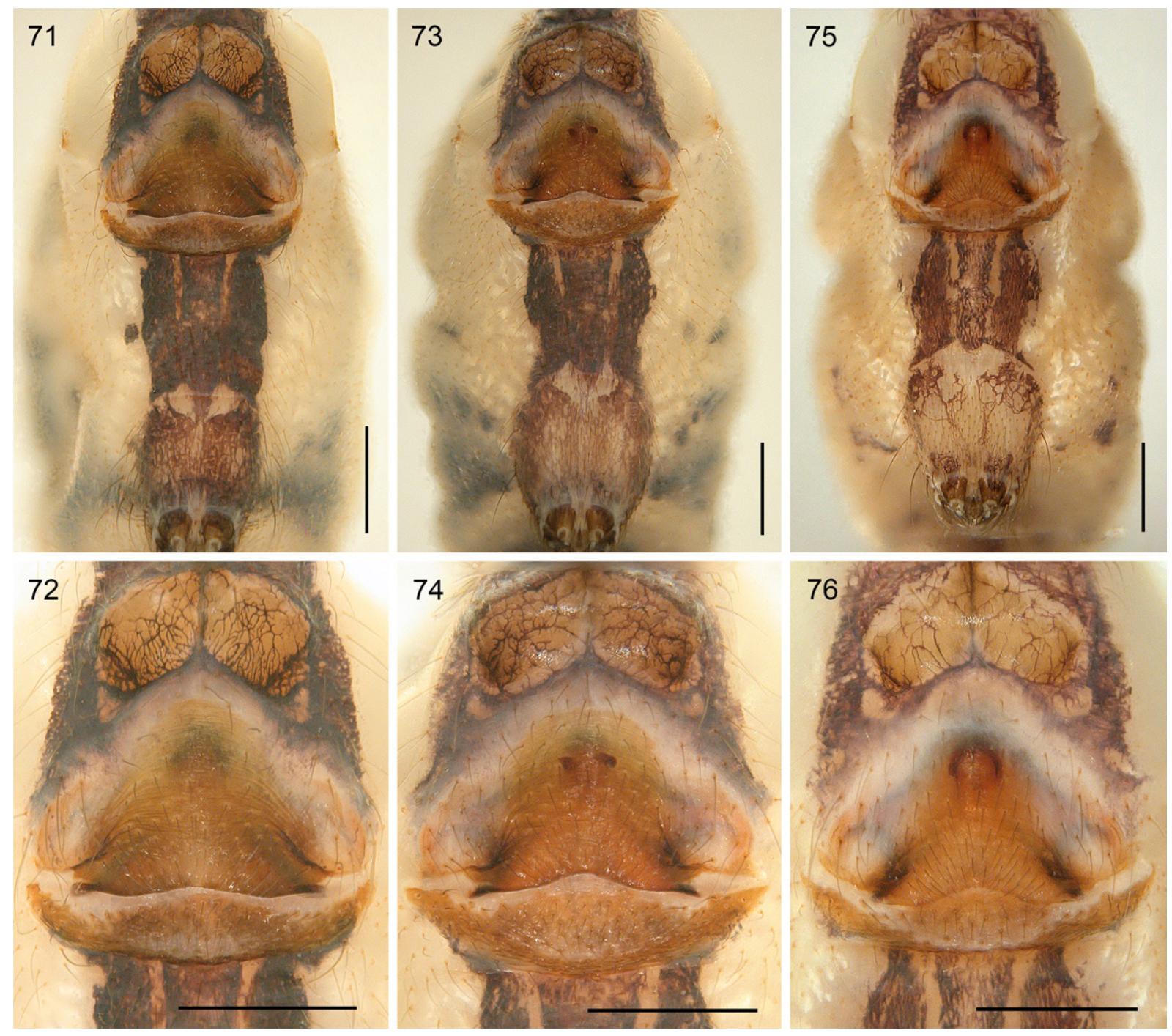

Figs 71-76. Holocnemus reini (C. Koch, 1873) comb. nov.; female abdomens and epigyna, ventral views. 71-72. Morocco, SW of Bab Boudir (ZFMK Ar 22346). 73-74. Morocco, near El Ksiba, Kehf d'bouba (ZFMK Ar 22336). 75-76. Morocco, W of Tamtetoucht (ZFMK Ar 22341). Scale bars = $0.5 \mathrm{~mm}$. 
560 m a.s.1.; 17 Apr. 2012; R. Bosmans leg.; stones in grassland; CRB $\bullet 2$ $\widehat{\jmath}, 1$ ○; near El Ksiba, Kehf d'bouba; $32.5165^{\circ} \mathrm{N}, 6.0679^{\circ} \mathrm{W} ; 1640 \mathrm{~m}$ a.s.1.; 24 Sep. 2018; B.A. Huber leg.; among rocks at cave entrance and near cave; ZFMK Ar 22336 1 i (in pure ethanol); same collection data as for preceding;

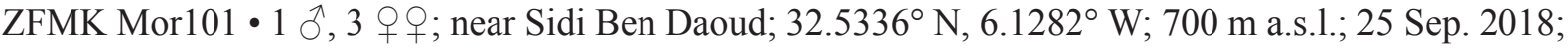
B.A. Huber leg.; among cacti and in small cave; ZFMK Ar 22337 • 1 ○े, 1 q; N of Imilchil, near R317; $32.300^{\circ} \mathrm{N}, 5.660^{\circ} \mathrm{W} ; 1585 \mathrm{~m}$ a.s.1.; 25 Sep. 2018; B.A. Huber leg.; among rocks near ground in dry riverbed; ZFMK Ar $22338 \cdot 1$ o ; SE of El Ksiba, near R317; $32.486^{\circ} \mathrm{N}, 5.942^{\circ} \mathrm{W} ; 1315 \mathrm{~m}$ a.s.l.;

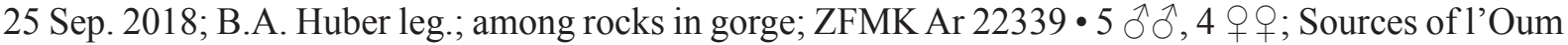
er Rbia; $33.051^{\circ} \mathrm{N}, 5.412^{\circ} \mathrm{W}-33.049^{\circ} \mathrm{N}, 5.411^{\circ} \mathrm{W} ; 1270-1330 \mathrm{~m}$ a.s.1.; 21 Sep. 2018; B.A. Huber leg.; in small cave and among rocks above waterfall; ZFMK Ar 22342 - 1 क (in pure ethanol); same collection data as for preceding; ZFMK Mor99. - Drâa-Tafilalet 1 juv. (in pure ethanol); Timoula; $31.791^{\circ} \mathrm{N}, 5.463^{\circ} \mathrm{W} ; 2000 \mathrm{~m}$ a.s.l.; 19 Sep. 2018; B.A. Huber leg.; under rocks at roadside; ZFMK Mor93 - 1 ô, 1 क; E of Tamtetoucht; $31.686^{\circ} \mathrm{N}, 5.521^{\circ} \mathrm{W} ; 1775 \mathrm{~m}$ a.s.1.; 19 Sep. 2018; B.A. Huber leg.; at rocks near spring; ZFMK Ar 22340 • 1 क (in pure ethanol); same collection data as for preceding;

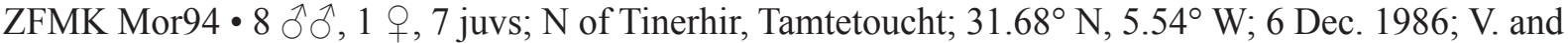

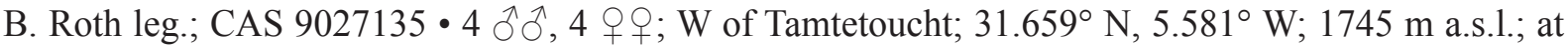
rocks; 19 Sep. 2018; B.A. Huber leg.; ZFMK Ar 22341 • 1 q (in pure ethanol); same collection data as
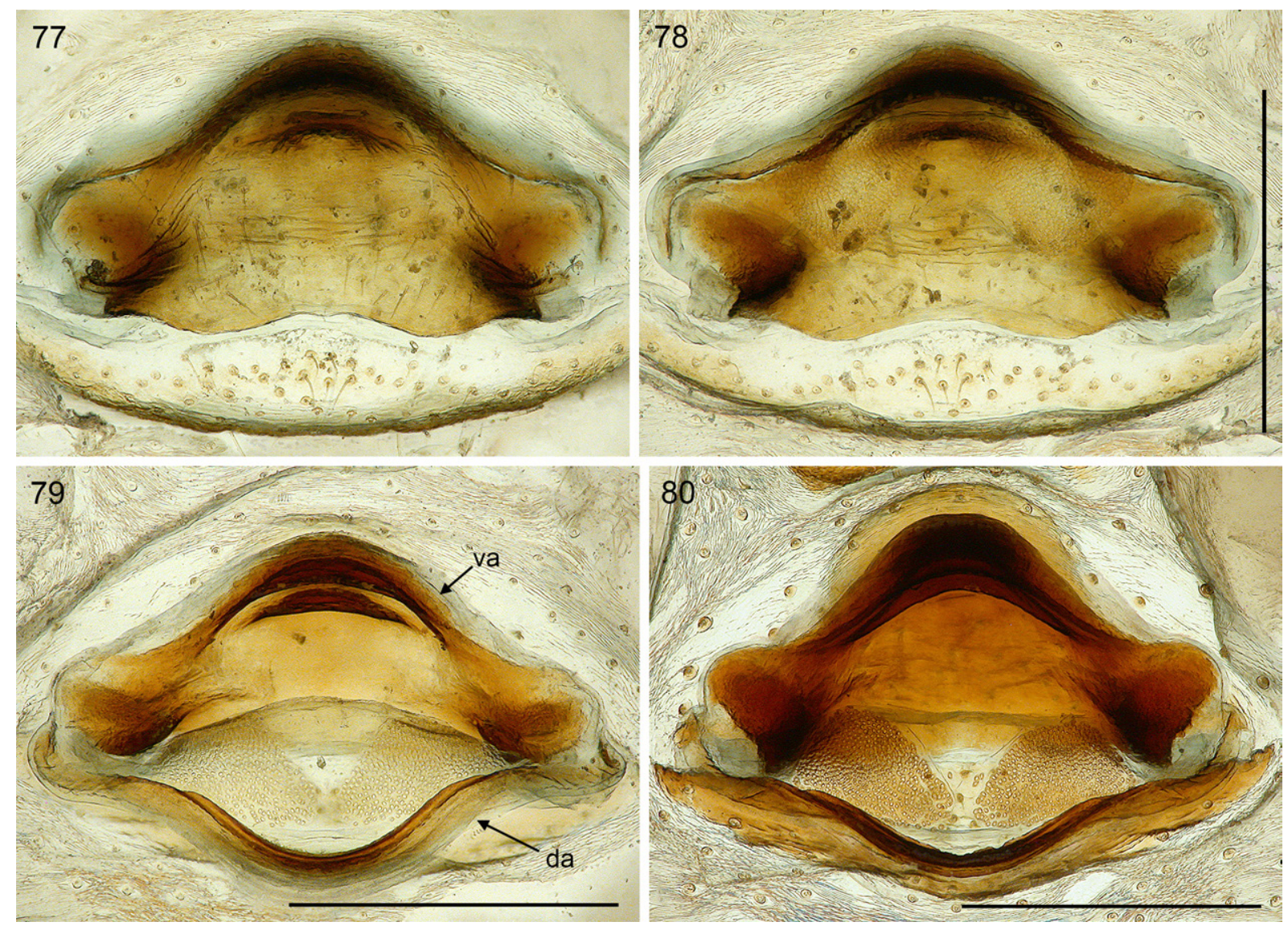

Figs 77-80. Holocnemus reini (C. Koch, 1873) comb. nov. 77-79. Female from Morocco, Al Kamoun (CAS 9027140); internal genitalia, ventral view, regular dorsal view, and dorsal view with dorsal arc tilted backwards. 80. Female from Morocco, near Tilamizene (ZFMK 22319); internal genitalia, dorsal view with dorsal arc tilted backwards. Abbreviations: da $=$ dorsal arc; va $=$ ventral arc. Scale bars $=0.5$ $\mathrm{mm}$. 
for preceding; ZFMK Mor95 • 1 ○ं; SE of Zebzat; $32.625^{\circ} \mathrm{N}$, $4.540^{\circ} \mathrm{W}$; $1675 \mathrm{~m}$ a.s.1.; 20 Sep. 2018; B.A. Huber leg.; among rocks outside of cave; ZFMK Ar 22343 • 1 ग, 1 o ; near Tizi n’Tairhemt; $32.593^{\circ} \mathrm{N}, 4.526^{\circ} \mathrm{W} ; 1855 \mathrm{~m}$ a.s.1.; 20 Sep. 2018; B.A. Huber leg.; among rocks in pine forest; ZFMK Ar $22344 \cdot 1$ juv. (in pure ethanol); same collection data as for preceding; ZFMK Mor97. - Rabat-SaléKénitra 11 đ, 2 juvs; 2 km N of Sidi Allal El Bahraoui, Forêt de la Maâmora; $34.03^{\circ}$ N, $6.55^{\circ} \mathrm{W} ; 8$ Feb. 1996; R. Bosmans leg.; litter in Quercus suber forest; CRB • 1 ô, 2 우; Oued Beht; $33.88^{\circ} \mathrm{N}, 5.93^{\circ} \mathrm{W}$; 7 Feb. 1996; J. Van Keer leg.; Pinus plantation, stones; CJVK • 1 क; same locality as for preceding; 7

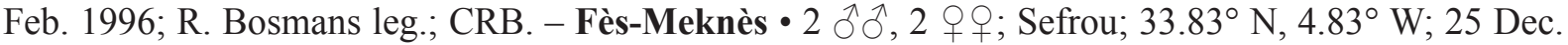

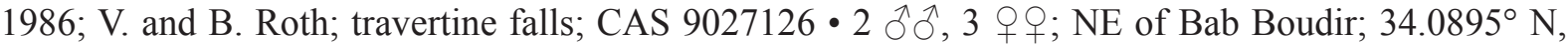

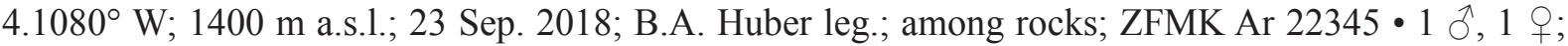
SW of Bab Boudir; $34.0618^{\circ} \mathrm{N}, 4.1317^{\circ} \mathrm{W} ; 1570 \mathrm{~m}$ a.s.l.; 23 Sep. 2018; B.A. Huber leg.; among rocks; ZFMK Ar 22346 • 1 ô, 1 q; Grotte Kef el Ghar (= Rhar); $34.4788^{\circ}$ N, 4.2766 W; 600 m a.s.1.; 22 Sep. 2018; B.A. Huber leg.; among rocks outside of cave; ZFMK Ar 22347. - Tanger-Tetouan-Al Hoceima

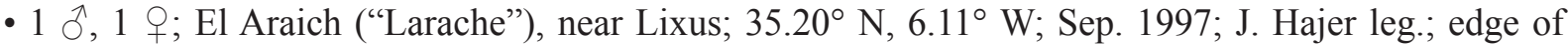

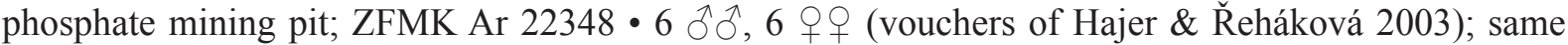
collection data as for preceding; ZFMK Ar 22349. - Laâyoune-Sakia El Hamra • 2 ${ }^{\lambda}, 2$, $q$, 1 juv.; Laayoune, $40 \mathrm{~km} \mathrm{~S}$ of Tarfaya; $27.70^{\circ} \mathrm{N}, 12.95^{\circ} \mathrm{W} ; 11 \mathrm{Feb} .2007$; R. Bosmans leg.; stony desert, stones along road; CRB.

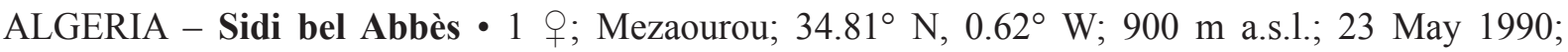

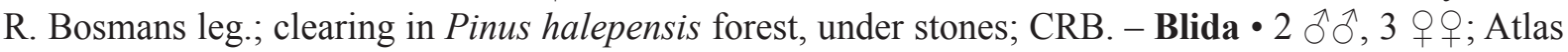
Blidéen, W of Chréa, Pic. E Abdelkader; $36.42^{\circ}$ N, 2.86 E; 1520 m a.s.1.; 20 Jun. 1987-9 May 1988; R. Bosmans leg.; open Cedrus forest with Berberis; CRB. - M'Sila • 1 + , 1 juv.; Djebel Maadid towards south; $35.80^{\circ} \mathrm{N}, 4.79^{\circ} \mathrm{E} ; 1350 \mathrm{~m}$ a.s.1.; 29 Apr. 1988; R. Bosmans leg.; along river, under rocks; CRB

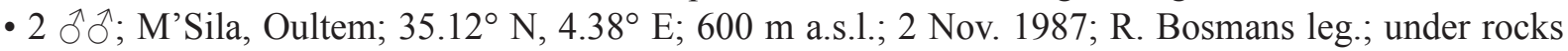
in steppe; CRB. - Sétif - 1 \& (only cleared epigynum); N of Magra, along Oued Nakhar; $35.727^{\circ} \mathrm{N}$, 5.161 ${ }^{\circ}$ E; 850 m a.s.1.; 2 Nov. 1988; R. Bosmans leg.; litter; CRB. - Batna 1 o+; S of Tazoult Lambese,

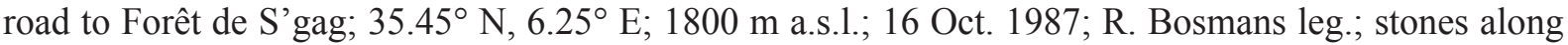
dry rivulet in grassland; CRB. - Biskra 1 ㄱ, 2 우 (without abdomens), 19 juvs; Biskra; $34.84^{\circ} \mathrm{N}$, 5.73 ${ }^{\circ}$ E; date and collector unknown; Simon collection \#12283; MNHN Ar 10325.

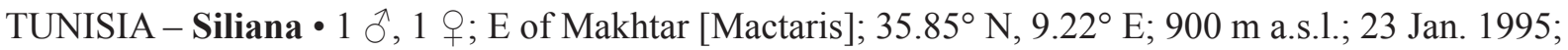
R. Bosmans leg.; stones along oued; CRB. - Sidi Bouzid • 1 ð , 1 + ; Djebel Bou Hedma National Park; 34.5 N, 9.6 $6^{\circ}$ E; 25-29 Mar. 2001; U. Moldrzyk leg.; CRB. - Gafsa • 1 \%; near Bou Omrane; $34.35^{\circ} \mathrm{N}$,

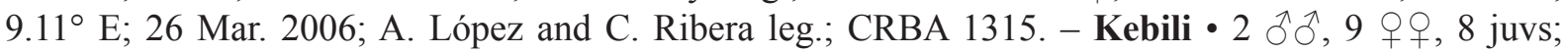
"Nefzana" [presumably Nefzaoua]; $33.5^{\circ} \mathrm{N}, 9.0^{\circ} \mathrm{E}$; date and collector unknown; Simon collection \#4890; MNHN Ar 10338.

\section{Redescription}

Male (Morocco, near Tilamizene, ZFMK Ar 22319)

Measurements. Total length 6.7, carapace width 1.75. Distance PME-PME $120 \mu \mathrm{m}$; diameter PME $120 \times 140 \mu \mathrm{m}$; distance PME-ALE $50 \mu \mathrm{m}$; diameter AME $100 \mu \mathrm{m}$; distance AME-AME $25 \mu \mathrm{m}$. Leg 1: $38.4(11.7+0.8+10.0+13.6+2.3)$, tibia $2: 6.9$, tibia $3: 5.5$, tibia $4: 6.5$; tibia $1 \mathrm{~L} / \mathrm{d}: 47$; femora $1-4$ diameters: $0.31,0.25,0.22,0.23$.

CoLOR (in ethanol). Carapace ochre-yellow; ocular area posteriorly with light brown V-mark; sternum dark brown; legs ochre-yellow, femora and tibiae with light tips and indistinct darker subdistal rings, with black lines on femora and (few) on tibiae, very few also on metatarsi; abdomen with distinct pattern of dark marks and whitish marks dorsally and laterally; ventrally with distinct black median band, partly disrupted, behind gonopore divided by two thin longitudinal light lines. 
Body. Habitus as in Fig. 7. Ocular area slightly raised. Deep thoracic pit and pair of shallow furrows diverging from pit toward posterior margin. Clypeus unmodified but rim more sclerotized than in female. Sternum wider than long (1.10/0.85), with distinct indentations between consecutive leg coxae and between coxae 4 (cf. Fig. 81). Abdomen strongly elongated beyond spinnerets, dorso-posteriorly pointed. Gonopore with seven epiandrous spigots (Fig. 93); ALS with one widened spigot and one pointed spigot (Fig. 94).

Chelicerae. As in Figs 64-65, with pair of low proximal frontal humps and pair of frontal lateral apophyses, each with two large modified cone-shaped hairs; distance between tips of modified hairs: $520 \mu \mathrm{m}$; several 'regular' frontal hairs with small but distinct processes at basis; lateral stridulatory ridges very fine (Fig. 84; distances between ridges: $2-3 \mu \mathrm{m}$ ), barely visible in dissecting microscope.

PALPS. As is Figs 59-61; coxa with rounded retrolateral hump; trochanter barely modified; femur slightly curved towards dorsal, distally widened, with rounded ventral protrusion, proximally with prolateral stridulatory pick (Fig. 86), with barely visible retrolateral transversal line, without retrolateral proximal process; femur-patella joints shifted toward prolateral side; tibia very large compared to femur, tibia-tarsus joints shifted toward retrolateral side; tarsus without macrotrichia; procursus (Figs 62-63) straight, few hairs slightly curved upwards; tarsal organ capsulate (Fig. 87); proximally on prolateral side with strong hump free of hairs, procursus tip with distinctive flat membranous element retrolaterally (arrow in Fig. 63) covering proximal part of ventral sclerite, with dorsal transversal flap and complex retrolateral elements (Figs 89-91); genital bulb (Figs 66-68) with simple basal sclerite connected to distal (main) sclerite, sperm duct opening in membranous area prolaterally at basis of distal sclerite (Fig. 92); distal sclerite with one large apophysis (fold) and two smaller apophyses on prolateral side; with indistinct retrolateral parallel ridges (Fig. 68).

Legs. Femur 1 with single row of $\sim 24$ ventral spines; without curved hairs; few vertical hairs; retrolateral trichobothrium of tibia 1 at $3 \%$; prolateral trichobothrium absent on tibia 1, present on other leg tibiae; tarsal pseudosegments indistinct and irregular except 4-5 distally.

Male (variation)

Tibia 1 in 73 males: 7.6-12.8 (mean 10.6). Chelicerae maximum width $(\mathrm{N}=57)$ : $0.56-0.80$ (mean $0.67)$; distance between tips of cheliceral apophyses: $0.41-0.58$; cheliceral apophyses sometimes with 3 modified hairs, sometimes asymmetric ( 2 and 3 hairs, respectively; Figs 82-83). Procursus length $(\mathrm{N}=58)$ : 0.82-1.16 (mean 1.03). Coloration of fresh specimens slightly variable, usually with dark median band on carapace. Posterior elongation of abdomen variably long, $\sim 0.5-1.0 \times$ distance pedicelspinnerets. Gonopore with 6-8 epiandrous spigots.

\section{Female}

In general similar to male (Fig. 8) but without spines on legs; often with slightly longer dorsal abdominal elongation. Without stridulatory apparatus between carapace and abdomen; with fine cheliceral stridulatory ridges as in male (Fig. 85; distances between ridges $\sim 3 \mu \mathrm{m}$ ). Tibia 1 in 53 females: 6.412.5 (mean 9.2). Epigynum as in Figs 71-76 and 96, main epigynal plate triangular to trapezoidal, weakly protruding; only posteriorly laterally strongly sclerotized; with pair of variably distinct pockets (distance between pockets $0.5-0.6$ ); internal sclerotized arc and median round structure variably visible in uncleared specimens; posterior epigynal plate large but simple; pair of low but distinct elevations in front of epigynum. Internal genitalia (Figs 69-70, 77-80) with triangular pore plates converging anteriorly, dorsal arc simple and slender, ventral arc laterally strongly widened, medially strong, with simple median pouch. 

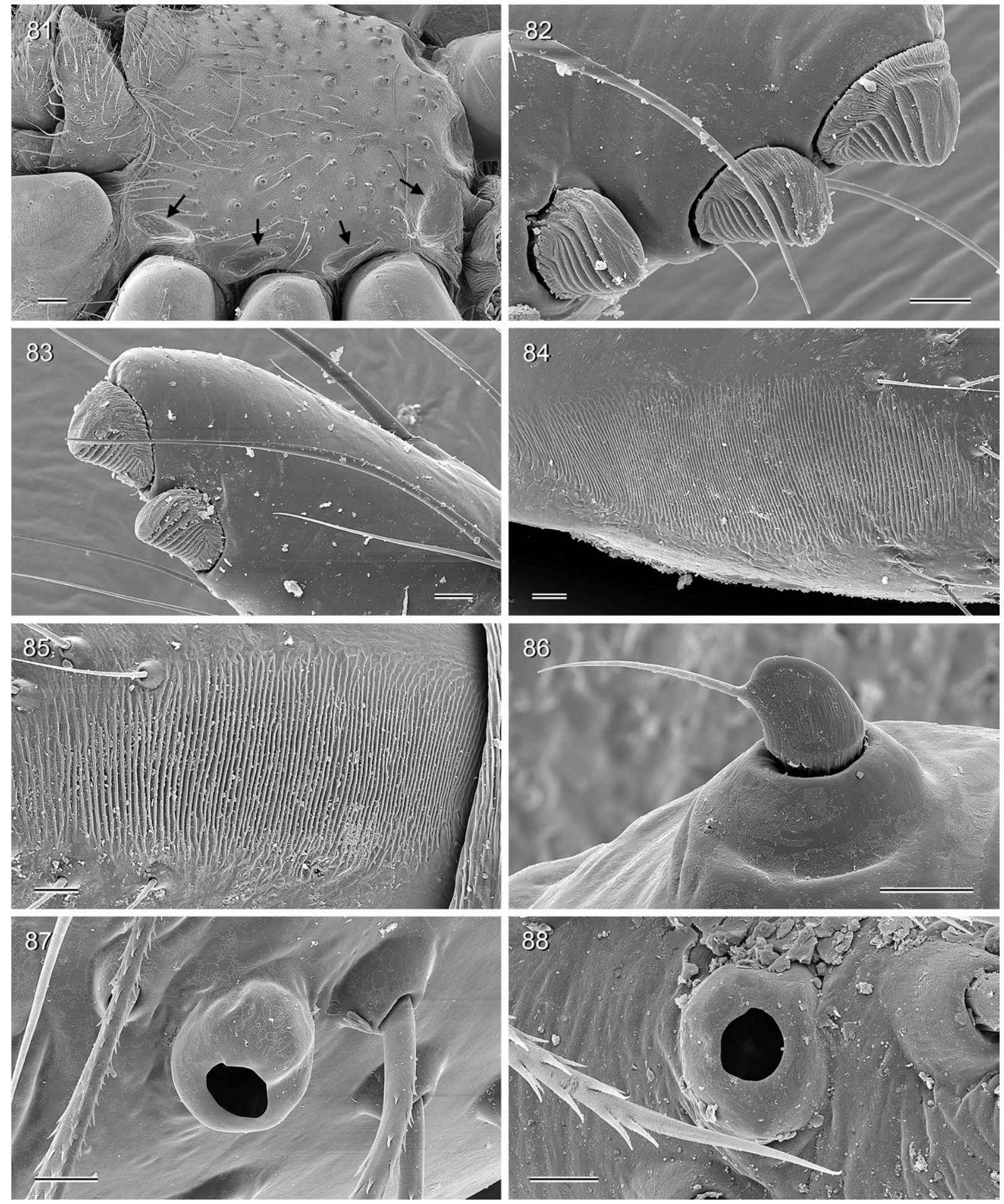

Figs 81-88. Holocnemus reini (C. Koch, 1873) comb. nov.; male and female from Morocco, near Tilamizene (ZFMK Ar 22319). 81. Female sternum; arrows: indentations. 82-83. Modified hairs on male cheliceral apophyses (from same specimen). 84-85. Male and female cheliceral stridulatory files. 86. Stridulatory pick on male palpal femur. 87-88. Male and female palpal tarsal organs. Scale bars: $81=100 \mu \mathrm{m} ; 82-83,86-88=10 \mu \mathrm{m} ; 84-85=20 \mu \mathrm{m}$. 

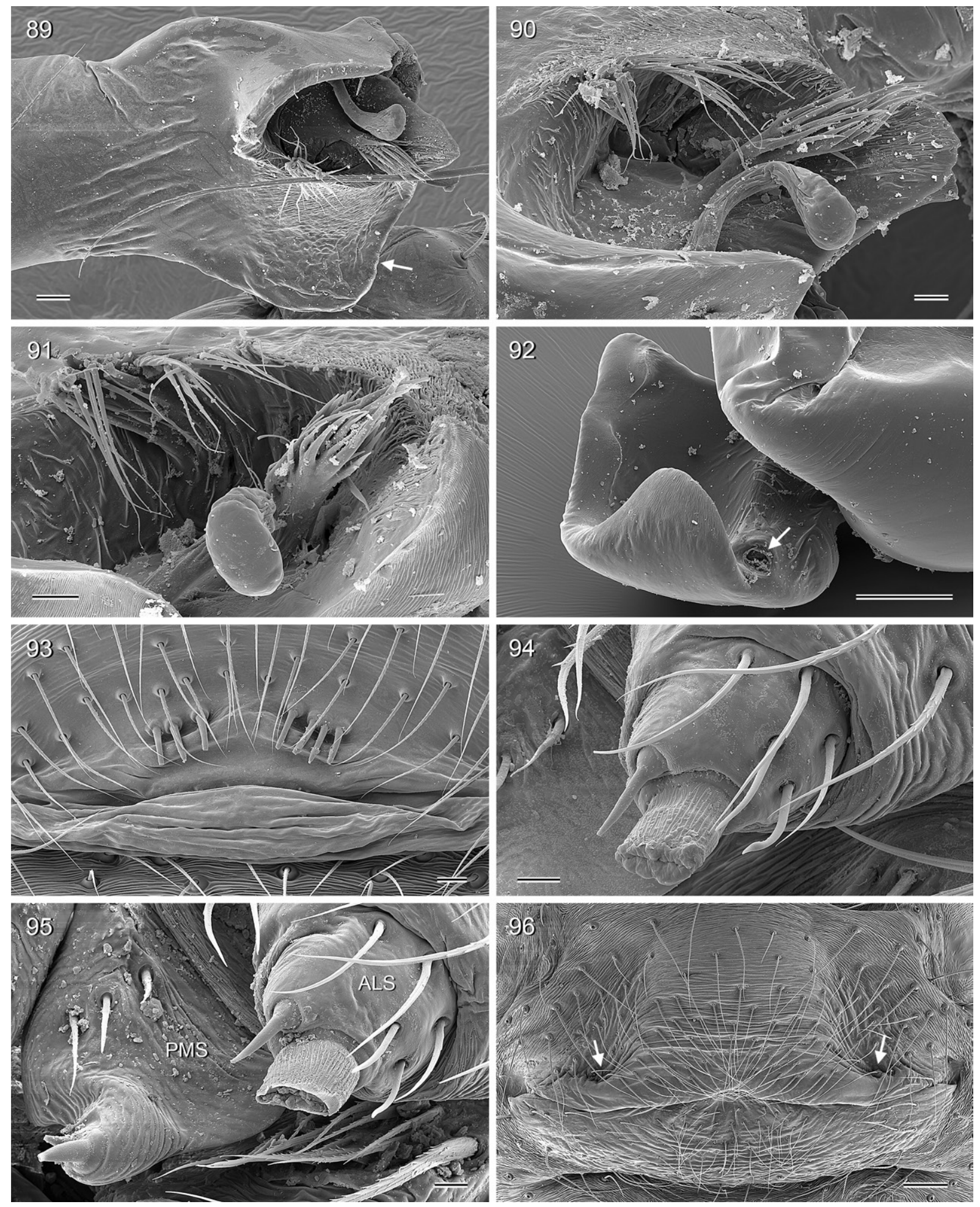

Figs 89-96. Holocnemus reini (C. Koch, 1873) comb. nov.; male and female from Morocco, near Tilamizene (ZFMK Ar 22319). 89-91. Tips of right (89) and left (90-91) procursi, retrolateral and slightly distal views; arrow: retrolateral membrane. 92. Left genital bulb, prolateral view; arrow: sperm duct opening. 93. Male gonopore. 94. Male ALS. 95. Female spinnerets. 96. Epigynum; arrows: pockets. Scale bars: $89,93=20 \mu \mathrm{m} ; 90-91,94-95=10 \mu \mathrm{m} ; 92,96=100 \mu \mathrm{m}$. 


\section{Natural history}

This species lives in a variety of habitats, ranging from shady pine forests and river valleys to very dry arid slopes. Most specimens were found in sheltered spaces among and under rocks. The large webs (diameter $\sim 30 \mathrm{~cm}$ ) were often exposed to direct sunlight, but during the day the spiders rested on the shady rock surface. On some occasions, the species was present outside of caves while the caves were occupied by a different species (e.g., SE of Zebzat: Maghreba aurouxi gen. nov.; Grotte Kef el Ghar: Micropholcus sp.). On several occasions, H. reini was found on rocks very close to undescribed representatives of Micropholcus, occupying much the same microhabitat (Paradise Valley; Imi n'Ifri; near Ksiba). Near Sidi Ben Daoud, the spiders were extremely abundant in a very dense field of cacti. The domed webs transformed to distinct funnels that led into the barely accessible space among the cacti. High abundances were also reported by Hajer \& Řeháková (2003), who observed "communities of up to several hundred individuals" (vouchers of their "Crossopriza sp." were reexamined; see above).

Hajer \& Řeháková (2003) also described web construction and the production of silk puffs, which were in principle identical to those of $H$. pluchei (see above). Unlike, H. pluchei, however, mated females built a more open spotted dome (rather than a sphere) within the main web and left this dome for attacking prey. Also, females tolerated the presence of males inside the dome and copulated repeatedly while still holding the egg sac. After the second molt of the spiderlings, females destroyed the dome and the spiderlings dispersed (Hajer \& Řeháková 2003).

\section{Distribution}

The species is widely distributed in northwestern Africa, ranging from Morocco to Tunisia (Fig. 3). The MNHN has a female with a dubious label saying "Espagne", while the original label is no longer readable.

Holocnemus caudatus (Dufour, 1820)

Figs 3, 97-128

Pholcus caudatus Dufour, 1820: 208, pl. 76 fig. 2a-b.

Pholcus caudatus - Walckenaer 1837: 654 (description copied from Dufour 1820). — Simon 1866: 123, pl. 2, figs 12-13.

Holocnemus caudatus - Simon 1873: 49. - Franganillo 1925: 35; 1926c: 71. — Wiehle 1933: 244, fig. 2b. - Brignoli 1971a: 84; 1971c: 256 ("Holocnemus prope caudatus"). - Melic 1994: 15. — Ribera et al. 2003: 8. — Benhadi-Marín et al. 2013: 75, fig. 2a(1-3). — Breitling 2020: 352, fig. 26a-d.

Dubious records (see Distribution below)

Holocnemus caudatus - Barrientos \& Ferrández 1982: 82. — Ferrández et al. 2006: 76.

\section{Misidentifications}

Barrientos et al. 2019: 12 (see H. reini).

\section{Remark}

Holocnemus acuminatus Franganillo, 1925 from Spain, Málaga, is currently considered a nomen dubium (World Spider Catalog 2021). It is probably a synonym of H. caudatus but could also be a senior synonym of $H$. hispanicus Wiehle, 1933. The World Spider Catalog (2021) considers Franganillo's (1925) publication in the Boletín de la Sociedad Entomológica de España as the original publication for this name. However, in that paper, Franganillo writes "Holocnemus acuminatus Frang.", i.e., without 
adding "sp. nov." as he did in other occasions, suggesting that the species had been described before. In fact, in the introduction he cites a series of publications from which the listed records are taken. I suspect that $H$. acuminatus was originally intended to be described in Franganillo's contribution to the Proceedings volume of the Tercer Congreso Científico Panamericano (Lima, 22 Dec. 1924-5 Jan. 1925), under the title "Arácnidos de Andalucía". However, that proceedings volume was never published. Thus, Holocnemus acuminatus Franganillo, 1925 is here considered a nomen nudum.

\section{Diagnosis}

Easily distinguished from most known Smeringopinae (except $H$. reini) by distinct indentations on sternum (Fig. 112; in males and females; deepest pair between coxae 4), by shape of procursus (cf. Figs 62-63, 117; distinctive retrolateral membrane), by shape of genital bulb (cf. Figs 66-68; distal bulbal sclerite triangular in prolateral view, with one large and two small rounded processes); from known congeners and representatives of Crossopriza also by 2-3 modified hairs on each male cheliceral apophysis (rather than one; Fig. 115); from H. reini only by slightly smaller distances between male cheliceral apophyses (50-60\% of cheliceral maximum width) and between female epigynal pockets $(<0.4 \mathrm{~mm})$.

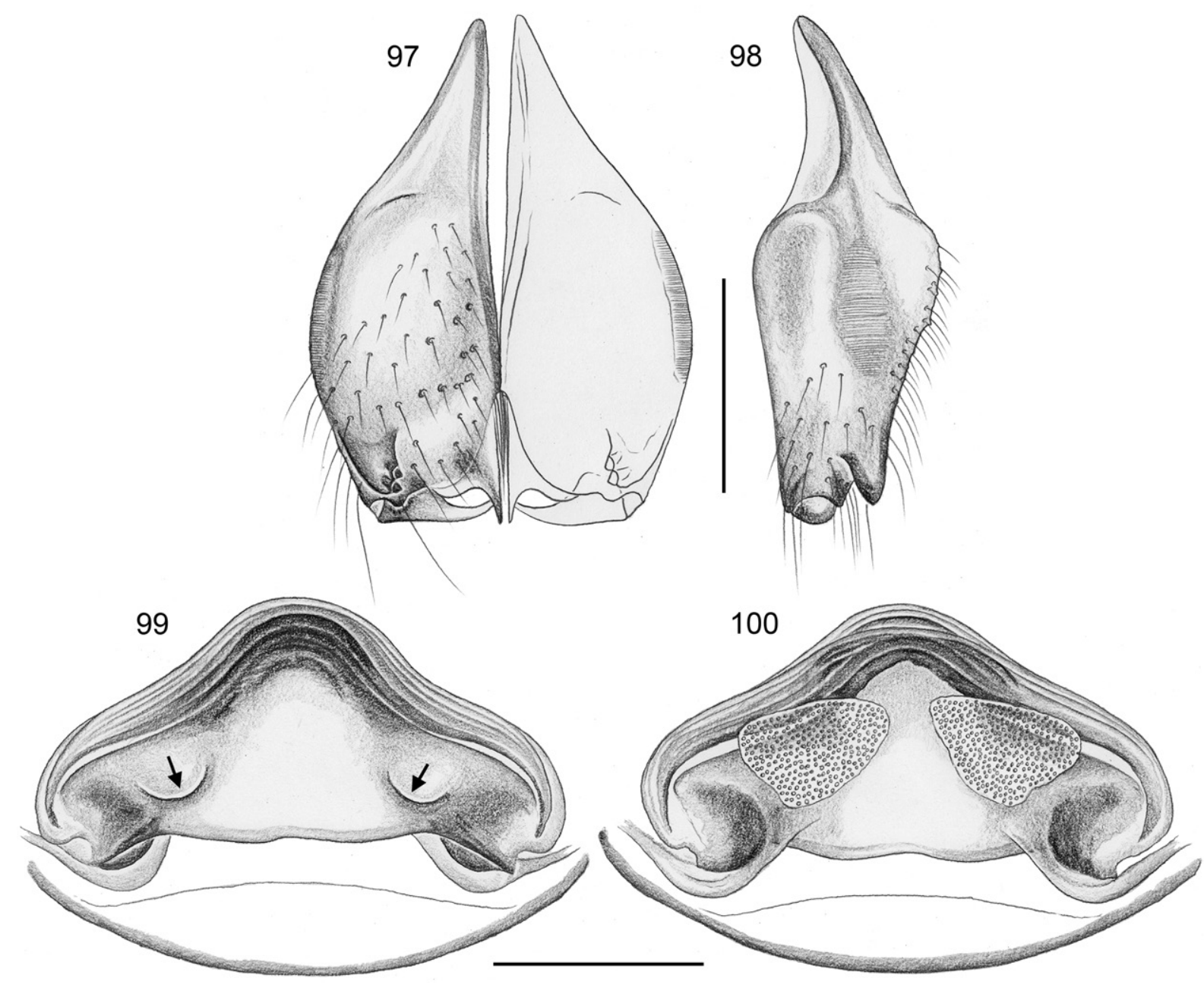

Figs 97-100. Holocnemus caudatus (Dufour, 1820); male and female from Spain, Valencia, Montroy (MHNG). 97-98. Male chelicerae, frontal and lateral views. 99-100. Cleared female genitalia, ventral and dorsal views; arrows: pockets. Scale bars $=0.3 \mathrm{~mm}$. 


\section{Type material}

\section{Syntypes}

SPAIN - Unspecified number; Valencia, Mogente/Moixent ("Moxente"); $38.88^{\circ} \mathrm{N}, 0.76^{\circ} \mathrm{W}$; date unknown; L. Dufour leg.; probably lost.

\section{Material examined}

SPAIN - Valencian Community • 1 đ; Alicante, Cova del Rull near Val de Ebo; $38.812^{\circ} \mathrm{N}, 0.177^{\circ} \mathrm{W}$;

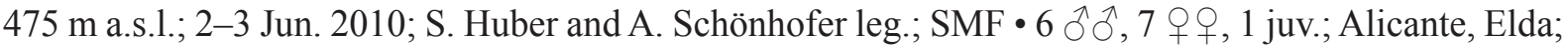
$38.48^{\circ} \mathrm{N}, 0.79^{\circ} \mathrm{W} ; 19$ Jun. 1971; A. Senglet leg.; MHNG • 3 ○े $^{\lambda}, 3$ 우; Valencia, Ayora; $39.06^{\circ} \mathrm{N}$,

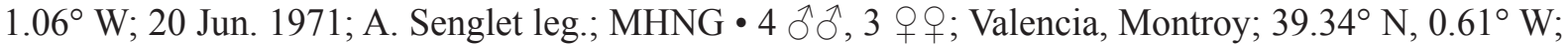

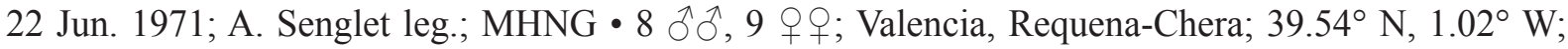
23 Jun. 1971; A. Senglet leg.; MHNG • 1 juv.; Castellón, Cabanes, "Avenc d'en Soria"; $40.15^{\circ}$ N,

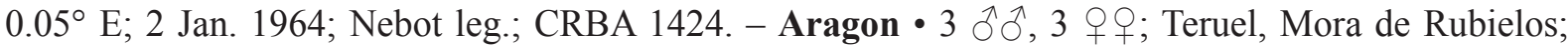
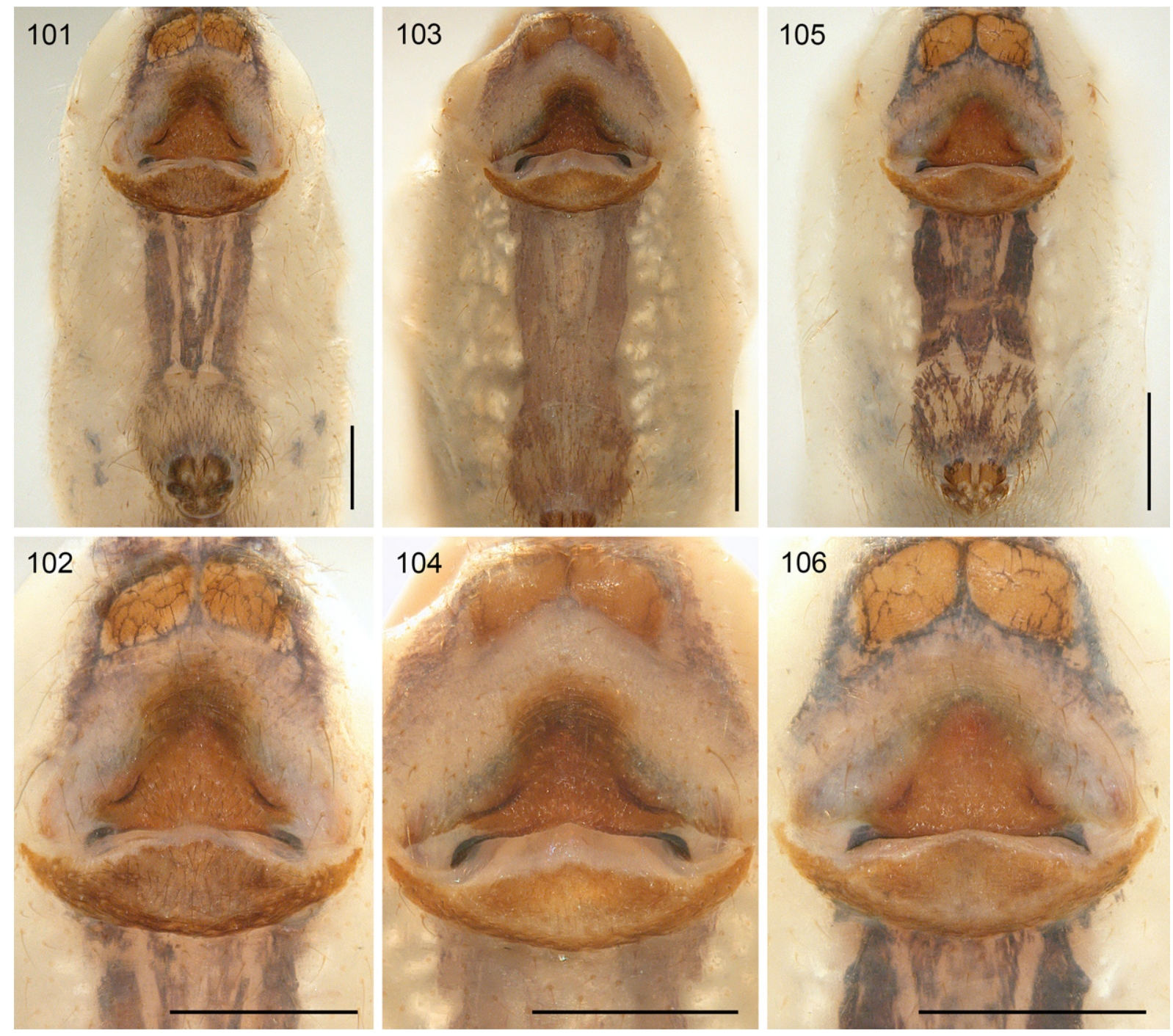

Figs 101-106. Holocnemus caudatus (Dufour, 1820); female abdomens and epigyna, ventral views. 101-102. Spain, Albacete, S of Tarazona de La Mancha (CRB). 103-104. Spain, Granada, Trevélez (MHNG). 105-106. Spain, Granada, Motril (MMUE Murphy \#14441). Scale bars $=0.5 \mathrm{~mm}$. 


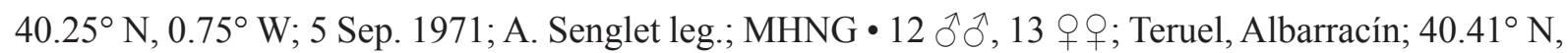
$1.44^{\circ}$ W; 3 Sep. 1971; A. Senglet leg.; MHNG • 1 + , 1 juv.; Teruel, Aguaviva, along Río Bergantes;

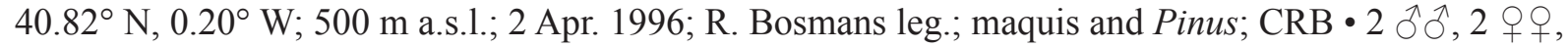
1 juv.; Teruel (the label says "Lerida" but Castelnou is not in Lérida), Castelnou; $41.23^{\circ} \mathrm{N}, 0.36^{\circ} \mathrm{W}$; 8 Jun. 1955; A. Comellini leg.; MHNG. - Castilla-La Mancha - $3 \widehat{\jmath}, 6$ q $q$; Cuenca, Sta Cruz de Moya; 39.955 N, $1.255^{\circ}$ W; 24 Jun. 1971; A. Senglet leg.; MHNG • 3 o 9 , 3 juvs; Albacete, S of

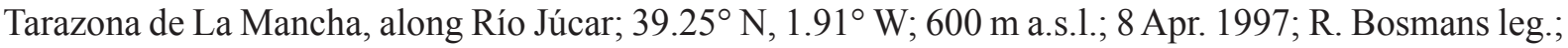
degraded Quercus ilex forest; CRB 11 ○े, 3 우, 3 juvs; Albacete, La Gineta; $39.11^{\circ}$ N, $2.00^{\circ} \mathrm{W}$; 28 Jun.

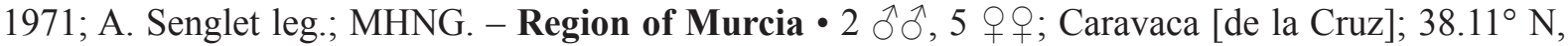

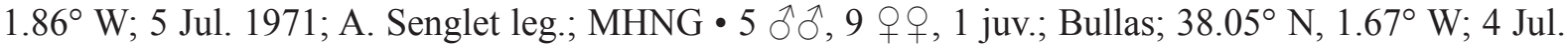
1971; A. Senglet leg.; MHNG $\bullet 2 \overbrace{}^{\Uparrow} \partial^{\lambda}, 2$ 우 (partly used for SEM); Mazarrón; $37.60^{\circ} \mathrm{N}, 1.32^{\circ} \mathrm{W}$; 1 May 2001; J. Van Keer leg.; CJVK • 1 q; Mazarrón, Cueva del Agua; 37.576 N, $1.219^{\circ}$ W; 18 Feb.

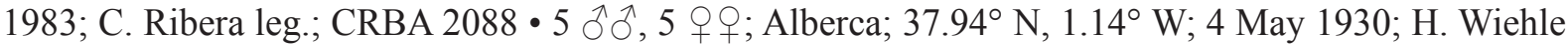
leg.; SMF 11442, 11443, 19430. - Catalonia • 2 우; Barcelona, Parque Natural del Garraf; $41.28^{\circ} \mathrm{N}$, $1.84^{\circ}$ E; 6 Sep. 2006; R. Bosmans leg.; CRB • 1 ô; $^{\top}$ Lleida, Bor, Cova de la Fou de Bor; $42.339^{\circ} \mathrm{N}$, $1.801^{\circ}$ E; 28 Sep. 1965; C. Ribera leg.; CRBA 1409. - Andalusia • 1 đ, 3 q $q$; Granada, Puebla de
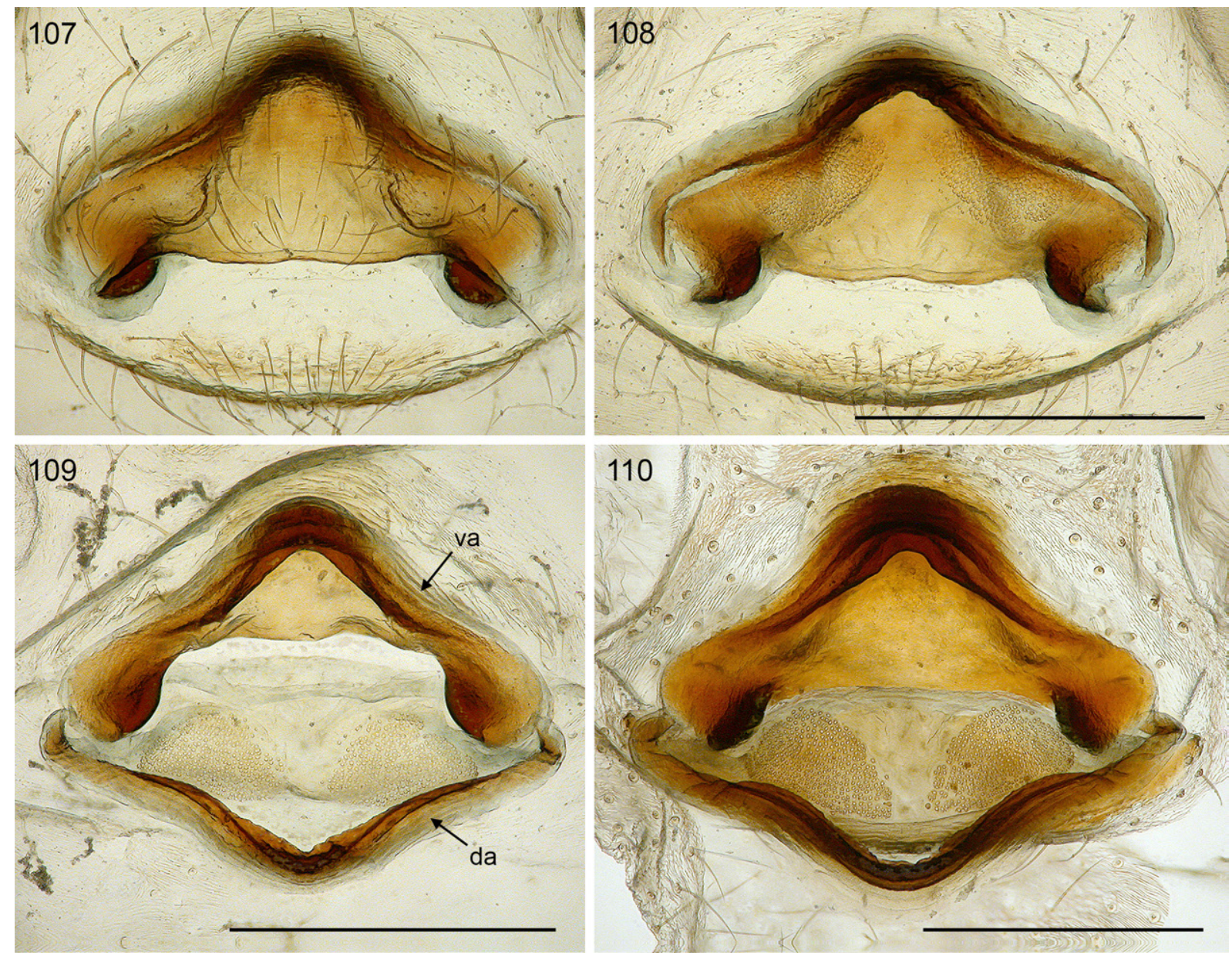

Figs 107-110. Holocnemus caudatus (Dufour, 1820). 107-109. Female from Spain, Valencia, Montroy (MHNG); internal genitalia, ventral view, regular dorsal view, and dorsal view with dorsal arc tilted backwards. 110. Female from Spain, Albacete, S of Tarazona de La Mancha (CRB); internal genitalia, dorsal view with dorsal arc tilted backwards. Abbreviations: da $=$ dorsal arc; va $=$ ventral arc. Scale bars $=0.5 \mathrm{~mm}$. 
Don Fadrique; $37.96^{\circ}$ N, 2.44 W; 1000-1200 m a.s.1.; 6 Jul. 1971; A. Senglet leg.; MHNG • 1 万,

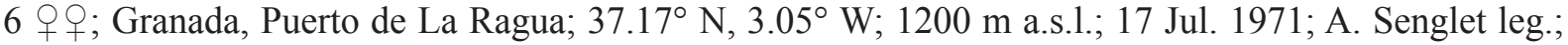

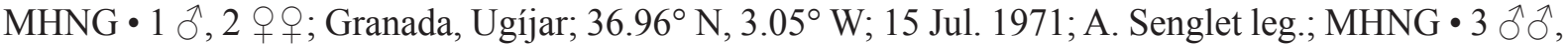

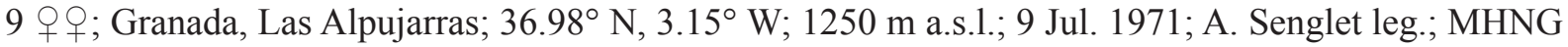

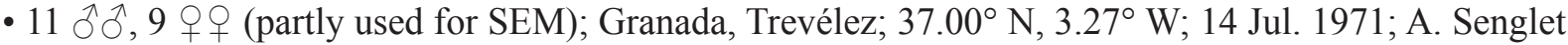
leg.; MHNG • 3 §ิô, 2 우; Granada, Sierra Nevada, Alto del Chorrillo; $37.01^{\circ} \mathrm{N}, 3.31^{\circ} \mathrm{W} ; 2100$ 2800 m a.s.l.; 11 Jul. 1971; A. Senglet leg.; MHNG • 1 q; Granada, La Mamola; 36.75 N, $3.28^{\circ}$ W; 50 m a.s.l.; 13 Aug. 1991; R. Bosmans leg.; CRB - 1 ơ; Granada (province or city?); Aug. 1924;

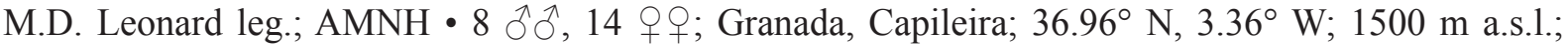
10 Jul. 1971; A. Senglet leg.; MHNG • 9 तै ô, 6 우; Granada, Pampaneira/Órgiva; $36.92^{\circ}$ N, $3.38^{\circ}$ W; 1200 m a.s.l.; 10 Jul. 1971; A. Senglet leg.; MHNG - 2 우; Granada, Motril; 36.75 ${ }^{\circ}$ N, $3.52^{\circ}$ W; 50 m a.s.l.; 3 Apr. 1987; J. Murphy leg.; garrigue, stones; MMUE Murphy \#14441 • 1 o, 1 क; Granada, Sierra Nevada, Albergue Universitario; $37.094^{\circ} \mathrm{N}, 3.387^{\circ} \mathrm{W} ; 2500 \mathrm{~m}$ a.s.l.; date and collector not

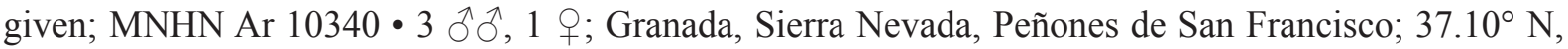
$3.39^{\circ} \mathrm{W}$; date not given; H. Janetschek leg.; MNHN Ar 10346 • 3 우, 1 juv.; Almería, Cabo de Gata; $36.78^{\circ} \mathrm{N}, 2.24^{\circ} \mathrm{W} ; 50 \mathrm{~m}$ a.s.1.; 5 Apr. 1996; R. Bosmans leg.; stones in dunes; CRB ・ 1 ○, 1 ㅇ (in pure ethanol); same locality as for preceding; $36.7389^{\circ} \mathrm{N}, 2.2014^{\circ} \mathrm{W}$; Jul.-Aug. 2021; J. Moya-Laraño leg.; subdesert grassland; ZFMK G154, G155 • 1 đ; Almería, Sierra de Gádor, Sima de los Locos; $36.856^{\circ}$ N,

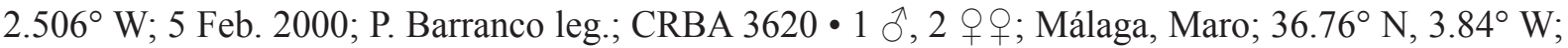
100 m a.s.l.; 3 Apr. 1987; J. Murphy leg.; stones, litter; MMUE Murphy \#14318 - 2 đ̊̊̄; Málaga, Frigiliana; $36.79^{\circ} \mathrm{N}$, $3.89^{\circ} \mathrm{W} ; 20 \mathrm{~m}$ a.s.1.; 6 Apr. 1987; J. Murphy leg.; stones, litter; MMUE Murphy

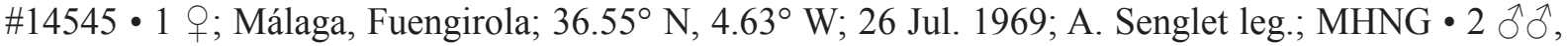
4 우, 2 juvs; Málaga, Marbella; $36.52^{\circ} \mathrm{N}, 4.88^{\circ} \mathrm{W}$; 12 Aug. 1991; R. Bosmans leg.; stones in Pinus forest; CRB • 1 q; Málaga, San Pedro de Alcántara; $36.49^{\circ}$ N, $4.99^{\circ}$ W; 23 Jul. 1969; A. Senglet leg.;

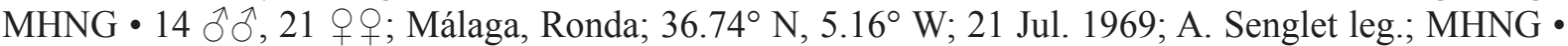
1 Јै, 2 우우 same locality as for preceding; 20-23 Jul. 1969; A. Senglet leg.; MMUE Murphy \#12790 • 1 ; same locality as for preceding; 750 m a.s.l.; 12 Aug. 1991; R. Bosmans leg.; stones; CRB.

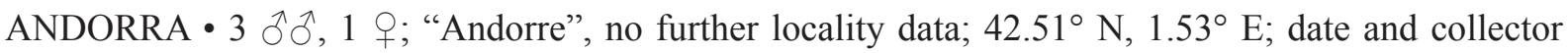
unknown; MNHN Ar 10328.

\section{Redescription}

Male (Granada, Trevélez, MHNG)

Measurements. Total length 5.5, carapace width 1.6. Distance PME-PME $110 \mu \mathrm{m}$; diameter PME $110 \times$ $130 \mu \mathrm{m}$; distance PME-ALE $50 \mu \mathrm{m}$; diameter AME $70 \mu \mathrm{m}$; distance AME-AME $25 \mu \mathrm{m}$. Leg 1: 33.4 $(9.7+0.8+8.8+12.1+2.0)$, tibia $2: 6.3$, tibia $3: 4.7$, tibia $4: 5.7$; tibia $1 \mathrm{~L} / \mathrm{d}: 49$; femora $1-4$ diameters: $0.32,0.24,0.21,0.22$.

Color (in ethanol). Carapace ochre-yellow; ocular area, carapace pit and posterior median area light brown; sternum brown, with dark brown radial marks; legs ochre-yellow, without darker rings, with black lines on femora and (few) on tibiae; abdomen ochre-gray, with some dark marks dorsally and laterally; ventrally with distinct black median band, partly disrupted, with three parallel longitudinal marks behind gonopore.

Body. Habitus very similar to $H$. reini (cf. Fig. 7). Ocular area slightly raised. Deep thoracic pit and pair of shallow furrows diverging from pit toward posterior margin. Clypeus unmodified but rim more sclerotized than in female. Sternum wider than long (1.05/0.85), with distinct indentations between consecutive leg coxae and between coxae 4 . Abdomen strongly elongated beyond spinnerets, dorsoposteriorly pointed. Gonopore with six epiandrous spigots (Fig. 118); ALS with one widened spigot and one pointed spigot, PMS with two pointed spigots (Fig. 125). 

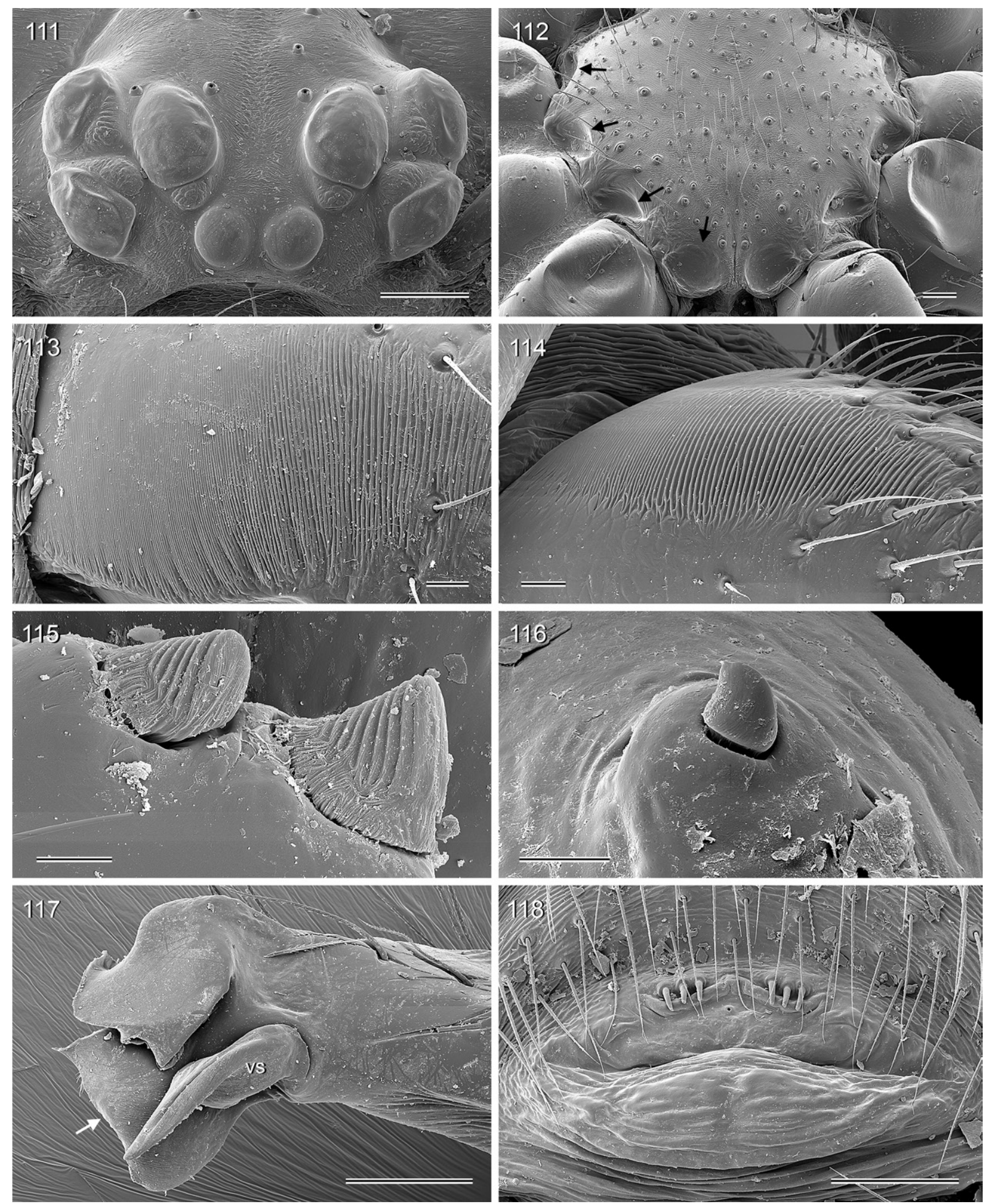

Figs 111-118. Holocnemus caudatus (Dufour, 1820); male and female from Spain, Granada, Trevélez (MHNG). 111. Female ocular area. 112. Male sternum; arrows: indentations. 113-114. Male and female cheliceral stridulatory files. 115. Modified hairs on male cheliceral apophysis. 116. Stridulatory pick on male palpal femur. 117. Right procursus tip, prolateral view; arrow: retrolateral membrane. 118. Male gonopore. Abbreviation: vs $=$ ventral sclerite. Scale bars: $111-112,117-118=100 \mu \mathrm{m} ; 113-114=$ $20 \mu \mathrm{m} ; 115-116=10 \mu \mathrm{m}$. 
Chelicerae. As in Figs 97-98, with pair of low proximal frontal humps and pair of frontal lateral apophyses, each with two large modified cone-shaped hairs (Fig. 115); distance between tips of modified hairs: $320 \mu \mathrm{m}$; several 'regular' frontal hairs with small but distinct processes at basis; lateral stridulatory ridges very fine (Fig. 113; distances between ridges proximally $\sim 1.5 \mu \mathrm{m}$, distally $\sim 4 \mu \mathrm{m}$ ), barely visible in dissecting microscope.

PALPS. In general apparently indistinguishable from H. reini (cf. Figs 59-61); coxa with rounded retrolateral hump; trochanter barely modified; femur slightly curved towards dorsal, distally widened, with rounded ventral protrusion, proximally with prolateral stridulatory pick (Fig. 116), with barely visible retrolateral transversal line, without retrolateral proximal process; femur-patella joints shifted toward prolateral side; tibia very large compared to femur, tibia-tarsus joints shifted toward retrolateral side; tarsus without macrotrichia; tarsal organ capsulate (Fig. 121); procursus (cf. Figs 62-63) straight, few hairs slightly curved upwards; proximally on prolateral side with strong hump free of hairs, procursus tip with distinctive flat membranous element retrolaterally (arrow in Fig. 117) covering proximal part of ventral sclerite in retrolateral view, with dorsal transversal flap; genital bulb (cf. Figs 66-68) with simple basal sclerite connected to distal (main) sclerite, sperm duct opening in membranous area prolaterally at basis of distal sclerite; distal sclerite with one large apophysis (fold) and two smaller apophyses on prolateral side; with indistinct retrolateral parallel ridges.

LEGs. Femur 1 with single row of $\sim 23$ ventral spines; without curved hairs; few vertical hairs (Figs 119120); retrolateral trichobothrium of tibia 1 at $4 \%$; prolateral trichobothrium absent on tibia 1, present on other leg tibiae; tarsal pseudosegments indistinct except 2-3 distally; tarsal organs capsulate, with round or slightly irregular rim (Figs 122-123).

\section{Male (variation)}

Tibia 1 in 82 males: 6.8-9.9 (mean 8.5). Chelicerae maximum width $(\mathrm{N}=43)$ : $0.46-0.62$ (mean 0.55); procursus length $(\mathrm{N}=46)$ : $0.86-1.00$ (mean 0.92 ). Coloration of fresh specimens very similar to H. reini (cf. Figs 7-8), with distinct pattern on abdomen. Ventral dark band on abdomen variably distinct, section behind gonopore with $2-4$ longitudinal bands.

\section{Female}

In general similar to male (cf. Fig. 8) but without spines on legs. Without stridulatory apparatus between carapace and abdomen; with fine cheliceral stridulatory ridges as in male (Fig. 114; distances between ridges $3 \mu \mathrm{m}$ ). Tibia 1 in 103 females: 5.1-8.8 (mean 6.9). Epigynum as in Figs 101-106 and 127-128, main epigynal plate triangular to semicircular, weakly protruding; only posteriorly laterally strongly sclerotized; with pair of variably distinct pockets (distance between pockets $0.32-0.35$ ); internal sclerotized arc and median round structure variably visible in uncleared specimens; posterior epigynal plate large but simple; pair of low but distinct elevations in front of epigynum. Internal genitalia (Figs 99-100, 107-110) with triangular pore plates converging anteriorly, dorsal arc simple and slender, ventral arc laterally strongly widened, medially strong, with simple median pouch.

\section{Natural history}

The biology of this common Spanish spider has apparently been little studied. According to Wiehle (1933) it occupies similar habitats as H. hispanicus (i.e., wall niches, rock cavities, etc.). The domed web was described as having a surprisingly regular mesh; the size of the dome is similar to H. hispanicus (i.e., approximately $35-40 \mathrm{~cm}$ ), but the silk puffs are not as close together as in $H$. hispanicus. Females lay up to 50 eggs per egg-sac and produce up to three egg-sacs per season (Wiehle 1933). 

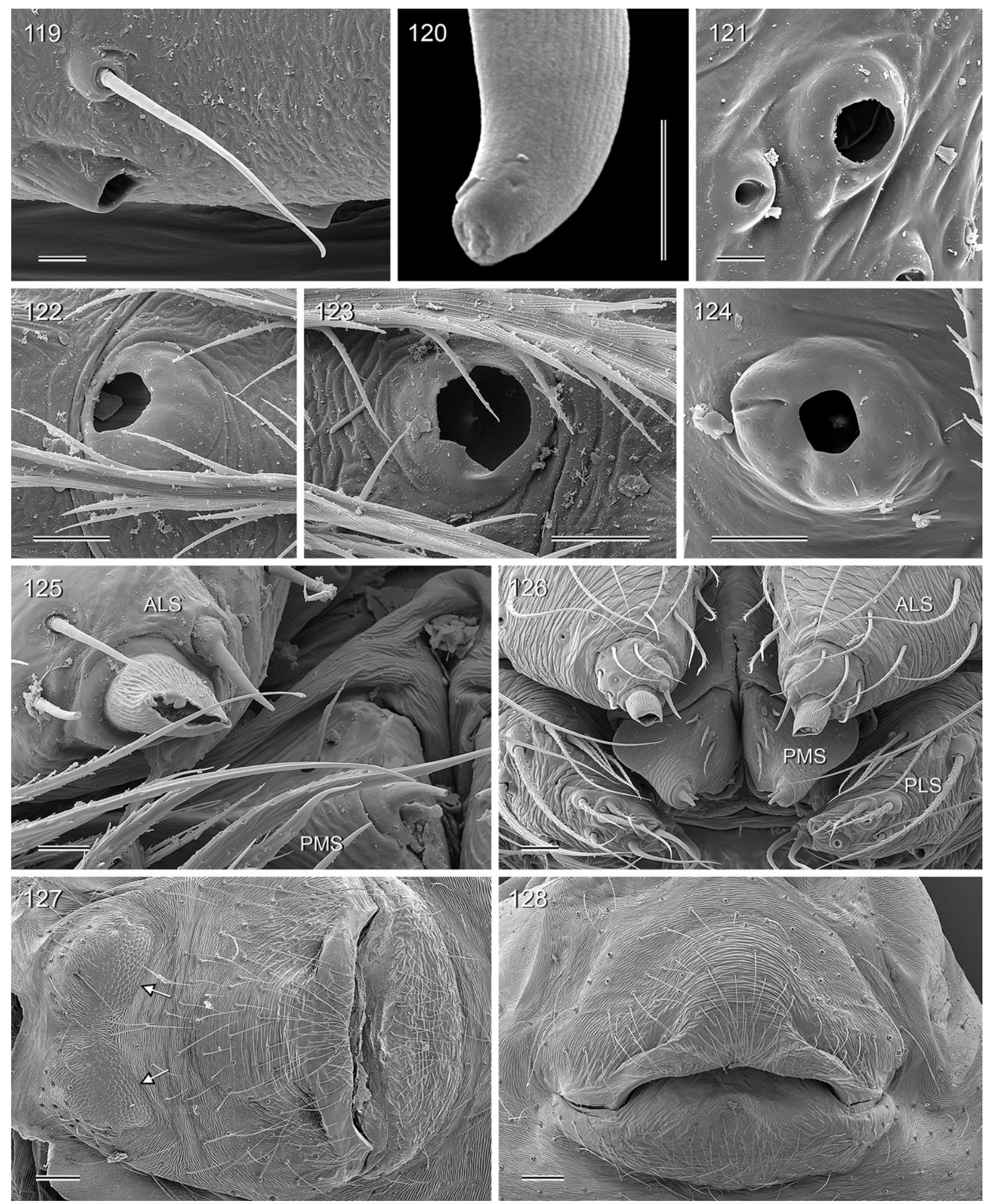

Figs 119-128. Holocnemus caudatus (Dufour, 1820); male and female from Spain, Granada, Trevélez (MHNG). 119. 'Short vertical hair' on male metatarsus 4. 120. Tip of hair from preceding figure. 121. Male palpal tarsal organ. 122-123. Tarsal organs on male tarsi 1 and 4. 124. Female palpal tarsal organ. 125-126. Male and female spinnerets. 127-128. Epigyna and anterior epigynal humps (arrows). Scale bars: $119,121-125=10 \mu \mathrm{m} ; 120=1 \mu \mathrm{m} ; 126=20 \mu \mathrm{m} ; 127-128=100 \mu \mathrm{m}$. 


\section{Distribution}

Widely distributed in southern and eastern Spain (Fig. 3), where it occurs from sea level up to $2500 \mathrm{~m}$ a.s.l. (Albergue Universitario). I have not seen any specimens from central-western Spain (Castile and León, Extremadura, and Madrid), and consider all previous records from this region (Fernández Galiano 1910 in Ferrández et al. 2006; Barrientos \& Ferrández 1982; Ferrández et al. 2006) dubious. I also follow Brignoli (1971a) in considering Simon's (1873) statement about the presence of H. caudatus in Sicily as dubious to highly improbable.

Holocnemus hispanicus Wiehle, 1933

Figs 4, 129-162

Holocnemus hispanicus Wiehle, 1933: 241, figs 1, 2c, 4-6, 7a-b, 8.

Holocnemus hispanicus - Senglet 1972: 315. — Timm 1976: 70, figs 3-4. - Huber 2000: figs 5, 97. Morano 2001: 68. - Barriga et al. 2006: 135. — Cardoso et al. 2009: 54. — Benhadi-Marín et al. 2013: 75, fig. 2 (B1-3). — Ribera 2013: 59. — Breitling 2020: 352, fig. 26e-f.

\section{Remark}

Wiehle (1933) did not mention type specimens in the original description, and none of the presumed syntypes listed below is labeled as type. However, most or all of these specimens originate from the type locality, and most were collected in May 1930, which is the date mentioned in the original description. Wiehle kept and reared specimens for several generations, and some specimens listed under non-type material may actually be descendants of the specimens he collected in May 1930. Some were preserved between June and November 1930, suggesting that these may partly be specimens he collected in May 1930, partly the first reared generation that became adult in November and December 1930 (Wiehle 1933); these are listed under types. Several SMF labels seem to be erroneous: it seems very unlikely that specimens were collected in the field on exactly the same days that others were preserved after being reared in the lab (25 Apr. 1936, 15 Jun. 1936).

\section{Diagnosis}

Distinguished from nominal congeners and from representatives of Crossopriza by shape of procursus (Figs 136-138; distal prolateral transparent process, ventral sclerite proximally wide and partly membranous), by shape of distal bulbal sclerite (Figs 139-141; trapezoidal in prolateral view, with one rounded process on prolateral side), and by epigynum with anteriorly converging furrows and pair of internal sclerites usually visible in uncleared specimens (Figs 132-134); from the two only syntopic Smeringopinae (H. pluchei, H. caudatus) also by combination of: unmodified sternum in males and females, only one modified hair on each male cheliceral apophysis (Figs 142, 150), and slender female palp.

Type material (see Remark above)

\section{Syntypes}

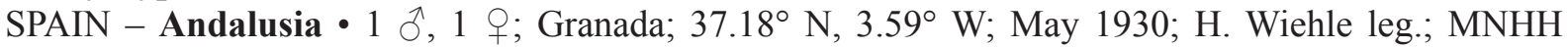
Ar 10335 • 1 क; same collection data as for preceding; preserved on 25 Jun. 1930; SMF $19314 \bullet 1$; presumably same collection data as for preceding; preserved on 4 Nov. 1930; SMF $19313 \cdot 1$, mislabeled as "Crossopriza semicaudata"; same collection data as for preceding; preserved on 5 Nov. 1930; SMF 19311 • 1 q; same locality as for preceding; 1928; H. Wiehle leg.; SMF $19306 \bullet 1$ ¿ ; same collection data as for preceding; preserved on 19 Aug. 1930; SMF 19312. 


\section{Other material examined}

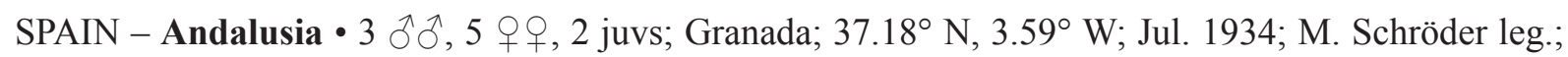
SMF 11472-73, 19309-19310, 19315• 1 O; same locality as for preceding; 19 Jan. 1935; collector unknown; SMF 11476 - 1 ô; same locality as for preceding; reared and preserved on 25 Apr. 1936; collector unknown; SMF $19307 \cdot 1$ o ; same locality as for preceding; reared and preserved on 1 Apr. 1936; collector unknown; SMF 19305 • 1 q; Granada; 16 Aug. 1924; M.D. Leonard leg.; AMNH • 1 Oे, 2 우; Granada, Guadix (Paulenca de Guadix); 37.293 N, 3.168 ${ }^{\circ}$ W; 18 Jul. 1971; A. Senglet leg.;

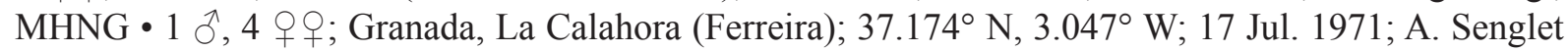

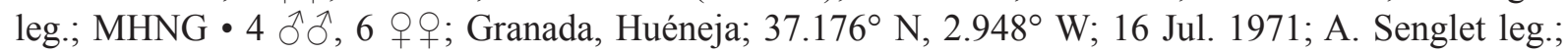

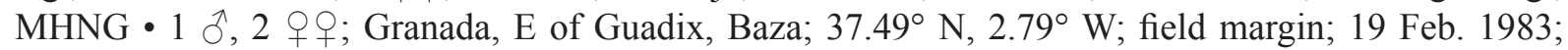

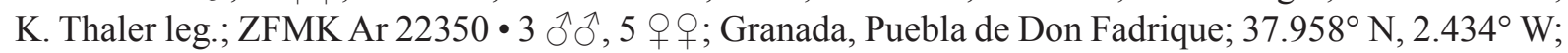
1000-1200 m a.s.l.; 6 Jul. 1971; A. Senglet leg.; MHNG • 1 क; Granada, "Sierra Havana" (locality not identified); 1000 m a.s.l.; 29 Sep. 1953; collector unknown; SMF 8802 • 1 क; Granada, Sima Rica de Alhama; $37.055^{\circ} \mathrm{N}, 4.105^{\circ} \mathrm{W} ; 1190 \mathrm{~m}$ a.s.1.; 10 Dec. 1983; C. Ribera leg.; CRBA $2062 \bullet 1$ ơ; Granada, Piñar, Cueva de Pagarrecio; $37.442^{\circ}$ N, 3.416 W; 1035 m a.s.1.; 25 May 2013; “GEG” leg.; CRBA 5132 - 1 + ; Málaga, between Periana and Ventas Zafarrayas; $36.944^{\circ}$ N, $4.131^{\circ}$ W; 30 Oct. 1983; A. RussellSmith; under stones in rocky fallow; ZFMK Ar 23862 • 1 §, 2 juvs; Málaga, $10 \mathrm{~km} \mathrm{~N}$ of Málaga, Río

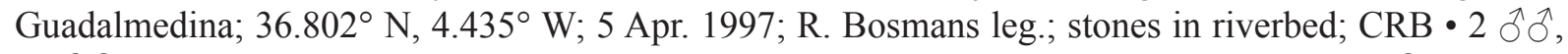
15 우; Málaga, Antequera; 37.02 N, 4.56 W; 1-2 Aug. 1969; A. Senglet leg.; MHNG • 1 O; Málaga, Pizarra, along Río Guadalhorce; $36.77^{\circ} \mathrm{N}, 4.71^{\circ} \mathrm{W} ; 17$ Dec. 1997; R. Bosmans leg.; grassland; CRB •

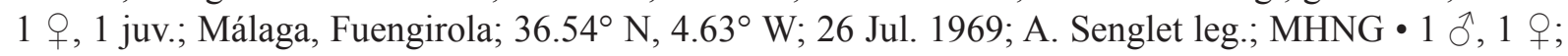

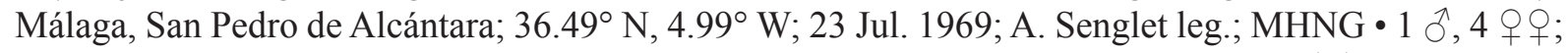
Málaga, Ronda; $36.74^{\circ} \mathrm{N}, 5.16^{\circ} \mathrm{W}$; 20-23 Jul. 1969; A. Senglet leg.; MHNG • 3 đ̊̊, 4 우우; same collection data as for preceding; 21 Jun. 1969; MHNG • 1 क; Málaga, Ronda; $36.74^{\circ} \mathrm{N}, 5.16^{\circ} \mathrm{W}$;

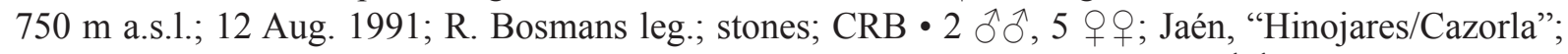

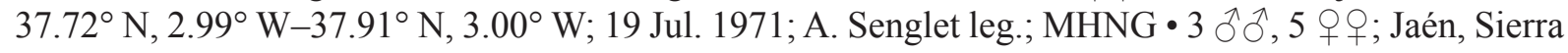
de Cazorla, El Sagreo; 37.946 ${ }^{\circ}$, $2.945^{\circ}$ W; 22 Jul. 1971; A. Senglet leg.; MHNG • 1 क; Jaén, Cazorla, Ruta Río Borosa; 37.950 N, $2.947^{\circ}$ W; 9 May 1919; A. Pérez leg.; ZFMK Ar 22351 • 1 क; Jaén, Santo Tomé, "Cueva GEV 2"; $38.031^{\circ} \mathrm{N}, 2.992^{\circ} \mathrm{W}$; 31 Dec. 2006; collector unknown; CRBA 1636 • 1 ○,,

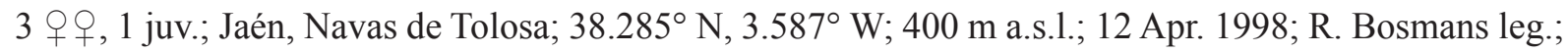
stones in planted Pinus forest; CRB - 1 ð̄; Jaén, Santiago de la Espada-Pontones, Cueva del Jabalí; $38.118^{\circ} \mathrm{N}, 2.647^{\circ} \mathrm{W} ; 1530 \mathrm{~m}$ a.s.1.; 14 Apr. 2002; "GEV” leg.; CRBA $3867 \bullet 1$ đ, 2 우, 1 juv.; Jaén,

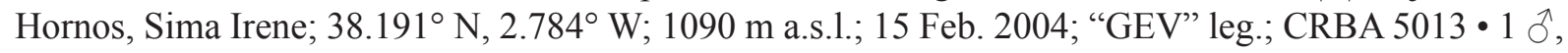

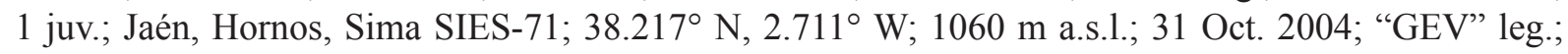
CRBA 5028 • 2 우, 2 juvs; Córdoba, Villaviciosa; $38.076^{\circ}$ N, 5.014 W; 29 Jun. 1969; A. Senglet leg.; MHNG • 1 đ̊, 7 우; Córdoba, Peñarroya; $38.30^{\circ}$ N, 5.27 W; 30 Jun. 1969; A. Senglet leg.; MHNG •

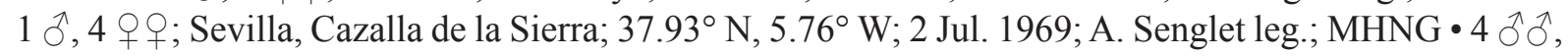

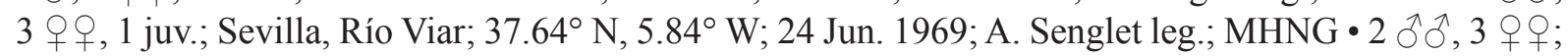

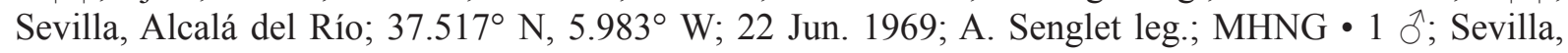
Sevilla; $37.38^{\circ} \mathrm{N}, 5.99^{\circ} \mathrm{W}$; 2 Jul. 1999; T. Lyen leg.; in building; CAS 9027125 • 1 đ , 3 o 9 , 1 juv.; Sevilla, E of Ronquillo, Embalse de Cala; $37.71^{\circ} \mathrm{N}, 6.13^{\circ} \mathrm{W}$; 6 Apr. 1994; R. Bosmans leg.; CRB • 1 \%;

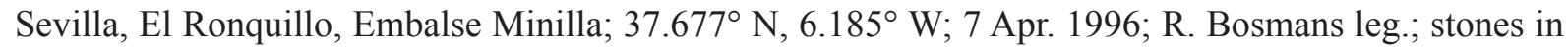
Quercus suber forest; CRB $\bullet 1$ ग , 6 q 9,4 juvs; same locality as for preceding [W of Ronquillo]; 5 Apr. 1994; R. Bosmans leg.; CRB • 1 J, 6 o 9 ; Huelva, Santa Olalla [del Cala]; $37.906^{\circ}$ N, $6.230^{\circ}$ W; 4 Jul. 1969; A. Senglet leg.; MHNG • 2 우, 1 juv.; Huelva, SW of Higuera de la Sierra; $37.83^{\circ} \mathrm{N}, 6.44^{\circ} \mathrm{W}$;

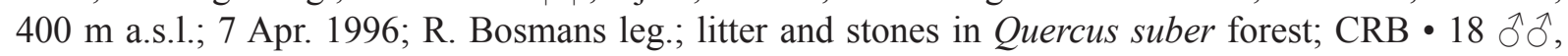
23 우 (partly used for SEM); Huelva, “Alájar/Aracena”; $37.88^{\circ} \mathrm{N}, 6.66^{\circ} \mathrm{W}-37.89^{\circ} \mathrm{N}, 6.56^{\circ} \mathrm{W} ; 7 \mathrm{Jul}$. 1969; A. Senglet leg.; MHNG $\bullet 2 \AA \widehat{\partial}, 2$ क्+; same collection data as for preceding; MMUE Murphy $\# 12791 \cdot 2 \widehat{\partial^{\lambda}}, 2$ 우, 1 juv.; Huelva, S of Valverde del Camino; $37.555^{\circ} \mathrm{N}, 6.761^{\circ} \mathrm{W} ; 270 \mathrm{~m}$ a.s.1.; 2 


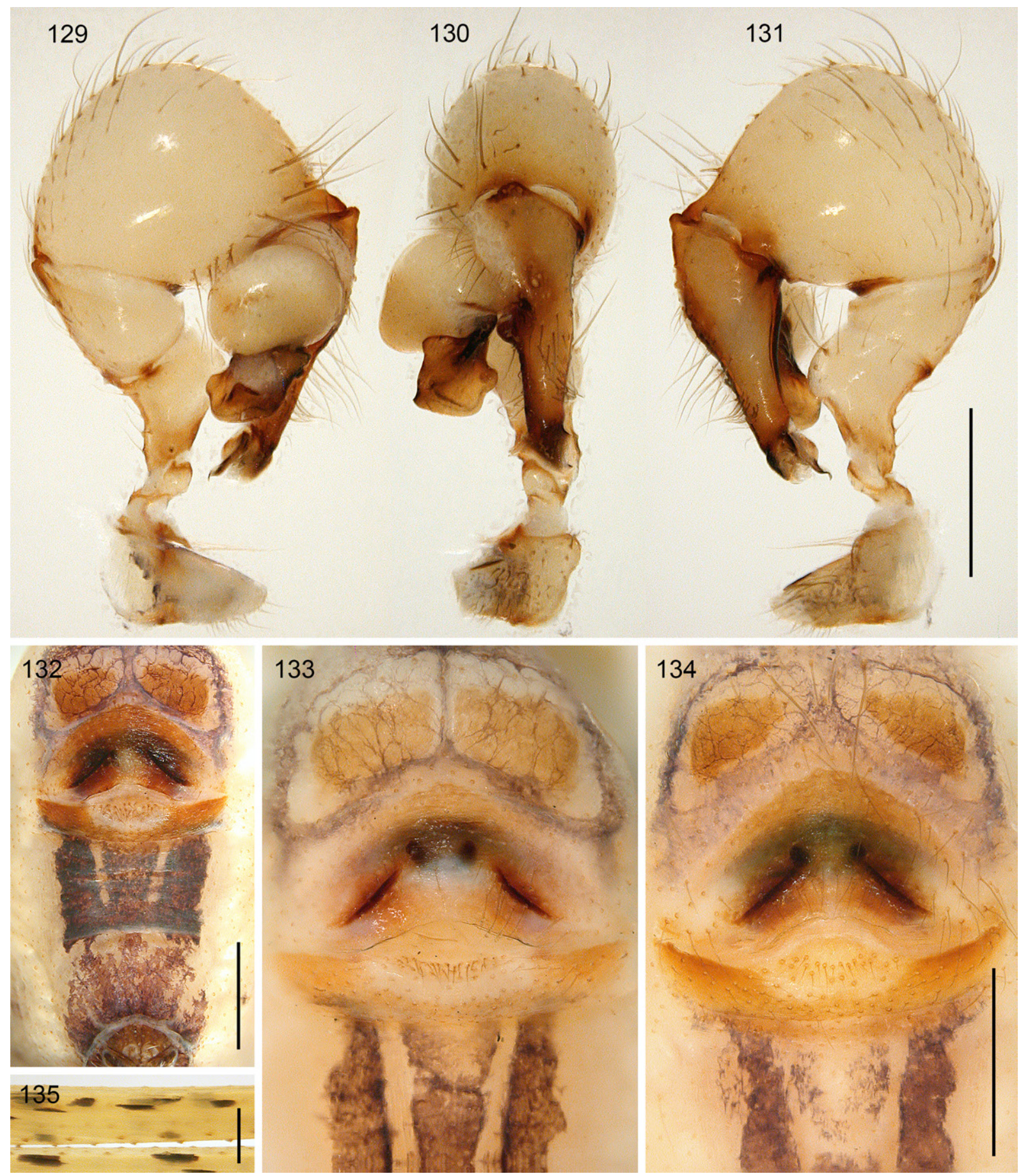

Figs 129-135. Holocnemus hispanicus Wiehle, 1933. 129-131. Male from Spain, Cáceres, Conquista de la Sierra (CRB); left palp, prolateral, dorsal, and retrolateral views. 132. Female from Spain, Málaga, Ronda (CRB); abdomen, ventral view. 133. Female from Spain, Granada, "Sierra Havana" (SMF 8802); epigynum, ventral view. 134. Female from Spain, Málaga, between Periana and Ventas Zafarrayas (ZFMK Ar 23862); epigynum, ventral view. 135. Female from Spain, Badajoz, SW of Zafra (CRB); detail of female left femora 3 and 4, retrolateral-ventral view. Scale bars: 129-134 $=0.5 \mathrm{~mm} ; 135=$ $0.2 \mathrm{~mm}$. 


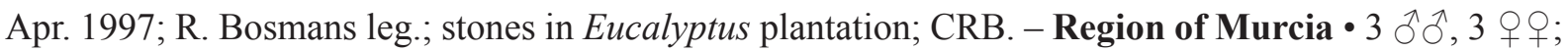

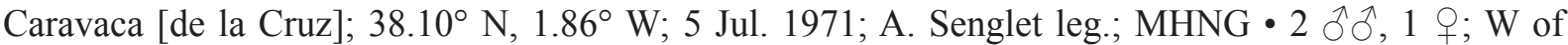
Alhama de Murcia, Sierra Espuña; $37.856^{\circ}$ N, $1.571^{\circ}$ W; 1235 m a.s.1.; 4 Jun. 2010; S. Huber and A. Schönhofer leg.; Pinus forest, open slopes; SMF • 1 q; Jumilla, Sierra de las Nieves, Cueva del Portachuelo; $38.47^{\circ} \mathrm{N}, 1.32^{\circ} \mathrm{W} ; 5$ Apr. 1983; C. Ribera leg.; CRBA 2098. - Castilla-La Mancha • 3 ổ $^{\lambda} 4$ o O ; Ciudad Real, Fuencaliente; $38.406^{\circ}$ N, $4.304^{\circ}$ W; 3 Aug. 1969; A. Senglet leg.; MHNG •

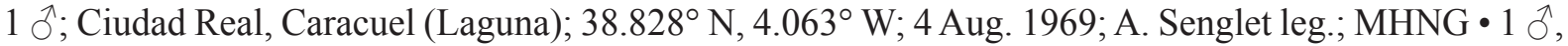

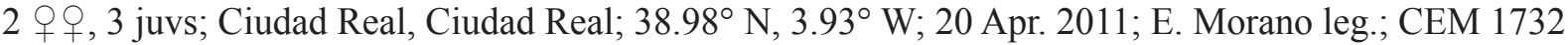

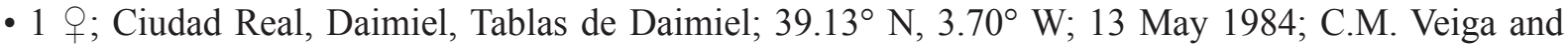

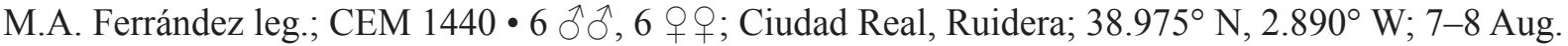
1969; A. Senglet leg.; MHNG • 1 क ; Ciudad Real, Campo de Criptana; $39.40^{\circ}$ N, 3.12 ${ }^{\circ}$ W; 650 m a.s.l.; 13 Apr. 1998; R. Bosmans leg.; stones in open Quercus suber forest; CRB • 4 우; Albacete, Almansa;

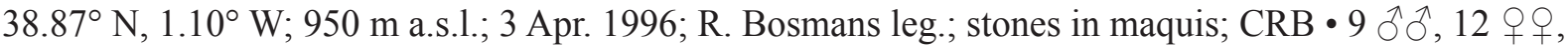
9 juvs; Toledo, Escalona del Alberche; $40.167^{\circ}$ N, 4.400 W; 14 Jun. 1969; A. Senglet leg.; MHNG • 1 q; Toledo, near Aranjuez, Seseña; $40.10^{\circ}$ N, 3.70 W; 10 Jun. 1952; Franz leg.; SMF $19436 \bullet 2$ đo $\sigma^{\circ}$,

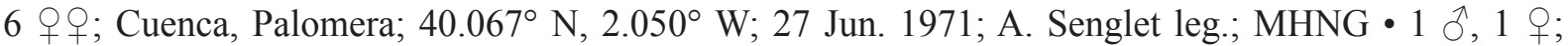
Guadalajara; $40.63^{\circ} \mathrm{N}$, 3.15 ${ }^{\circ} \mathrm{W}$; Jun. 1907; collector unknown; MNHN Ar 10315. - Extremadura •

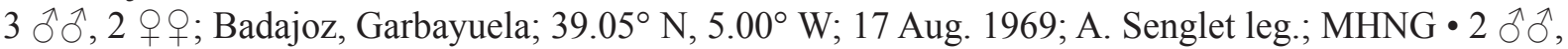
2 우; Badajoz, Puebla de Alcocer; $38.98^{\circ}$ N, 5.26 W; 12 Apr. 1994; R. Bosmans leg.; among stones in

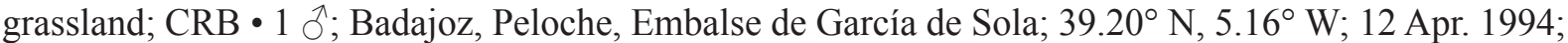

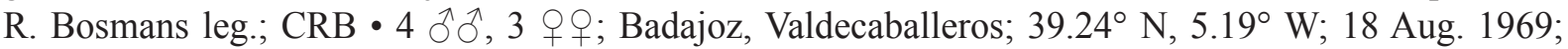

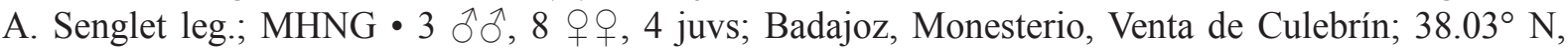
$6.22^{\circ}$ W; 19 Jun. 1969; A. Senglet leg.; MHNG • 3 o 9 ; Badajoz, SW of Zafra, valley of Río Bodión; $38.410^{\circ} \mathrm{N}, 6.425^{\circ} \mathrm{W} ; 2$ Apr. 1997; R. Bosmans leg.; stones; CRB • 1 ô, 1 ; Cáceres, Conquista de la Sierra; $39.350^{\circ} \mathrm{N}, 5.735^{\circ} \mathrm{W}$; 14 Apr. 1994; R. Bosmans leg.; open Quercus suber forest; CRB • 1 万, 4 우; Cáceres, Jarandilla [de la Vera]; $40.126^{\circ}$ N, 5.658 ${ }^{\circ}$ W; 22-23 Aug. 1969; A. Senglet leg.; MHNG

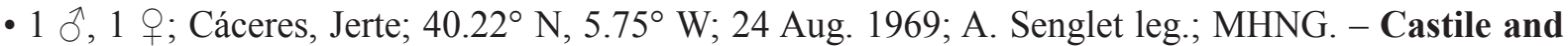
León - 2 우; Ávila, El Barco de Ávila; $40.36^{\circ}$ N, 5.52 ${ }^{\circ}$ W; 25 Aug. 1969; A. Senglet leg.; MHNG •

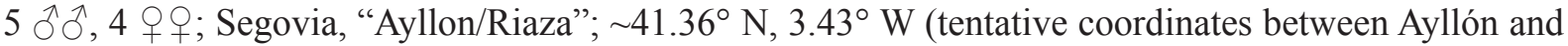
Riaza); 1 Sep. 1971; A. Senglet leg.; MHNG • 1 Oे, 1 q; same collection data as for preceding; "von

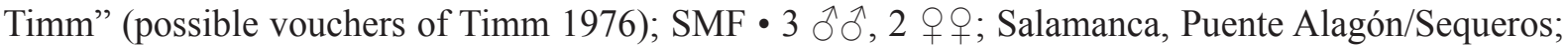

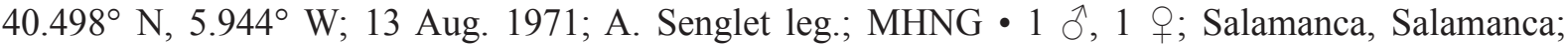

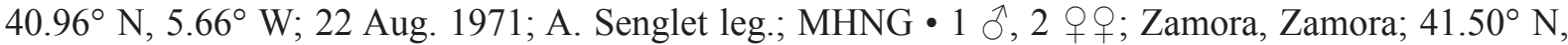
$5.75^{\circ}$ W; 25 Aug. 1971; A. Senglet leg.; MHNG • 1 đ̃, 1 q, 2 juvs; Zamora, near Sandín de Carballeda; $42.00^{\circ} \mathrm{N}, 6.48^{\circ} \mathrm{W} ; 750 \mathrm{~m}$ a.s.1.; Jul. 1979; P. Haymoz leg.; MHNG.

SPAIN - no further locality data $-2 \hat{\partial} \bar{\delta}$ (in pure ethanol), vouchers of Ávila Herrera et al. 2021;

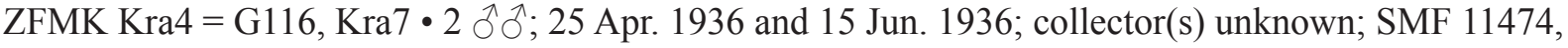
$11475 \cdot 1 \sigma^{\Uparrow}$ (reared specimen); killed on 15 Jun. 1936, no further data; SMF 19308.

PORTUGAL - Faro・ $1 \delta^{\lambda}$; Quarteira; $37.07^{\circ} \mathrm{N}, 8.10^{\circ} \mathrm{W}$; Aug. 1982; J. Murphy leg.; MMUE Murphy

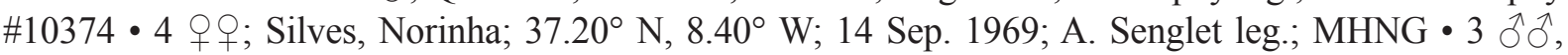
9 ㅇ; ; Barragem do Arrade; $37.238^{\circ}$ N, $8.376^{\circ} \mathrm{W}$; 16 Feb. 2006; R. Bosmans leg.; stones in Pinus forest;

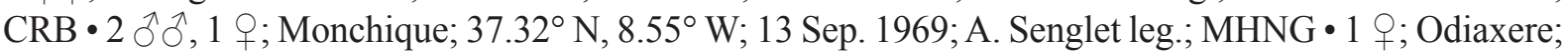
$37.15^{\circ} \mathrm{N}, 8.66^{\circ} \mathrm{W}$; 13 Feb. 2006; R. Bosmans leg.; stones in wet grassland; CRB • 1 ô, 1 q; Salema, Boca do Rio; $37.066^{\circ} \mathrm{N}, 8.825^{\circ} \mathrm{W} ; 14$ Feb. 2006; R. Bosmans leg.; stones around castle ruins; CRB. - Beja • 1 क; Moura, Sobral da Adiça; $38.03^{\circ}$ N, 7.29 W; 27 Mar. 2013; J. Dolansky leg.; CJL 8784 •

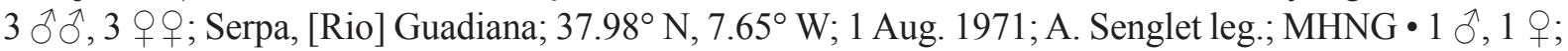
Mértola; $37.64^{\circ} \mathrm{N}, 7.66^{\circ} \mathrm{W} ; 30$ Mar. 2013; J. Dolansky leg.; CJL 8776 • 1 § , 1 juv.; São Teotónio W; $37.51^{\circ} \mathrm{N}, 8.72^{\circ} \mathrm{W} ; 16$ Apr. 2011; R. Bosmans leg.; CRB. - Sétubal • $3 \overbrace{}^{\lambda} \partial^{\lambda}, 5$ $q$; ; Santiago, Azinheira 


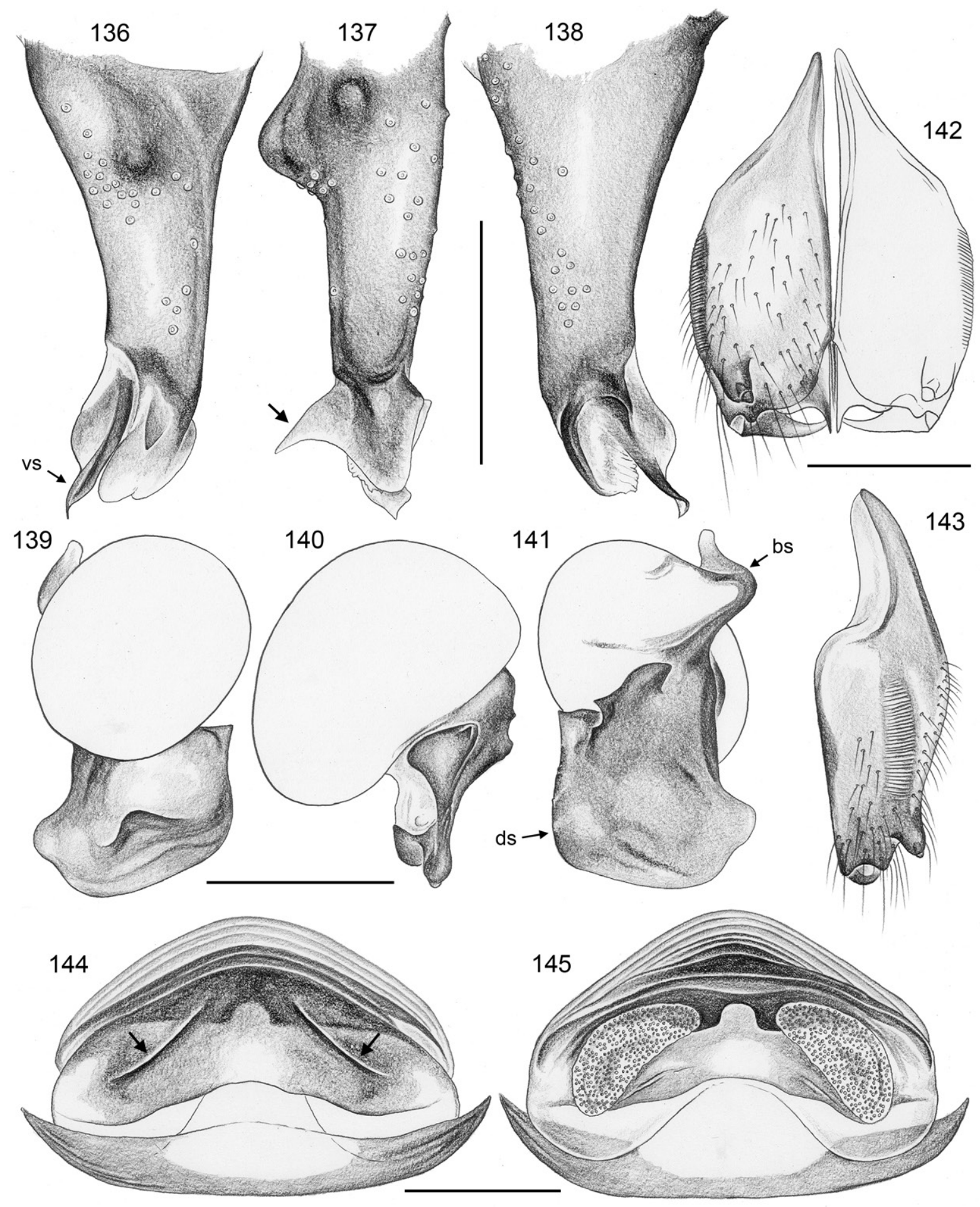

Figs 136-145. Holocnemus hispanicus Wiehle, 1933; male from Spain, Cáceres, Conquista de la Sierra (CRB), female from Spain, Málaga, Ronda (CRB). 136-138. Left procursus, prolateral, dorsal, and retrolateral views; arrow: prolateral transparent process. 139-141. Left genital bulb, prolateral, dorsal, and retrolateral views. 142-143. Male chelicerae, frontal and lateral views. 144-145. Cleared female genitalia, ventral and dorsal views; arrows: pockets. Abbreviations: bs = basal sclerite; ds $=$ distal sclerite; vs = ventral sclerite. Scale bars $=0.3 \mathrm{~mm}$. 

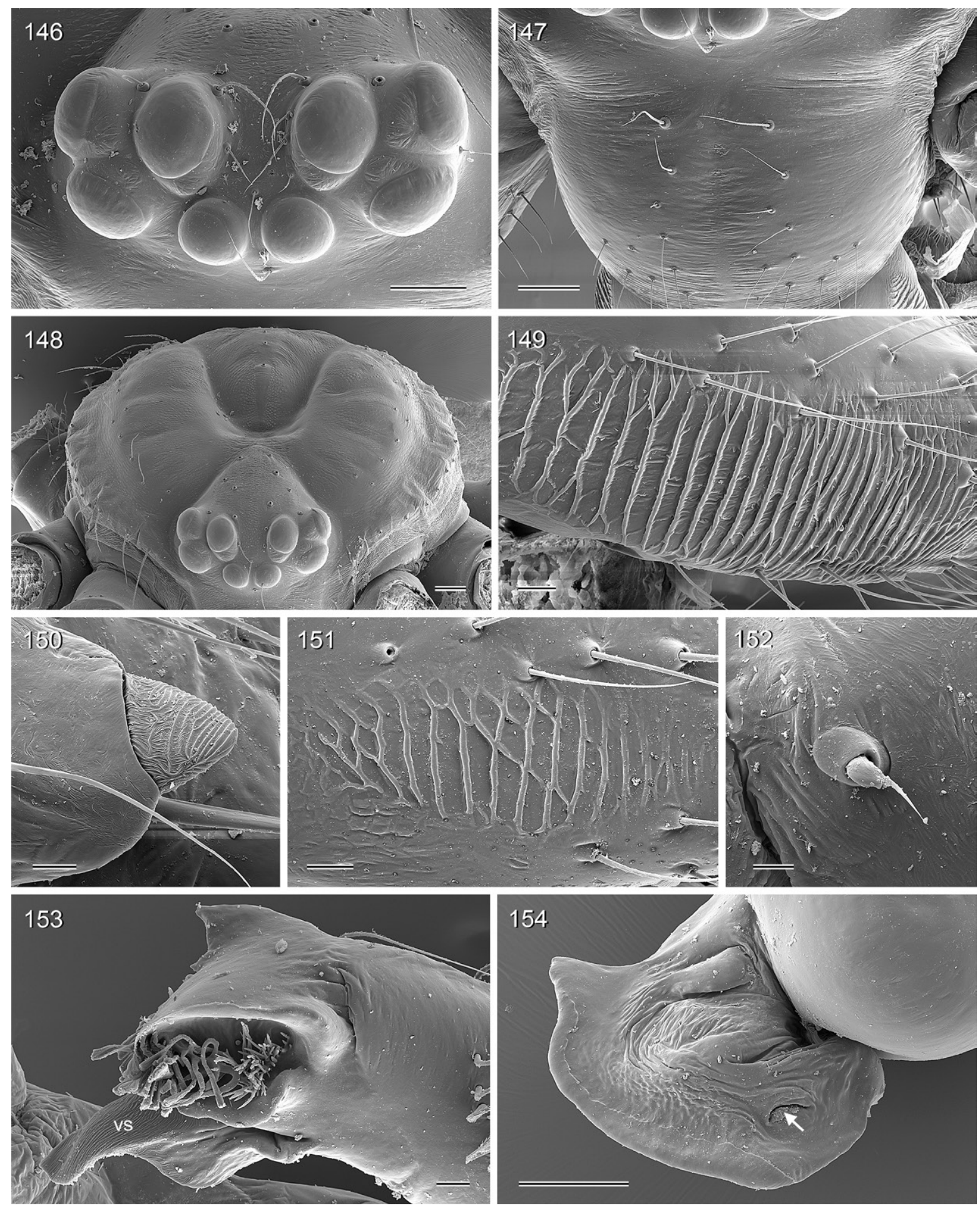

Figs 146-154. Holocnemus hispanicus Wiehle, 1933; male and female from Spain, Huelva, "Alájar/ Aracena" (MHNG). 146. Male ocular area. 147. Male clypeus. 148. Female carapace and ocular area. 149. Male cheliceral stridulatory file. 150. Tip of male cheliceral apophysis. 151. Female cheliceral stridulatory file. 152. Stridulatory pick on female palpal femur. 153. Left procursus, retrolateral-ventral view. 154. Distal (main) sclerite of left genital bulb, prolateral (slightly distal) view; arrow: sperm duct opening. Abbreviation: vs = ventral sclerite. Scale bars: $146-148,154=100 \mu \mathrm{m} ; 149,151,153=$ $20 \mu \mathrm{m} ; 150,152=10 \mu \mathrm{m}$. 
dos Barros; 38.07 N, 8.42 ${ }^{\circ}$ W; 2 Aug. 1971; A. Senglet leg.; MHNG. - Leiria • 1 क; near Grutas de San António; $39.537^{\circ}$ N, 8.742 ${ }^{\circ}$ W; 1-3 May 1991; V.D. Roth leg.; CAS $9027123 \bullet 1$ q; Mira de Aire,

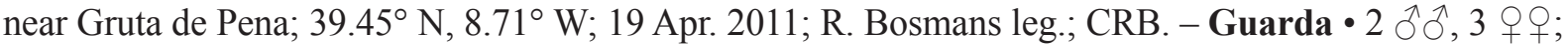
Fornos de Algodres, Maceira; 40.69 N, 7.47 W; 9 Aug. 1971; A. Senglet leg.; MHNG. - Bragança •

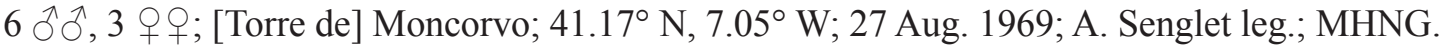

\section{Redescription}

Male (10 km N of Málaga, CRB)

MeAsurements. Total length 3.9, carapace width 1.4. Distance PME-PME $105 \mu \mathrm{m}$; diameter PME $100 \times$ $130 \mu \mathrm{m}$; distance PME-ALE $30 \mu \mathrm{m}$; diameter AME $80 \mu \mathrm{m}$; distance AME-AME $40 \mu \mathrm{m}$. Leg 1: 31.2 $(9.0+0.6+8.1+11.4+2.1)$, tibia $2: 5.6$, tibia $3: 4.2$, tibia $4: 5.0$; tibia $1 \mathrm{~L} / \mathrm{d}: 54$; femora $1-4$ diameters: $0.21,0.19,0.17,0.18$.

COLOR (in ethanol). Carapace ochre-yellow; ocular area, carapace pit and posterior median area slightly darker, with brown V-mark behind ocular area; sternum brown, with dark brown radial marks; legs ochre-yellow, without darker rings, with black lines on femora and tibiae; abdomen ochre-gray, with some dark marks dorsally and laterally; ventrally with distinct black median band, partly disrupted, with three parallel longitudinal marks behind gonopore.

Body. Habitus similar to H. caudatus (cf. Fig. 7) but abdomen less elongated above spinnerets (rather as in Crossopriza tiwi sp. nov.; cf. Fig. 394). Ocular area slightly raised. Deep thoracic pit and pair of shallow furrows diverging from pit toward posterior margin (cf. Fig. 148). Clypeus unmodified but rim more sclerotized than in female. Sternum wider than long (1.00/0.70), unmodified, without indentations as in $H$. caudatus and $H$. reini. Abdomen slightly elongated, dorso-posteriorly angular. Gonopore with six epiandrous spigots (Fig. 161); ALS with one widened spigot and one pointed spigot (Fig. 159).

Chelicerae. As in Figs 142-143, with pair of frontal lateral apophyses, each with one large modified cone-shaped hair (Fig. 150); distance between tips of modified hairs: $310 \mu \mathrm{m}$; without proximal frontal protrusion; lateral stridulatory ridges distinct (Fig. 149); distances between ridges proximally $\sim 14 \mu \mathrm{m}$, distally $\sim 6 \mu \mathrm{m}$.

PALPS. As is Figs 129-131; coxa with rounded retrolateral hump; trochanter barely modified; femur curved towards dorsal, distally widened, with rounded ventral protrusion, proximally with prolateral stridulatory pick, without retrolateral transversal line, without retrolateral proximal process; femur-patella joints slightly shifted toward prolateral side; tibia very large compared to femur, tibia-tarsus joints shifted toward retrolateral side; tarsus without macrotrichia; palpal tarsal organ capsulate (Fig. 155); procursus (Figs 136-138) straight, few dorsal hairs slightly curved upwards; proximally on prolateral side with strong hump free of hairs, with brush of hairs distal of hump, procursus tip with strong ventral sclerite slightly spiraling and proximally wide and partly semitransparent, on prolateral side with semitransparent pointed process (arrow in Fig. 137); genital bulb (Figs 139-141) with simple basal sclerite connected to distal (main) sclerite; distal sclerite large and mostly flat, with distinctive prolateral rounded process; sperm duct opening prolaterally (arrow in Fig. 154; not visible in dissecting microscope).

LEGs. Femur 1 with single row of $\sim 26$ ventral spines; without curved hairs; few vertical hairs; retrolateral trichobothrium of tibia 1 at $3.5 \%$; prolateral trichobothrium absent on tibia 1, present on other leg tibiae; tarsal pseudosegments indistinct except $2-3$ distally.

Male (variation)

Tibia 1 in 134 males: 6.1-11.8 (mean 8.7). Most specimens with pair of brown marks behind carapace pit, close to median line. Abdomen often higher posteriorly than anteriorly; posterior elongation of 

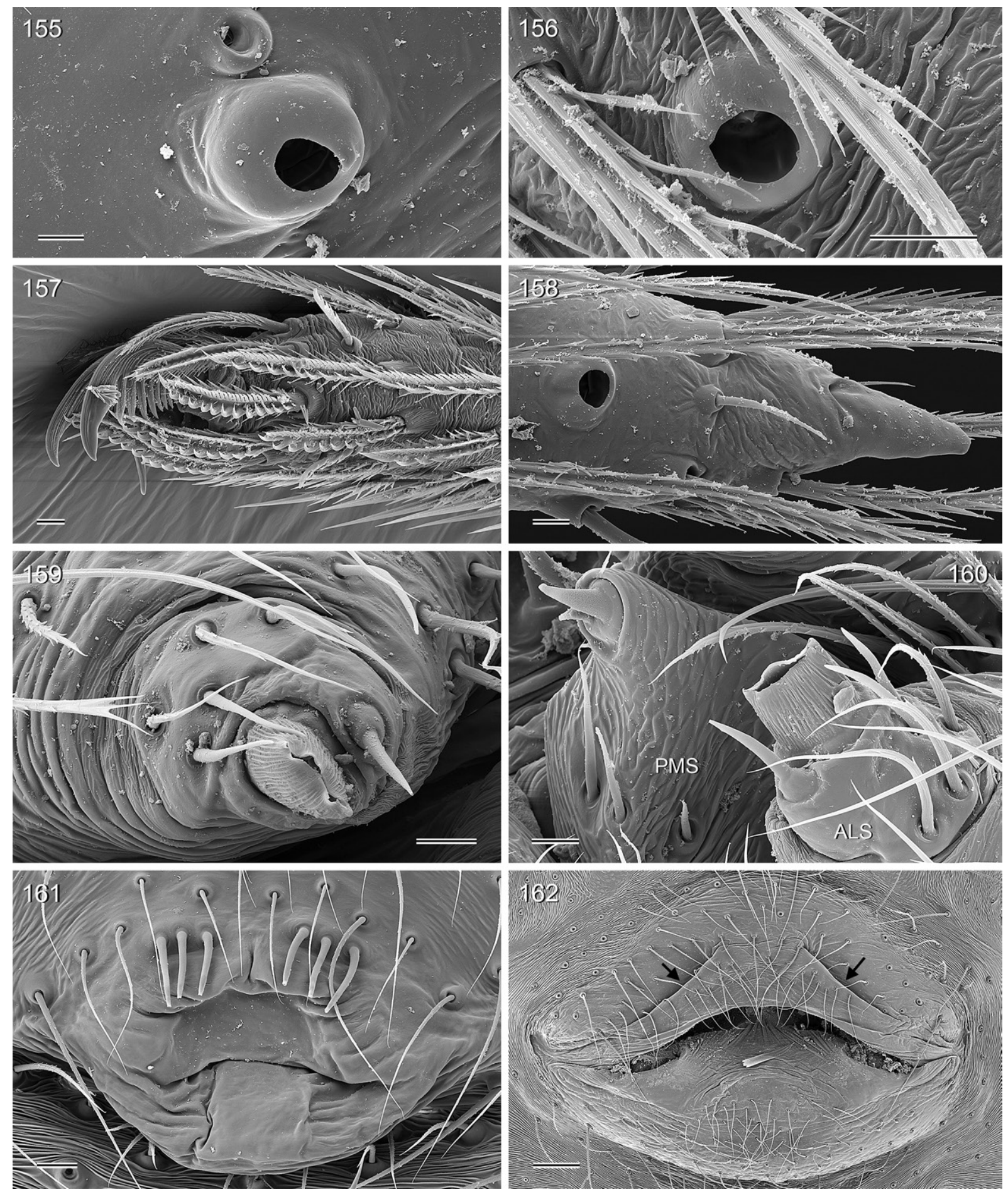

Figs 155-162. Holocnemus hispanicus Wiehle, 1933; male and female from Spain, Huelva, "Alájar/ Aracena" (MHNG). 155. Male palpal tarsal organ. 156. Tarsal organ on male tarsus 3. 157. Tip of male right tarsus 4, showing comb-hairs. 158. Tip of female palp with tarsal organ. 159. Male ALS. 160. Female spinnerets. 161. Male gonopore. 162. Epigynum; arrows: pockets. Scale bars: $155-160=$ $10 \mu \mathrm{m} ; 161=20 \mu \mathrm{m} ; 162=100 \mu \mathrm{m}$. 
abdomen variably long; abdomen usually with many whitish marks. Difference in diameter between femur 1 and other femora stronger in larger males (e.g., large male from Ronda with tibia 1 length of 10.2: femur 1 diameter 0.38 ; femora $2-4$ diameters $0.29-0.31$ ). Rarely with spines on femur 2 (only when leg 1 on corresponding side is missing). Distance between tips of modified hairs on cheliceral apophyses: $\sim 285-375 \mu \mathrm{m}$.

\section{Female}

In general similar to male but without spines on legs, with stridulatory apparatus between carapace and abdomen consisting of pale processes posteriorly on carapace (distance $\sim 0.66$ ) and barely visible plates anteriorly on abdomen; with cheliceral stridulatory ridges similar to male but in much smaller file (Fig. 151; distances between ridges $\sim 12 \mu \mathrm{m}$ ). Tibia 1 in 212 females: 4.3-10.4 (mean 7.5). Epigynum as in Figs 132-134, main epigynal plate bean-shaped, weakly protruding, with distinctive pair of furrows converging anteriorly (distance of closest/widest sections of furrows: $\sim 240 / 440 \mu \mathrm{m}$ ), internal arc and pair of dark round structures usually visible in uncleared specimens; posterior epigynal plate large but simple, anterior median part usually lighter; pair of low but distinct elevations in front of epigynum. Internal genitalia (Figs 144-145) with elongate pore plates converging anteriorly, dorsal arc relatively simple [Wiehle's (1933) "Receptaculum seminis" is just an elongated sclerotized fold along the dorsal arc], ventral arc medially widened and heavily sclerotized, with distinctive indentation posteriorly; apparently without median ventral pouch but with pair of small ventral processes.

\section{Natural history}

This species is common in wall niches, rock crevices, and under rocks, generally less exposed than H. pluchei (Wiehle 1933; Benhadi-Marín et al. 2013). The webs have a diameter of approximately 35$40 \mathrm{~cm}$ and a relatively regularly-woven inner dome. They are sometimes provided with small silk puffs, closer together than in webs of $H$. caudatus (Wiehle 1933). Males undergo six or seven molts before adulthood, females one more (Wiehle 1933). In southern Spain (Granada), adult males do not seem to overwinter (only females and juveniles were present in May; Wiehle 1933). Wiehle (1933) gave a brief description of copulation, and Senglet (2001) observed a case where a male almost completely destroyed the female web after mating. Egg-sacs contained approximately 20-25 eggs; mean egg diameter was $682 \mu \mathrm{m}$ (Wiehle 1933). In two cases, Wiehle (1933) counted nine days from copulation to egg-laying, and 37 days from egg-laying to eclosion; females produced two egg-sacs per season; Senglet (1972) observed that H. hispanicus and Spermophorides petraea (Senglet, 1972) seem to compete for the same microhabitat; in Barco de Ávila, he suspected that S. petraea had largely replaced H. hispanicus; in Jerte, $S$. petraea seemed to prefer piles of rocks, while $H$. hispanicus occupied holes in rock walls.

\section{Distribution}

Widely distributed on the Iberian Peninsula (Fig. 4). I have not seen any specimens from north of approximately $\sim 42.5^{\circ} \mathrm{N}$, which agrees with the observation that the species seems to be absent from the Cantabrian Range (C.E. Prieto, pers. com., 9 Feb. 2021).

\section{Maghreba gen. nov. urn:1sid:zoobank.org:act:948E7573-BBF2-435C-BB9B-BDE8DE507173}

Figs 9-12, 163-350

\section{Type species}

Maghreba amezyan gen. et sp. nov.

\section{Diagnosis}

Males are easily distinguished from other representatives of the spotted-leg clade of Smeringopinae by numerous details of the palp (coxa with distinct retrolateral-ventral process, Fig. 205; femur with 
small dorsal apophysis, Fig. 205; procursus tip bent towards dorsal, Fig. 166; membranous transparent process accompanying ventral sclerite of procursus, Fig. 166; bulbal sclerite with deep retrolateral pocket, Fig. 169). Females are easily distinguished from Holocnemus reini (which occurs in the same geographic region) by the much shorter abdomen that is only weakly angular rather than pointed posteriorly (Figs 9-10, 195-202); females closely resemble certain representatives of Crossopriza (which shares at least parts of Algeria with Maghreba gen. nov.) and cannot be assigned with confidence to any of the two genera if not accompanied by males; usually the distance between the eye triads is larger in Maghreba gen. nov. than in Crossopriza (distance PME-PME usually $\geq 1.1 \times$ PME diameter in Maghreba gen. nov. vs $\leq 1.1$ in Crossopriza).

\section{Etymology}

The genus name is derived from the Maghreb, the western part of North Africa and the Arab World; gender feminine.

\section{Description}

\section{Male}

BoDy. Total body length $\sim 3.0-4.5$; carapace width $\sim 1.2-1.7$. Carapace with deep central pit and pair of shallow furrows diverging from posterior side of pit toward posterior rim (cf. Figs 305, 319); ocular area slightly raised, eye triads relatively far apart (distance PME-PME usually 1.1-1.5 × PME diameter, in the slightly troglomorphic $M$. kahfa gen. et sp. nov. 2.1), each secondary eye (especially PME) accompanied by indistinct elevation ("pseudo-eyes"; cf. Huber 2009a), PME oval, AME large ( $\sim 60-105 \%$ of PME small diameter). Clypeus high, unmodified. Abdomen oval, dorsally posteriorly usually slightly angular (e.g., Figs 9, 196, 258). Male gonopore with 4-6 epiandrous spigots (sometimes asymmetric: 2+3; Figs 316, 320-321), ALS with only two spigots each: one large widened spigot and one pointed spigot (cf. Figs 317, 329); PMS with two spigots each; PLS without spigots.

CoLor. In general ochre-yellow to light brown. Carapace mostly pale, with darker median mark, without lateral marks (Figs 195-202); sternum light to dark brown, with darker radial marks. Legs without or with indistinct darker rings, with oval to short longitudinal line-shaped dark marks on femora and tibiae, sometimes also a few on metatarsi (Figs 188-189, 206-207). Abdomen usually with distinct dorsal and ventral patterns: dark heart-mark and further dark and whitish marks dorsally and laterally, ventral median band variably distinct.

Chelicerae. Chelicerae with one large modified (cone-shaped) hair on each cheliceral apophysis (Figs 170, 306, 323); usually with fine stridulatory ridges (Figs 307, 324), distances between ridges in M. amezyan gen. et sp. nov. and M. aurouxi gen. nov. $\sim 3.5 \mu \mathrm{m}$; with more distinct ridges in M. kahfa gen. et sp. nov., apparently without ridges in M. gharbija gen. et sp. nov.

PALPS. In general as in Figs 203-205; coxa with distinct retrolateral-ventral process; trochanter barely modified, slightly protruding ventrally; femur widened distally, dorsal line straight except for small dorsal apophysis, with proximal retrolateral process, without or with very indistinct transversal dark line on retrolateral side, with stridulatory pick (modified hair) on prolateral side; femur-patella joints shifted toward prolateral side (arrows \#3 in Fig. 203); tibia-tarsus joints shifted toward retrolateral side (arrows \#4 in Fig. 205); palpal tarsus without dorsal macrotrichia, palpal tarsal organ capsulate (Figs 311, 327); procursus dorsally with straight or weakly curved hairs; procursus (e.g., Figs 164-166, 309) with hairless prolateral process, without ventral 'knee', distally with ventral sclerite accompanied by membranous transparent to semitransparent process, procursus tip curved towards dorsal, with one or few transparent hair-like processes on retrolateral side (barely visible in light microscope); genital bulb (e.g., Figs 167169, 310) with basal sclerite (bs in Fig. 169) connecting to tarsus; basal sclerite sometimes with distinct dorsal (slightly retrolateral) apophysis (e.g., Fig. 212); distal (main) bulbal sclerite with strong dorsal 
process, variable number of ventral teeth, and large retrolateral pocket (arrow in Fig. 169); sperm duct opening in membranous area on prolateral side of distal bulbal sclerite (arrow in Fig. 310).

Legs. Legs long and relatively thin, leg 1 length $\sim 20-45$, tibia 1 length $\sim 6-12$, tibia 2 longer than tibia $4(\sim 1.1-1.2 \times)$. Tibia $1 \mathrm{~L} / \mathrm{d}$ usually $\sim 50-65,80$ in the slightly troglomorphic $M$. kahfa gen. et sp. nov. Femur 1 usually thicker than other femora; femur 1 with one row of ventral spines (Fig. 206), rarely (in very small males) without spines; spines proximally gradually transforming into regular setae; spines never present on femur 2 or on tibia 1; legs without curved hairs; in most species with slightly higher than usual density of short vertical hairs prolaterally-dorsally on one or more tibiae; retrolateral trichobothrium in proximal position (at 2-4\% of tibia length in tibia 1), prolateral trichobothrium absent on tibia 1, present on other tibiae. Tarsal pseudosegments very indistinct except $\sim 2-5$ distally, proximally with indistinct irregular platelets rather than distinct rings. Tarsal organs of legs capsulate, with round or weakly undulating rim (Figs 313-315, 328).

\section{Female}

In general very similar to male; chelicerae with even less distinct and smaller stridulatory files or without stridulatory files (Figs 308, 325); legs slightly shorter than in male, without spines. Usually with pair of indistinct processes posteriorly on carapace (arrows in Figs 305, 319) acting against pair of poorly visible plates on abdomen. Epigynum usually consisting of large, simple anterior plate and short but wide posterior plate (e.g., Figs 175-178); anterior plate with pair of low pockets (pits) (Figs 318, 330), in some species with pair of variably distinct processes (e.g., Fig. 238); without bulging areas in front of anterior plate. Internal genitalia (e.g., Figs 179-181) with sclerotized arc that consists of dorsal and ventral parts (da and va in Fig. 179) and is variably visible in uncleared specimens; uterus externus sometimes with small median ventral structure (pouch or pocket?), sometimes visible as round structure in untreated specimens (e.g., Figs 178, 220); pore plates large, flat, widening towards median line, left and right plates usually clearly separated except in M. kahfa gen. et sp. nov. (Fig. 187); pores usually in groups rather than homogeneously distributed.

\section{Distribution}

Most records are from the Moroccan Atlas (Fig. 163), but the genus ranges into Algeria and may in fact be more diverse in the Algerian Atlas as well. A single male specimen from the Algerian Hoggar Mountains, Tamanrasset, In [Ain] Zbib $\left(22.748^{\circ} \mathrm{N}, 5.575^{\circ} \mathrm{E}\right.$ ) deposited in CRB (shown in Fig. 163 but not formally described) suggests that the genus actually has a much wider distribution in NW Africa. The spiders have been found from sea level up to over $2000 \mathrm{~m}$ a.s.l.

\section{Relationships}

Together with Holocnemus, Stygopholcus, and Crossopriza, Maghreba gen. nov. is clearly a representative of the spotted-leg clade (Fig. 1) but beyond that relationships remain obscure. The sister-group relationship with Stygopholcus suggested by the cladistic analyses using equal character weights and successive character weighting is supported by a single homoplastic character and is thus unconvincing. Geographically, the genus is closer to Crossopriza with which it also shares a greater general similarity than with Stygopholcus. In fact, implied character weighting with conc $=1$ and conc $=2$ suggested a trichotomy including Maghreba gen. nov., Holocnemus hispanicus, and Crossopriza. However, no synapomorphy has been found to support a sister-group relationship between Maghreba gen. nov. and Crossopriza, or between Maghreba gen. nov. and H. hispanicus.

\section{Composition}

The genus currently includes eight named species, all of which are treated below: seven new species plus M. aurouxi gen. nov. that is newly transferred from Holocnemus. In the examined collections, only 
one further species was seen, represented by a single male specimen from Algeria deposited in CRB (see Distribution above). The palp of this species strongly resembles the Moroccan species described below; it is easily distinguished from all Moroccan species by the presence of two pairs of proximal processes on the male chelicerae; it further differs by a distinct subdistal dorsal process on the procursus and by strongly developed cheliceral stridulatory ridges.
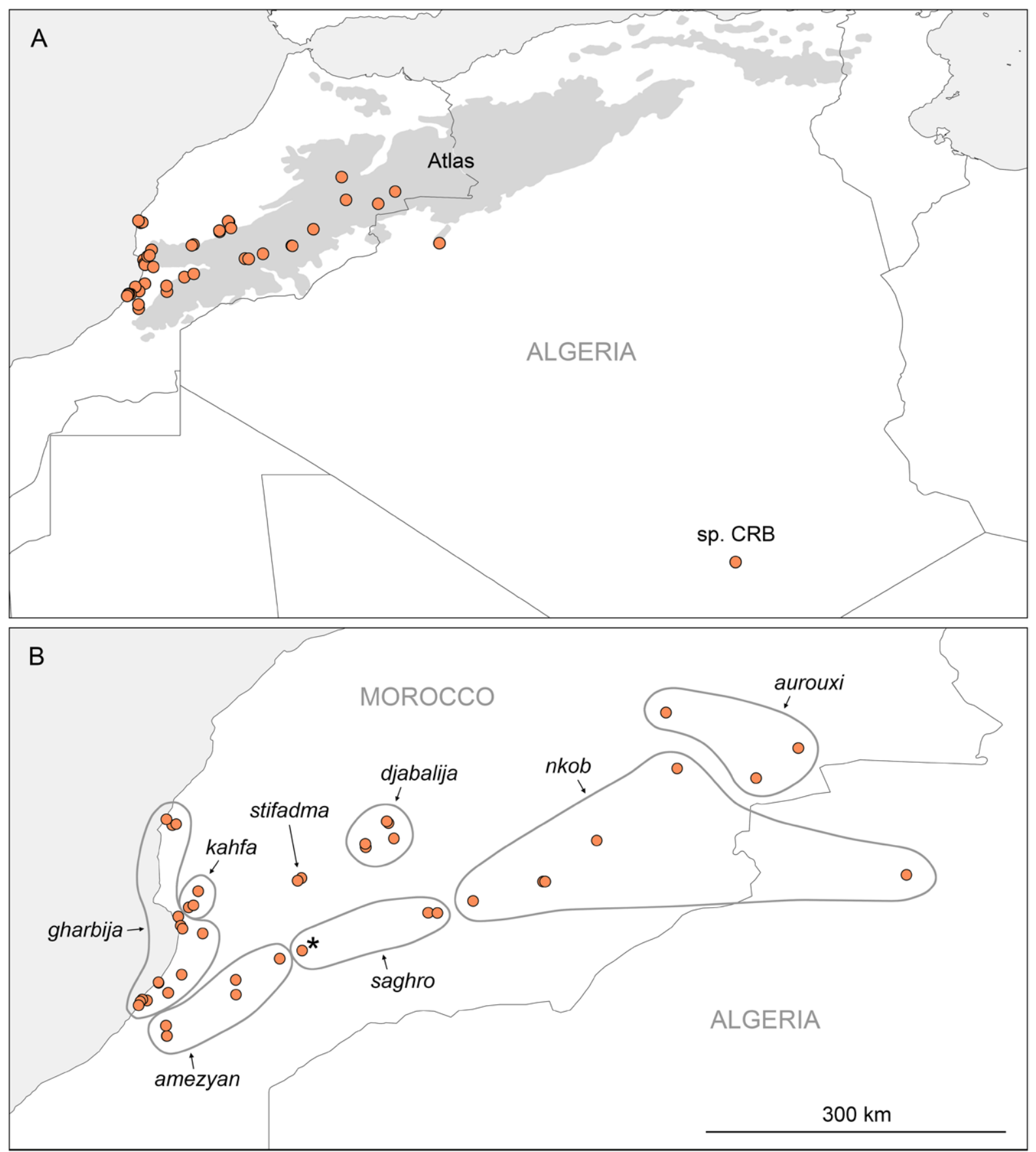

Fig. 163. Known distribution of Maghreba gen. nov. A. Complete known distribution, including an undescribed Algerian species in CRB; the approximate extent of the Atlas Mountains is shown $(1000 \mathrm{~m}$ altitude isolines, taken from https://www.britannica.com/). B. Known distributions of named species. Asterisk: specimens tentatively assigned to $M$. aurouxi gen. nov. 


\section{Natural history}

Different species seem to prefer different microhabitats: some appear restricted to caves (twilight area and slightly beyond), others occupy sheltered spaces among and under rocks and small cavities in the ground, and some live quite exposed among the leaves of palms and other objects close to the ground. Webs usually consist of a domed sheet and are often modified at the center to include a further bellshaped dome that is provided with silk puffs (Figs 11-12). At disturbance the spiders vibrate or run towards the back; whether this behavior is species-specific or not is unknown. For further details on individual species, see natural history sections below.

\section{Identification key}

1. Dorsally-directed tip of procursus short and wide (Fig. 245); distal bulbal sclerite ventrally with 3-4 large teeth and several tiny teeth (Figs 246, 248); epigynum triangular rather than semicircular (Fig. 251) M. nkob gen. et sp. nov.

- Dorsally-directed tip of procursus long and slender (e.g., Figs 166, 210); distal bulbal sclerite ventrally either with two or with many ( 14) large teeth (e.g., Figs 167, 211); epigynum semicircular or oval rather than triangular (e.g., Figs 178, 218)

2. Distal bulbal sclerite ventrally with $\sim 14$ large teeth (Fig. 211); epigynum relatively short and wide, posteriorly slightly indented, medially lighter than laterally, with dark internal structure visible anteriorly in untreated specimens (Figs 218-220) M. saghro gen. et sp. nov.

- Distal bulbal sclerite ventrally with two large teeth; epigynum different 3

3. Procursus tip with dark process between ventral sclerite and transparent process (Figs 267, 336); epigynum with pair of small processes and dorsal arc of internal female genitalia without pair of posterior processes

- Procursus tip without process between ventral sclerite and transparent process; female genitalia different (i.e., either epigynum without processes or dorsal arc of internal female genitalia with pair of posterior processes; e.g., Figs 181, 303)

4. Ventral teeth on distal bulbal sclerite long (Figs 270, 273); female genitalia as in Figs 276-280, 282-284 M. gharbija gen. et sp. nov.

- Ventral teeth on distal bulbal sclerite short (Figs 339, 342); female genitalia as in Figs 345-350 ....

.M. djabalija gen. et sp. nov.

5. Dorsal process of distal bulbal sclerite only slightly longer than ventral part (Fig. 232); epigynum with pair of large whitish processes (Fig. 238) M. stifadma gen. et sp. nov.

- Dorsal process of distal bulbal sclerite much longer than ventral part (Figs 167, 184, 295); epigynum with pair of small processes or without processes

6. Slightly troglomorphic spider with reduced eye size (distance PME-PME $=2.1 \times$ PME diameter) and very thin legs (male tibia $1 \mathrm{~L} / \mathrm{d} 80$ ); dorsal arc of internal female genitalia without or with very indistinct posterior processes (Figs 187, 194)

M. $k a h f a$ gen. et sp. nov.

- Eye size not reduced (distance PME-PME $=1.1-1.5 \times$ PME diameter) and legs thicker (male tibia $1 \mathrm{~L} / \mathrm{d} \sim 60-65$ ); dorsal arc of internal female genitalia with small to distinct posterior processes (Figs 181, 303)

7. Large size (body size $\sim 4.0$, carapace width $\sim 1.7$ ), eye triads far apart (distance PME-PME $=1.5 \times$ PME diameter); dorsal arc of internal female genitalia with small posterior processes (Fig. 181) ....

M. amezyan gen. et sp. nov. 
- Smaller size (body size $\sim 3.0$, carapace width $\sim 1.2$ ), eye triads closer together (distance PME$\mathrm{PME}=1.1 \times$ PME diameter); dorsal arc of internal female genitalia with distinct posterior processes (Fig. 303) M. aurouxi (Barrientos, 2019) gen. et comb. nov.

\author{
Maghreba amezyan gen. et sp. nov. \\ urn:lsid:zoobank.org:act:CFA70C27-960B-436B-B01E-153F61575912 \\ Figs 9-12, 163B, 164-181, 305-318
}

\title{
Diagnosis
}

Distinguished from known congeners by combination of: procursus with long and straight ventral sclerite and slender dorsally-directed tip (Fig. 166), distal bulbal sclerite with two ventral teeth of equal size and strongly protruding dorsal process (Fig. 167), epigynum without processes (Figs 175-178), dorsal arc of internal female genitalia with pair of small posterior protrusions (Fig. 181); from most congeners (except $M$. saghro gen. et sp. nov.) also by large body size and long legs (male tibia 1 length $>11.0$, female tibia 1 length $>9.5$ ).

\section{Etymology}

The species name is derived from the Berber name for Anti-Atlas, Atlas Amezyan; noun in apposition.

\section{Type material}

\section{Holotype}

MOROCCO - Guelmim-Oued Noun - ${ }^{\top}$; between Lakhssas and Bouizakarne, "cave 1"; $29.2488^{\circ} \mathrm{N}$, 9.7435 ${ }^{\circ}$ W; 1000 m a.s.1.; 15 Sep. 2018; B.A. Huber leg.; ZFMK Ar 22352.

\section{Other material examined}

MOROCCO - Guelmim-Oued Noun • $11 \hat{\partial} \hat{\partial}, 14$ $q$ q, 2 juvs (partly used for SEM); same collection data as for holotype; ZFMK Ar 22353, Ar 22354 1 \&, 3 juvs (in pure ethanol); same collection data as for holotype; ZFMK Mor83 • 2 우, 1 juv.; same locality as for preceding ("Kef Hman, Tleta el

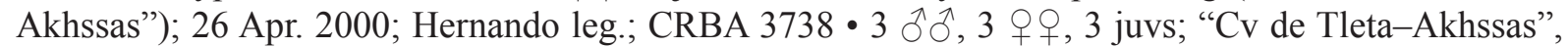
near Bouizakarne; $29.358^{\circ} \mathrm{N}, 9.754^{\circ} \mathrm{W} ; 12$ Mar. 2007; C. Ribera, Txasko, and A. Lopez leg.; CRBA.

Assigned tentatively (see variation below)

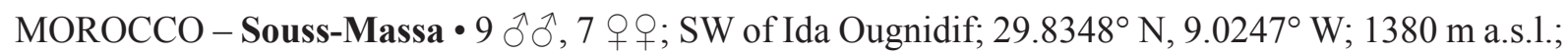

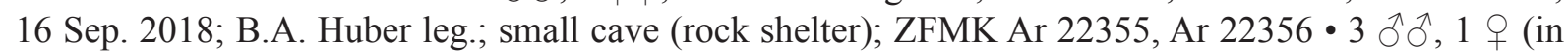
pure ethanol); same collection data as for preceding; ZFMK Mor85 $\bullet 3 \hat{\jmath} \widehat{\jmath}, 2$ ㅇq; same locality as for preceding ("near Ait Baha"); 14 Mar. 2007; C. Ribera, Txasko, and A. Lopez leg.; CRBA 1 ऽ , 4 q $ᄋ$; near Tafraoute; $29.681^{\circ}$ N, $9.023^{\circ}$ W; $1060 \mathrm{~m}$ a.s.l.; 13 Mar. 2007; C. Ribera, Txasko, and A. Lopez leg.; CRBA 1 P; Ifri Tafounast-Tatawt-Taroudant; $30.058^{\circ} \mathrm{N}, 8.568^{\circ} \mathrm{W} ; 22$ Jul. 2006; Auroux, Comas, and Fadrique leg.; CRBA.

\section{Description}

\section{Male (holotype)}

Measurements. Total length 4.1, carapace width 1.7. Distance PME-PME $140 \mu \mathrm{m}$; diameter PME $120 \times 130 \mu \mathrm{m}$; distance PME-ALE $20 \mu \mathrm{m}$; diameter AME $90 \mu \mathrm{m}$; distance AME-AME $20 \mu \mathrm{m}$. Leg 1: $44.8(13.2+0.7+11.9+16.3+2.7)$, tibia $2: 7.9$, tibia $3: 5.9$, tibia $4: 6.8$; tibia $1 \mathrm{~L} / \mathrm{d}: 66$; femora $1-4$ diameters: $0.27,0.24,0.22,0.20$.

COLOR (in ethanol). Carapace pale gray, medially partly darker (brown V-mark behind ocular area; posterior part of pit darkened); sternum dark brown; legs ochre-yellow, with indistinct darker rings on 


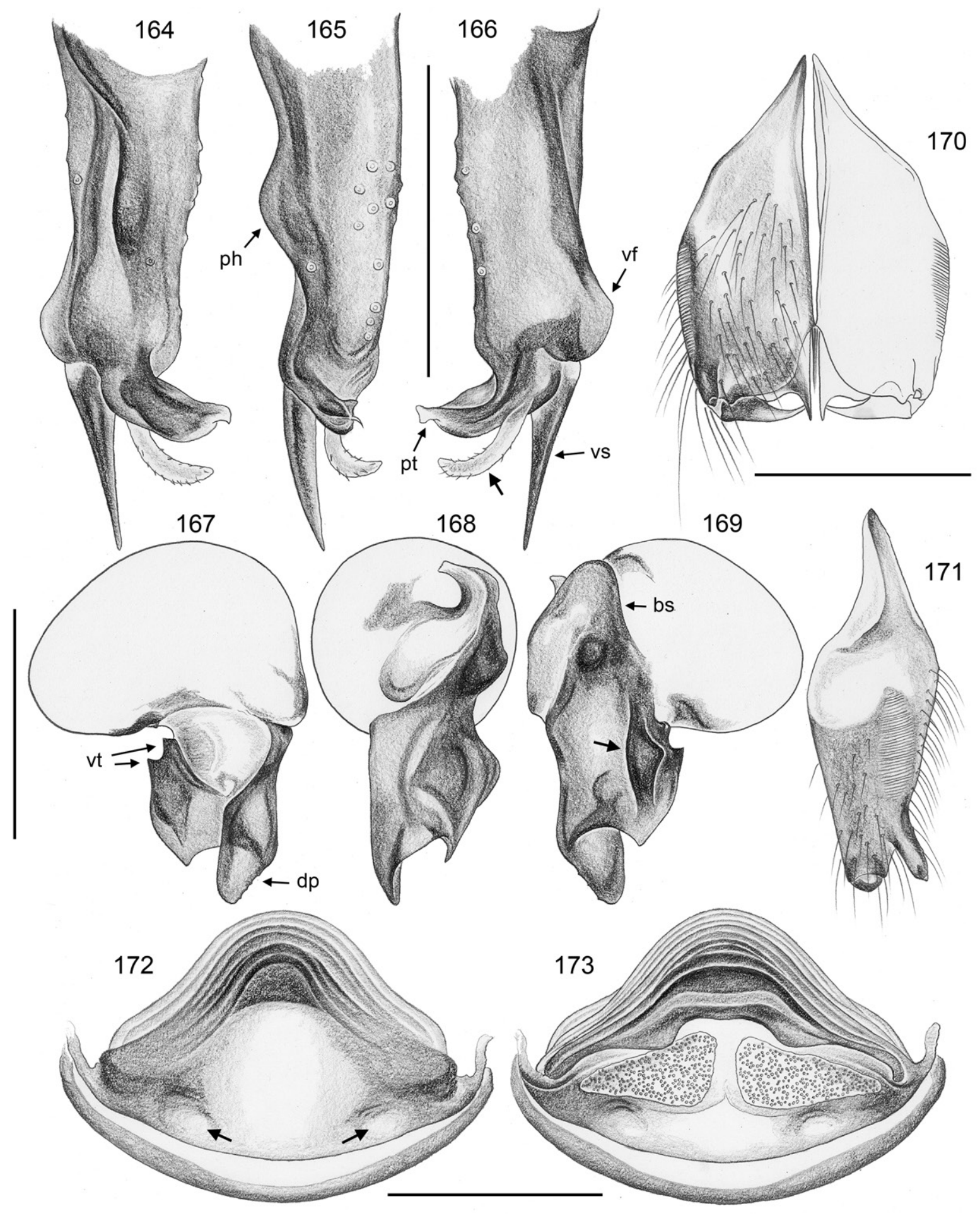

Figs 164-173. Maghreba amezyan gen. et sp. nov.; male and female from Morocco, between Lakhssas and Bouizakarne (ZFMK Ar 22353, Ar 22354). 164-166. Left procursus, prolateral, dorsal, and retrolateral views; arrow: transparent process. 167-169. Left genital bulb, prolateral, dorsal, and retrolateral views; arrow: deep pocket. 170-171. Male chelicerae, frontal and lateral views. 172-173. Cleared female genitalia, ventral and dorsal views; arrows: pockets (pits). Abbreviations: $b s=$ basal sclerite; $d p=$ dorsal process; $\mathrm{ph}=$ prolateral hump; $\mathrm{pt}=$ procursus tip; $\mathrm{vf}=$ ventral flap; $\mathrm{vs}=$ ventral sclerite; $\mathrm{vt}=$ ventral teeth. Scale bars $=0.5 \mathrm{~mm}$. 
femora subdistally, patellae + tibiae proximally, and tibiae subdistally, tips of femora and tibiae whitish, with black lines on femora and tibiae, very few also on metatarsi; abdomen gray, with whitish marks dorsally and laterally, few indistinct darker marks posteriorly; ventrally with large brown mark in front of gonopore, median dark band and pair of lateral oval marks behind gonopore, dark brown area at spinnerets.

Body. Habitus as in Fig. 9. Ocular area slightly raised. Deep thoracic pit and pair of shallow furrows diverging from pit toward posterior margin (cf. Fig. 305). Clypeus unmodified, rim not more sclerotized than in female. Sternum wider than long (1.15/0.85), unmodified. Abdomen slightly elongated, dorsoposteriorly weakly angular. Gonopore with five epiandrous spigots (Fig. 316); ALS with one widened spigot and one pointed spigot (cf. Fig. 317).

Chelicerae. As in Figs 170-171, with pair of frontal lateral apophyses, each with one large modified cone-shaped hair (Fig. 306); distance between tips of modified hairs: $440 \mu \mathrm{m}$; without proximal frontal protrusion; lateral stridulatory ridges fine (Fig. 307; distance between ridges $\sim 2.5-3.0 \mu \mathrm{m}$ ) but clearly visible in dissecting microscope.

PALPS. In general as in congeners (cf. Figs 203-205, 224-226); coxa with small retrolateral-ventral process; trochanter barely modified; femur not curved towards dorsal, distally widened and with very low rounded ventral protrusion, proximally with prolateral stridulatory pick (modified hair), with very indistinct retrolateral transversal line, with distinct retrolateral-ventral proximal process and small but distinct dorsal process; femur-patella joints slightly shifted toward prolateral side; tibia relatively long and slender, tibia-tarsus joints shifted toward retrolateral side; tarsus without macrotrichia; tarsal organ capsulate (Fig. 311); procursus (Figs 164-166) straight, some dorsal hairs slightly curved upwards; on prolateral side with distinct hairless hump; distally with small weakly sclerotized ventral flap; procursus tip strongly curved towards dorsal, with strong ventral sclerite, semitransparent to light brown process, and hair-like retrolateral process (Fig. 309); genital bulb (Figs 167-169) basal sclerite with indistinct dorsal apophysis; distal (main) sclerite large, with deep retrolateral pocket, strong dorsal process with very small teeth, ventral part with two ventral teeth, sperm duct opening on prolateral side in membranous basal area (arrow in Fig. 310).

Legs. Femur 1 with single row of $\sim 30$ ventral spines (Fig. 174); without curved hairs; with usual low number of short vertical hairs; retrolateral trichobothrium of tibia 1 at $2 \%$; prolateral trichobothrium absent on tibia 1, present on other leg tibiae; tarsal pseudosegments irregular and indistinct except $\sim 5-8$ distally; tarsal organs capsulate with round to slightly irregular margins (Figs 313-315).

Male (variation)

Tibia 1 in 13 other males from type locality and other localities near Bouizakarne: 11.2-13.2 (mean 12.1). Distance between tips of cheliceral apophyses $\sim 410-460 \mu \mathrm{m}$. Ventral abdominal pattern slightly variable (pair of lateral oval marks usually fused to median band); white abdominal marks variably distinct.

Males from SW of Ida Ougnidif differ very slightly in several respects and are therefore assigned tentatively (see also females below): slightly shorter legs (tibia 1 in 11 males 9.6-12.3, mean 11.0); slightly wider procursus ( $250 \mu \mathrm{m}$ at half length, vs $210 \mu \mathrm{m}$ in males from type locality); dorsal process of distal bulbal sclerite slightly larger (length $200 \mu \mathrm{m}$, vs $160 \mu \mathrm{m}$ in males from type locality); distal section of ventral part of main bulbal sclerite more rounded; abdominal coloration slightly darker (including dark line around heart area).

Male from near Tafraoute (legs 1 missing) with slightly smaller dorsal process of distal bulbal sclerite (length $140 \mu \mathrm{m}$, vs $160 \mu \mathrm{m}$ in males from type locality); slightly narrower procursus $(190 \mu \mathrm{m}$, vs 

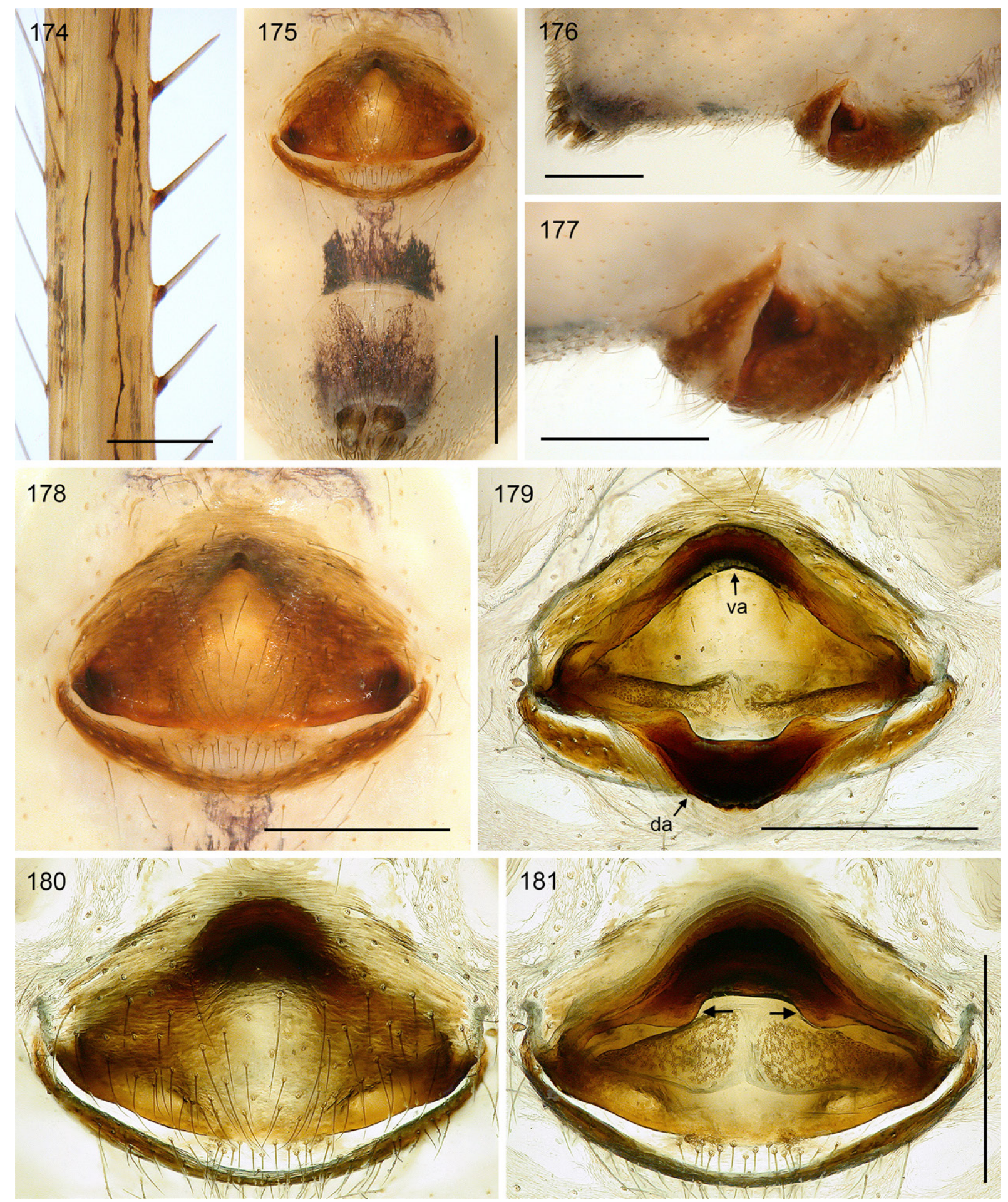

Figs 174-181. Maghreba amezyan gen. et sp. nov.; male and female from Morocco, between Lakhssas and Bouizakarne (ZFMK Ar 22353, AR 22354). 174. Male right femur 1, retrolateral view. 175178. Female abdomen and epigynum, ventral and lateral views. 179-181. Cleared female genitalia, dorsal view with dorsal arc tilted backwards, ventral view, and regular dorsal view; arrows: posterior processes of dorsal arc. Abbreviations: $d a=$ dorsal arc; va $=$ ventral arc. Scale bars: $174=0.3 \mathrm{~mm}$; $175-181=0.5 \mathrm{~mm}$. 
$210 \mu \mathrm{m}$ in males from type locality); distal section of ventral part of main bulbal sclerite more rounded; abdominal coloration slightly darker (including dark line around heart area).

\title{
Female
}

In general similar to male (Fig. 10) but without spines on legs. With poorly developed stridulatory apparatus between prosoma and abdomen: posterior carapace margin with pair of low processes (Fig. 305), and abdomen apparently with pair of areas with thicker cuticle (visible in shrunken abdomens). With fine cheliceral stridulatory ridges as in male but wider apart $(\sim 5-6 \mu \mathrm{m})$ (Fig. 308). Tibia 1 in 18 females from type locality and other localities near Bouizakarne: 9.9-12.4 (mean 11.1). Epigynum as in Figs $175-178$, main epigynal plate triangular to semicircular, weakly protruding, without processes (in some females slightly angular in lateral view), medially weakly sclerotized; with pair of indistinct round pockets (Fig. 318; distance $\sim 450 \mu \mathrm{m}$ ); internal sclerotized arc variably visible in uncleared specimens; posterior plate short and wide, simple; indistinct plate in front of epigynum, not elevated. Internal genitalia (Figs 172-173, 179-181) with triangular pore plates; dorsal and ventral arcs medially strongly sclerotized, ventral arc with very indistinct ventral process (pocket?) directly below (i.e., dorsal of) epigynal plate; dorsal arc with pair of small posterior protrusions (arrows in Fig. 181).

Females from SW of Ida Ougnidif differ very slightly and are therefore assigned tentatively (see also males above): slightly shorter legs (tibia 1 in 8 females 8.7-10.3, mean 9.6); epigynum with pair of very low whitish humps.

Females from near Tafraoute very similar to females from type locality but with shorter legs (tibia 1: 8.3, 8.9, 8.9), and with darker abdominal pattern. Female form Ifri Tafounast poorly preserved.

\section{Natural history}

At the type locality, this species was very abundant within the cave. In the entrance area, only juveniles were found, and no specimens were seen outside the cave nor in deeper parts of the cave (beyond approximately 10-15 $\mathrm{m}$ from the entrance). When disturbed, they vibrated and ran towards the rock, then dropped from the web if the disturbance persisted. Webs were sometimes provided with silk puffs. Near Ida Ougnidif, the spiders were resting very flat on the rock wall, on small mats of silk (diameter $\sim 4 \mathrm{~cm}$ ) that were mostly provided with silk puffs (Figs 11-12); from these small silk mats, larger domed sheets extended that were not set with silk puffs.

\section{Distribution}

Known from several localities in the Anti-Atlas, Morocco (Fig. 163B). Note, however, that all specimens other than those from near Bouizakarne are assigned tentatively.

\author{
Maghreba kahfa gen. et sp. nov. \\ urn:1sid:zoobank.org:act:3EA692AD-0EFD-4A81-82D0-421F5A261AC7
}

Figs 163B, 182-194

\section{Diagnosis}

Distinguished from known congeners by combination of: procursus with long and straight ventral sclerite and slender dorsally-directed tip (identical to M. aurouxi gen. nov., cf. Figs 290-292; very similar to M. amezyan gen. et sp. nov. but smaller), distal bulbal sclerite with two small ventral teeth of different sizes and strongly protruding dorsal process (Figs 182-185; very similar to M. aurouxi and M. amezyan), epigynum without processes (Fig. 191; unlike M. aurouxi), dorsal arc of internal female genitalia without or with very indistinct posterior protrusions (Figs 187, 194; unlike M. aurouxi and M. amezyan); from all congeners also by pore plates in female internal genitalia (Fig. 187; pores in groups, right and left sides not clearly separated) and by slight troglomorphism: reduced eye size 
(diameter PME/distance PME-PME: 0.46; in other species: >0.60), thin legs (tibia $1 \mathrm{~L} / \mathrm{d}$ : 80; in other species: <70), and long legs (male tibia 1 length/carapace width: 7.8; in other species 5.3-7.0).

\section{Etymology}

The species name is an adjective derived from the Arabian ' $k a h f$ ' = 'cave'.

\section{Type material}

\section{Holotype}

MOROCCO - Souss-Massa • ○’; Agadir, Grotte des Chauves-souris, "812”; $30.6129^{\circ}$ N, $9.4671^{\circ}$ W; 780 m a.s.l.; 30 Oct. 2019; J. Lips leg.; ZFMK Ar 22357.

\section{Other material examined}

MOROCCO - Souss-Massa • 2 $q \circ$; same collection data as for holotype, "822", "782"; ZFMK

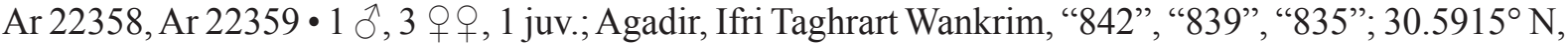

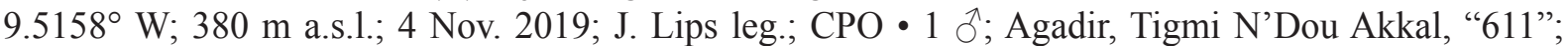

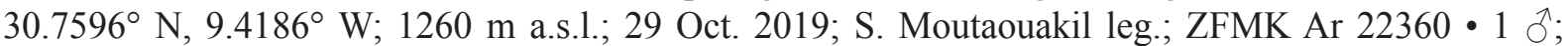
Agadir, Imi Ougoug, “543”; 30.6123 N, 9.4671 W; 770 m a.s.1.; 28 Oct. 2019; S. Moutaouakil leg.; ZFMK Ar 22361.

\section{Description}

Male (holotype)

MEAsurements. Total length 3.3, carapace width 1.3. Distance PME-PME $140 \mu \mathrm{m}$; diameter PME $65 \times$ $80 \mu \mathrm{m}$; distance PME-ALE $25 \mu \mathrm{m}$; diameter AME $40 \mu \mathrm{m}$; distance AME-AME $40 \mu \mathrm{m}$. Leg 1: $10.3+$ $0.5+10.2$, metatarsus broken; tibia $2: 6.4$, tibia $3: 4.8$, tibia $4: 5.3$; tibia $1 \mathrm{~L} / \mathrm{d}: 80$; femora $1-4$ diameters: $0.18,0.17,0.17,0.17$.

CoLOR (in ethanol). Carapace pale ochre to whitish, with light brown median Y-mark; sternum brown with dark brown radial marks; legs pale ochre-yellow, without dark rings, with very thin black lines on femora and tibiae (Figs 188-189); abdomen pale gray, with numerous whitish marks, few dark dorsal marks posteriorly only; ventrally with very indistinct spots in front of gonopore and between gonopore and spinnerets, only around spinnerets slightly more distinct dark mark.

Body. Habitus similar to M. aurouxi gen. nov. (cf. Fig. 260-261). Ocular area slightly raised. Deep thoracic pit and pair of shallow furrows diverging from pit toward posterior margin. Clypeus unmodified, rim not more sclerotized than in female. Sternum wider than long $(0.95 / 0.65)$, unmodified. Abdomen slightly elongated, dorso-posteriorly weakly angular.

Chelicerae. As in M. aurouxi gen. nov. and M. stifadma gen. et sp. nov. (cf. Fig. 233), with pair of low whitish proximal humps and pair of frontal lateral apophyses, each with one cone-shaped modified hair; distance between tips of modified hairs: $360 \mu \mathrm{m}$; lateral stridulatory ridges distinct, clearly visible in dissecting microscope.

PALPS. In general as in M. aurouxi gen. nov. (cf. Figs 285-287); coxa with rounded hump and small but distinct retrolateral-ventral process; trochanter barely modified (low ventral rounded hump); femur not curved towards dorsal, distally widened and with very low rounded ventral protrusion, proximally with prolateral stridulatory pick (modified hair), without retrolateral transversal line, with distinct retrolateral-ventral proximal process and distinct dorsal process; femur-patella joints shifted toward prolateral side; tibia relatively long and slender, tibia-tarsus joints shifted toward retrolateral side; tarsus without macrotrichia; procursus apparently identical to M. aurouxi gen. nov. (cf. Figs 290-292), some dorsal hairs slightly curved upwards; on prolateral side with proximal ridge followed by distinct 
hairless hump at half length; distally with low ventral flap; procursus tip with strong ventral sclerite and semitransparent process, main branch strongly curved towards dorsal, with semitransparent hairlike process and wide tip divided by retrolateral ridge; genital bulb (Figs 182-185) basal sclerite with indistinct dorsal apophysis; distal (main) sclerite large, with deep retrolateral pocket, strong dorsal process without teeth, ventral part with two ventral teeth of different sizes, sperm duct opening not seen.

LEGS. Femur 1 with single row of $\sim 25$ ventral spines; without curved hairs; few vertical hairs; retrolateral trichobothrium of tibia 1 at $2.5 \%$; prolateral trichobothrium absent on tibia 1, present on other leg tibiae; tarsal pseudosegments proximally irregular and indistinct, distally $\sim 5$ fairly distinct.

Male (variation)

Tibia 1 in two other males: 7.8, 10.0. Abdomen shape variable, sometimes more globular. Dark pigment slightly variable (abdomen sometimes monochromous whitish).

\section{Female}

In general similar to male but without spines on legs, with pair of indistinct whitish (almost transparent) humps posteriorly on carapace, and pair of corresponding indistinct plates frontally on abdomen;
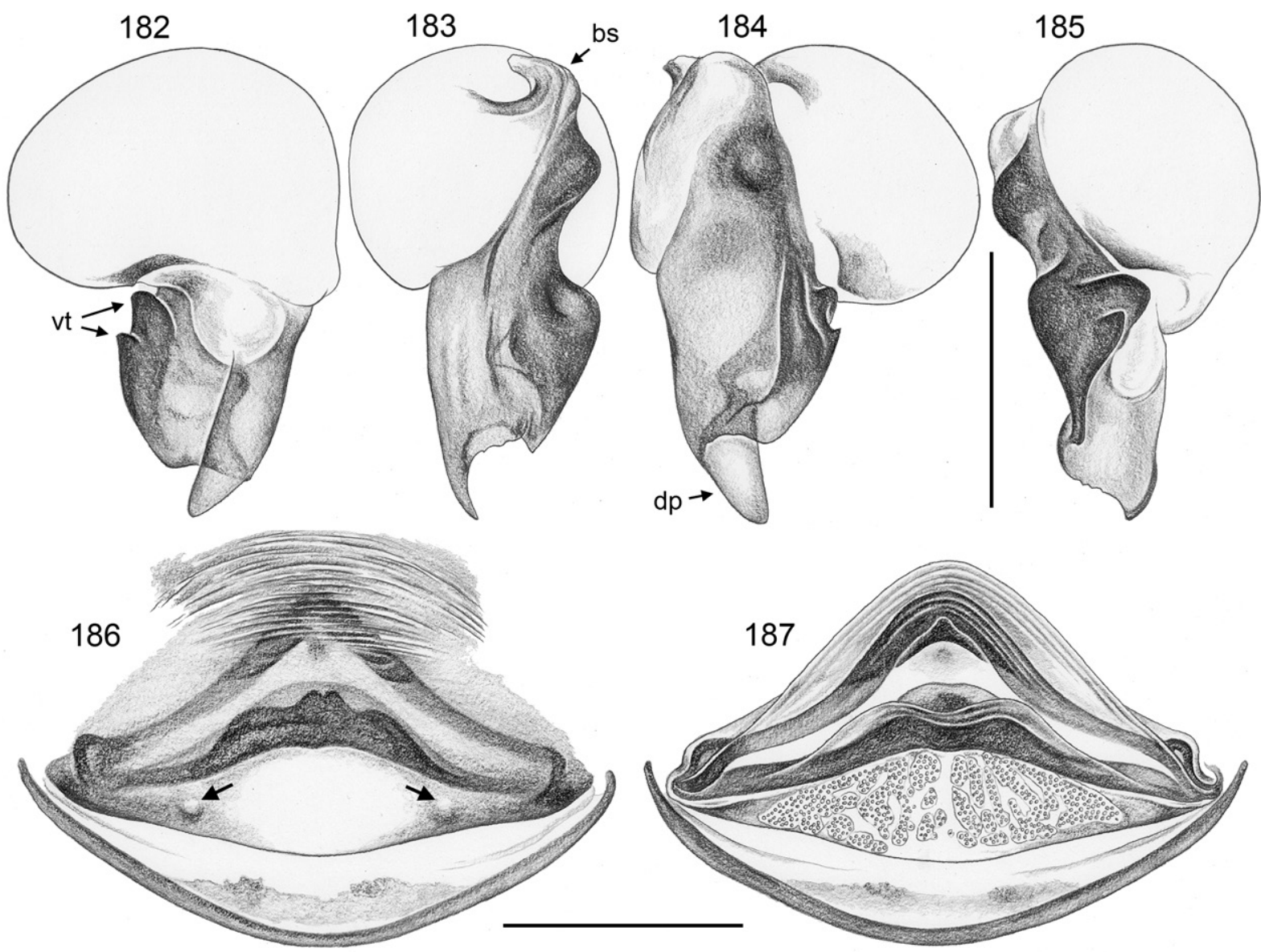

Figs 182-187. Maghreba kahfa gen. et sp. nov.; male and female from Morocco, Agadir, Ifri Taghrart Wankrim (CPO). 182-185. Left genital bulb, prolateral, dorsal, retrolateral, and ventral views. 186187. Cleared female genitalia, ventral and dorsal views; arrows: pockets (pits). Abbreviations: bs = basal sclerite; $\mathrm{dp}=$ dorsal process; $\mathrm{vt}=$ ventral teeth. Scale bars $=0.3 \mathrm{~mm}$. 
cheliceral stridulatory ridges not seen in dissecting microscope but apparently present (possible stridulatory pick present on palpal femur). Tibia 1 in four females: 8.8, 9.6, 10.8, 10.9. Epigynum as in Fig. 191, main epigynal plate triangular, weakly protruding, without processes; with pair of indistinct round pits (distance $330 \mu \mathrm{m}$ ); posterior plate short and wide, simple; indistinct plate (or brown area) in front of epigynum, not elevated. Internal genitalia (Figs 186-187, 192-194) with contiguous pore plates medially 'dissolved' into groups of pores; dorsal and ventral arcs both strongly developed, ventral arc with very indistinct ventral median process (pocket?).

\section{Natural history}

The specimens were found among rocks and on the cave wall (rather than under rocks) near the cave entrance but in complete darkness (J. Lips, pers. com. Jun. 2021).
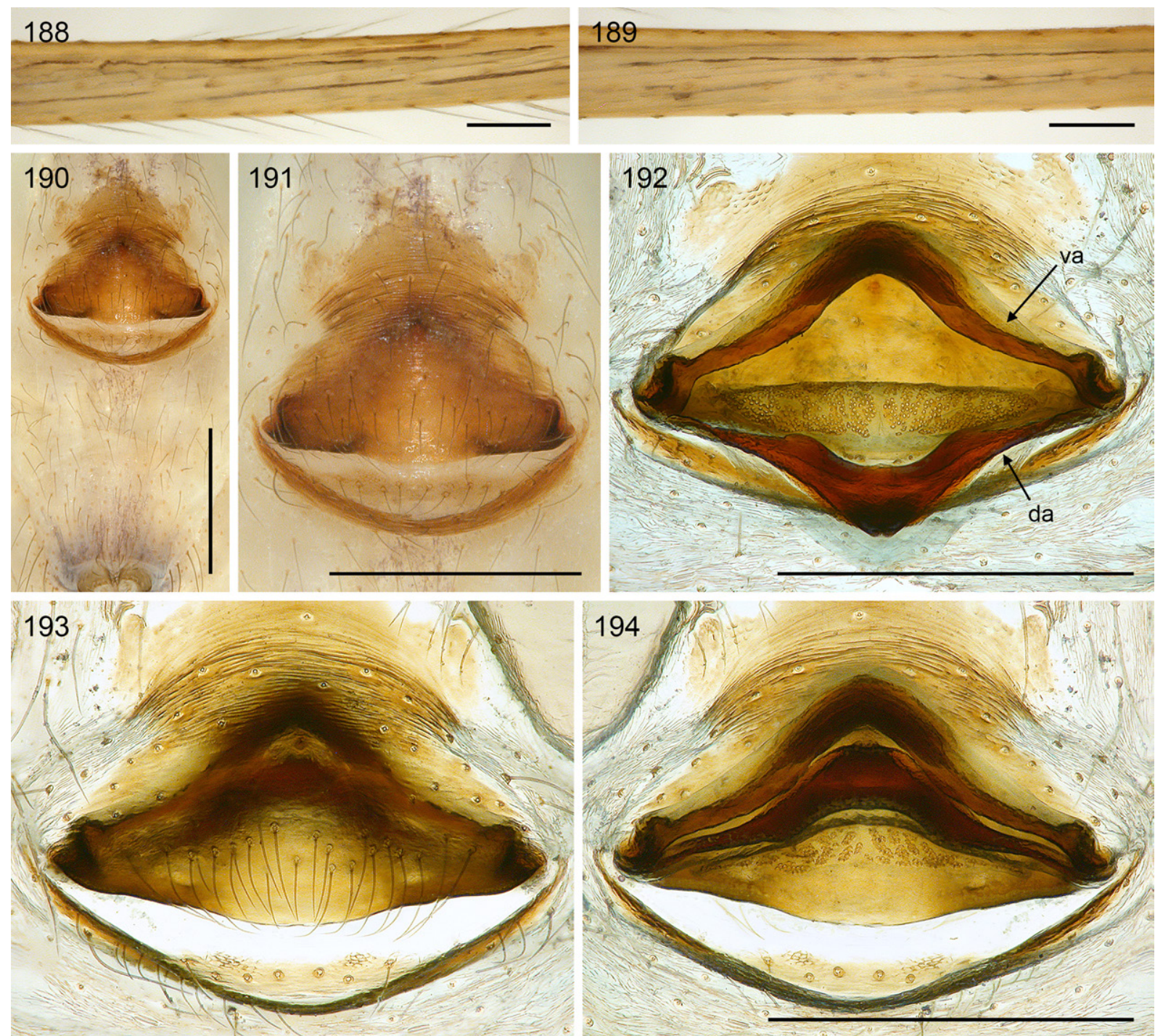

Figs 188-194. Maghreba kahfa gen. et sp. nov.; male and female from Morocco, Agadir, Ifri Taghrart Wankrim (CPO). 188-189. Male right femora 3 and 4, ventral views. 190-191. Female abdomen and epigynum, ventral views. 192-194. Cleared female genitalia, dorsal view with dorsal arc tilted backwards, ventral view, and regular dorsal view. Abbreviations: da = dorsal arc; va $=$ ventral arc. Scale bars: $188-189=0.2 \mathrm{~mm} ; 190-194=0.5 \mathrm{~mm}$. 


\section{Distribution}

Known from several neighboring localities in the western-most High Atlas, Morocco (Fig. 163B).

Maghreba saghro gen. et sp. nov. urn:lsid:zoobank.org:act:6CB9CA32-D460-48FA-BBA7-419353040F8F

Figs 163B, 195, 203-223

\section{Diagnosis}

Easily distinguished from known congeners by serrated ventral margin of distal bulbal sclerite (Figs 211, 213); also by combination of: procursus with short ventral sclerite (Fig. 210); epigynum short and wide, without processes (Figs 218-220), dorsal arc of internal female genitalia without pair of posterior protrusions (Fig. 222); from most known congeners (except M. amezyan gen. et sp. nov. and M. kahfa gen. et sp. nov.) also by long legs (male tibia 1 length $>9.0$; female tibia 1 length $>8.5$ ).

\section{Etymology}

The species name is derived from Djebel (Jbel) Saghro (see Distribution below); noun in apposition.

\section{Type material}

\section{Holotype}

MOROCCO - Souss-Massa ${ }^{\top}$; between Irherm and Tiferki, near road R106; 30.1406 N, $8.3337^{\circ} \mathrm{W}$; 1745 m a.s.l.; 16 Sep. 2018; B.A. Huber leg.; among rocks and boulders; ZFMK Ar 22362.

\section{Other material examined}

MOROCCO - Souss-Massa $・ 2 \hat{\partial}, 6$ 9 ; ; same collection data as for holotype; ZFMK Ar 22363, $\operatorname{Ar} 22364 \bullet 2$ 우 (in pure ethanol); same collection data as for holotype; ZFMK Mor86・1 $ð, 2$ 우 우 SE of Tazenakht; $30.534^{\circ} \mathrm{N}, 7.020^{\circ} \mathrm{W}$; $1200 \mathrm{~m}$ a.s.l.; 17 Sep. 2018; B.A. Huber leg.; along dry riverbed; ZFMK Ar 22365 • 3 우 (in pure ethanol); same collection data as for preceding; ZFMK Mor89 • 1 웅 between Tazenakht and Agdz, near road R108; 30.531 ${ }^{\circ} \mathrm{N}, 6.923^{\circ} \mathrm{W} ; 1365 \mathrm{~m}$ a.s.1.; 17 Sep. 2018; B.A. Huber leg.; under rock; ZFMK Ar 22366.

\section{Description}

\section{Male (holotype)}

Measurements. Total length 4.6, carapace width 1.65. Distance PME-PME $140 \mu \mathrm{m}$; diameter PME $110 \times 130 \mu \mathrm{m}$; distance PME-ALE $30 \mu \mathrm{m}$; diameter AME $100 \mu \mathrm{m}$; distance AME-AME $25 \mu \mathrm{m}$. Leg 1: $39.1(11.6+0.8+10.5+13.9+2.3)$, tibia $2: 7.3$, tibia $3: 5.7$, tibia $4: 6.4$; tibia $1 \mathrm{~L} / \mathrm{d}: 58$; femora $1-4$ diameters: $0.30,0.24,0.21,0.22$.

Color (in ethanol). Carapace pale ochre-yellow, medially darker (brown); sternum dark ochre, with darker radial marks; legs ochre-yellow, without dark rings, with black lines on femora and tibiae, very few also on metatarsi; abdomen gray, with distinct dorsal mark around heart area and indistinct dorsal and lateral marks; ventrally with large dark brown mark in front of gonopore, median dark band behind gonopore not reaching spinnerets, dark brown mark around spinnerets.

BoDy. Habitus as in Fig. 195. Ocular area slightly raised. Deep thoracic pit and pair of shallow furrows diverging from pit toward posterior margin. Clypeus unmodified but rim more sclerotized than in female. Sternum wider than long (1.20/0.75), unmodified. Abdomen slightly elongated, dorso-posteriorly weakly angular. 

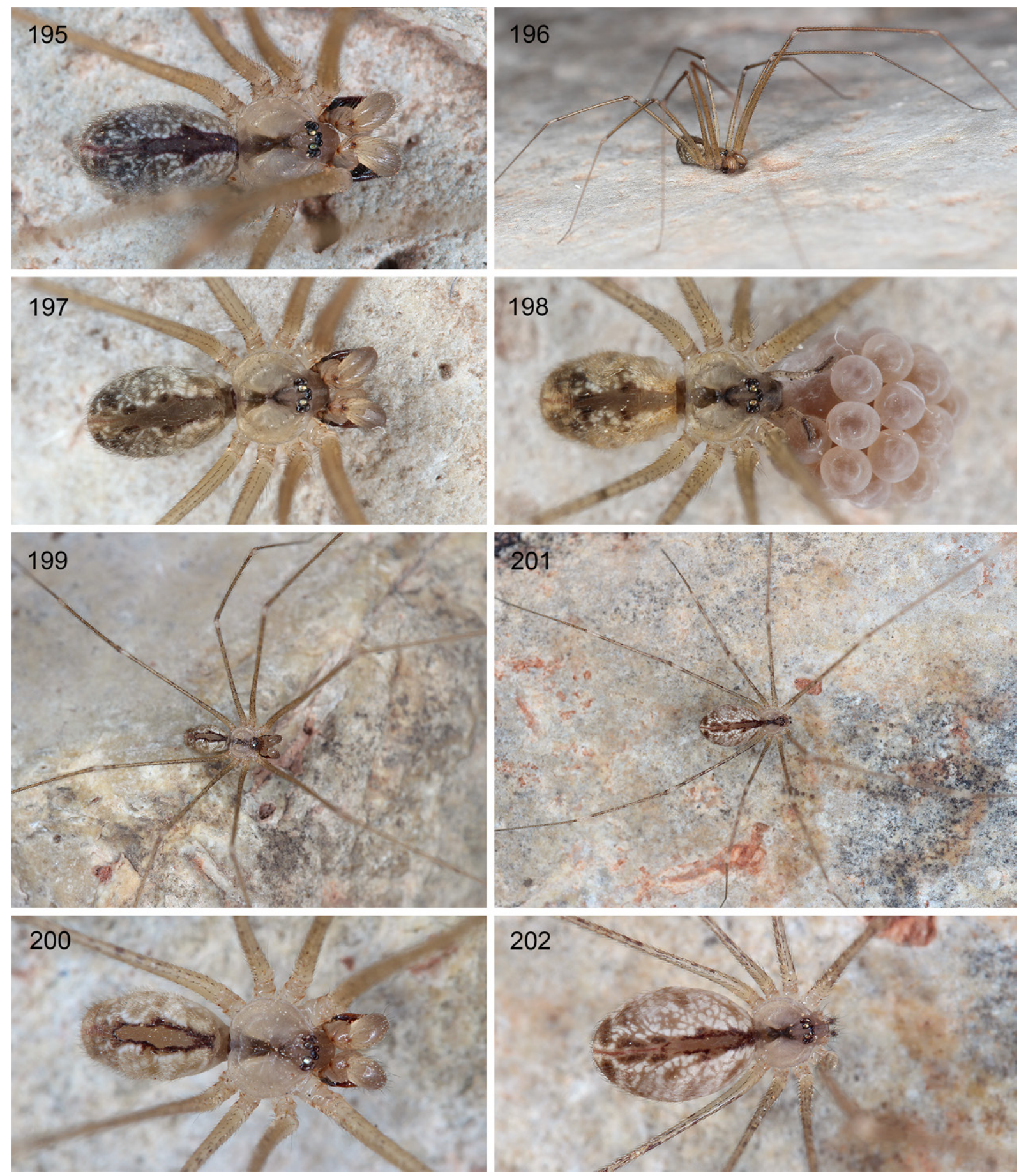

Figs 195-202. Maghreba gen. nov., live specimens. 195. M. saghro gen. et sp. nov., male from Morocco, between Irherm and Tiferki. 196-198. M. stifadma gen. et sp. nov., male and female from Morocco, Setti-Fatma. 199-202. M. nkob gen. et sp. nov., male and female from Morocco, E of Nkob. 
Chelicerae. As in Figs 214-215, with low frontal hump proximally and pair of frontal lateral apophyses distally, each with one large modified cone-shaped hair at tip; distance between tips of modified hairs: $580 \mu \mathrm{m}$; lateral stridulatory ridges very fine, barely visible in dissecting microscope.

PalPs. As is Figs 203-205; coxa with distinct retrolateral-ventral process; trochanter barely modified, slightly protruding ventrally; femur not curved towards dorsal, distally widened with very low rounded ventral protrusion, proximally with prolateral stridulatory pick (modified hair), with barely visible retrolateral transversal line, with distinct retrolateral-ventral proximal process and small dorsal process; femur-patella joints slightly shifted toward prolateral side; tibia relatively long and slender, tibia-tarsus joints shifted toward retrolateral side; tarsus without macrotrichia; procursus (Figs 208-210) with few hairs slightly curved upwards; on prolateral side with strong hairless hump, procursus tip strongly curved towards dorsal, with strong ventral sclerite and semitransparent process; genital bulb (Figs 211-213) basal sclerite with distinct dorsal apophysis; distal (main) sclerite large, with deep retrolateral pocket, distinct ventral serration; sperm duct opening not seen.

LEGS. Femur 1 with single row of $\sim 25$ ventral spines; without curved hairs; with more than usual vertical hairs on all tibiae (dorsally and laterally); retrolateral trichobothrium of tibia 1 at $3.5 \%$; prolateral trichobothrium absent on tibia 1, present on other leg tibiae; tarsal pseudosegments irregular, very indistinct.

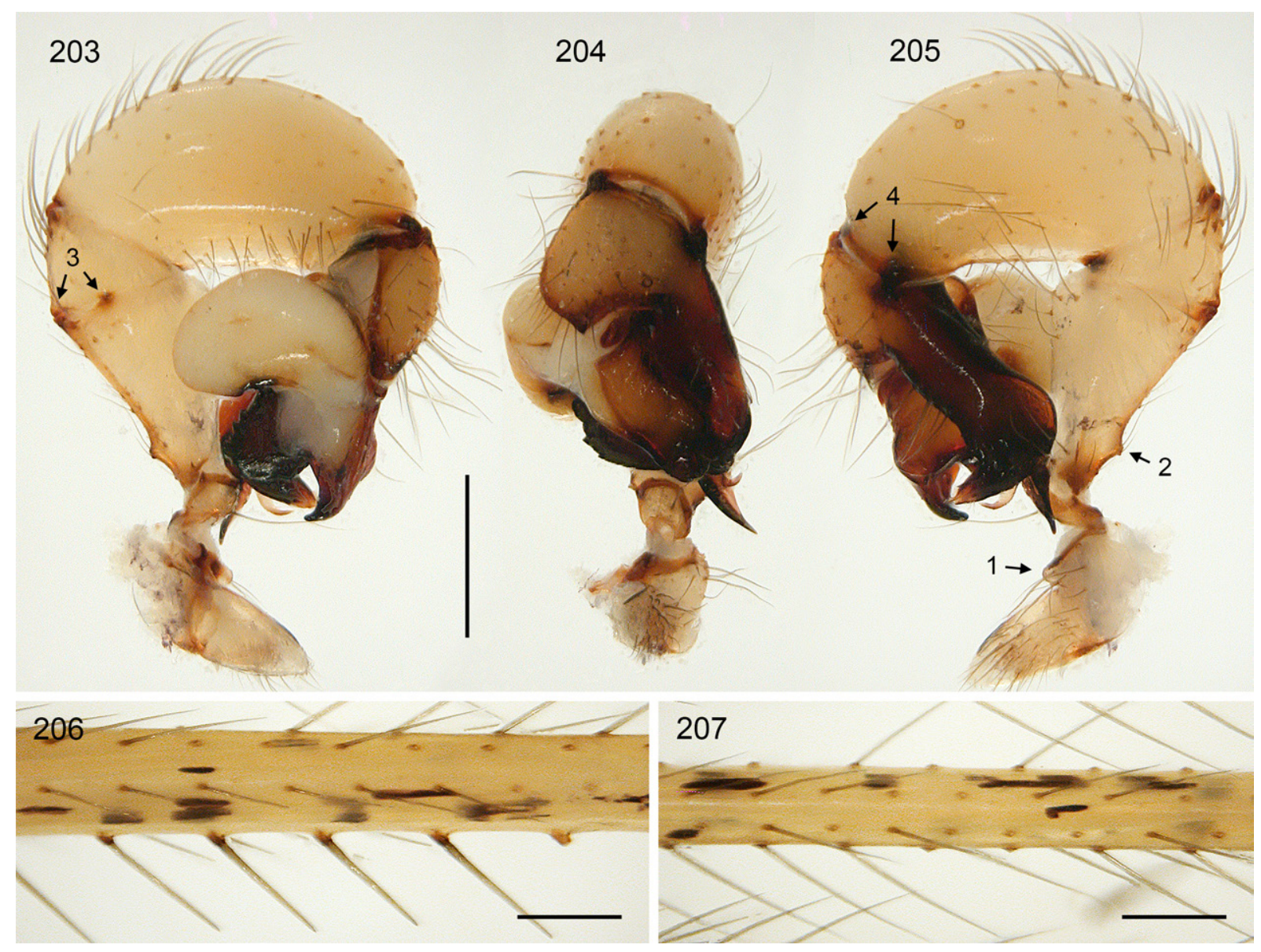

Figs 203-207. Maghreba saghro gen. et sp. nov.; male from Morocco, between Irherm and Tiferki (ZFMK Ar 22363). 203-205. Left male palp, prolateral, dorsal, and retrolateral views; arrow 1: coxa apophysis; arrow 2: dorsal femur apophysis; arrows 3: femur-patella joints; arrows 4: tibia-tarsus joints. 206-207. Male femora 1 and 2. Scale bars: 203-205 $=0.5 \mathrm{~mm} ; 206-207=0.3 \mathrm{~mm}$. 

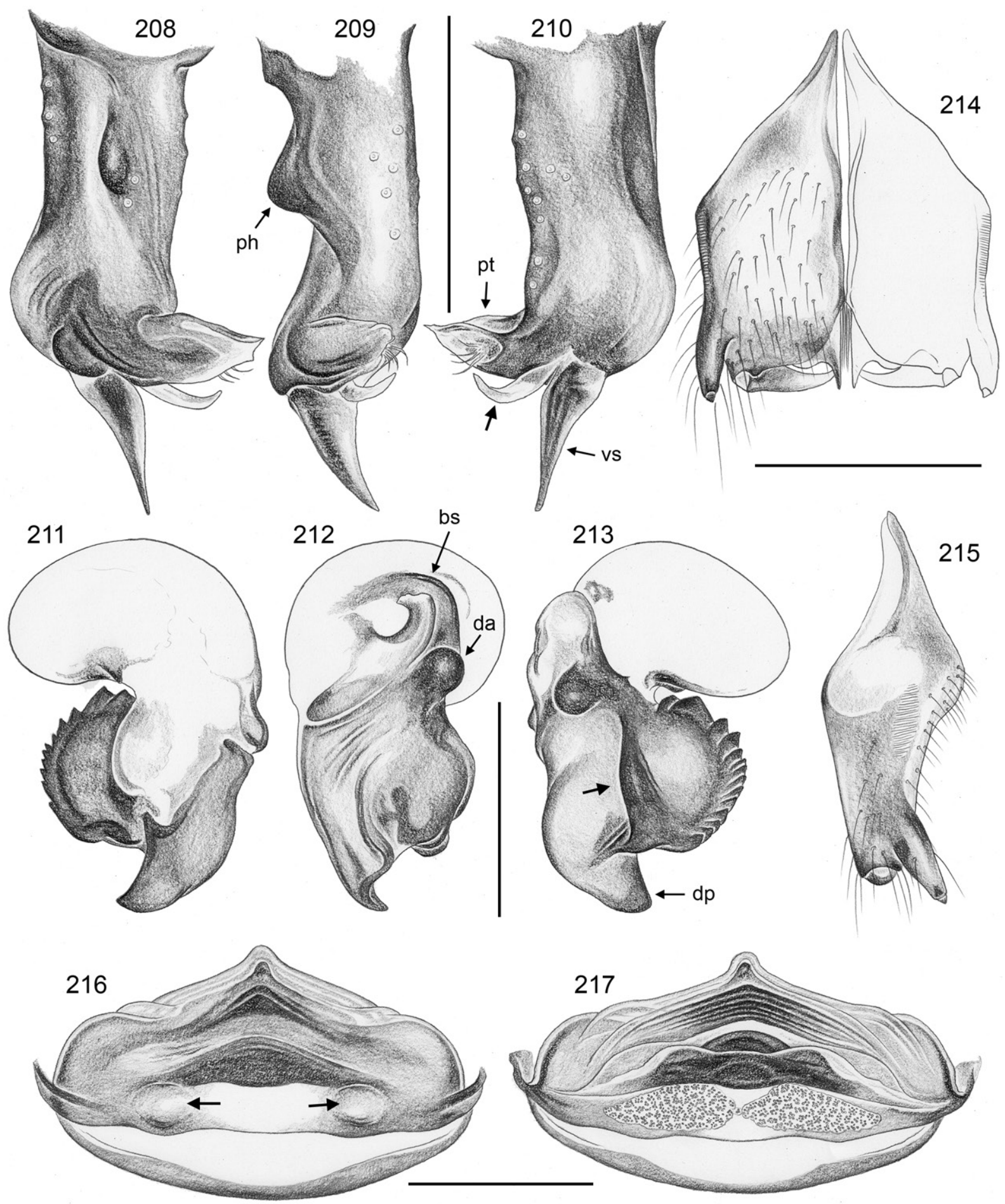

Figs 208-217. Maghreba saghro gen. et sp. nov.; male and female from Morocco, between Irherm and Tiferki (ZFMK Ar 22363, Ar 22364). 208-210. Left procursus, prolateral, dorsal, and retrolateral views; arrow: semitransparent process. 211-213. Left genital bulb, prolateral, dorsal, and retrolateral views; arrow: deep pocket. 214-215. Male chelicerae, frontal and lateral views. 216-217. Cleared female genitalia, ventral and dorsal views; arrows: pockets (pits). Abbreviations: $b s=$ basal sclerite; $d a=$ dorsal apophysis; $\mathrm{dp}=$ dorsal process; $\mathrm{ph}=$ prolateral hump; $\mathrm{pt}=$ procursus tip; $\mathrm{vs}=$ ventral sclerite. Scale bars $=0.5 \mathrm{~mm}$. 
Male (variation)

Tibia 1 in three other males: 9.3, 10.1, 10.5.

\section{Female}

In general similar to male but without spines on legs, with usual very low number of short vertical hairs on leg tibiae. With pair of very indistinct light humps posteriorly on carapace, corresponding plates frontally on abdomen not seen; with fine cheliceral stridulatory ridges as in male. Tibia 1 in nine females:
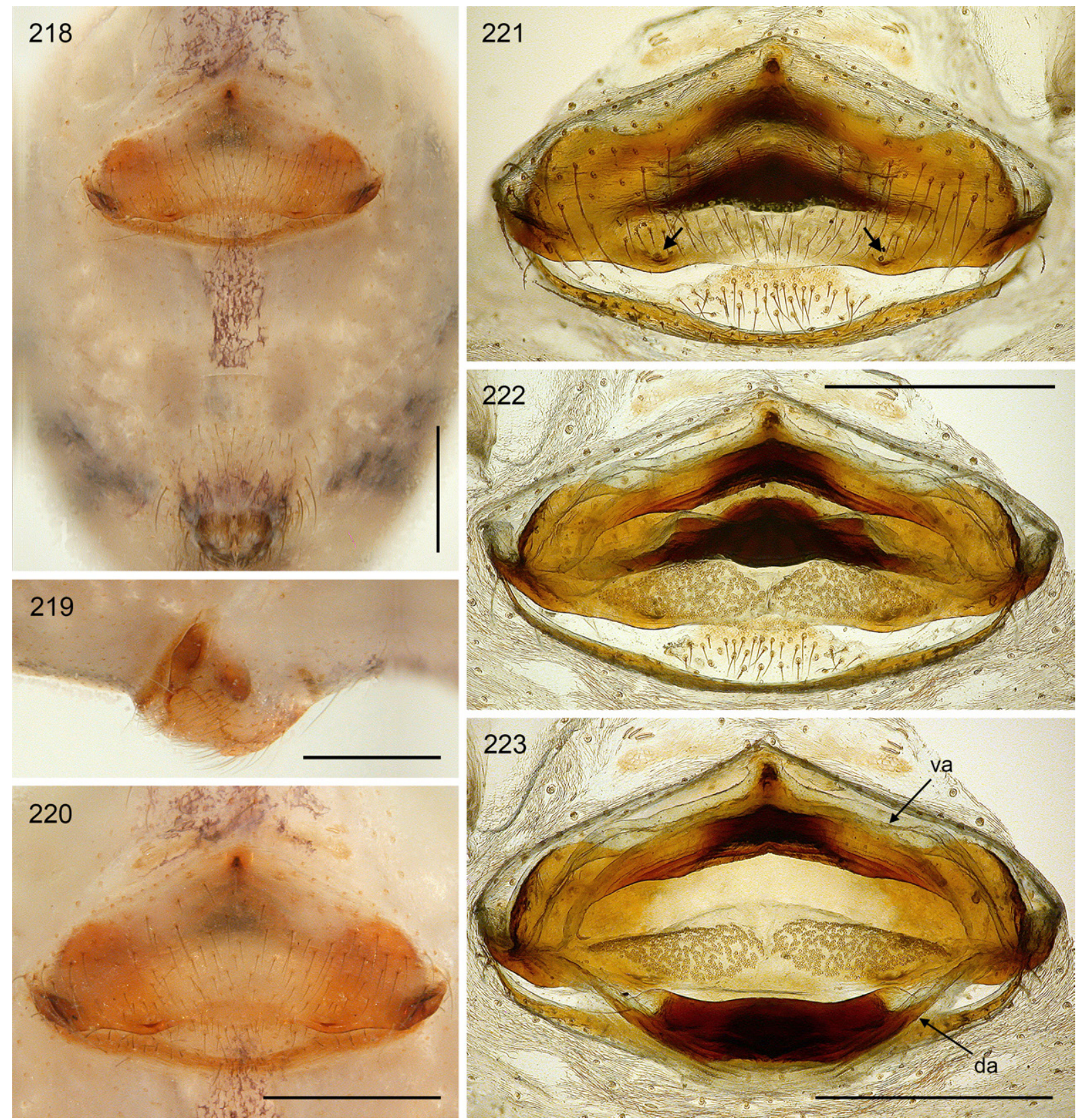

Figs 218-223. Maghreba saghro gen. et sp. nov.; female from Morocco, between Irherm and Tiferki (ZFMK Ar 22364). 218. Abdomen, ventral view. 219-220. Epigynum, lateral and ventral views. 221223. Cleared female genitalia, ventral view, regular dorsal view, and dorsal view with dorsal arc tilted backwards; arrows: pockets (pits). Abbreviations: $\mathrm{da}=$ dorsal arc; va $=$ ventral arc. Scale bars $=0.5 \mathrm{~mm}$. 
8.7-10.0 (mean 9.3). Epigynum as in Figs 218-220, main epigynal plate large relative to abdomen, wider than long, weakly protruding, without processes; medially weakly sclerotized except small brown plate anteriorly; with pair of variably distinct pockets (distance between pockets $500-530 \mu \mathrm{m}$ ); internal sclerotized arc and median round structure variably visible in uncleared specimens; posterior plate short and wide, simple; pair of indistinct narrow plates in front of epigynum, not elevated. Internal genitalia (Figs 216-217, 221-223) with elongated pore plates, contiguous and in transversal position; dorsal and ventral arcs medially strongly sclerotized, ventral arc with simple ventral process (pocket?) directly 'below' (i.e., dorsal of) epigynal plate.

\section{Natural history}

Between Irherm and Tiferki, this species was found among large rocks and boulders on a dry slope and in a small canyon; at the same place, $M$. aurouxi gen. nov. occupied a small cave. In other places, the spiders were found in washed-out sand cavities along a dry riverbed (SE of Tazenakht) and under a rock (between Tazenakht and Agdz).

\section{Distribution}

Known from three localities in the Djebel (Jbel) Saghro range of the Moroccan Atlas (Fig. 163B).

Maghreba stifadma gen. et sp. nov. urn:Isid:zoobank.org:act:9F617A8F-D984-47DE-8D55-01C12B6A2E66

Figs 163B, 196-198, 224-242

\section{Diagnosis}

Distinguished from known congeners by distinctive pair of epigynal processes (Fig. 238); also by combination of: slender procursus (Fig. 229; in contrast to M. gharbija gen. et sp. nov.) with long dorsallydirected tip (in contrast to $M$. nkob gen. et sp. nov., M. djabalija gen. et sp. nov.); distal bulbal sclerite with two short ventral teeth (Fig. 232; in contrast to M. saghro gen. et sp. nov., M. nkob, M. gharbija, M. djabalija) and relatively short dorsal process (in contrast to M. amezyan gen. et sp. nov., M. kahfa gen. et sp. nov., M. aurouxi gen. nov.); epigynum only slightly wider than long (Fig. 239; in contrast to M. saghro); dorsal arc of female internal genitalia without pair of posterior processes (Fig. 236; in contrast to M. aurouxi); no troglomorphism (in contrast to M. kahfa).

\section{Etymology}

The species name refers to the type locality; noun in apposition.

\section{Type material}

\section{Holotype}

MOROCCO - Marrakesh-Safi • Oे $^{\wedge}$ Setti-Fatma (= Sti Fadma); 31.221 ${ }^{\circ} \mathrm{N}, 7.671^{\circ} \mathrm{W}-31.219^{\circ} \mathrm{N}$, 7.669 ${ }^{\circ}$ W; 1600-1690 m a.s.l.; 11 Sep. 2018; B.A. Huber leg.; on overhanging rocks; ZFMK Ar 22367.

\section{Other material examined}

MOROCCO - Marrakesh-Safi $• 5$ $\widehat{\partial}, 16$ 우, 1 juv.; same collection data as for holotype; ZFMK $\operatorname{Ar} 22368$, Ar 22369 • 4 q , 1 juv. (in pure ethanol); same collection data as for holotype; ZFMK Mor71 • 1 q; Zaouia Sti Fatma; approximately $31.255^{\circ} \mathrm{N}, 7.675^{\circ} \mathrm{W}$ (label: $31.217,-7.700$ ); 1 Dec. 1987; V.D. and

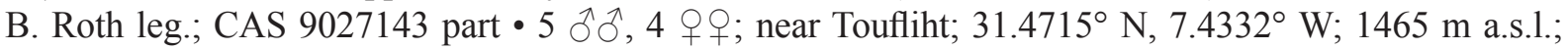
27 Sep. 2018; B.A. Huber leg.; under rocks in ravine; ZFMK Ar 22370 • 2 q + , 1 juv. (in pure ethanol); same collection data as for preceding; ZFMK Mor106 • 1 đ, 3 q 9 ; Ait Barka; $31.49^{\circ} \mathrm{N}, 7.45^{\circ} \mathrm{W}$;

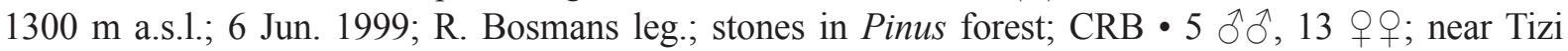
n'Tichka; $31.3120^{\circ}$ N, 7.3752 ${ }^{\circ}$ W; 2035 m a.s.1.; 27 Sep. 2018; B.A. Huber leg.; under rocks; ZFMK Ar 22371, Ar 22372 • 4 우 (in pure ethanol); same collection data as for preceding; ZFMK Mor109. 


\section{Description}

Male (holotype)

MeAsurements. Total length 2.9, carapace width 1.2. Distance PME-PME $90 \mu \mathrm{m}$; diameter PME $80 \times$ $100 \mu \mathrm{m}$; distance PME-ALE $25 \mu \mathrm{m}$; diameter AME $65 \mu \mathrm{m}$; distance AME-AME $20 \mu \mathrm{m}$. Leg 1: 22.7 $(6.5+0.5+6.3+7.8+1.6)$, tibia $2: 4.1$, tibia $3: 3.0$, tibia $4: 3.5$; tibia $1 \mathrm{~L} / \mathrm{d}: 53$; femora $1-4$ diameters: $0.23,0.18,0.18,0.18$.

COLOR (in ethanol). Carapace pale ochre-yellow, medially darker brown (including posterior part of ocular area); sternum brown with darker and lighter radial marks; legs ochre-yellow, without dark rings, with elongate black marks on femora and tibiae; abdomen ochre-gray, with distinct dorsal mark around heart area and dorsal and lateral dark and whitish marks; ventrally with large dark brown mark in front of gonopore, pair of small marks behind gonopore, dark brown area at spinnerets.

Body. Habitus as in Figs 196-197. Ocular area slightly raised. Deep thoracic pit and pair of shallow furrows diverging from pit toward posterior margin. Clypeus unmodified, rim not more sclerotized than in female. Sternum wider than long (0.90/0.55), unmodified. Abdomen slightly elongated, dorsoposteriorly weakly angular.

Chelicerae. As in Figs 233-234, with pair of low whitish proximal humps and pair of frontal lateral apophyses, each with one cone-shaped modified hair; distance between tips of modified hairs: $330 \mu \mathrm{m}$; lateral stridulatory ridges very fine, barely visible in dissecting microscope.

Palps. As in Figs 224-226; coxa with distinct retrolateral-ventral process; trochanter barely modified; femur not curved towards dorsal, distally widened and with very low rounded ventral protrusion, proximally with prolateral stridulatory pick (modified hair), without retrolateral transversal line, with distinct retrolateral-ventral proximal process and distinct dorsal process; femur-patella joints shifted toward prolateral side; tibia relatively long and slender, tibia-tarsus joints shifted toward retrolateral side;

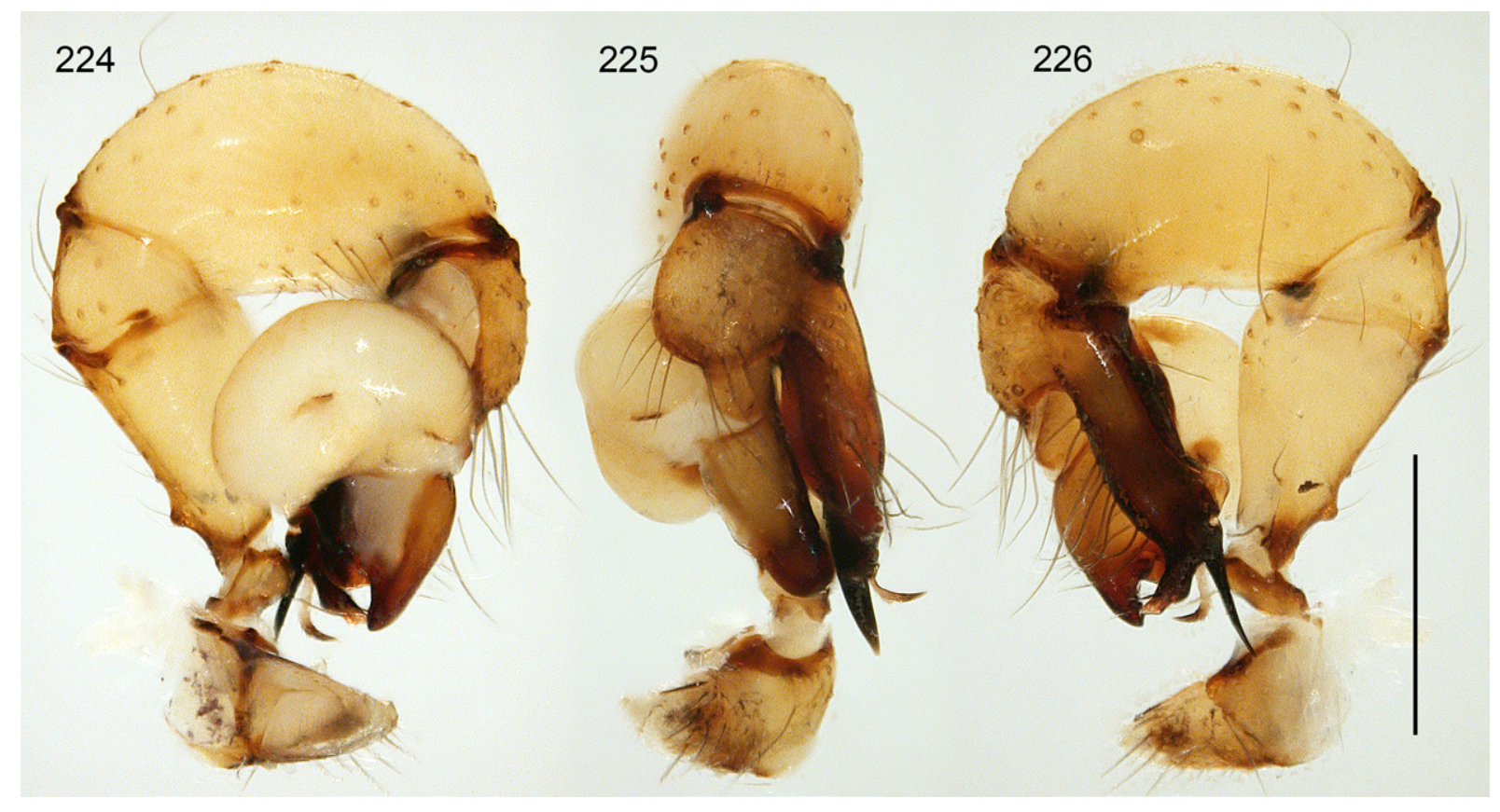

Figs 224-226. Maghreba stifadma gen. et sp. nov.; male from Morocco, Setti-Fatma (ZFMK Ar 22368); left palp, prolateral, dorsal, and retrolateral views. Scale bar $=0.5 \mathrm{~mm}$. 


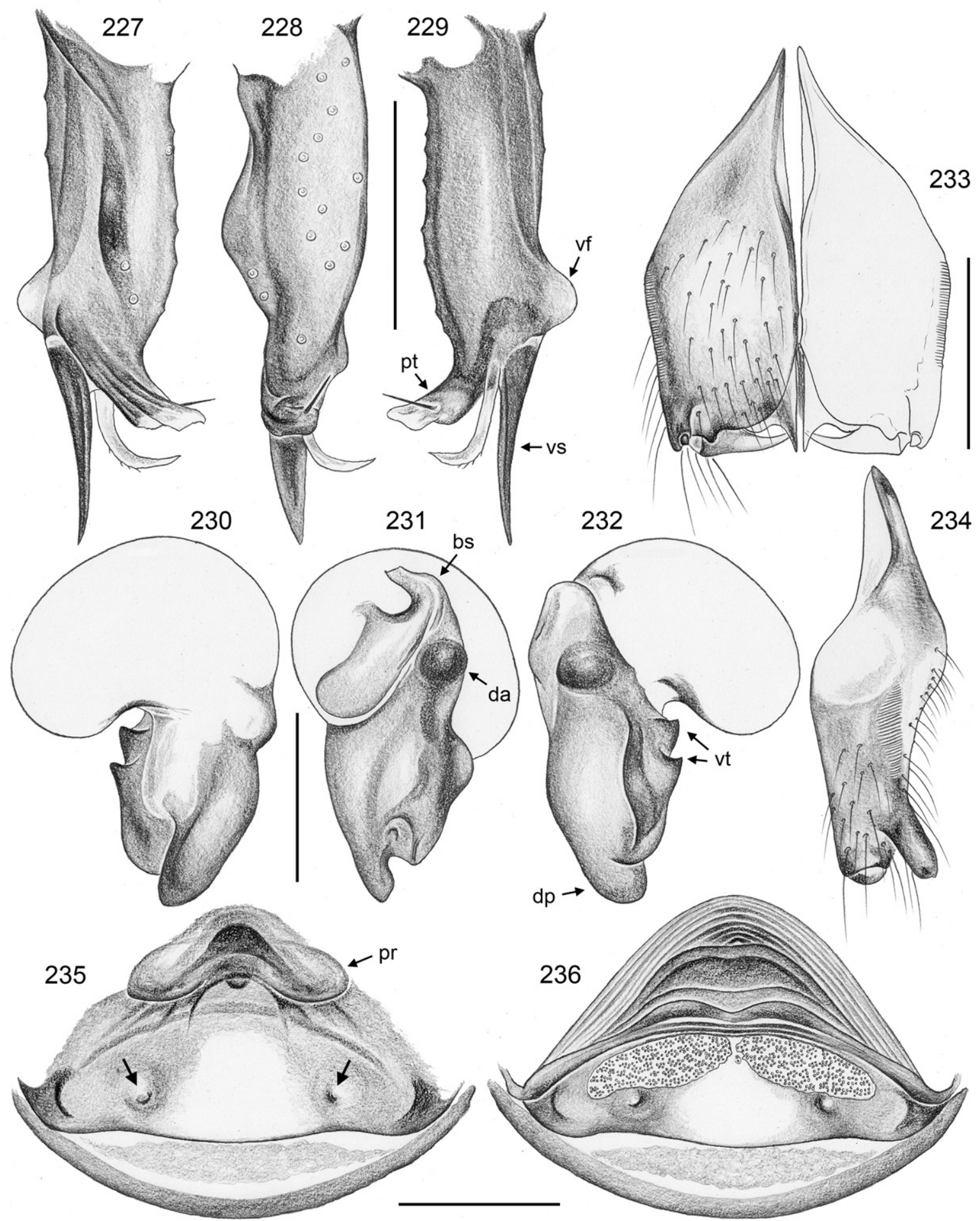

Figs 227-236. Maghreba stifadma gen. et sp. nov.; male and female from Morocco, Setti-Fatma (ZFMK Ar 22368, Ar 22369). 227-229. Left procursus, prolateral, dorsal, and retrolateral views. 230-232. Left genital bulb, prolateral, dorsal, and retrolateral views. 233-234. Male chelicerae, frontal and lateral views. 235-236. Cleared female genitalia, ventral and dorsal views; arrows: pockets (pits). Abbreviations: bs = basal sclerite; $\mathrm{da}=$ dorsal apophysis; $\mathrm{dp}=$ dorsal process; $\mathrm{pr}=$ epigynal process; $\mathrm{pt}=$ procursus tip; $\mathrm{vf}=$ ventral flap; $v s=$ ventral sclerite; $v t=$ ventral teeth. Scale bars $=0.3 \mathrm{~mm}$. 

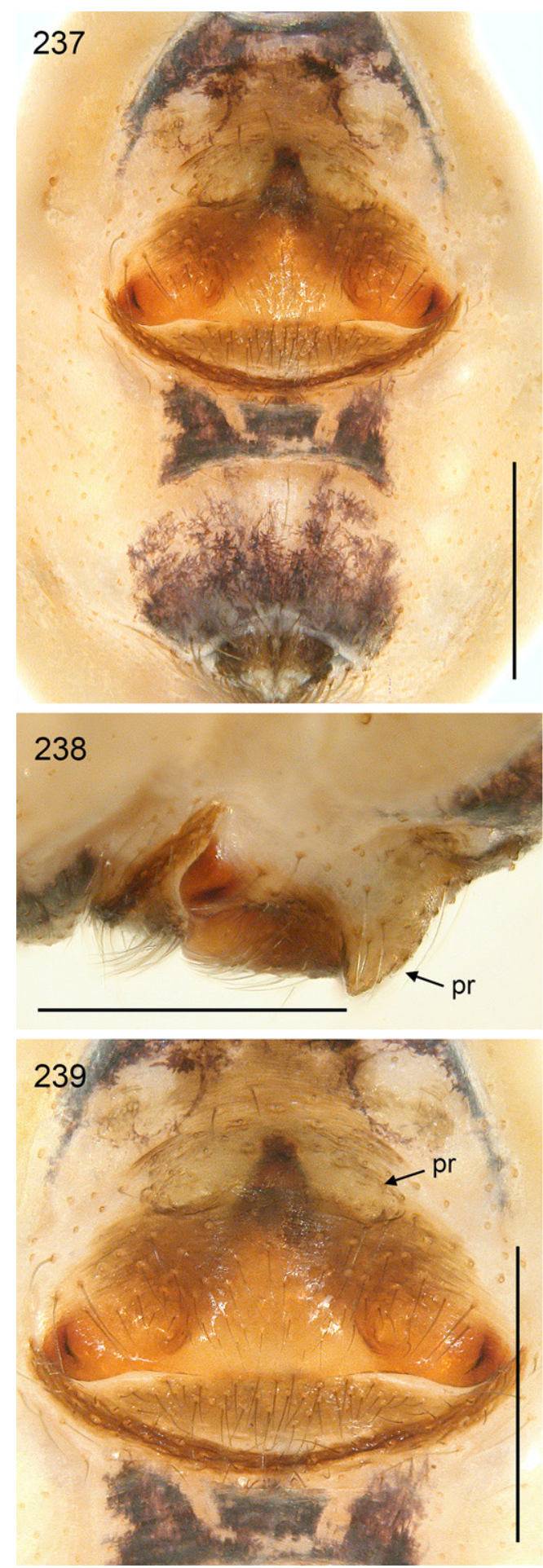
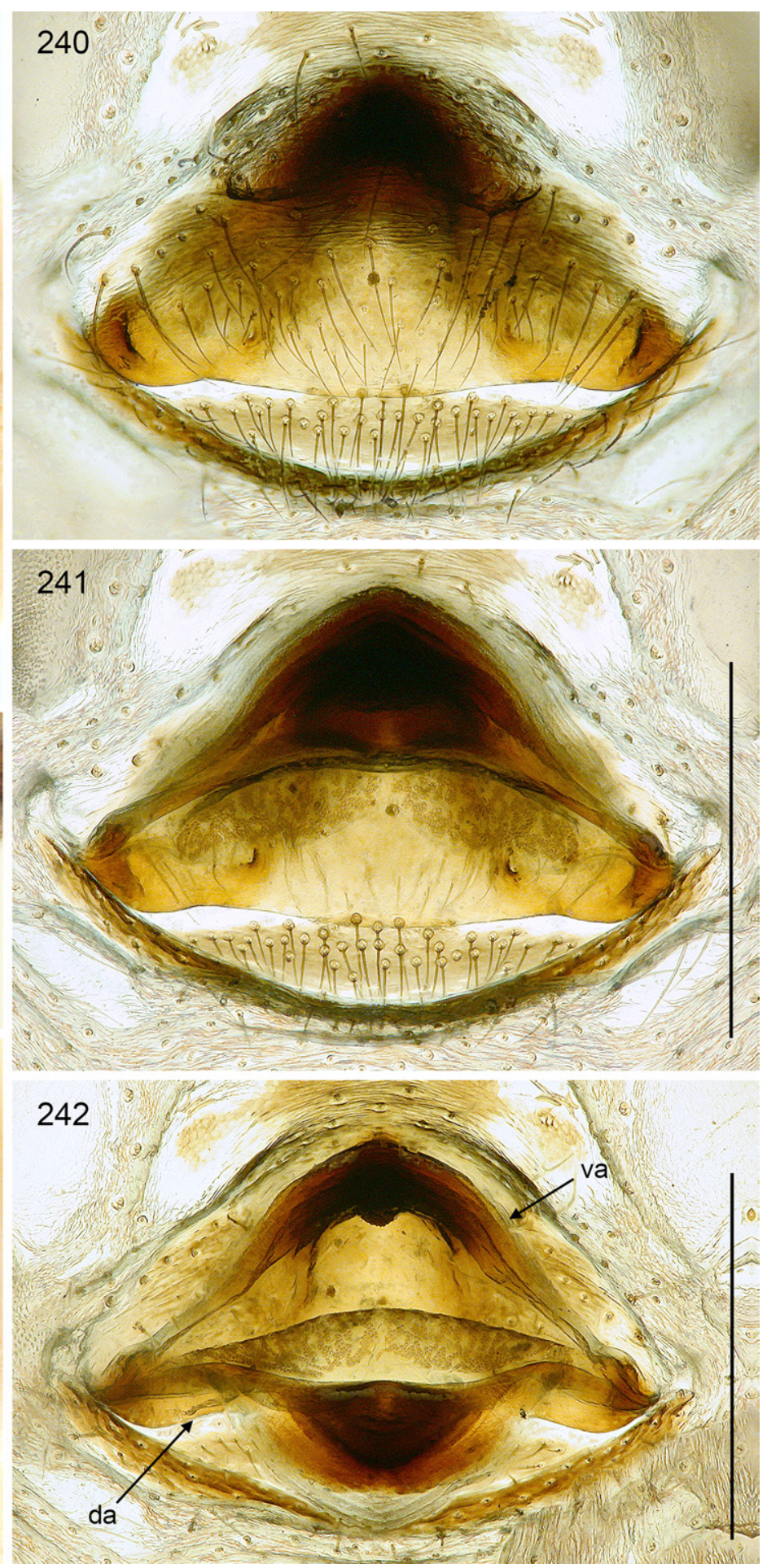

Figs 237-242. Maghreba stifadma gen. et sp. nov.; female from Morocco, Setti-Fatma (ZFMK Ar 22369). 237. Abdomen, ventral view. 238-239. Epigynum, lateral and ventral views. 240-242. Cleared female genitalia, ventral view, regular dorsal view, and dorsal view with dorsal arc tilted backwards. Abbreviations: $\mathrm{da}=$ dorsal arc; $\mathrm{pr}=$ epigynal process; $\mathrm{va}=$ ventral arc. Scale bars $=0.5 \mathrm{~mm}$. 
tarsus without macrotrichia; procursus (Figs 227-229) with few dorsal hairs slightly curved upwards; on prolateral side with low proximal ridge and more prominent hump (ridge) at half length, both hairless; with semitransparent ventral flap; procursus tip strongly curved towards dorsal, with distinct hair-like process, with strong and long ventral sclerite and semitransparent process; genital bulb (Figs 230-232) basal sclerite with distinct dorsal apophysis; distal (main) sclerite large, with deep retrolateral pocket, with two ventral teeth, without serration, short dorsal process, sperm duct opening not seen.

LEGs. Femur 1 with single row of $\sim 20$ ventral spines; without curved hairs; with barely more than usual vertical hairs on tibiae (only proximally); retrolateral trichobothrium of tibia 1 at $4 \%$; prolateral trichobothrium absent on tibia 1, present on other leg tibiae; tarsal pseudosegments proximally irregular and indistinct, distally $\sim 5-10$ fairly distinct.

Male (variation)

Tibia 1 in 17 males (including holotype): 5.5-7.0 (mean 6.2). Distance between tips of cheliceral apophyses $\sim 320-370 \mu \mathrm{m}$. Ventral abdominal band variably distinct, median part sometimes quite 'complete', i.e., with three parallel bands, sometimes reduced to small irregular marks.

\section{Female}

In general similar to male (Fig. 198) but without spines on legs, with usual very low number of short vertical hairs on leg tibiae. With pair of indistinct whitish humps posteriorly on carapace, and pair of corresponding indistinct plates frontally on abdomen, the latter in some females not visible; with fine cheliceral stridulatory ridges as in male. Tibia 1 in 34 females: 5.0-6.7 (mean 5.7). Epigynum as in Figs 237-239, main epigynal plate trapezoidal, wider than long, weakly protruding, anteriorly with pair of distinct whitish processes, tips of processes variably rather rounded or pointed; with small brown median plate between whitish processes; with pair of indistinct round pits (distance 300-340 $\mu \mathrm{m}$ ); posterior plate short and wide, simple; indistinct plate in front of epigynum, not elevated. Internal genitalia (Figs 235-236, 240-242) with elongated pore plates in transversal position, almost contiguous; dorsal and ventral arcs medially strongly sclerotized, ventral arc with very indistinct ventral process (pocket?) directly 'below' (i.e., dorsal of) small median plate between whitish processes.

\section{Natural history}

At Setti-Fatma, the spiders were abundant on the undersides of rocks, often very close to specimens of an undescribed species of Pholcus Walckenaer, 1805. Only juveniles had distinctly domed sheets, while older webs did not seem to have a clear shape. Some webs included a very narrow dome and had small whitish silk puffs. At disturbance, the spiders rarely vibrated but rather ran away and then dropped to the ground. Near Toufliht, most spiders were found under rocks on the ground of a ravine, while Pholcus occupied suitable niches higher on the rocks. Near Tizi n'Tichka, at over $2000 \mathrm{~m}$ a.s.l., the spiders were abundant under rocks on a dry slope.

\section{Distribution}

Known from several localities in the High Atlas, Morocco (Fig. 163B). All records are from between 1300 and $2035 \mathrm{~m}$ a.s.1.

Maghreba nkob gen. et sp. nov. urn:lsid:zoobank.org:act:D21218A6-089B-4264-BE1A-7BF13DB40B52

Figs 163B, 199-202, 243-256

\section{Diagnosis}

Easily distinguished from known congeners by details of male palp (Figs 243-248; dorsally-directed tip of procursus short and wide, procursus ventral sclerite short, procursus ventral flap distinct; dorsal 
process of distal bulbal sclerite only slightly longer than ventral part, ventral part with series of distinctive teeth) and epigynum (Fig. 253; triangular and relatively long, with pair of small processes).

\section{Etymology}

The species name refers to the type locality; noun in apposition.

\section{Type material}

\section{Holotype}

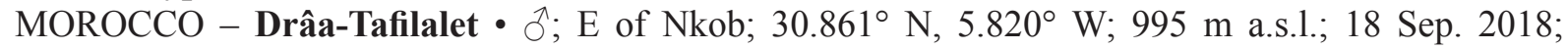
B.A. Huber leg.; at bases of palm trees; ZFMK Ar 22373.

\section{Other material examined}

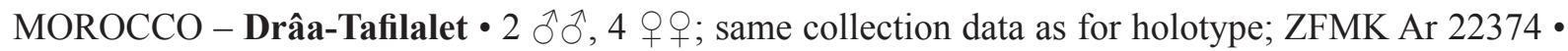
2 우, 1 juv. (in pure ethanol); same collection data as for holotype; ZFMK Mor91 1 § ; "road NekobMellal" [Nkob, Mlal]; $30.86^{\circ} \mathrm{N}, 5.80^{\circ} \mathrm{W}$; $940 \mathrm{~m}$ a.s.1.; 22 Apr. 2012; R. Bosmans leg.; stones in palm

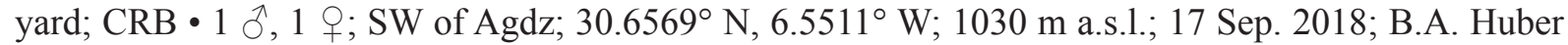
leg.; at bases of palm trees; ZFMK Ar $22375 \bullet 2 \circ \circ, 1$ juv. (in pure ethanol); same collection data as

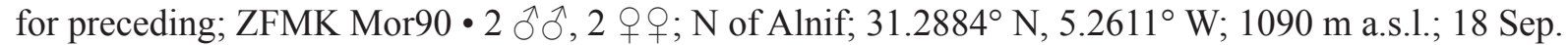
2018; B.A. Huber leg.; at rocks in dry riverbed; ZFMK Ar 22376 • 2 9 9,1 juv. (in pure ethanol); same

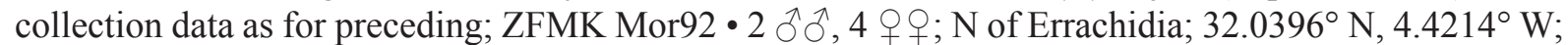
1180 m a.s.l.; 20 Sep. 2018; B.A. Huber leg.; dry ravine, among rocks near ground; ZFMK Ar 22377 • 2 우 (in pure ethanol); same collection data as for preceding; ZFMK Mor96.

ALGERIA -2 우 (1 prosoma missing); Wil. Bechar, Taghit; $30.93^{\circ} \mathrm{N}, 2.03^{\circ} \mathrm{W} ; 630 \mathrm{~m}$ a.s.1.; 3 Apr. 1989; R. Bosmans leg.; palm gardens; CRB.

\section{Description}

\section{Male (holotype)}

Measurements. Total length 3.2, carapace width 1.2. Distance PME-PME $100 \mu \mathrm{m}$; diameter PME $80 \times$ $90 \mu \mathrm{m}$; distance PME-ALE $20 \mu \mathrm{m}$; diameter AME $75 \mu \mathrm{m}$; distance AME-AME $20 \mu \mathrm{m}$. Leg 1: 26.5 $(7.7+0.5+7.2+9.3+1.8)$, tibia $2: 5.0$, tibia $3: 3.8$, tibia $4: 4.4$; tibia $1 \mathrm{~L} / \mathrm{d}: 58$; femora $1-4$ diameters: $0.20,0.16,0.14,0.14$.

COLOR (in ethanol). Carapace pale ochre-yellow, medially darker (brown; except ocular area); sternum ochre-yellow, darker posteriorly and with darker radial marks; legs ochre-yellow, without dark rings, with black lines on femora and tibiae, very few also on metatarsi; abdomen gray, with distinct dorsal mark around heart area and dorsal and lateral whitish marks; ventrally with large dark brown mark in front of gonopore, median dark band behind gonopore divided into three parallel bands, dark brown area at spinnerets.

Body. Habitus as in Figs 199-200. Ocular area slightly raised. Deep thoracic pit and pair of shallow furrows diverging from pit toward posterior margin. Clypeus unmodified, rim barely more sclerotized than in female. Sternum wider than long $(0.85 / 0.60)$, unmodified. Abdomen slightly elongated, dorsoposteriorly weakly angular.

Chelicerae. Shape as in M. saghro gen. et sp. nov. (cf. Figs 214-215), but smaller (maximum width: $460 \mu \mathrm{m}$ ) and pair of frontal lateral apophyses more cylindrical in lateral view (rather as in M. stifadma gen. et sp. nov.; cf. Fig. 234); distance between tips of modified hairs: $380 \mu \mathrm{m}$; lateral stridulatory ridges very fine, barely visible in dissecting microscope. 


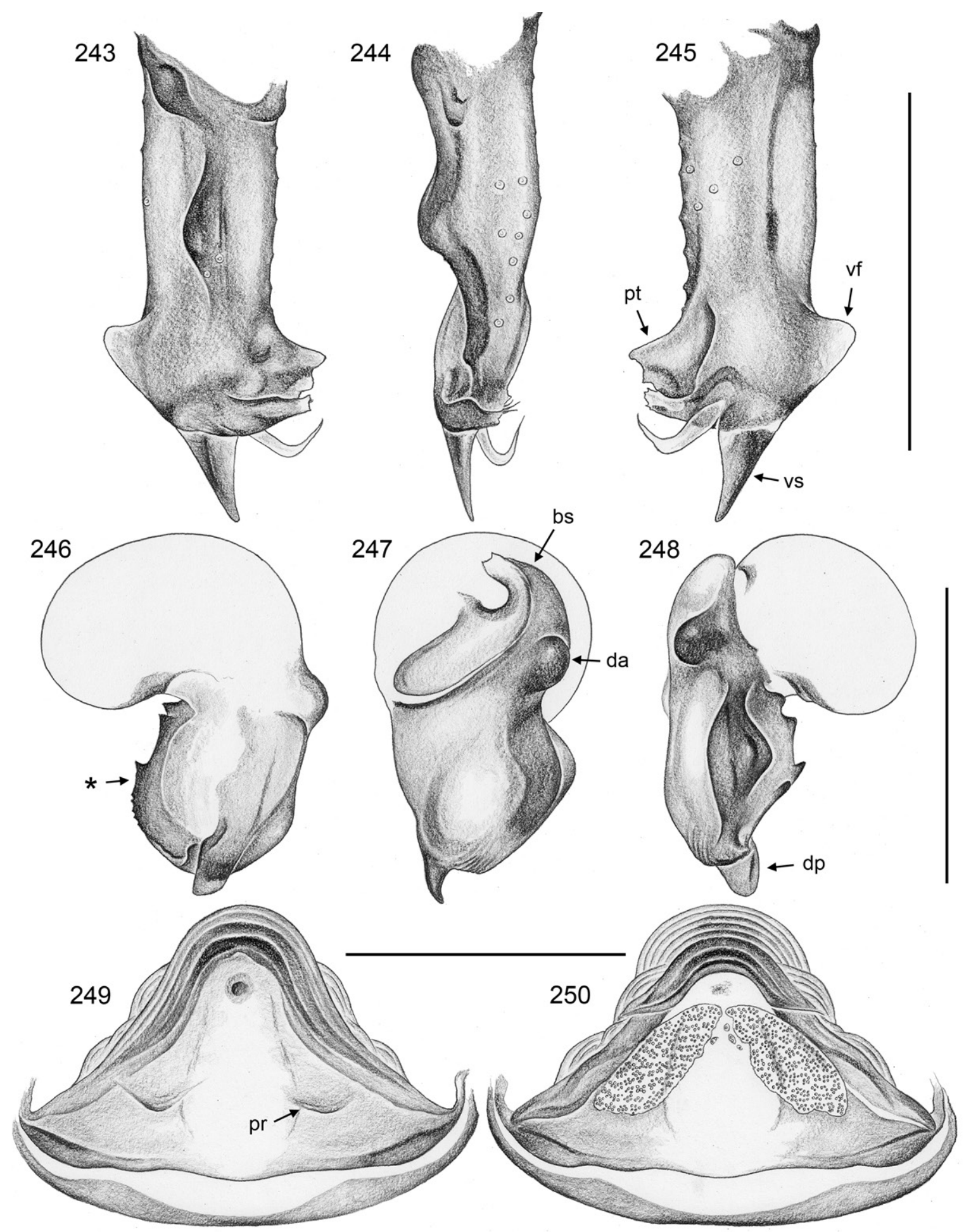

Figs 243-250. Maghreba nkob gen. et sp. nov.; male and female from Morocco, E of Nkob (ZFMK Ar 22374). 243-245. Left procursus, prolateral, dorsal, and retrolateral views. 246-248. Left genital bulb, prolateral, dorsal, and retrolateral views; asterisk: one or two additional small teeth present in males from $\mathrm{N}$ of Alnif and $\mathrm{N}$ of Errachidia. 249-250. Cleared female genitalia, ventral and dorsal views. Abbreviations: $\mathrm{bs}=$ basal sclerite; $\mathrm{da}=$ dorsal apophysis; $\mathrm{dp}=$ dorsal process; $\mathrm{pr}=$ epigynal process; $\mathrm{pt}=$ procursus tip; $\mathrm{vf}=$ ventral flap; $\mathrm{vs}=$ ventral sclerite. Scale bars $=0.5 \mathrm{~mm}$. 

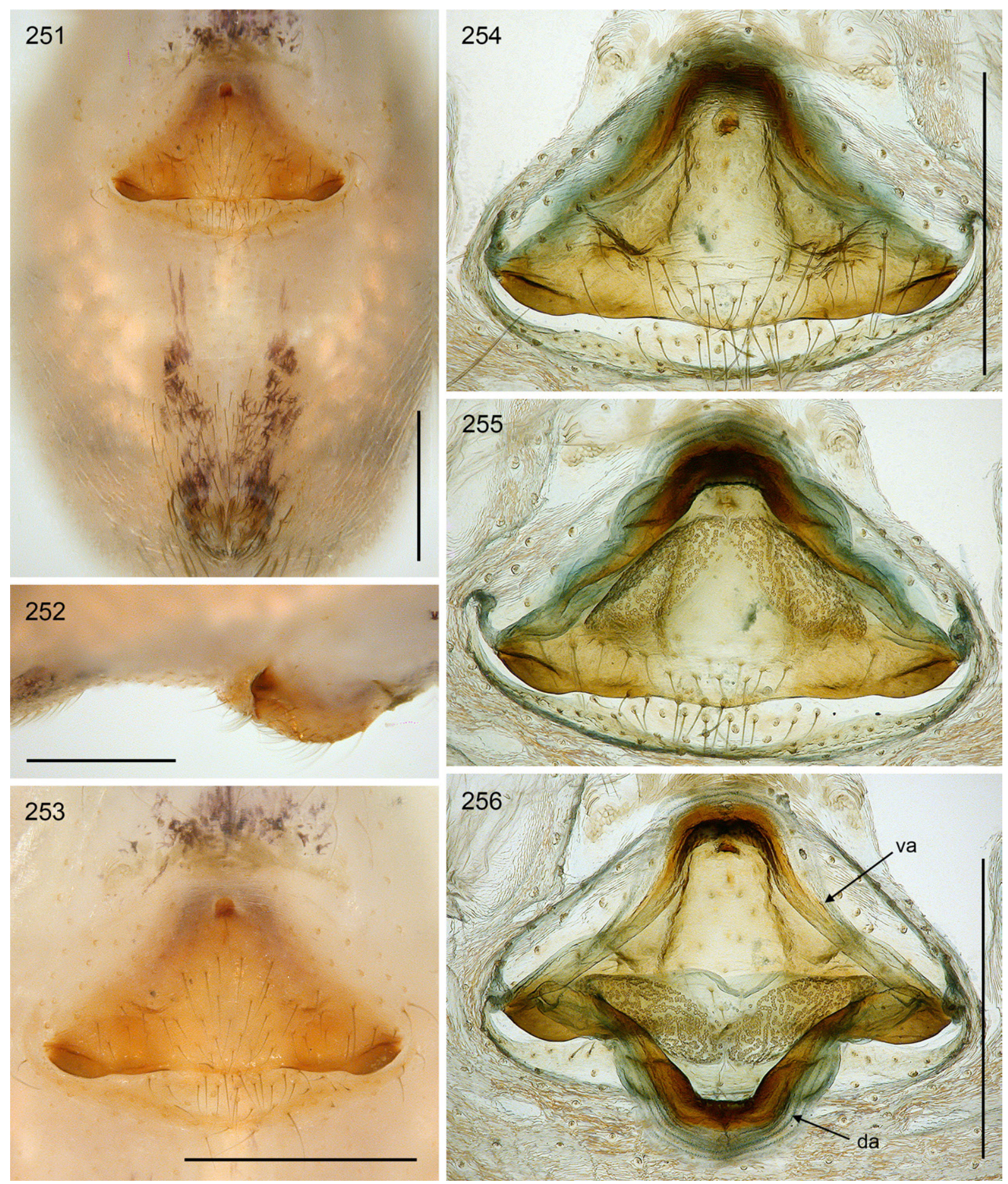

Figs 251-256. Maghreba nkob gen. et sp. nov.; female from Morocco, E of Nkob (ZFMK Ar 22374). 251. Abdomen, ventral view. 252-253. Epigynum, lateral and ventral views. 254-256. Cleared female genitalia, ventral view, regular dorsal view, and dorsal view with dorsal arc tilted backwards. Abbreviations: $\mathrm{da}=$ dorsal arc; $\mathrm{va}=$ ventral arc. Scale bars $=0.5 \mathrm{~mm}$. 
PALPS. In general similar to congeners (cf. Figs 203-205, 224-226); coxa with low retrolateral-ventral process; trochanter barely modified; femur not curved towards dorsal, distally widened and with low rounded ventral protrusion, proximally with prolateral stridulatory pick (modified hair), without retrolateral transversal line, with distinct retrolateral-ventral proximal process and very indistinct dorsal process; femur-patella joints slightly shifted toward prolateral side; tibia relatively long and slender, tibia-tarsus joints shifted toward retrolateral side; tarsus without macrotrichia; procursus (Figs 243-245) with few dorsal hairs slightly curved upwards; on prolateral side with two humps, both hairless; with semitransparent ventral flap; procursus tip strongly curved towards dorsal but ending abruptly, with strong but short ventral sclerite and semitransparent process; genital bulb (Figs 246-248) basal sclerite with dorsal apophysis; distal (main) sclerite large, with deep retrolateral pocket, with three ventral teeth and fine serration distally, short dorsal process, sperm duct opening not seen.

Legs. Femur 1 with single row of $\sim 28$ ventral spines; without curved hairs; with more than usual vertical hairs on all tibiae (mostly prolaterally); retrolateral trichobothrium of tibia 1 at $3.5 \%$; prolateral trichobothrium absent on tibia 1, present on other leg tibiae; tarsal pseudosegments irregular and indistinct except $\sim 3-5$ distally.

Male (variation)

Tibia 1 in ten males (including holotype): 7.2-8.8 (mean 7.9). Distance between tips of cheliceral apophyses in larger males $\sim 400-420 \mu \mathrm{m}$. Ventral abdominal band variably distinct, median part sometimes reduced to small irregular marks. Males from $\mathrm{N}$ of Alnif with one additional ventral tooth on distal bulbal sclerite (asterisk in Fig. 246); males from $\mathrm{N}$ of Errachidia with two additional teeth in same area.

\section{Female}

In general similar to male (Figs 201-202) but without spines on legs, with usual very low number of short vertical hairs on leg tibiae. With pair of whitish but distinct humps posteriorly on carapace, and pair of corresponding indistinct plates frontally on abdomen; with fine cheliceral stridulatory ridges as in male. Tibia 1 in nine females: 6.4-8.5 (mean 7.5). Epigynum as in Figs 251-253, main epigynal plate triangular, weakly protruding, with pair of very low processes (in some females barely visible), medially weakly sclerotized except small brown plate anteriorly; with pair of indistinct round pockets (distance 380-420 $\mu \mathrm{m}$ ); internal sclerotized arc and median round structure variably visible in uncleared specimens; posterior plate short and wide, simple; indistinct plate in front of epigynum, not elevated. Internal genitalia (Figs 249-250, 254-256) with elongated pore plates, converging anteriorly, almost contiguous; dorsal and ventral arcs medially strongly sclerotized, ventral arc with very indistinct ventral process (pocket?) directly 'below' (i.e., dorsal of) dark median area of epigynal plate.

\section{Natural history}

Several records are associated with palms, but the species occurs in a variety of near-ground microhabitats. Near Nkob, most specimens were found at the bases of palm trees, but some also at the bases of other trees, under a dead palm trunk on the ground, and one specimen in a small stable made of mud. Near Agdz, the spiders built relatively large webs at the bases of palm leaves near the ground. They were very difficult to catch (i.e., possibly well protected from certain predators) because of the long spines on the hard palm leaves and because the spiders fled rapidly at the slightest disturbance. Near Alnif and Errachidia, the spiders were collected at large rocks at the side of a dry riverbed and among rocks and overhangs near the ground in a dry ravine.

\section{Distribution}

Widely distributed along the southern slopes of the eastern Moroccan and western Algerian Atlas range (Fig. 163B). 


\section{Maghreba gharbija gen. et sp. nov. urn:lsid:zoobank.org:act:134E8E3D-7C0C-45ED-B2DD-78475A180514}

Figs 163B, 257-259, 265-284

\section{Diagnosis}

Easily distinguished from known congeners by long teeth on distal bulbal sclerite (prolateral and ventral views; Figs 270, 273); from most species (except $M$. djabalija gen. et sp. nov.) also by small sclerite on procursus tip between ventral sclerite and transparent process (arrow in Fig. 267). From the geographically neighboring M. amezyan gen. et sp. nov. also by smaller size (carapace width: $1.3 \mathrm{vs}$ 1.7; male tibia 1 length $<9$ vs $>11$; female tibia 1 length $<8$ vs $>9$ ); from the geographically neighboring M. kahfa gen. et sp. nov. also by larger eyes (AME diameter 95 vs $40 \mu \mathrm{m}$ ) and thicker legs (male tibia 1 L/d 60 vs 80$)$.

\section{Etymology}

The species name is an adjective derived from the Arab 'gharbija' = 'western'.

\section{Type material}

\section{Holotype}

MOROCCO - Marrakesh-Safi • ${ }^{\top} ; 4$ km E of Ghazoua; 31.449 $\mathrm{N}, 9.688^{\circ} \mathrm{W}$; $60 \mathrm{~m}$ a.s.1.; 29 Sep. 2018; B.A. Huber leg.; in small ravines; ZFMK Ar 22378.

\section{Other material examined}

MOROCCO - Marrakesh-Safi • $3 \widehat{\partial}, 10$ q $\propto$; same collection data as for holotype; ZFMK Ar 22379, $\operatorname{Ar} 22380 \bullet 3$ 우 (in pure ethanol); same collection data as for holotype; ZFMK Mor111 • 3 $\widehat{\partial}, 9$ 우; $8 \mathrm{~km}$ E of Ghazoua; $31.459^{\circ} \mathrm{N}$, 9.646 ${ }^{\circ} \mathrm{W} ; 100 \mathrm{~m}$ a.s.1.; 29 Sep. 2018; B.A. Huber leg.; under rocks and at tree bases; ZFMK Ar 22381, Ar 22382 - 3 우, 1 juv. (in pure ethanol); same collection data as for

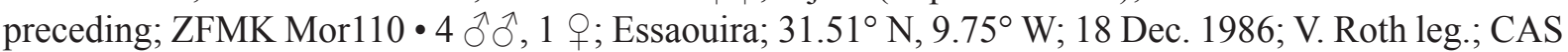
9027136. - Souss Massa • 1 ; $12 \mathrm{~km} \mathrm{~N}$ of Agadir, $2 \mathrm{~km} \mathrm{E} \mathrm{of} \mathrm{Aourir} \mathrm{[Awrir];} 30.496^{\circ} \mathrm{N}, 9.626^{\circ} \mathrm{W}$; 75 m a.s.1.; 3 Feb. 1996; R. Bosmans leg.; stones near river; CRB • 1 J, 3 q $\circ$; Agadir; $30.4^{\circ} \mathrm{N}, 9.6^{\circ} \mathrm{W}$; May 1939; L. Berland leg.; MNHN Ar $10321 ・ 1$ ô, 1 q; Agadir S; $30.37^{\circ}$ N, $9.58^{\circ}$ W; 16 Feb. 2007; R. Bosmans leg.; stones bordering salt marsh; CRB • 1 क , 1 juv.; Forêt d'Ademime; $30.32^{\circ} \mathrm{N}, 9.37^{\circ} \mathrm{W}$; May 1939; L. Berland leg.; MNHN Ar 10312 • 1 क; same collection data as for preceding; Ar $10317 \bullet$

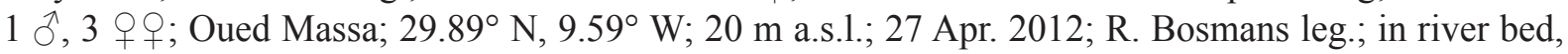
litter in Eucalyptus forest; CRB 11 q; Tiznit; $29.70^{\circ} \mathrm{N}, 9.73^{\circ} \mathrm{W}$; May 1939; L. Berland leg.; MNHN Ar $10329 \bullet 1$ Ō, 7 क $O$; Aglou; $29.806^{\circ}$ N, $9.828^{\circ}$ W; 10 m a.s.1.; 14 Sep. 2018; B.A. Huber leg.; among rocks near ground; ZFMK Ar 22383 - 3 우 (in pure ethanol); same collection data as for preceding; ZFMK Mor79 • $1{ }^{\text {T}}$; Aglou beach; 29.81 ${ }^{\circ} \mathrm{N}, 9.83^{\circ} \mathrm{W} ; 25 \mathrm{~m}$ a.s.1.; 26 Apr. 2012; R. Bosmans leg.; stony steppe; CRB • 1 O, 7 우; same collection data as for preceding; J. Van Keer leg.; CJVK・ 3 o $9 ; 5 \mathrm{~km}$ E of Gourizim; $29.62^{\circ}$ N, $9.95^{\circ}$ W; 26 Apr. 2012; J. Van Keer leg.; stones in Argania steppe; CJVK •

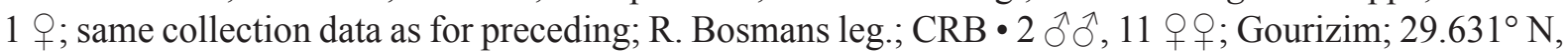
$10.003^{\circ} \mathrm{W} ; 20 \mathrm{~m}$ a.s.1.; 14 Sep. 2018; B.A. Huber leg.; among rocks near ground and in vertical bank of dry riverbed; ZFMK Ar 22384 • 4 우 (in pure ethanol); same collection data as for preceding; ZFMK Mor80 • 1 o; Gourizim; $29.63^{\circ} \mathrm{N}, 10.00^{\circ} \mathrm{W} ; 10 \mathrm{~m}$ a.s.1.; 26 Apr. 2012; J. Van Keer leg.; salt marsh, near sea; CJVK • 1 ๙ , 2 o 9 ; between Gourizim and Mirleft; $29.61^{\circ} \mathrm{N}, 10.02^{\circ} \mathrm{W} ; 9$ Feb. 2007; R. Bosmans leg.; stones in flooded fields near coast; CRB • 1 o, 4 o 9 ; Mirleft; $29.57^{\circ} \mathrm{N}, 10.04^{\circ} \mathrm{W}$; 25 m a.s.l.; 26 Apr. 2012; R. Bosmans leg.; litter and stones in valley near sea; CRB • 4 qo+; same collection data as for preceding; J. Van Keer leg.; CJVK. 

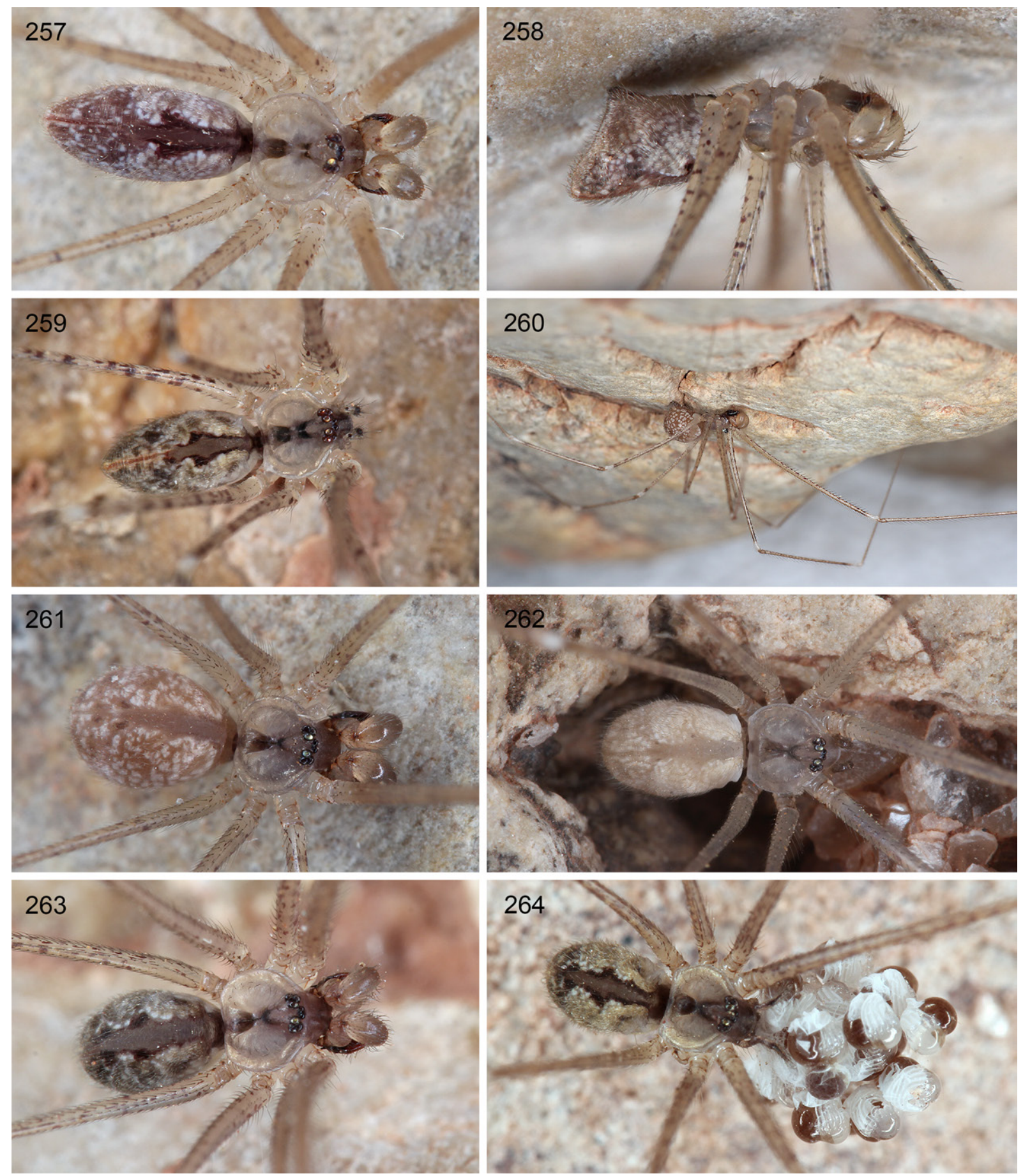

Figs 257-264. Maghreba gen. nov., live specimens. 257-259. M. gharbija gen. et sp. nov., male and female from Morocco, E of Ghazoua. 260-262. M. aurouxi (Barrientos, 2019) gen. nov., male and female from Morocco, SE of Zebzat. 263-264. M. djabalija gen. et sp. nov., male and female with eggsac from Morocco, NE of Tizi n'Test. 


\section{Description}

Male (holotype)

Measurements. Total length 3.9, carapace width 1.3. Distance PME-PME $120 \mu \mathrm{m}$; diameter PME $90 \times$ $120 \mu \mathrm{m}$; distance PME-ALE $30 \mu \mathrm{m}$; diameter AME $95 \mu \mathrm{m}$; distance AME-AME $20 \mu \mathrm{m}$. Leg 1: 31.4 $(9.4+0.5+8.6+11.0+1.9)$, tibia $2: 5.7$, tibia $3: 4.2$, tibia $4: 5.0$; tibia $1 \mathrm{~L} / \mathrm{d}: 61$; femora $1-4$ diameters: $0.21,0.19,0.18,0.19$.

COLOR (in ethanol). Carapace pale grey, medially partly darker (brown V-mark behind ocular area; posterior part of pit darkened); clypeus with pair of black vertical bands; sternum dark ochre, laterally lighter; legs ochre-yellow, without darker rings, with black marks on femora and tibiae (cf. Fig. 281), very few also on metatarsi; abdomen gray, with distinct dark pattern around heart area, indistinct whitish marks dorsally and laterally; ventrally with large brown mark in front of gonopore, three indistinct bands behind gonopore, dark brown area at spinnerets.

Body. Habitus as in Figs 257-258. Ocular area slightly raised. Deep thoracic pit and pair of shallow furrows diverging from pit toward posterior margin. Clypeus unmodified, rim not more sclerotized than in female. Sternum wider than long (1.0/0.6), unmodified. Abdomen distinctly elongated beyond spinnerets.

Chelicerae. As in Figs 268-269, with pair of frontal lateral apophyses, each with one large modified cone-shaped hair; distance between tips of modified hairs: $410 \mu \mathrm{m}$; with very low proximal frontal protrusion; lateral stridulatory ridges not visible in dissecting microscope.

PALPS. In general similar to congeners (cf. Figs 203-205, 224-226); coxa with distinct retrolateral-ventral process; trochanter barely modified (low ventral rounded hump); femur not curved towards dorsal, distally widened and with very low rounded ventral protrusion, proximally with prolateral stridulatory pick (modified hair), with very indistinct retrolateral transversal line, with distinct retrolateral-ventral proximal process and distinct dorsal process; femur-patella joints slightly shifted toward prolateral side; tibia relatively long and slender, tibia-tarsus joints shifted toward retrolateral side; tarsus without macrotrichia; procursus (Figs 265-267) relatively short and wide, dorsal hairs barely curved; on prolateral side with proximal ridge followed distally by distinct hump, both hairless; distally with weakly sclerotized ventral flap; procursus tip with strong ventral sclerite, semitransparent process, and small apophysis in-between (arrow in Fig. 267); main branch strongly curved towards dorsal, distinctly bifid with thin semitransparent hair-like process; genital bulb (Figs 270-273) basal sclerite with dorsal apophysis; distal (main) sclerite large, with deep retrolateral pocket, strong dorsal process without teeth, ventral part with two distinctively long ventral teeth, sperm duct opening not seen.

LEGS. Femur 1 with single row of $\sim 30$ ventral spines; without curved hairs; with higher than usual density of short vertical hairs on all tibiae (mainly proximally and prolaterally); retrolateral trichobothrium of tibia 1 at $3.5 \%$; prolateral trichobothrium absent on tibia 1, present on other leg tibiae; tarsal pseudosegments irregular and indistinct except $\sim 3-5$ distally.

Male (variation)

Tibia 1 in 19 males (incl. holotype): 6.3-8.6 (mean 7.2). Distance between tips of cheliceral apophyses $\sim 390-410 \mu \mathrm{m}$. Ventral abdominal pattern slightly variable (three bands behind gonopore variably 'complete' or reduced to irregular marks); white abdominal marks variably distinct; abdomen in some males dorsally and laterally with dense pattern of dark (internal) abdominal marks. Retrolateral transversal line on palpal femur sometimes not visible.

\section{Female}

In general similar to male (Fig. 259) but without spines on legs; with stridulatory apparatus between prosoma and abdomen: distinct whitish processes on carapace and very indistinct light brown areas frontally on 


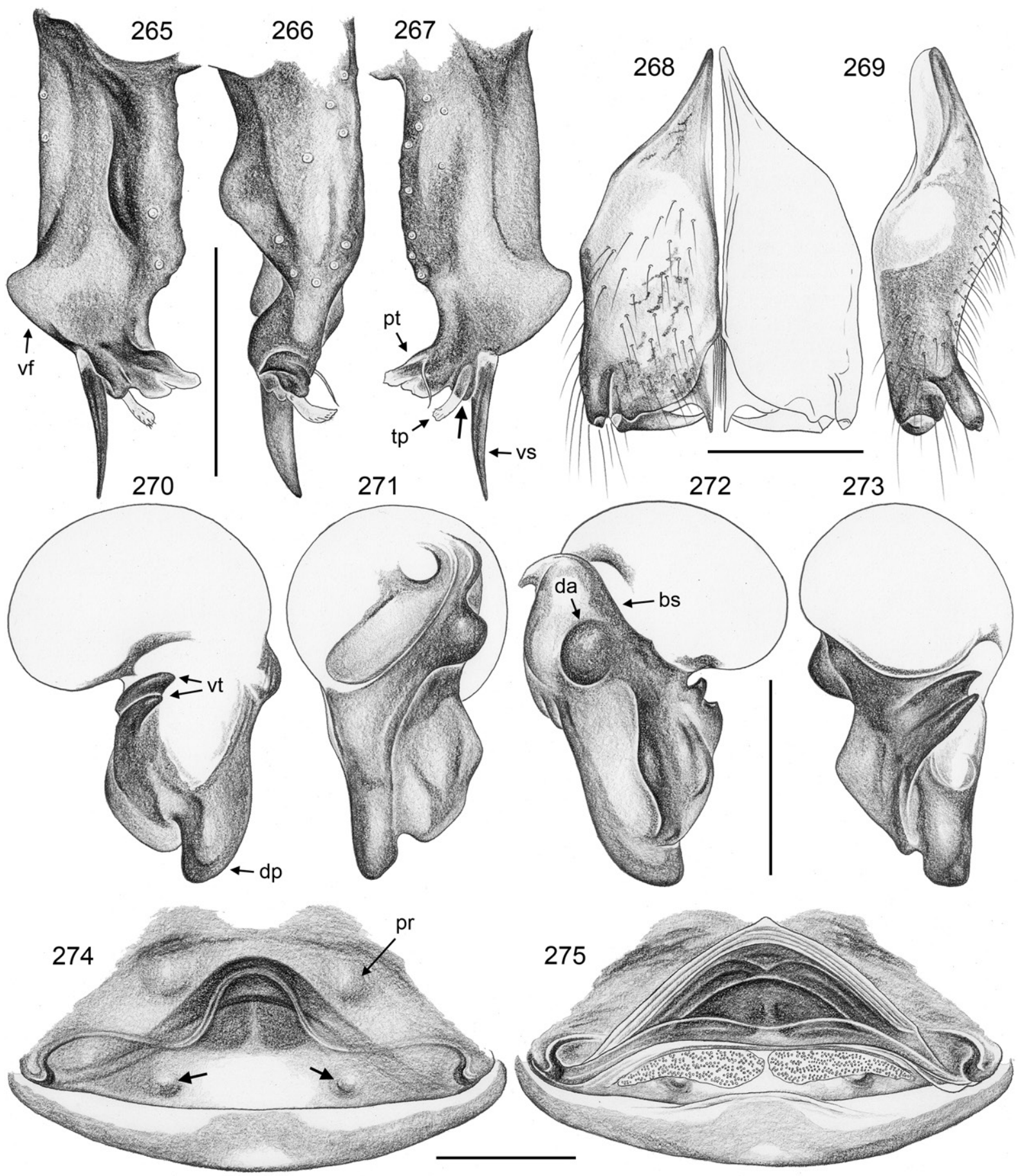

Figs 265-275. Maghreba gharbija gen. et sp. nov.; male and female from Morocco, $4 \mathrm{~km}$ E of Ghazoua (ZFMK Ar 22379, Ar 22380). 265-267. Left procursus, prolateral, dorsal, and retrolateral views; arrow: dark process between ventral sclerite and transparent process. 268-269. Male chelicerae, frontal and lateral views. 270-273. Left genital bulb, prolateral, dorsal, retrolateral, and ventral views. 274275. Cleared female genitalia, ventral and dorsal views; arrows: pockets (pits). Abbreviations: bs $=$ basal sclerite; $\mathrm{da}=$ dorsal apophysis; $\mathrm{dp}=$ dorsal process; $\mathrm{pr}=$ epigynal process; $\mathrm{pt}=$ procursus tip; $\mathrm{tp}=$ transparent process; $\mathrm{vf}=$ ventral flap; $\mathrm{vs}=$ ventral sclerite; $\mathrm{vt}=$ ventral teeth. Scale bars $=0.3 \mathrm{~mm}$. 
abdomen; dark clypeus marks usually more distinct than in male, sometimes fused to large mark including anterior part of ocular area; ventral abdominal bands usually distinct. Apparently without cheliceral stridulatory ridges as in male. Tibia 1 in 63 females: 5.0-7.5 (mean 6.2). Epigynum as in Figs 276-280, main epigynal plate semicircular, variably protruding, with pair of low processes; with pair of indistinct round
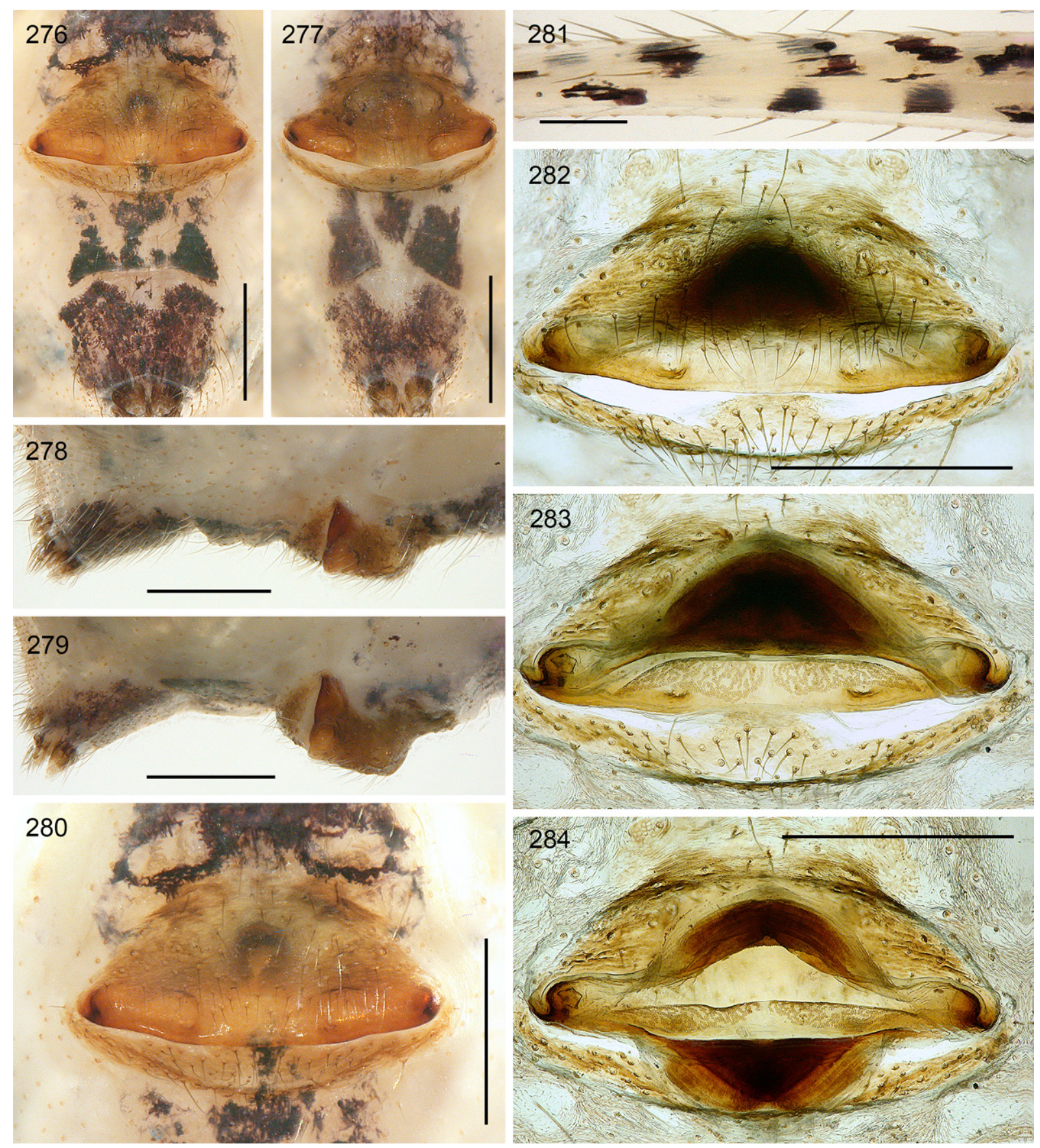

Figs 276-284. Maghreba gharbija gen. et sp. nov.; females from Morocco, $4 \mathrm{~km}$ E of Ghazoua (276, 278, 280-284; ZFMK Ar 22380) and from Morocco, Gourizim (277, 279; ZFMK Ar 22384). 276279. Abdomens, ventral and lateral views. 280. Epigynum, ventral view. 281. Right femur 2, ventral view. 282-284. Cleared female genitalia, ventral view, regular dorsal view, and dorsal view with dorsal arc tilted backwards. Scale bars: $276-280,282-284=0.5 \mathrm{~mm} ; 281=0.2 \mathrm{~mm}$. 
pockets (distance $\sim 360-390 \mu \mathrm{m}$ ); internal median round structure variably visible in uncleared specimens; posterior plate short and wide, simple; indistinct plate in front of epigynum, not elevated. Internal genitalia (Figs 274-275, 282-284) with elongated pore plates in transversal position, widening medially; dorsal and ventral arcs medially strongly sclerotized, ventral arc with indistinct ventral rounded pocket.

\section{Natural history}

The spiders were collected from webs close to the ground, among and under rocks (Aglou), in holes at the basis of river-banks (Gourizim, near Ghazoua), and at the basis of trees and in small piles of rocks (near Ghazoua). Webs usually consisted of a relatively large domed sheet and a dense, bell-shaped part directly attached to the rock. When disturbed, the spiders vibrated strongly and then retreated towards the back (i.e., towards the rock).

\section{Distribution}

Widely distributed along the coast where the Moroccan Atlas range meets the Atlantic Ocean (Fig. 163B).

Maghreba aurouxi (Barrientos, 2019) gen. et comb. nov. Figs 163B, 260-262, 285-304, 319-330

Holocnemus aurouxi Barrientos in Barrientos et al. 2019: 5, figs 2-5, 13a-b (ふ)).

\section{Diagnosis}

Easily distinguished from known congeners by dorsal arc of internal female genitalia with pair of distinct posterior protrusions (Fig. 303); from the geographically neighboring M. nkob gen. et sp. nov. also by procursus with long and straight ventral sclerite and longer dorsally-directed tip (Fig. 292), distal (main) bulbal sclerite with two small ventral teeth of equal size and strongly protruding dorsal process (Figs 293-296), epigynum short, triangular to semi-circular (Figs 299-301); from the morphologically very similar M. amezyan gen. et sp. nov. also by smaller size (e.g., carapace width $<1.5$ vs $>1.5$; palpal tibia length: $<0.95$ vs $>0.95$ ); from the morphologically very similar $M$. kahfa gen. et sp. nov. also by stronger legs (male tibia $1 \mathrm{~L} / \mathrm{d} 60$ vs 80) and larger eyes (AME diameter $65 \mu \mathrm{m}$ vs $40 \mu \mathrm{m}$ ).

\section{Type material}

Holotype (not examined, see Remark below)

MOROCCO - L'Oriental • Oे $^{\wedge}$ Figuig, Beni Bassia, Ifri Lala Jama; $32.252^{\circ} \mathrm{N}, 3.159^{\circ} \mathrm{W} ; 1105 \mathrm{~m}$ a.s.1.; 1 Jun. 2001; F. Fadrique and O. Escolá leg.; Museo de Zoología de Barcelona 2001-0424 (= Museu de Ciències Naturals de Barcelona).

\section{Remark}

I could not examine the type specimen because a loan request was denied (B. Caballero, pers. com. 19 Feb. 2021). However, Jorge Mederos kindly prepared photographs of the distinctive features and these were compared with newly collected specimens from three localities; see variation below.

\section{Material examined}

MOROCCO - Drâa-Tafilalet $\cdot 7 \hat{\partial} \hat{\partial}, 10$ q $\propto$ (partly used for SEM); SE of Zebzat; $32.625^{\circ} \mathrm{N}$, $4.540^{\circ} \mathrm{W} ; 1675 \mathrm{~m}$ a.s.1.; 20 Sep. 2018; B.A. Huber leg.; in small cave above road, near ground; ZFMK Ar 22385, Ar 22386 • 1 र, 3 우 (in pure ethanol); same collection data as for preceding;

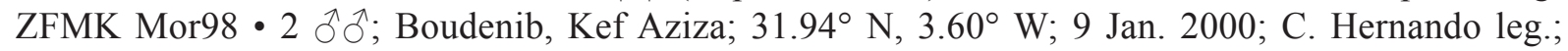
CRBA $3638 \bullet 2$ 우; same collection data as for preceding; CRBA $3639 \bullet 1$ ㅇ same collection data as for preceding; CRBA 3736. 
Assigned tentatively (see Distribution below)

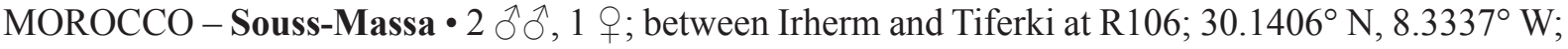
1745 m a.s.l.; 16 Sep. 2018; B.A. Huber leg.; in small cave-like shelter made of large rocks; ZFMK $\operatorname{Ar} 22387 \bullet 1$ (in pure ethanol); same collection data as for preceding; ZFMK Mor87.

\section{Redescription}

Male (ZFMK Ar 22385)

MeAsurements. Total length 2.8, carapace width 1.2. Distance PME-PME $130 \mu \mathrm{m}$; diameter PME $85 \times$ $105 \mu \mathrm{m}$; distance PME-ALE $25 \mu \mathrm{m}$; diameter AME $65 \mu \mathrm{m}$; distance AME-AME $20 \mu \mathrm{m}$. Leg 1: 26.4 $(7.6+0.5+7.3+9.2+1.8)$, tibia $2: 4.8$, tibia $3: 3.7$, tibia $4: 4.2$; tibia $1 \mathrm{~L} / \mathrm{d}: 61$; femora $1-4$ diameters: $0.19,0.17,0.17,0.17$.

Color (in ethanol). Carapace pale ochre to whitish, medially slightly darker; clypeus not darkened; sternum ochre with darker radial marks; legs pale ochre-yellow, without darker rings, with black lines on femora and tibiae (Figs 288-289); abdomen pale gray, with few small dark marks dorsally, many whitish marks; ventrally with diffuse dark marks anterior of gonopore and around spinnerets; with only two small dark marks between gonopore and spinnerets.

Body. Habitus as in Figs 260-261. Ocular area slightly raised. Deep thoracic pit and pair of shallow furrows diverging from pit toward posterior margin (cf. Fig. 319). Clypeus unmodified, rim not more sclerotized than in female. Sternum wider than long (0.85/0.60), unmodified. Abdomen posteriorly rounded, high but not elongated beyond spinnerets.

Chelicerae. Very similar to M. stifadma gen. et sp. nov. (cf. Figs 233-234), with pair of frontal lateral apophyses, each with one large modified cone-shaped hair (Fig. 323); distance between tips of modified hairs: $380 \mu \mathrm{m}$; with very low proximal frontal protrusion; lateral stridulatory ridges fine (Fig. 324; distances between ridges $3.0-3.5 \mu \mathrm{m}$ ), poorly visible in dissecting microscope.

PALPS. As is Figs 285-287; coxa with distinct retrolateral-ventral process (arrow in Fig. 287); trochanter barely modified (low ventral rounded hump); femur not curved towards dorsal, distally widened and with very low rounded ventral protrusion, proximally with prolateral stridulatory pick (modified hair), without retrolateral transversal line, with distinct retrolateral-ventral proximal process and distinct dorsal process; femur-patella joints slightly shifted toward prolateral side; tibia relatively long and slender, tibia-tarsus joints shifted toward retrolateral side; tarsus without macrotrichia; tarsal organ capsulate (Fig. 327); procursus (Figs 290-292) with some dorsal hairs slightly curved upwards; on prolateral side with proximal ridge followed by distinct hump at half length, both hairless; distally with very low ventral flap; procursus tip with strong ventral sclerite and semitransparent process, main branch strongly curved towards dorsal, with semitransparent hair-like process and wide tip divided by retrolateral ridge; genital bulb (Figs 293-296) basal sclerite with dorsal apophysis; distal (main) sclerite large, with deep retrolateral pocket, strong dorsal process without teeth, ventral part with two teeth of similar sizes, sperm duct opening in membranous area on prolateral side (arrow in Fig. 322).

Legs. Femur 1 with single row of $\sim 27$ ventral spines; without curved hairs; with slightly higher than usual density of short vertical hairs prolaterally on all tibiae; retrolateral trichobothrium of tibia 1 at $4 \%$; prolateral trichobothrium absent on tibia 1, present on other leg tibiae; tarsal pseudosegments irregular and indistinct except $\sim 3-5$ distally; tarsal organs capsulate, sometimes with weakly undulating rim (Fig. 328).

Male (variation)

Tibia 1 in seven males from SE of Zebzat: 6.9-7.9 (mean 7.4); abdomen pattern slightly variable, some males with slightly more dark marks dorsally, with more 'complete' ventral pattern behind gonopore 
(three parallel bands). Males from between Irherm and Tiferki differ very slightly from those from SE of Zebzat: tip of procursus narrower; ventral teeth of distal bulbal sclerite slightly smaller and closer together; dorsal process on distal bulbal sclerite slightly narrower and longer in lateral views; tibia 1 in these two males: 7.1, 7.3. The holotype seems identical to the males from SE of Zebzat regarding the procursus, but more similar to the males from between Irherm and Tiferki with respect to the bulb. The two males from Boudenib are in very poor condition but their palps appear identical to those from between Irherm and Tiferki; tibia 1 in one of these males: 8.3 (missing in second male). Gonopore with 4-6 epiandrous spigots, sometimes asymmetric (2+3; Figs 320-321).

\section{Female}

In general similar to male (Fig. 262) but without spines on legs; with very indistinct stridulatory apparatus between prosoma and abdomen: barely visible whitish processes on carapace (arrow in Fig. 319) and light brown areas frontally on abdomen; without cheliceral stridulatory ridges but with rugose area proximally (Fig. 325). Tibia 1 in 11 females from SE of Zebzat: 5.9-8.2 (mean 7.1); in female from Irherm and Tiferki: 6.5. Epigynum as in Figs 299-301 and 330, main epigynal plate wider than long, triangular to semicircular, weakly protruding, with pair of very low processes; with pair of indistinct round pockets (distance $\sim 310 \mu \mathrm{m}$ ); posterior plate short and wide, simple; indistinct plate in front of epigynum, not elevated. Internal genitalia (Figs 297-298, 302-304) with elongated pore

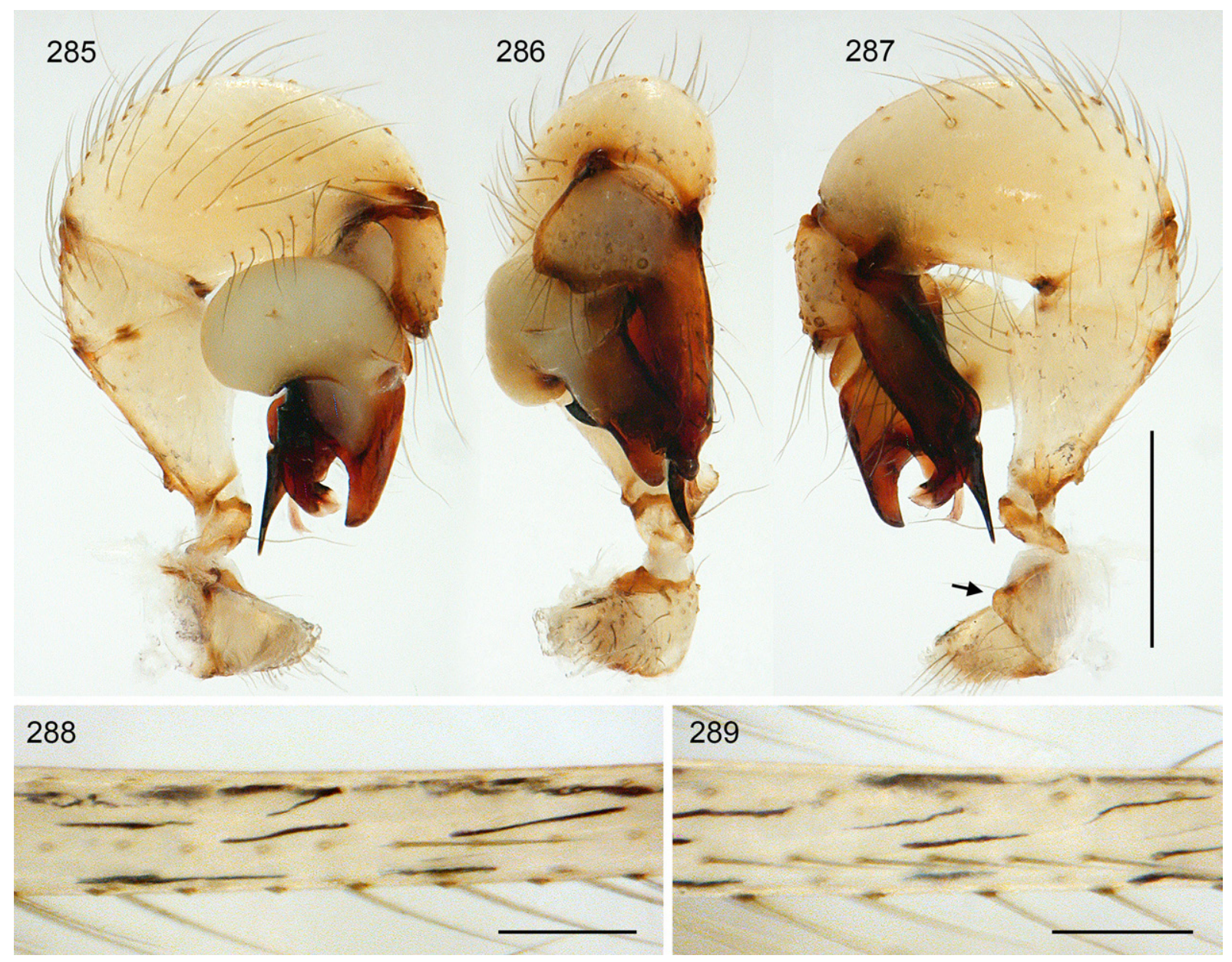

Figs 285-289. Maghreba aurouxi (Barrientos, 2019) gen. nov.; male from Morocco, SE of Zebzat (ZFMK Ar 22385). 285-287. Left palp, prolateral, dorsal, and retrolateral views; arrow: process on coxa. 288-289. Femora 3 and 4, ventral views. Scale bars: $285-287=0.5 \mathrm{~mm} ; 288-289=0.2 \mathrm{~mm}$. 


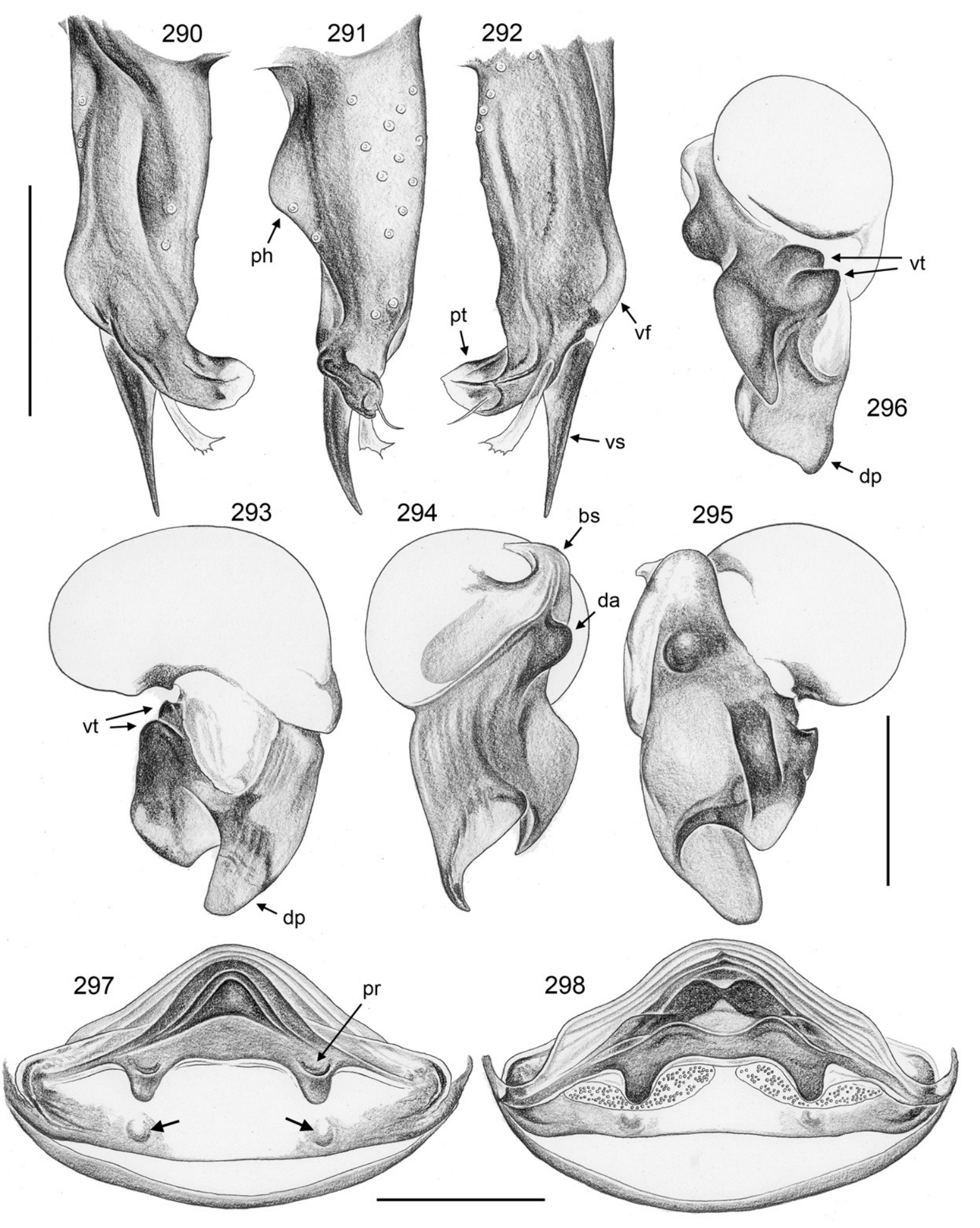

Figs 290-298. Maghreba aurouxi (Barrientos, 2019) gen. nov.; male and female from Morocco, SE of Zebzat (ZFMK Ar 22385, Ar 22386). 290-292. Left procursus, prolateral, dorsal, and retrolateral views. 293-296. Left genital bulb, prolateral, dorsal, retrolateral, and ventral views. 297-298. Cleared female genitalia, ventral and dorsal views; arrows: pockets (pits). Abbreviations: $b s=$ basal sclerite; $d a=$ dorsal apophysis; $\mathrm{dp}=$ dorsal process; $\mathrm{ph}=$ prolateral hump; $\mathrm{pr}=$ epigynal process; $\mathrm{pt}=$ procursus tip; $\mathrm{vf}=$ ventral flap; vs $=$ ventral sclerite; $v \mathrm{t}=$ ventral teeth. Scale bars $=0.3 \mathrm{~mm}$. 

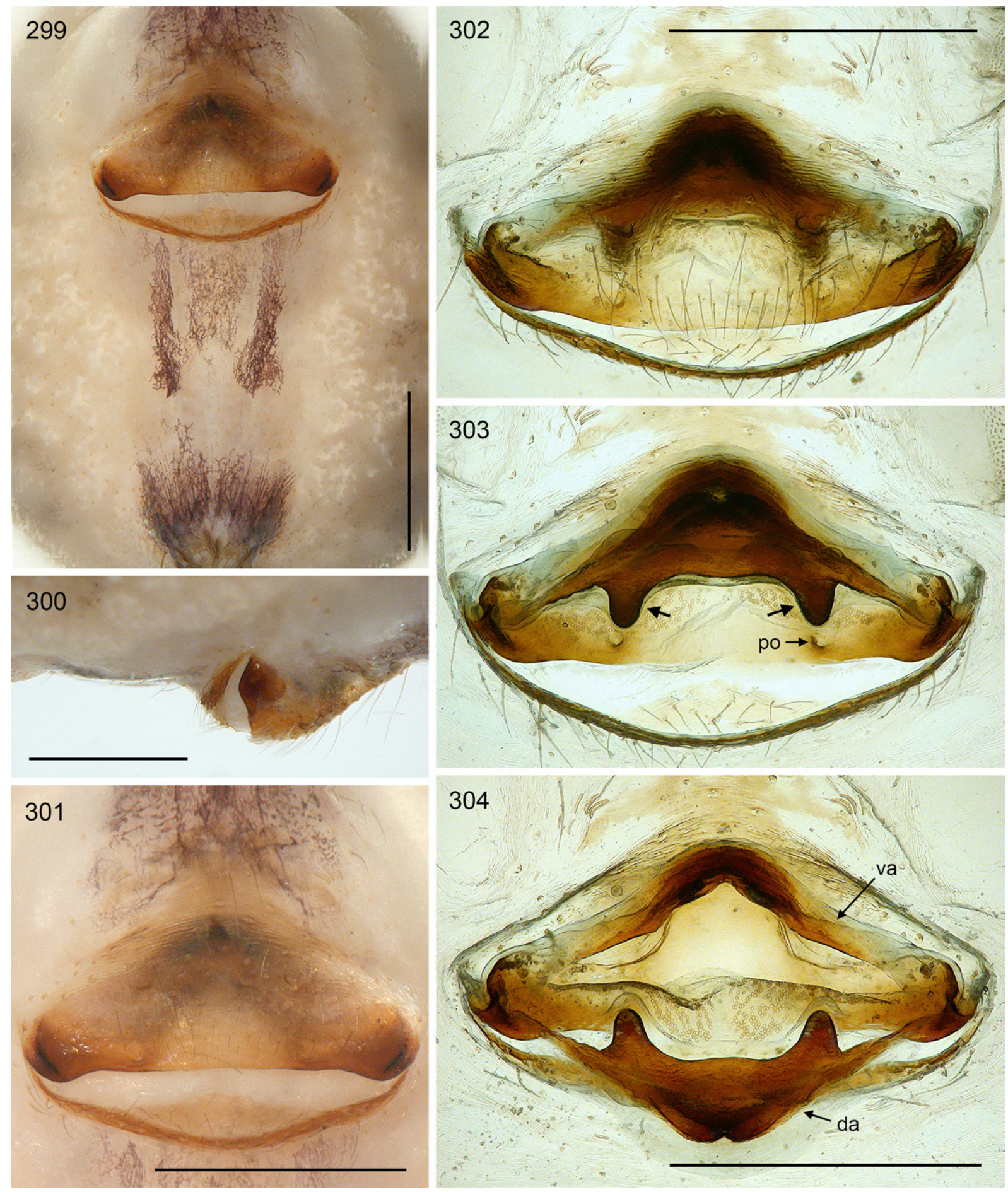

Figs 299-304. Maghreba aurouxi (Barrientos, 2019) gen. nov.; female from Morocco, SE of Zebzat (ZFMK Ar 22386). 299. Abdomen, ventral view. 300-301. Epigynum, lateral and ventral views. 302304. Cleared female genitalia, ventral view, regular dorsal view, and dorsal view with dorsal arc tilted backwards; arrows: posterior processes on dorsal arc. Abbreviations: da $=$ dorsal arc; po = pocket; va $=$ ventral arc. Scale bars $=0.5 \mathrm{~mm}$. 

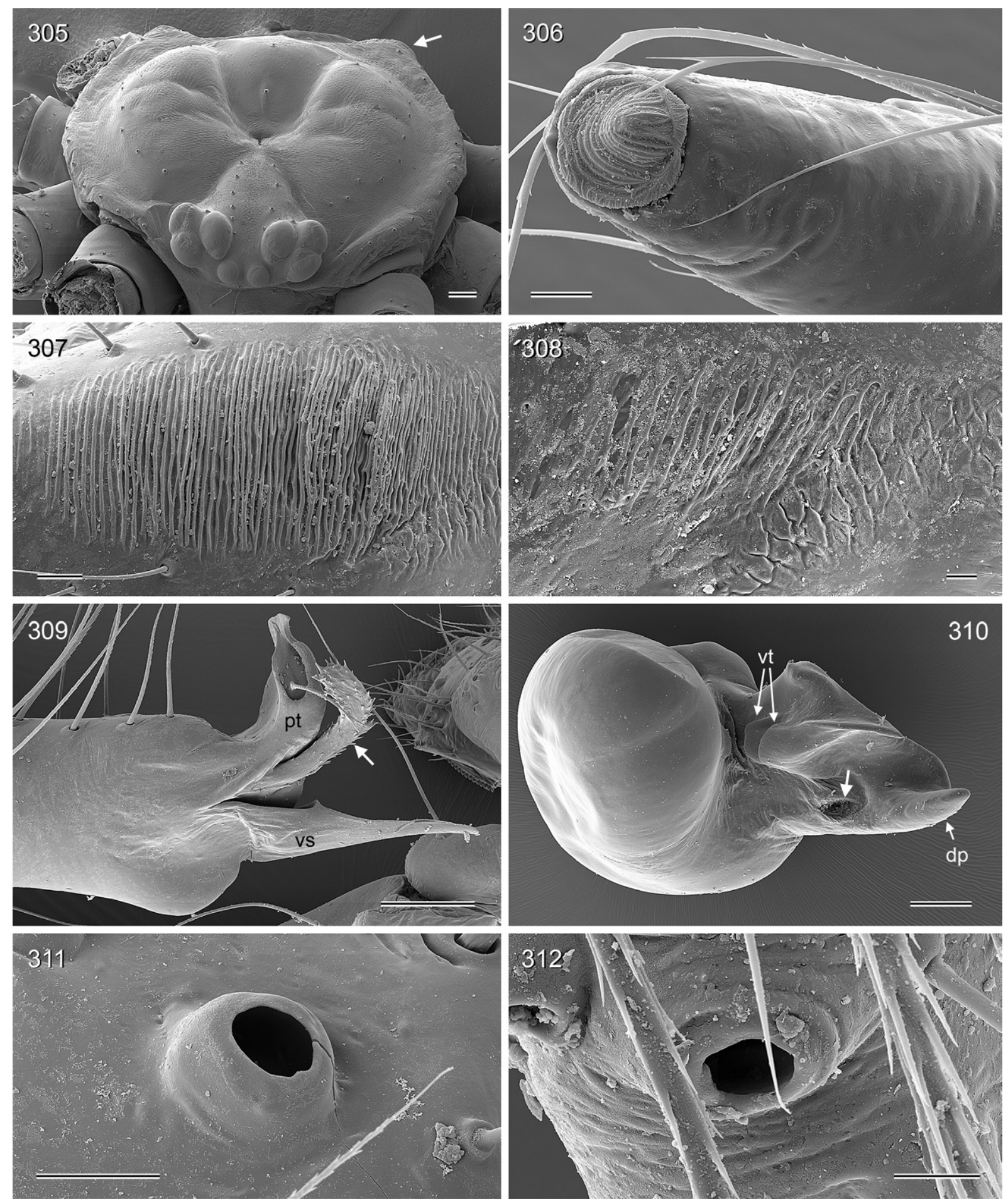

Figs 305-312. Maghreba amezyan gen. et sp. nov.; male and female from Morocco, between Lakhssas and Bouizakarne (ZFMK Ar 22353, Ar 22354). 305. Female ocular area and carapace; arrow: posterior process. 306. Male cheliceral apophysis. 307-308. Male and female cheliceral stridulatory files. 309. Right procursus, retrolateral view; arrow: transparent process. 310. Right genital bulb, prolateral (slightly ventral) view; arrow: sperm duct opening. 311-312. Male and female palpal tarsal organs. Abbreviations: $\mathrm{dp}=$ dorsal process; $\mathrm{pt}=$ procursus tip; $\mathrm{vs}=$ ventral sclerite; $\mathrm{vt}=$ ventral teeth. Scale bars: $305,309-310=100 \mu \mathrm{m} ; 306,308,312=10 \mu \mathrm{m} ; 307,311=20 \mu \mathrm{m}$. 

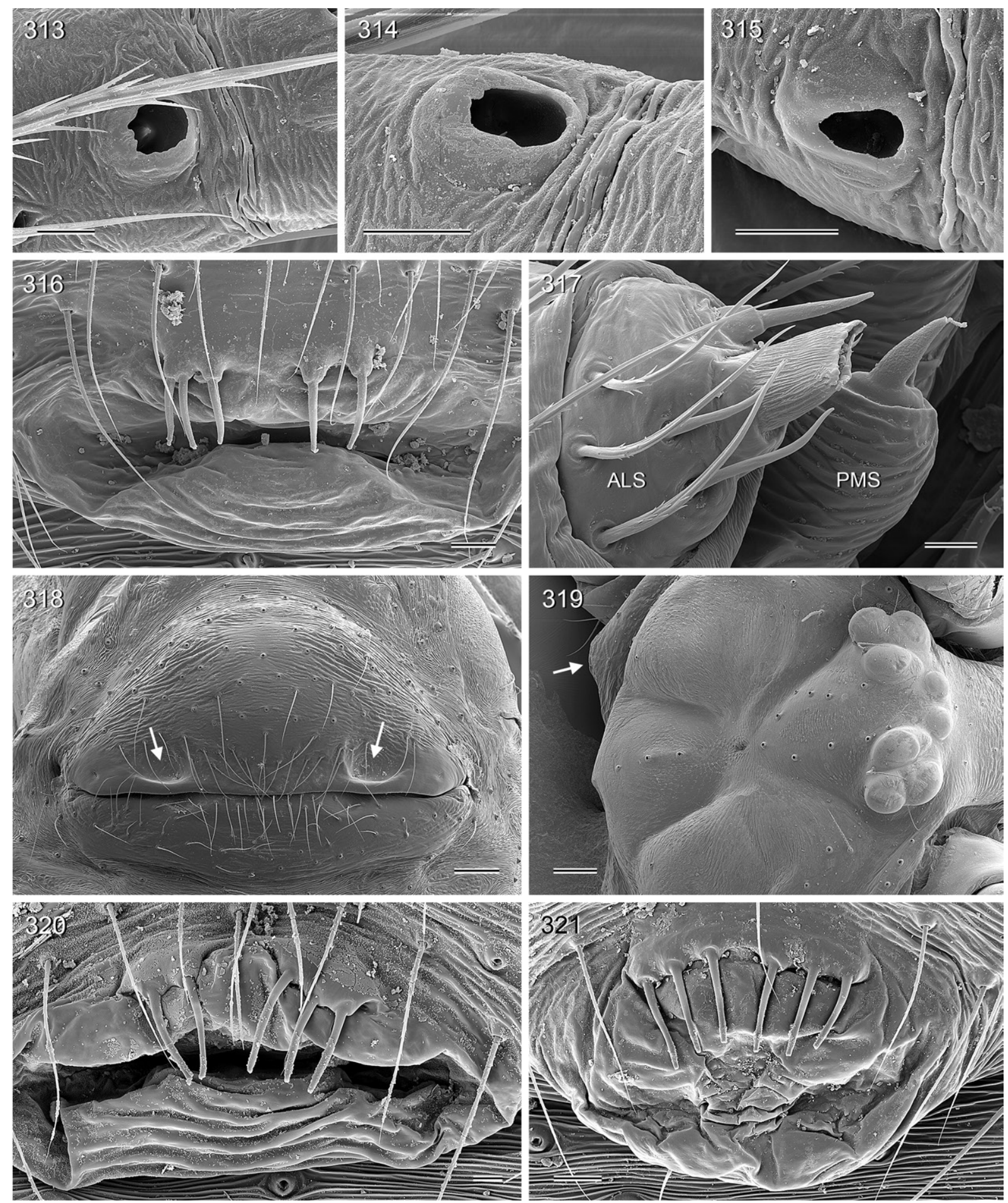

Figs 313-321. 313-318. Maghreba amezyan gen. et sp. nov.; male and female from Morocco, between Lakhssas and Bouizakarne (ZFMK Ar 22353, Ar 22354). 313-315. Tarsal organs on male tarsi 1, 2, and 4. 316. Male gonopore. 317. Female spinnerets. 318. Epigynum; arrows: pockets (pits). 319321. Maghreba aurouxi (Barrientos, 2019) gen. nov.; males and female from Morocco, SE of Zebzat (ZFMK Ar 22385, Ar 22886). 319. Female carapace; arrow: posterior process. 320-321. Gonopores of two male specimens. Scale bars: 313-315, 317, 320 = $10 \mu \mathrm{m} ; 316,321=20 \mu \mathrm{m} ; 318-319=100 \mu \mathrm{m}$. 

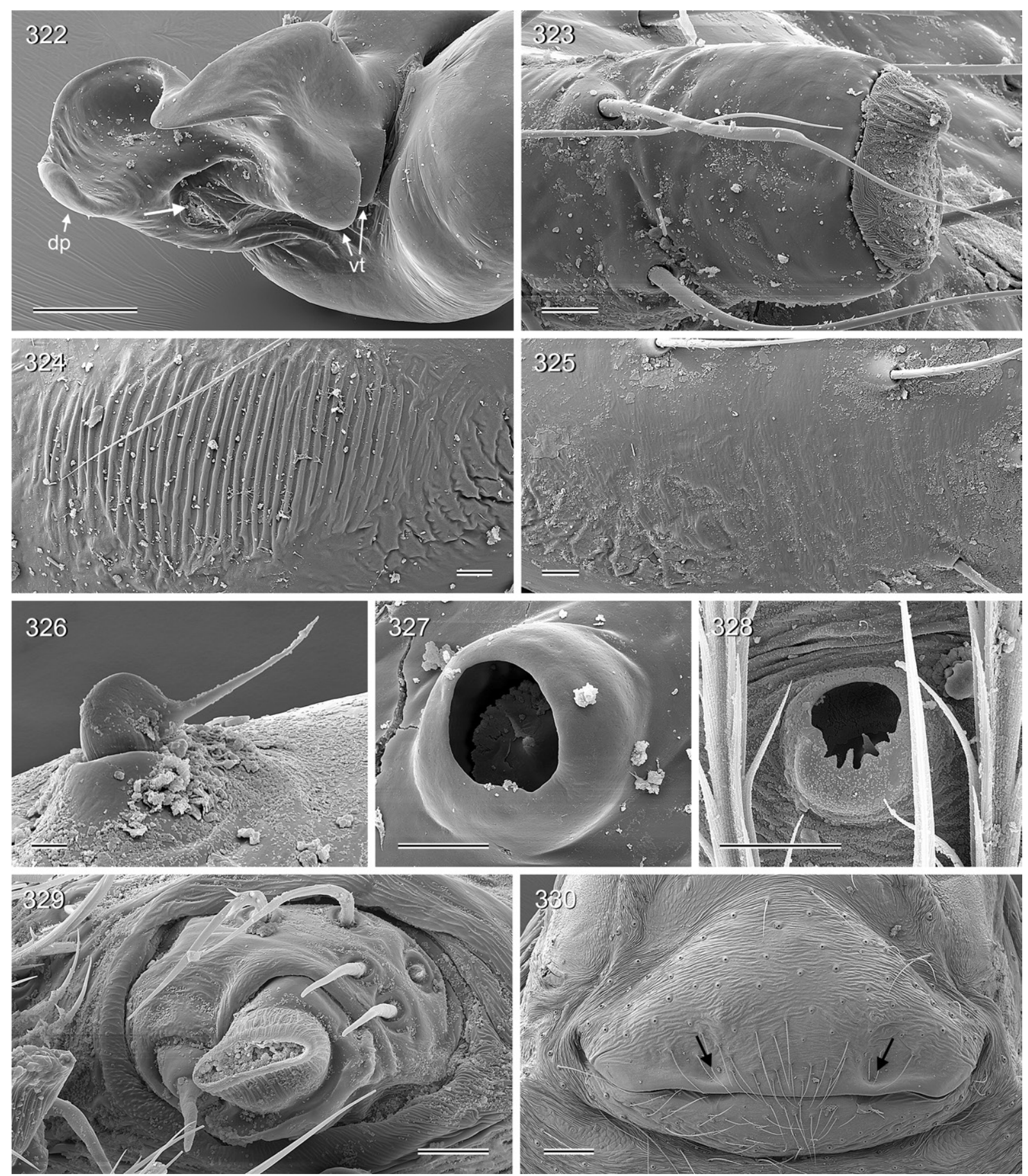

Figs 322-330. Maghreba aurouxi (Barrientos, 2019) gen. nov.; male and female from Morocco, SE of Zebzat (ZFMK Ar 22385, Ar 22386). 322. Left genital bulb, ventral (slightly distal) view; arrow: sperm duct opening. 323. Male cheliceral apophysis. 324-325. Male stridulatory file and corresponding largely smooth female cheliceral side. 326. Stridulatory pick on female palpal femur. 327. Male palpal tarsal organ. 328. Tarsal organ on male tarsus 3. 329. Female ALS. 330. Epigynum; arrows: pockets (pits). Abbreviations: $\mathrm{dp}=$ dorsal process; $\mathrm{vt}=$ ventral teeth. Scale bars: $322,330=100 \mu \mathrm{m} ; 323-325$, $327-329=10 \mu \mathrm{m} ; 326=2 \mu \mathrm{m}$. 
plates in transversal position, widening medially; dorsal arc medially heavily sclerotized with distinctive posterior processes, ventral arc apparently without ventral pocket.

The females from Boudenib are larger and have much longer legs (tibia 1: 9.6, 10.1; missing in third female), and the epigynal humps are barely visible, but they share the distinctive posterior processes of the internal dorsal arc (one female cleared).

\section{Natural history}

All specimens were collected in caves or small cave-like shelters. Near Zebzat, the spiders were abundant in a shallow cave, with their domed webs close to the ground. They were hanging in their webs rather than sitting on the rock surface. Between Irherm and Tiferki, the spiders were only found in a small cave-like shelter of large rocks, while M. saghro gen. et sp. nov. occupied a more epigean microhabitat, i.e., spaces among and under rocks.

\section{Distribution}

The type locality and two further localities are in the eastern Moroccan Atlas (Fig. 163B). The specimens from between Irherm and Tiferki are from almost $500 \mathrm{~km}$ further west and therefore assigned tentatively.

Maghreba djabalija gen. et sp. nov. urn:1sid:zoobank.org:act:933904E7-301A-4E9E-8B07-7EE5D21DD8EA

Figs 163B, 263-264, 331-350

\section{Diagnosis}

Distinguished from most known congeners (except $M$. gharbija gen. et sp. nov.) by sclerite on procursus tip between ventral sclerite and transparent process (arrow in Fig. 336); from M. gharbija and other species also by the combination of: procursus with long and slightly curved ventral sclerite (Fig. 336); dorsal process of distal (main) bulbal sclerite only slightly longer than ventral part, ventral part with three teeth of different sizes (Figs 339-342); epigynum short trapezoidal and with pair of whitish processes (Figs 345-347).

\section{Etymology}

The species name is an adjective derived from the Arab 'djabalija' = 'mountain-dwelling'.

\section{Type material}

Holotype

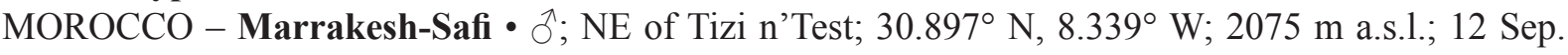
2018; B.A. Huber leg.; among rocks near ground; ZFMK Ar 22388.

\section{Other material examined}

MOROCCO - Marrakesh-Safi • $3 \widehat{\partial} \widehat{\partial}, 9$ ㅇ; same collection data as for holotype; ZFMK Ar 22389,

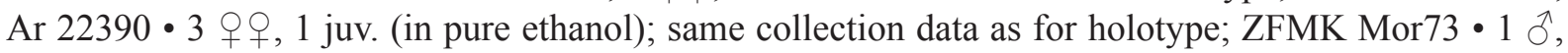

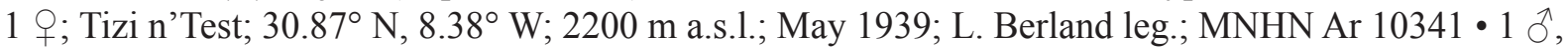
1 juv.; same collection data as for preceding; MNHN Ar 10342.

\section{Description}

Male (holotype)

MEAsurements. Total length 3.2, carapace width 1.3. Distance PME-PME $110 \mu \mathrm{m}$; diameter PME $95 \times$ $115 \mu \mathrm{m}$; distance PME-ALE $25 \mu \mathrm{m}$; diameter AME $70 \mu \mathrm{m}$; distance AME-AME $20 \mu \mathrm{m}$. Leg 1: 25.3 $(7.2+0.5+7.0+8.8+1.8)$, tibia $2: 4.8$, tibia $3: 3.6$, tibia $4: 4.2$; tibia $1 \mathrm{~L} / \mathrm{d}: 58$; femora $1-4$ diameters: $0.25,0.22,0.21,0.21$. 
Color (in ethanol). Carapace pale ochre-yellow, medially partly darker (brown V-mark behind ocular area; posterior part of pit darkened); clypeus not darkened; sternum dark brown, with black radial marks; legs ochre-yellow, without darker rings, with black marks on femora and tibiae, few also on metatarsi; abdomen gray, densely covered with dark and whitish marks; ventrally with wide dark brown band, band behind gonopore divided by two light longitudinal lines.

Body. Habitus as in Fig. 263. Ocular area slightly raised. Deep thoracic pit and pair of shallow furrows diverging from pit toward posterior margin. Clypeus unmodified, rim not more sclerotized than in female. Sternum wider than long (0.90/0.65), unmodified. Abdomen posteriorly rounded, barely elongated beyond spinnerets.

Chelicerae. As in Figs 337-338, with pair of frontal lateral apophyses, each with one large modified cone-shaped hair; distance between tips of modified hairs: $360 \mu \mathrm{m}$; with pair of light proximal frontal protrusions set with brushes of long hairs; lateral stridulatory ridges barely visible in dissecting microscope.

PALPS. As is Figs 331-333; coxa with distinct retrolateral-ventral process; trochanter barely modified (low ventral rounded hump); femur not curved towards dorsal, distally widened and with low rounded ventral protrusion, proximally with prolateral stridulatory pick (modified hair), without retrolateral transversal line, with distinct retrolateral-ventral proximal process and distinct dorsal process; femur-patella joints slightly shifted toward prolateral side; tibia relatively long and slender, tibia-tarsus joints shifted toward retrolateral side; tarsus without macrotrichia; procursus (Figs 334-336) with dorsal hairs slightly curved; on prolateral side with proximal ridge followed distally by distinct hump, free of hairs; distally with small ventral flap; procursus tip with strong ventral sclerite, semitransparent process, and distinctive apophysis inbetween (arrow in Fig. 336), main branch strongly curved towards dorsal, with semitransparent hairlike process and simple tip; genital bulb (Figs 339-342) basal sclerite with dorsal apophysis; distal (main) sclerite large, with deep retrolateral pocket, strong dorsal process without teeth, ventral part with three teeth of different sizes, sperm duct opening in membranous area on prolateral side.
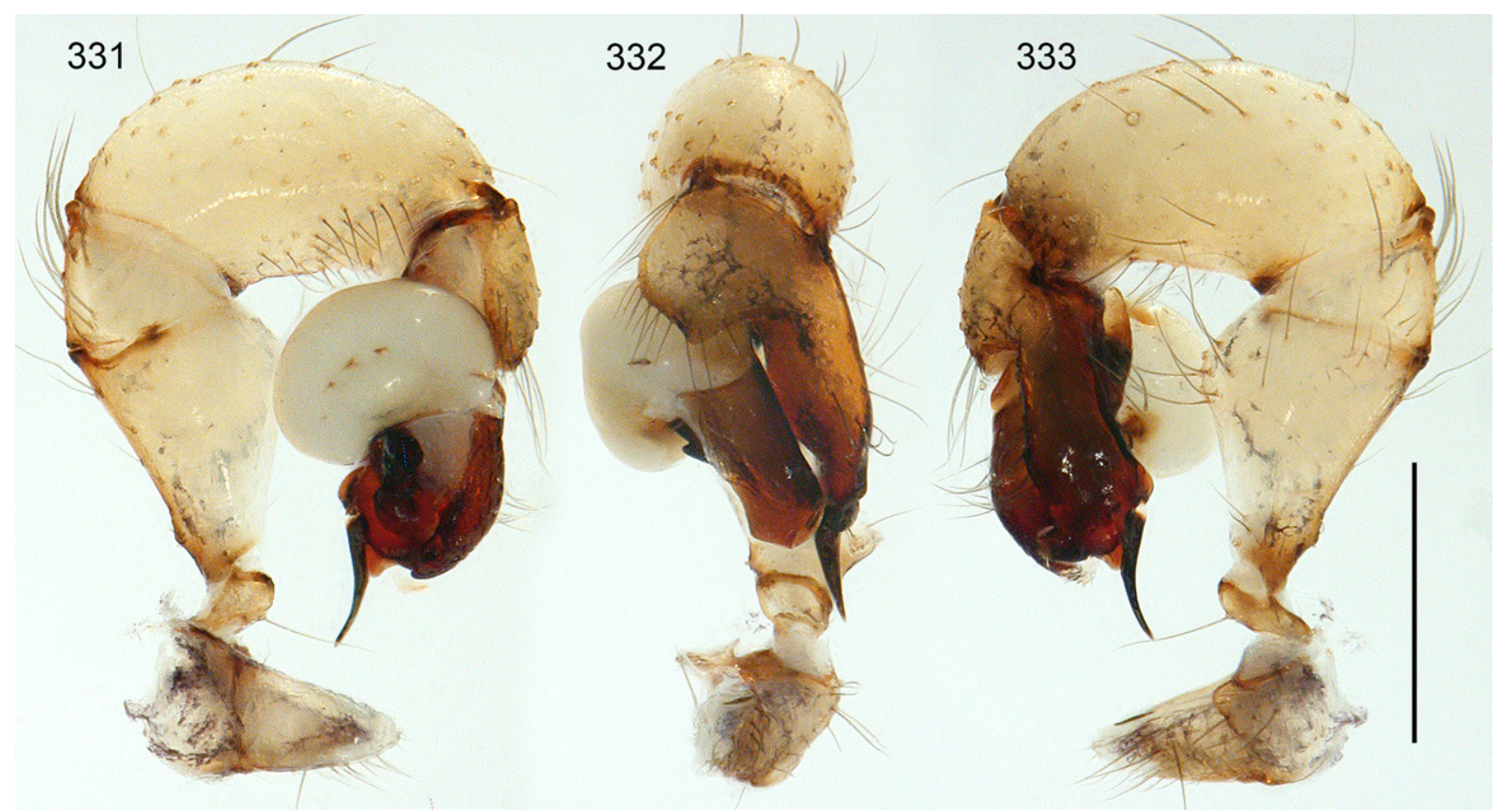

Figs 331-333. Maghreba djabalija gen. et sp. nov.; male from Morocco, NE of Tizi n'Test (ZFMK Ar 22389); left palp, prolateral, dorsal, and retrolateral views. Scale bar $=0.5 \mathrm{~mm}$. 


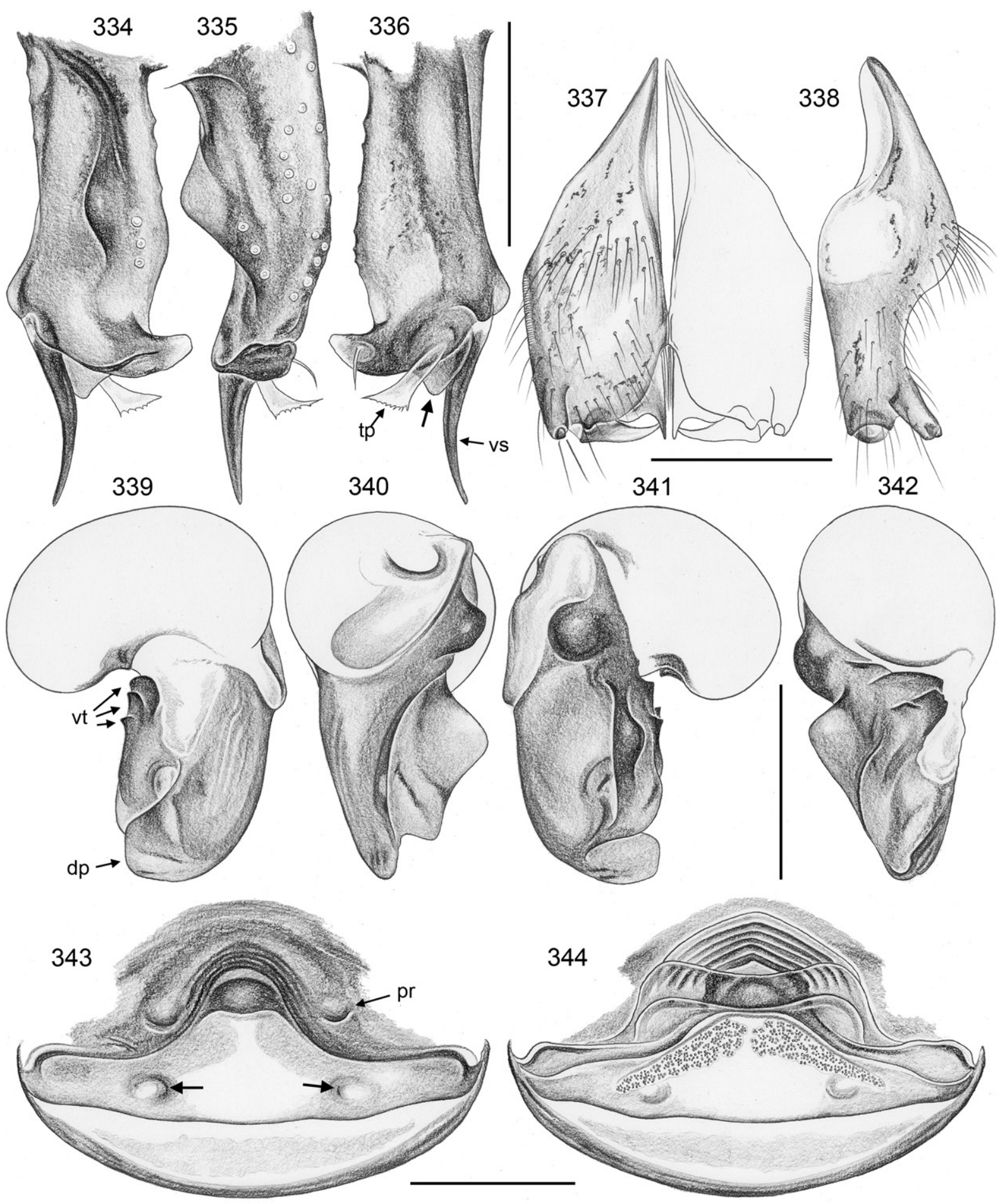

Figs 334-344. Maghreba djabalija gen. et sp. nov.; male and female from Morocco, NE of Tizi n'Test (ZFMK Ar 22389, Ar 22390). 334-336. Left procursus, prolateral, dorsal, and retrolateral views; arrow: dark process between ventral sclerite and transparent process. 337-338. Male chelicerae, frontal and lateral views. 339-342. Left genital bulb, prolateral, dorsal, retrolateral, and ventral views. 343344. Cleared female genitalia, ventral and dorsal views; arrows: pockets (pits). Abbreviations: $d p=$ dorsal process; $\mathrm{pr}=$ epigynal process; $\mathrm{tp}=$ transparent process; $\mathrm{vs}=$ ventral sclerite; $\mathrm{vt}=$ ventral teeth. Scale bars $=0.3 \mathrm{~mm}$. 

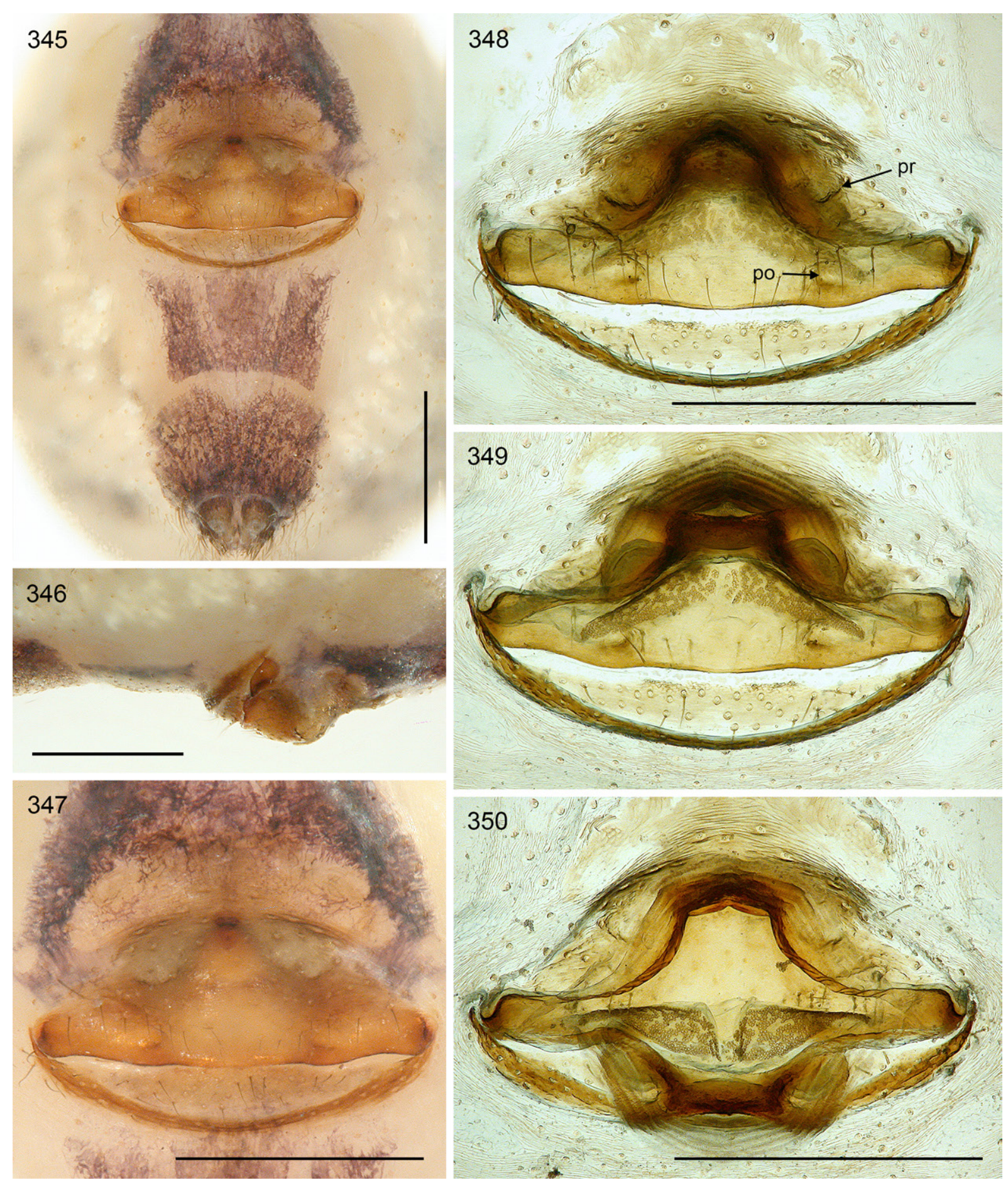

Figs 345-350. Maghreba djabalija gen. et sp. nov.; female from Morocco, NE of Tizi n'Test (ZFMK Ar 22390). 345. Abdomen, ventral view. 346-347. Epigynum, lateral and ventral views. 348-350. Cleared female genitalia, ventral view, regular dorsal view, and dorsal view with dorsal arc tilted backwards. Abbreviations: $\mathrm{po}=$ pocket; $\mathrm{pr}=$ epigynal process. Scale bars $=0.5 \mathrm{~mm}$. 
Legs. Femur 1 with single row of $\sim 20$ ventral spines; without curved hairs; with slightly higher than usual density of short vertical hairs prolaterally on tibiae 3-4; retrolateral trichobothrium of tibia 1 at $3.5 \%$; prolateral trichobothrium absent on tibia 1, present on other leg tibiae; tarsal pseudosegments irregular and indistinct except $\sim 5-8$ distally.

Male (variation)

Tibia 1 in six males (incl. holotype): 5.7-7.0 (mean 6.3).

\section{Female}

In general similar to male (Fig. 264) but without spines on legs; with indistinct stridulatory apparatus between prosoma and abdomen: barely visible whitish processes on carapace and light brown areas frontally on abdomen; cheliceral stridulatory ridges not seen. Tibia 1 in ten females: 5.4-7.2 (mean 6.0). Epigynum as in Figs 345-347, main epigynal plate trapezoidal, weakly protruding, with pair of low processes; with pair of indistinct round pockets (distance $\sim 340 \mu \mathrm{m}$ ); posterior plate short and wide, simple; indistinct plate in front of epigynum, not elevated. Internal genitalia (Figs 343-344, 348-350) with elongate pore plates in transversal position, widening medially; dorsal arc with distinctive median elements, ventral arc with indistinct ventral rounded pocket.

\section{Natural history}

The specimens were found under large rocks along a dry riverbed. They were very fast at escaping, very different from the otherwise similar M. stifadma gen. et sp. nov. at Setti-Fatma.

\section{Distribution}

Known from the Tizi n'Test area only, in the Moroccan High Atlas (Fig. 163B).

\section{Genus Crossopriza Simon, 1893}

Crossopriza Simon, 1893: 476 (type species: Artema pristina Simon, 1890).

Ceratopholcus Spassky, 1934: 361 (type species: C. maculipes Spassky, 1934). Synonymized in Huber et al. 2014a: 420.

Tibiosa González-Sponga, 2006: 10 (type species: T. caracensis González-Sponga 2006). Synonymized in Huber 2009b: 65; see also Huber \& Villarreal 2020: 57.

Crossopriza - Wiehle 1933: 241. - Huber et al. 1999: 7. — Deeleman-Reinhold \& van Harten 2001: 194.

\section{Diagnosis}

Long-legged pholcids with spotted leg femora and tibiae (e.g., Figs 558, 577, 709), abdomen dorsoposteriorly often angular or pointed (e.g., Figs 394, 398, 699), females with paired stridulatory apparatus between carapace and abdomen (Figs 468, 595, 660, 797). Distinguished from similar genera (Maghreba gen. nov., Stygopholcus, Holocnemus) by combination of: male chelicerae with only one pair of modified (cone-shaped) hairs at tips of main cheliceral apophyses (e.g., Figs 598, 845, 859; in contrast to Stygopholcus, H. reini, and H. caudatus); male palpal coxa with rounded retrolateral hump, without distinct process (in contrast to Maghreba); male palpal femur without retrolateral proximal process (in contrast to Stygopholcus and Maghreba); male palpal femur without dorsal process (in contrast to Maghreba); male palpal tarsal organ capsulate (e.g., Figs 426, 602, 849, 862; in contrast to Stygopholcus); procursus usually with distinct prolateral hump set with numerous hairs (e.g., Figs 357, 376, 616, 628); procursus tip not strongly bent towards dorsal (in contrast to Maghreba); procursus usually without transparent membranous process at tip (in contrast to Maghreba; present in C. soudanensis); genital bulb without slender dorsal process (in contrast to Stygopholcus); distal (main) bulbal sclerite without deep retrolateral pocket (in contrast to Maghreba). 


\section{Description}

Male

BoDy. Total body length $\sim 2.5-6.5$; carapace width $\sim 1.0-2.4$. Carapace with deep central pit and pair of shallow furrows diverging from posterior side of pit toward posterior rim of carapace (cf. Figs 468, 595); ocular area slightly raised, eye triads relatively close together (distance PME-PME $=0.5-1.2 \times$ PME diameter), each secondary eye (especially PME) accompanied by small elevation (arrows in Fig. 467; "pseudo-eyes"; cf. Huber 2009a), sometimes very distinct (i.e., reflecting light), e.g., in C. miskin sp. nov. PME oval; AME large ( $70-110 \%$ of PME small diameter). Clypeus high, usually unmodified, in C. sengleti sp. nov. with rounded median process (Fig. 768), in C. johncloudsleyi Deeleman-Reinhold \& van Harten, 2001 with pair of small hooked processes (Deeleman-Reinhold \& van Harten 2001: fig. 14; Huber 2009a). Abdomen oval, dorsally posteriorly usually angular (Fig. 394), sometimes rounded (Fig. 476) or pointed (Fig. 699). Male gonopore with 4-6 epiandrous spigots (sometimes asymmetric: 2+3; Figs 420, 507, 606, 854), ALS with only two spigots each: one large widened spigot and one pointed spigot (Figs 424, 505, 607); PMS with two spigots each (Figs 424, 505, 607); PLS without spigots.

COLOR. In general ochre-yellow to light brown. Carapace mostly pale, with darker median mark, without lateral marks (cf. Figs 391, 481, 702); sternum light brown to dark brown, with darker radial marks. Legs without or with indistinct darker rings, with oval to short longitudinal line-shaped dark marks on femora and tibiae (cf. Figs 558, 577, 709), sometimes also a few on metatarsi, rarely on femora only. Abdomen usually with distinct dorsal and ventral patterns: dark heart-mark and further dark and whitish marks dorsally and laterally, ventral median band variably distinct, rarely absent (e.g., C. sengleti sp. nov.). Cave-dwelling species slightly lighter/paler (see C. moqal sp. nov.; C. kittan sp. nov.).

Chelicerae. Chelicerae with one large modified (cone-shaped) hair on each main cheliceral apophysis (Figs 598, 845, 859); main cheliceral apophyses usually in lateral position, in some species moved to frontal position; in latter case sometimes with additional pair of lateral apophyses (without modified hairs; e.g., Figs 731, 774, 814). With fine to distinct stridulatory ridges (Figs 421, 500, 599, 841), distances between ridges $\sim 3.5-8.0 \mu \mathrm{m}$, sometimes variable within file (distances proximally larger than distally; see C. sahtan sp. nov., C. maculipes).

PALPS. Coxa with rounded retrolateral-ventral hump, without distinct apophysis; trochanter barely modified, slightly protruding ventrally; femur distally widened, on ventral side usually protruding, without dorsal apophysis, without proximal retrolateral process, without or with indistinct transversal dark line on retrolateral side, with stridulatory pick (modified hair) on prolateral side (Fig. 842); femurpatella joints shifted toward prolateral side (arrows in Fig. 354); tibia-tarsus joints shifted toward retrolateral side (arrows in Fig. 356); palpal tarsus without dorsal macrotrichia, palpal tarsal organ capsulate (Figs 426, 602, 849, 862); procursus dorsally with straight or weakly curved hairs; procursus with prolateral process set with numerous long hairs, without ventral 'knee', distally usually with ventral sclerite (absent in C. miskin sp. nov.), usually without membranous transparent process (present in C. soudanensis; Fig. 358), tip of procursus not curved towards dorsal, with one or more transparent hair-like or spine-like processes on retrolateral side (Figs 417, 506, 596, 847, 857); genital bulb with basal sclerite connecting to tarsus (bs in Fig. 361), and distal (main) sclerite often with distinctive set of prolateral (slightly ventral) apophyses and ridges (e.g., Figs 406, 440, 716, 789), without retrolateral pocket; sperm duct opening in membranous area on prolateral side of distal bulbal sclerite (arrows in Figs 419, 597, 848, 858).

LeGs. Long and relatively thin, leg 1 length $\sim 20-65$, tibia 1 length $\sim 5.5-18$, tibia 2 longer than tibia 4 $(\sim 1.1-1.3 \times)$. Tibia $1 \mathrm{~L} / \mathrm{d}$ usually $\sim 50-80,>80$ in the slightly troglomorphic $C$. kittan sp. nov. Femur 1 usually thicker than other femora; femur 1 with spines ventrally in one row, in very small males rarely without spines; spines proximally gradually transforming into regular setae; spines rarely present 
on femur 2 (C. sanaa sp. nov.); legs without curved hairs; with few short vertical hairs; retrolateral trichobothrium in proximal position (at $2-5 \%$ of tibia length in tibia 1), prolateral trichobothrium absent on tibia 1, present on other tibiae. Tarsal pseudosegments very indistinct except a few ( 2-3) distally, proximally with indistinct irregular platelets rather than distinct rings. Tarsal organs of legs capsulate, with round or weakly undulating rim (Figs 428-431, 863).

\section{Female}

In general similar to male; chelicerae either with indistinct and small stridulatory files (with slightly larger distances between ridges than in males; 4.0-8.5 $\mu \mathrm{m}$ ) or without stridulatory files (Figs 422, 469, $501,600,843,861)$; legs slightly shorter than in male, without spines. Usually with pair of variably distinct processes posteriorly on carapace (arrows in Figs 468, 595) acting against pair of variably distinct plates on abdomen (Figs 660, 797), absent in C. johncloudsleyi. Epigynum usually consisting of large, simple anterior plate and short but wide posterior plate; anterior plate usually with pair of distinct pockets, sometimes close together on median elevated ridge, sometimes more like furrows than pockets; epigynum usually without processes, only in C. johncloudsleyi with two pairs of distinct apophyses (Deeleman-Reinhold \& van Harten 2001: fig. 11; Huber 2009a: fig. 30); without bulging areas in front of anterior plate. Internal genitalia with sclerotized arc that consists of dorsal and ventral parts (da and va in Figs 388, 447) and is variably visible in uncleared specimens; uterus externus sometimes with small median ventral structure (pouch or pocket?), sometimes visible as round structure in untreated specimens (e.g., Figs 463, 551, 612, 837); pore plates large, flat, of variable shape and position, pores either homogeneously distributed or in groups.

\section{Distribution}

All species except $C$. lyoni are restricted to an area that ranges from Mali to India and from Kenya to Kazakhstan (Fig. 351). The type species C. lyoni has expanded around the globe (Fig. 351), apparently since approximately the second half of the $19^{\text {th }}$ century (see below).

\section{Relationships}

Together with Holocnemus, Stygopholcus, and Maghreba gen. nov., Crossopriza is an unambiguous representative of the spotted-leg clade, but beyond that the cladistic analysis provides only weak evidence for inter-generic relationships. Even the monophyly of Crossopriza is poorly supported by a single character of questionable strength (Appendix 3, char. 18). Within Crossopriza, a group of 14 species (including the type species) is reasonably well supported by two functionally related characters (medially-directed male cheliceral apophyses and medially positioned female epigynal pockets). Within this group, a further sub-group of eight species (again including the type species) share a second pair of male cheliceral apophyses. Finally, $C$. lyoni shares with three other species a ventral sclerite on the procursus provided with a retrolateral side branch (arrows in Figs 769, 788, 810, 828). The few remaining sister-group relationships suggested by the cladogram are either weakly supported or of little relevance, or both.

\section{Composition}

The genus now includes 24 named species, of which 18 are newly described. All named species are treated below except for C. johncloudsleyi which was redescribed in Huber (2009a). Several additional undescribed species are present in collections. The ZMMU has an undescribed species from Pakistan $\left(\mathrm{N}\right.$ of Islamabad, $33.75^{\circ} \mathrm{N}, 73.06^{\circ} \mathrm{E}$ ), which is not formally described because the only available male is in very poor condition. The ZFMK (Ar 22391) has two females from Iran (Hormozgan, Siyahu; $27.75^{\circ} \mathrm{N}, 56.34^{\circ} \mathrm{E}$ ) that resemble $C$. miskin sp. nov. in habitus, size, and epigynum, but with the epigynal pockets wider apart. For further undescribed species that are very similar to species (re)described herein, see under $C$. dhofar sp. nov., C. khayyami sp. nov., and C. maculipes. 


\section{Natural history}

Most species have been collected in sheltered spaces under rocks, in small cavities of the ground, and in the twilight area of caves. A few species build their webs in more exposed habitats, among rocks and on plants (e.g., C. tiwi sp. nov.). Others are occasionally or regularly found in houses (e.g., C. semicaudata, C. pristina, C. maculata, C. lyoni). Little is known about the biology of Crossopriza beyond these basic microhabitat data (see individual natural history sections below). Only the cosmopolitan C. lyoni has been studied in some detail, including development and prey capture (see below).

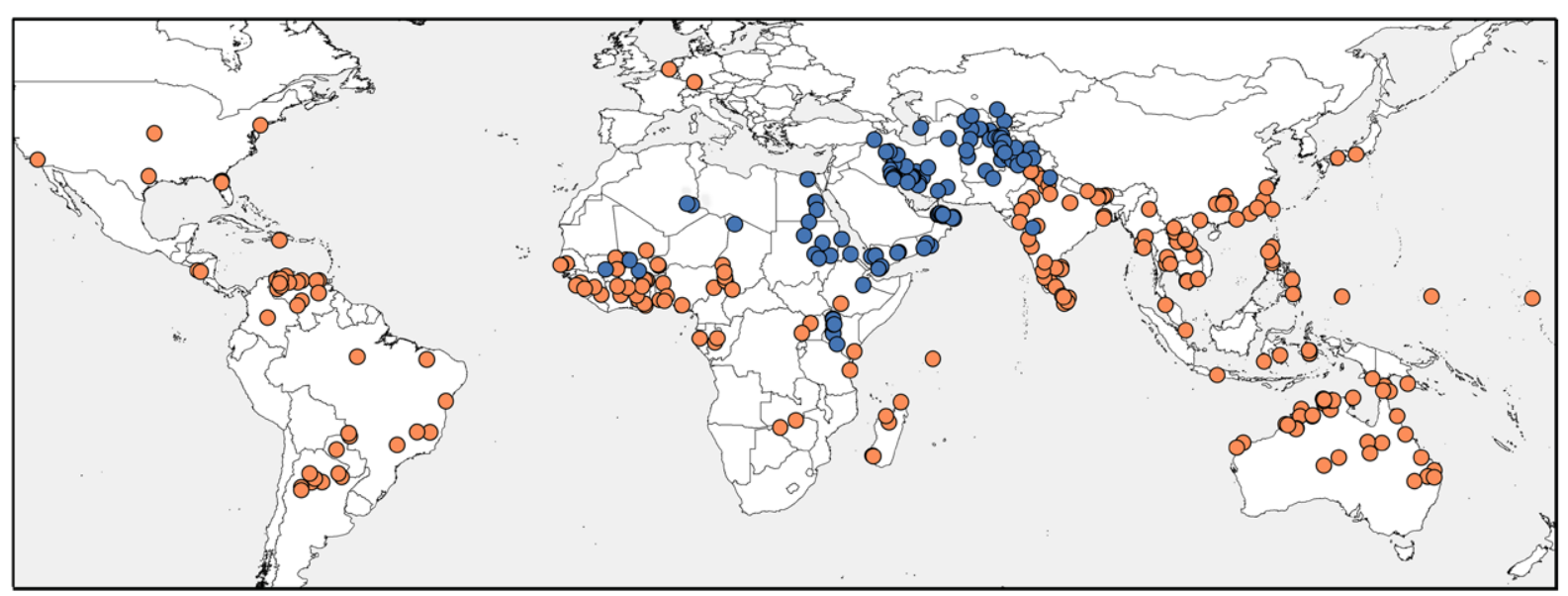

Fig. 351. Known distribution of Crossopriza Simon, 1893. Orange: C. lyoni (Blackwall, 1867); blue: all other species (cf. Figs 352-353).

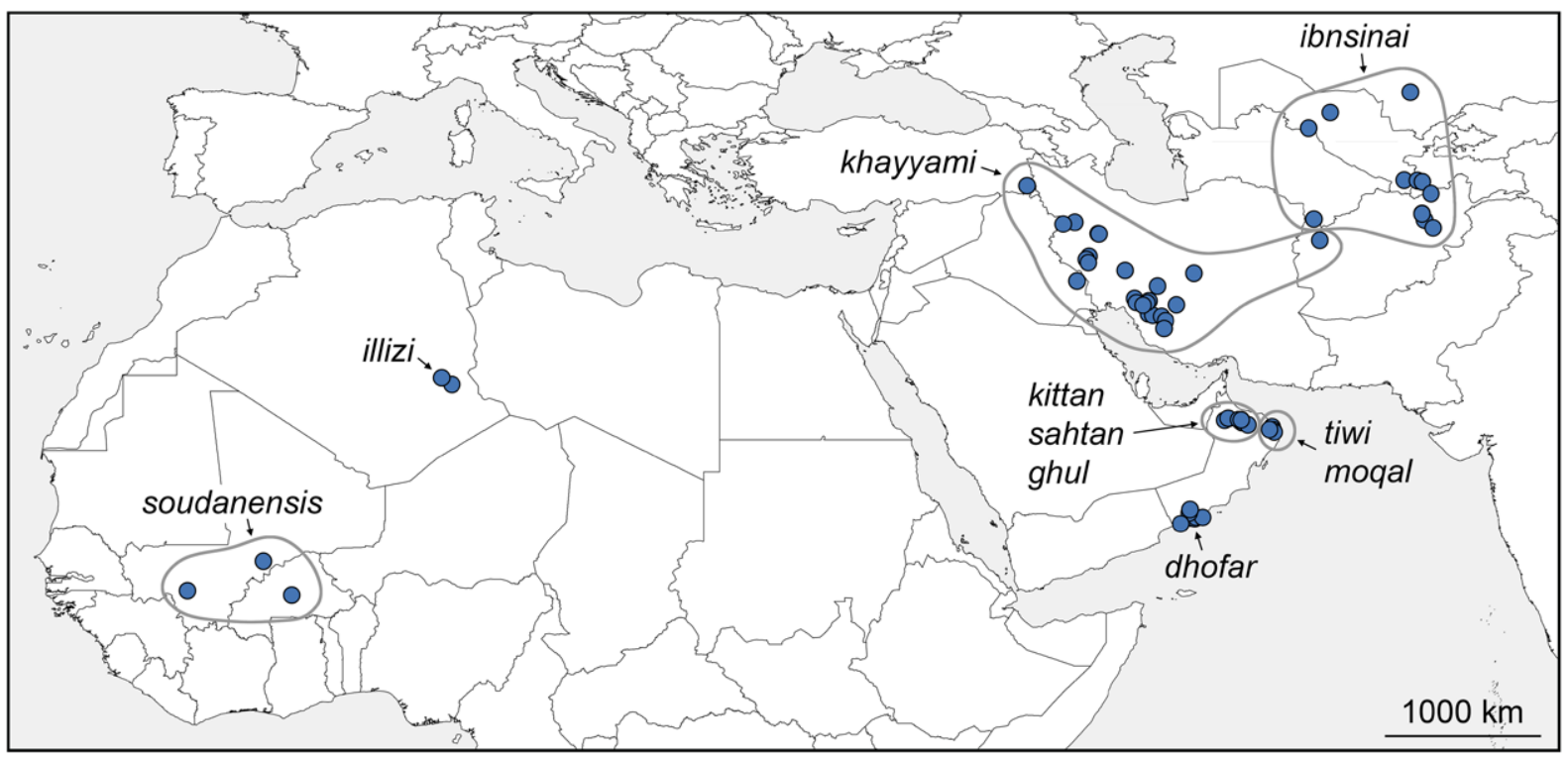

Fig. 352. Distribution of Crossopriza Simon, 1893; autochthonous species part 1 (cf. Fig. 353): species with main cheliceral apophyses in lateral position. 


\section{Identification}

A key that works for both males and females proved difficult to construct. At the same time, a combination of geography (Figs 351-353) and diagnostic characters (mainly male palp, male chelicerae, female epigynal plate) usually makes identification relatively quick and easy.
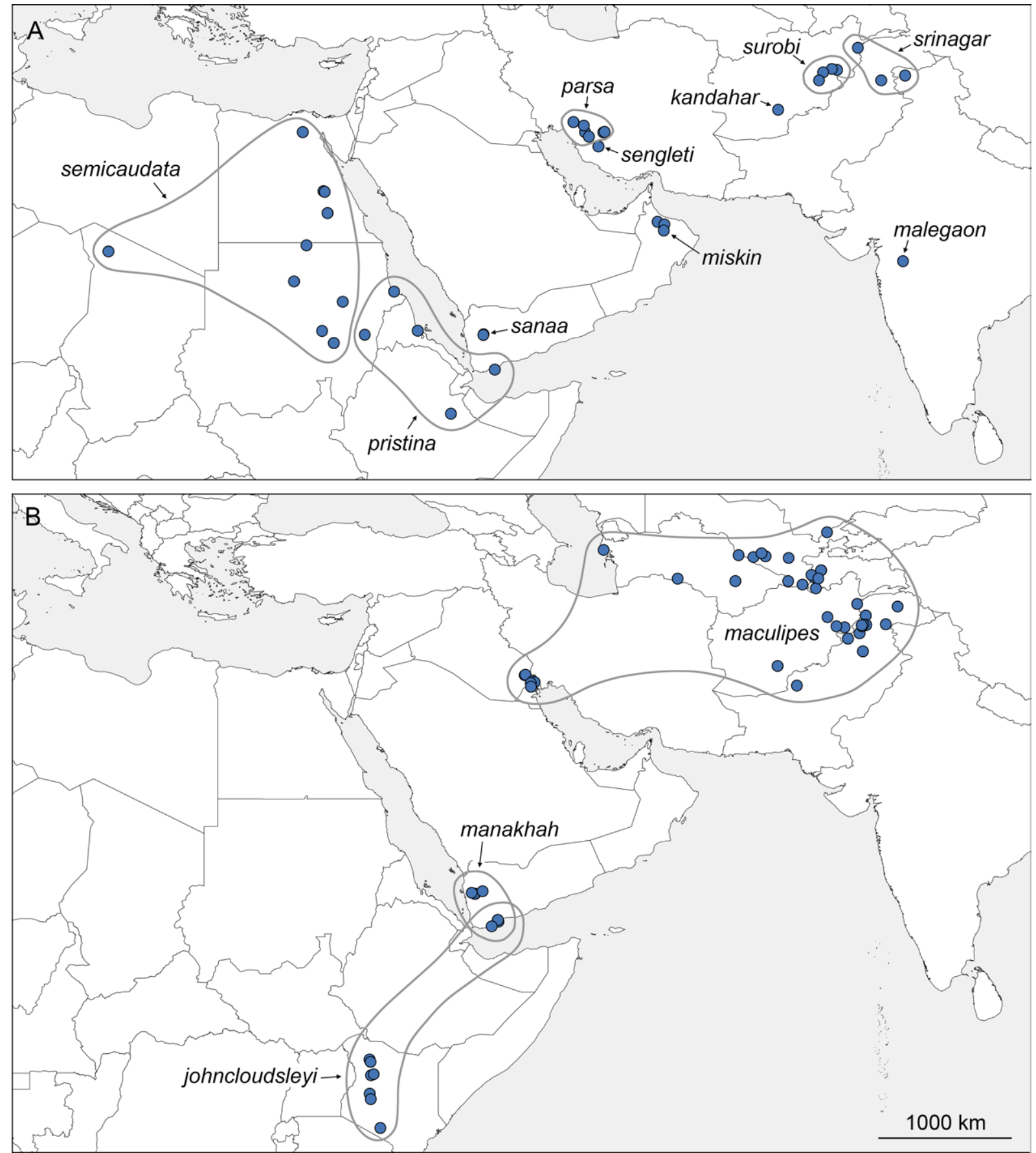

Fig. 353. Known distribution of Crossopriza Simon, 1893; autochthonous species part 2 (cf. Fig. 352): species with main cheliceral apophyses in frontal position and epigynal pockets close together. The figure is split into two to avoid too much overlap; the split is not meant to reflect any natural grouping of species. The single record of C. maculipes from China is not shown (see text). 
Crossopriza soudanensis Millot, 1941

Figs 352, 354-372

Crossopriza soudanensis Millot, 1941: 28, fig. 11a-1.

\section{Diagnosis}

Distinguished from known congeners by details of male palp (Figs 357-361; procursus tip with fringed transparent process; shape of distal bulbal sclerite); from the only geographically close congener (C. illizi sp. nov.) also by male chelicerae without modified hairs on frontal face (Fig. 362); from many congeners also by female genitalia (Figs 364-372; epigynum with elongate pockets; roundish pore plates far apart; similar to C. illizi).

\section{Type material}

Lectotype (examined; designated herein)

BURKINA FASO • ${ }^{\text {; }}$; Ouagadougou; $12.36^{\circ}$ N, $1.52^{\circ}$ W; Sep. 1937; J. Millot leg.; MNHN Ar 10520.

Paralectotypes (examined)

BURKINA FASO • 1 क ; same collection data as for lectotype; MNHN Ar 10520.

MALI • 3 우, 2 juvs; Bamako; $12.63^{\circ}$ N, 7.99 W; Oct. 1937; J. Millot leg.; MNHN Ar $10512 \bullet 1$; Sangha [Sanga]; $14.46^{\circ}$ N, 3.30 W; Oct. 1937; J. Millot leg.; MNHN Ar 10518.

\section{Other material examined}

None.

\section{Redescription}

Male (lectotype)

MeAsurements. Total length 3.5, carapace width 1.45. Distance PME-PME $80 \mu \mathrm{m}$; diameter PME $100 \times 140 \mu \mathrm{m}$; distance PME-ALE $50 \mu \mathrm{m}$; diameter AME $95 \mu \mathrm{m}$; distance AME-AME $20 \mu \mathrm{m}$. Leg 1: $42.0(12.0+0.7+11.1+15.9+2.3)$, tibia $2: 7.5$, tibia $3: 5.5$, tibia $4: 6.1$; tibia $1 \mathrm{~L} / \mathrm{d}: 72$; femora $1-4$ diameters: $0.21,0.18,0.17,0.18$.

CoLOR (in ethanol). Entire specimen very pale, bleached; original coloration (Millot 1941) apparently similar to other epigean congeners.

Body. Habitus similar to C. sahtan sp. nov. and C. ghul sp. nov. (cf. Figs 391, 481). Ocular area slightly raised. Deep thoracic pit and pair of furrows diverging from pit toward posterior margin. Clypeus unmodified, only rim more sclerotized than in female. Sternum wider than long (1.05/0.65), unmodified. Abdomen slightly elongated, dorso-posteriorly barely elevated, rounded.

CheliCerae. As in Figs 362-363, distally with pair of lateral apophyses provided with one large modified cone-shaped hair each; distance between tips of modified hairs: $390 \mu \mathrm{m}$; lateral stridulatory ridges fine but visible in dissecting microscope.

PALPS. As in Figs 354-356; coxa with strong retrolateral hump; trochanter barely modified; femur small (relative to tibia), distally on ventral side widened, proximally with prolateral stridulatory pick, without retrolateral transversal line, without retrolateral proximal process; femur-patella joints slightly shifted toward prolateral side (arrows in Fig. 354); tibia-tarsus joints shifted toward retrolateral side (arrows in Fig. 356); tarsus without macrotrichia; procursus (Figs 357-358) short and straight, proximally on prolateral side with hump set with numerous hairs, dorsal hairs straight or curved downwards, procursus 
tip with ventral sclerite with short dorsal branch, with distinctive process ending in fringed transparent membrane; genital bulb (Figs 359-361) with simple basal sclerite connected to distal (main) sclerite, connecting sclerite with ventral projection, sperm duct opening not seen; distal sclerite with retrolateral ridge, with two small prolateral apophyses and distal rim widely curved towards prolateral.

LEGS. Femur 1 with single row of $\sim 26$ ventral spines; without curved hairs; few vertical hairs; retrolateral trichobothrium of tibia 1 at 3\%; prolateral trichobothrium absent on tibia 1, present on other leg tibiae; tarsal pseudosegments not seen.

\section{Female}

In general similar to male but without spines on legs, apparently without stridulatory files on chelicerae, and with stridulatory organ consisting of pair of small whitish processes posteriorly on carapace and pair of small light brown plates anteriorly on abdomen. Tibia 1 in female from Ouagadougou: 9.1. Epigynum as in Fig. 366, main epigynal plate oval to semicircular, with pair of distinct elongate pockets (distance between pockets $\sim 310 \mu \mathrm{m}$ ); posterior plate more strongly developed laterally than medially. The only female specimen from Ouagadougou was not cleared.

Females from Mali possibly not conspecific with lectotype: very similar epigyna but pockets in female from Sangha slightly closer together (Fig. 367; distance $250 \mu \mathrm{m}$ ), in females from Bamako considerably farther apart (Fig. 370; distance $\sim 450 \mu \mathrm{m}$ ). Internal genitalia (Figs 371-372; specimen from Bamako) with almost round pore plates, simple dorsal and ventral arcs, ventral arc medially barely modified. Tibia 1 in 2 females from Bamako: 7.8, 8.7 (missing in other females from Mali).

\section{Distribution}

Known from three localities in Burkina Faso and southern Mali (Fig. 352).

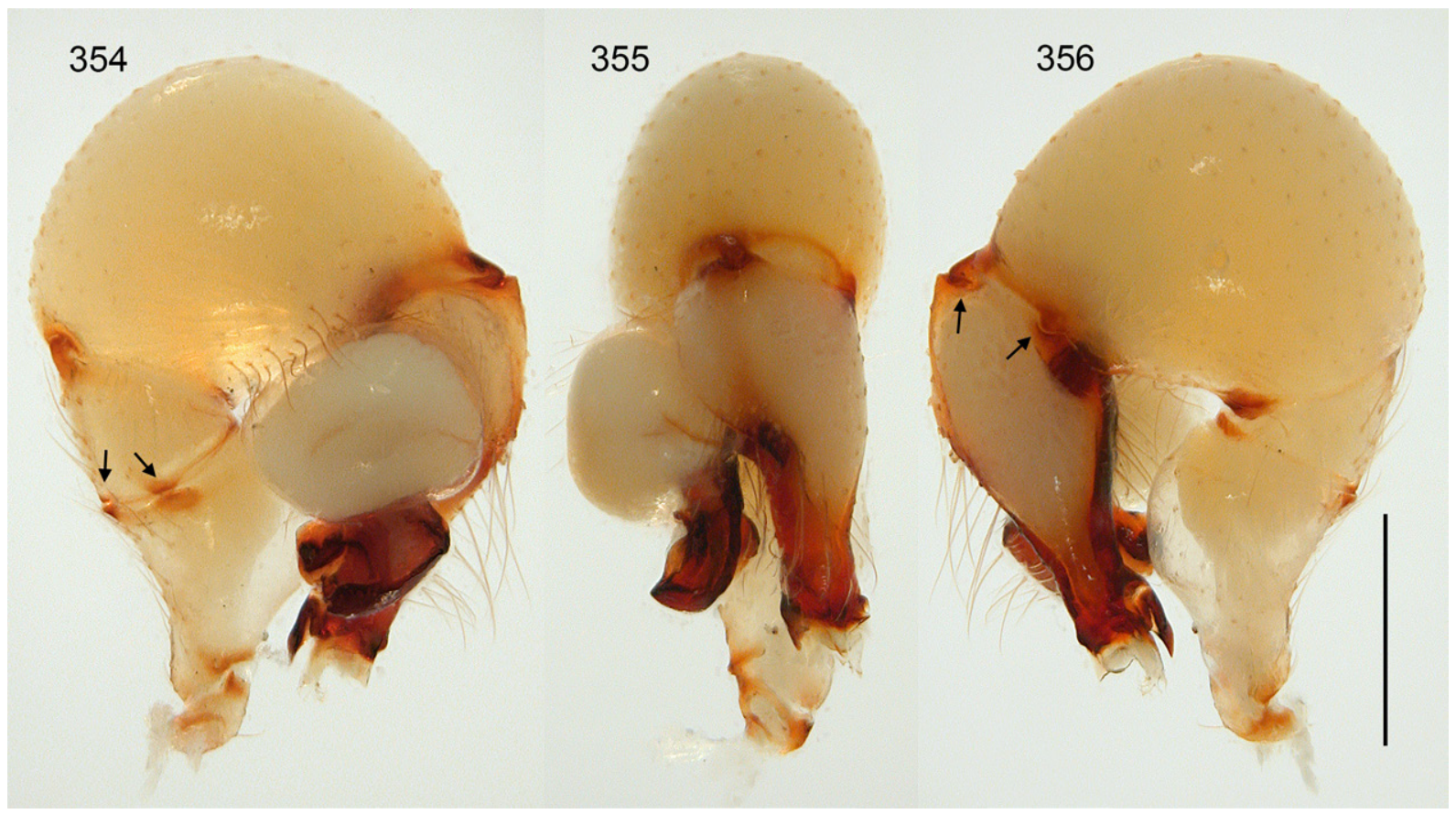

Figs 354-356. Crossopriza soudanensis Millot, 1941; lectotype male from Burkina Faso, Ouagadougou (MNHN Ar 10520); mirror images of right palp, prolateral, dorsal, and retrolateral views; arrows: joints between femur and patella and between tibia and tarsus. Scale bar $=0.5 \mathrm{~mm}$. 


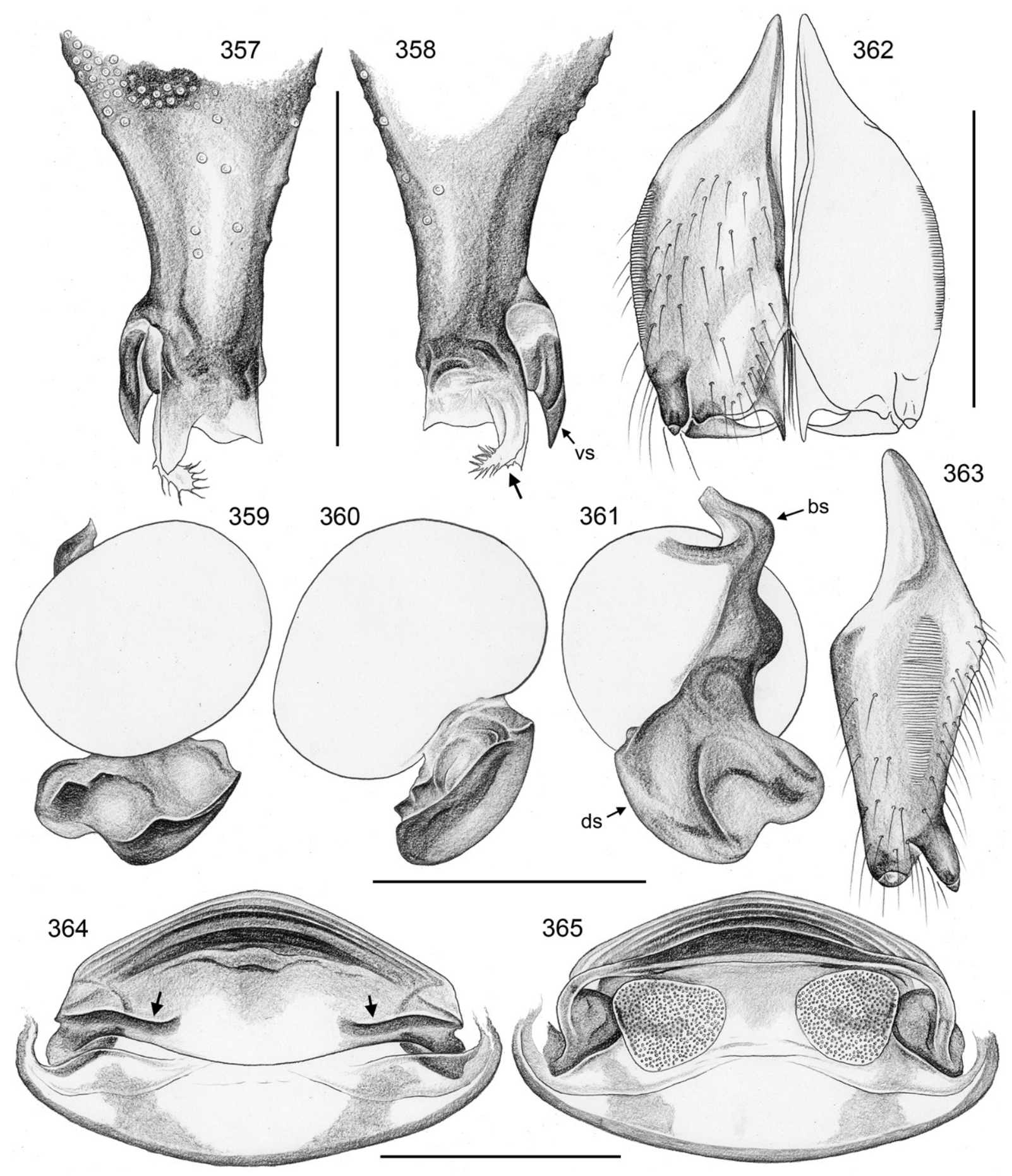

Figs 357-365. Crossopriza soudanensis Millot, 1941; lectotype male from Burkina Faso, Ouagadougou (MNHN Ar 10520), paralectotype female from Mali, Bamako (MNHN Ar 10512). 357-358. Mirror images of right procursus, prolateral and retrolateral views; arrow: transparent process. 359-361. Mirror images of right genital bulb, prolateral, dorsal, and retrolateral views. 362-363. Male chelicerae, frontal and lateral views. 364-365. Cleared female genitalia, ventral and dorsal views; arrows: pockets. Abbreviations: $\mathrm{bs}=$ basal sclerite; ds $=$ distal sclerite; vs $=$ ventral sclerite. Scale bars $=0.5 \mathrm{~mm}$. 

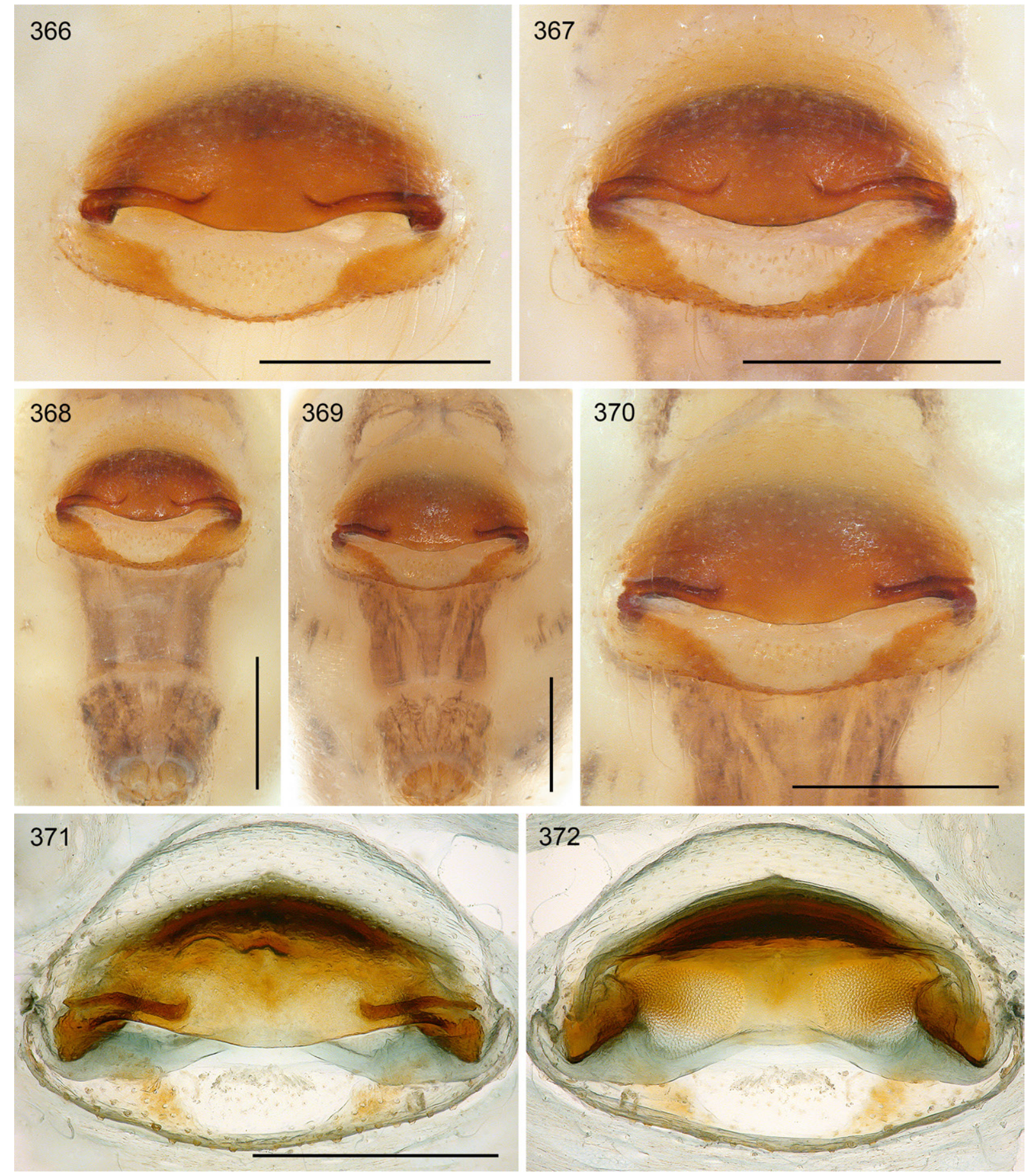

Figs 366-372. Crossopriza soudanensis Millot, 1941, paralectotypes. 366-367. Epigyna, ventral views, females from Burkina Faso, Ouagadougou (366; MNHN Ar 10520) and from Mali, Sangha (367; MNHN Ar 10518). 368-369. Abdomens, ventral views, females from Burkina Faso, Ouagadougou (368; MNHN Ar 10520) and from Mali, Bamako (369; MNHN Ar 10512). 370. Epigynum, ventral view, female from Mali, Bamako (MNHN Ar 10512). 371-372. Cleared female genitalia, ventral and dorsal views, female from Mali, Bamako (MNHN Ar 10512). Scale bars $=0.5 \mathrm{~mm}$. 


\section{Crossopriza illizi sp. nov. \\ urn:1sid:zoobank.org:act:8A2A2B20-5BD3-48A4-948C-E3EB160601F9}

Figs 352, 373-390

\section{Diagnosis}

Distinguished from known congeners by shape of distal bulbal sclerite (Fig. 379; distinctive ridges on prolateral side), by male chelicerae with modified hairs on frontal face (Figs 382-383; also present but more distal in C. dhofar sp. nov.); from many congeners also by female genitalia (Figs 386-390; epigynum with elongate pockets; roundish pore plates far apart; similar to C. soudanensis).

\section{Etymology}

The species name refers to the type locality; noun in apposition.

\section{Type material}

\section{Holotype}

ALGERIA - Illizi • ${ }^{\wedge}$; Iherir; $25.41^{\circ}$ N, $8.41^{\circ}$ E; 29 Dec. 1986; K. de Smet leg.; in Typha litter, among stones and grasses; ZFMK Ar 22392.

\section{Other material examined}

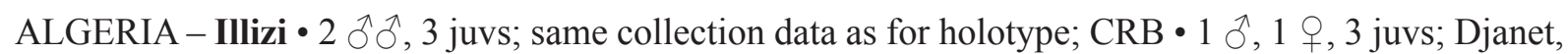
Aguelmane ti-n-Azzaret, "Adjé”; 25.83 N, 7.75 E; 17 Apr. 1979; J. Mertens leg.; CRB.

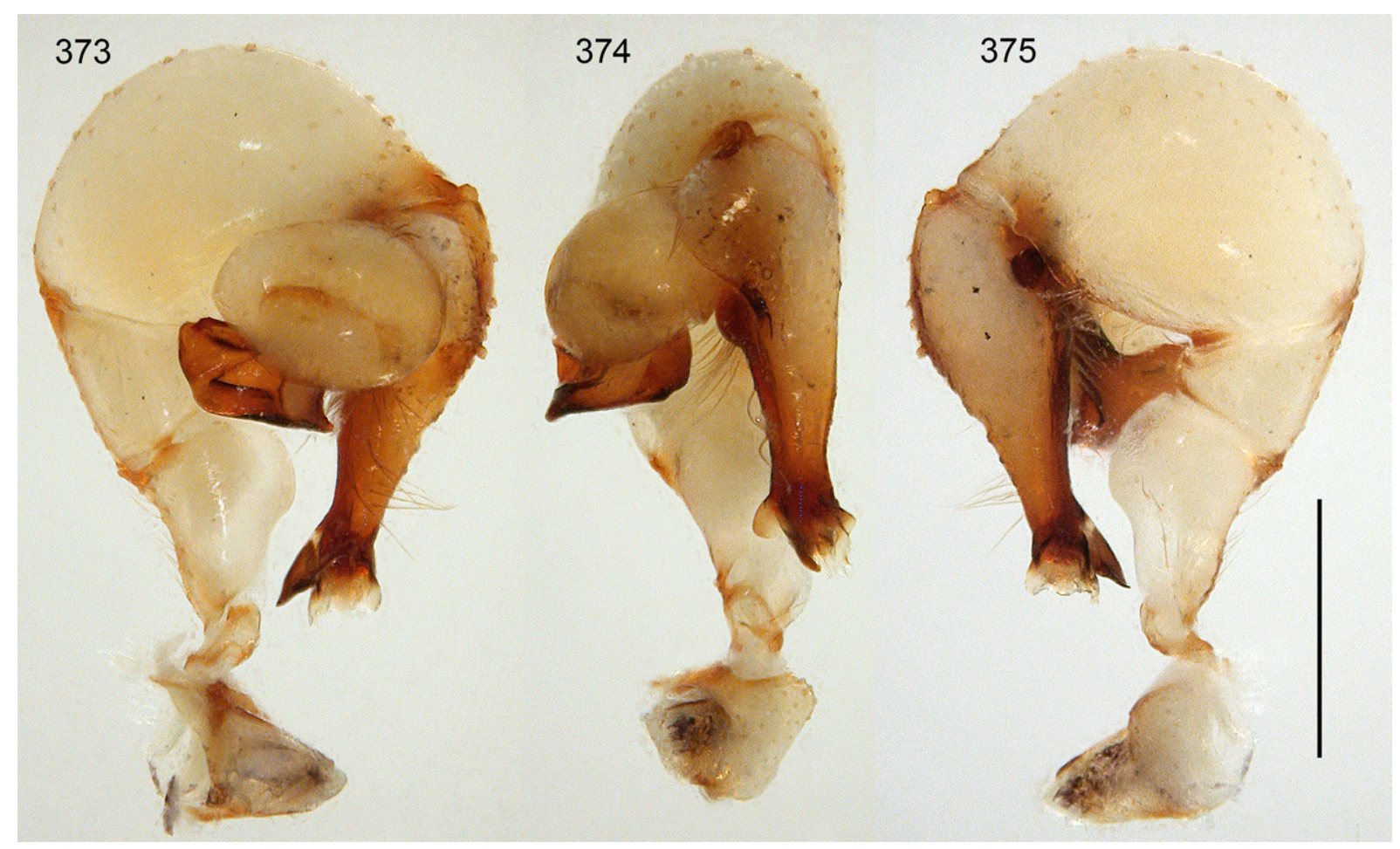

Figs 373-375. Crossopriza illizi sp. nov.; male from Algeria, Iherir (CRB); left palp, prolateral, dorsal, and retrolateral views. Scale bar $=0.5 \mathrm{~mm}$. 

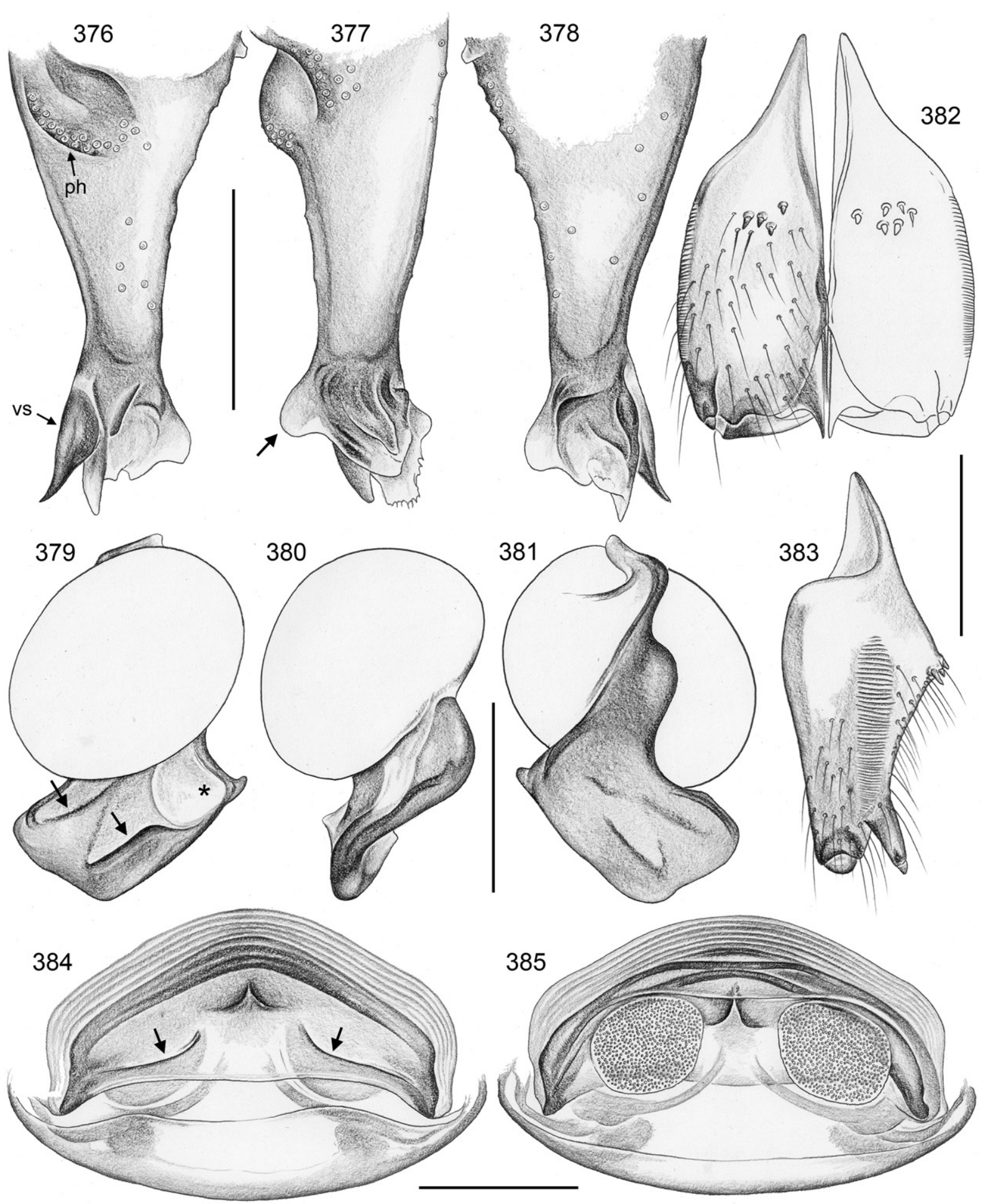

Figs 376-385. Crossopriza illizi sp. nov.; male from Algeria, Iherir (CRB), female from Algeria, Aguelmane ti-n-Azzaret (CRB). 376-378. Left procursus, prolateral, dorsal, and retrolateral views; arrow: prolateral process. 379-381. Left genital bulb, prolateral, dorsal, and retrolateral views; arrows: distinctive ridges; asterisk: putative position of sperm duct opening. 382-383. Male chelicerae, frontal and lateral views. 384-385. Cleared female genitalia, ventral and dorsal views; arrows: pockets. Abbreviations: $\mathrm{ph}=$ prolateral hump; $\mathrm{vs}=$ ventral sclerite. Scale bars $=0.3 \mathrm{~mm}$. 


\section{Description}

Male (holotype)

Measurements. Total length 3.6, carapace width 1.25. Distance PME-PME $80 \mu \mathrm{m}$; diameter PME $100 \times 120 \mu \mathrm{m}$; distance PME-ALE $25 \mu \mathrm{m}$; diameter AME $90 \mu \mathrm{m}$; distance AME-AME $35 \mu \mathrm{m}$. Leg 1: $10.9+0.6+9.9$, metatarsus damaged; tibia $2: 6.9$, tibia $3: 5.1$, tibia $4: 5.7$; tibia $1 \mathrm{~L} / \mathrm{d}: 70$; femora $1-4$ diameters: $0.18,0.17,0.16,0.16$.

CoLOR (in ethanol). Carapace pale ochre-yellow, medially darker (brown; ocular area only posteriorly brown); sternum light brown with dark brown radial marks; legs pale ochre-yellow, without dark rings, with small black marks on femora and tibiae; abdomen pale ochre-gray, with small dark marks dorsally, especially at posterior tip; ventrally with large dark brown mark in front of gonopore, three longitudinal marks behind gonopore, and dark brown area at spinnerets.

Body. Habitus similar to C. tiwi sp. nov. (cf. Figs 393-394). Ocular area slightly raised. Deep thoracic pit and pair of shallow furrows diverging from pit toward posterior margin. Clypeus unmodified, rim not more sclerotized than in female. Sternum wider than long (0.95/0.70), unmodified. Abdomen slightly elongated above spinnerets, dorso-posteriorly slightly pointed.

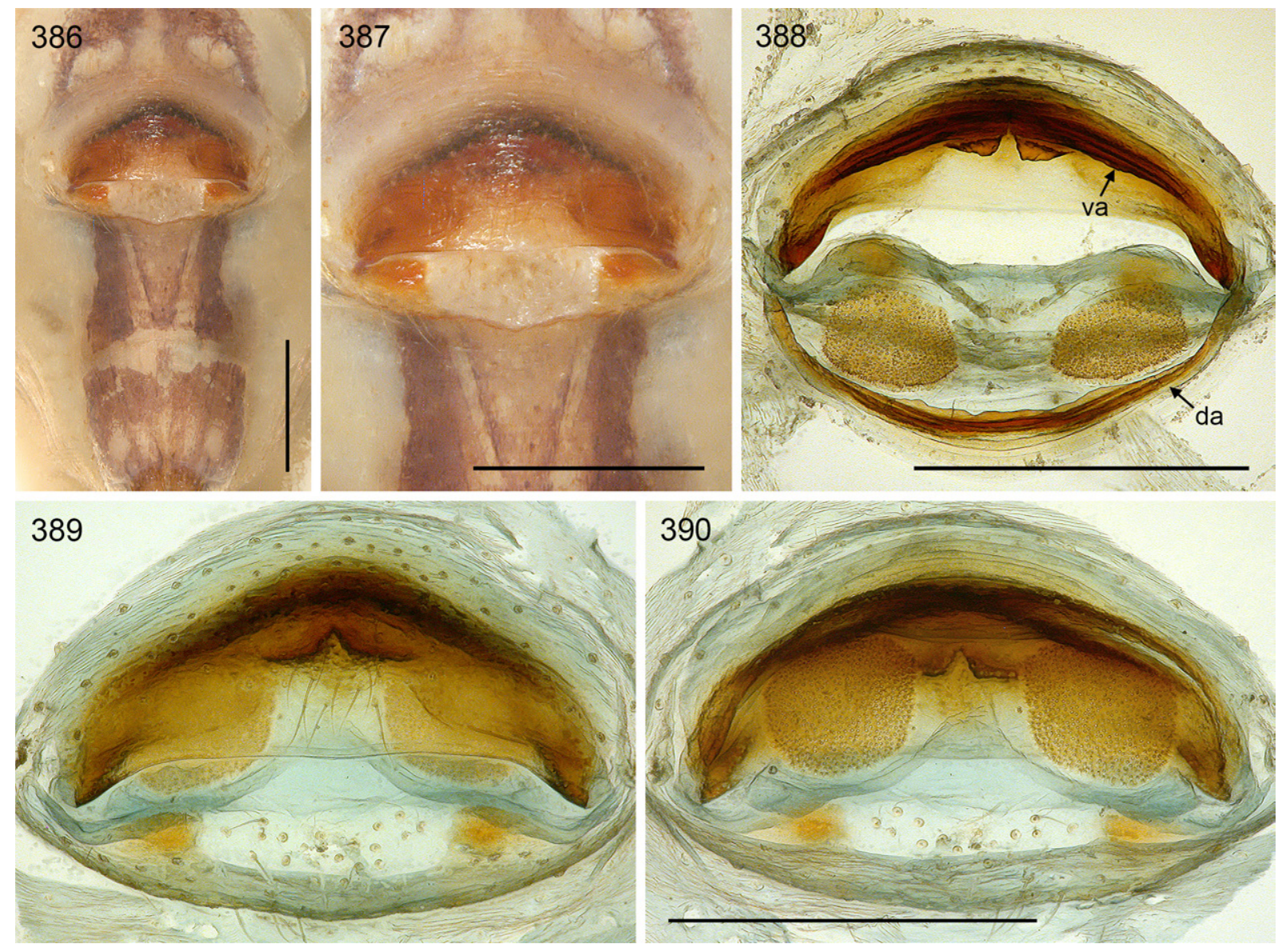

Figs 386-390. Crossopriza illizi sp. nov.; female from Algeria, Aguelmane ti-n-Azzaret (CRB). 386387. Abdomen and epigynum, ventral views. 388-390. Female internal genitalia, dorsal view (with dorsal arc tilted backwards), ventral view, and regular dorsal view. Abbreviations: da $=$ dorsal arc; va $=$ ventral arc. Scale bars $=0.5 \mathrm{~mm}$. 
Chelicerae. As in Figs 382-383, with pair of low whitish proximal humps, each provided with 5-6 small, modified (cone-shaped) hairs, and pair of frontal lateral apophyses, each with one large cone-shaped hair; distance between tips of modified hairs at tips of lateral apophyses: $370 \mu \mathrm{m}$; lateral stridulatory ridges fine but visible in dissecting microscope.

PALPS. As is Figs 373-375; coxa with rounded retrolateral-ventral hump; trochanter barely modified; femur slightly curved towards dorsal (dorso-distal line), distally widened and with distinct rounded ventral protrusion, proximally with prolateral stridulatory pick (modified hair), without retrolateral transversal line, without retrolateral-ventral proximal process, without dorsal process; femur-patella joints slightly shifted toward prolateral side; tibia strongly widened, tibia-tarsus joints shifted toward retrolateral side; tarsus without macrotrichia; procursus (Figs 376-378) straight, dorsal hairs not curved upwards; on prolateral side with proximal hump provided with numerous hairs; procursus tip with short ventral sclerite, distinctive semitransparent prolateral process, and membranous elements; genital bulb (Figs 379-381) with simple basal sclerite connected to distal (main) sclerite; distal sclerite flat and rectangular, with small dorsal process and distinctive prolateral ridges; sperm duct opening not seen (presumably in membranous prolateral field; asterisk in Fig. 379).

LEGS. Femur 1 with single row of $\sim 22$ ventral spines; without curved hairs; few vertical hairs; retrolateral trichobothrium of tibia 1 at $3 \%$; prolateral trichobothrium absent on tibia 1, present on other leg tibiae; tarsal pseudosegments not seen.

Male (variation)

Tibia 1 in two other males: 9.5, 9.6. Two males with many whitish marks on abdomen.

\section{Female}

In general similar to male but without spines on legs; with pair of prominent whitish humps posteriorly on carapace, and pair of corresponding indistinct plates frontally on abdomen; cheliceral stridulatory ridges not seen (very fine or absent). Tibia 1: 7.0. Epigynum as in Figs 386-387, main epigynal plate semicircular, wider than long, weakly protruding, posteriorly with whitish median area; with pair of grooves rather than pockets (distance $\sim 330-400 \mu \mathrm{m}$ ); posterior plate short and wide, only laterally sclerotized; area in front of epigynum not elevated. Internal genitalia (Figs 384-385, 388-390) with large rounded pore plates; dorsal arc weak, ventral arc medially strongly developed, with distinctive pair of median sclerites and indistinct small pouch ventral of these sclerites.

\section{Distribution}

Known from two localities in south-eastern Algeria (Illizi Province) (Fig. 352).

Crossopriza sahtan sp. nov. urn:1sid:zoobank.org:act:51F747A4-2F7D-4873-92BF-0D1939DDE70B

Figs 352, 391-392, 399-432

\section{Diagnosis}

Distinguished from known congeners by details of male palp (Figs 403, 406; procursus with two distal elements of similar shape; distal bulbal sclerite with single prolateral tooth); from the similar C. tiwi sp. nov. also by male chelicerae (Figs 404-405; distal apophyses closer together relative to width of chelicerae; proximal frontal processes more distinct) and by shape of epigynum (Figs 411-412; shorter, anteriorly not evenly curved).

\section{Etymology}

The species name refers to the type locality; noun in apposition. 


\section{Type material}

\section{Holotype}

OMAN - Al Batinah South • ${ }^{\wedge}$; mountain village above Wadi Sahtan; $3.220^{\circ}$ N, $57.316^{\circ}$ E; $950 \mathrm{~m}$ a.s.1.; 26 Mar. 2017; B.A. Huber leg.; among rocks in wadi; ZFMK Ar 22393.

\section{Other material examined}

OMAN - Al Batinah South • 3 ô, 6 우; same collection data as for holotype; ZFMK Ar $22394 \cdot$ 1 q, 3 juvs (in pure ethanol); same collection data as for holotype; ZFMK Om37. - Ad Dakhiliya • 1 ㄱ, 3 우, 1 juv. (in pure ethanol); Misfah (Misfat); 23.140 ${ }^{\circ}$, 57.311 ${ }^{\circ}$ E; 940 m a.s.1.; 24 Mar. 2017; B.A. Huber leg.; among rocks in wadi; ZFMK Om32 - 2 우; Wadi Hoota near upper entrance of Al Hoota cave; $23.102^{\circ} \mathrm{N}, 57.373^{\circ}$ E; 1000 m a.s.1.; 16 Feb. 2018; B.A. Huber leg.; ZFMK Ar $22395 \bullet$ $1 \mathrm{O}^{\top}$; Al Hamra; $23.123^{\circ} \mathrm{N}, 57.293^{\circ}$ E; $670 \mathrm{~m}$ a.s.l.; 24 Mar. 2017; B.A. Huber leg.; cavities in rocks;

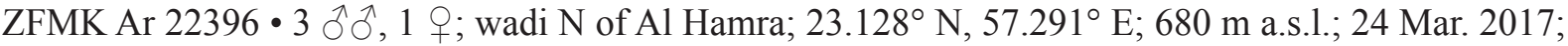
B.A. Huber leg.; cavities in rocks; ZFMK Ar 22397 1 q (in pure ethanol); same collection data as for preceding; ZFMK Om31 • $4 \hat{\jmath} \hat{\jmath}, 4$ + $\bigcirc$ (partly used for SEM); between Tanuf and Al Hamra, Al Ghubrat cave, lower entrance; $23.0718^{\circ} \mathrm{N}, 57.3680^{\circ}$ E; $665 \mathrm{~m}$ a.s.1.; 15 Feb. 2018; B.A. Huber leg.; cave entrance area; ZFMK Ar 22398 1 1 , 1 क (in pure ethanol); same collection data as for preceding; ZFMK Om103 • 1 +, 1 juv.; same locality as for preceding; 18 Nov. 2001; H. Sattmann leg.; NHMW

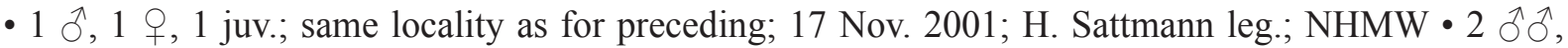
1 q; Wadi Ghul, 'site 2'; $23.233^{\circ}$ N, 57.150 E; 1430 m a.s.l.; 15 Feb. 2018; B.A. Huber leg.; ZFMK

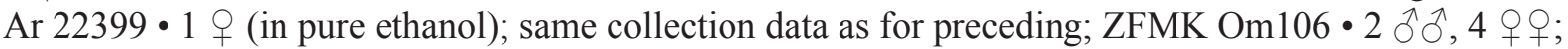
Izki, Jarnan Cave; $22.924^{\circ}$ N, $57.764^{\circ}$ E; 540 m a.s.1.; 24 Mar. 2017; B.A. Huber leg.; in cave and nearby overhangs; ZFMK Ar 22400.

\section{Description}

Male (holotype)

MeAsurements. Total length 5.5, carapace width 2.0. Distance PME-PME $110 \mu \mathrm{m}$; diameter PME $130 \times 150 \mu \mathrm{m}$; distance PME-ALE $40 \mu \mathrm{m}$; diameter AME $100 \mu \mathrm{m}$; distance AME-AME $40 \mu \mathrm{m}$. Leg 1: $60.6(17.9+0.8+15.1+23.6+3.2)$, tibia $2: 10.9$, tibia $3: 7.6$, tibia $4: 8.9$; tibia $1 \mathrm{~L} / \mathrm{d}: 66$; femora $1-4$ diameters: $0.33,0.27,0.24,0.26$.

Color (in ethanol). Carapace and clypeus ochre-yellow, anteriorly in median pit light brown; sternum dark brown with black radial marks; legs ochre-yellow, without darker rings, with many black lines on femora, few on tibiae, very few on metatarsi; abdomen ochre-gray, with dark marks dorsally, laterally, and posteriorly above spinnerets and with many whitish marks; ventrally with distinct black median band, partly disrupted, with three parallel longitudinal marks behind gonopore.

Body. Habitus as in Fig. 391. Ocular area slightly raised. Deep thoracic pit and pair of furrows diverging from pit toward posterior margin. Clypeus unmodified, only rim slightly more sclerotized than in female. Sternum wider than long (1.2/0.9), unmodified. Abdomen slightly elongated, dorso-posteriorly angular. Gonopore with four epiandrous spigots (Fig. 420); ALS with one widened spigot and one pointed spigot, PMS with two pointed spigots (Fig. 424).

Chelicerae. As in Figs 404-405, proximally with pair of pale frontal conical protrusions, distally with pair of lateral apophyses provided with one large modified cone-shaped hair each; distance between tips of modified hairs: $345 \mu \mathrm{m}$; lateral stridulatory ridges distinct (Fig. 421; distances between ridges proximally $\sim 8 \mu \mathrm{m}$, distally $\sim 6.5 \mu \mathrm{m}$ ), clearly visible in dissecting microscope.

PALPS. As in Figs 399-401; coxa with rounded retrolateral hump; trochanter barely modified; femur distally strongly widened, with rounded ventral protrusion, proximally with prolateral stridulatory 

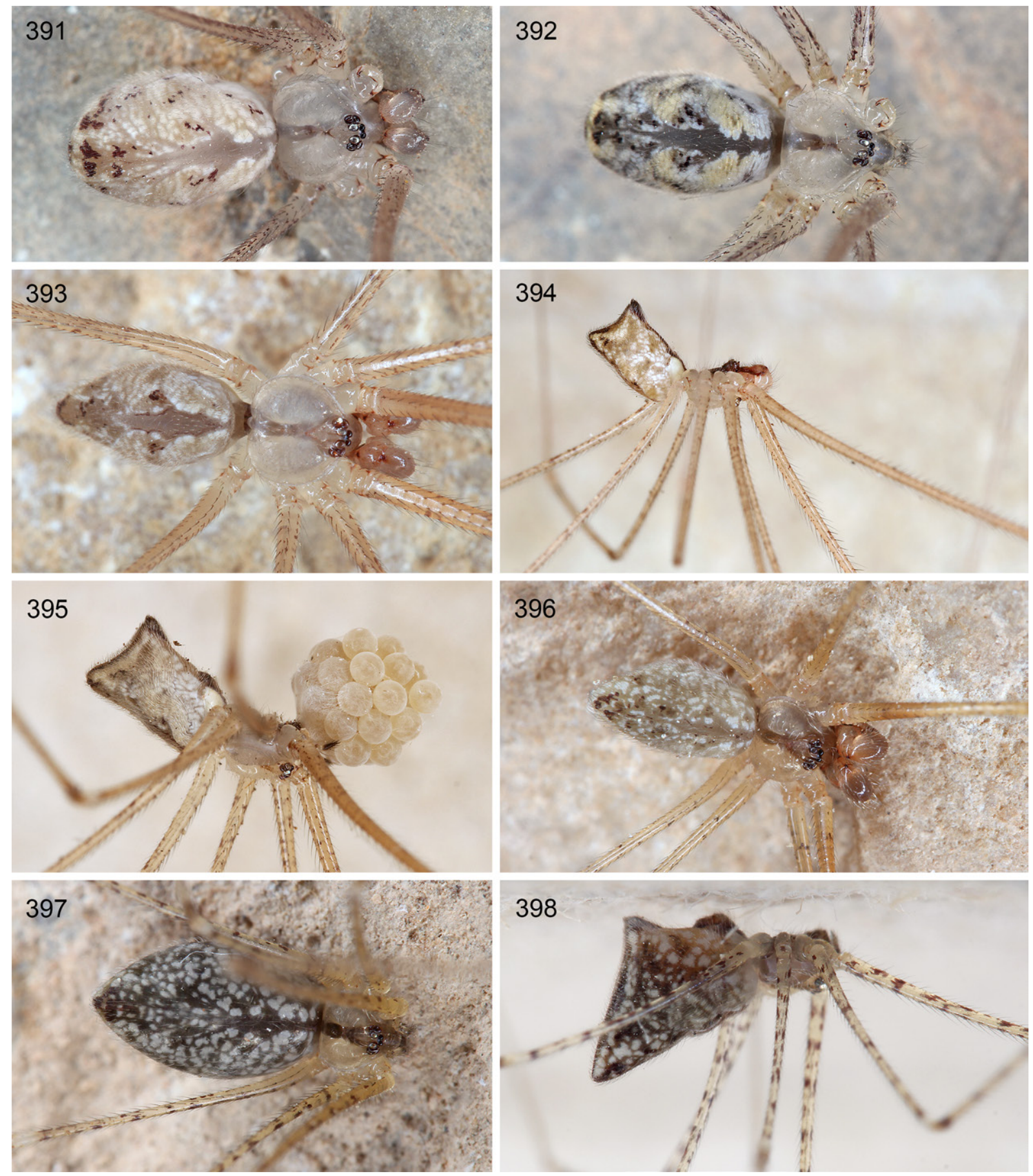

Figs 391-398. Crossopriza Simon, 1893, live specimens. 391-392. C. sahtan sp. nov., male and female from Oman, Wadi Ghul. 393-395. C. tiwi sp. nov., males and female with egg-sac from Oman, Wadi Tiwi. 396-398. C. dhofar sp. nov., male and female from Oman, Ain Razad, and female from Oman, Wadi Nahiz. 
pick, without retrolateral transversal line, without retrolateral proximal process; femur-patella joints shifted toward prolateral side; tibia-tarsus joints slightly shifted toward retrolateral side; tarsus without macrotrichia; tarsal organ capsulate (Fig. 426); procursus straight, proximal prolateral hump set with numerous long hairs, long dorsal hairs partly weakly curved upwards, procursus tip (Figs 402-403) with strong ventral sclerite and distinctive dorsal sclerite narrowing distally, with single hair-like process (arrow in Fig. 417); genital bulb (Figs 406-408, 418-419) with simple basal sclerite connected to distal (main) sclerite, sperm duct opening basally on prolateral side (arrow in Fig. 419); distal sclerite with sharp retrolateral ridge and single pointed prolateral tooth.

LEGS. Femur 1 with single row of $\sim 40$ ventral spines; without curved hairs; few vertical hairs; retrolateral trichobothrium of tibia 1 at 3\%; prolateral trichobothrium absent on tibia 1, present on other leg tibiae; tarsi without regular pseudosegments except 2-3 at tip; tarsal organs capsulate, with round or slightly irregular rim (Figs 428-431).

Male (variation)

Tibia 1 in 15 males (incl. holotype): 12.5-16.5 (mean 14.3). Smaller males with fewer spines on femur 1 $(\sim 30)$. Dark marks on abdomen always distinct but in variably 'complete' pattern.

\section{Female}

In general similar to male (Fig. 392) but without spines on legs; without stridulatory files on chelicerae (Fig. 422), corresponding hair on palpal femur only weakly modified (Fig. 423; slightly stronger than usual); with stridulatory organ consisting of pair of weakly sclerotized but distinct processes posteriorly on carapace and pair of indistinct light brown or non-darkened plates anteriorly on abdomen. Tibia 1 in 18 females: 11.9-15.3 (mean 13.1). Epigynum as in Figs 412 and 432, main epigynal plate short but wide

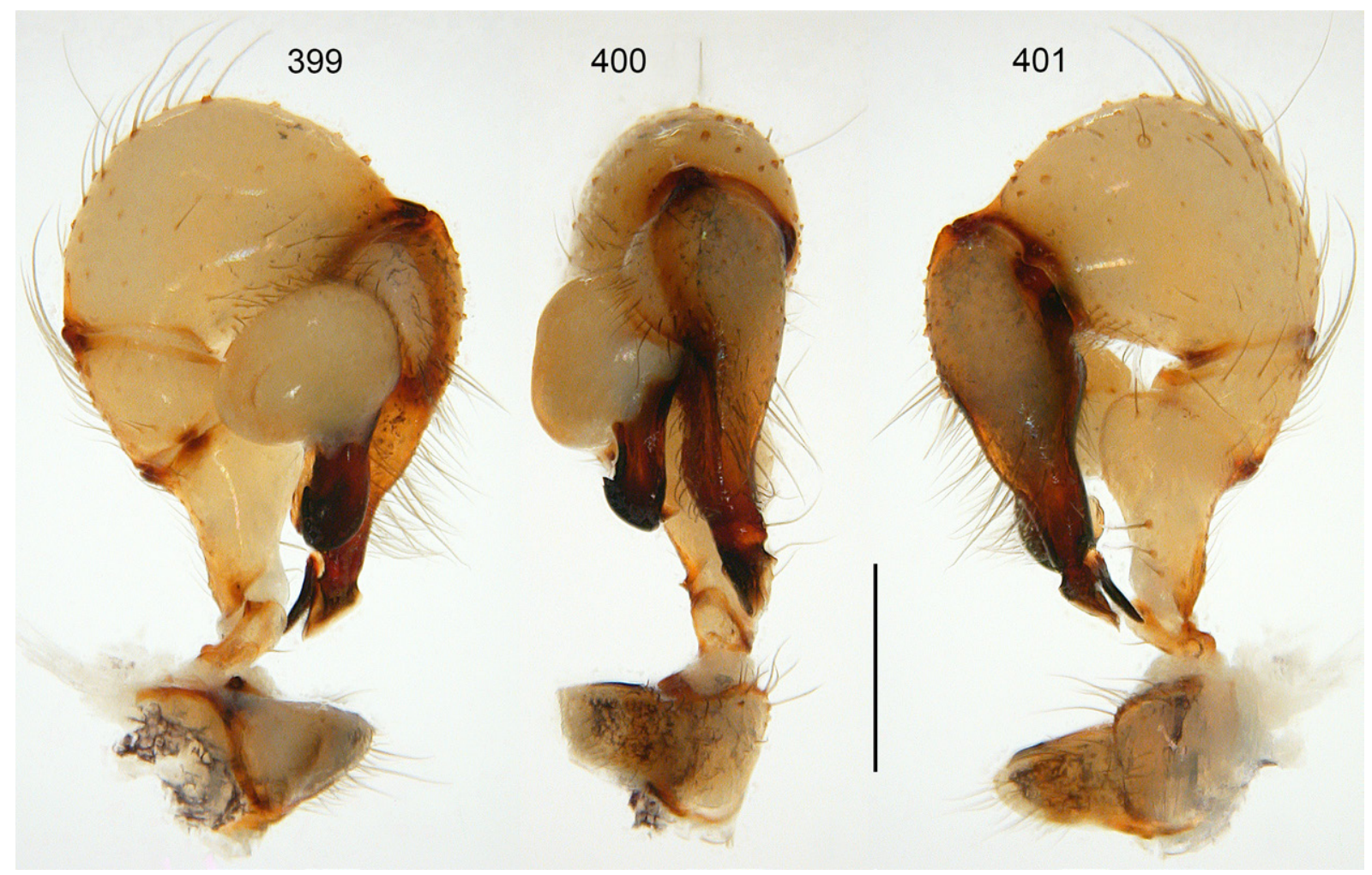

Figs 399-401. Crossopriza sahtan sp. nov.; male from Oman, above Wadi Sahtan (ZFMK Ar 22394); left palp, prolateral, dorsal, and retrolateral views. Scale bar $=0.5 \mathrm{~mm}$. 


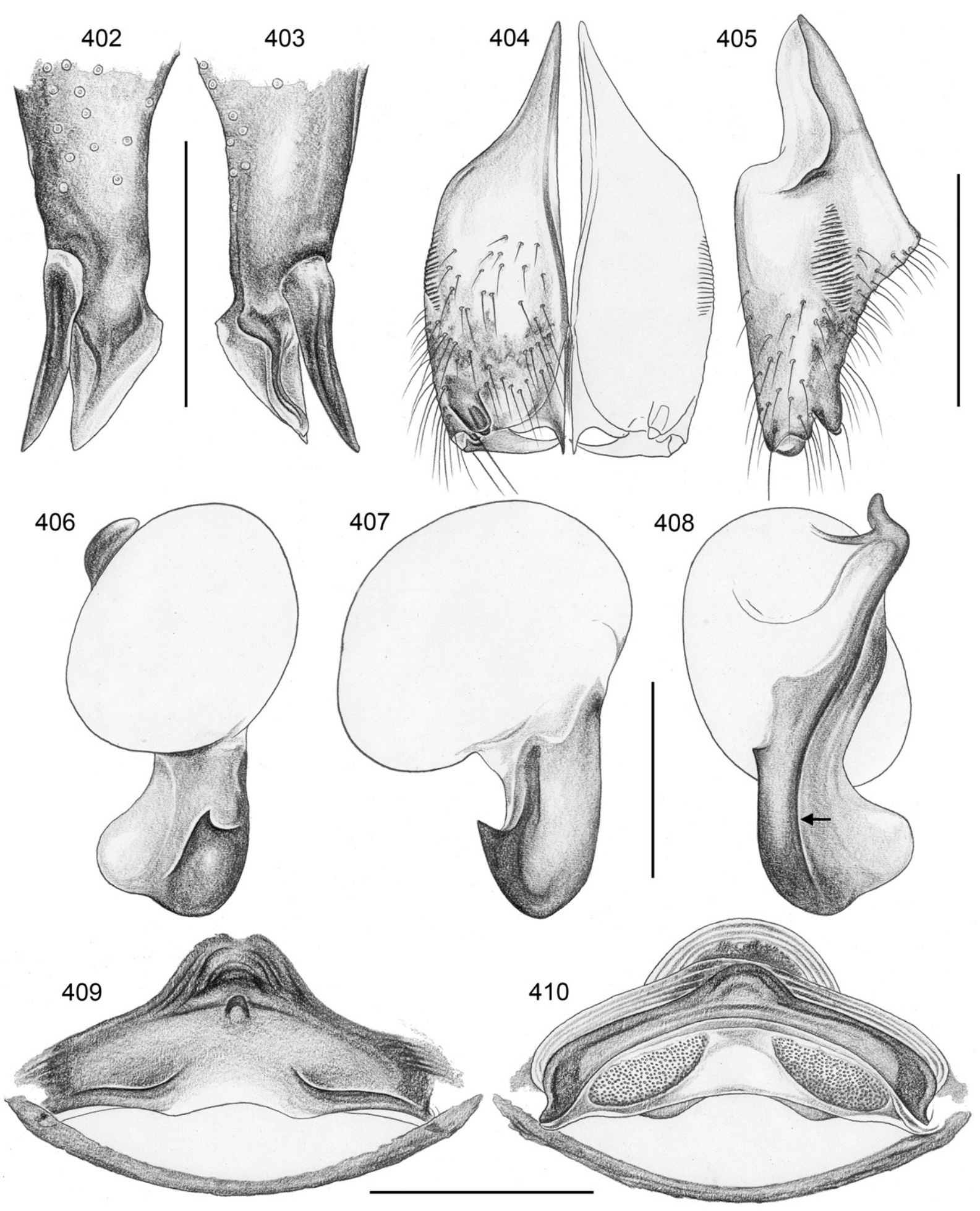

Figs 402-410. Crossopriza sahtan sp. nov.; male and female from Oman, above Wadi Sahtan (ZFMK Ar 22394). 402-403. Left procursus tip, prolateral and retrolateral views. 404-405. Male chelicerae, frontal and lateral views. 406-408. Left genital bulb, prolateral, dorsal, and retrolateral views; arrow: retrolateral ridge. 409-410. Cleared female genitalia, ventral and dorsal views. Scale bars: 402-403, $406-408=0.3 \mathrm{~mm} ; 404-405,409-410=0.5 \mathrm{~mm}$. 
trapezoidal, weakly protruding; with pair of shallow pockets (rather furrows) $\sim 365 \mu \mathrm{m}$ apart; internal sclerotized arc and median round structure visible in uncleared specimens; posterior plate short but wide. Internal genitalia (Figs 409-410, 413-415) with large oval pore plates converging anteriorly, dorsal arc strong but simple, ventral arc with median pouch or pocket of variable size and unknown function.

\section{Natural history}

This species was sometimes abundant in cavities in and under rocks, sometimes in very dry environments (e.g., the very shallow Jarnan Cave). In one particularly suitable habitat above Wadi Sahtan (a large rock suspended above a small stream) the density was extremely high: approximately 100 large specimens and many small specimens were estimated on a rock surface of approximately $2 \mathrm{~m}^{2}$. At Al Ghubrat Cave, the spiders were only found at the cave entrance, while the interior of the cave was occupied by Artema ghubrat Huber, 2019.

\section{Distribution}

Known from several localities in north eastern Oman (Al Batinah South and Ad Dakhiliya Provinces) (Fig. 352).
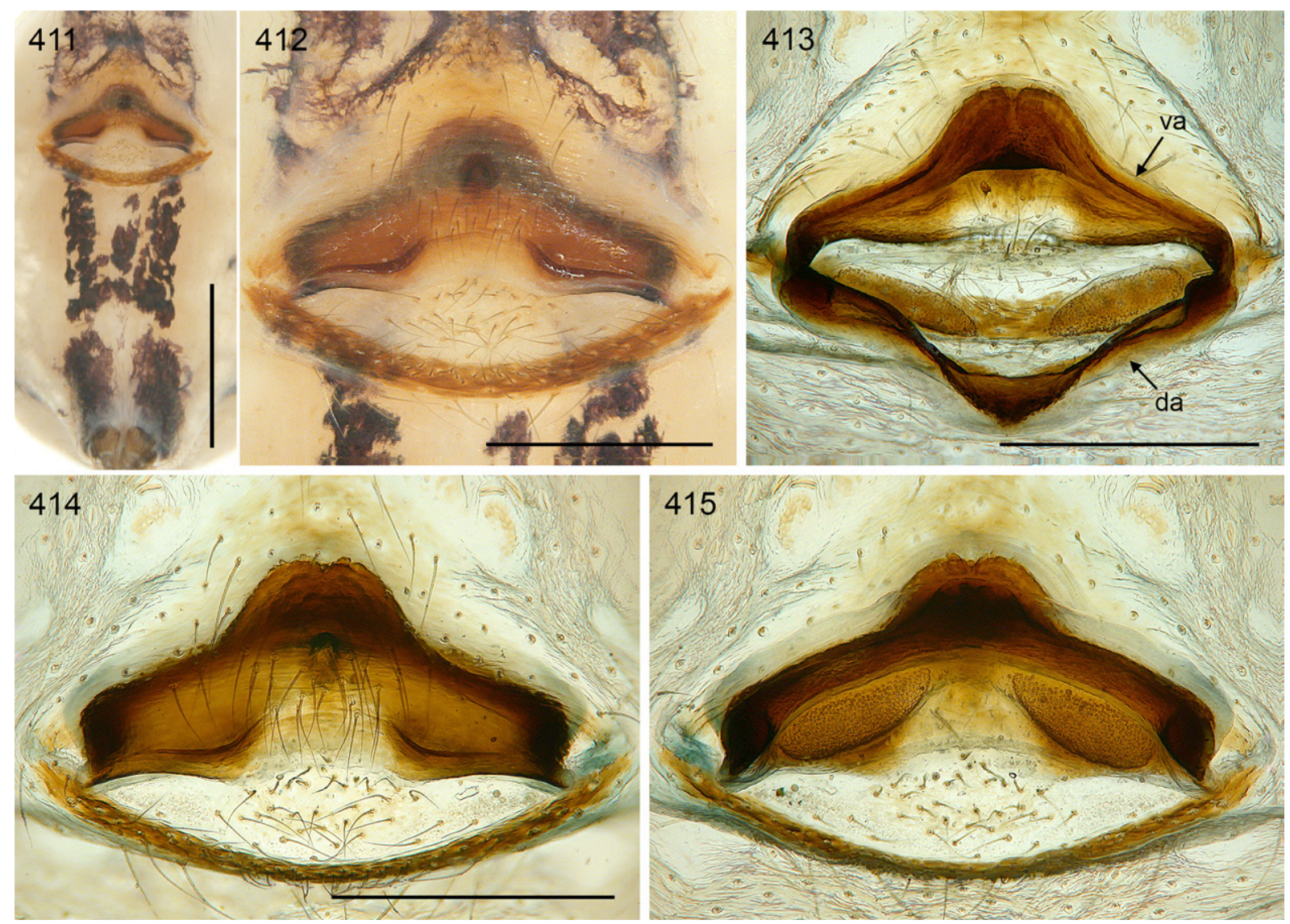

Figs 411-415. Crossopriza sahtan sp. nov.; female from Oman, above Wadi Sahtan (ZFMK Ar 22394). 411-412. Abdomen and epigynum, ventral views. 413-415. Female internal genitalia, dorsal view (with dorsal arc tilted backwards), ventral view, and regular dorsal view. Abbreviations: da $=$ dorsal arc; va $=$ ventral arc. Scale bars: $411=1.0 \mathrm{~mm} ; 412-415=0.5 \mathrm{~mm}$. 

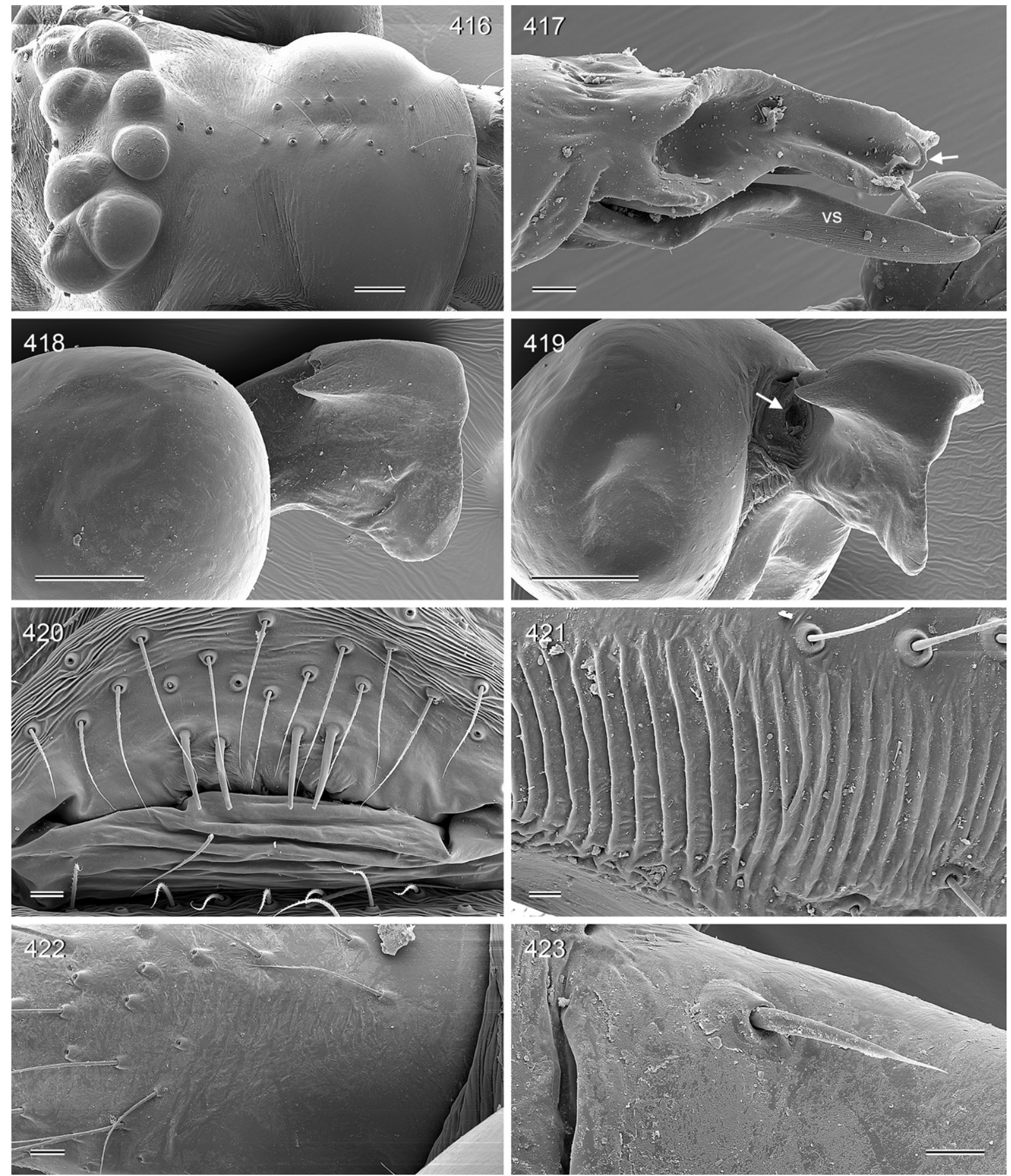

Figs 416-423. Crossopriza sahtan sp. nov.; male and female from Oman, Al Ghubrat cave (ZFMK Ar 22398). 416. Male ocular area and clypeus. 417. Right procursus, retrolateral-ventral view; arrow: hair-like process. 418-419. Left genital bulbs, prolateral and prolateral-distal views; arrow: sperm duct opening. 420. Male gonopore. 421-422. Male stridulatory ridges and corresponding smooth female cheliceral side. 423. Prolateral proximal hair on female palpal femur. Abbreviation: vs $=$ ventral sclerite. Scale bars: $416,418-419=100 \mu \mathrm{m} ; 417,420,422=20 \mu \mathrm{m} ; 421,423=10 \mu \mathrm{m}$. 

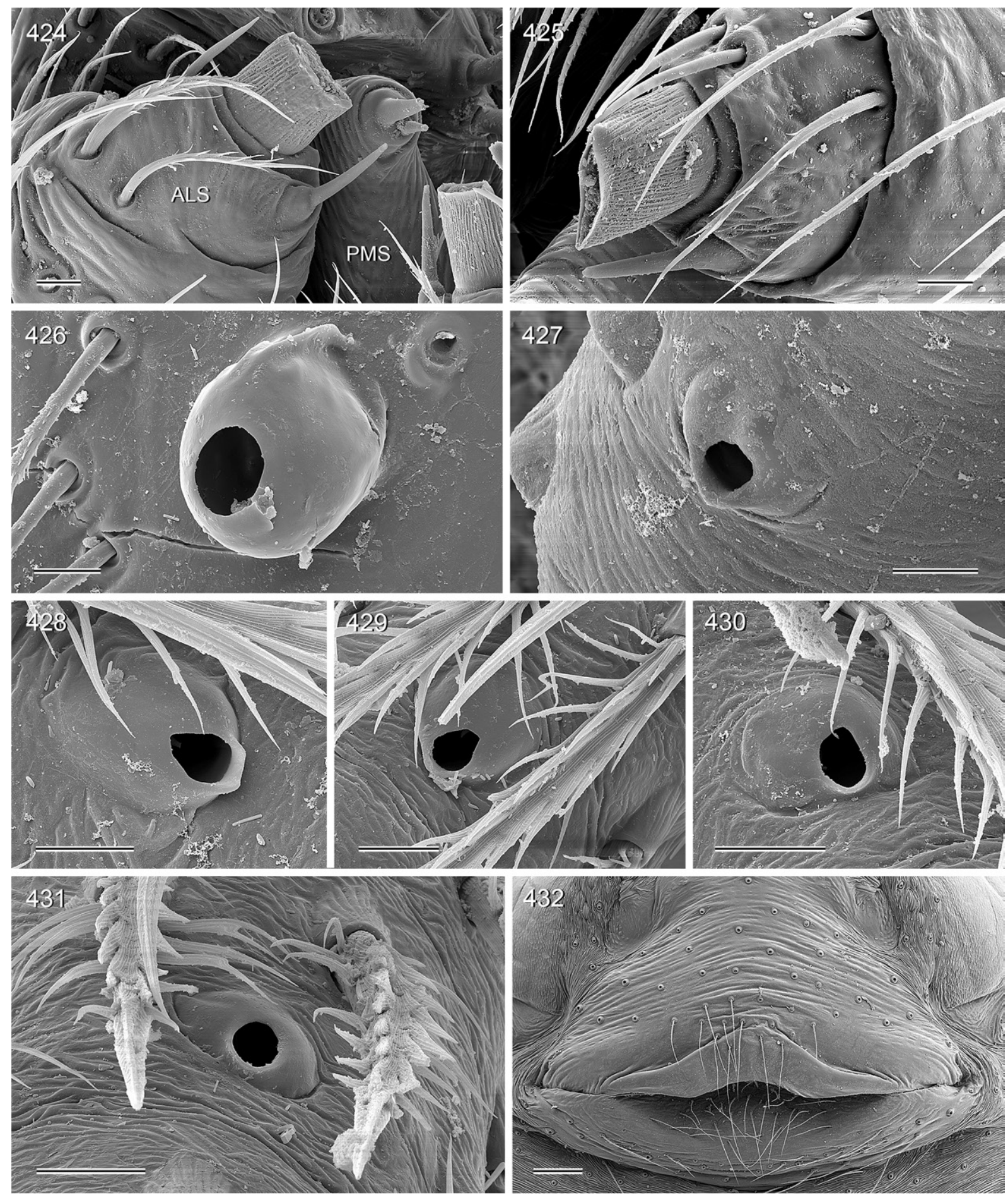

Figs 424-432. Crossopriza sahtan sp. nov.; male and female from Oman, Al Ghubrat cave (ZFMK Ar 22398). 424. Male spinnerets. 425. Female ALS. 426-427. Male and female palpal tarsal organs. 428-431. Tarsal organs on male tarsi 1-4. 432. Epigynum. Scale bars: $424-431=10 \mu \mathrm{m} ; 432=100 \mu \mathrm{m}$. 
Crossopriza tiwi sp. nov. urn:1sid:zoobank.org:act:740EB862-E768-4F8A-8E25-84A54D43403E

Figs 352, 393-395, 433-449

\section{Diagnosis}

Distinguished from known congeners by details of male palp (Figs 437, 440; procursus tip with short ventral sclerite and wide dorsal membranous element; distal bulbal sclerite with distinctive set of prolateral teeth and ridges); from the similar C. sahtan sp. nov. also by male chelicerae (Figs 438 439; distal apophyses wider apart relative to width of chelicerae; proximal frontal processes much less distinct) and by shape of epigynum (Fig. 446; longer, anteriorly evenly curved).

\section{Etymology}

The species name refers to the type locality; noun in apposition.

\section{Type material}

\section{Holotype}

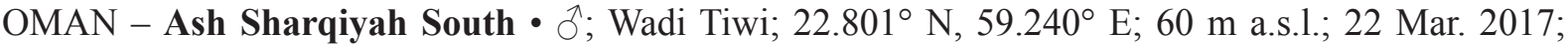
B.A. Huber leg.; ZFMK Ar 22401.

\section{Other material examined}

OMAN - Ash Sharqiyah South $10 \hat{\partial} \hat{\partial}, 7$ 우; same collection data as for holotype; ZFMK Ar 22402,

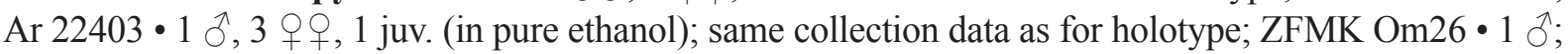
8 km NNW of Wadd; $22.6136^{\circ}$ N, $59.2919^{\circ}$ E; 430 m a.s.1.; 18 Feb. 2018; B.A. Huber leg.; ZFMKAr 22404 - 1 juv. (in pure ethanol); same collection data as for preceding; ZFMK Om119 1 \%; between Sur and Al Kamil; 22.463 ${ }^{\circ}$ N, 59.387 E; 90 m a.s.l.; 23 Mar. 2017; B.A. Huber leg.; in wadi; ZFMK Ar $22405 \bullet$

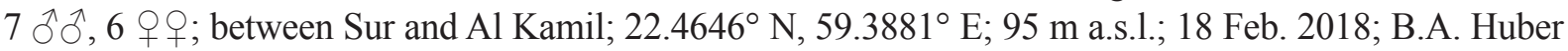
leg.; palm garden; ZFMK Ar 22406, Ar 22407•3 우, 2 juvs (in pure ethanol); same collection data as for preceding; ZFMK Om117. - Ash Sharqiyah North • 3 우우 ; Wadi Bani Khalid, outside of Mukal (Moqal) Cave; 22.624 N, 59.097 E; 700 m a.s.1.; 23 Mar. 2017; B.A. Huber leg.; ZFMK Ar 22408.

\section{Description}

\section{Male (holotype)}

Measurements. Total length 4.1, carapace width 1.7. Distance PME-PME $75 \mu \mathrm{m}$; diameter PME $130 \times$ $150 \mu \mathrm{m}$; distance PME-ALE $30 \mu \mathrm{m}$; diameter AME $100 \mu \mathrm{m}$; distance AME-AME $20 \mu \mathrm{m}$. Leg 1: 49.7 $(14.9+0.7+12.9+18.5+2.7)$, tibia $2: 9.1$, tibia $3: 6.5$, tibia $4: 7.7$; tibia $1 \mathrm{~L} / \mathrm{d}: 65$; femora $1-4$ diameters: $0.31,0.25,0.22,0.24$.

Color (in ethanol). Carapace ochre-yellow, anteriorly in median pit light brown; sternum dark brown with black radial marks; legs ochre-yellow, without darker rings, with black lines on femora and (few) on tibiae; abdomen pale gray, with some dark marks dorsally and posteriorly above spinnerets; ventrally with distinct black pattern, with three parallel longitudinal marks behind gonopore.

Body. Habitus as in Figs 393-394. Ocular area slightly raised. Deep thoracic pit and pair of furrows diverging from pit toward posterior margin. Clypeus unmodified, only rim slightly more sclerotized than in female. Sternum wider than long (1.2/0.8), unmodified. Abdomen slightly elongated, dorsoposteriorly angular.

Chelicerae. As in Figs 438-439, with pair of latero-distal apophyses provided with one large modified cone-shaped hair each; distance between tips of modified hairs: $360 \mu \mathrm{m}$; lateral stridulatory ridges clearly visible in dissecting microscope. 
PaLPS. As in Figs 433-435; coxa with rounded retrolateral hump; trochanter barely modified; femur distally strongly widened, with rounded ventral protrusion, proximally with prolateral stridulatory pick, with indistinct retrolateral transversal line, without retrolateral proximal process; femur-patella joints shifted toward prolateral side; tibia-tarsus joints slightly shifted toward retrolateral side; tarsus without macrotrichia; procursus straight, with prolateral proximal hump set with many long hairs, long dorsal hairs not or only weakly curved, procursus tip (Figs 436-437) with strong ventral sclerite and further distinctive membranous and sclerotized elements; genital bulb (Figs 440-442) with simple basal sclerite connected to distal (main) sclerite, sperm duct opening at basis of distal sclerite; distal sclerite with sharp retrolateral ridge and distinctive set of prolateral apophyses and ridges.

LEGS. Femur 1 with single row of $\sim 40$ ventral spines; without curved hairs; few vertical hairs; retrolateral trichobothrium of tibia 1 at 3.5\%; prolateral trichobothrium absent on tibia 1, present on other leg tibiae; tarsi with indistinct platelets rather than regular pseudosegments.

Male (variation)

Tibia 1 in 16 males (incl. holotype): 9.9-13.1 (mean 11.3). Smaller males with fewer spines on femur 1 ( 10-20). Abdomen often also with white marks. Ventral parallel marks on abdomen variably fused or separate.

\section{Female}

In general similar to male (Fig. 395) but without spines on legs, with small and poorly visible stridulatory files on chelicerae, and with stridulatory organ consisting of pair of weakly sclerotized but distinct processes posteriorly on carapace and pair of distinct light brown plates anteriorly on abdomen. Tibia 1 in 17 females: 7.1-11.7 (mean 9.8). Epigynum as in Figs 445-446, main epigynal plate semicircular to

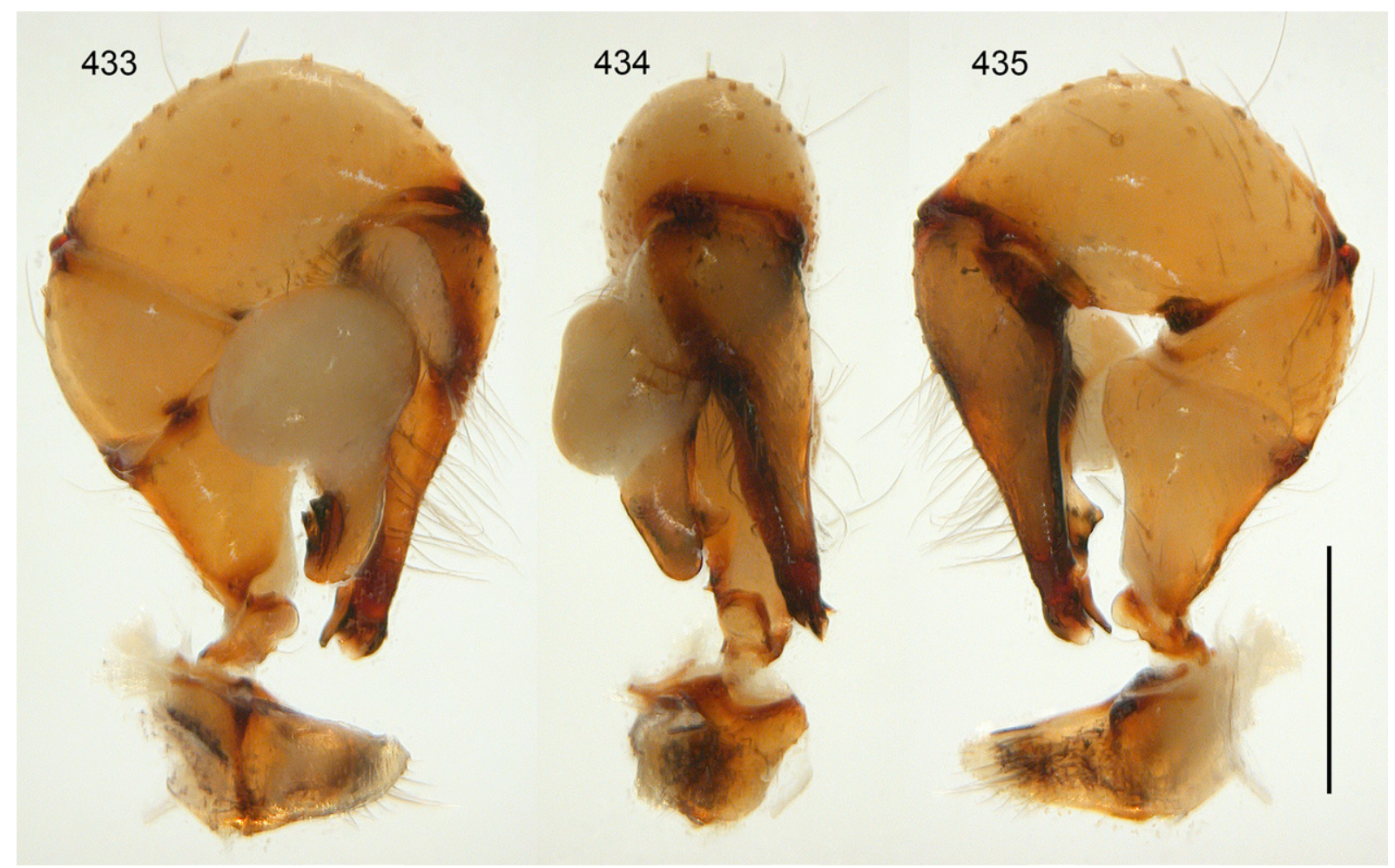

Figs 433-435. Crossopriza tiwi sp. nov.; male from Oman, Wadi Tiwi (ZFMK Ar 22402); left palp, prolateral, dorsal, and retrolateral views. Scale bar $=0.5 \mathrm{~mm}$. 


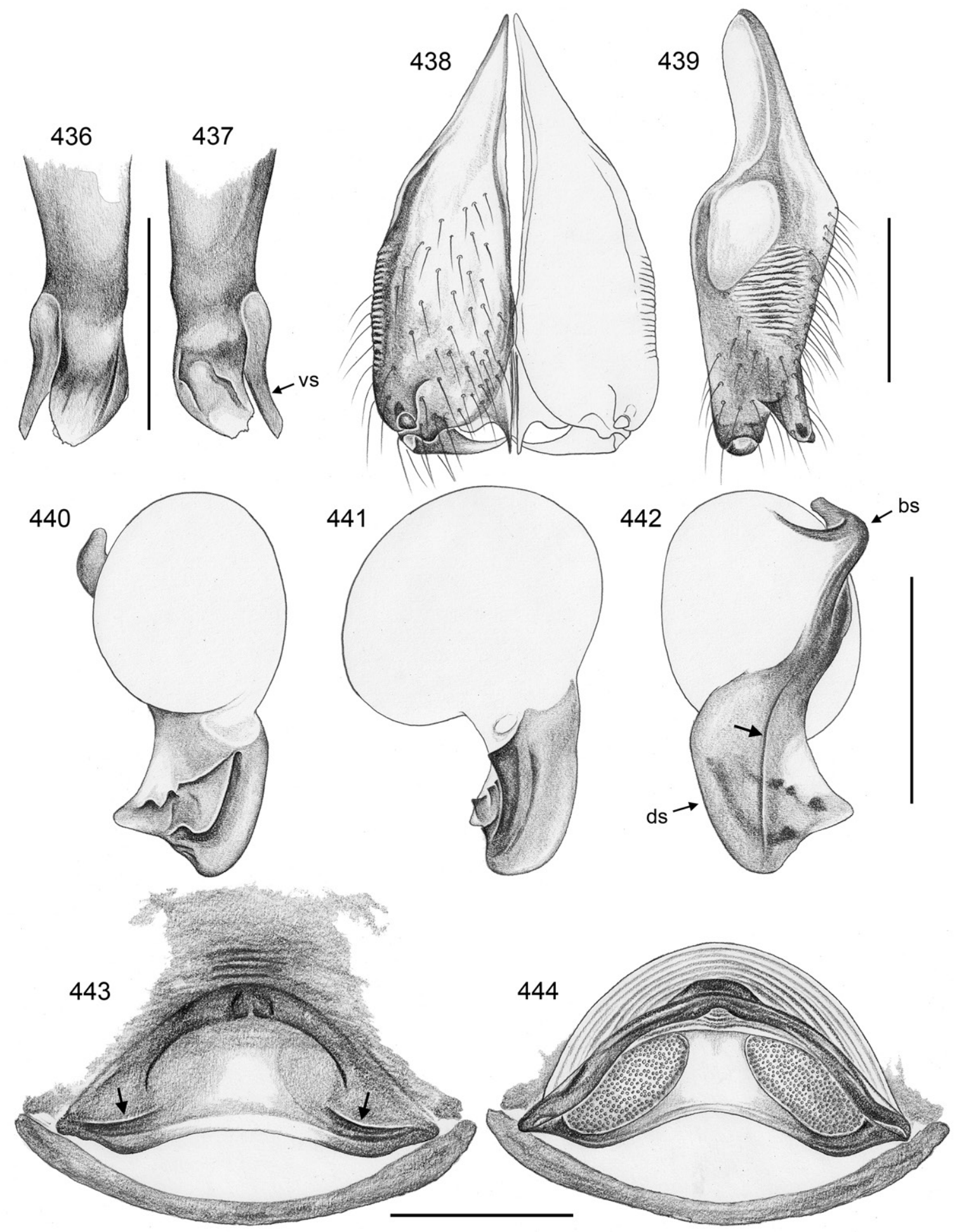

Figs 436-444. Crossopriza tiwi sp. nov.; male and female from Oman, Wadi Tiwi (ZFMK Ar 22402, Ar 22403). 436-437. Left procursus tip, prolateral and retrolateral views. 438-439. Male chelicerae, frontal and lateral views. 440-442. Left genital bulb, prolateral, dorsal, and retrolateral views; arrow: retrolateral ridge. 443-444. Cleared female genitalia, ventral and dorsal views; arrows: pockets. Abbreviations: bs $=$ basal sclerite; $d s=$ distal sclerite; vs $=$ ventral sclerite. Scale bars: $436-437=$ $0.2 \mathrm{~mm} ; 438-444=0.3 \mathrm{~mm}$. 
triangular, weakly protruding, only posteriorly laterally heavily sclerotized; with pair of shallow furrows $\sim 390 \mu \mathrm{m}$ apart; internal sclerotized arcs visible in uncleared specimens; posterior plate short but wide. Internal genitalia (Figs 443-444, 447-449) with large oval pore plates converging anteriorly, dorsal arc strong but simple, ventral arc with median modification of unknown function.

\section{Natural history}

This species was often very abundant, both in villages (in and on abandoned buildings; in fairly exposed webs at the basis of banana and other plants) and in natural environments (under rocks, in small cavities). At Mukal Cave, it was only found outside the cave, while the cave itself was occupied by C. moqal sp. nov. Between Sur and Al Kamil, the species was abundant in a palm garden surrounded by large rocks; while large specimens were mostly restricted to the rocks, smaller specimens were also found on the palms, even among leaves of young palms, at least up to $1.5 \mathrm{~m}$ above the ground.

\section{Distribution}

Known from several localities in the Eastern Hajars of NE Oman (Fig. 352).
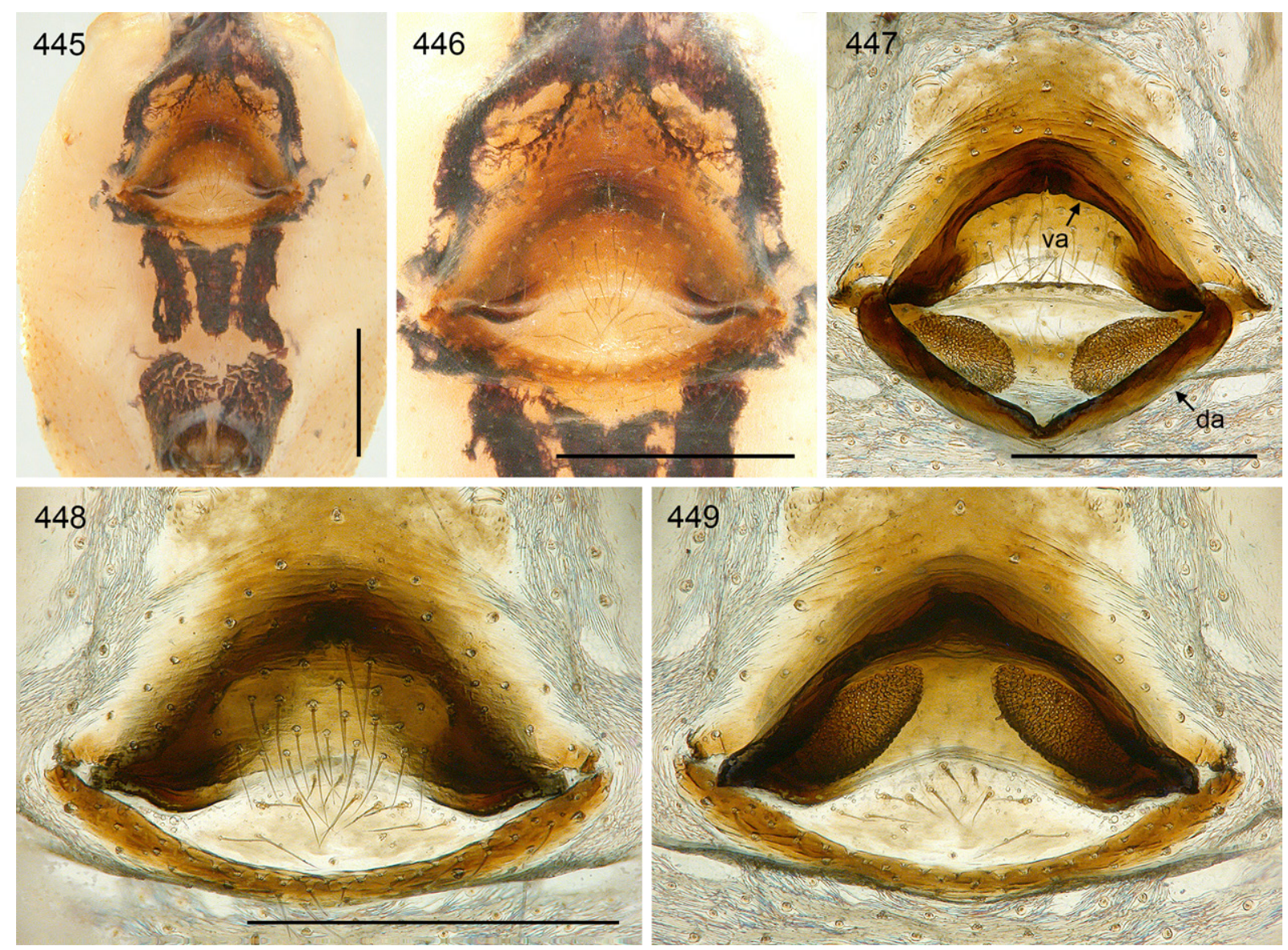

Figs 445-449. Crossopriza tiwi sp. nov.; female from Oman, Wadi Tiwi (ZFMK Ar 22403). 445446. Abdomen and epigynum, ventral views. 447-449. Female internal genitalia, dorsal view (with dorsal arc tilted backwards), ventral view, and regular dorsal view. Abbreviations: da $=$ dorsal arc; va $=$ ventral arc. Scale bars $=0.5 \mathrm{~mm}$. 


\section{Crossopriza dhofar sp. nov. urn:1sid:zoobank.org:act:52766710-5651-40D5-8657-3E484C5AB946}

Figs 352, 396-398, 450-474

\section{Diagnosis}

Easily distinguished from all described congeners by details of male palp (Figs 453-457; procursus tip with ventral sclerite and two further pointed processes; bulbal sclerite with two simple pointed processes), by male chelicerae (Figs 458-459; modified hairs on frontal face), and female genitalia (Figs 460-466, 473-474; pair of large pockets; large internal median sclerite; pair of lateral internal sclerites) (see also Remark below).

\section{Remark}

A very similar undescribed species occurs in Yemen (Seyoun, $15.95^{\circ} \mathrm{N}, 48.78^{\circ} \mathrm{E}$ ). It shares the distinctive configuration of the distal procursus and bulb elements as well as the presence of modified hairs proximally on the male chelicerae. However, the shapes and positions of these elements differ from C. dhofar sp. nov. The species is not formally described because the female is unknown. A single male specimen and one juvenile are deposited in ZFMK Ar 22409-10.

\section{Etymology}

The species name refers to the type locality; noun in apposition.

\section{Type material}

\section{Holotype}

OMAN - Dhofar • Oे $^{\prime}$ Ain Razad cave; $17.1301^{\circ}$ N, 54.2364 E; 115 m a.s.1.; 22 Feb. 2018; B.A. Huber leg.; in cave; ZFMK Ar 22411.

\section{Other material examined}

OMAN - Dhofar • 1 ภ, 2 + $\odot$; same collection data as for holotype; ZFMK Ar 22412・1 + , 1 juv. (in pure ethanol); same collection data as for holotype; ZFMK Om130 • 12 우, 3 juvs (partly used for

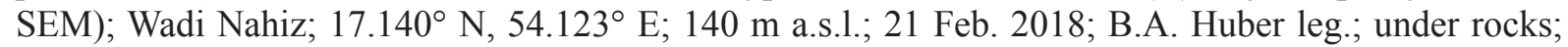
ZFMK Ar 22413 • 1 \&, 4 juvs (in pure ethanol); same collection data as for preceding; ZFMK Om125 •

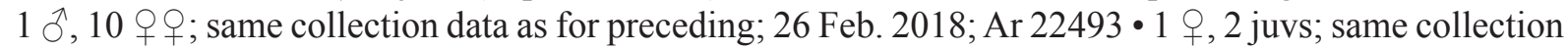
data as for preceding; ZFMK Om143・1 ${ }^{\lambda}, 3$ q $O$; Ain Athoom; $17.116^{\circ} \mathrm{N}, 54.369^{\circ} \mathrm{E} ; 290 \mathrm{~m}$ a.s.l.; 28 Feb. 2018; B.A. Huber leg.; under rocks in wadi; ZFMK Ar 22414 • 1 q (in pure ethanol); same collection data as for preceding; ZFMK Om148 • 4 우; Wadi Darbat; $17.09^{\circ} \mathrm{N}, 54.45^{\circ} \mathrm{E} ; 200 \mathrm{~m}$ a.s.l.; 23 Feb. 2018; B.A. Huber leg.; ZFMK Ar 22415 1 ㅇ (in pure ethanol); same collection data as for preceding; ZFMK Om132 • 1 O; Tayq Cave; $17.182^{\circ} \mathrm{N}, 54.534^{\circ} \mathrm{E} ; 790 \mathrm{~m}$ a.s.1.; 23 Feb. 2018; B.A. Huber leg.; among rocks near cave; ZFMK Ar 22416 • 1 q (in pure ethanol); same collection data as for preceding; ZFMK Om134 • 1 ; ; Wadi Shalyon; $17.1844^{\circ} \mathrm{N}, 54.9538^{\circ}$ E; $360 \mathrm{~m}$ a.s.1.; 1 Mar. 2018; B.A. Huber leg.; under rocks in wadi; ZFMK Ar 22417 • 2 q 9 ; NE of Rawiyyah; $17.4733^{\circ} \mathrm{N}$, 54.1195 ${ }^{\circ}$ E; 540 m a.s.1.; 27 Feb. 2018; B.A. Huber leg.; among palms near ground; ZFMK Ar 22418 - 1 ㅇ, 1 juv. (in pure ethanol); same collection data as for preceding; ZFMK Om145 3 juvs (in pure ethanol); E of Thumrait; $17.670^{\circ} \mathrm{N}, 54.163^{\circ} \mathrm{E}$; $460 \mathrm{~m}$ a.s.1.; 27 Feb. 2018; B.A. Huber leg.; among rocks and in low palms near ground; ZFMK Om144 1 ภ, 2 우, 1 juv.; Shaat sinkhole, in wadis leading to sinkhole; $16.774^{\circ}$ N, 53.587 E; 850 m a.s.l.; 24 Feb. 2018; B.A. Huber leg.; ZFMK Ar $22419 \bullet 1$ ô, 2 웅, 1 juv.; same collection data as for preceding; 25 Feb. 2018; Ar 23863.

\section{Description}

Male (holotype)

MEASUREMEnTS. Total length 3.3, carapace width 1.1. Distance PME-PME $80 \mu \mathrm{m}$; diameter PME $80 \times$ $90 \mu \mathrm{m}$; distance PME-ALE $30 \mu \mathrm{m}$; diameter AME $75 \mu \mathrm{m}$; distance AME-AME $20 \mu \mathrm{m}$. Leg 1: 28.3 
$(7.9+0.5+7.8+10.3+1.8)$, tibia $2: 5.1$, tibia $3: 4.1$, tibia $4: 4.4$; tibia $1 \mathrm{~L} / \mathrm{d}: 74$; femora $1-4$ diameters: $0.14,0.11,0.11,0.11$.

COLOR (in ethanol). Carapace ochre-yellow, with light brown median band including ocular area; clypeus not darkened; sternum densely covered with dark brown spots; legs ochre-yellow, without darker rings, with black marks on femora and (few) on tibiae; abdomen gray, with dorsal pattern of dark marks among many internal whitish marks; ventrally with distinct black pattern (disrupted median band), three longitudinal bands behind gonopore.

Body. Habitus as in Fig. 396. Ocular area slightly raised. Shallow but wide thoracic pit and pair of furrows diverging from pit toward posterior margin. Clypeus unmodified, only rim slightly more sclerotized than in female. Sternum wider than long $(0.80 / 0.55)$, unmodified. Abdomen elongated and pointed above spinnerets.

Chelicerae. As in Figs 458-459, with pair of distal lateral apophyses provided with one modified cone-shaped hair each, two pairs of large conical hairs frontally near median line, and pair of smaller modified hairs more proximally (slightly asymmetric); distance between tips of modified hairs on lateral apophyses: $405 \mu \mathrm{m}$; with distinct lateral stridulatory ridges.

PALPS. As in Figs 450-452; coxa with very low retrolateral hump; trochanter barely modified; femur distally strongly widened, with rounded ventral protrusion, proximally with prolateral stridulatory pick, without retrolateral transversal line, without retrolateral proximal process; femur-patella joints shifted toward prolateral side; tibia large relative to femur; tibia-tarsus joints slightly shifted toward retrolateral side; tarsus without macrotrichia; procursus (Figs 453-454) slightly curved towards ventral, short, long dorsal hairs not curved, with two strong humps proximally on prolateral side, ventral hump densely set

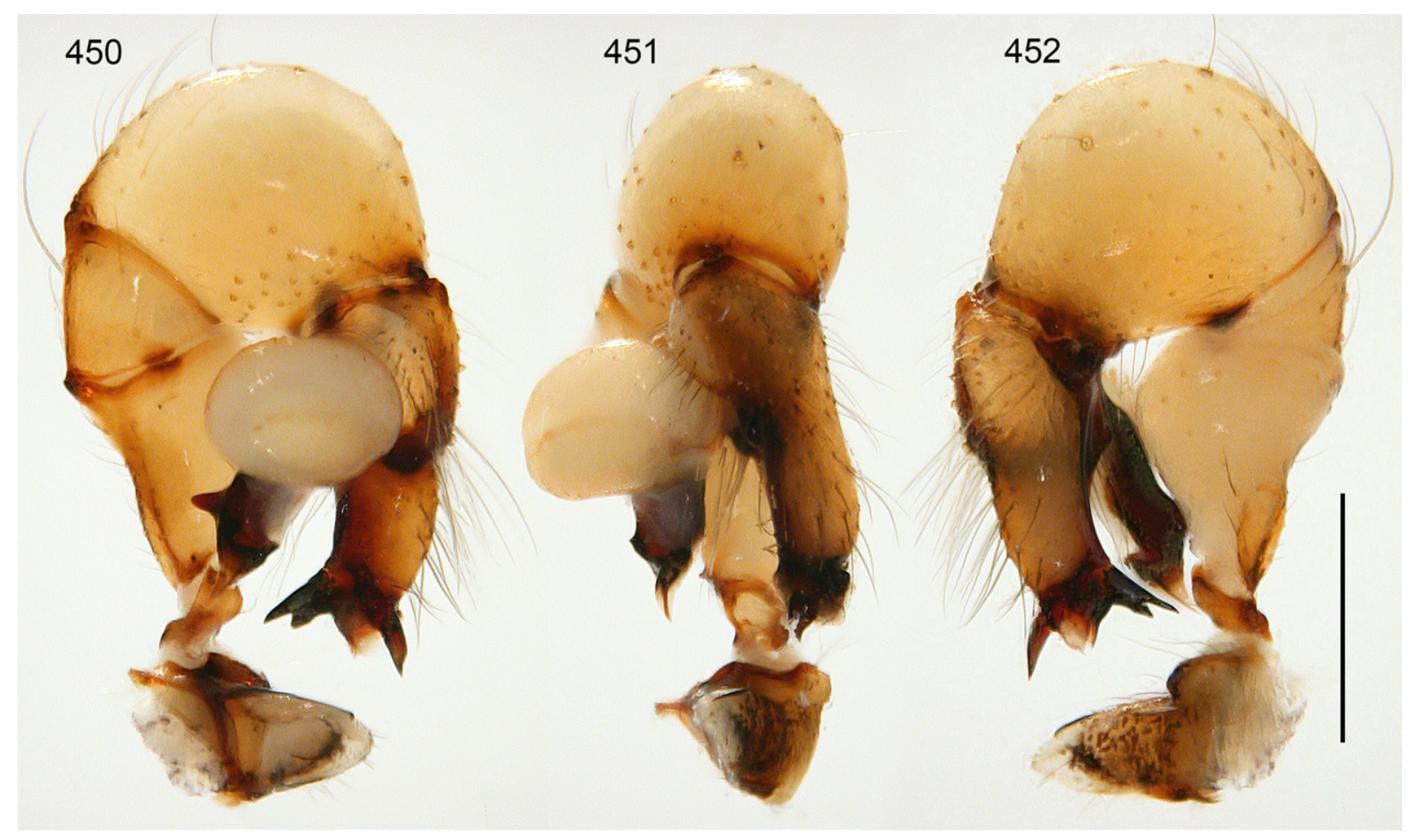

Figs 450-452. Crossopriza dhofar sp. nov.; male from Oman, Ain Razad cave (ZFMK Ar 22412); left palp, prolateral, dorsal, and retrolateral views. Scale bar $=0.5 \mathrm{~mm}$. 


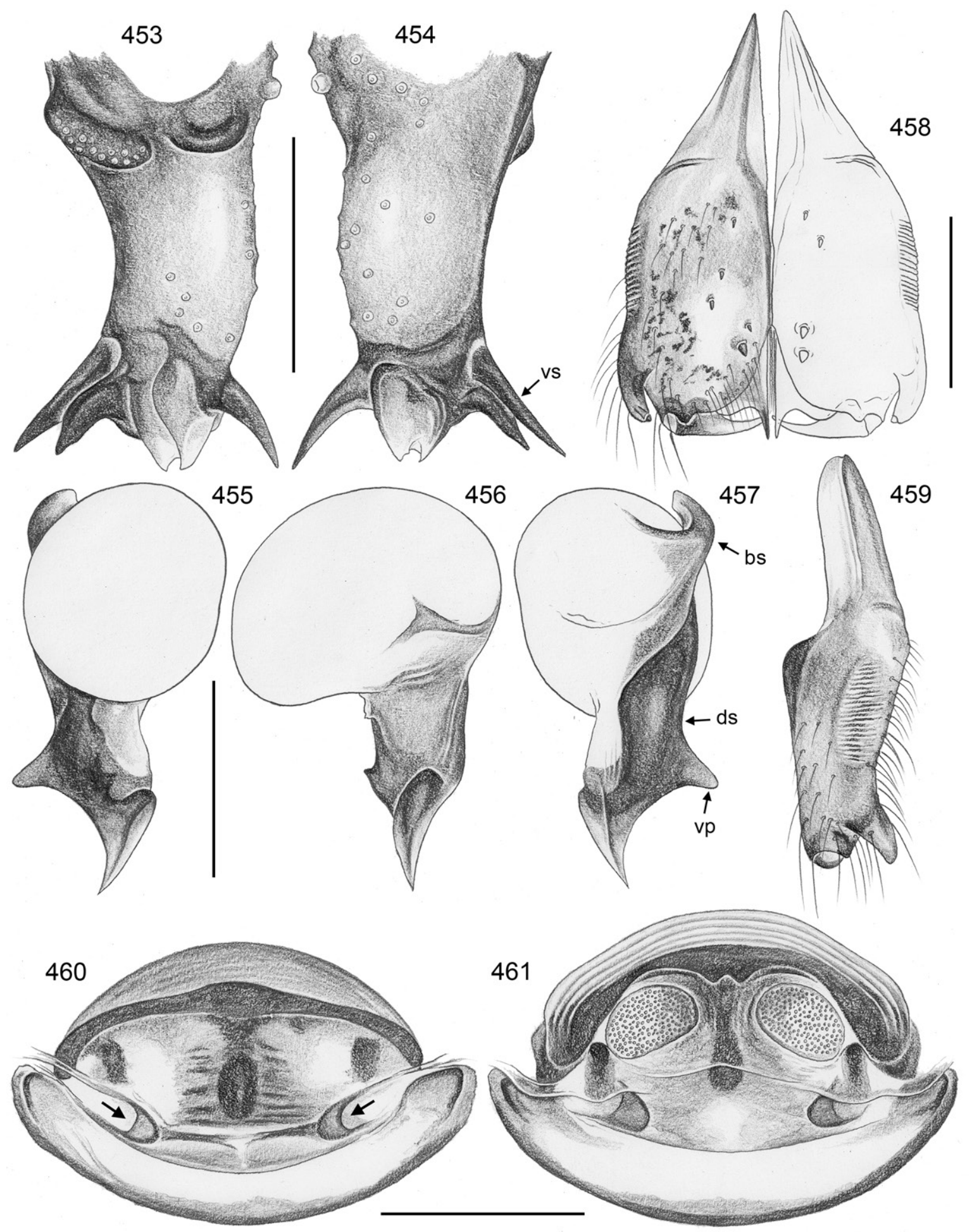

Figs 453-461. Crossopriza dhofar sp. nov.; male from Oman, Ain Razad cave (ZFMK Ar 22412), female from Oman, Wadi Nahiz (ZFMK Ar 22413). 453-454. Left procursus, prolateral and retrolateral views. 455-457. Left genital bulb, prolateral, dorsal, and retrolateral views. 458-459. Male chelicerae, frontal and lateral views. 460-461. Cleared female genitalia, ventral and dorsal views; arrows: pockets. Abbreviations: $\mathrm{bs}=$ basal sclerite; $\mathrm{ds}=$ distal sclerite; $\mathrm{vp}=$ ventral process (of distal sclerite); $\mathrm{vs}=$ ventral sclerite. Scale bars $=0.3 \mathrm{~mm}$. 
with long hairs; procursus tip complex, with ventral hinged sclerite accompanied by flat parallel sclerite, pointed dorsal sclerite, and median partly membranous elements; genital bulb (Figs 455-457) with simple basal sclerite connected to distal (main) sclerite, sperm duct opening not seen; distal sclerite with distinctive ventral process, smaller prolateral process, and partly semitransparent pointed tip.

Legs. Femur 1 with single row of $\sim 10$ ventral spines; without curved hairs; few vertical hairs; retrolateral trichobothrium of tibia 1 at $2.5 \%$; prolateral trichobothrium absent on tibia 1, present on other leg tibiae; tarsi without regular pseudosegments (except for 2-3 at tip).

Male (variation)

Tibia 1 in six males (incl. holotype): 6.4-9.9 (mean 8.2). Larger males with more spines of femur 1 ( 23 in largest male); sternum sometimes uniformly dark, almost black; ventral abdominal pattern slightly variable, three longitudinal bands sometimes fused; frontal hairs on chelicerae slightly variable, but usually with two pairs of strong hairs and 1-3 more proximal pairs of smaller hairs, often asymmetric. Males from Shaat sinkhole with slightly shorter dorsal process at tip of procursus.

\section{Female}

In general similar to male (Figs 397-398) but without spines on legs, with very indistinct stridulatory files on chelicerae (Fig. 469; barely visible in dissecting microscope), and with stridulatory organ consisting
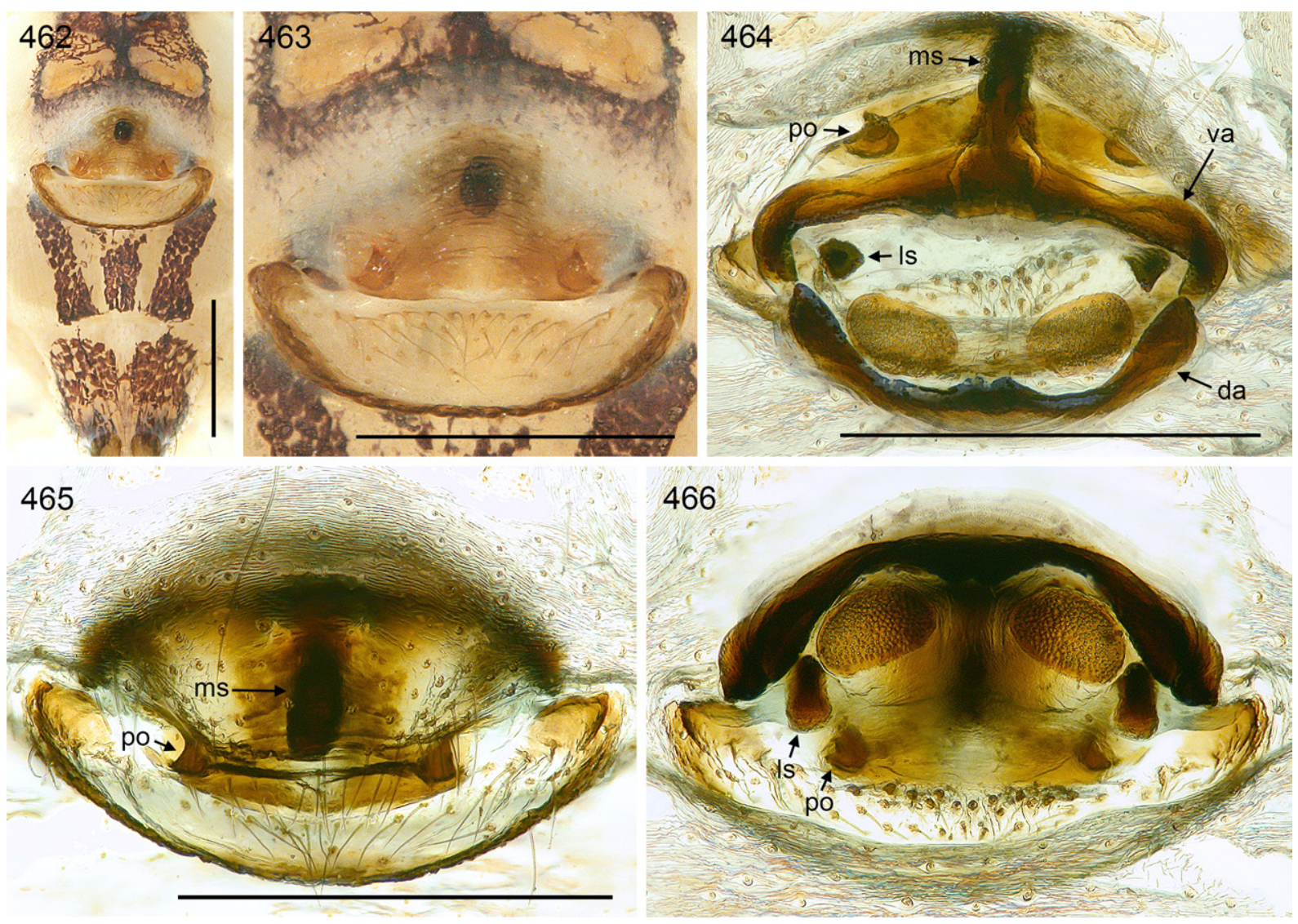

Figs 462-466. Crossopriza dhofar sp. nov.; female from Oman, Wadi Nahiz (ZFMK Ar 22413). 462463. Abdomen and epigynum, ventral views. 464-466. Female internal genitalia, dorsal view (with dorsal arc tilted backwards), ventral view, and regular dorsal view. Abbreviations: da $=$ dorsal arc; $\mathrm{ms}=$ median (internal) sclerite; po $=$ pocket; $1 \mathrm{~s}=$ lateral (internal) sclerite; va $=$ ventral arc. Scale bars $=$ $0.5 \mathrm{~mm}$. 

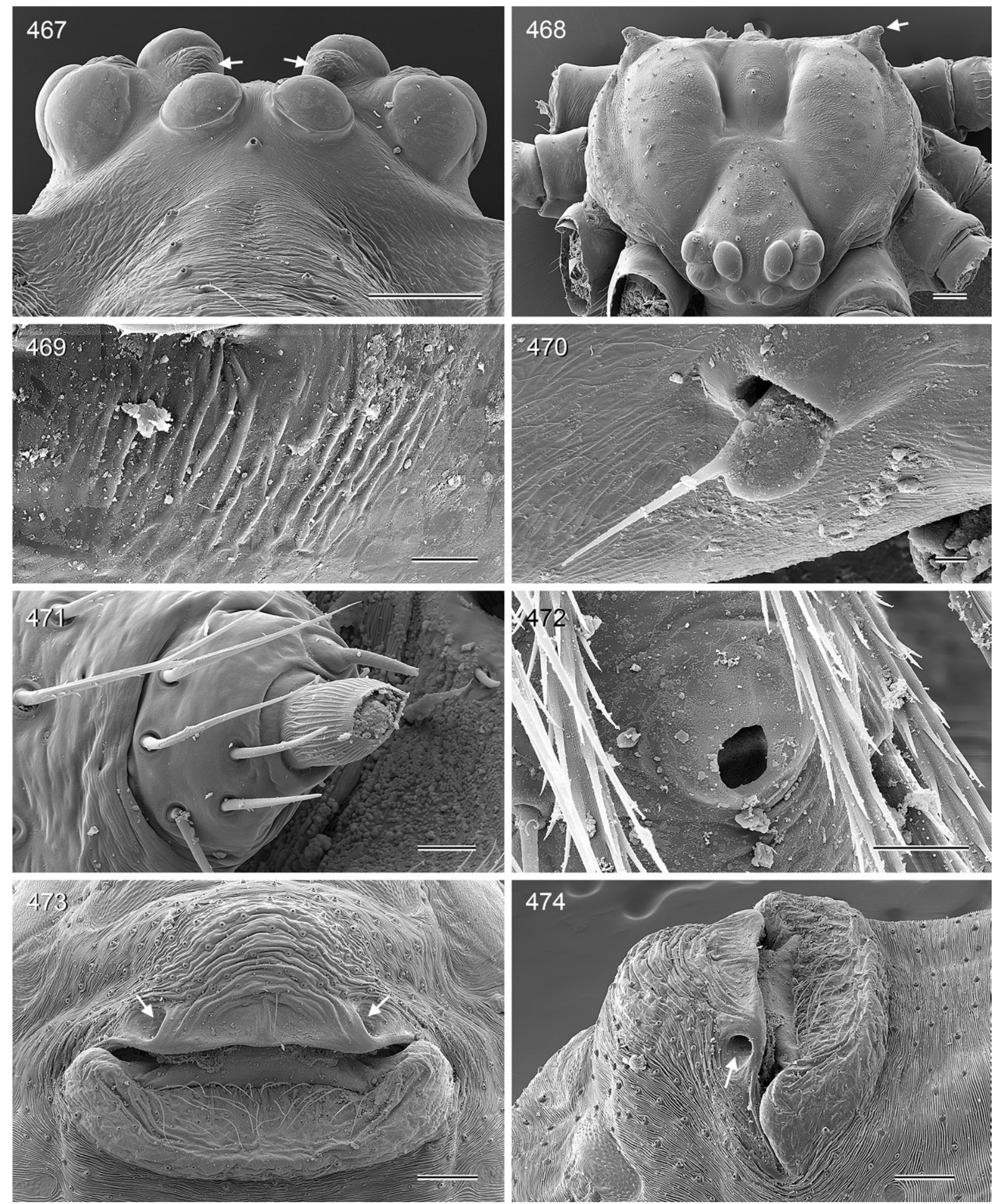

Figs 467-474. Crossopriza dhofar sp. nov.; female from Wadi Nahiz (ZFMK Ar 22413). 467. Ocular area and clypeus; arrows: 'pseudo lenses'. 468. Ocular area and carapace; arrow: posterior process. 469. Cheliceral stridulatory file. 470. Stridulatory pick on palpal femur. 471. ALS. 472. Palpal tarsal organ. 473-474. Epigynum, ventral and lateral-ventral views; arrows: pockets. Scale bars: 467-468, $473-474=100 \mu \mathrm{m} ; 469,471-472=10 \mu \mathrm{m} ; 470=2 \mu \mathrm{m}$. 
of pair of weakly sclerotized but distinct processes posteriorly on carapace (arrow in Fig. 468) and pair of light brown plates anteriorly on abdomen. Tarsal organ capsulate (Fig. 472); ALS with one widened spigot and one pointed spigot (Fig. 471). Tibia 1 in 36 females: 5.2-8.7 (mean 6.5). Epigynum as in Figs 462-463 and 473-474, protruding area in anterior part whitish, posterior part with triangular brown plate; with pair of large pockets $(250 \mu \mathrm{m}$ apart); internal median sclerite (pouch?) clearly visible in untreated specimens; posterior plate short but wide. Internal genitalia (Figs 460-461, 464-466) with oval pore plates converging anteriorly, with pair of distinct lateral sclerites embedded in membrane, dorsal arc simple, ventral arc with strong median sclerite (pouch?) of unknown function.

\title{
Natural history
}

Most specimens were found on the undersides of rocks on the floor. In Wadi Nahiz, they shared this microhabitat with an undescribed Buitinga (generic assignment tentative) and an undescribed Micropholcus. Relatively few males were found (Wadi Nahiz: one male vs 24 females; all other localities combined: five males vs 22 females), suggesting that the species might be seasonal. At Ain Razad, this species shared a small cavity in the deepest part of a shallow cave with Smeringopus lineiventris Simon, 1890 and Artema dhofar Huber, 2019. At Shaat sinkhole, the spiders were found in small holes in the karstic rocks, sitting on small silk mats directly on the rock. In the desert NE of Rawiyyah and E of Thumrait, the spiders were found in rock crevices and hidden among the spiny leafs of palm bushes close to the ground.

\section{Distribution}

Widespread in the Dhofar Region of Oman, where it is the only known representative of Crossopriza (Fig. 352).

\author{
Crossopriza moqal sp. nov. \\ urn:1sid:zoobank.org:act:207D5CBF-F03A-4F86-AE29-7FAA4227D1B6
}

Figs 352, 475-478, 483-508

\section{Diagnosis}

Distinguished from similar congeners (C. kittan sp. nov., C. ghul sp. nov.) by details of procursus (Figs 486-487; ventral hump at basis of ventral sclerite; elongated membranous element at tip), and distal bulbal sclerite (Fig. 490; distinctive series of prolateral apophyses); from C. kittan also by more slender male cheliceral apophyses (Figs 488-489) and shorter epigynum (Fig. 496); from C. ghul also by smaller distance between pore plates in female internal genitalia (Fig. 494).

\section{Etymology}

The species name refers to the type locality; noun in apposition.

\section{Type material}

\section{Holotype}

OMAN - Ash Sharqiyah North • Oे $^{\wedge}$; Wadi Bani Khalid, Mukal (Moqal) Cave; $22.624^{\circ} \mathrm{N}, 59.097^{\circ} \mathrm{E}$; 700 m a.s.1.; 23 Mar. 2017; B.A. Huber leg.; in cave; ZFMK Ar 22420.

\section{Other material examined}

OMAN - Ash Sharqiyah North $• 5 \hat{\partial} \hat{\partial}, 12$ $q \propto, 1$ juv. (partly used for SEM); same collection data as for holotype; ZFMK Ar 22421, Ar 22422 • 3 우 (in pure ethanol); same collection data as for holotype; ZFMK Om30.

\section{Description}

Male (holotype)

Measurements. Total length 3.1, carapace width 1.35. Distance PME-PME $80 \mu \mathrm{m}$; diameter PME $70 \mu \mathrm{m}$; distance PME-ALE $20 \mu \mathrm{m}$; diameter AME $70 \mu \mathrm{m}$; distance AME-AME $20 \mu \mathrm{m}$. Leg 1: 35.8 

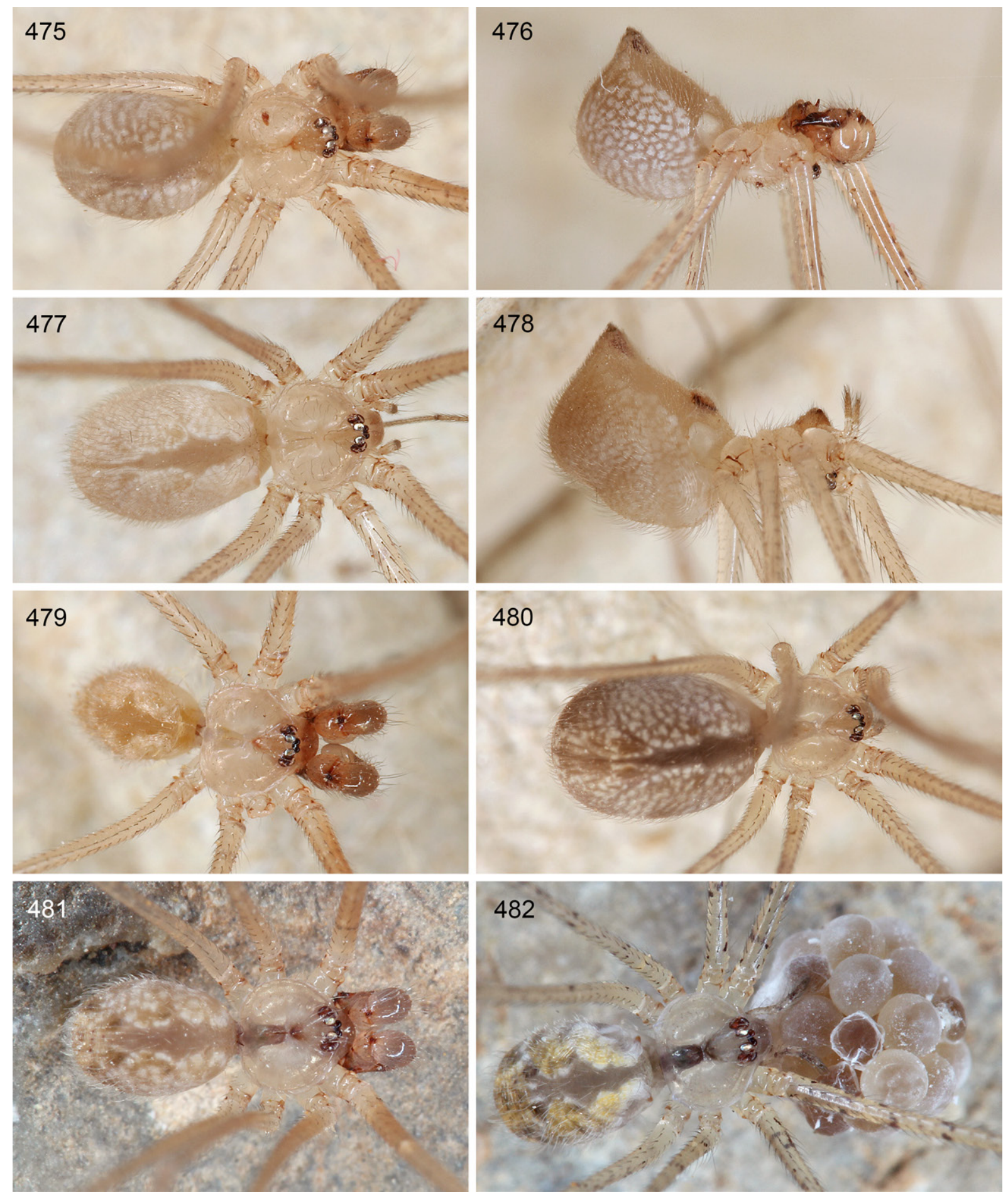

Figs 475-482. Crossopriza Simon, 1893, live specimens. 475-478. C. moqal sp. nov., male and female from Oman, Moqal Cave. 479-480. C. kittan sp. nov., male and female from Oman, Ibri, Al Kittan Cave. 481-482. C. ghul sp. nov., male and female with egg-sac from Oman, Wadi Ghul. 
$(10.2+0.6+10.2+12.9+1.9)$, tibia $2: 6.9$, tibia $3: 4.8$, tibia $4: 5.3$; tibia $1 \mathrm{~L} / \mathrm{d}: 79$; femora $1-4$ diameters: $0.22,0.19,0.16,0.17$.

Color (in ethanol). In general paler than epigean congeners (i.e., slightly troglomorphic). Carapace ochre-yellow, anteriorly in median pit light brown; sternum ochre-yellow with brown anterior margins; legs ochre-yellow, without darker rings, with black lines on femora and (few) on tibiae; abdomen pale gray, with many indistinct internal whitish marks and few indistinct dark marks near spinnerets.

BoDy. Habitus as in Figs 475-476. Ocular area slightly raised. Deep thoracic pit and pair of furrows diverging from pit toward posterior margin. Clypeus unmodified (but rim more strongly sclerotized than in female). Sternum wider than long (0.95/0.60), unmodified. Abdomen oval, dorso-posteriorly rounded, tapering at spinnerets. Gonopore with four epiandrous spigots (Fig. 507); ALS with one widened spigot and one pointed spigot, PMS with two small spigots (Fig. 505).

Chelicerae. As in Figs 488-489, with pair of latero-distal apophyses provided with one large modified cone-shaped hair each (Fig. 502); distance between tips of modified hairs: $430 \mu \mathrm{m}$; lateral stridulatory ridges indistinct (Fig. 500; distances between ridges $\sim 6.5 \mu \mathrm{m}$ ) but visible in dissecting microscope.

Palps. As in Figs 483-485; coxa with rounded retrolateral hump; trochanter barely modified; femur distally strongly widened, with rounded ventral protrusion, proximally with prolateral stridulatory pick, with indistinct retrolateral transversal line, without retrolateral proximal process; femur-patella joints shifted toward prolateral side; tibia-tarsus joints slightly shifted toward retrolateral side; tarsus without macrotrichia; tarsal organ capsulate (Fig. 503); procursus straight, proximal prolateral hump set with numerous long hairs, long dorsal hairs not or only weakly curved, procursus tip (Figs 486-487) with strong ventral sclerite and further distinctive membranous and sclerotized elements, with one hair-like

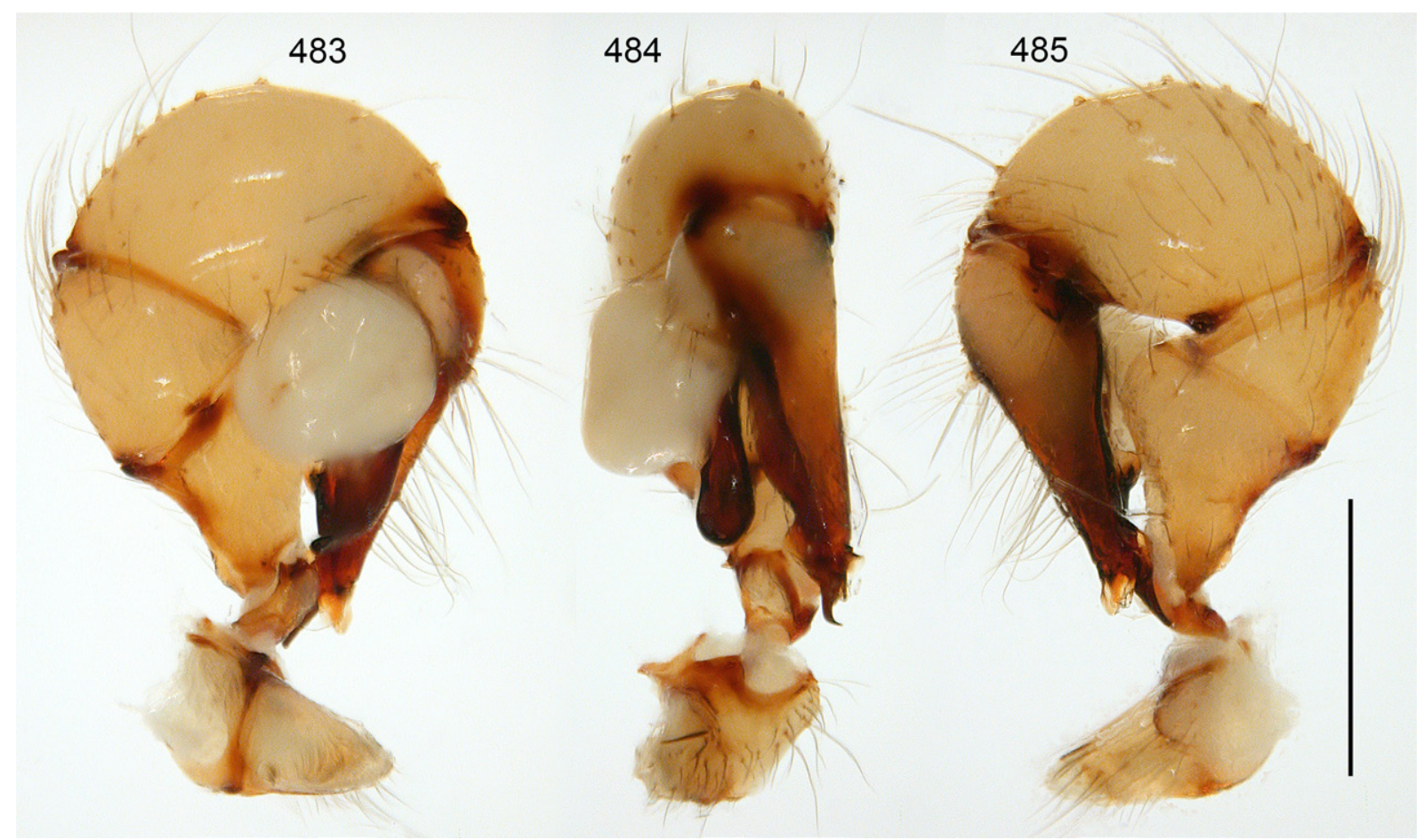

Figs 483-485. Crossopriza moqal sp. nov.; male from Oman, Moqal Cave (ZFMK Ar 22421); left palp, prolateral, dorsal, and retrolateral views. Scale bar $=0.5 \mathrm{~mm}$. 


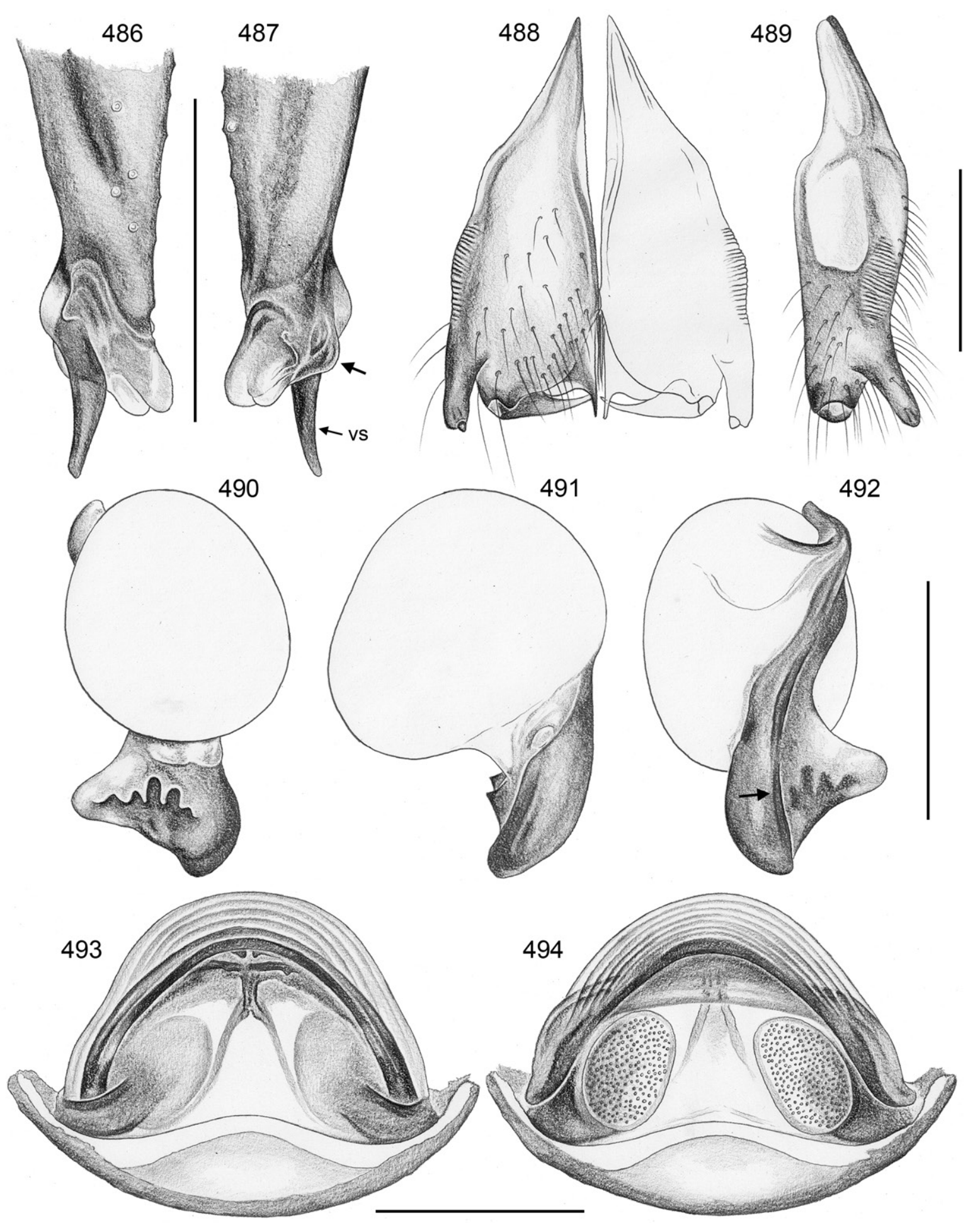

Figs 486-494. Crossopriza moqal sp. nov.; male and female from Oman, Moqal Cave (ZFMK Ar 22421, Ar 22422). 486-487. Left procursus, prolateral and retrolateral views; arrow: distinctive ventral hump. 488-489. Male chelicerae, frontal and lateral views. 490-492. Left genital bulb, prolateral, dorsal, and retrolateral views; arrow: retrolateral ridge. 493-494. Cleared female genitalia, ventral and dorsal views. Abbreviation: $\mathrm{vs}=$ ventral sclerite. Scale bars $=0.3 \mathrm{~mm}$. 
process on retrolateral side (arrow in Fig. 506); genital bulb (Figs 490-492) with simple basal sclerite connected to distal (main) sclerite, sperm duct opening at basis of distal sclerite; distal sclerite with retrolateral ridge and series of distinctive prolateral apophyses.

Legs. Femur 1 with single row of $\sim 24$ ventral spines; without curved hairs; few vertical hairs; retrolateral trichobothrium of tibia 1 at 3\%; prolateral trichobothrium absent on tibia 1, present on other leg tibiae; tarsi without regular pseudosegments but with indistinct platelets.

Male (variation)

Tibia 1 in six males (incl. holotype): 8.7-10.3 (mean 9.6).

\section{Female}

In general similar to male (Figs 477-478) but without spines on legs, with rudimentary stridulatory files on chelicerae (Fig. 501; distances between ridges $\sim 8.5 \mu \mathrm{m}$; not visible in dissecting microscope), and with stridulatory organ consisting of pair of weakly sclerotized but distinct processes posteriorly on carapace and pair of light brown plates anteriorly on abdomen. Tibia 1 in ten females: 7.8-10.0 (mean 9.0). Epigynum as in Figs 495-496 and 508, main epigynal plate semicircular, weakly protruding, only posteriorly laterally heavily sclerotized; with pair of pockets (shallow furrows) $\sim 410 \mu \mathrm{m}$ apart; internal sclerotized arcs clearly visible in untreated specimens; posterior plate short but wide. Internal genitalia

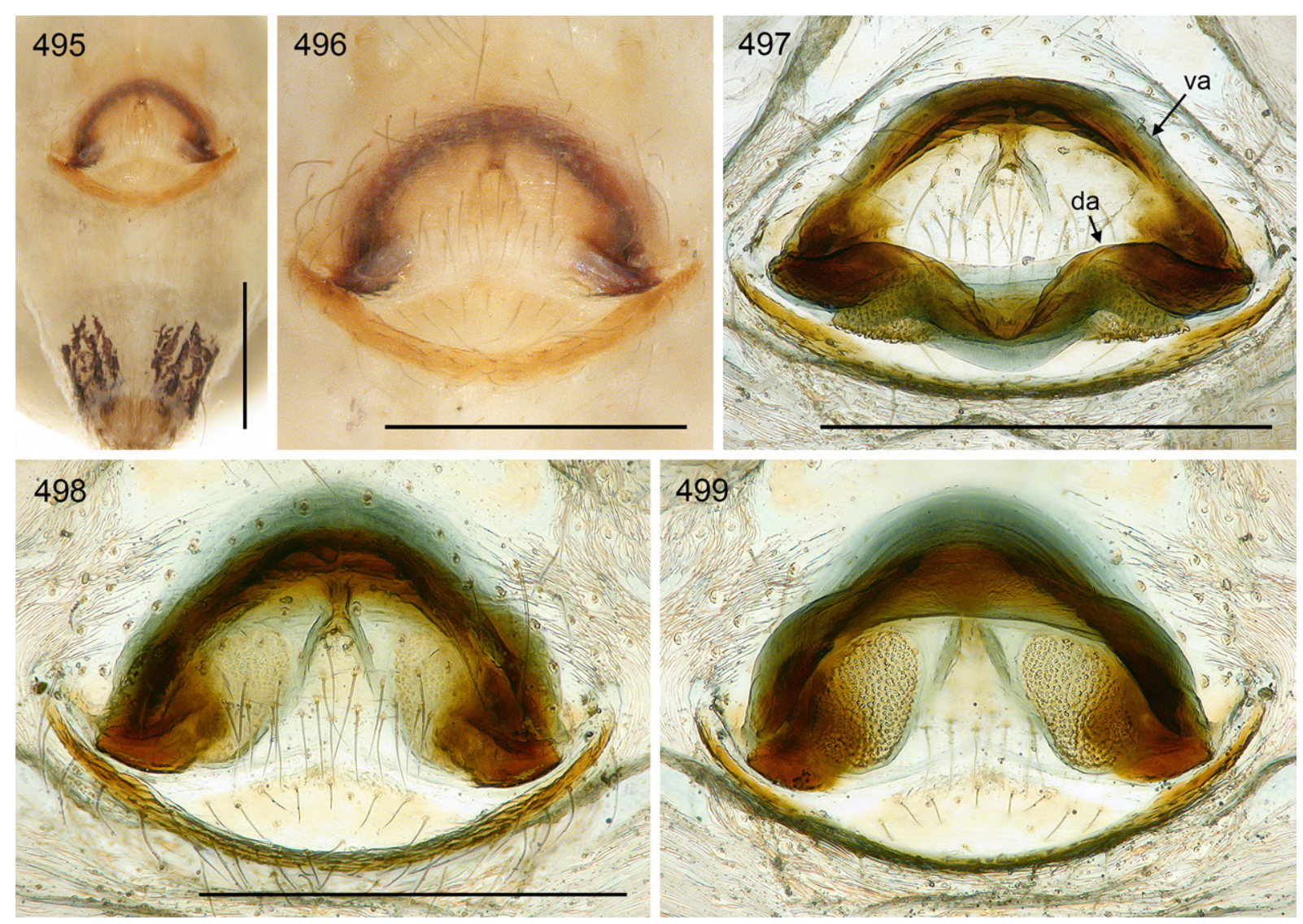

Figs 495-499. Crossopriza moqal sp. nov.; female from Oman, Moqal Cave (ZFMK Ar 22422). 495496. Abdomen and epigynum, ventral views. 497-499. Female internal genitalia, dorsal view (with dorsal arc tilted backwards), ventral view, and regular dorsal view. Abbreviations: da $=$ dorsal arc; va $=$ ventral arc. Scale bars $=0.5 \mathrm{~mm}$. 

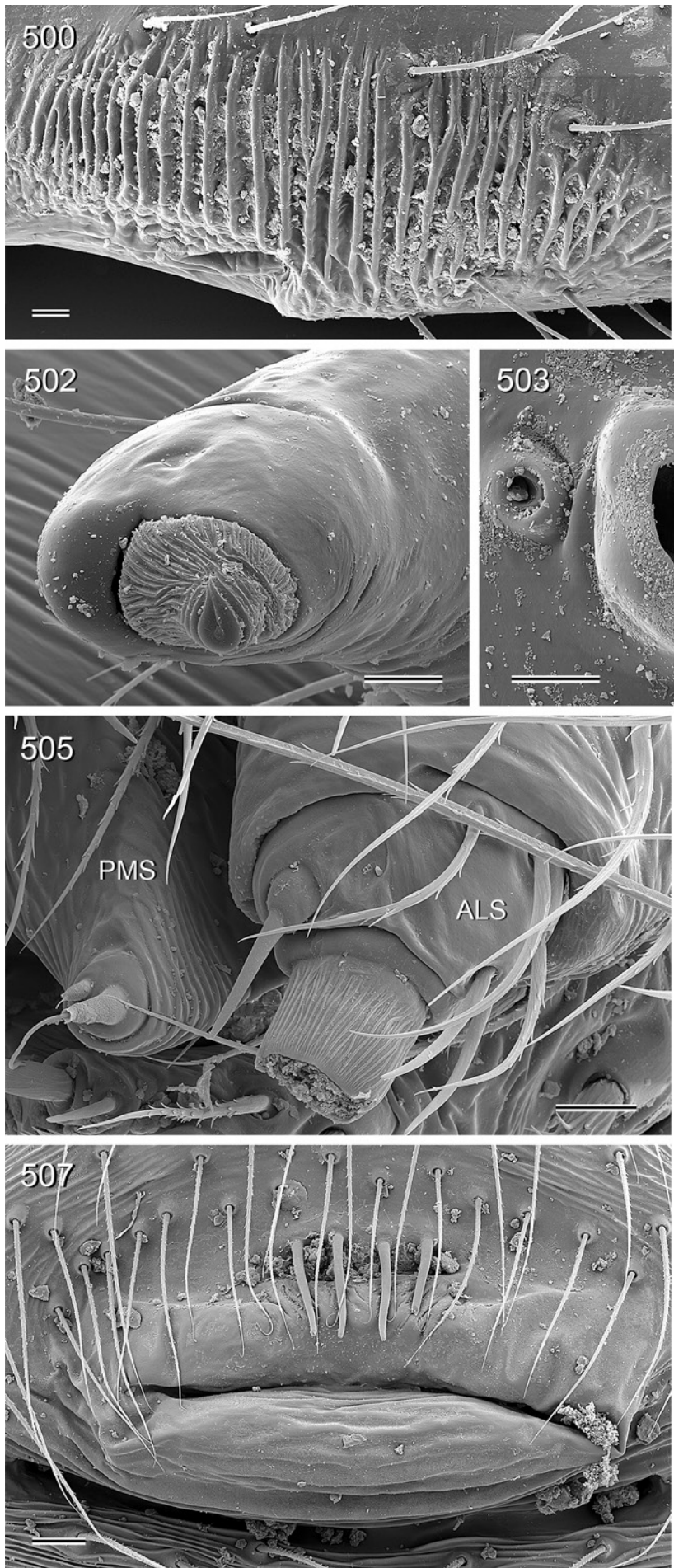
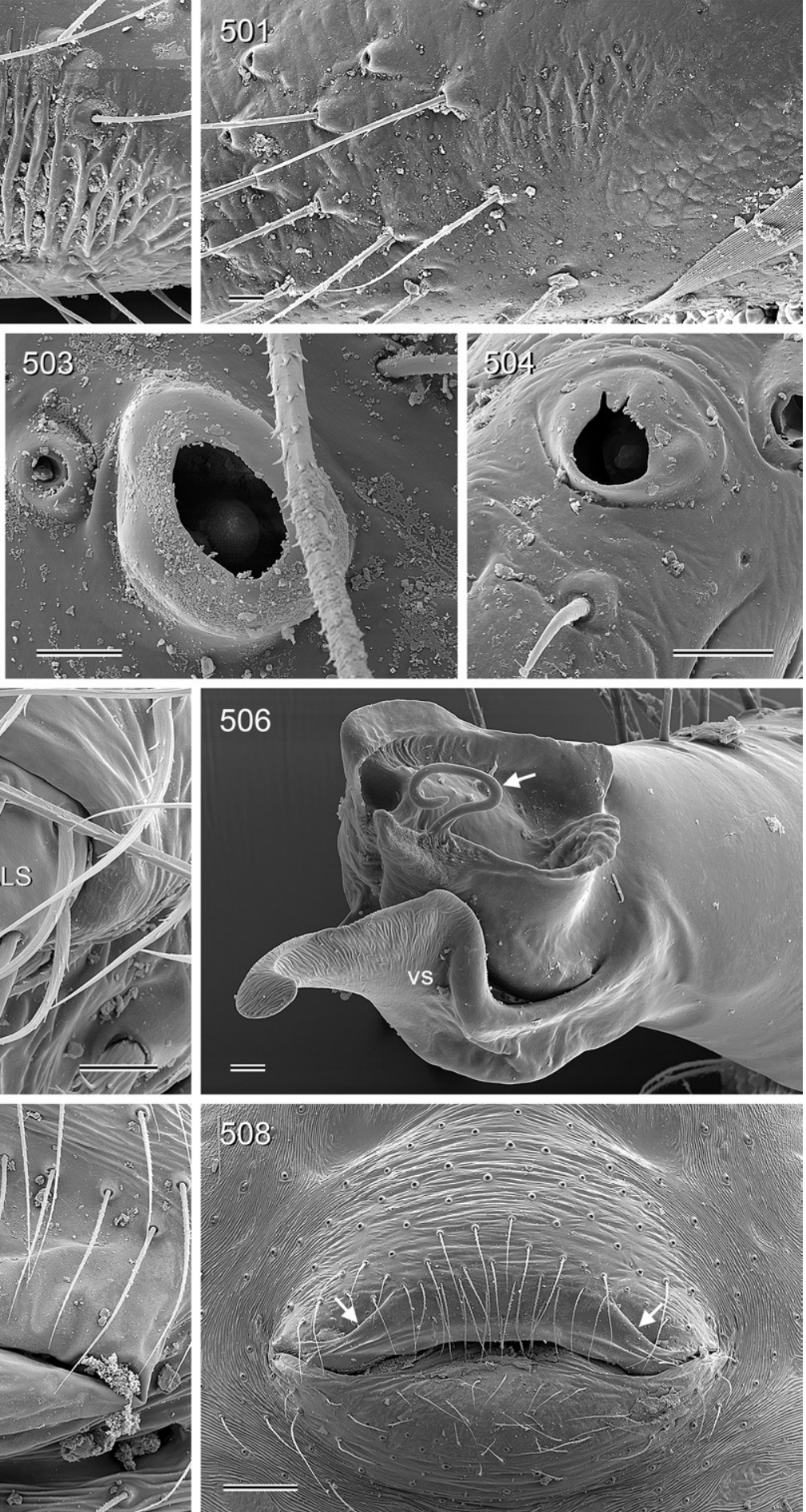

Figs 500-508. Crossopriza moqal sp. nov.; male and female from Oman, Moqal Cave (ZFMK Ar 22421, Ar 22422). 500-501. Male and female cheliceral stridulatory files. 502. Tip of male cheliceral apophysis. 503-504. Male and female palpal tarsal organs. 505. Male spinnerets. 506. Left procursus tip, retrolateral-distal view; arrow: hair-like process. 507. Male gonopore. 508. Epigynum; arrows: pockets. Abbreviation: vs $=$ ventral sclerite. Scale bars: 500-506 $=10 \mu \mathrm{m} ; 507=20 \mu \mathrm{m} ; 508=100 \mu \mathrm{m}$. 
(Figs 493-494, 497-499) with large oval pore plates converging anteriorly, dorsal arc wide but simple, ventral arc with indistinct median modification of unknown function.

\section{Natural history}

The spiders were found in the twilight area of the cave, in small exposed webs mostly close to the floor, rarely slightly higher; no spiders were seen in the deeper, very hot parts of the cave (beyond $\sim 15 \mathrm{~m}$ from the entrance). Outside of the cave, suitable spaces among rocks were occupied by C. tiwi sp. nov.

\section{Distribution}

Known from type locality only (Oman, Ash Sharqiyah North; Fig. 352).

Crossopriza kittan $\mathrm{sp}$. nov. urn:1sid:zoobank.org:act:46030F38-742D-4B37-ADC2-D52F5B91570A

Figs 352, 479-480, 509-525

\section{Diagnosis}

Easily distinguished from similar congeners (C. moqal sp. nov., C. ghul sp. nov.) by longer epigynum (Fig. 522); also by details of procursus (Fig. 513; distinctive retrolateral apophysis), bulbal sclerite (Fig. 516; two prolateral apophyses connected by sclerotized ridge), slightly stronger male cheliceral apophyses (Figs 514-515), and by elongate pore plates converging and narrowing anteriorly (Fig. 520).

\section{Etymology}

The species name refers to the type locality; noun in apposition.

\section{Type material}

\section{Holotype}

OMAN - Ad Dhahira - O’; Ibri, Al Kittan Cave; $23.298^{\circ}$ N, 56.511 E E; 410 m a.s.1.; 25 Mar. 2017; B.A. Huber leg.; in cave; ZFMK Ar 22423.

\section{Other material examined}

OMAN - Ad Dhahira $\bullet 4 \hat{\jmath}, 7$ $q$, 2 juvs; same collection data as for holotype; ZFMK Ar 22424, $\operatorname{Ar} 22425 \cdot 3$ 우, 1 juv. (in pure ethanol); same collection data as for holotype; ZFMK Om34 1 ô, 4 우, 1 juv. (4 vials); same locality as for holotype; 13 Jul. 2009; H. Steiner leg.; SMF • 1 ô, 1 क ; Ibri, near Al Kittan Cave; $23.310^{\circ}$ N, 56.517 ${ }^{\circ}$ E; 390 m a.s.1.; 25 Mar. 2017; B.A. Huber leg.; under rock overhang; ZFMK Ar 22426 • 1 juv. (in pure ethanol); same collection data as for preceding; ZFMK Om35.

\section{Description}

Male (holotype)

MEASUREMEnTs. Total length 3.6, carapace width 1.4. Distance PME-PME $90 \mu \mathrm{m}$; diameter PME $90 \times$ $100 \mu \mathrm{m}$; distance PME-ALE $20 \mu \mathrm{m}$; diameter AME $65 \mu \mathrm{m}$; distance AME-AME $30 \mu \mathrm{m}$. Leg 1: 45.5 $(12.9+0.6+12.5+16.8+2.7)$, tibia $2: 8.4$, tibia $3: 6.0$, tibia $4: 6.7$; tibia $1 \mathrm{~L} / \mathrm{d}: 83$; femora $1-4$ diameters: $0.20,0.19,0.17,0.18$.

CoLor (in ethanol). In general paler than epigean congeners (i.e., slightly troglomorphic). Carapace ochre-yellow, anteriorly in median pit light brown; sternum with star-shaped dark median mark and slightly darker margins; legs ochre-yellow, without darker rings, with black lines on femora and (few) on tibiae; abdomen pale gray, with some indistinct dark marks above spinnerets; ventrally mostly monochromous, few darker specks near pedicel and near spinnerets. 
Body. Habitus as in Fig. 479. Ocular area slightly raised. Deep thoracic pit and pair of furrows diverging from pit toward posterior margin. Clypeus unmodified but rim slightly more sclerotized than in female. Sternum wider than long (1.05/0.75), unmodified. Abdomen oval, dorso-posteriorly rounded, tapering at spinnerets.

Chelicerae. As in Figs 514-515, with pair of latero-distal apophyses provided with one large modified cone-shaped hair each; distance between tips of modified hairs: $390 \mu \mathrm{m}$; lateral stridulatory ridges indistinct but visible in dissecting microscope.

PALPS. As in Figs 509-511; coxa with rounded retrolateral hump; trochanter barely modified; femur distally strongly widened, with rounded ventral protrusion, proximally with prolateral stridulatory pick, with indistinct retrolateral transversal line, without retrolateral proximal process; femur-patella joints shifted toward prolateral side; tibia-tarsus joints slightly shifted toward retrolateral side; tarsus without macrotrichia; procursus straight, proximal prolateral hump set with numerous long hairs, long dorsal hairs not or only weakly curved, procursus tip (Figs 512-513) with strong ventral sclerite and further distinctive membranous and sclerotized elements; genital bulb (Figs 516-518) with simple basal sclerite connected to distal (main) sclerite, sperm duct opening at basis of distal sclerite; distal sclerite with retrolateral ridge and two distinctive prolateral apophyses connected by sclerotized ridge.

LEGS. Femur 1 with single row of $\sim 28$ ventral spines; without curved hairs; few vertical hairs; retrolateral trichobothrium of tibia 1 at $2.5 \%$; prolateral trichobothrium absent on tibia 1, present on other leg tibiae; tarsi without regular pseudosegments but with indistinct platelets.

Male (variation)

Tibia 1 in seven males (incl. holotype): 10.0-12.9 (mean 11.7). Smaller males with fewer spines on femur $1(\sim 5-10)$. Abdomen often also with white marks.

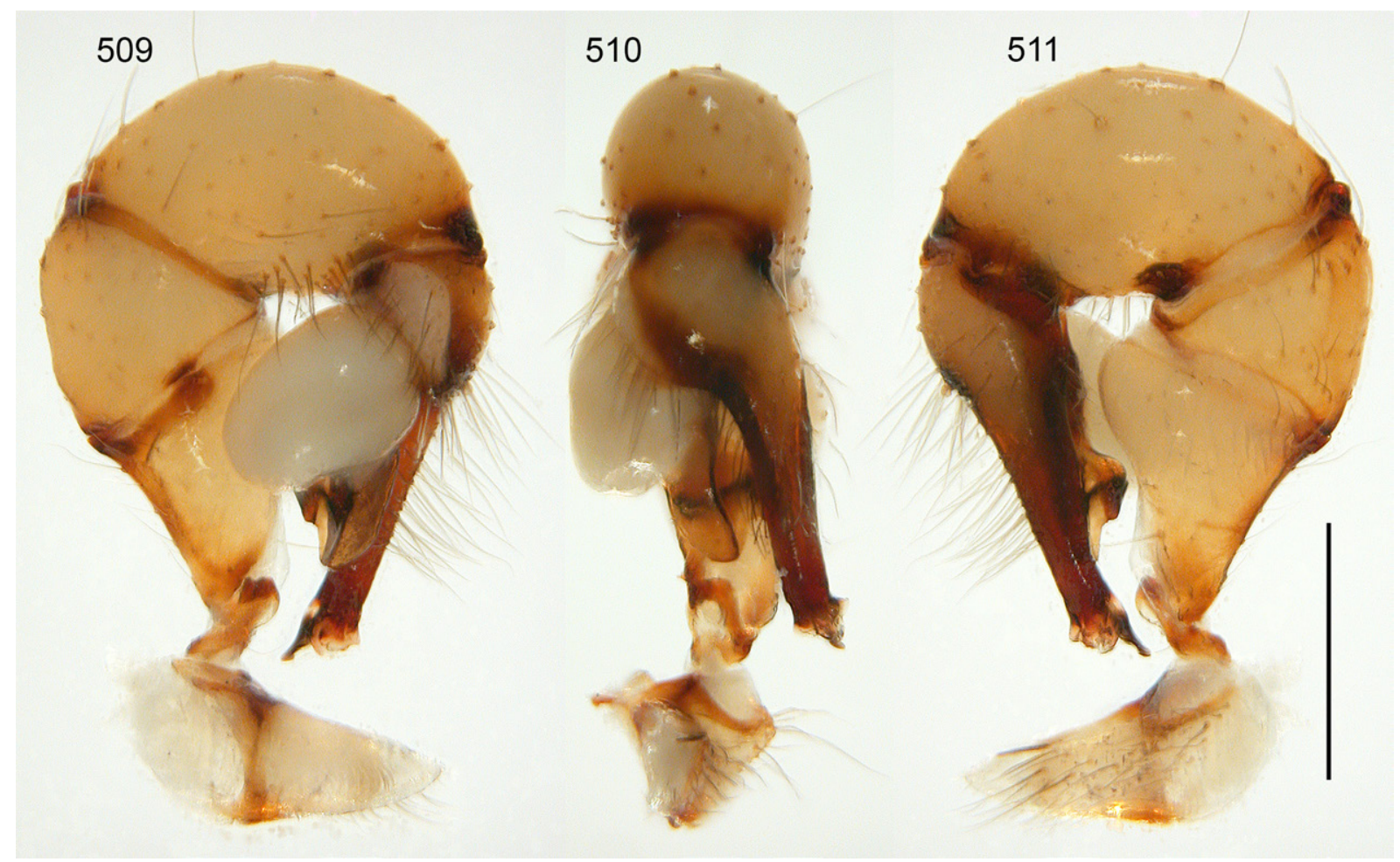

Figs 509-511. Crossopriza kittan sp. nov.; male from Oman, Ibri, Al Kittan Cave (ZFMK Ar 22424); left palp, prolateral, dorsal, and retrolateral views. Scale bar $=0.5 \mathrm{~mm}$. 


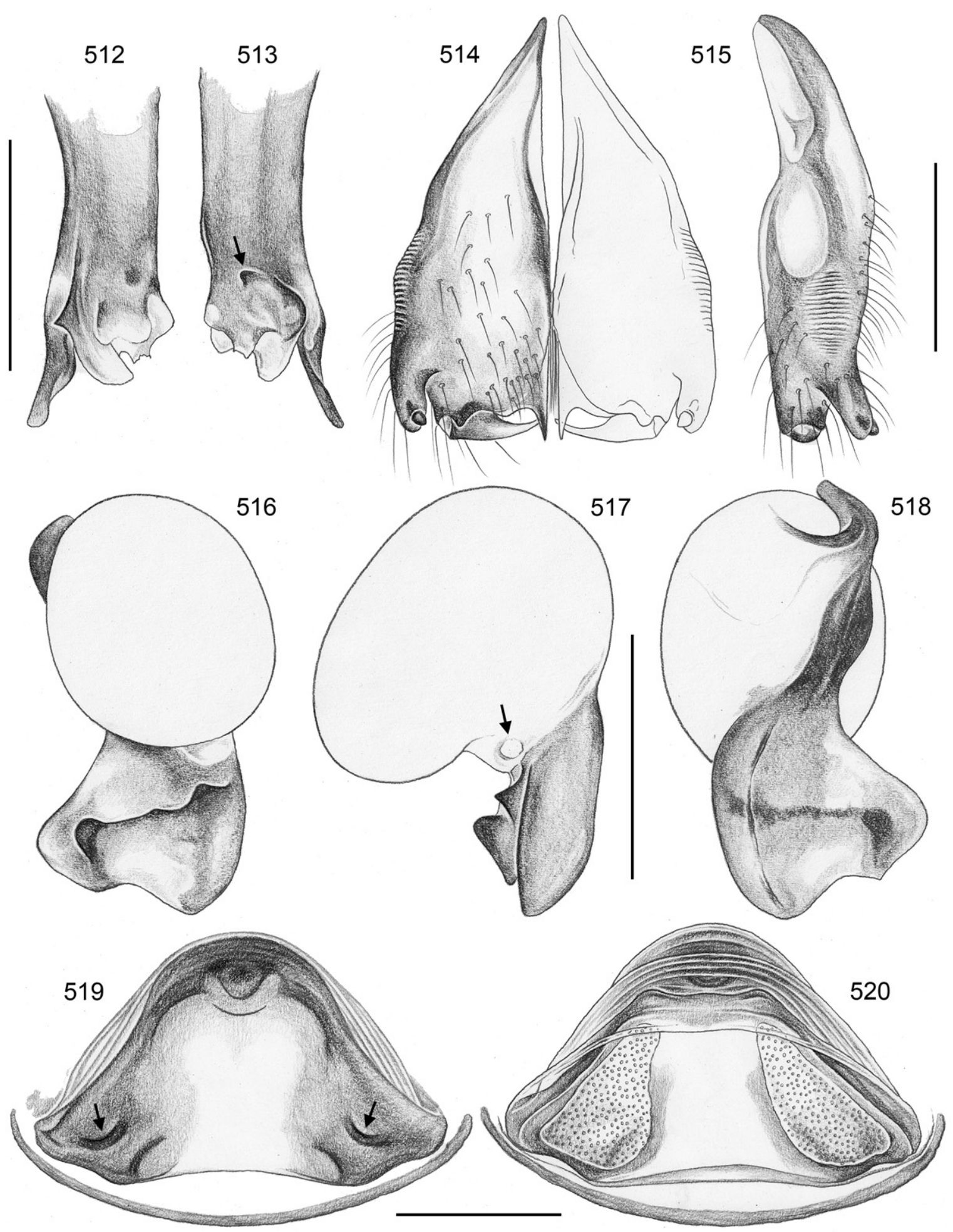

Figs 512-520. Crossopriza kittan sp. nov.; male and female from Oman, Al Kittan Cave (ZFMK Ar 22424, Ar 22425). 512-513. Left procursus tip, prolateral and retrolateral views; arrow: distinctive retrolateral apophysis. 514-515. Male chelicerae, frontal and lateral views. 516-518. Left genital bulb, prolateral, dorsal, and retrolateral views; arrow: sperm duct opening. 519-520. Cleared female genitalia, ventral and dorsal views; arrows: pockets. Scale bars: $512-513=0.2 \mathrm{~mm} ; 514-520=0.3 \mathrm{~mm}$. 


\section{Female}

In general similar to male (Fig. 480) but without spines on legs, apparently without stridulatory files on chelicerae, and with stridulatory organ consisting of pair of weakly sclerotized but distinct processes posteriorly on carapace and pair of distinct light brown plates anteriorly on abdomen. Tibia 1 in ten females: 8.9-11.5 (mean 10.5). Epigynum as in Figs 521-522, main epigynal plate semicircular to triangular, weakly protruding, only posteriorly laterally heavily sclerotized; with pair of shallow pockets $450 \mu \mathrm{m}$ apart; internal sclerotized arcs clearly visible in untreated specimens; posterior plate very short but wide. Internal genitalia (Figs 519-520, 523-525) with large pore plates converging and narrowing anteriorly, dorsal arc weak and simple, ventral arc with round median modification of unknown function.

\section{Natural history}

This species was abundant in Al Kittan Cave, were it lived in exposed and relatively large webs (diameter $30-50 \mathrm{~cm}$ ) close to the floor, while the ceiling was occupied by Artema bahla Huber, 2019; barely any other macroscopic life was observed in the dry cave. None of the two pholcids was found in the deeper parts of the cave, beyond approximately $10-15 \mathrm{~m}$. The species was also found near the cave under a large overhang (or rock shelter), suggesting it is probably more widespread in the area and not bound to caves.
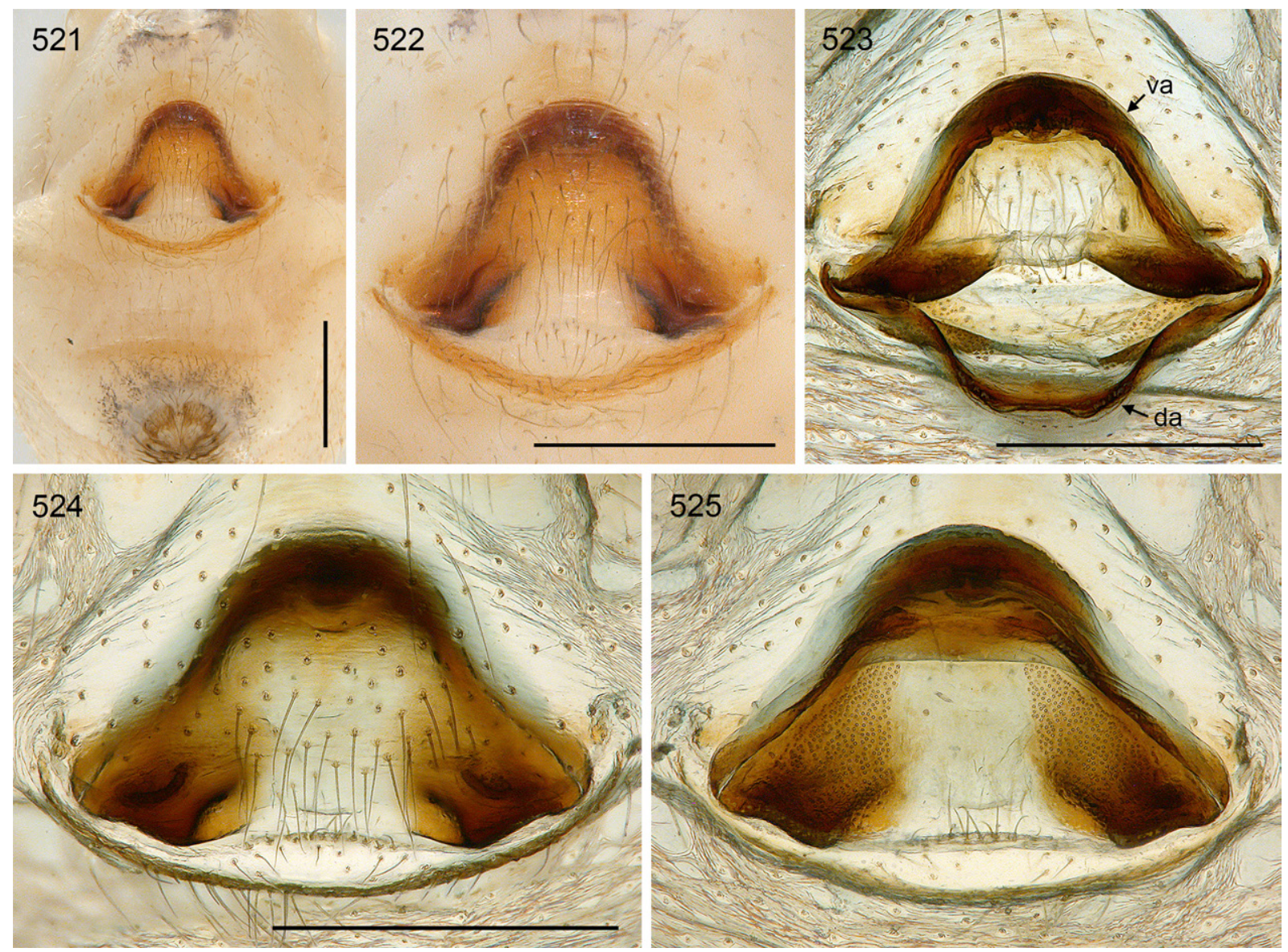

Figs 521-525. Crossopriza kittan sp. nov.; female from Oman, Ibri, Al Kittan Cave (ZFMK Ar 22425). 521-522. Abdomen and epigynum, ventral views. 523-525. Female internal genitalia, dorsal view (with dorsal arc tilted backwards), ventral view, and regular dorsal view. Abbreviations: da $=$ dorsal arc; va $=$ ventral arc. Scale bars $=0.5 \mathrm{~mm}$. 


\title{
Distribution
}

Known from two neighboring localities in north-eastern Oman (Ad Dhahira Province) (Fig. 352).

\author{
Crossopriza ghul sp. nov. \\ urn:1sid:zoobank.org:act:DBDAE29C-0C1C-4B8A-B062-0AB9790BEDA1
}

Figs 352, 481-482, 526-537

\section{Diagnosis}

Distinguished from the very similar C. moqal sp. nov. by distinctive set of apophyses prolaterally on distal bulbal sclerite (Fig. 528), by slightly wider distance between pore plates in female internal genitalia (Fig. 532), by pair of shallow epigynal furrows closer together ( 365 $\mu \mathrm{m}$ apart), and by female internal median structure different (W-shaped structure in 'valve'; no Y-shaped structure behind 'valve');

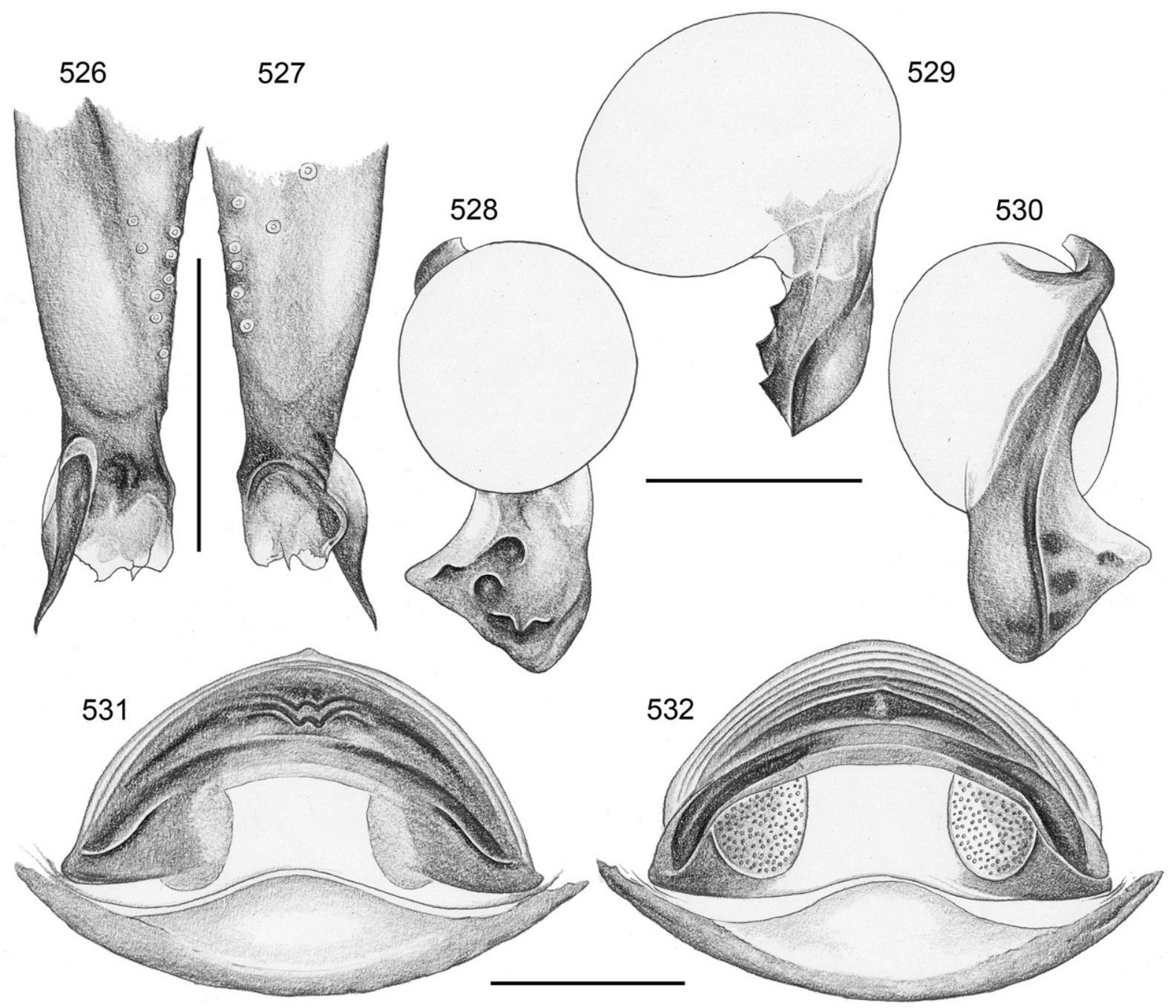

Figs 526-532. Crossopriza ghul sp. nov.; holotype male (ZFMK Ar 22427) and female from Oman, Wadi Ghul (ZFMK Ar 22428). 526-527. Left procursus, prolateral and retrolateral views. 528-530. Left genital bulb, prolateral, dorsal, and retrolateral views. 531-532. Cleared female genitalia, ventral and dorsal views. Scale bars $=0.2 \mathrm{~mm}$. 
from geographically closer $C$. kittan sp. nov. by apophyses prolaterally on distal bulbal sclerite and by relatively shorter epigynum (Fig. 534).

\section{Etymology}

The species name refers to the type locality; noun in apposition.

\section{Type material}

\section{Holotype}

OMAN - Ad Dakhiliyah • ${ }^{7}$; Wadi Ghul, 'site 1'; $23.234^{\circ}$ N, 57.150 E; 1440 m a.s.1.; 15 Feb. 2018; B.A. Huber leg.; ZFMK Ar 22427.

\section{Other material examined}

OMAN - Ad Dakhiliyah • 2 q $q$; same collection data as for holotype; ZFMK Ar $22428 \bullet 4 \circ q$ (in pure ethanol); same collection data as for holotype; ZFMK Om105. - Al Batinah South - 1 क; hill above mountain village above Wadi Sahtan; 23.218 $\mathrm{N}, 57.315^{\circ}$ E; $980 \mathrm{~m}$ a.s.1.; 26 Mar. 2017; B.A. Huber leg.; ZFMK Ar 22429.

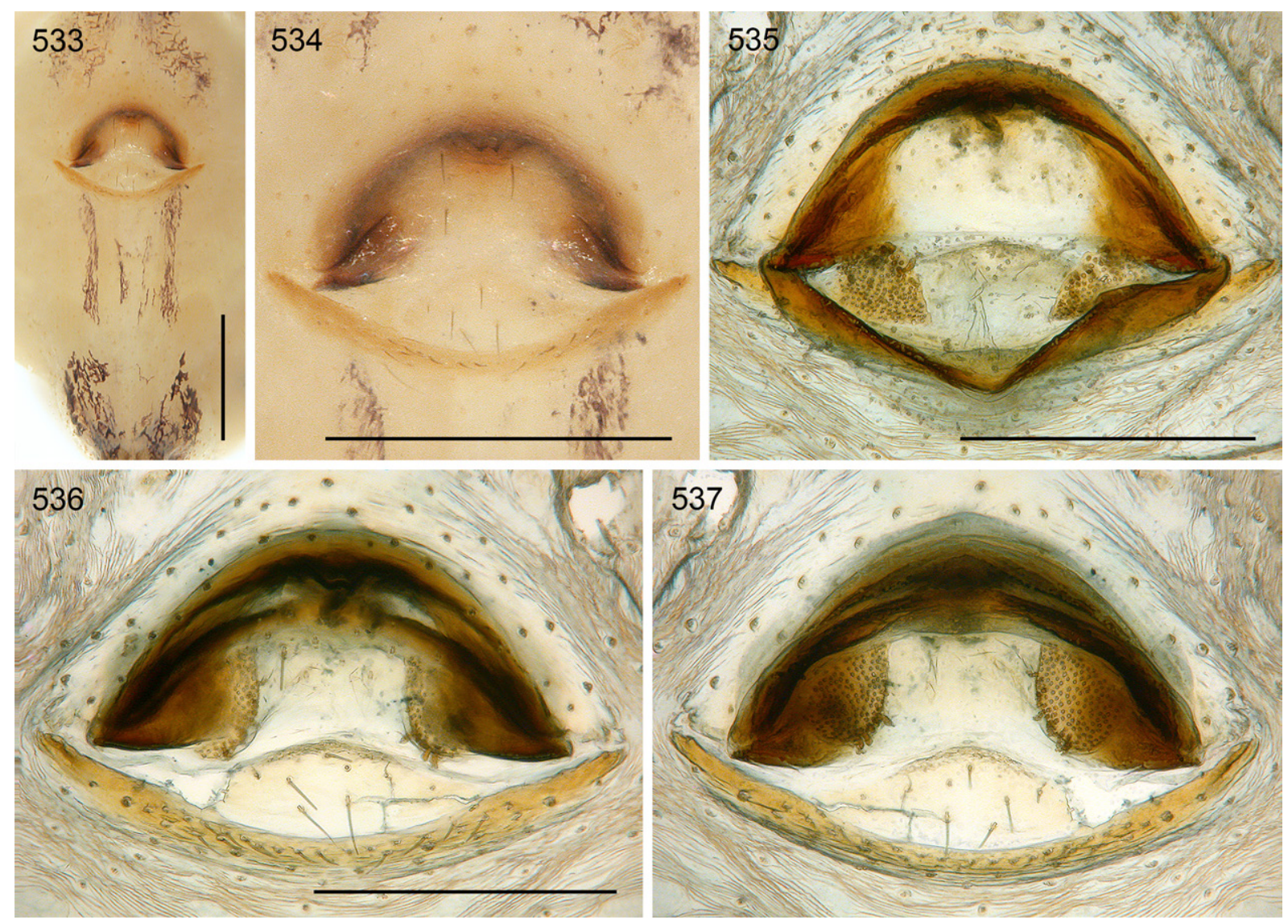

Figs 533-537. Crossopriza ghul sp. nov.; female from Oman, Wadi Ghul (ZFMK Ar 22428). 533534. Abdomen and epigynum, ventral views. 535-537. Female internal genitalia, dorsal view (with dorsal arc tilted backwards), ventral view, and regular dorsal view. Scale bars: $533-534=0.5 \mathrm{~mm}$; $535-537=0.3 \mathrm{~mm}$. 


\section{Description}

Male (holotype)

Measurements. Total length 2.6, carapace width 1.0. Distance PME-PME $70 \mu \mathrm{m}$; diameter PME $60 \times$ $80 \mu \mathrm{m}$; distance PME-ALE $20 \mu \mathrm{m}$; diameter AME $65 \mu \mathrm{m}$; distance AME-AME $20 \mu \mathrm{m}$. Leg 1: 26.8 $(7.5+0.5+7.6+9.5+1.7)$, tibia $2: 4.8$, tibia $3: 3.5$, tibia $4: 4.3$; tibia $1 \mathrm{~L} / \mathrm{d}: 76$; femora $1-4$ diameters: $0.16,0.13,0.13,0.13$.

COLOR (in ethanol). Carapace pale ochre-yellow, anteriorly in median pit light brown; sternum laterally light ochre-yellow, medially with wide, dark brown band; legs ochre-yellow, without darker rings, with black lines on femora and (few) on tibiae; abdomen pale gray, with few indistinct dark marks dorsally, mainly posteriorly above spinnerets, ventrally with darker marks near pedicel and near spinnerets and pair of indistinct longitudinal bands behind gonopore.

Body. Habitus as in Fig. 481. Ocular area slightly raised. Wide but shallow thoracic pit and pair of furrows diverging from pit toward posterior margin. Clypeus unmodified (but rim more strongly sclerotized than in female). Sternum wider than long (0.80/0.55), unmodified. Abdomen oval, dorso-posteriorly slightly angular, tapering at spinnerets.

Chelicerae. As in C. moqal sp. nov. (cf. Figs 488-489) but smaller: maximum width $405 \mu$ m; distance between tips of modified hairs: $335 \mu \mathrm{m}$; stridulatory files clearly visible in dissecting microscope.

PaLPs. Proximal segments as in C. moqal sp. nov. (cf. Figs 483-485) but smaller: femur dorsal length $270 \mu \mathrm{m}$; tibia maximum length $570 \mu \mathrm{m}$; procursus tip (Figs 526-527) with strong ventral sclerite (more pointed in lateral view than in C. moqal sp. nov.), and further membranous and sclerotized elements (very similar to C. moqal sp. nov.); genital bulb (Figs 528-530) with simple basal sclerite connected to distal (main) sclerite, sperm duct opening at basis of distal sclerite; distal sclerite with retrolateral ridge and several distinctive prolateral apophyses (arrangement different from C. moqal sp. nov.).

Legs. Femur 1 with single row of $\sim 20$ ventral spines; without curved hairs; few vertical hairs; retrolateral trichobothrium of tibia 1 at 3.5\%; prolateral trichobothrium absent on tibia 1, present on other leg tibiae; tarsi without regular pseudosegments but with indistinct platelets.

\section{Female}

In general similar to male (Fig. 482) but without spines on legs, apparently without stridulatory files on chelicerae, and with stridulatory organ consisting of pair of weakly sclerotized but distinct processes posteriorly on carapace and pair of light brown plates anteriorly on abdomen; sternum coloration as in male. Tibia 1 in three females: 6.0, 6.2, 7.2. Epigynum as in Figs 533-534, pair of pockets (shallow furrows) $\sim 365 \mu \mathrm{m}$ apart; internal median structure W-shaped.

\section{Natural history}

All specimens were collected by turning rocks on the ground in the wadi and on the hill.

\section{Distribution}

Known from two localities in the Hajar Mountains of north-eastern Oman (Fig. 352).

Crossopriza ibnsinai sp. nov. urn:lsid:zoobank.org:act:7F98CE47-8276-444D-A2FC-E0B5BD965E1F

Figs 352, 538-554

Holocnemus pluchei (misidentification) - Roewer 1960: 40 (all specimens except juvenile from RigRevan, which is a Pholcus). 


\section{Diagnosis}

Easily distinguished from geographically close species (C. maculipes, $C$. srinagar sp. nov., C. surobi sp. nov.) by male chelicerae with single pair of apophyses in lateral position (Fig. 543) and corresponding pockets on female epigynal plate far apart (Figs 548, 551); from some very similar species on the Arabian Peninsula (especially C. sahtan sp. nov., C. tiwi sp. nov.) by details of male palp (Figs 542, 545; ventral sclerite at tip of procursus long, much longer than dorsal element; distal bulbal sclerite with distinctive prolateral ridge and single apophysis) and by medially light epigynal plate with distinct semicircular internal median structure (Fig. 551).

\section{Etymology}

The name honors Ibn Sina (also known as Avicenna; 980-1037), a Persian polymath, physician, astronomer, and thinker.

\section{Type material}

\section{Holotype}

UZBEKISTAN - Surkhandarya • ${ }^{\lambda}$; Baison Distr., E foothills of Dzhetymkalyas Mt Range, ca $4.5 \mathrm{~km}$ SE of Sarykamysh, Pul'khakim River valley, Karadara Boundary; $38.0750^{\circ}$ N, $67.4425^{\circ}$ E; 705 m a.s.1.; 3 May 2002; A.V. Gromov leg.; ZMMU.

\section{Other material examined}

UZBEKISTAN - Surkhandarya 1 1 ; same collection data as for holotype; ZMMU (together with holotype) • 1 O; Babatagh Mt Range, near Ak-Machit [Ak-Mechet']; $38.032^{\circ} \mathrm{N}, 68.295^{\circ} \mathrm{E} ; 12-20 \mathrm{Apr}$. 1994; O. Lyakhov leg.; ZMMU • 1 đ , 1 क; Uzun Distr., E slope of Babatagh Mt Range, $\sim 7 \mathrm{~km}$ W of Akmechet [Ak-Mechet']; 38.05 ${ }^{\circ}$ N, 68.24 E; 1095 m a.s.1.; 1 May 2002; A.V. Gromov leg.; ZMMU. - Choresmien - $2 \widehat{\partial}, 1$ क 1 juv.; Amudarya River middle flow, Kyzylkum Reserve, "cliff on tugai

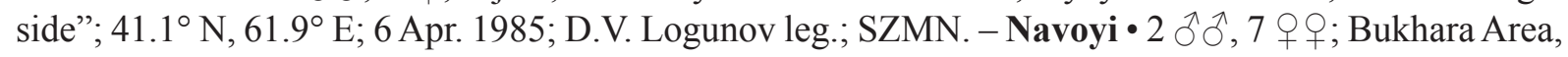
$\sim 70 \mathrm{~km}$ W of Uch-Kuduk, Mynbulak hollow, near Dzhyra-Kuduk; $42.26^{\circ} \mathrm{N}, 62.86^{\circ}$ E; 11 Sep. 1989; D.V. Logunov leg.; small cave in clayey cliff; SZMN.

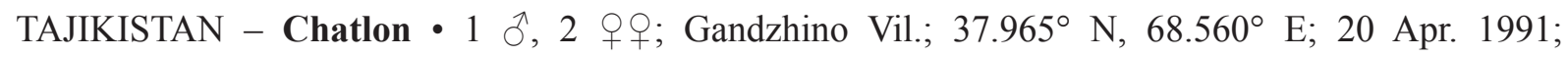
S. Ovtchinnikov leg.; ZMMU • 2 $q$; same locality as for preceding ("near Gandzhino Vil.”); 19 Apr. 1990; S.L. Zonstein leg.; ZMMU • 1 q; same locality as for preceding; 13-15 Apr. 1986; S.L. Zonstein leg.; ZMMU • 2 qक; same locality as for preceding; 7-12 Apr. 1987; S.L. Zonstein and A. Zor'kin leg.; ZMMU • 1 ô, 2 o+o; Pandzh, Karatau Mt Range, Astana; 37.23 N, 69.11 E; 22 Apr. 1991; S. Ovtchinnikov leg.; ZMMU.

TURKMENISTAN - Mary • 2 우; Kushka Distr., $\sim 18 \mathrm{~km} \mathrm{~S}$ of Kyzyldzhar Kordon, $\sim 1 \mathrm{~km} \mathrm{ESE} \mathrm{of}$ Eroilandaz; $35.661^{\circ} \mathrm{N}, 61.837^{\circ}$ E; 375 m a.s.1.; 7 Apr. 2002; A.V. Gromov leg.; saline soil; ZMMU.

KAZAKHSTAN - Chimkent 1 1 ; Kyzylkum Distr., Kyzylkum Desert, Karaktau mountain massif, Karamola Mt; $43.5^{\circ}$ N, $67.8^{\circ}$ E; 8 Jun. 1989; A.A. Zyuzin leg.; ZMMU.

AFGHANISTAN • 1 q, 2 juvs; Sorkh-Kotal [Surkh Kotal], "bei Tehachmeh Cher”, "A361”; 36.006 N, $68.544^{\circ}$ E; 10 Oct. 1957; K. Lindberg leg.; in pile of leaves; NHMG • 1 क , 1 juv.; Douchi [Doshi], “A465"; $35.61^{\circ} \mathrm{N}, 68.68^{\circ} \mathrm{E}$; 11 Nov. 1957; K. Lindberg leg.; NHMG • 1 juv. (assigned tentatively); $\mathrm{N}$ of Pol-Khomri [Pol-e-Khomri], unnamed cave of "Tschachméh Cher", "A195"; 35.980 N, 68.544 E; 10 Oct. 1957; K. Lindberg leg.; NHMG • 2 juvs (assigned tentatively); Salang valley, Djebel os-Siradj [Jabal-os-Saraj], “A532”; 35.12 N, 69.23 E; 26 Jul. 1957; K. Lindberg leg.; NHMG. 


\section{Description}

Male (holotype)

MeAsurements. Total length 4.0, carapace width 1.6. Distance PME-PME $55 \mu \mathrm{m}$; diameter PME $100 \times$ $130 \mu \mathrm{m}$; distance PME-ALE $30 \mu \mathrm{m}$; diameter AME $90 \mu \mathrm{m}$; distance AME-AME $30 \mu \mathrm{m}$. Leg 1: 35.8 $(10.2+0.7+9.6+12.9+2.4)$, tibia $2: 6.8$, tibia $3: 5.6$, tibia $4: 6.4$; tibia $1 \mathrm{~L} / \mathrm{d}: 56$; femora $1-4$ diameters: $0.26,0.22,0.21,0.22$.

COLOR (in ethanol). Carapace ochre-yellow, anteriorly in median pit with distinct brown mark; sternum brown with dark brown radial marks; legs ochre-yellow, without darker rings, with small black lines on femora only; abdomen gray, with whitish internal marks, with few and indistinct dorsal marks; ventrally with broken dark band, with indistinct parallel longitudinal marks behind gonopore.

Body. Habitus similar to C. sahtan sp. nov. (cf. Fig. 391). Ocular area slightly raised. Deep thoracic pit and pair of shallow furrows diverging from pit toward posterior margin. Clypeus unmodified, only rim more sclerotized than in female. Sternum wider than long (1.2/0.7), unmodified. Abdomen slightly elongated, dorso-posteriorly angular to conical.

Chelicerae. As in Figs 543-544 (very similar to C. moqal sp. nov.), with pair of latero-distal apophyses provided with one large modified cone-shaped hair each; distance between tips of modified hairs: $430 \mu \mathrm{m}$; lateral stridulatory ridges clearly visible in dissecting microscope.

PALPS. As in Figs 538-540; coxa with rounded retrolateral hump; trochanter barely modified; femur distally strongly widened, with rounded ventral protrusion, proximally with prolateral stridulatory pick, with indistinct retrolateral transversal line, without retrolateral proximal process but retrolateral-ventral

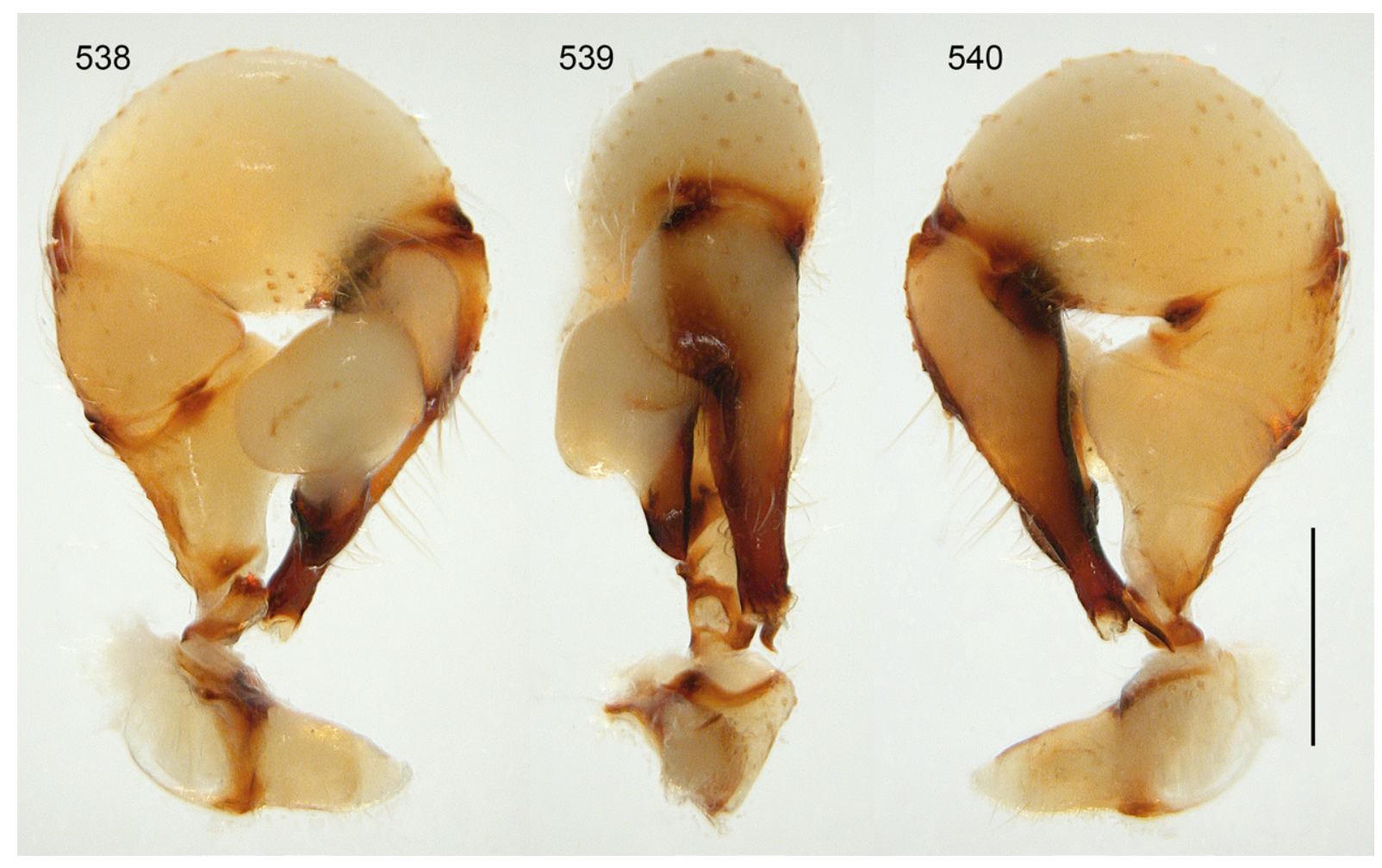

Figs 538-540. Crossopriza ibnsinai sp. nov.; male from Uzbekistan, near Dzhyra-Kuduk (SZMN); mirror images of right palp, prolateral, dorsal, and retrolateral views. Scale bar $=0.5 \mathrm{~mm}$. 


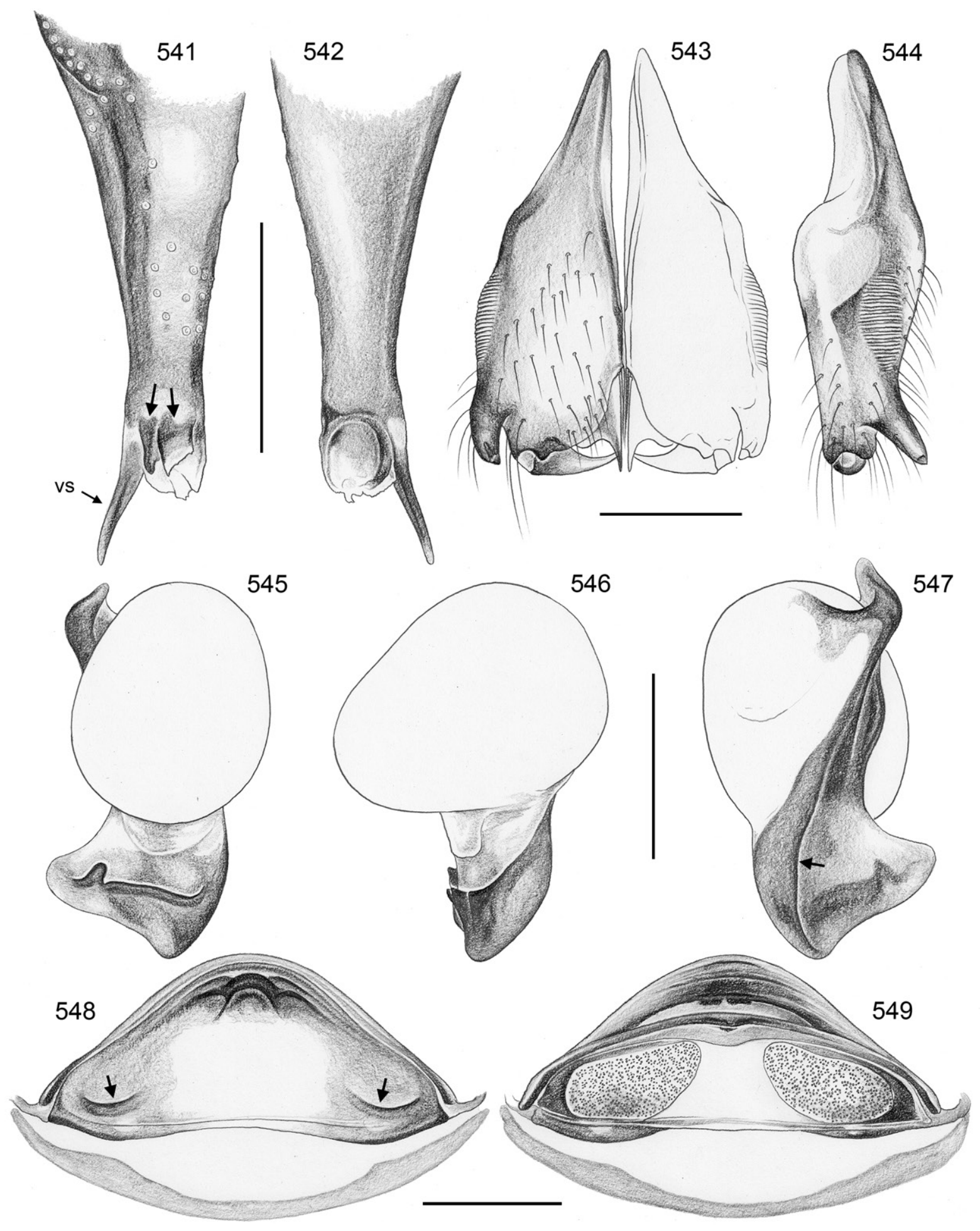

Figs 541-549. Crossopriza ibnsinai sp. nov.; male and female from Uzbekistan, near Dzhyra-Kuduk (SZMN). 541-542. Left procursus, prolateral and retrolateral views; arrows: distinctive prolateral sclerites. 543-544. Male chelicerae, frontal and lateral views. 545-547. Left genital bulb, prolateral, dorsal, and retrolateral views; arrow: retrolateral ridge. 548-549. Cleared female genitalia, ventral and dorsal views; arrows: pockets. Abbreviation: vs $=$ ventral sclerite. Scale bars $=0.3 \mathrm{~mm}$. 
ridge with some slightly stronger hair-bases; femur-patella joints shifted toward prolateral side; tibiatarsus joints shifted toward retrolateral side; tarsus without macrotrichia; procursus (Figs 541-542) straight, narrowing distally, with low prolateral hump proximally set with numerous long hairs, followed distally by thick ridge, long dorsal hairs mostly straight or weakly curved, procursus tip with long ventral sclerite and two distinctive prolateral sclerites; genital bulb (Figs 545-547) with simple basal sclerite connected to distal (main) sclerite, sperm duct opening not seen; distal sclerite with retrolateral ridge, with distinctive prolateral transversal ridge and small apophysis on ventral semitransparent flap.

LEGS. Femur 1 with single row of $\sim 20$ ventral spines; without curved hairs; few vertical hairs; retrolateral trichobothrium of tibia 1 at 3\%; prolateral trichobothrium absent on tibia 1, present on other leg tibiae; tarsal pseudosegments indistinct and irregular, only at distal tip $\sim 3-4$ regular pseudosegments.

Male (variation)

Tibia 1 in seven males (incl. holotype): 7.2-9.7 (mean 8.8). Some males with distinct dark and whitish marks on abdomen; dark lines sometimes also on leg tibiae.

\section{Female}

In general similar to male but without spines on legs, apparently without stridulatory files on chelicerae, and with stridulatory organ consisting of pair of weakly sclerotized but distinct processes posteriorly on carapace and pair of small but distinct light brown plates anteriorly on abdomen. Tibia 1 in 21 females:
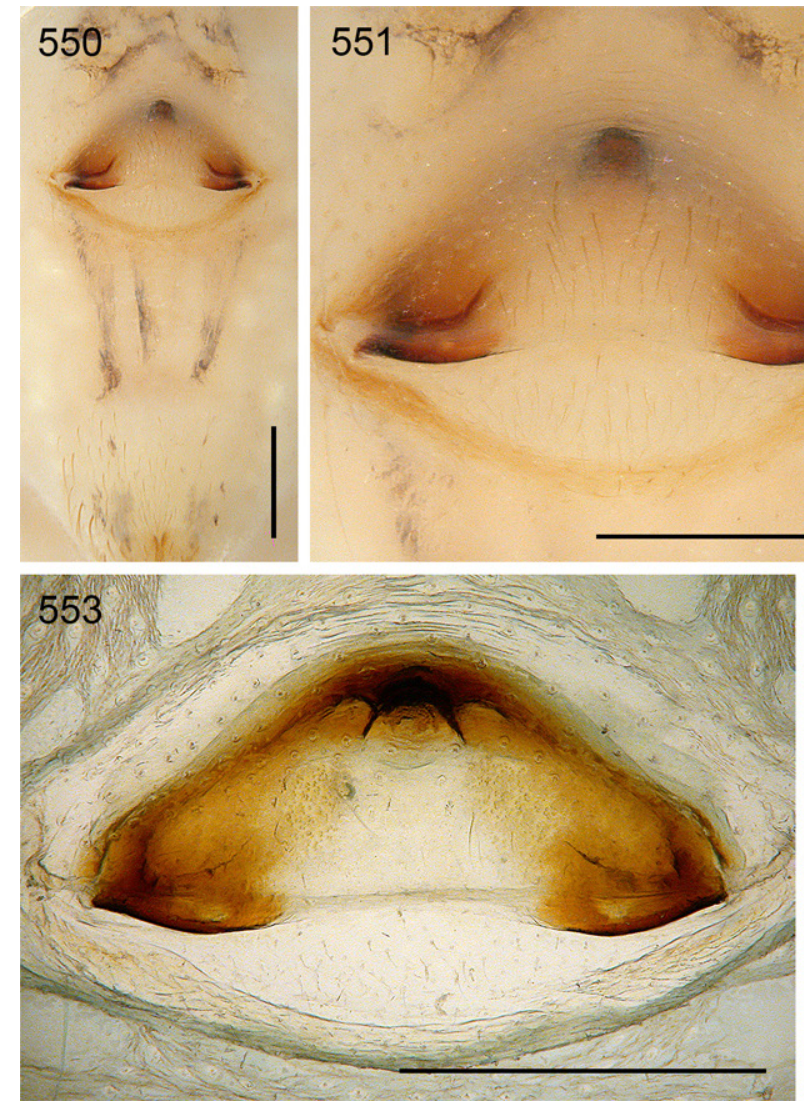
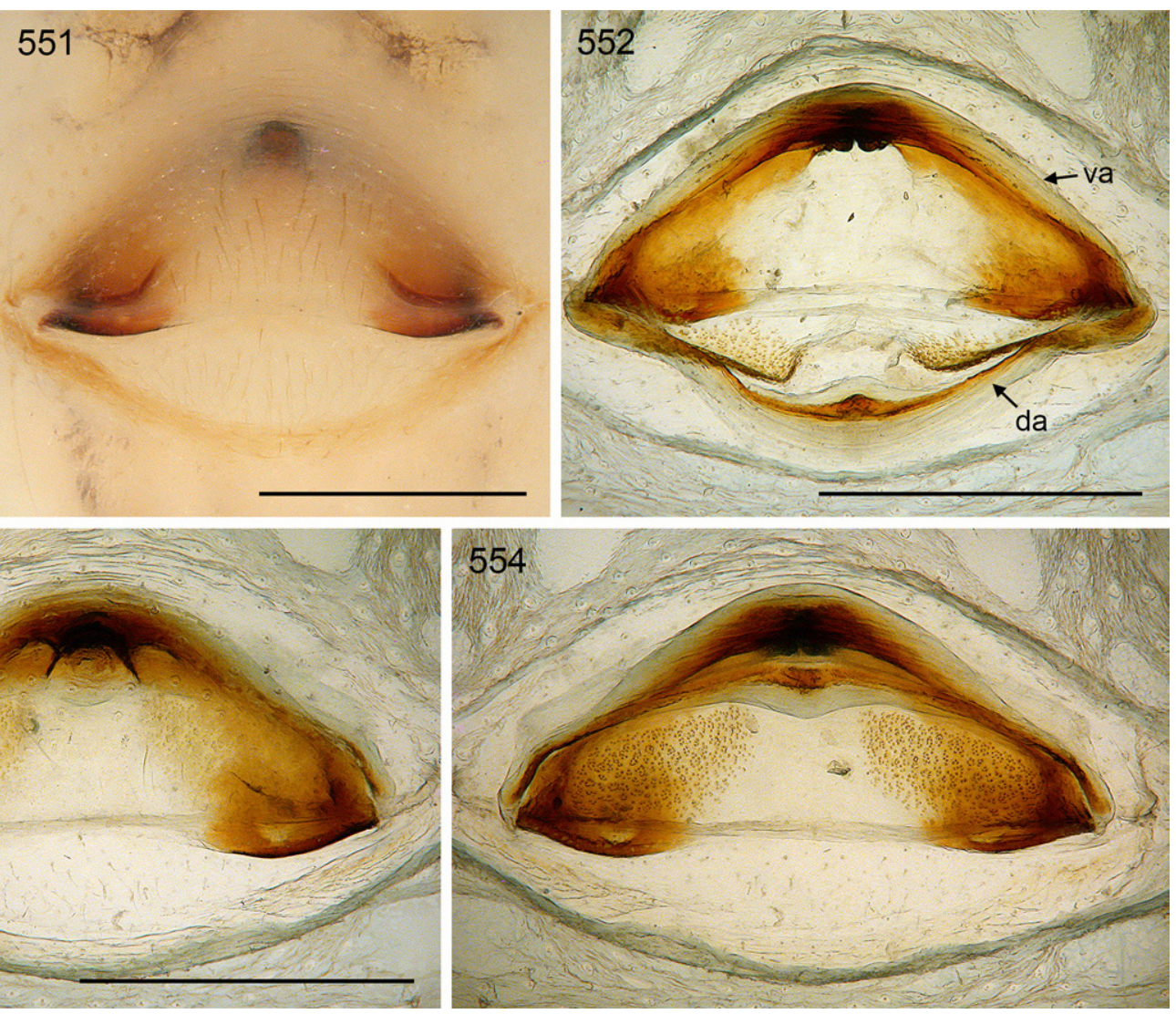

Figs 550-554. Crossopriza ibnsinai sp. nov.; female from Uzbekistan, near Dzhyra-Kuduk (SZMN). 550-551. Abdomen and epigynum, ventral views. 552-554. Female internal genitalia, dorsal view (with dorsal arc tilted backwards), ventral view, and regular dorsal view. Abbreviations: da $=$ dorsal arc; $v a=$ ventral arc. Scale bars $=0.5 \mathrm{~mm}$. 
6.3-10.3 (mean 8.6). Epigynum as in Figs 550-551, main epigynal plate semicircular, barely protruding, medially posteriorly light, laterally heavily sclerotized with pair of large but narrow pockets (distance $560 \mu \mathrm{m}$ ); internal median structure visible in uncleared specimens; posterior plate light brown, short but wide. Internal genitalia (Figs 548-549, 552-554) with large elongate pore plates converging anteriorly, dorsal arc slender, ventral arc with median pouch-like modification.

\section{Distribution}

Widely distributed In Central Asia (Uzbekistan, Tajikistan, Turkmenistan, Kazakhstan, Afghanistan) (Fig. 352).

$$
\begin{gathered}
\text { Crossopriza khayyami sp. nov. } \\
\text { urn:Isid:zoobank.org:act:5EF0299D-0FBC-49BF-AFAA-F62087037056 }
\end{gathered}
$$

Figs 352, 555-573

\section{Diagnosis}

Distinguished from named congeners with only one pair of apophyses laterally on male chelicerae (Fig. 562) by details of male palp (Figs 561, 564; procursus with ventral sclerite accompanied by strong flat sclerite; genital bulb distal sclerite strongly protruding ventrally, with distinctive set of prolateral apophyses); females differ from very similar species (C. sahtan sp. nov., C. ibnsinai sp. nov.) by details of epigynum and internal genitalia (Figs 567, 573; anterior epigynal margin evenly curved; distance between epigynal pockets). A very similar unnamed species is known from Iran, Kerman (see Remark below).

\section{Remark}

The CBSU has a very similar species from Iran (only $1{ }^{\AA}, 2$ 2 \% ; Kerman: Jiroft, $28.519^{\circ} \mathrm{N}, 58.171^{\circ} \mathrm{E}$; Jefriz, $29.492^{\circ} \mathrm{N}, 50.470^{\circ} \mathrm{E}$ ) that shares the general distinctive shape of the bulbal process but is distinguished by a different configuration of the apophyses on this process and by several further minor details: presence of distinct prolateral sclerite on procursus tip; cheliceral apophyses more strongly bent inwards; and epigynal pockets rounder. A single male in SMF is probably conspecific with the specimens from Kerman, but the label information is dubious and possibly erroneous ("Anotalia: Taurus", no further data).

\section{Etymology}

The name honors Omar Khayyam (1048-1131), a Persian polymath, mathematician, astronomer, philosopher, and poet.

\section{Type material}

\section{Holotype}

IRAN - Fars - ${ }^{\wedge}$; Izad Khast; $31.52^{\circ} \mathrm{N}, 52.15^{\circ} \mathrm{E}$ (on accuracy of Senglet's label coordinates see Remarks under C. parsa sp. nov.); 16 Aug. 1973; A. Senglet leg.; MHNG.

\section{Other material examined}

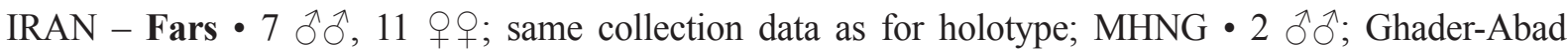
[Qaderabad]; $30.35^{\circ} \mathrm{N}, 53.32^{\circ} \mathrm{E}$ [or rather $30.28^{\circ} \mathrm{N}, 53.27^{\circ}$ E?]; 17 Aug. 1973; A. Senglet leg.; MHNG

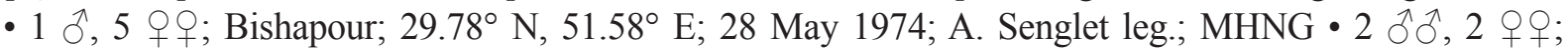

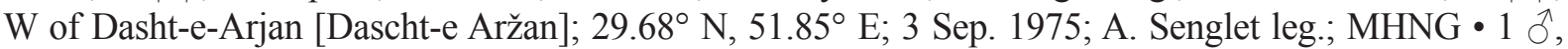
1 甲; Shiraz; $29.62^{\circ}$ N, 52.38 E; 2 Jun. 1974; A. Senglet leg.; MHNG • 1 Oे, 1 juv.; 35 km S of Shiraz, "Bahakisecula"; $29.34^{\circ} \mathrm{N}, 52.63^{\circ} \mathrm{E}$; date unknown; Bilek leg.; NHMW 29568 • 2 ô ô, 7 q q (2 vials); Firouzabad; $28.87^{\circ}$ N, 52.53 E; 6 Jun. 1974; A. Senglet leg.; MHNG. - Kohgiluyeh and Boyer-Ahmad • 


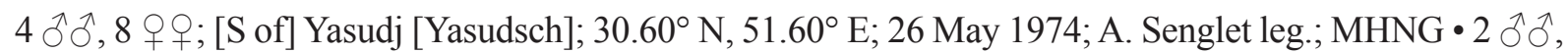
1 ㅇ; "route de Yasudj"; $30.47^{\circ} \mathrm{N}, 51.50^{\circ} \mathrm{E}$ (the label says "Kohkiluyeh" but the coordinates indicate a point slightly outside of the province); 25 May 1974; A. Senglet leg.; MHNG 11 ; Charam [Choram]; $30.73^{\circ}$ N, 50.73 E; 23 May 1974; A. Senglet leg.; MHNG • 1 Oे, 4 우; "route de Charam"; $30.47^{\circ} \mathrm{N}$, $50.83^{\circ}$ E; 22 May 1974; A. Senglet leg.; MHNG • 1 क; Basht; $30.33^{\circ} \mathrm{N}, 51.25^{\circ} \mathrm{E}$ [or rather $30.36^{\circ} \mathrm{N}$, $51.16^{\circ} \mathrm{E}$ ?, the coordinates on the label indicate a point slightly outside of Kohkliluyeh]; 25 May 1974; A. Senglet leg.; MHNG. - Bakhtiyari • $2{ }^{\lambda}{ }^{\lambda}, 2$ o $q$; Kuhrang [Koohrang]; $32.47^{\circ}$ N, $50.13^{\circ}$ E; 19 Jun.

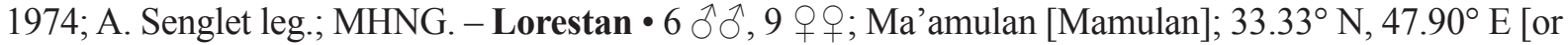

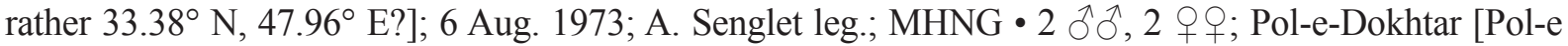

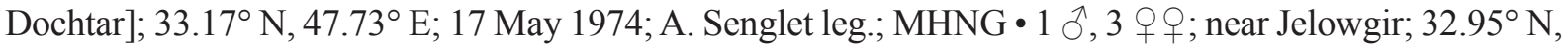

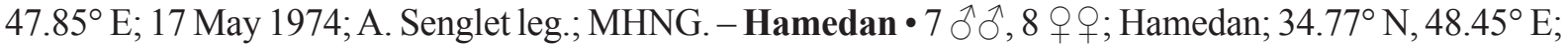
29 Jul. 1973; A. Senglet leg.; MHNG • 1 क; Ganznameh/Hamedan [Ganjameh]; $34.73^{\circ} \mathrm{N}, 48.50^{\circ} \mathrm{E}$ [or rather $34.76^{\circ} \mathrm{N}, 48.44^{\circ}$ E?]; $2100 \mathrm{~m}$ a.s.l.; 4 Jul. 1974; A. Senglet leg.; MHNG. - Kordestan • 3 § ${ }^{\lambda}$, 4 우; N of Sanandajd [Sanandadsch]; $35.47^{\circ}$ N, $47.02^{\circ}$ E; 22 Jun. 1975; A. Senglet leg.; MHNG • 4 § ${ }^{\lambda}$, 1 q; “Kal'eh Dju” [Qellacê]; 35.35 N, 46.28 E; 15 Sep. 1975; A. Senglet leg.; MHNG.

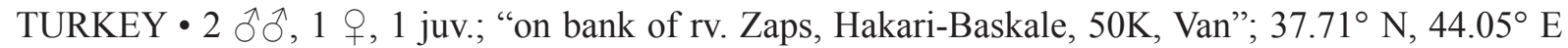
(these estimated coordinates are in Hakkari Province because the Great Zab river does not enter Van); 30 Aug. 1956; collector unknown; HECO.

Assigned tentatively (see Variation below)

IRAN - Yazd・ 1 \%; Shaddad; $32.294^{\circ}$ N, $54.411^{\circ}$ E; 2015; M.S. Tahami leg.; CBSU.

IRAQ • 1 ก, 1 क; Amarah (“Amara, Mesopot."); $31.83^{\circ} \mathrm{N}, 47.15^{\circ} \mathrm{E}$; date and collector unknown; NHMW.

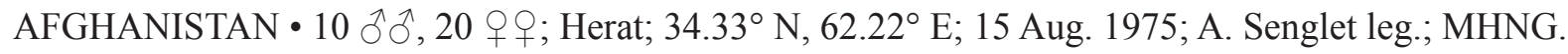

\section{Description}

\section{Male (holotype)}

Measurements. Total length 4.1, carapace width 1.4. Distance PME-PME $90 \mu \mathrm{m}$; diameter PME $90 \times$ $110 \mu \mathrm{m}$; distance PME-ALE $30 \mu \mathrm{m}$; diameter AME $85 \mu \mathrm{m}$; distance AME-AME $20 \mu \mathrm{m}$. Leg 1: 34.2 $(10.1+0.6+9.3+11.9+2.3)$, tibia $2: 6.6$, tibia $3: 5.1$, tibia $4: 6.0$; tibia $1 \mathrm{~L} / \mathrm{d}: 64$; femora $1-4$ diameters: $0.23,0.19,0.18,0.18$.

Color (in ethanol). Carapace ochre-yellow, anteriorly in median pit light brown; sternum light brown with darker brown radial marks; legs ochre-yellow, without darker rings, with indistinct black lines on femora and (few) on tibiae (cf. Figs 558-559); abdomen pale gray, with whitish internal marks, without dark dorsal marks; ventrally with broken dark band, with indistinct parallel longitudinal marks behind gonopore.

Body. Habitus similar to C. sahtan sp. nov. (cf. Fig. 391). Ocular area slightly raised. Deep thoracic pit and pair of furrows diverging from pit toward posterior margin. Clypeus unmodified, rim apparently as in female. Sternum wider than long (1.05/0.65), unmodified. Abdomen slightly elongated, dorsoposteriorly angular to conical.

Chelicerae. As in Figs 562-563, laterally much darker than frontally, with one pair of apophyses distally, each with one large modified hair at tip; distance between tips of modified hairs: $255 \mu \mathrm{m}$; lateral stridulatory ridges fine but visible in dissecting microscope.

PALPS. As in Figs 555-557; coxa with rounded retrolateral hump; trochanter barely modified; femur distally strongly widened, with rounded ventral protrusion, proximally with prolateral stridulatory pick, 
with retrolateral transversal line, without retrolateral proximal process but retrolateral-ventral ridge with some small sclerotized processes (hair-bases?); femur-patella joints shifted toward prolateral side; tibia-tarsus joints shifted toward retrolateral side; tarsus without macrotrichia; procursus (Figs 560-561) slightly curved towards ventral, strongly narrowing distally, with low prolateral hump proximally set with many hairs, long dorsal hairs mostly straight, only $2-3$ hairs weakly curved, procursus tip with ventral sclerite accompanied by strong flat sclerite (prominent in retrolateral view; arrow in Fig. 561); genital bulb (Figs 564-566) with simple basal sclerite connected to distal (main) sclerite, sperm duct opening not seen; distal sclerite without retrolateral ridge, with distinctive set of prolateral apophyses, with transparent prolateral structure of unknown function.

LEGS. Femur 1 with single row of $\sim 20$ ventral spines; without curved hairs; few vertical hairs; retrolateral trichobothrium of tibia 1 at $3.5 \%$; prolateral trichobothrium absent on tibia 1, present on other leg tibiae; tarsal pseudosegments not seen except 1-2 at tip.

Male (variation)

Tibia 1 in 35 males from Iran and Turkey: 6.4-9.7 (mean 7.8). Males (and females) from some localities (e.g., Izad Khast, Pol-e Dochtar) seem to be significantly larger than specimens from other localities

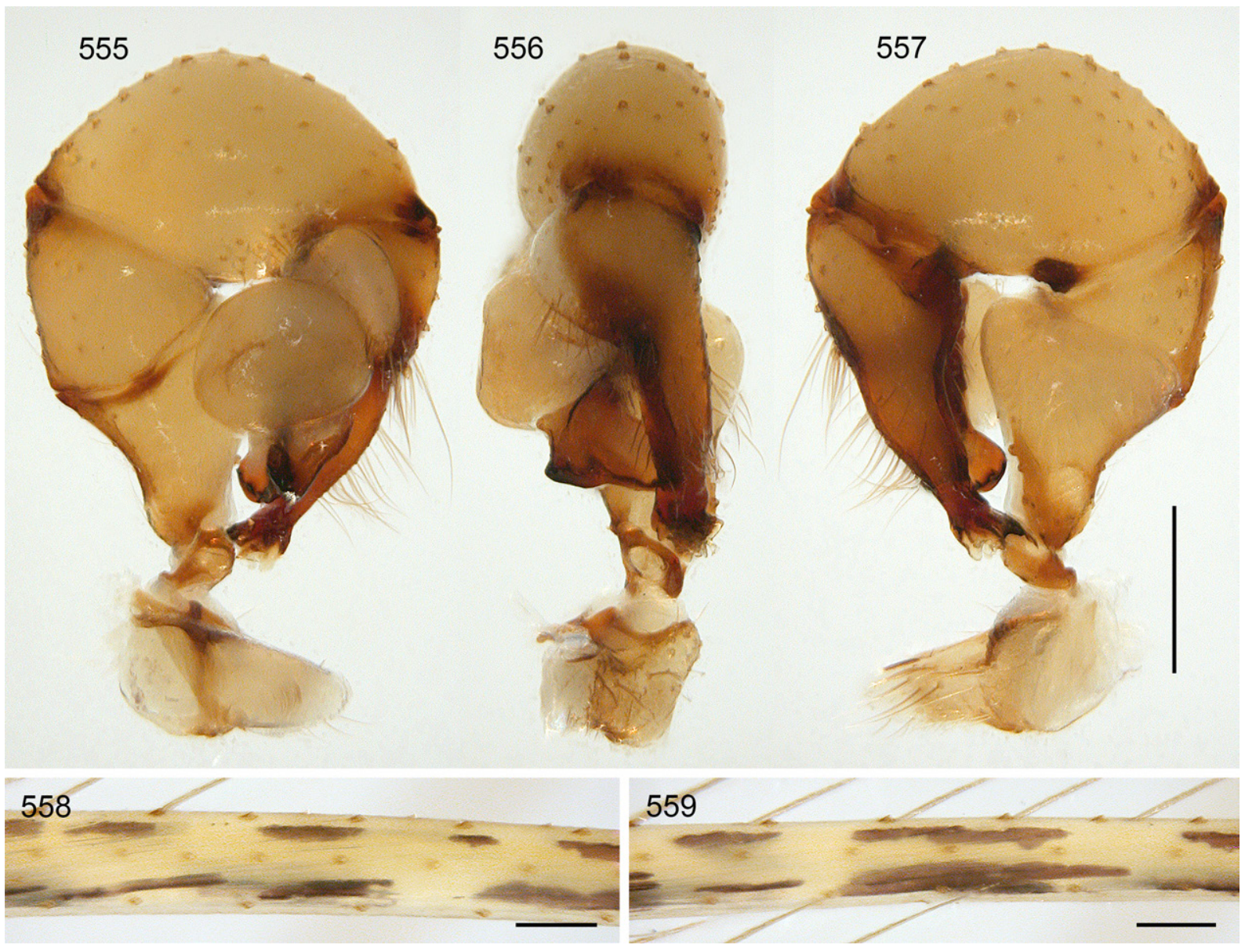

Figs 555-559. Crossopriza khayyami sp. nov., non-type male and female from Iran, Fars, Izad Khast (MHNG). 555-557. Left male palp, prolateral, dorsal, and retrolateral views. 558-559. Female femora 1 and 2, retrolateral views. Scale bars: $555-557=0.5 \mathrm{~mm} ; 558-559=0.2 \mathrm{~mm}$. 


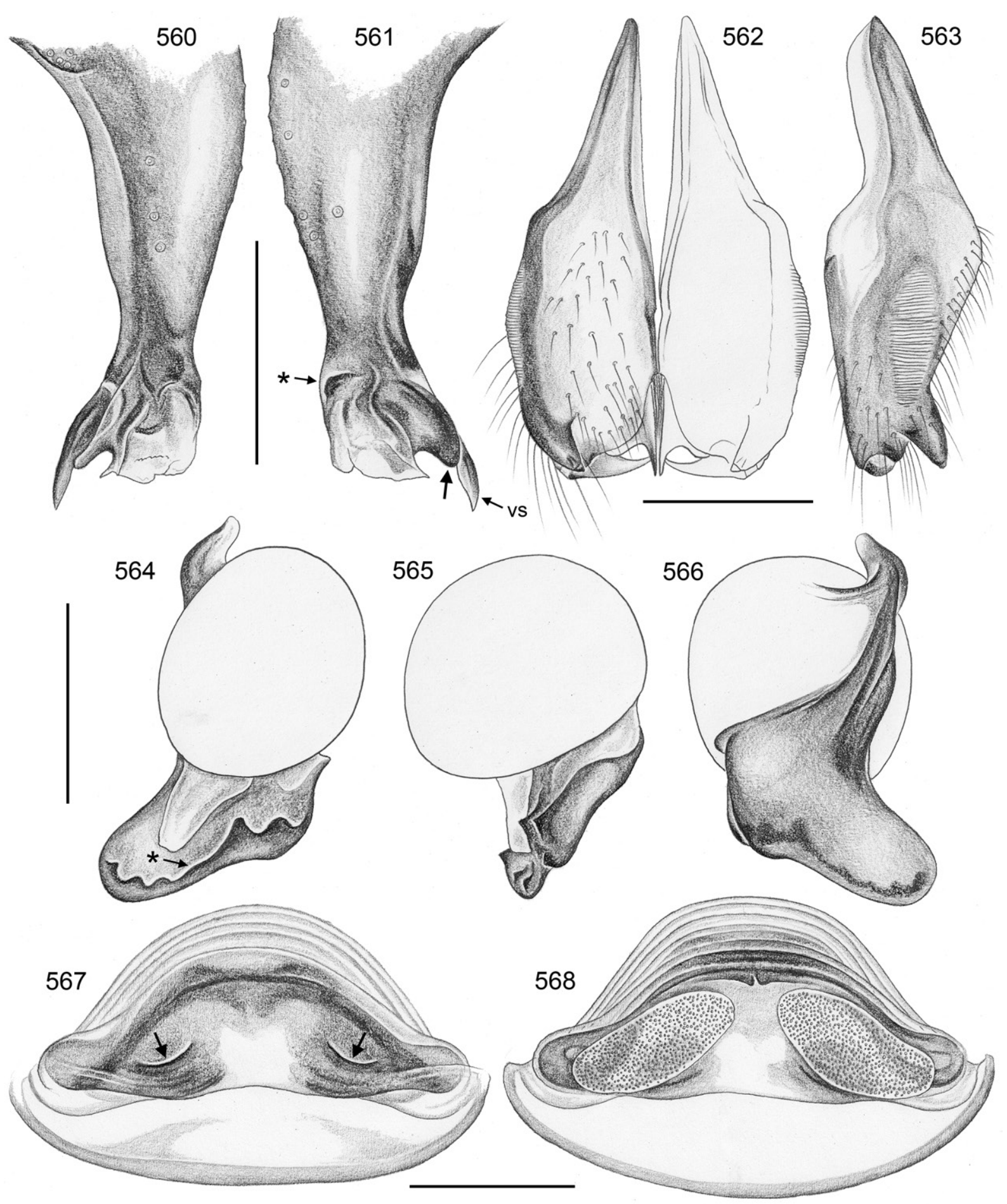

Figs 560-568. Crossopriza khayyami sp. nov., non-type male and female from Iran, Fars, Izad Khast (MHNG). 560-561. Left procursus, prolateral and retrolateral views; asterisk: process that is larger in specimens from Afghanistan; arrow: distinctive sclerite parallel to ventral sclerite. 562-563. Male chelicerae, frontal and lateral views. 564-566. Left genital bulb, prolateral, dorsal, and retrolateral views; asterisk: ridge where specimens from Afghanistan have two additional small apophyses. 567568. Cleared female genitalia, ventral and dorsal views; arrows: pockets. Abbreviation: vs = ventral sclerite. Scale bars $=0.3 \mathrm{~mm}$. 
(e.g., S of Yasudsch, Mamulan) (small sample sizes). Some males with distinct dark and whitish marks on abdomen; dark lines on leg femora and tibiae variably distinct.

Specimens from Iraq and Afghanistan are assigned tentatively. The single male from Iraq appears identical in shape to the males from Iran but the female differs (see below); the male is also unusually large (tibia 1: 11.1). The males from Afghanistan show tiny but consistent differences in the procursus tip and in the bulbal ventral apophyses (asterisks in Figs 561 and 564); they are also considerably larger than the males from Iran and Turkey (tibia 1 in 10 males: 9.6-11.4; mean: 10.9).

\section{Female}

In general similar to male but without spines on legs, apparently without stridulatory files on chelicerae, and with stridulatory organ consisting of pair of weakly sclerotized but distinct processes posteriorly on carapace and pair of small but distinct light brown plates anteriorly on abdomen. Tibia 1 in 55 females from Iran and Turkey: 5.3-9.1 (mean 6.7). Epigynum as in Figs 569-570, main epigynal plate wider than long, weakly protruding, medially posteriorly light, anteriorly with variably distinct brown mark, laterally heavily sclerotized with pair of large pockets (distance $\sim 290 \mu \mathrm{m}$ ); internal sclerotized arcs and simple median structure visible in uncleared specimens; posterior plate light brown, short but wide. Internal genitalia (Figs 567-568, 571-573) with large elongate pore plates converging anteriorly, dorsal arc strongly
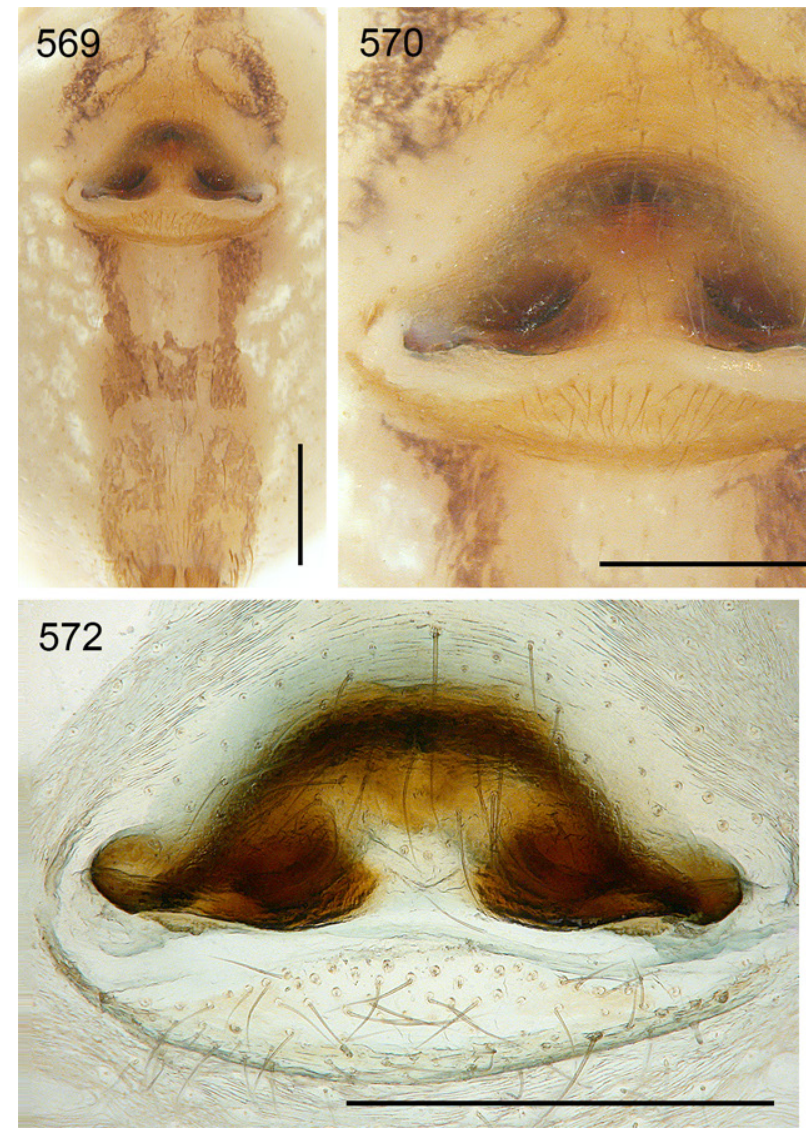
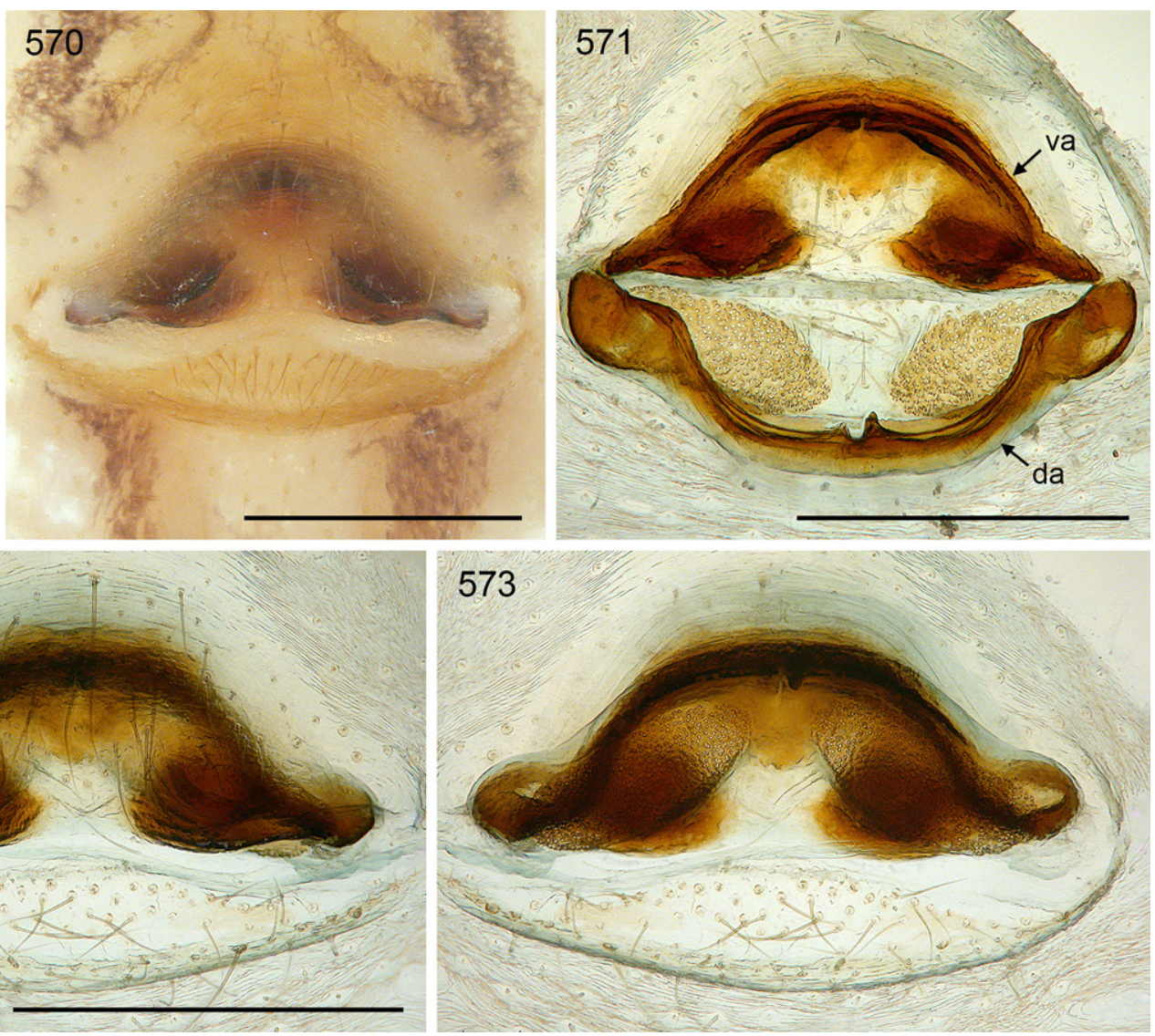

Figs 569-573. Crossopriza khayyami sp. nov., female from Iran, Fars, Izad Khast (MHNG). 569570. Abdomen and epigynum, ventral views. 571-573. Female internal genitalia, dorsal view (with dorsal arc tilted backwards), ventral view, and regular dorsal view. Abbreviations: da = dorsal arc; va = ventral arc. Scale bars $=0.5 \mathrm{~mm}$. 
widened laterally, ventral arc with small and simple median modification (not pouch or pocket) apparently asymmetric, with counterpart in dorsal arc also asymmetric (apparently not an artifact of preparation).

The single female from Yazd Province is assigned tentatively because the epigynal pockets are slightly closer to the epigynal margin and no male is available from this province. The single female from Iraq (not cleared) seems to have a more prominent median internal structure in the ventral arc; tibia 1: 8.6. In females from Afghanistan the epigynum is slightly longer (length/width: $0.51 \mathrm{vs} 0.45$ in females from Iran); they are also significantly larger (tibia 1 in 16 females: 8.3-11.2; mean: 9.9).

\section{Distribution}

Widely distributed in the Zagros Mountains of western and south-western Iran (Fig. 352). Specimens from Iraq and Afghanistan are assigned tentatively (see above).

Crossopriza semicaudata (O. Pickard-Cambridge, 1876)

Figs 353A, 574-607

Pholcus semicaudatus O. Pickard-Cambridge, 1876: 565.

Crossopriza semicaudata - Simon 1893: 477. — Wiehle 1933: 244, fig. 3b.

Holocnemus semicaudatus - Simon 1907: 5.

Probable misidentification (see Remarks below)

Crossopriza semicaudata - Denis 1945: 8, fig. 9.

\section{Remarks}

Pickard-Cambridge (1876) noted that this species was common in ruins near Cairo and Thebes (= Luxor) but did not specify the number of specimens actually available to him. The only specimen presumably originating from the type series I could locate is the lectotype below. The label says "lectotype" but this designation was never published; therefore, the lectotype is formally designated herein. The exact geographic origin of this particular specimen (Cairo or Luxor) is unknown.

Denis (1945) published a credible record from Egypt, Luxor, but I could not locate his specimens (they do not seem to be at the MNHN) and his only figure (of a palp) does not show C. semicaudata. Confusingly, this same figure appears again as figure 10 in Deeleman-Reinhold \& van Harten (2001), supposedly showing the palp of C. pristina (which is also wrong).

The specimens from Chad below are here thought to originate from Bardaï, even though the labels say only "massif du Tibesti". In 1965, the collector Max-Yves Brandily accompanied the ethnomusicologist Monique Brandily who made recordings in the Tibesti Mountains. According to the locality data attached to each recording (Centre de Recherche en Ethnomusicologie 2020) they worked in Bardaï in July and in October 1965.

\section{Diagnosis}

Easily distinguished from congeners by details of male palp (Figs 579-584; procursus with distinctive prolateral process, ventral sclerite with proximal ventral process; distal bulbal sclerite simple and flat, without ventral apophysis); also by male chelicerae (Figs 585-586; medially directed apophyses and enlarged hair bases) and female genitalia (Figs 587-593; epigynum short and wide as in C. pristina, but without distinct median internal structure and pore plates farther apart). 


\section{Type material}

Lectotype (designated herein)

EGYPT 1 1 0 , examined; Cairo or Luxor (see Remarks above); label data: "Pholcus (= Crossopriza) semicaudata OPC, Egypt, Lectotype $\widehat{O}$, loan 3846, B 53619 [or 536A?]”; Jan.-Apr. 1864; O. PickardCambridge leg.; HECO.

\section{Other material examined}

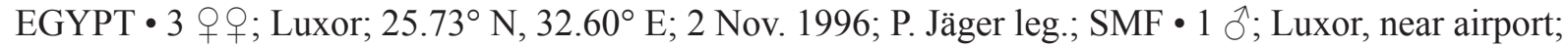
15 Nov. 1996; P. Jäger leg.; in house; SMF • $3{ }^{\widehat{\partial}} \partial^{\lambda}, 4$ + 9 , 1 juv.; $5 \mathrm{~km} \mathrm{~N}$ of Aswan; $24.13^{\circ} \mathrm{N}, 32.89^{\circ} \mathrm{E}$; 8 Jan. 1987; V. and B. Roth leg.; on sand dunes; CAS 9027139.

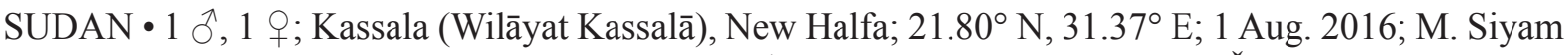
leg.; in house; ZFMK Ar 22430, Ar 22431 • 1 ô, 1 O; Northern (Wilāyat aš-Šamāliyya), Dongola;

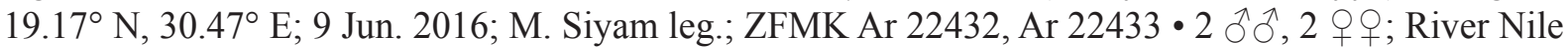
(Wilāyat Nahr an-Nīl), Atbara; $17.70^{\circ} \mathrm{N}, 34.00^{\circ} \mathrm{E}$; 29-31 Oct. 2016; M. Siyam leg.; in house; ZFMK Ar 22434 to Ar 22437 • 1 đ̊; Khartoum (Wilāyat Karțūm), Khartoum; $15.6^{\circ}$ N, 32.5 E; Nov. 1964; J.S. Cloudsely-Thompson leg.; MRAC $127505 \bullet 1$ \&; same collection data as for preceding; Jan.-Mar.

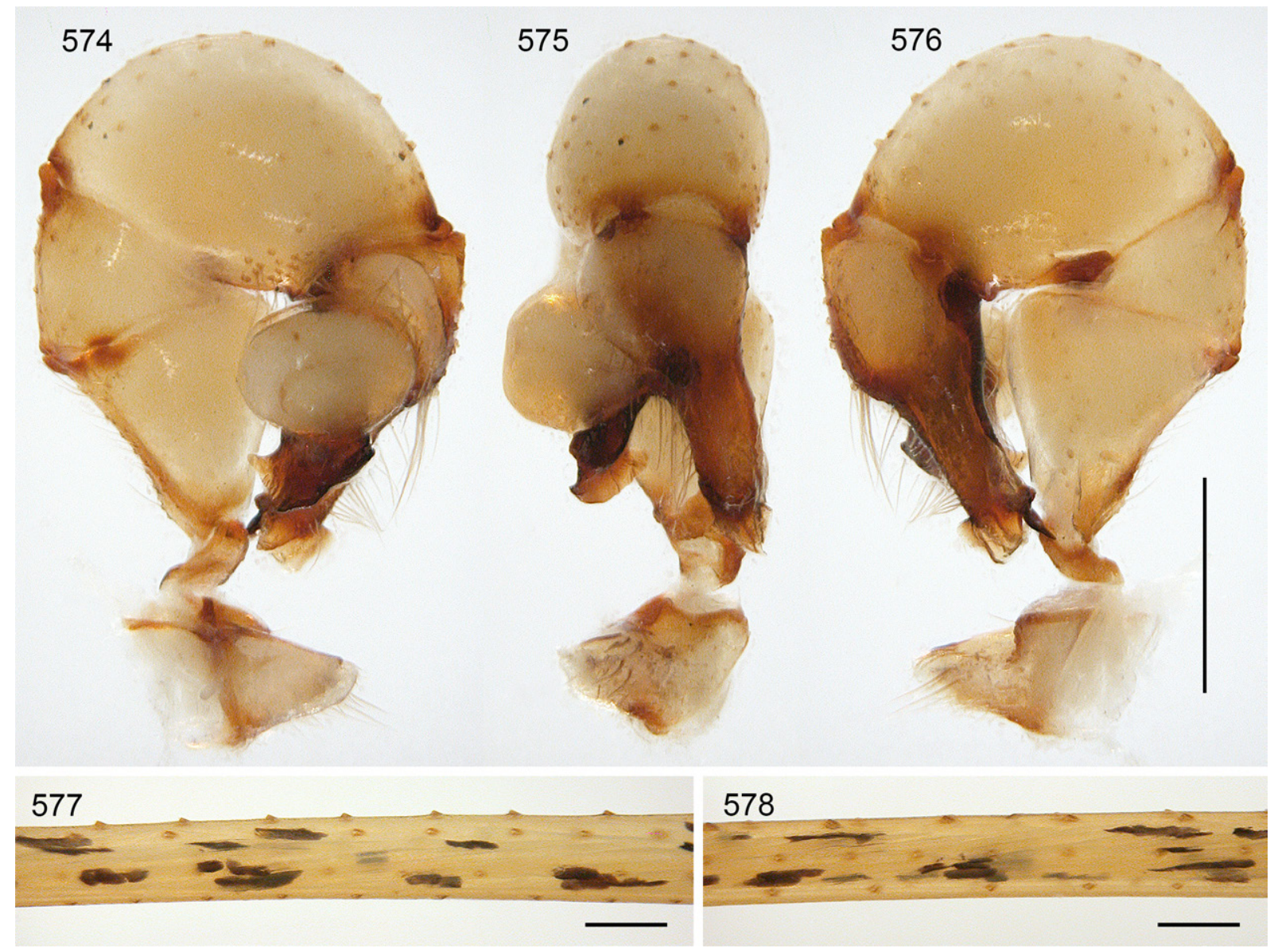

Figs 574-578. Crossopriza semicaudata (O. Pickard-Cambridge, 1876); male from Egypt, $5 \mathrm{~km} \mathrm{~N}$ of Aswan (CAS 9027139), and female from Egypt, Luxor (SMF). 574-576. Left male palp, prolateral, dorsal, and retrolateral views. 577-578. Female right femora 2 and 3, dorsal views. Scale bars: 574 $576=0.5 \mathrm{~mm} ; 577-578=0.2 \mathrm{~mm}$. 

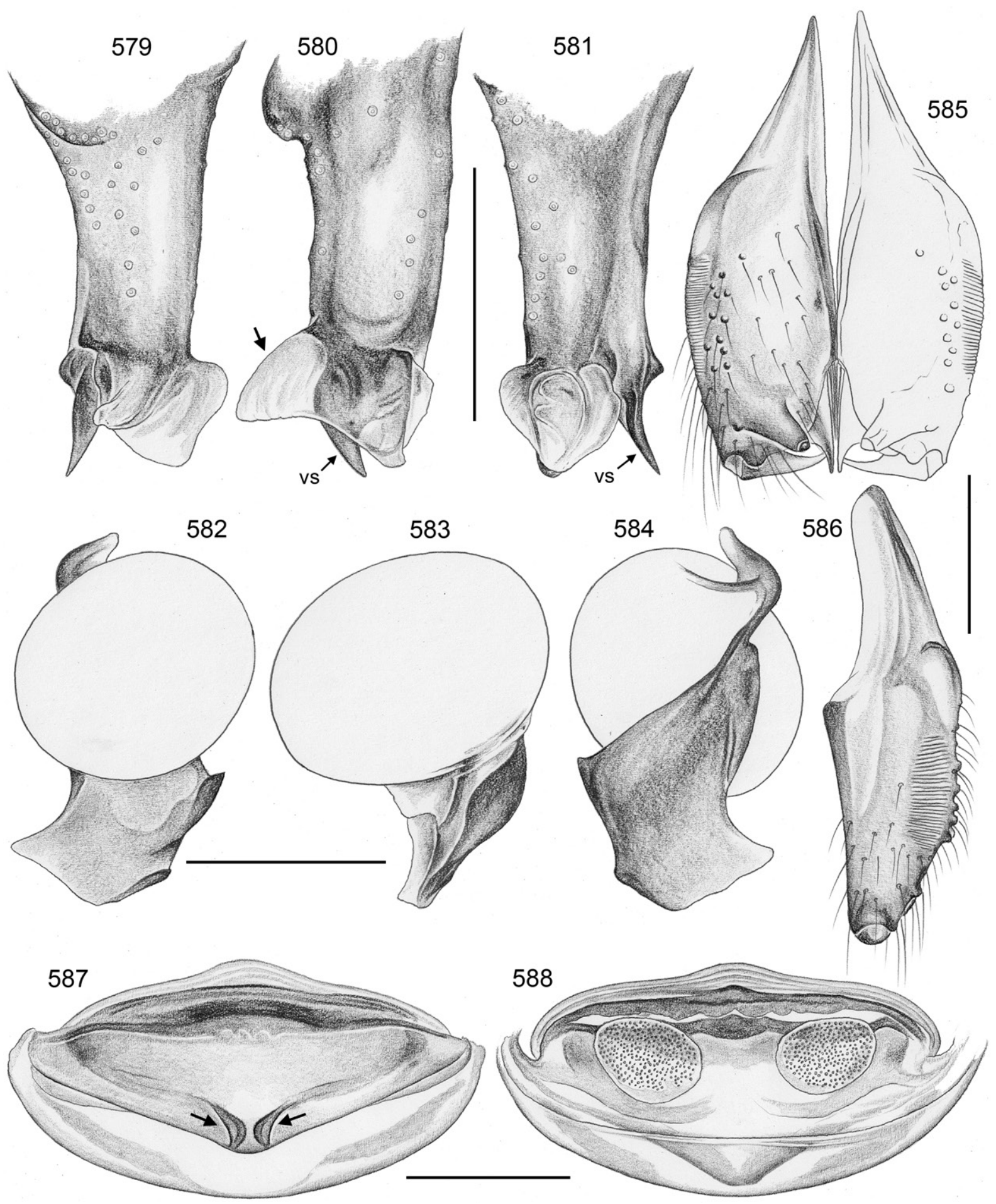

Figs 579-588. Crossopriza semicaudata (O. Pickard-Cambridge, 1876); male and female from Egypt, $5 \mathrm{~km} \mathrm{~N}$ of Aswan (CAS 9027139). 579-581. Left procursus, prolateral, dorsal, and retrolateral views; arrow: distinctive prolateral process. 582-584. Left genital bulb, prolateral, dorsal, and retrolateral views. 585-586. Male chelicerae, frontal and lateral views. 587-588. Cleared female genitalia, ventral and dorsal views; arrows: pockets. Abbreviation: vs $=$ ventral sclerite. Scale bars $=0.3 \mathrm{~mm}$. 
1962; MRAC 121719 • 1 đ’; Gezira (Wilāyat al-Jazīra), Hasaheisa; $14.73^{\circ}$ N, 33.35 E; 14 Apr. 2007; M. Siyam leg.; ZFMK Ar 22438.

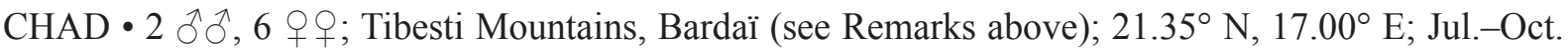

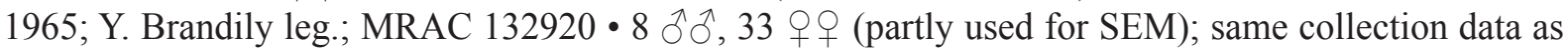
for preceding; MRAC 132959• 6 우, 16 juvs; same collection data as for preceding; MARC 132967.

\section{Redescription}

Male (Khartoum, MRAC 127505)

MEAsuREMENTs. Total length 3.4, carapace width 1.4. Distance PME-PME $80 \mu \mathrm{m}$; diameter PME $100 \times$ $120 \mu \mathrm{m}$; distance PME-ALE $30 \mu \mathrm{m}$; diameter AME $95 \mu \mathrm{m}$; distance AME-AME $30 \mu \mathrm{m}$. Leg 1: 35.7 $(10.3+0.6+9.4+13.3+2.1)$, tibia $2: 6.3$, tibia $3: 4.7$, tibia $4: 5.6$; tibia $1 \mathrm{~L} / \mathrm{d}: 59$; femora $1-4$ diameters: $0.20,0.18,0.17,0.17$.

CoLOR (in ethanol). Carapace ochre-yellow; carapace pit anteriorly light brown; sternum brown with darker radial marks; legs ochre-yellow, without darker rings, with black lines on femora and tibiae (cf. Figs 577-578); abdomen ochre-gray, with few indistinct dark marks dorsally; ventrally with black median band, partly disrupted.
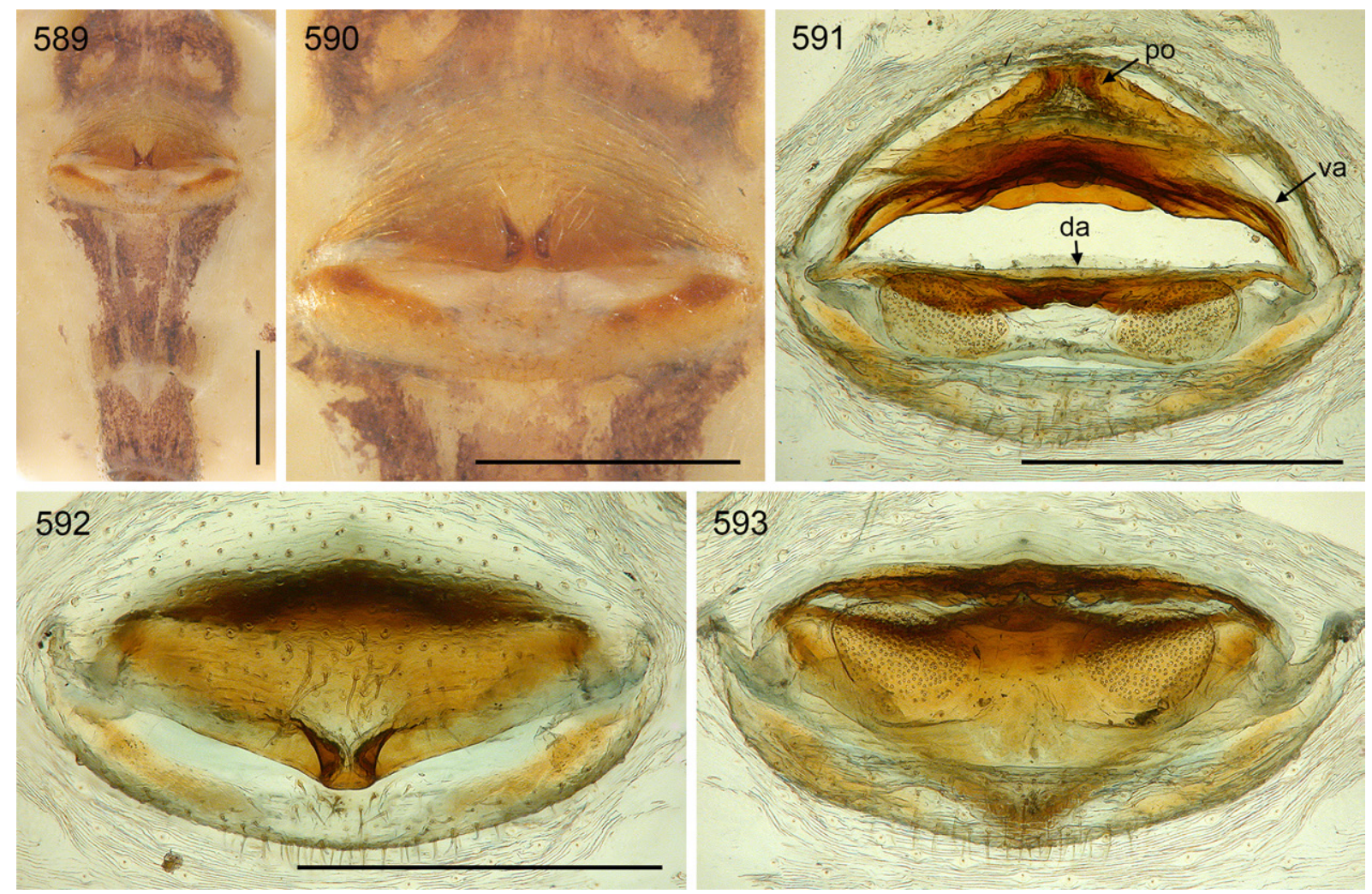

Figs 589-593. Crossopriza semicaudata (O. Pickard-Cambridge, 1876); female from Egypt, $5 \mathrm{~km} \mathrm{~N}$ of Aswan (CAS 9027139). 589-590. Abdomen and epigynum, ventral views. 591-593. Female internal genitalia, dorsal view (with dorsal arc tilted backwards and epigynal plate accidentally flipped forward), ventral view, and regular dorsal view. Abbreviations: $\mathrm{da}=$ dorsal arc; $\mathrm{po}=$ pocket; va = ventral arc. Scale bars $=0.5 \mathrm{~mm}$. 

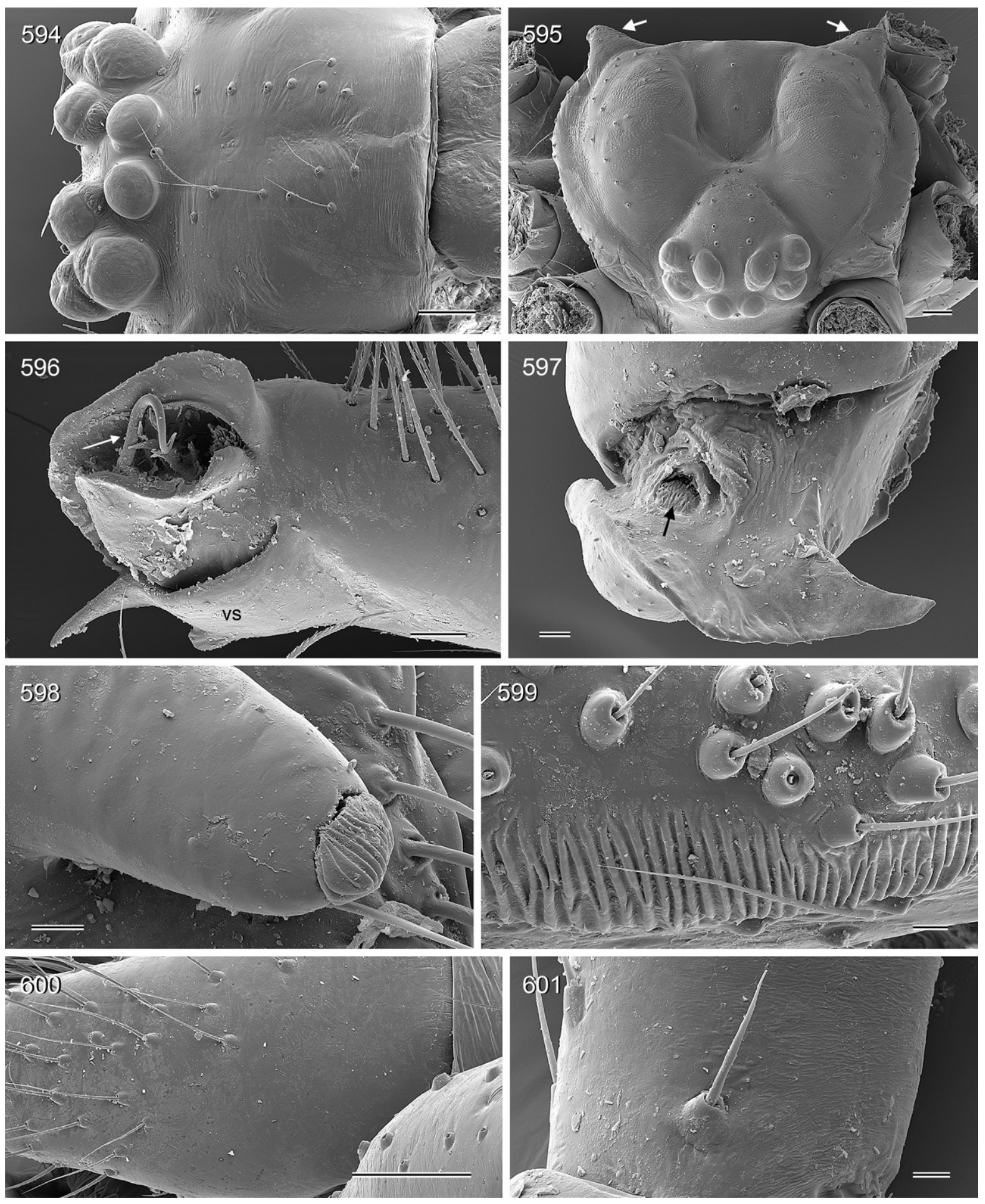

Figs 594-601. Crossopriza semicaudata (O. Pickard-Cambridge, 1876); male and female from Chad, Bardaï (MRAC 132959). 594. Male ocular area and clypeus. 595. Female ocular area and carapace; arrows: stridulatory processes. 596. Left procursus tip, retrolateral view; arrow: hair-like process. 597. Distal sclerite of right genital bulb, prolateral (slightly distal) view; arrow: sperm duct opening. 598. Tip of male cheliceral apophysis. 599-600. Male stridulatory file and corresponding smooth side of female chelicera. 601. Proximal prolateral hair on female palpal femur. Abbreviation: vs $=$ ventral sclerite. Scale bars: 594-595, $600=100 \mu \mathrm{m} ; 596=30 \mu \mathrm{m} ; 597=20 \mu \mathrm{m} ; 598-599,601=10 \mu \mathrm{m}$. 
Body. Habitus very similar to C. sahtan sp. nov. (cf. Fig. 391). Ocular area slightly raised. Deep thoracic pit and pair of furrows diverging from pit toward posterior margin. Clypeus unmodified, only rim more sclerotized than in female. Sternum wider than long (1.0/0.7), unmodified. Abdomen slightly elongated, dorso-posteriorly angular. Gonopore with four epiandrous spigots (Fig. 606); ALS with one widened spigot and one pointed spigot; PMS with two pointed spigots (Fig. 607).

Chelicerae. As in Figs 585-586, distally with pair of frontal apophyses provided with one large modified cone-shaped hair each (Fig. 598); distance between tips of modified hairs $90 \mu \mathrm{m}$; with $\sim 12$ enlarged hair-bases on each side (Fig. 599); lateral stridulatory ridges distinct (Fig. 599; distances between ridges $\sim 4.5 \mu \mathrm{m}$ ), clearly visible in dissecting microscope.
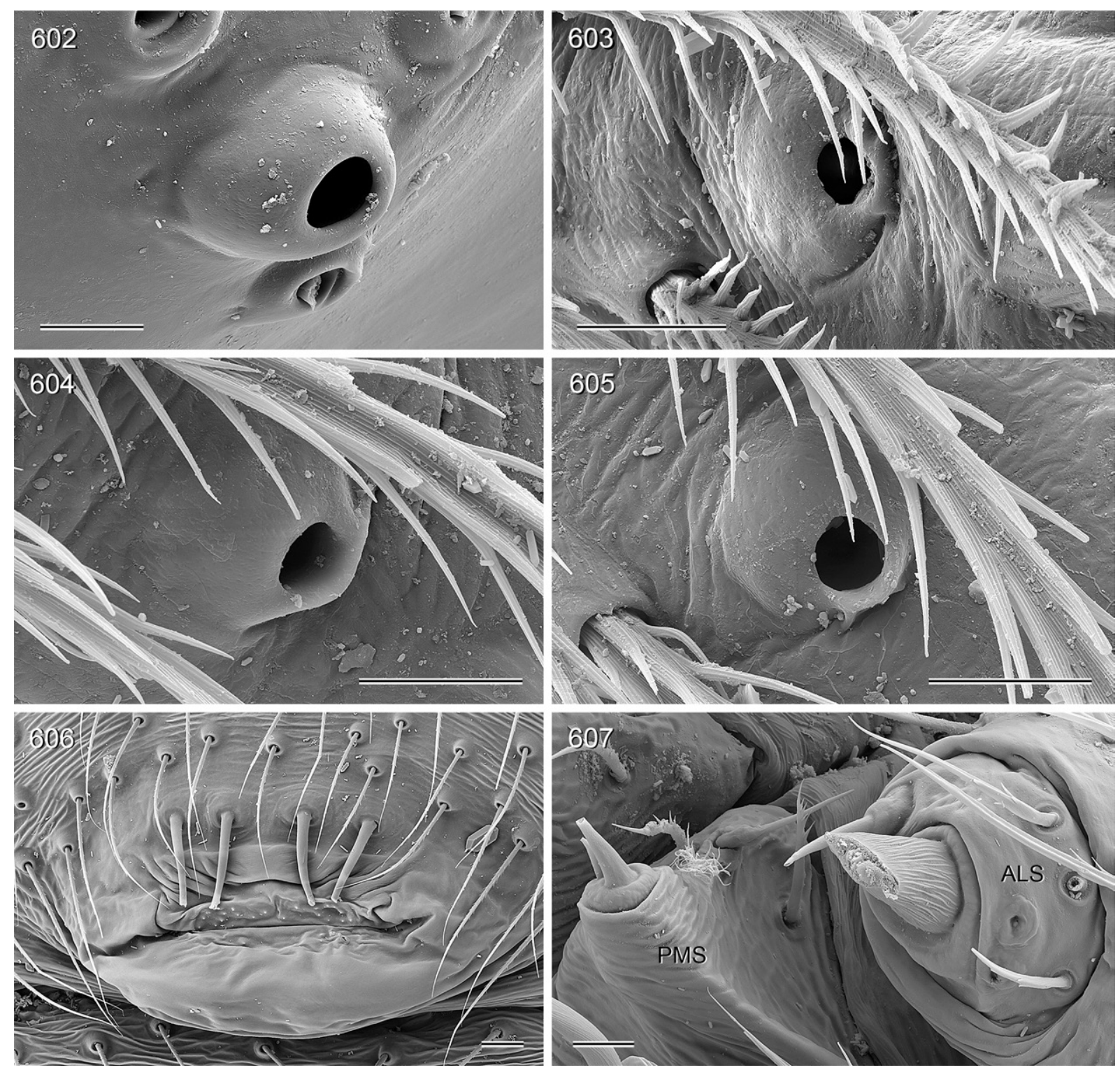

Figs 602-607. Crossopriza semicaudata (O. Pickard-Cambridge, 1876); male and female from Chad, Bardaï (MRAC 132959). 602. Male palpal tarsal organ. 603-605. Tarsal organs on female tarsi 1, 2, and 4. 606. Male gonopore. 607. Male spinnerets. Scale bars: 602-605, $607=10 \mu \mathrm{m} ; 606=20 \mu \mathrm{m}$. 
PALPS. As in Figs 574-576; coxa with low retrolateral hump; trochanter barely modified; femur distally strongly widened, with rounded ventral protrusion, proximally with prolateral stridulatory pick, with very indistinct retrolateral transversal line, without retrolateral proximal process; femur-patella joints close together, barely shifted toward prolateral side; tibia-tarsus joints shifted toward retrolateral side; tarsus without macrotrichia; tarsal organ capsulate (Fig. 602); procursus (Figs 579-581) short and straight, proximally on prolateral side with strong hump set with numerous long hairs, dorsal hairs mostly straight, some weakly curved, procursus tip with ventral sclerite with proximal ventral process, distinctive prolateral process, and semitransparent retrolateral-dorsal flap; with hair-like process on retrolateral side (arrow in Fig. 596); genital bulb (Figs 582-584) with simple basal sclerite connected to distal (main) sclerite, sperm duct opening on prolateral side (arrow in Fig. 597); distal sclerite simple and flat, with semitransparent ventral process, without retrolateral ridge, without prolateral apophysis or ridge.

LEGS. Femur 1 with single row of $\sim 25$ ventral spines; without curved hairs; few vertical hairs; retrolateral trichobothrium of tibia 1 at 4\%; prolateral trichobothrium absent on tibia 1, present on other leg tibiae; tarsal pseudosegments very indistinct, irregular except 1-2 at tip.

Male (variation)

Tibia 1 in 16 males (incl. lectotype): 8.3-12.1 (mean 10.0); lectotype smaller (carapace width 1.25), with shortest tibia (8.3) and fewer spines on femur $1(\sim 18)$. More recently collected specimens with distinct pattern of dark and whitish marks dorsally on abdomen (similar C. sahtan sp. nov., cf. Fig. 391); ventral pattern behind gonopore variably distinct, with 2-4 longitudinal bands often not clearly separate.

\section{Female}

In general similar to male but without spines on legs, without stridulatory files on chelicerae (Fig. 600), and with stridulatory organ consisting of pair of weakly sclerotized but distinct processes posteriorly on carapace (arrows in Fig. 595) and pair of small light brown plates anteriorly on abdomen. Tibia 1 in 46 females: 7.0-10.2 (mean 8.8). Epigynum as in Figs 589-590; main epigynal plate semicircular, medially protruding; with pair of large pockets close to median line (distance between pockets $40 \mu \mathrm{m}$ ); internal sclerotized arc variably visible in uncleared specimens; posterior plate relatively large but mostly weakly sclerotized except frontally laterally. Internal genitalia (Figs 587-588, 591-593) with oval pore plates, dorsal arc weakly sclerotized laterally, ventral arc medially barely modified.

\section{Natural history}

Several specimens were collected in houses, suggesting that this is at least partly a synanthropic species. At Dongola, spiders were collected from webs among palm trees (M. Siyam, pers. com., Apr. 2021).

\section{Distribution}

Widespread in NW Africa (Egypt, Chad, Sudan) (Fig. 353A).

\section{Crossopriza pristina (Simon, 1890)}

Figs 353A, 608-624

Artema pristina Simon, 1890: 93.

Pholcus rivulatus (misidentification) - L. Koch 1875: 25 (only specimens from Massaua, see Remarks below).

Crossopriza pristina - Simon 1893: 475, fig. 465. — Timm 1976: 73, figs 5-6 (see Remarks below). Pérez González 1996: 432. - Siyam et al. 2015: 265, figs 2-6.

Crossoprisa [sic] pristina - Wiehle 1933: 244, fig. 3a. 


\section{Misidentifications}

Crossopriza pristina - Franganillo 1925: 33 (see H. hispanicus); 1926a: 49 (see C. lyoni); 1926b: 11 (see C. lyoni); 1926c: 70 (see H. hispanicus); 1936a: 46 (see C. lyoni); 1936b: 77 (see C. lyoni). — Deeleman-Reinhold \& van Harten 2001: 195, figs 1-4, 7-10 (see C. sanaa sp. nov. and C. manakhah sp. nov.).

\section{Remarks}

L. Koch (1875) assigned two juvenile specimens from Massaua (Massawa) to "Pholcus rivulatus", a name that was later synonymized with Holocnemus pluchei. Photos of these specimens (deposited in Museo Civico di Storia Naturale Giacomo Doria, Genova, Italy) were kindly provided by M. Tavano (Jan. 2014) and the relatively short and posteriorly angular abdomen strongly suggests that this is C. pristina rather than $H$. pluchei.

Timm (1976) illustrated the male palp without providing any data about the origin of the specimens. It seems likely that he used the specimens that L. Fage loaned to H. Wiehle in the 1930s (Wiehle 1933: 243 , footnote) and that are now deposited in SMF. The labels with the SMF specimens do not specify the origin of the spiders. Considering the very large series of $C$. pristina from Massaua in MNHN, it seems likely that L. Fage took the specimens from that series rather than from the smaller type series from Aden. The SMF specimens below are thus thought to originate from Massaua.

The locality "Didi Davvs" (also published for Artema kochi in Aharon et al. 2014) is here thought to be a misspelling of Dire Dawa in Ethiopia. The collector, Barnum Brown, accompanied the so-called Dudley Expedition, which in 1920 conducted geologic surveys in Harrar Province between Harar and Jijigga. Brown was in Aden on June 16, 1920, and reached Addis Ababa on July 1, 1920 (Peter Purcell, pers. com., Dec. 2020). It is thus very plausible that he was in Dire Dawa on the days specified on the label (June 20-26).

\section{Diagnosis}

Easily distinguished from known congeners by details of male palp (Figs 617-618; short and thick procursus with short ventral sclerite and distinctive retrolateral process; distinctive distal bulbal sclerite), male chelicerae (Figs 621-622; low sclerotized humps near median line), and female genitalia (Figs 612, 623-624; short but wide epigynum as in $S$. semicaudata but with large median internal structure and pore plates closer together).

\section{Type material}

\section{Syntypes}

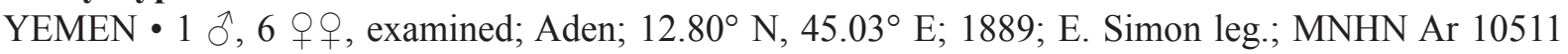
(E. Simon collection number 10764).

\section{Other material examined}

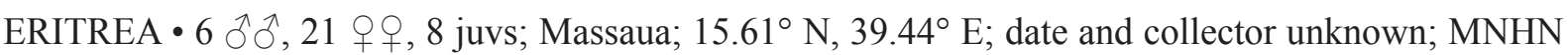

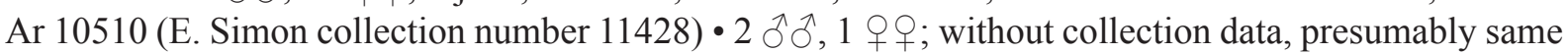
collection data as for preceding (i.e., taken from MNHN Ar 10510 and loaned by L. Fage to H. Wiehle); SMF 19429/1, 19433/3 • 1 ô, 1 क; Massaua; date unknown; L. Vincentini leg.; MRAC 131273, 131274.

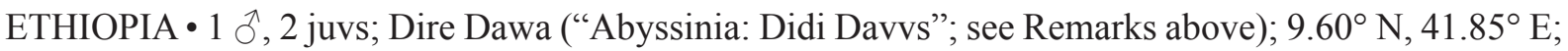
1200 m a.s.1.; 20 and 26 Jun. 1920; B. Brown leg.; AMNH. 
SUDAN • 1 đ̆, 1 क; Kassala State, New Halfa; $15.317^{\circ}$ N, $35.600^{\circ}$ E (coordinates in Siyam et al. 2015 wrong); 26 Jun. 2013; M. Siyam leg.; ZMB • 1 ภ, 1 क (abdomen), 1 juv. (prosoma) (in pure ethanol); same locality as for preceding; 10 Aug. 2014; M. Siyam leg.; ZFMK Siy4, Siy5.

Credible published records (not examined)

SUDAN • 1 đ, 1 क; Kassala, New Halfa; 26 Jun. 2013; M. Siyam leg.; Siyam et al. 2015; ZMB 48672 • 1 ô, 1 क; same locality as for preceding; 10 Aug. 2014; M. Siyam leg.; Siyam et al. 2015; ZMB 48674 - 1 q; Red Sea State, Tokar (Towkar); $18.452^{\circ}$ N, 37.727 ${ }^{\circ}$ E (coordinates in Siyam et al. 2015 wrong); 9 Oct. 2011; Siyam et al. 2015; ZMB 48671.

\section{Redescription}

Male (Massaua, MNHN Ar 10510)

MEASUREMEnTs. Total length 4.5, carapace width 1.6. Distance PME-PME $90 \mu \mathrm{m}$; diameter PME $120 \times$ $140 \mu \mathrm{m}$; distance PME-ALE $35 \mu \mathrm{m}$; diameter AME $105 \mu \mathrm{m}$; distance AME-AME $40 \mu \mathrm{m}$. Leg 1: 49.1 $(13.5+0.7+12.7+19.3+2.9)$, tibia $2: 8.5$, tibia $3: 6.1$, tibia $4: 6.9$; tibia $1 \mathrm{~L} / \mathrm{d}: 67$; femora $1-4$ diameters: $0.28,0.23,0.23,0.23$.

COLOR (in ethanol). Bleached specimen mostly pale ochre-yellow; carapace pit anteriorly light brown; sternum posteriorly slightly darkened, with radial marks; legs ochre-yellow, without darker rings, black lines on femora and tibiae barely visible; abdomen ochre-gray, with few indistinct dark marks dorsally; ventrally with distinct black median band, partly disrupted, with three parallel longitudinal marks behind gonopore.

Body. Habitus very similar to C. sahtan sp. nov. (cf. Fig. 391). Ocular area slightly raised. Deep thoracic pit and pair of furrows diverging from pit toward posterior margin. Clypeus unmodified, only rim more sclerotized than in female. Sternum wider than long (1.15/0.75), unmodified. Abdomen slightly elongated, dorso-posteriorly angular.

Chelicerae. As in Figs 621-622, proximally with low sclerotized humps near median line, distally with pair of frontal apophyses provided with one large modified cone-shaped hair each; distance between tips of modified hairs: $50 \mu \mathrm{m}$; lateral stridulatory ridges fine but visible in dissecting microscope.

PALPS. As in Figs 608-610; coxa barely modified, with low retrolateral hump; trochanter barely modified; femur short, distally strongly widened, with rounded ventral protrusion, proximally with prolateral stridulatory pick, retrolateral-ventral rim with row of sclerotized hair-bases, with indistinct retrolateral transversal line, without retrolateral proximal process; femur-patella joints very close together, not shifted toward prolateral side; tibia very large relative to femur, tibia-tarsus joints slightly shifted toward retrolateral side; tarsus without macrotrichia; procursus (Figs 616-617) short and straight, proximally on prolateral side with strong hump set with numerous long hairs, dorsal hairs not curved, procursus tip with short ventral sclerite and distinctive dorsal and retrolateral processes; genital bulb (Figs 618-620) with simple basal sclerite connected to distal (main) sclerite, sperm duct opening on prolateral-dorsal side at basis of distal sclerite (arrow in Fig. 619); distal sclerite with distinctive ventral process and prolateral ridge.

LEGS. Femur 1 with single row of $\sim 30$ ventral spines; without curved hairs; few vertical hairs; retrolateral trichobothrium of tibia 1 at 4\%; prolateral trichobothrium absent on tibia 1, present on other leg tibiae; tarsal pseudosegments not seen.

Male (variation)

Tibia 1 in 11 males: 11.6-13.6 (mean 12.7). Specimens from Sudan (collected between 2013 and 2015) with distinct pattern of dark and whitish marks on abdomen (similar C. sahtan sp. nov., cf. Fig. 391), 


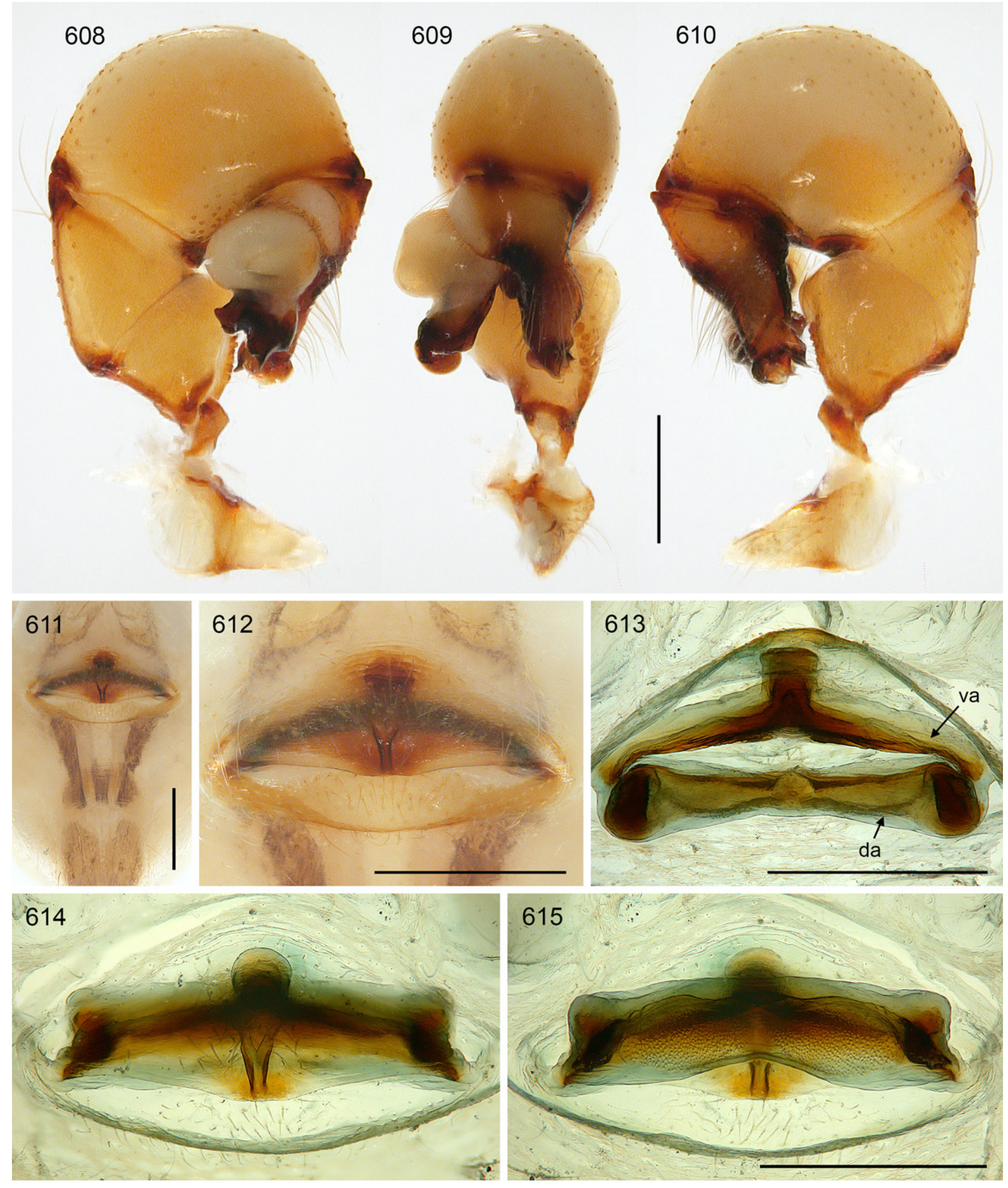

Figs 608-615. Crossopriza pristina (Simon, 1890); male from Eritrea, Massaua (MNHN Ar 10510), syntype female from Yemen, Aden (MNHN Ar 10511). 608-610. Left male palp, prolateral, dorsal, and retrolateral views. 611-612. Female abdomen and epigynum, ventral views. 613-615. Female internal genitalia, dorsal view (with dorsal arc tilted backwards), ventral view, and regular dorsal view. Abbreviations: $\mathrm{da}=$ dorsal arc; $\mathrm{va}=$ ventral arc. Scale bars $=0.5 \mathrm{~mm}$. 


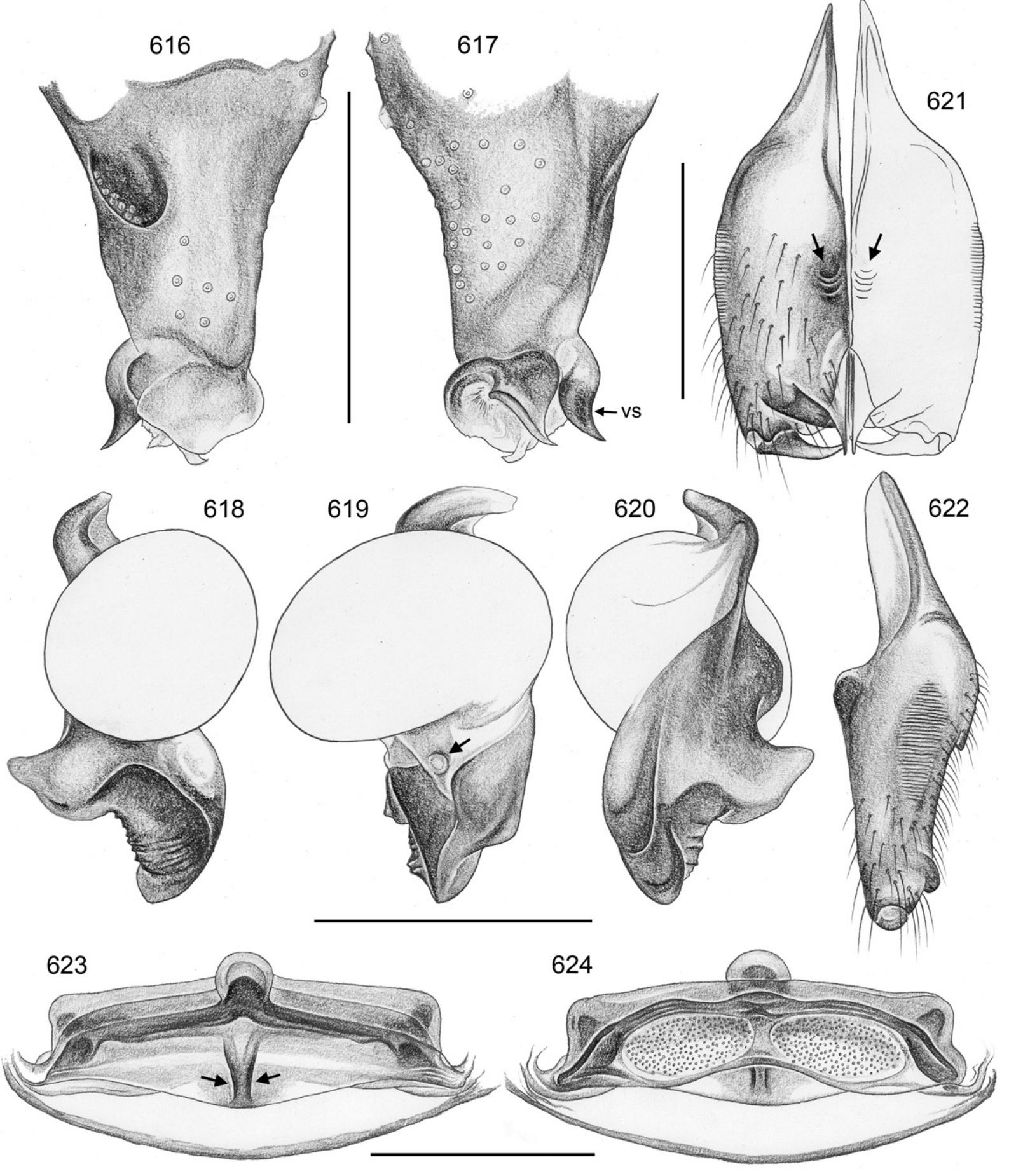

Figs 616-624. Crossopriza pristina (Simon, 1890); male from Eritrea, Massaua (MNHN Ar 10510), syntype female from Yemen, Aden (MNHN Ar 10511). 616-617. Left procursus, prolateral and retrolateral views. 618-620. Left genital bulb, prolateral, dorsal, and retrolateral views; arrow: sperm duct opening. 621-622. Male chelicerae, frontal and lateral views; arrows: low sclerotized humps. 623624. Cleared female genitalia, ventral and dorsal views; arrows: pockets. Abbreviation: vs = ventral sclerite. Scale bars $=0.5 \mathrm{~mm}$. 
with distinct black lines on femora and tibiae, and with dark brown sternum. All other specimens are much older (100 years and more) and probably artificially pale.

\section{Female}

In general similar to male but without spines on legs, apparently without stridulatory files on chelicerae, and with stridulatory organ consisting of pair of weakly sclerotized but distinct processes posteriorly on carapace and pair of indistinct light brown or non-darkened plates anteriorly on abdomen. Tibia 1 in 26 females: 10.0-13.6 (mean 11.9). Epigynum as in Figs 611-612, main epigynal plate short but wide, weakly protruding; with pair of shallow pockets very close to median line on both sides of median ridge (distance between pockets $40 \mu \mathrm{m}$ ); internal sclerotized arc and median round structure visible in uncleared specimens; posterior plate indistinct, short but wide. Internal genitalia (Figs 613-615, 623624) with large oval pore plates in transversal position and close together, dorsal arc strongly sclerotized laterally, ventral arc with median pouch or pocket of unknown function.

\section{Natural history}

At the type locality (Aden), Simon (1890) collected this species in houses, together with "Artema mauricia" (=A. atlanta Walckenaer, 1837).

\section{Distribution}

Known from NW Africa (Sudan, Eritrea, Ethiopia) and the Arabian Peninsula (Yemen) (Fig. 353A).

Crossopriza manakhah sp. nov. urn:1sid:zoobank.org:act:96A574A1-6DEA-42AF-9AF0-F88916EF8A04

Figs 353B, 625-642

Crossopriza pristina (misidentification) - Deeleman-Reinhold \& van Harten 2001: 195 (part; see Remark under C. sanaa sp. nov.).

\section{Diagnosis}

Easily distinguished from known congeners by details of male palp (Figs 628-633; procursus with distinctive prolateral process; distal sclerite of genital bulb with two simple pointed processes); also by male chelicerae (Figs 634-635; pair of very strong medially-directed apophyses originating from dark sclerotized U-shaped area) and female genitalia (Fig. 639; epigynum triangular, with strong median ridge).

\section{Etymology}

The species name refers to the type locality; noun in apposition.

\section{Type material}

\section{Holotype}

YEMEN • ${ }^{\top} ; 12 \mathrm{~km}$ NW of Manakhah, “1521”; $15.12^{\circ}$ N, $43.68^{\circ}$ E; 28 Aug. 2001; A. van Harten leg.; ZFMK Ar 22439.

\section{Other material examined}

YEMEN • 1 \% ; same collection data as for holotype; ZFMK Ar 22440 • 1 o; same locality as for holotype, "1742"; 27 Mar.-5 May 2002; A. van Harten leg.; ZFMK Ar 22441 • 1 क; "Manakhah x Khamis Bani Sa'd", "1428”; $15.12^{\circ}$ N, $43.63^{\circ}$ E; 3 Jul. 2001; A. van Harten leg.; ZFMK Ar 22442 • 1 ㅇ; Khamis Bani Sa'd, "788”; $15.18^{\circ}$ N, 43.51 ${ }^{\circ}$ E; 11 Oct. 1999; A. van Harten leg.; NMNL • 1 ; same locality as for preceding, "760"; 27 Jul. 1999; A. van Harten leg.; NMNL・ 1 ภ, 1 क; locality uncertain (Khamis Bani Sa'd area?), “960”; 1999; A. van Harten leg.; NMNL ARA 15212 • 1 ठૈ; near Sana'a,

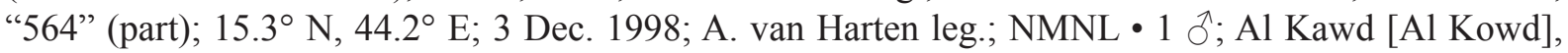

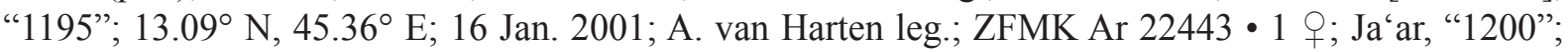


13.22 ${ }^{\circ}$ N, 45.30 E; 15 Jan. 2001; A. van Harten leg.; ZFMK Ar 22444 • 3 qo, “Aden x Little Aden”, “461”; $12.75^{\circ}$ N, $44.86^{\circ}$ E; 24 Mar. 1998; A. van Harten leg.; NMNL.

\section{Remark}

The coordinates of Khamis Bani Sa'd in Deeleman-Reinhold \& van Harten (2001) are approximately $10 \mathrm{~km} \mathrm{~W}$ of Khamis Bani Sa'd (coordinates above). The actual collecting point is not clear.

\section{Description}

\section{Male (holotype)}

Measurements. Total length 3.4, carapace width 1.3. Distance PME-PME $75 \mu \mathrm{m}$; diameter PME $90 \times$ $105 \mu \mathrm{m}$; distance PME-ALE $30 \mu \mathrm{m}$; diameter AME $90 \mu \mathrm{m}$; distance AME-AME $30 \mu \mathrm{m}$. Leg 1: 33.2 $(9.3+0.6+8.7+12.4+2.2)$, tibia $2: 5.6$, tibia $3: 4.0$, tibia 4 missing; tibia $1 \mathrm{~L} / \mathrm{d}: 70$; femora $1-3$ diameters: $0.21,0.18,0.18$.

COLOR (in ethanol). Carapace ochre-yellow, median pit anteriorly light brown; sternum brown, with black radial marks; legs ochre-yellow, without darker rings, with distinct black lines on femora and tibiae; abdomen ochre-gray, with distinct dark and white marks dorsally and laterally; ventrally with distinct black median band, partly disrupted, with two parallel longitudinal marks behind gonopore.

Body. Habitus similar to C. sahtan sp. nov. (cf. Fig. 391). Ocular area slightly raised. Deep thoracic pit and pair of furrows diverging from pit toward posterior margin. Clypeus unmodified, only rim slightly more sclerotized than in female. Sternum wider than long (0.9/0.5), unmodified. Abdomen oval, dorsoposteriorly angular.

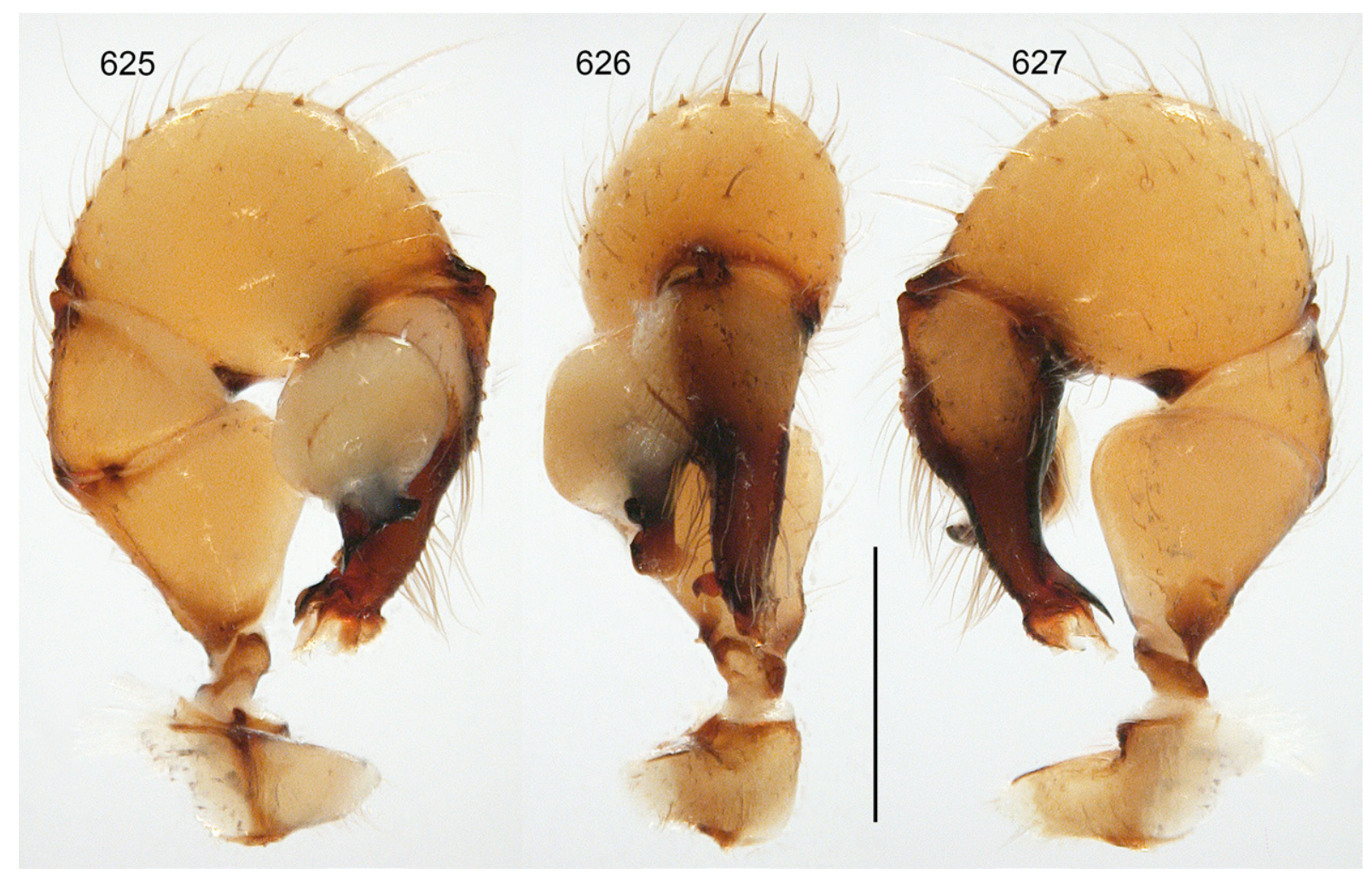

Figs 625-627. Crossopriza manakhah sp. nov.; male from Yemen, $12 \mathrm{~km}$ NW of Manakhah (ZFMK Ar 22441); left palp, prolateral, dorsal, and retrolateral views. Scale bar $=0.5 \mathrm{~mm}$. 


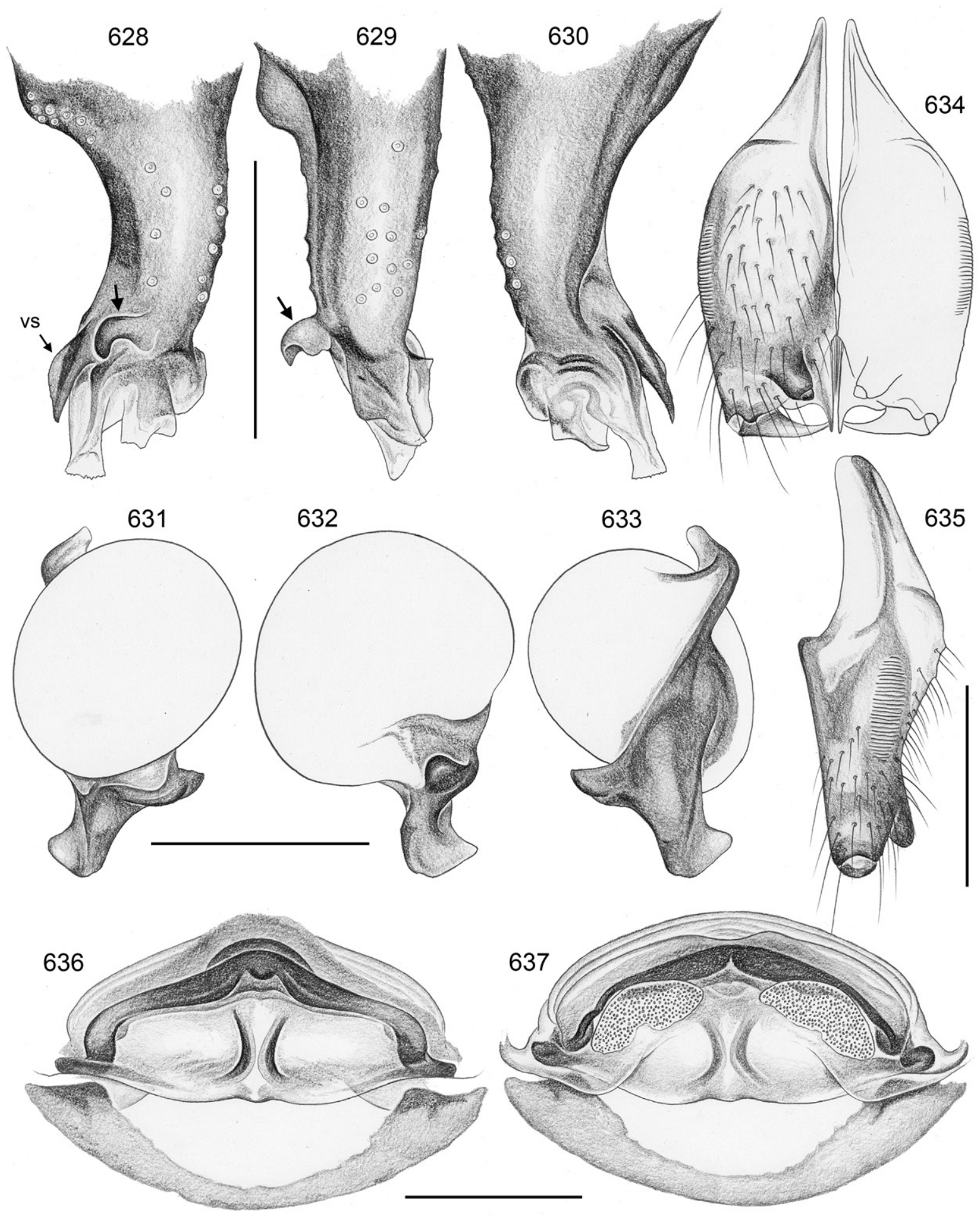

Figs 628-637. Crossopriza manakhah sp. nov.; male from Yemen, $12 \mathrm{~km}$ NW of Manakhah (ZFMK Ar 22441), female from Yemen, Ja'ar (ZFMK Ar 22444). 628-630. Left procursus, prolateral, dorsal, and retrolateral views; arrows: distinctive prolateral process. 631-633. Left genital bulb, prolateral, dorsal, and retrolateral views. 634-635. Male chelicerae, frontal and lateral views. 636-637. Cleared female genitalia, ventral and dorsal views. Abbreviation: vs $=$ ventral sclerite. Scale bars $=0.3 \mathrm{~mm}$. 
Chelicerae. As in Figs 634-635, with pair of strong frontal apophyses provided with one large modified cone-shaped hair each; distance between tips of modified hairs $60 \mu \mathrm{m}$; proximally partly whitish and slightly protruding; lateral stridulatory ridges fine but visible in dissecting microscope.

PALPS. As in Figs 625-627; coxa with rounded retrolateral hump; trochanter barely modified; femur distally strongly widened, with rounded ventral protrusion, proximally with prolateral stridulatory pick, with retrolateral transversal line, without retrolateral proximal process; femur-patella joints slightly shifted toward prolateral side; tibia-tarsus joints shifted toward retrolateral side; tarsus without macrotrichia; procursus (Figs 628-630) slightly curved towards ventral, proximally on prolateral side with strong hump set with numerous long hairs, dorsal hairs not curved, procursus tip with short ventral sclerite, distinctive prolateral process, and further membranous processes; genital bulb (Figs 631-633) with simple basal sclerite connected to distal (main) sclerite, sperm duct opening not seen; distal sclerite with two simple processes.

LEGS. Femur 1 with single row of $\sim 18$ ventral spines; without curved hairs; few vertical hairs; retrolateral trichobothrium of tibia 1 at $3.5 \%$; prolateral trichobothrium absent on tibia 1, present on other leg tibiae; tarsal pseudosegments not seen.

Male (variation)

Tibia 1 in three other males: 6.1, 7.7, 8.6. The only male from the three southern localities (from Al Kawd) with slightly different procursus tip: long transparent process shorter and prolateral process slightly less curved.
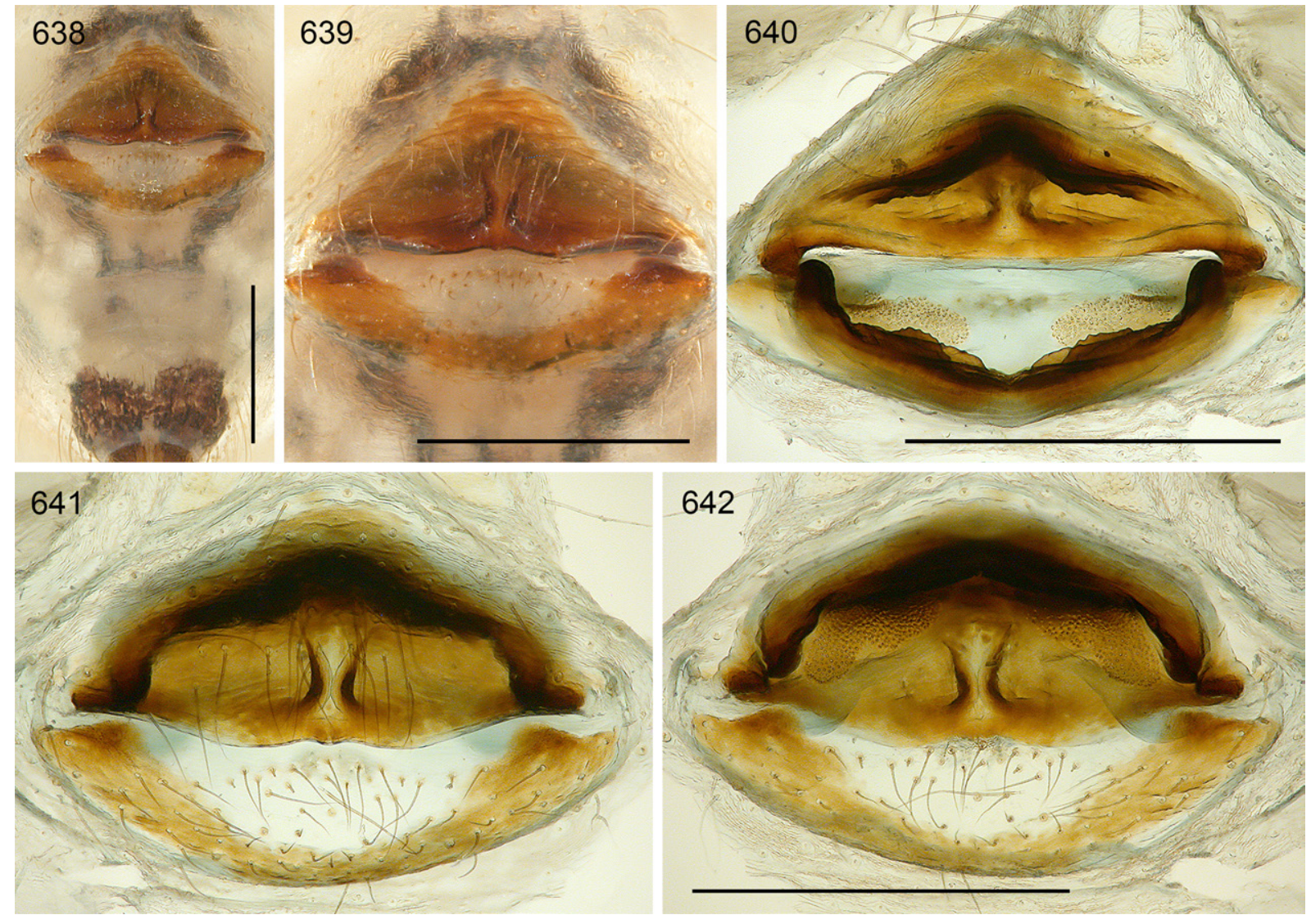

Figs 638-642. Crossopriza manakhah sp. nov.; female from Yemen, Ja'ar (ZFMK Ar 22444). 638639. Abdomen and epigynum, ventral views. 640-642. Female internal genitalia, dorsal view (with dorsal arc tilted backwards), ventral view, and regular dorsal view. Scale bars $=0.5 \mathrm{~mm}$. 
Female

In general similar to male but without spines on legs, apparently without stridulatory files on chelicerae, and with stridulatory organ consisting of pair of weakly sclerotized but distinct processes posteriorly on carapace and pair of barely visible plates anteriorly on abdomen. Tibia 1 in seven females: 6.3-7.0 (mean 6.7). Epigynum as in Figs 638-639, main epigynal plate roughly triangular, weakly protruding; with pair of shallow pockets close to median line on both sides of wide median ridge (distance between pockets $30 \mu \mathrm{m}$ ); internal sclerotized arc poorly visible in uncleared specimens; posterior plate large, widened laterally. Internal genitalia (Figs 636-637, 640-642) with relatively small crescent-shaped pore plates, dorsal arc strongly sclerotized, ventral arc only medially strongly sclerotized, apparently without median pouch or pocket.

\section{Distribution}

Known from several localities in western Yemen (Fig. 353B).

\section{Crossopriza sanaa sp. nov.}

urn:1sid:zoobank.org:act:BD71FF3D-A3B9-4830-ACCC-A1DB61C7BAD6

Figs 353A, 643-663

Crossopriza pristina (misidentification) - Deeleman-Reinhold \& van Harten 2001: 195 (part; see Remark below), figs 1-4, 7-9.

\section{Remark}

The redescription of C. pristina in Deeleman-Reinhold \& van Harten (2001) is based largely on specimens from Sana'a that are here identified as C. sanaa sp. nov. However, in their "Studied material" section

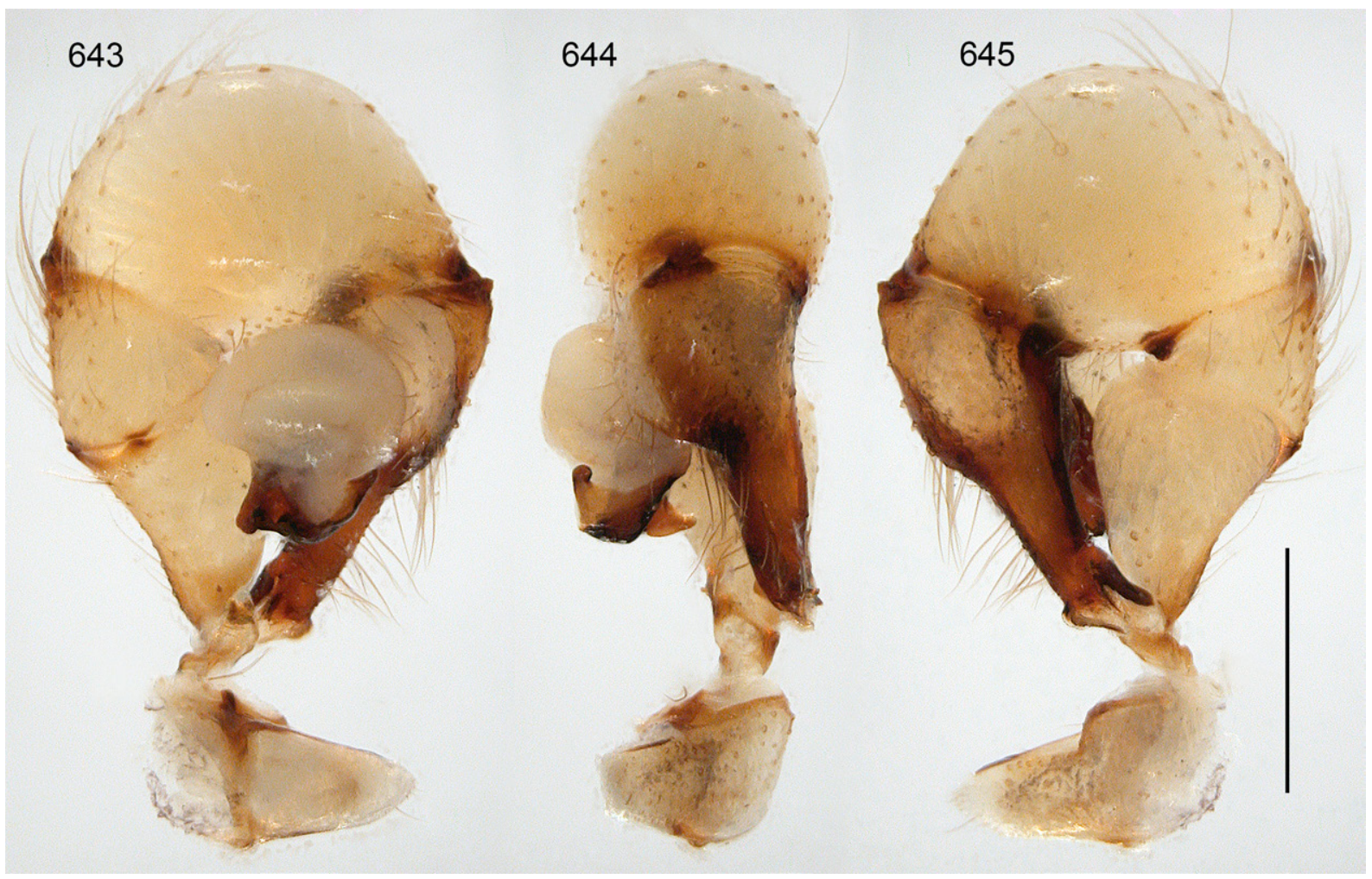

Figs 643-645. Crossopriza sanaa sp. nov.; non-type male from Yemen, near Sana'a (NMNL); left palp, prolateral, dorsal, and retrolateral views. Scale bar $=0.5 \mathrm{~mm}$. 


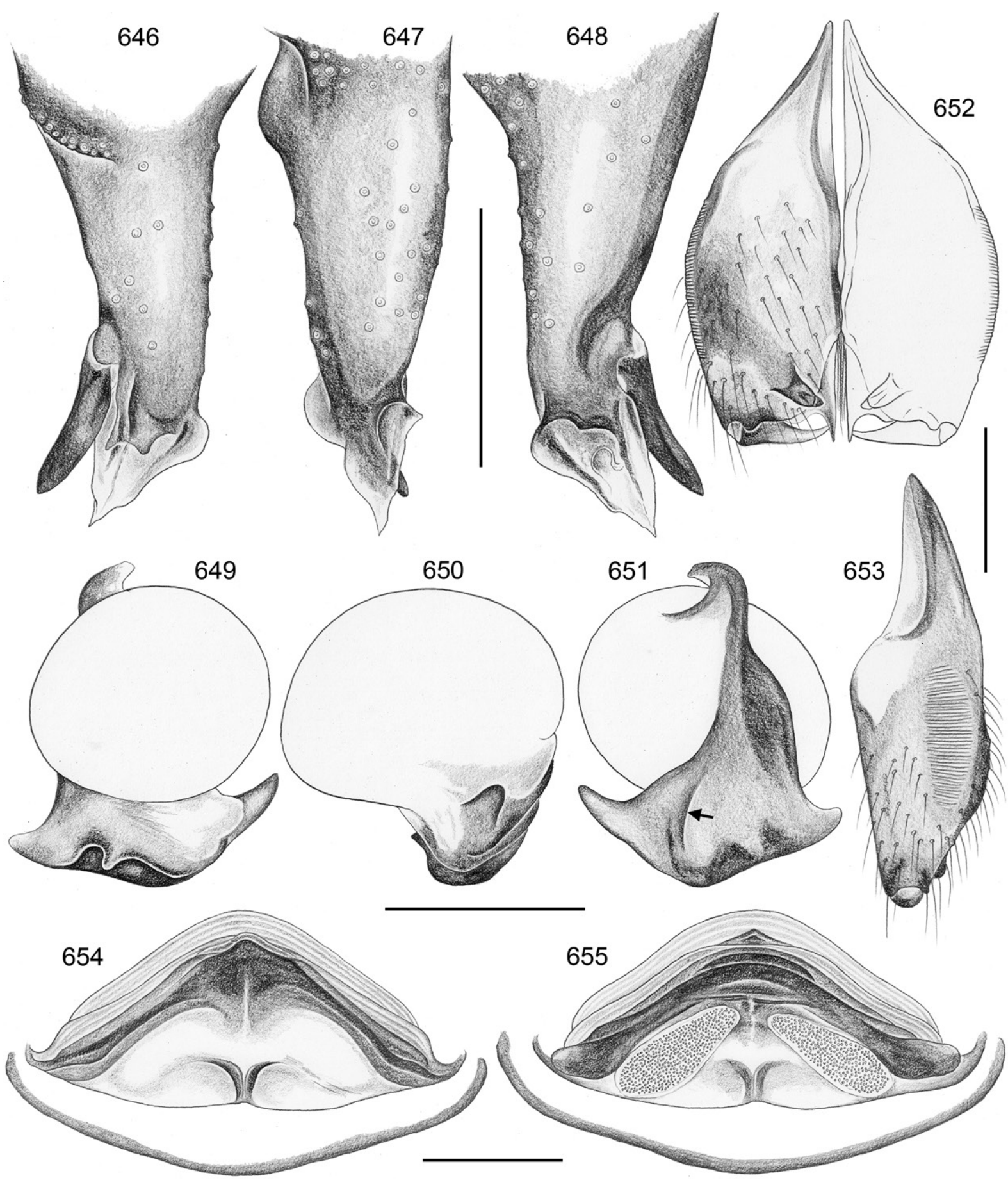

Figs 646-655. Crossopriza sanaa sp. nov.; holotype male from Yemen, Sana'a (646-651; NMNL ARA 15023), non-type male from near Sana'a (652-653; NMNL, unnumbered), and female from Sana'a (654-655; NMNL ARA 15215). 646-648. Left procursus, prolateral, dorsal, and retrolateral views. 649-651. Left genital bulb, prolateral, dorsal, and retrolateral views; arrow: retrolateral ridge. 652653. Male chelicerae, frontal and lateral views. 654-655. Cleared female genitalia, ventral and dorsal views. Scale bars $=0.3 \mathrm{~mm}$. 
they also list specimens from Khamis Bani Sa'd and Little Aden. These represent a different species, C. manakhah sp. nov. Most of the figures in Deeleman-Reinhold \& van Harten (2001) that are supposed to represent $C$. pristina do in fact represent $C$. sanaa sp. nov. The only exception is their figure 10, which is copied from Denis (1945), and which Denis thought represented C. semicaudata (which is also wrong).

\section{Diagnosis}

Distinguished from known congeners by details of male palp (Figs 646-651; procursus with strong ventral sclerite, distal bulbal sclerite with pointed dorsal and ventral tips and two prolateral apophyses), and by female genitalia (Figs 654-663; median ridge forming a ' $\mathrm{T}$ '); from many congeners also by male chelicerae (Figs 652-653; relatively small apophyses pointing toward median line, no lateral processes).

\section{Etymology}

The species name refers to the type locality; noun in apposition.

\section{Type material}

\section{Holotype}

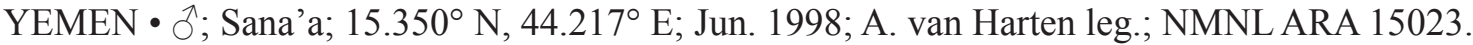

\section{Other material examined}

YEMEN • 3 đô; Sana'a, Plant Protection Department; $15.350^{\circ}$ N, $44.217^{\circ}$ E; 19 May 1999; NMNL ARA 15213 - 3 우; same locality as for preceding; Dec. 1997; A. van Harten leg.; NMNL ARA 15215 - 5 우; same locality as for preceding; Jan. 1998; A. van Harten leg.; NMNL ARA $15214 \bullet 1$ ภ, 1 ○;

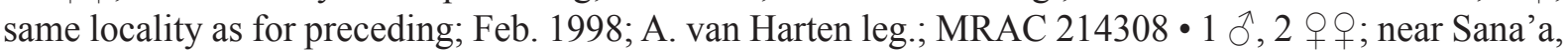
“564”; 3 Dec. 1998; A. van Harten leg.; NMNL.

\section{Description}

\section{Male (holotype)}

MEAsurements. Total length 4.2, carapace width 1.6. Distance PME-PME $120 \mu \mathrm{m}$; diameter PME $105 \times$ $120 \mu \mathrm{m}$; distance PME-ALE $40 \mu \mathrm{m}$; diameter AME $90 \mu \mathrm{m}$; distance AME-AME $40 \mu \mathrm{m}$. Leg 1: 32.9 $(9.6+0.7+9.1+11.4+2.1)$, tibia $2: 6.4$, tibia $3: 4.9$, tibia $4: 5.6$; tibia $1 \mathrm{~L} / \mathrm{d}: 52$; femora $1-4$ diameters: $0.26,0.24,0.24,0.24$.

COLOR (in ethanol). Carapace ochre-yellow, median pit and posterior part of ocular area light brown; sternum mostly dark brown, laterally lighter; legs ochre-yellow, without darker rings, with elongate black marks on femora and tibiae; abdomen pale gray, with few indistinct dark marks dorsally, ventrally with darker marks near pedicel and near spinnerets and three indistinct longitudinal bands behind gonopore.

Body. Habitus similar to $C$. ghul sp. nov. and C. sahtan sp. nov. (cf. Figs 391, 481). Ocular area slightly raised. Wide and deep thoracic pit and pair of furrows diverging from pit toward posterior margin. Clypeus unmodified but rim more strongly sclerotized than in female. Sternum wider than long $(1.15 / 0.75)$, unmodified. Abdomen dorso-posteriorly slightly angular.

Chelicerae. As in Figs 652-653, with pair of converging apophyses close to median line, each with one strong modified (cone-shaped) hair at tip, distance between tips of modified hairs $80 \mu \mathrm{m}$; with very fine stridulatory ridges and pair of low but dark frontal lateral humps.

PALPS. As in Figs 643-645; coxa with very low rounded retrolateral hump; trochanter barely modified; femur proximally with prolateral stridulatory pick (modified hair), without retrolateral process, with indistinct transversal retrolateral line, without dorsal process; distally widened, with low ventral 
protrusion; femur-patella joints close together and shifted towards prolateral; tibia large relative to femur; tibia-tarsus joints shifted towards retrolateral; procursus (Figs 646-648) proximally with strong prolateral hump with many hairs, dorsal hairs barely curved, procursus tip with strong ventral sclerite, rounded prolateral lamella, and further distinctive partly membranous elements; genital bulb (Figs 649651) with simple basal sclerite connected to distal (main) sclerite, sperm duct opening not seen; distal
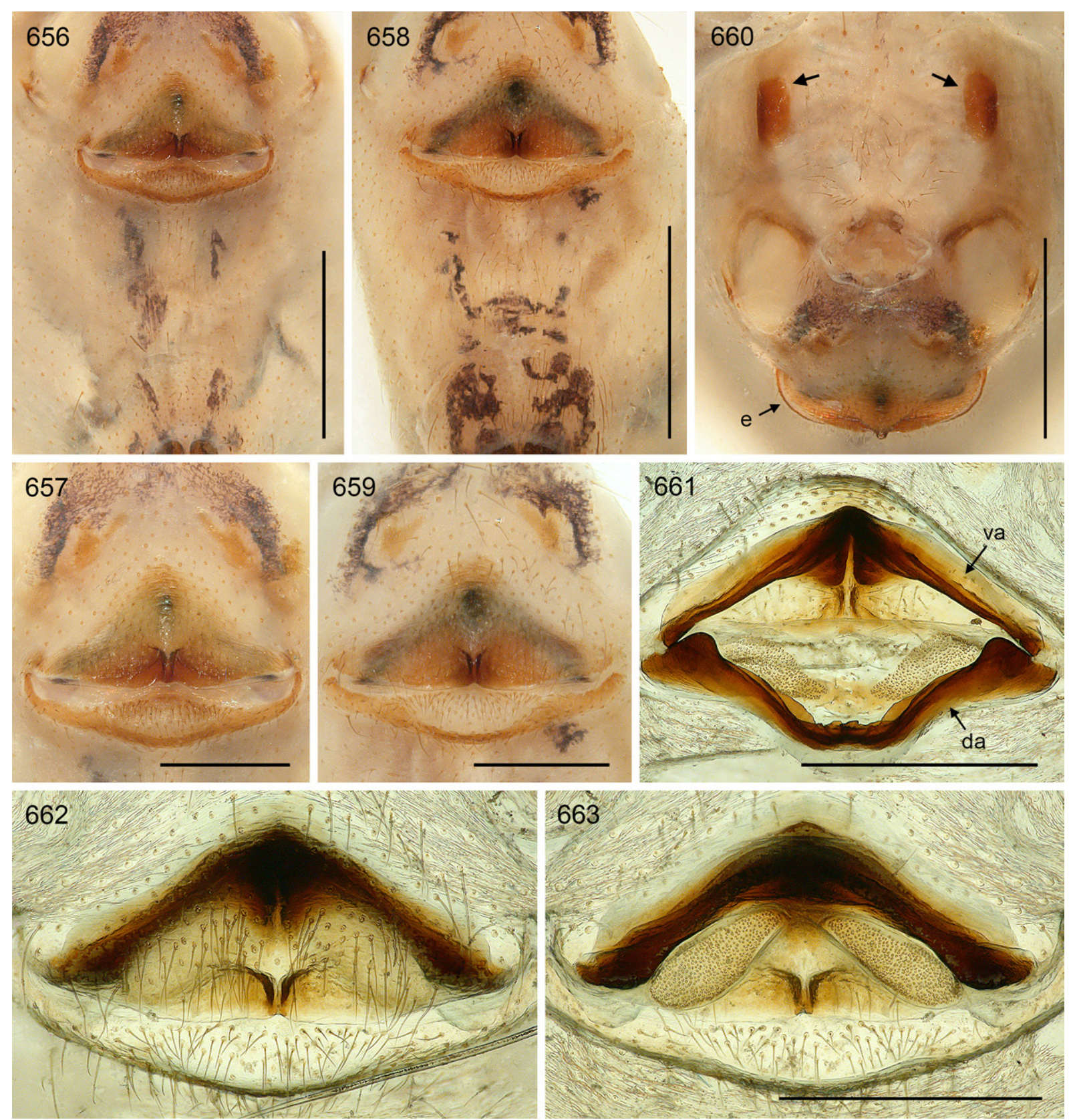

Figs 656-663. Crossopriza sanaa sp. nov.; females from Yemen, Sana'a (656-657, 660: NMNL ARA 15214; 658-659, 661-663: NMNL ARA 15215). 656-659. Abdomens and epigyna, ventral views. 660. Abdomen, frontal view; arrows: stridulatory plates. 661-663. Female internal genitalia, dorsal view (with dorsal arc tilted backwards), ventral view, and regular dorsal view. Abbreviations: da $=$ dorsal arc; $\mathrm{e}=$ epigynum; va $=$ ventral arc. Scale bars: $656,658,660=1.0 \mathrm{~mm} ; 657,659,661-663=0.5 \mathrm{~mm}$. 
sclerite with retrolateral ridge, two distinctive processes pointing in opposite directions and pair of distinctive prolateral apophyses.

LEGS. Femur 1 with single row of $\sim 20$ ventral spines, also femur 2 distally with $\sim 10$ stronger hairs in one ventral row; without curved hairs; few vertical hairs; retrolateral trichobothrium of tibia 1 at $4 \%$; prolateral trichobothrium absent on tibia 1, present on other leg tibiae; tarsi without regular pseudosegments but with indistinct platelets.

Male (variation)

Tibia 1 in five males (incl. holotype): 8.9-11.0 (mean 9.6). Ventral dark band on abdomen variably complete (cf. female, Figs 656, 658).

\section{Female}

In general similar to male but without spines on legs, apparently without (or with extremely fine) stridulatory files on chelicerae, and with stridulatory organ consisting of pair of weakly sclerotized but distinct processes posteriorly on carapace and pair of light brown plates anteriorly on abdomen (Fig. 660). Tibia 1 in eight females: 8.1-10.4 (mean 9.4). Epigynum as in Figs 656-659, main epigynal plate triangular, weakly protruding, with pockets very close together on both sides of median ridge (distance $\sim 45 \mu \mathrm{m}$ ); internal arcs and median anterior structure variably visible in uncleared specimens; posterior plate short but wide, simple; with pair of small brown plates in front of epigynum, not elevated. Internal genitalia (Figs 654-655, 661-663) with pair of elongate pore plates converging and narrowing anteriorly, ventral arc with distinctive median cleft and ventral median process (pocket?).

\section{Distribution}

Known from type locality only (Yemen, Sana'a) (Fig. 353A).

Crossopriza kandahar sp. nov. urn:1sid:zoobank.org:act:AC7041F8-BC5B-4600-A5E1-A8C05BE8673F

Figs 353A, 664-680

\section{Diagnosis}

Males are easily distinguished from known congeners by presence of small pair of apophyses frontally on male chelicerae (Fig. 669); also by details of male palp (Figs 668, 671; procursus tip with small dorsal flat process; distal bulbal sclerite with two large prolateral apophyses), and by shape of epigynum (Fig. 677; short and wide plate, pair of large pockets close together but not on median rim; similar to C. sengleti sp. nov.).

\section{Etymology}

The species name refers to the type locality; noun in apposition.

\section{Type material}

Holotype

AFGHANISTAN • ${ }^{\wedge}$; Kandahar Province, $\mathrm{W}$ of Kandahar; $31.62^{\circ} \mathrm{N}, 65.60^{\circ} \mathrm{E} ; 1000 \mathrm{~m}$ a.s.1.; $31 \mathrm{Jul}$. 1975; A. Senglet leg.; MHNG.

\section{Other material examined}

AFGHANISTAN • $10 \hat{\jmath} \widehat{\partial}, 15$ q,+ 4 juvs (2 vials); same collection data as for holotype; MHNG. 


\section{Description}

Male (holotype)

MeAsurements. Total length 5.3, carapace width 1.65. Distance PME-PME $70 \mu \mathrm{m}$; diameter PME $110 \times 150 \mu \mathrm{m}$; distance PME-ALE $30 \mu \mathrm{m}$; diameter AME $95 \mu \mathrm{m}$; distance AME-AME $30 \mu \mathrm{m}$. Leg 1: $37.5(10.8+0.7+10.3+13.3+2.4)$, tibia $2: 7.2$, tibia $3: 5.6$, tibia $4: 6.8$; tibia $1 \mathrm{~L} / \mathrm{d}: 61$; femora $1-4$ diameters: $0.26,0.23,0.22,0.24$.

CoLor (in ethanol). Carapace ochre-yellow, anteriorly in median pit light brown; sternum with many small brown spots partly arranged in radial marks; legs ochre-yellow, without darker rings, with small black lines on femora and (few) on tibiae; abdomen pale gray, with whitish internal marks and indistinct dark band posteriorly above spinnerets; ventrally with broken dark band, with three indistinct parallel longitudinal marks behind gonopore.

Body. Habitus similar to C. tiwi sp. nov. (cf. Figs 393-394). Ocular area slightly raised. Deep thoracic pit and pair of furrows diverging from pit toward posterior margin. Clypeus unmodified, only rim slightly more sclerotized than in female. Sternum wider than long (1.1/0.75), unmodified. Abdomen slightly elongated, dorso-posteriorly angular to conical.

Chelicerae. As in Figs 669-670, with two pairs of frontal apophyses; proximal pair small and simple; distal pair larger, at tip with one large modified cone-shaped hair each; distance between tips of modified hairs $180 \mu \mathrm{m}$; lateral stridulatory ridges clearly visible in dissecting microscope.

PALPS. As in Figs 664-666; coxa with rounded retrolateral hump; trochanter barely modified; femur distally strongly widened, with rounded ventral protrusion, proximally with prolateral stridulatory pick, without

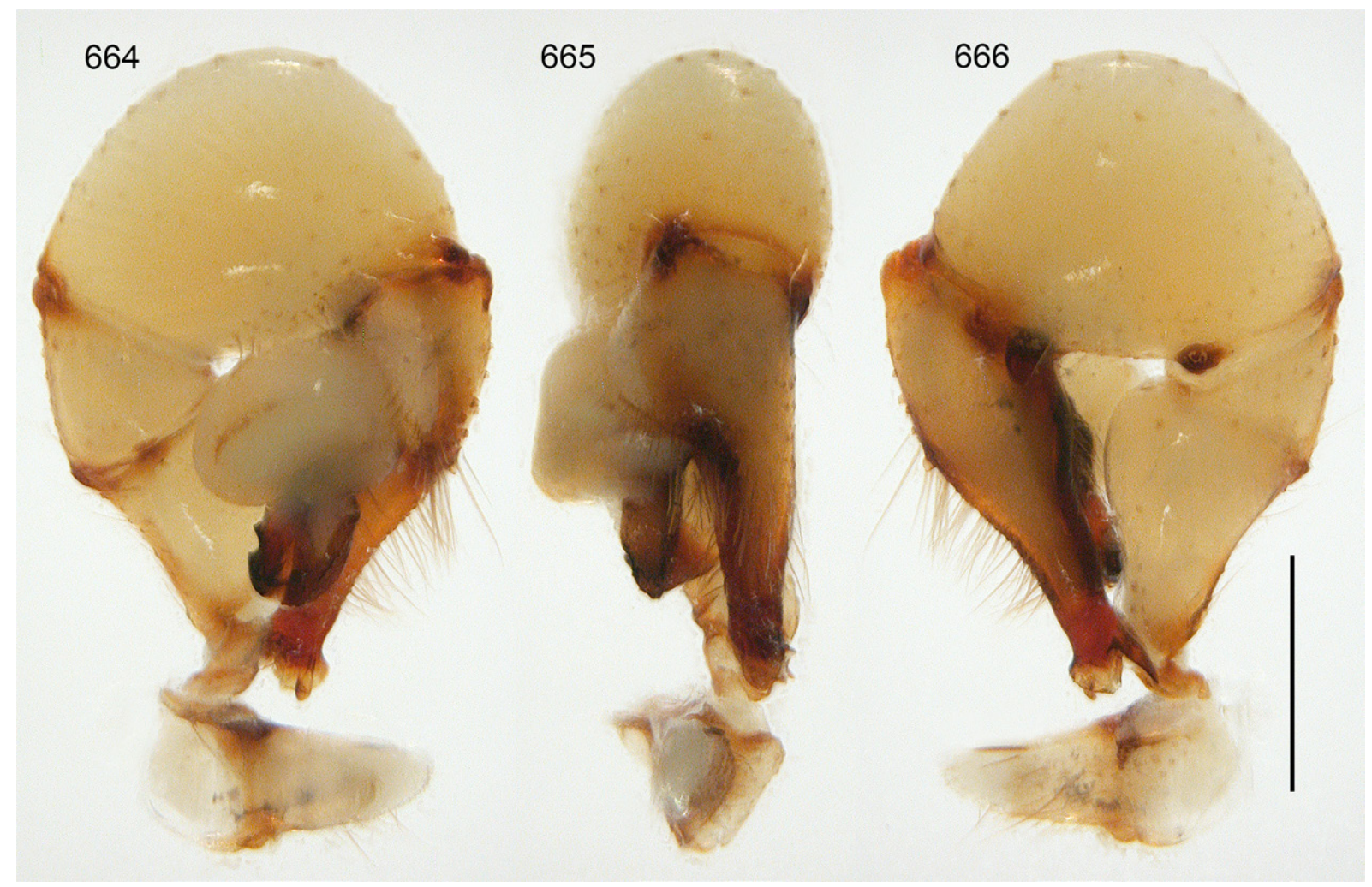

Figs 664-666. Crossopriza kandahar sp. nov.; non-type male from Afghanistan, W of Kandahar (MHNG); left palp, prolateral, dorsal, and retrolateral views. Scale bar $=0.5 \mathrm{~mm}$. 

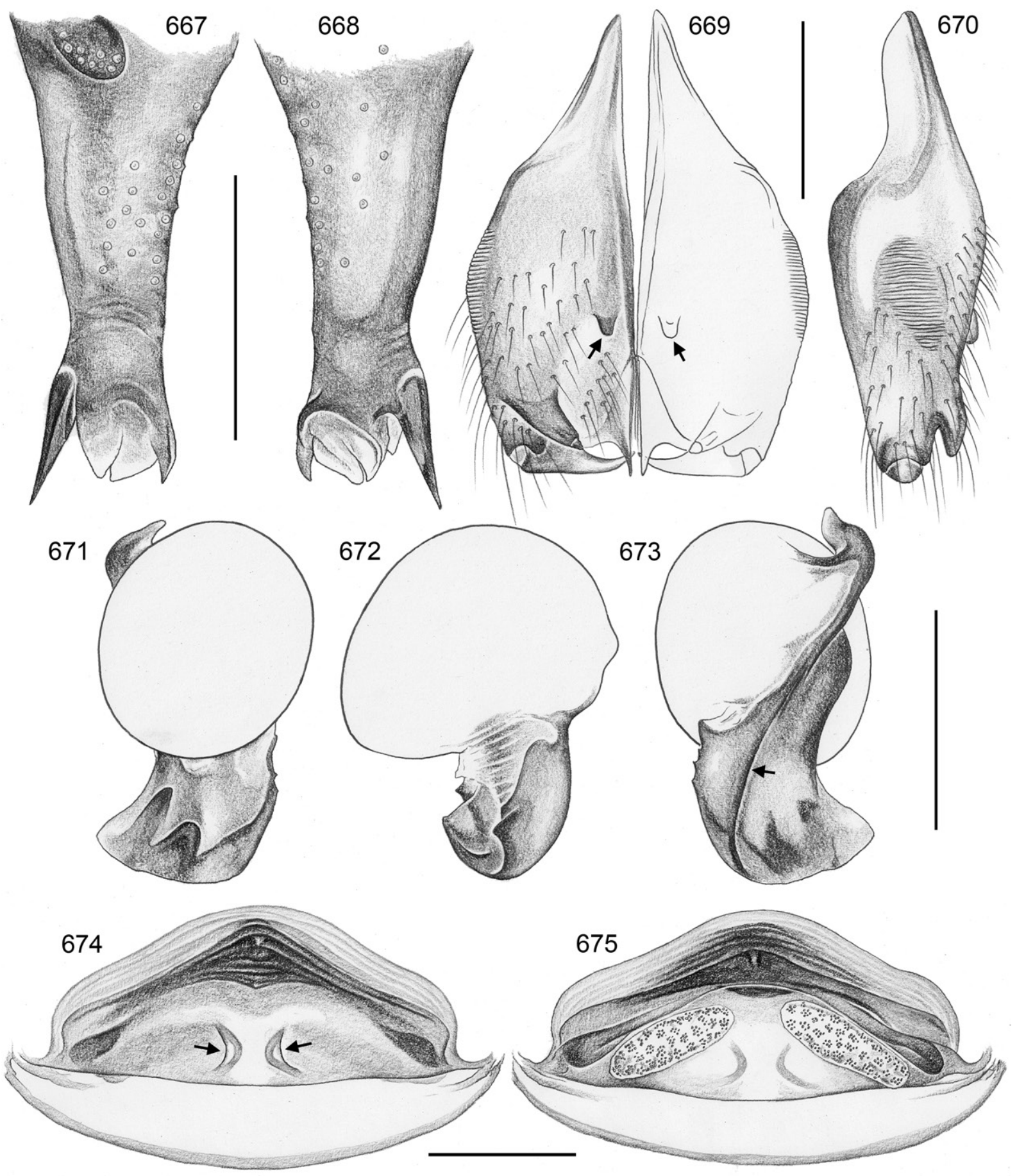

Figs 667-675. Crossopriza kandahar sp. nov.; non-type male and female from Afghanistan, W of Kandahar (MHNG). 667-668. Left procursus, prolateral and retrolateral views. 669-670. Male chelicerae, frontal and lateral views; arrows: distinctive pair of apophyses. 671-673. Left genital bulb, prolateral, dorsal, and retrolateral views; arrow: retrolateral ridge. 674-675. Cleared female genitalia, ventral and dorsal views; arrows: pockets. Scale bars $=0.3 \mathrm{~mm}$. 
retrolateral transversal line, without retrolateral proximal process; femur-patella joints shifted toward prolateral side; tibia-tarsus joints slightly shifted toward retrolateral side; tarsus without macrotrichia; procursus (Figs 667-668) straight, dorsal hairs not curved, prolateral proximal hump set with many hairs, procursus tip with strong ventral sclerite, small dorsal sclerite with prolateral-dorsal ridge, and membranous elements; genital bulb (Figs 671-673) with simple basal sclerite connected to distal (main) sclerite, sperm duct opening not seen; distal sclerite with retrolateral ridge and distinctive set of two prolateral apophyses and ridge.

LEGS. Femur 1 with single row of $\sim 18$ ventral spines; without curved hairs; few vertical hairs; retrolateral trichobothrium of tibia 1 at $3.5 \%$; prolateral trichobothrium absent on tibia 1, present on other leg tibiae; tarsal pseudosegments not seen.

Male (variation)

Tibia 1 in 11 males (incl. holotype): 9.6-10.8 (mean 10.0). Some males with few indistinct dark marks dorsally on abdomen; ventral abdominal marks behind gonopore variable (2-4 parallel bands).

\section{Female}

In general similar to male but without spines on legs, apparently without stridulatory files on chelicerae, and with stridulatory organ consisting of pair of weakly sclerotized but distinct processes posteriorly on carapace and pair of distinct light brown plates anteriorly on abdomen. Tibia 1 in 15 females: 8.1-10.0 (mean 9.1). Epigynum as in Figs 676-677, main epigynal plate triangular, short but wide, weakly protruding, only posteriorly heavily sclerotized; with pair of large pockets $80 \mu \mathrm{m}$ apart; internal
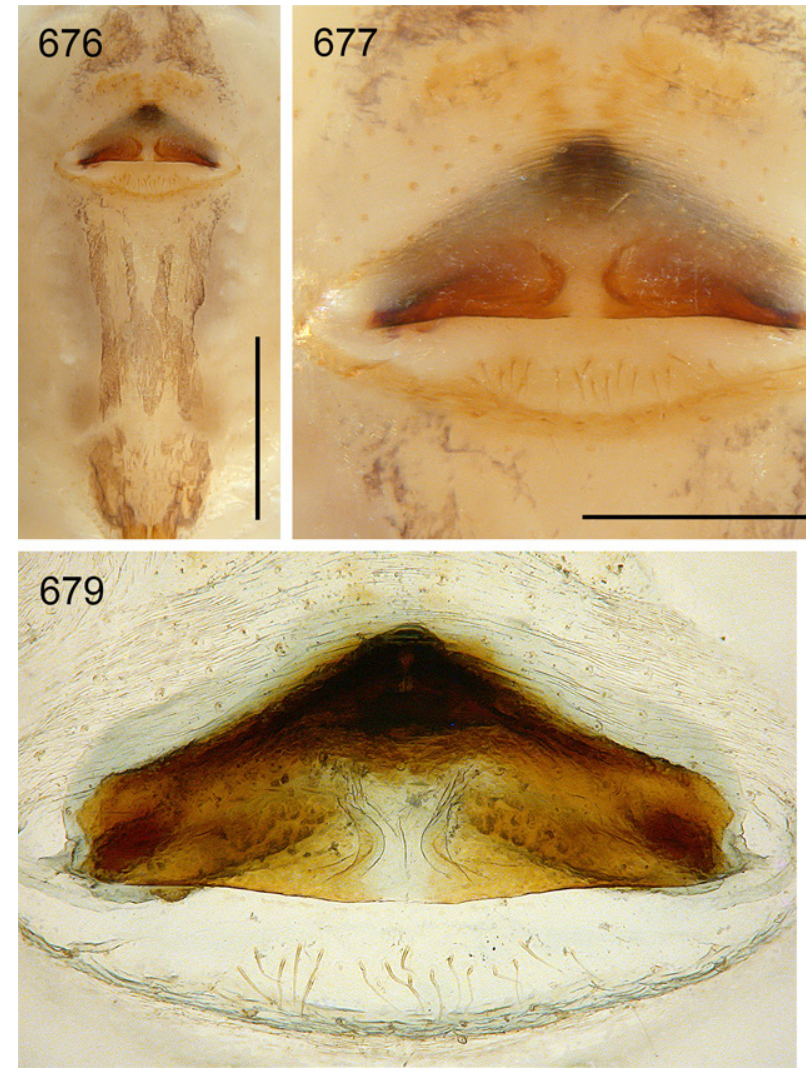
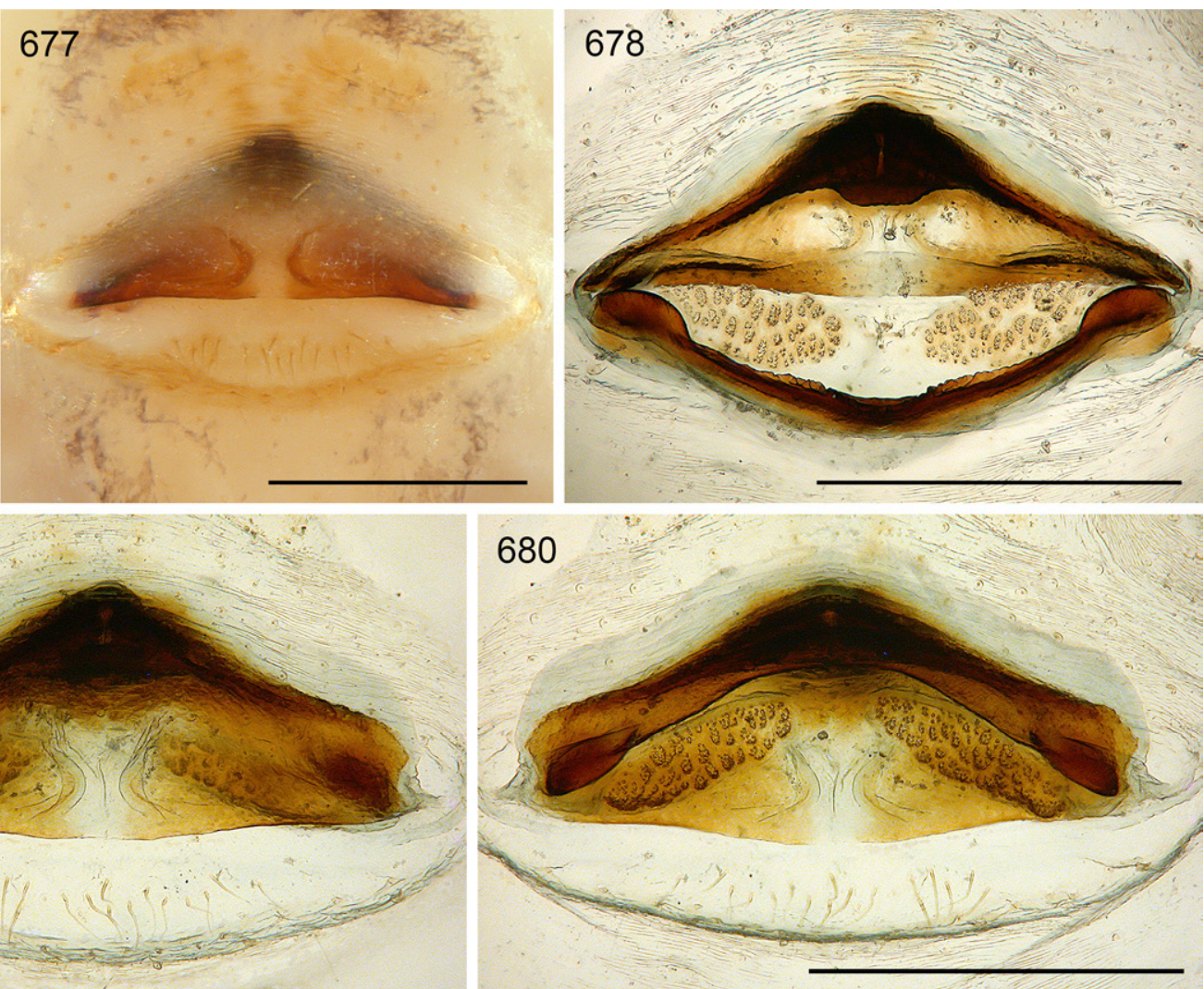

Figs 676-680. Crossopriza kandahar sp. nov.; female from Afghanistan, W of Kandahar (MHNG). 676-677. Abdomen and epigynum, ventral views. 678-680. Female internal genitalia, dorsal view (with dorsal arc tilted backwards), ventral view, and regular dorsal view. Scale bars: $676=1.0 \mathrm{~mm}$; $677-680=$ $0.5 \mathrm{~mm}$. 
sclerotized arcs and median anterior internal sclerite visible in uncleared specimens; posterior plate light brown, short but wide. Internal genitalia (Figs 674-675, 678-680) with large oval pore plates converging anteriorly, pores arranged in groups, dorsal arc strong but simple, ventral arc with median sclerite (rather than pouch or pocket) of unknown function.

\section{Distribution}

Known from type locality only, in Afghanistan, Kandahar Province (Fig. 353A).

\section{Crossopriza malegaon sp. nov. urn:1sid:zoobank.org:act:964FF86C-7766-4DF0-9187-83D8CA7D0FAD}

Figs 353A, 681-697

\section{Diagnosis}

Distinguished from known congeners by details of male palp (Figs 684-689; procursus tip with flat triangular ventral sclerite and simple dorsal elements; distal bulbal sclerite with prolateral sclerotized fold and weakly sclerotized ventral process), by armature of male chelicerae (Figs 690-691; one pair of small apophyses close to median line), and by female genitalia (Figs 692-697; epigynal plate strongly protruding, laterally whitish, median brown band widening anteriorly; pair of pockets close to median line; large roundish pore plates; ventral arc with elongated median modification).

\section{Etymology}

The species name refers to the type locality; noun in apposition.

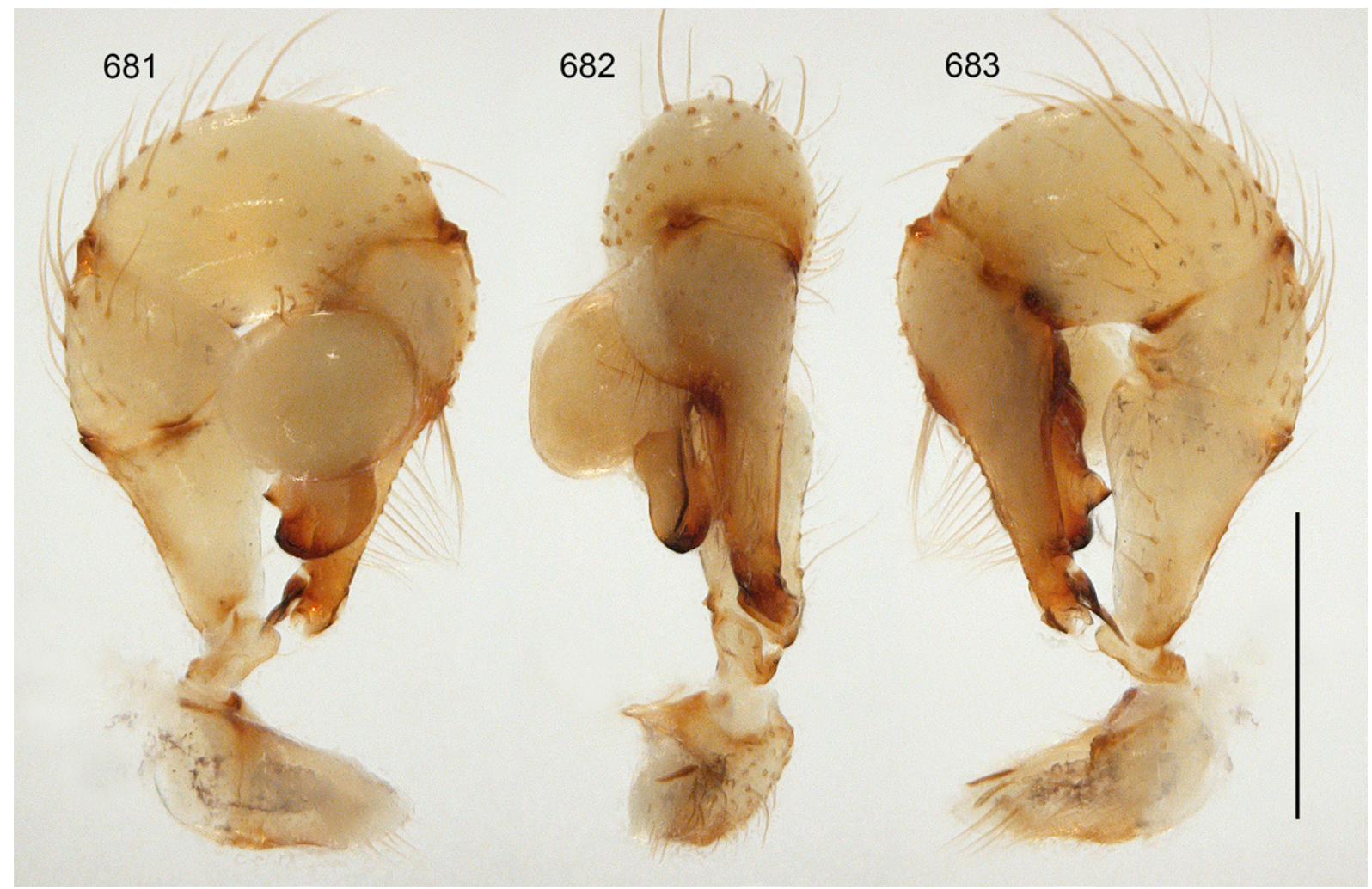

Figs 681-683. Crossopriza malegaon sp. nov.; holotype male from India, 17 mi NE of Malegaon (CAS); left palp, prolateral, dorsal, and retrolateral views. Scale bar $=0.5 \mathrm{~mm}$. 


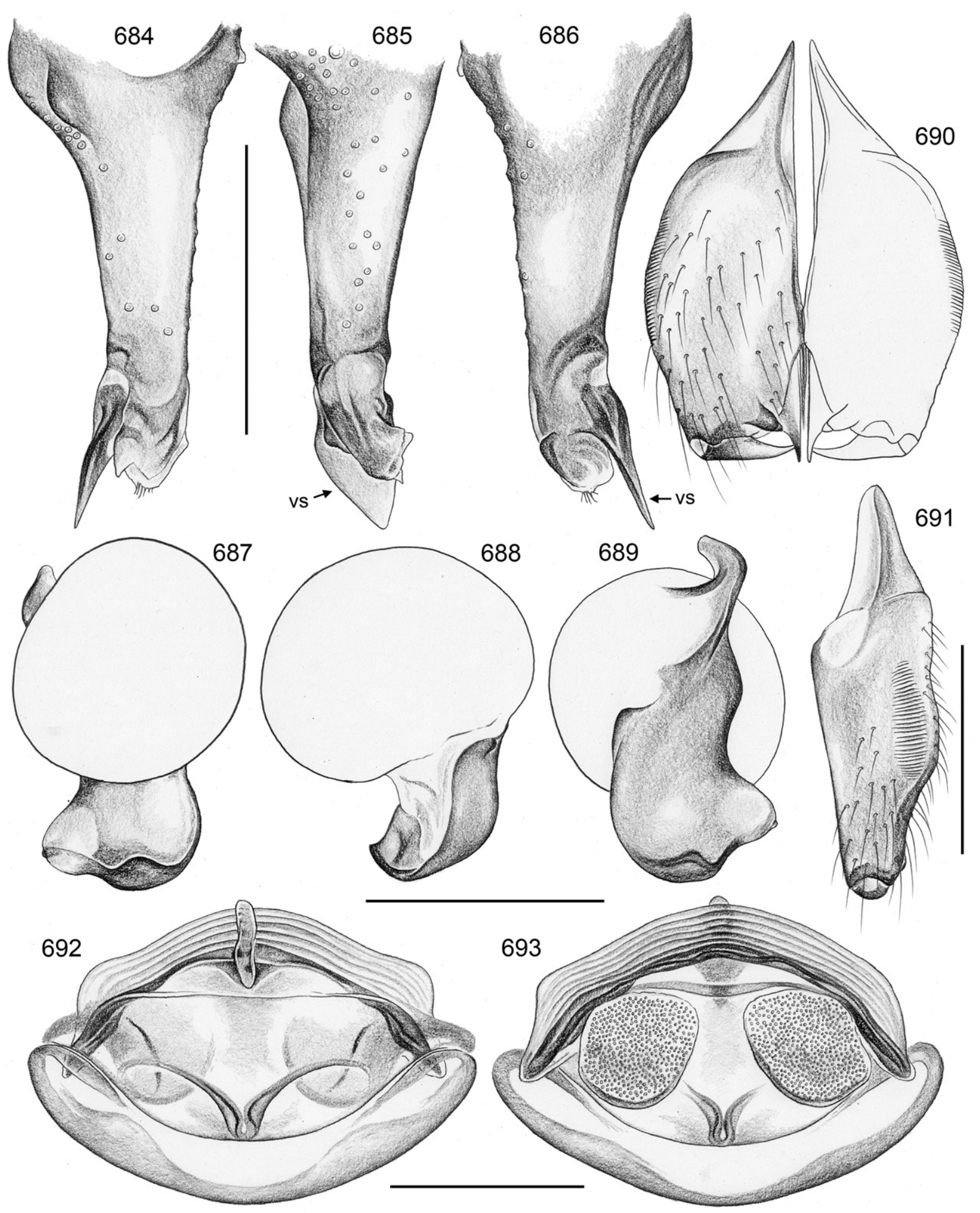

Figs 684-693. Crossopriza malegaon sp. nov.; holotype male, and female from India, $17 \mathrm{mi} \mathrm{NE}$ of Malegaon (CAS). 684-686. Left procursus, prolateral, dorsal, and retrolateral views. 687-689. Left genital bulb, prolateral, dorsal, and retrolateral views. 690-691. Male chelicerae, frontal and lateral views. 692-693. Cleared female genitalia, ventral and dorsal views. Abbreviation: vs = ventral sclerite. Scale bars $=0.3 \mathrm{~mm}$. 


\section{Type material}

\section{Holotype}

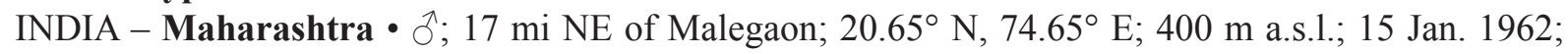
E.S. Ross and D.Q. Cavagnaro leg.; CAS.

\section{Other material examined}

INDIA - Maharashtra 1 1 ; same collection data as for holotype; CAS (together with holotype)

\section{Description}

\section{Male (holotype)}

MEAsurements. Total length 3.0, carapace width 1.25. Distance PME-PME $70 \mu \mathrm{m}$; diameter PME $80 \times$ $95 \mu \mathrm{m}$; distance PME-ALE $30 \mu \mathrm{m}$; diameter AME $70 \mu \mathrm{m}$; distance AME-AME $30 \mu \mathrm{m}$. Leg 1: 24.1 $(7.1+0.5+6.5+8.6+1.4)$, tibia $2: 4.5$, tibia $3: 3.4$, tibia $4: 3.8$; tibia $1 \mathrm{~L} / \mathrm{d}: 54$; femora $1-4$ diameters: $0.19,0.17,0.15,0.15$.
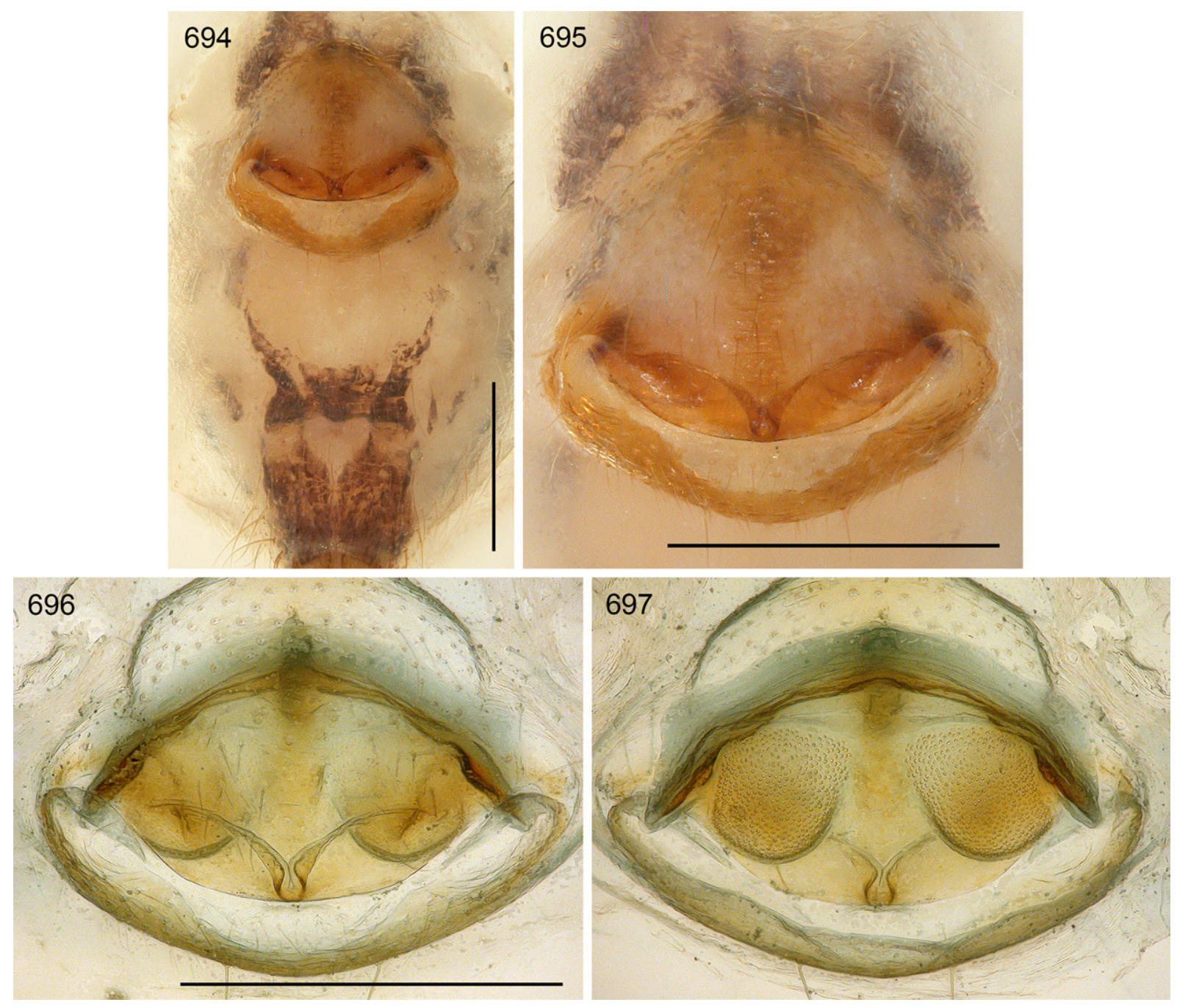

Figs 694-697. Crossopriza malegaon sp. nov.; female from India, $17 \mathrm{mi}$ NE of Malegaon (CAS). 694-695. Abdomen and epigynum, ventral views. 696-697. Female internal genitalia, ventral and dorsal views. Scale bars $=0.5 \mathrm{~mm}$. 
CoLoR (in ethanol). Carapace pale ochre-yellow, medially light brown; sternum light brown with dark brown radial marks; legs pale ochre-yellow, without darker rings, with small black marks (mostly round to oval) on femora and tibiae, very few on metatarsi; abdomen pale ochre-gray, with small dark marks dorsally and laterally; ventrally with distinct black pattern, with three parallel longitudinal marks behind gonopore.

Body. Habitus similar to C. tiwi sp. nov. (cf. Figs 393-394). Ocular area slightly raised. Deep thoracic pit and pair of furrows diverging from pit toward posterior margin. Clypeus unmodified, only rim more sclerotized than in female. Sternum wider than long (0.8/0.6), unmodified. Abdomen slightly elongated, dorso-posteriorly angular.

Chelicerae. As in Figs 690-691, with pair of small frontal apophyses close to median line and strongly converging, barely visible in lateral view, each provided with one large modified cone-shaped hair; distance between tips of modified hairs $50 \mu \mathrm{m}$; lateral stridulatory ridges clearly visible in dissecting microscope.

PaLps. As in Figs 681-683; coxa with rounded retrolateral hump with sclerotized ridge; trochanter barely modified (weakly projecting ventrally); femur distally widened, with very low rounded ventral protrusion, proximally with prolateral stridulatory pick, without retrolateral transversal line, without retrolateral proximal process; femur-patella joints shifted toward prolateral side; tibia-tarsus joints slightly shifted toward retrolateral side; tarsus without macrotrichia; procursus (Figs 684-686) straight, long dorsal hairs not or barely curved, prolateral proximal hump with many hairs, procursus tip with strong ventral sclerite and further membranous and sclerotized elements; genital bulb (Figs 687-689) with simple basal sclerite connected to distal (main) sclerite, sperm duct opening not seen, presumably prolaterally at basis of distal sclerite; distal sclerite without retrolateral ridge, with distinctive prolateral sclerotized fold and whitish ventral process.

LEGS. Femur 1 with single row of $\sim 18$ ventral spines; without curved hairs; with more than usual vertical hairs in one dorsal row each on tibiae 1 and 2; retrolateral trichobothrium of tibia 1 at 5\%; prolateral trichobothrium absent on tibia 1, present on other leg tibiae; tarsal pseudosegments not seen.

\section{Female}

In general similar to male but without spines on legs; stridulatory files on chelicerae not seen; with stridulatory organ consisting of pair of weakly sclerotized but distinct processes posteriorly on carapace and pair of indistinct light brown plates anteriorly on abdomen. Tibia 1: 5.4. Epigynum as in Figs 694695, main epigynal plate almost round, strongly protruding, laterally whitish, median brown band widening anteriorly, posteriorly heavily sclerotized with pair of distinct pockets close to median line, $20 \mu \mathrm{m}$ apart; internal sclerotized arcs not visible in uncleared specimen; posterior plate short but wide. Internal genitalia (Figs 692-693, 696-697) with large roundish pore plates, dorsal and ventral arcs weak, ventral arc with median modification (elongated pocket, duct, or fold?) of unknown function.

\section{Distribution}

Known from type locality only, in India, Maharashtra (Fig. 353A).

Crossopriza miskin sp. nov. urn:1sid:zoobank.org:act:6AED4DF0-17D0-485D-8959-71FF61131A34

Figs 353A, 698-701, 706-725

\section{Diagnosis}

Easily distinguished from known congeners by details of male palp (Figs 711-713, 716; procursus tip very simple, without ventral sclerite; distal bulbal sclerite with distinctive set of prolateral apophyses); also by male chelicerae (Figs 714-715; two pairs of apophyses in distinctive positions), and by female 
genitalia (Figs 719-720, 722; epigynum semicircular with large pockets close to median line; pore plates wide apart; ventral arc with elongate median modification); from most known congeners also by small body size (carapace width $\sim 1.0-1.1$ ) and by shape of abdomen (Figs 698-701; pointed elongation above spinnerets).
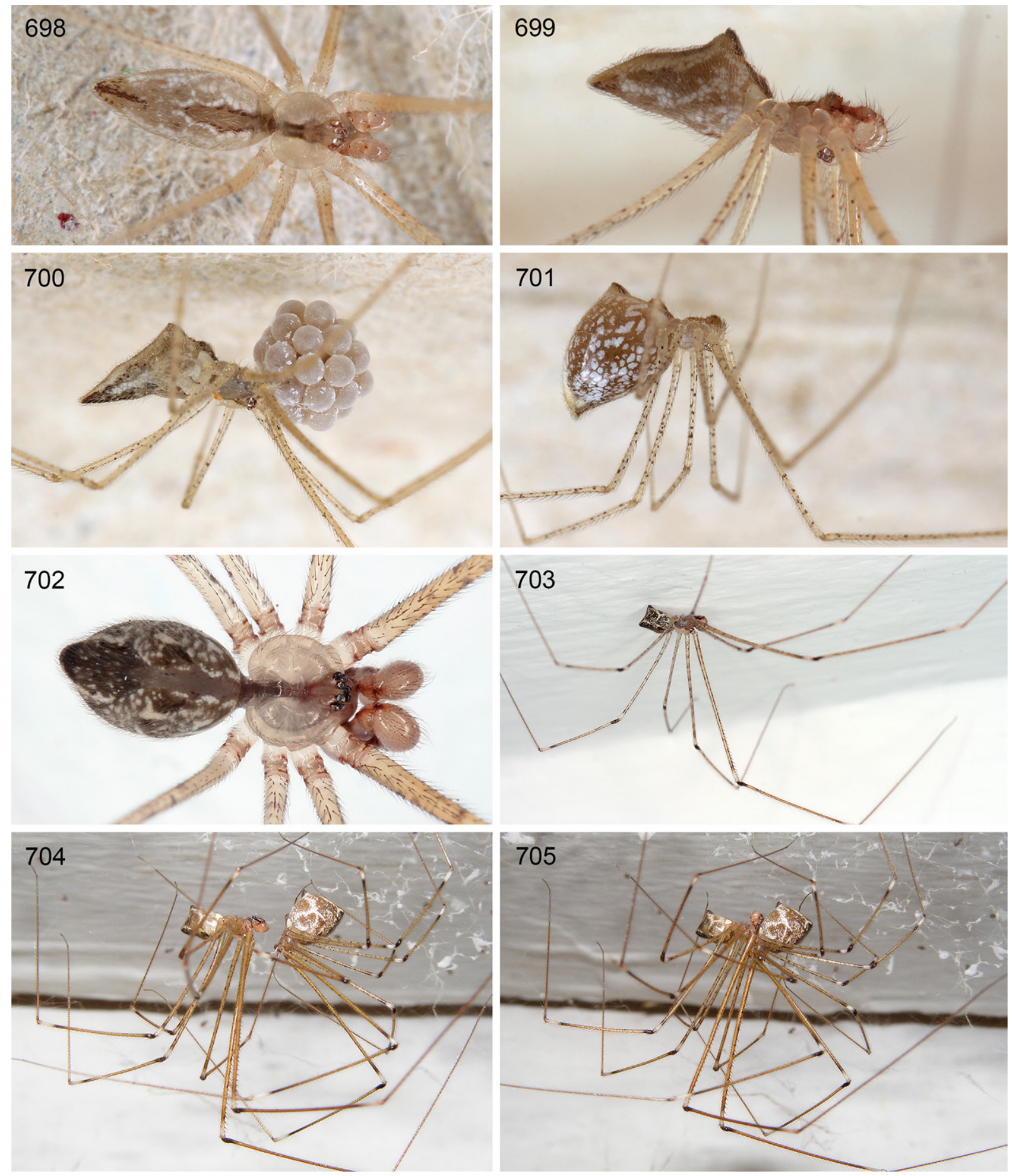

Figs 698-705. Crossopriza Simon, 1893, live specimens. 698-701. C. miskin sp. nov., from Oman, Ibri-Rusdaq; male in dorsal and lateral views; female with and without egg-sac. 702-705. C. lyoni (Blackwall, 1867), male from Singapore and courting/mating pair from Guinea. 


\section{Etymology}

The species name refers to the type locality; noun in apposition.

\section{Type material}

\section{Holotype}

OMAN - Ad Dhahira • ${ }^{\top}$; near Miskin (between Ibri and Rusdaq); $23.496^{\circ}$ N, 56.838 ${ }^{\circ}$ E; $660 \mathrm{~m}$ a.s.1.; under rocks; 25 Mar. 2017; B.A. Huber leg.; ZFMK Ar 22445.

\section{Other material examined}

OMAN - Ad Dhahira • 2 우; same collection data as for holotype; ZFMK Ar 22446 • 1 q (in pure ethanol); same collection data as for holotype; ZFMK Om36. - Al Batinah South • 1 ô, 3 $q$, 1 juv.; wadi near Fasah; $23.303^{\circ}$ N, 57.328 ${ }^{\circ}$ E; 660 m a.s.1.; 26 Mar. 2017; B.A. Huber leg.; under rocks; ZFMK Ar 22447 • 2 우 (in pure ethanol); same collection data as for preceding; ZFMK Om39. - Ad Dakhiliya • 1 q, 1 juv.; between Al Rawda and Al Hayema; 22.880 ${ }^{\circ}$, $57.293^{\circ}$ E; 690 m a.s.1.; 17 Feb. 2018; B.A. Huber leg.; ZFMK Ar $22448 \bullet 2$ juvs (in pure ethanol); same collection data as for preceding; ZFMK Om114.

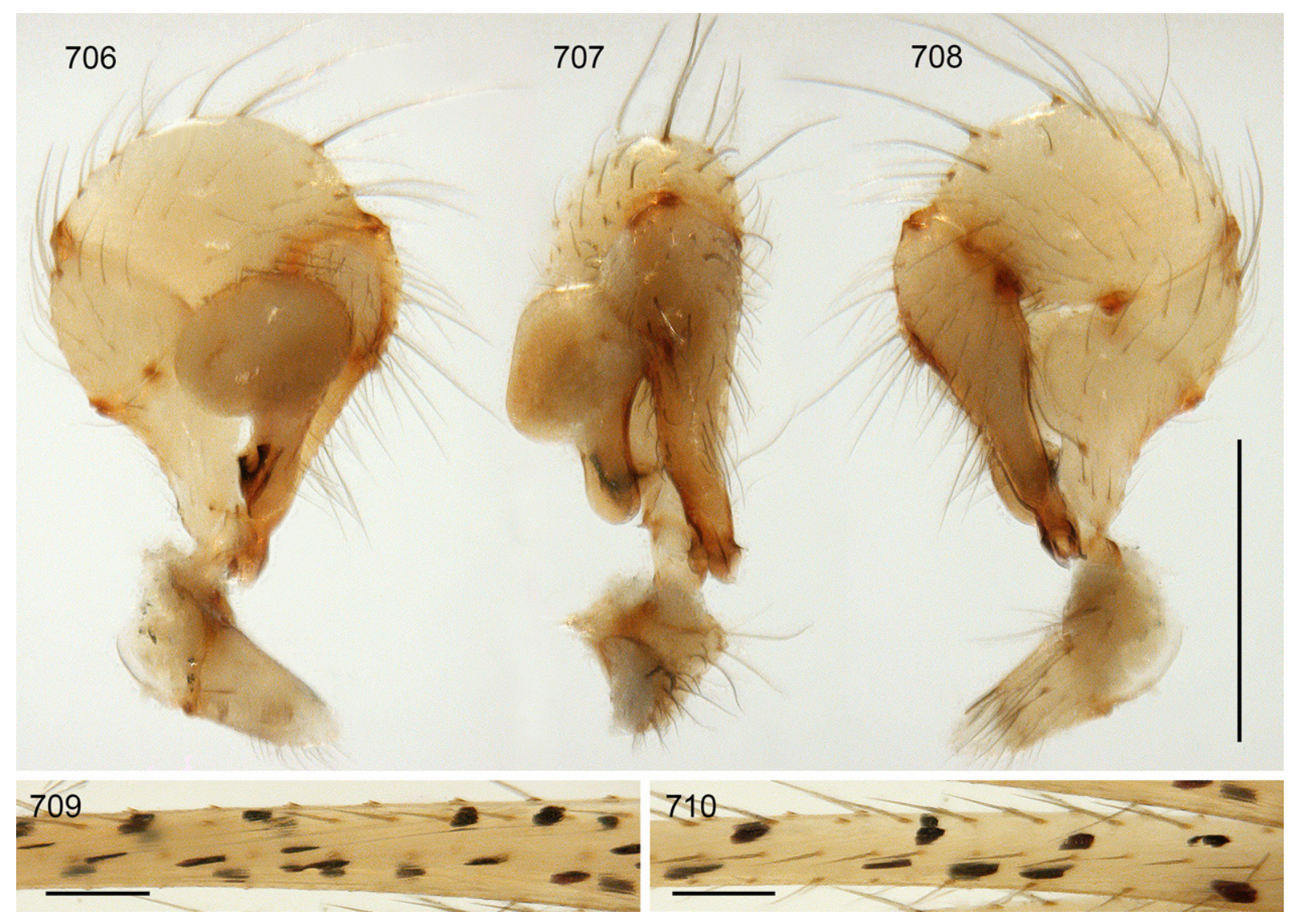

Figs 706-710. Crossopriza miskin sp. nov.; holotype male from Oman, near Miskin (ZFMK Ar 22445) and female from Oman, wadi near Fasah (ZFMK Ar 22447). 706-708. Left male palp, prolateral, dorsal, and retrolateral views. 709-710. Female right femora 2 and 3, prolateral views. Scale bars: 706-708 = $0.5 \mathrm{~mm} ; 709-710=0.2 \mathrm{~mm}$. 


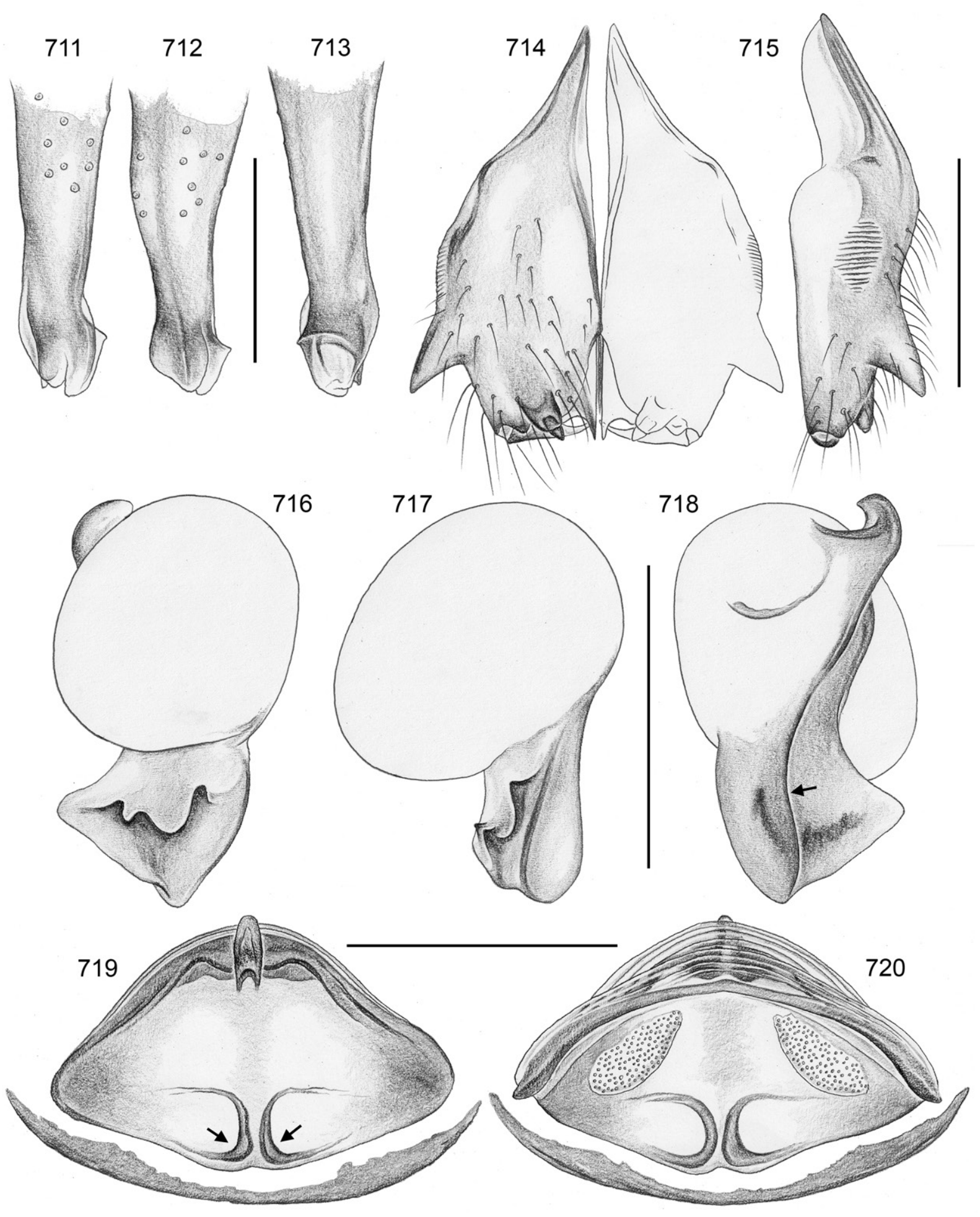

Figs 711-720. Crossopriza miskin sp. nov.; male and female from Oman, wadi near Fasah (ZFMK Ar 22447). 711-713. Left procursus, prolateral, dorsal, and retrolateral views. 714-715. Male chelicerae, frontal and lateral views. 716-718. Left genital bulb, prolateral, dorsal, and retrolateral views; arrow: retrolateral ridge. 719-720. Cleared female genitalia, ventral and dorsal views; arrows: pockets. Scale bars: $711-713=0.2 \mathrm{~mm} ; 714-720=0.3 \mathrm{~mm}$. 


\section{Description}

Male (holotype)

Measurements. Total length 3.0, carapace width 1.05. Distance PME-PME $60 \mu \mathrm{m}$; diameter PME $80 \times$ $100 \mu \mathrm{m}$; distance PME-ALE $30 \mu \mathrm{m}$; diameter AME $70 \mu \mathrm{m}$; distance AME-AME $30 \mu \mathrm{m}$. Leg 1: 23.2 $(6.5+0.4+6.2+8.4+1.7)$, tibia $2: 3.8$, tibia $3: 2.7$, tibia $4: 3.5$; tibia $1 \mathrm{~L} / \mathrm{d}: 59$; femora $1-4$ diameters: $0.17,0.14,0.13,0.14$.

Color (in ethanol). Carapace ochre-yellow, with slightly darker median band including ocular area; clypeus not darkened; sternum ochre-yellow with brown speckles; legs ochre-yellow, without darker rings, with black spots (rather than lines) on femora and (few) on tibiae (cf. Figs 709-710); abdomen pale gray, with dark lines around heart area and posterior median mark; ventrally with distinct black pattern (disrupted median band).

Body. Habitus as in Figs 698-699. Ocular area slightly raised. Shallow but wide thoracic pit and pair of furrows diverging from pit toward posterior margin. Clypeus unmodified, only rim slightly more sclerotized than in female. Sternum wider than long (0.75/0.60), unmodified. Abdomen strongly elongated and pointed above spinnerets.
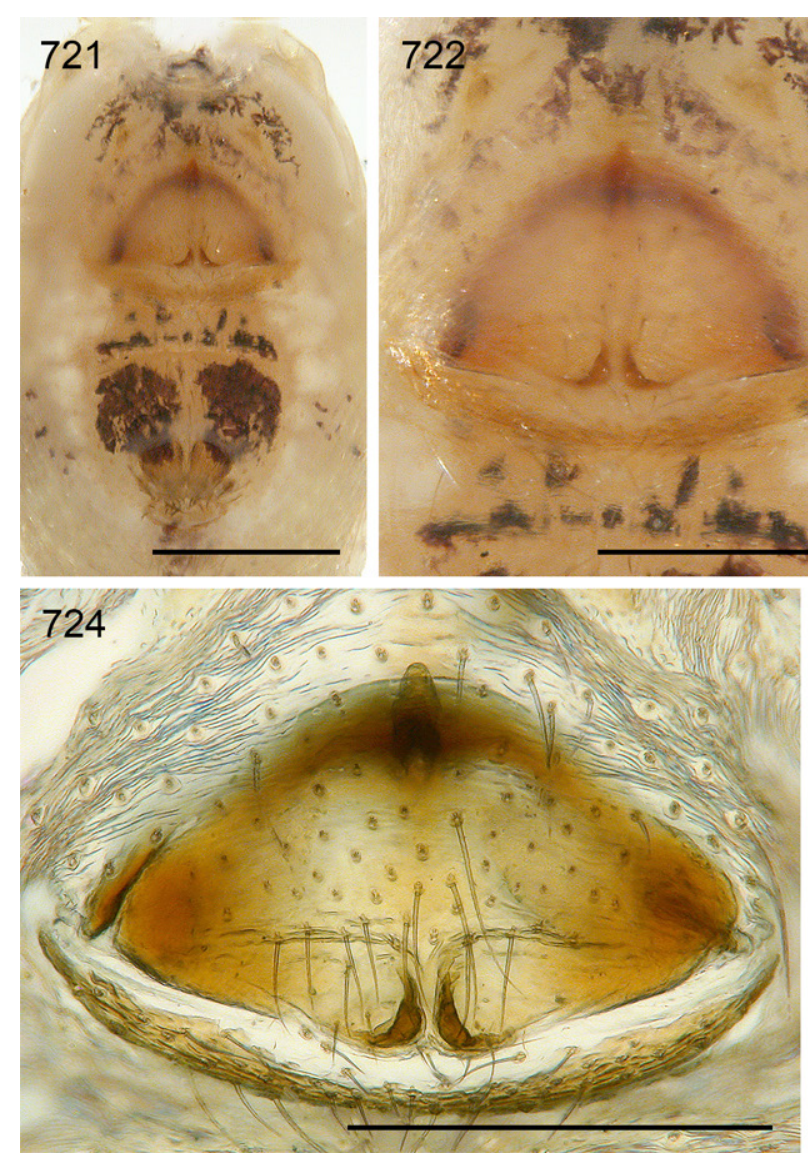
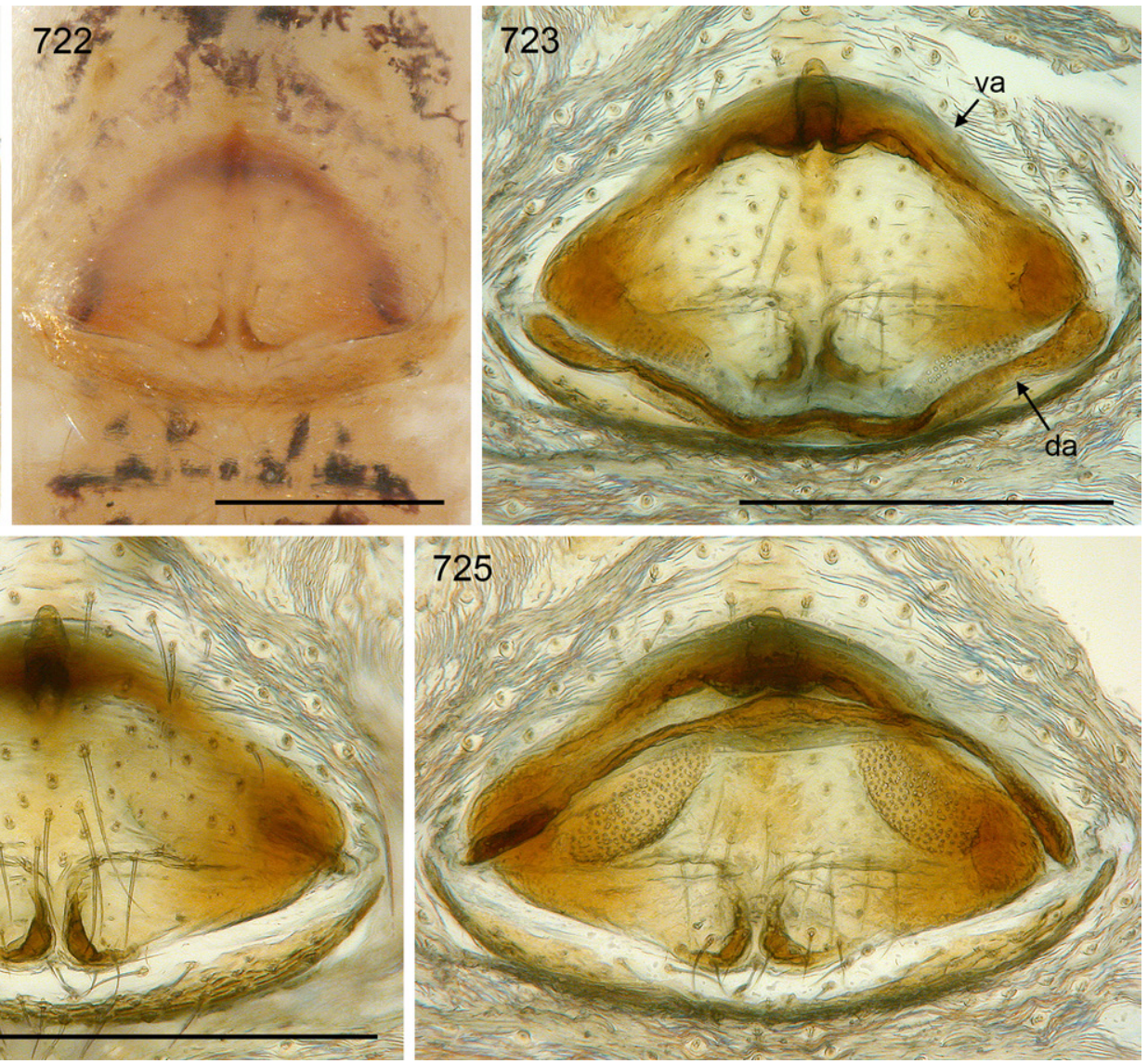

Figs 721-725. Crossopriza miskin sp. nov.; female from Oman, wadi near Fasah (ZFMK Ar 22447). 721-722. Abdomen and epigynum, ventral views. 723-725. Cleared female genitalia, dorsal view with dorsal arc tilted backwards, ventral view, and regular dorsal view. Scale bars: $721=0.5 \mathrm{~mm}$; 722-725= $0.3 \mathrm{~mm}$. 
Chelicerae. As in Figs 714-715, with pair of frontal distal apophyses provided with one large modified cone-shaped hair each, and pair of lateral apophyses directed towards lateral; distance between tips of modified hairs $85 \mu \mathrm{m}$; lateral stridulatory ridges barely visible in dissecting microscope.

PALPS. As in Figs 706-708; coxa with rounded retrolateral hump; trochanter barely modified; femur distally strongly widened, with rounded ventral protrusion, proximally with prolateral stridulatory pick, without retrolateral transversal line, without retrolateral proximal process; femur-patella joints shifted toward prolateral side; tibia-tarsus joints slightly shifted toward retrolateral side; tarsus without macrotrichia; procursus straight, prolateral proximal hump set with numerous long hairs, long dorsal hairs not curved, procursus tip (Figs 711-713) very simple, without (or with strongly reduced) ventral sclerite; genital bulb (Figs 716-718) with simple basal sclerite connected to distal (main) sclerite, sperm duct opening not seen; distal sclerite with retrolateral ridge and distinctive set of prolateral apophyses and ridges.

LEGS. Femur 1 with single row of $\sim 20$ ventral spines; without curved hairs; few vertical hairs; retrolateral trichobothrium of tibia 1 at $4 \%$; prolateral trichobothrium absent on tibia 1, present on other leg tibiae; tarsi without regular pseudosegments (except for 2-3 at tip).

Male (variation)

Tibia 1 in second male: 6.2 .

\title{
Female
}

In general similar to male (Figs 700-701) but without spines on legs, apparently without stridulatory files on chelicerae, and with stridulatory organ consisting of pair of weakly sclerotized but distinct processes posteriorly on carapace and pair of barely visible plates anteriorly on abdomen. Tibia 1 in six females: 4.1-4.9 (mean 4.4). Epigynum as in Figs 721-722, small relative to abdomen, main epigynal plate semicircular, weakly protruding; with pair of distinct pockets close together ( $25 \mu \mathrm{m}$ apart); internal sclerotized arc and median anterior structure visible in uncleared specimens; posterior plate short but wide. Internal genitalia (Figs 719-720, 723-725) with large pore plates wide apart and converging anteriorly, dorsal arc simple, ventral arc with elongate median modification (pouch?) of unknown function.

\section{Natural history}

All specimens were found on the undersides of rocks on the ground, where they were extremely well camouflaged and barely visible unless they moved. However, small parts of the webs usually extended from below the rock, indicating the presence of a spider. In all localities, the species lived in close proximity with Artema bahla Huber, 2019; both species were sometimes found on a single rock, only $10-20 \mathrm{~cm}$ apart.

\section{Distribution}

Known from several localities in north-eastern Oman (Ad Dhahira, Al Batinah South, Dakhiliya) (Fig. 353A).

\author{
Crossopriza parsa sp. nov. \\ urn:lsid:zoobank.org:act:03DA29E6-CDB9-46E6-82D7-DD5CA85571D9
}

Figs 353A, 726-744

\section{Diagnosis}

Distinguished from known congeners by details of male palp (Figs 729-730, 733-735; long and slender procursus slightly curved towards ventral, with prolateral process accompanying ventral sclerite; distal bulbal sclerite with prominent ventral process and distinctive set of prolateral apophyses; palp similar to 
C. srinagar sp. nov.), by male chelicerae (Figs 731-732; lateral apophyses prominent in frontal view, weakly protruding in lateral view), and by epigynum (Fig. 741; strongly sclerotized lateral elements; pockets close together).

\section{Etymology}

The species name is derived from the Old Persian name of Persepolis, 'pārsa'; noun in apposition.

\section{Type material}

\section{Holotype}

IRAN - Fars • đ̊; Persepolis; $29.983^{\circ}$ N, 52.900 E (see Remarks below); 18 Aug. 1973; A. Senglet leg.; MHNG.

\section{Other material examined}

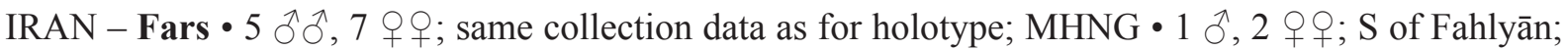
$30.000^{\circ}$ N, $51.583^{\circ}$ E; 4 Sep. 1975; A. Senglet leg.; MHNG.

\section{Material assigned tentatively (see Variation below)}

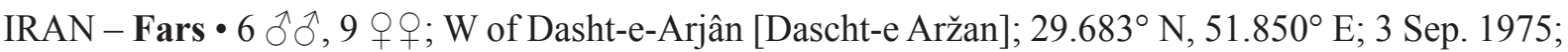
A. Senglet leg.; MHNG • 1 q; "Aliâbâd"; $30.017^{\circ}$ N, 53.000 E (see Remarks below); 9 Jun. 1974; A. Senglet leg.; MHNG • 4 우; "Barm-e-peere-Ghaibi” (see Remarks below); $28.898^{\circ}$ N, $52.540^{\circ}$ E; 29 May 2000; Y.M. Marusik and K. Elmi leg.; ZFMK Ar 5208. - Kohgiluyeh and Boyer-Ahmad • $4 \hat{\delta} \widehat{\partial}$,

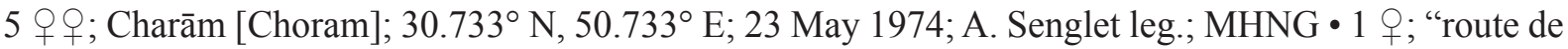
Yasudj”; $30.467^{\circ}$ N, $51.500^{\circ}$ E (see Remarks below); 25 May 1974; A. Senglet leg.; MHNG.

\section{Remarks}

The coordinates on A. Senglet's original labels are usually to the nearest minute (i.e., $\pm 2 \mathrm{~km}$ ), but in some cases the actual collecting spot seems to be even farther away than that. The type locality is possibly closer to $\sim 29.93^{\circ} \mathrm{N}, 52.89^{\circ} \mathrm{E}$ (ruins of Persepolis).

There are several "Aliâbâd" in Fars, none of them close to the coordinates on the original label; maybe the collecting site was Haji Abad $\left(29.99^{\circ} \mathrm{N}, 52.92^{\circ} \mathrm{E}\right)$.

The original coordinates of "route de Yasudj" define a spot in Fars Province, slightly outside of Kohgiluyeh and Boyer-Ahmad Province, but the label says Kohkiluyeh.

A locality with the name "Barm-e-peere-Ghaibi" is repeatedly cited in the arachnological literature (always referring to the same collecting event) but does apparently not exist (F. Mozaffarian, pers. com., Feb. 2021). The name possibly refers to Barm-e Peer, a small lake close to the Palace of Ardashir Pāpakan at approximately $28.898^{\circ} \mathrm{N}, 52.540^{\circ} \mathrm{E}$. Alternatively, an anonymous referee suggested that it may refer to a shrine called "Pir-e Qeybi" in the vicinities of Shiraz $\left(29.488^{\circ} \mathrm{N}, 52.497^{\circ} \mathrm{E}\right)$.

\section{Description}

Male (holotype)

MeAsurements. Total length 4.3, carapace width 1.7. Distance PME-PME $95 \mu \mathrm{m}$; diameter PME $105 \times 130 \mu \mathrm{m}$; distance PME-ALE $25 \mu \mathrm{m}$; diameter AME $100 \mu \mathrm{m}$; distance AME-AME $30 \mu \mathrm{m}$. Leg 1: $44.5(12.9+0.7+12.0+16.4+2.5)$, tibia $2: 8.9$, tibia $3: 6.1$, tibia $4: 6.7$; tibia $1 \mathrm{~L} / \mathrm{d}: 75$; femora $1-4$ diameters: $0.23,0.21,0.20,0.20$. 
Color (in ethanol). Carapace ochre-yellow, with distinct brown mark anteriorly in median pit; sternum dark brown to black; legs ochre-yellow, without darker rings, with small black lines on femora and (few) on tibiae; abdomen gray, with few and indistinct dorsal dark marks; ventrally with dark band limited to anterior and posterior part, absent from median part (behind gonopore).

Body. Habitus similar to C. sahtan sp. nov. (cf. Fig. 391). Ocular area slightly raised. Deep thoracic pit and pair of shallow furrows diverging from pit toward posterior margin. Clypeus unmodified, only rim slightly more sclerotized than in female. Sternum wider than long (1.1/0.7), unmodified. Abdomen slightly elongated, dorso-posteriorly angular to conical.

Chelicerae. As in Figs 731-732, with two pairs of apophyses; lateral pair set with small sclerotized scales; median pair with one large modified cone-shaped hair each; distance between tips of modified hairs $70 \mu \mathrm{m}$; lateral stridulatory ridges clearly visible in dissecting microscope.

Palps. As in Figs 726-728; coxa with rounded retrolateral hump; trochanter barely modified; femur distally strongly widened, with rounded ventral protrusion, proximally with prolateral stridulatory pick, without retrolateral transversal line, without retrolateral proximal process but some slightly stronger hairbases on retrolateral-ventral ridge; femur-patella joints shifted toward prolateral side; tibia-tarsus joints shifted toward retrolateral side; tarsus without macrotrichia; procursus (Figs 729-730) weakly curved towards ventral, with low prolateral hump proximally followed with thick ridge set with numerous long hairs, long dorsal hairs mostly straight or weakly curved, procursus tip with ventral sclerite accompanied by flat prolateral sclerite, with further membranous elements; genital bulb (Figs 733-735) with simple basal sclerite connected to distal (main) sclerite, sperm duct opening not seen; distal sclerite without retrolateral ridge, with two distinctive prolateral apophyses and long ventral process.

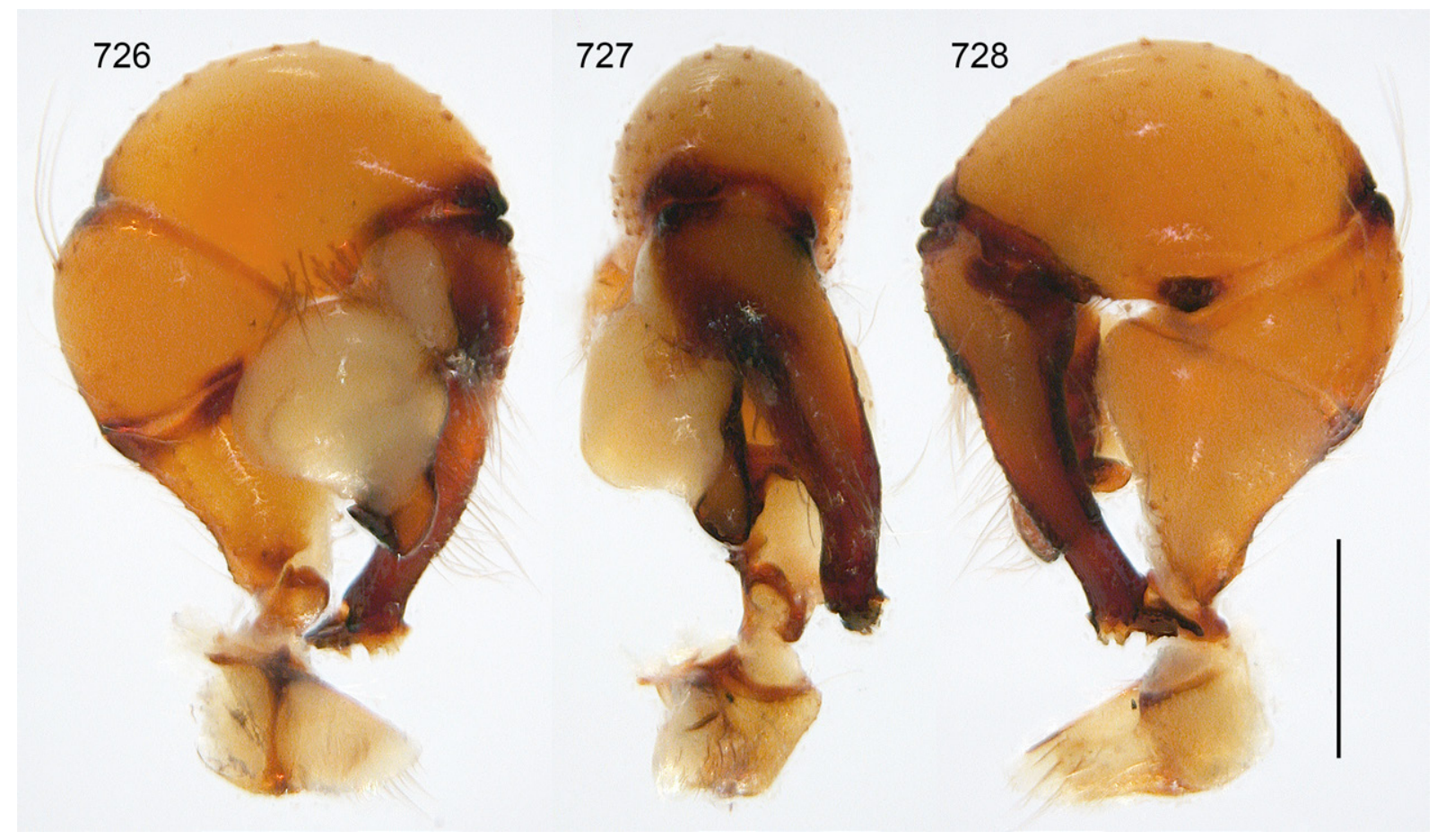

Figs 726-728. Crossopriza parsa sp. nov.; non-type male from Iran, Persepolis (MHNG); left palp, prolateral, dorsal, and retrolateral views. Scale bar $=0.5 \mathrm{~mm}$. 


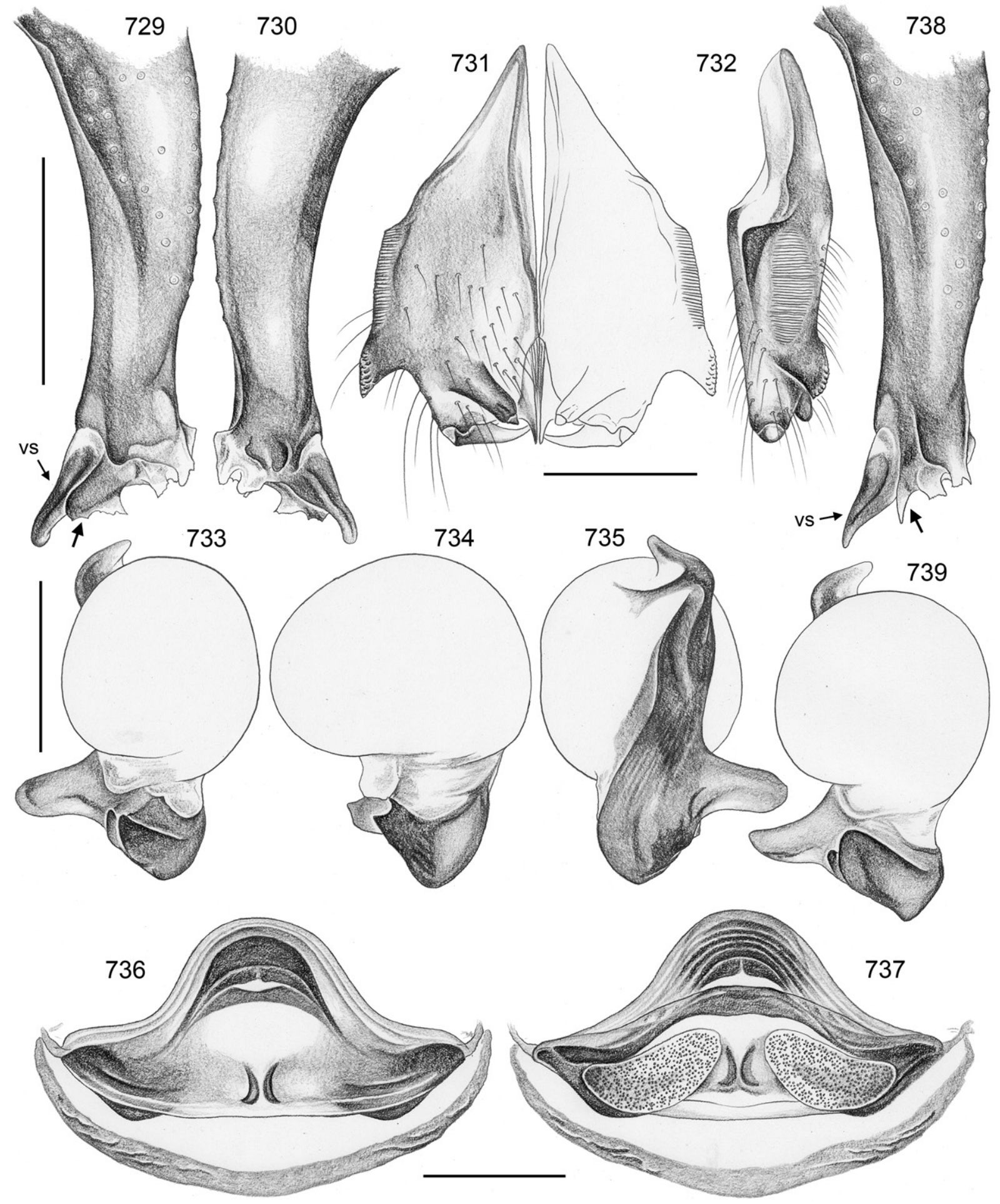

Figs 729-739. Crossopriza parsa sp. nov.; non-type male and female from Iran, Persepolis (MHNG) (729-737) and male from Iran, W of Dasht-e-Arjân (MHNG) (738-739). 729-730. Left procursus, prolateral and retrolateral views; arrow: prolateral process. 731-732. Male chelicerae, frontal and lateral views. 733-735. Left genital bulb, prolateral, dorsal, and retrolateral views. 736-737. Cleared female genitalia, ventral and dorsal views. 738. Left procursus, prolateral view; arrow: prolateral process. 739. Left genital bulb, prolateral view. Abbreviation: $v s=$ ventral sclerite. Scale bars $=0.3 \mathrm{~mm}$. 
LEGS. Femur 1 with single row of $\sim 28$ ventral spines; without curved hairs; few vertical hairs; retrolateral trichobothrium of tibia 1 at $4 \%$; prolateral trichobothrium absent on tibia 1, present on other leg tibiae; tarsal pseudosegments indistinct and irregular, only at distal tip $\sim 2-3$ regular pseudosegments.

Male (variation)

Tibia 1 in 12 males (incl. holotype): 9.9-12.4 (mean 11.3). Some males with few indistinct darker specks behind gonopore, but never with distinct dark pattern. All males other than those from type locality and from S of Fahlyān with slightly different procursus tip and slightly different processes on distal bulbal sclerite: in males from Dasht-e Arjân, the apophysis accompanying the ventral distal sclerite of the procursus is simple and weakly sclerotized (arrow in Fig. 738); the elements of the distal bulbal sclerite are slightly different in shape (Fig. 739). In males from Choram, the procursus tip appears intermediate between those from Persepolis and those from Dasht-e Arjân; the bulb appears identical to males from Persepolis. The chelicerae in all these males appear identical. They also share the 'incomplete' pattern ventrally on the abdomen, i.e., with almost no dark marks behind the gonopore.

\section{Female}

In general similar to male but without spines on legs, apparently without stridulatory files on chelicerae, and with stridulatory organ consisting of pair of weakly sclerotized but distinct processes posteriorly on carapace and pair of small but distinct light brown plates anteriorly on abdomen. Tibia 1 in 21 females: 8.8-12.5 (mean 10.0). Epigynum as in Figs 740-741, main epigynal plate roughly triangular, barely
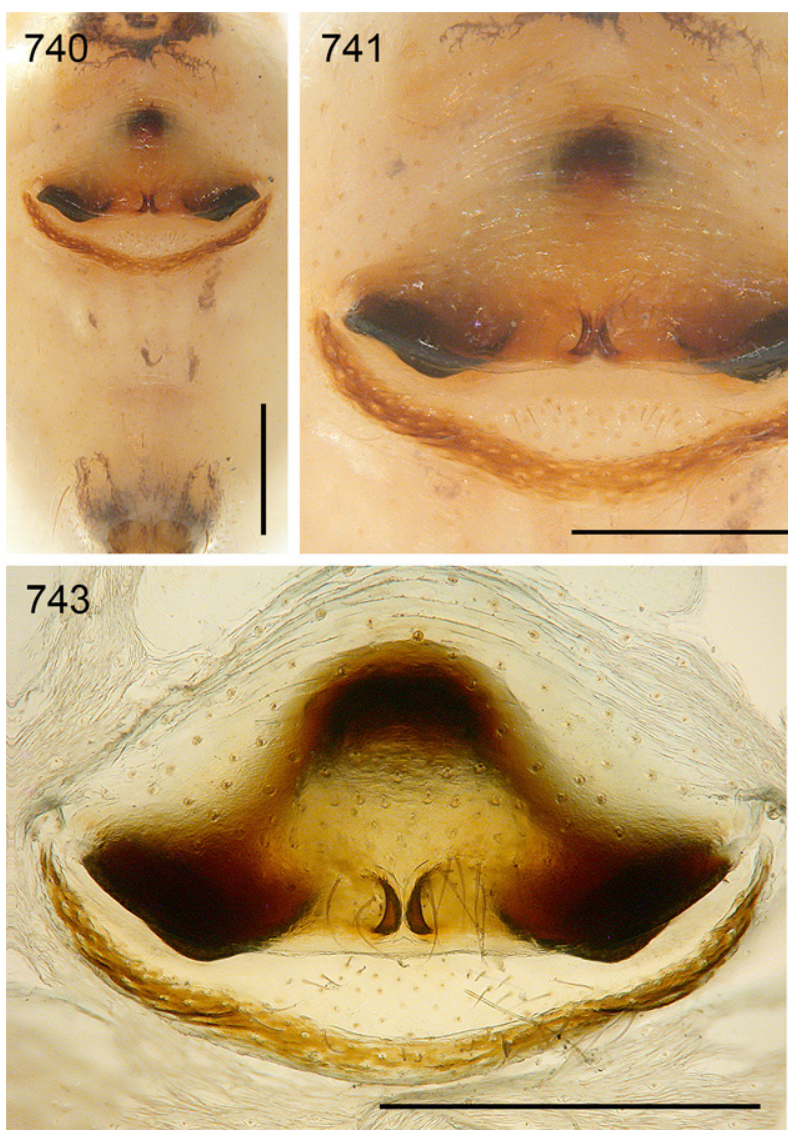
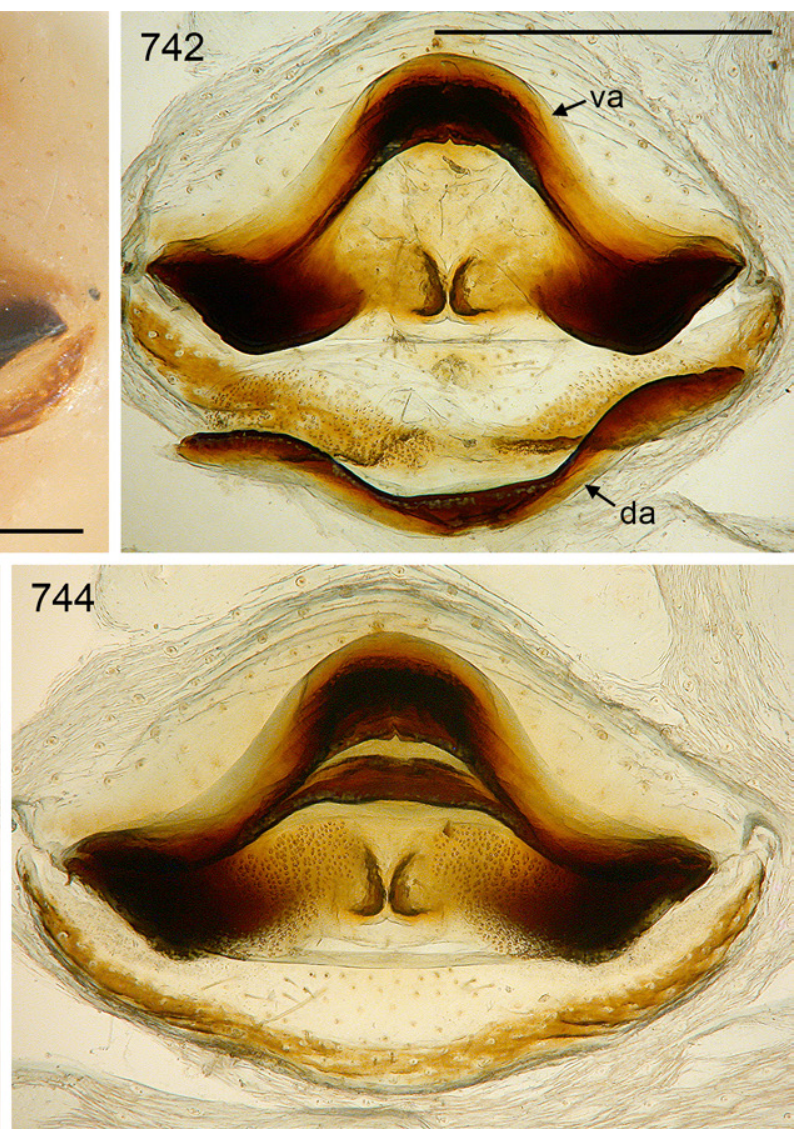

Figs 740-744. Crossopriza parsa sp. nov.; female from Iran, Persepolis (MHNG). 740-741. Abdomen and epigynum, ventral views. 742-744. Cleared female genitalia, dorsal view with dorsal arc tilted backwards, ventral view, and regular dorsal view. Scale bars $=0.5 \mathrm{~mm}$. 
protruding, laterally posteriorly heavily sclerotized with pair of low depressions, medially with pair of pockets on both sides of median rim (distance $30 \mu \mathrm{m}$ ); internal median structure visible in uncleared specimens; posterior plate light brown, short but wide. Internal genitalia (Figs 736-737, 742-744) with large elongate pore plates converging anteriorly, dorsal arc slender, ventral arc medially widened and strongly sclerotized, apparently without pouch-like modification.

Females from S of Fahlyān, Dasht-e Arjân, "Aliâbâd", and Choram appear identical to those from Persepolis. In females from "Barm-e-peere-Ghaibi" the lateral epigynal sclerites are less strongly developed.

\section{Distribution}

Known from several localities in Iran, Fars and Kohgiluyeh and Boyer-Ahmad Provinces (Fig. 353A).

\section{Crossopriza srinagar sp. nov.} urn:lsid:zoobank.org:act:A0DF1162-C8E6-457B-B627-028E156551C6

Figs 353A, 745-764

\section{Diagnosis}

Distinguished from known congeners by details of male palp (Figs 749, 752; long and slender procursus slightly curved towards ventral; distal bulbal sclerite with prominent ventral process and distinctive set of prolateral apophyses; palp similar to C. parsa sp. nov.), by male chelicerae (Figs 750-751; median pair of apophyses in rather lateral position, lateral pair accompanied by additional proximal pair of humps), and by epigynum (Fig. 758; relatively long, with pair of large pockets).

\section{Etymology}

The species name refers to the type locality; noun in apposition.

\section{Type material}

\section{Holotype}

INDIA - Jammu and Kashmir • ${ }^{\lambda}$; Srinagar; $34.10^{\circ}$ N, $74.80^{\circ}$ E; 1800 m a.s.1.; 3 Sep. 1962; E. Kullmann leg.; ZFMK Ar 22449.

\section{Other material examined}

INDIA - Jammu and Kashmir • 1 \&, 1 juv.; same collection data as for holotype; ZFMK Ar 22450.

Assigned tentatively (see description of female below)

PAKISTAN - Islamabad Capital Territory • 1 क; "N env. of Islamabad, National Park"; $33.75^{\circ} \mathrm{N}$, $73.06^{\circ}$ E; 800 m a.s.1.; 10 Jul. 2003; S.V. Ovchinnikov leg.; ZMMU. - Khyber Pakhtunkhwa • 1 \%; 7 km NE of Gobor-o-Bakh [Gobor Bakth]; $36.117^{\circ}$ N, $71.383^{\circ}$ E; 1 Aug. 2004; S.V. Ovchinnikov leg.; ZMMU.

\section{Description}

\section{Male (holotype)}

Measurements. Total length 3.4, carapace width 1.4. Distance PME-PME $115 \mu \mathrm{m}$; diameter PME $100 \times 110 \mu \mathrm{m}$; distance PME-ALE $50 \mu \mathrm{m}$; diameter AME $90 \mu \mathrm{m}$; distance AME-AME $40 \mu \mathrm{m}$. Leg 1: $29.0(8.4+0.6+7.8+10.2+2.0)$, tibia $2: 5.3$, tibia $3: 3.8$, tibia $4: 4.4$; tibia $1 \mathrm{~L} / \mathrm{d}: 52$; femora $1-4$ diameters: $0.25,0.20,0.19,0.21$. 
Color (in ethanol). Carapace ochre-yellow, anteriorly in median pit and posterior triangle light brown; sternum brown with dark brown radial marks; legs ochre-yellow, without darker rings, with black lines on femora and tibiae; abdomen pale gray, with some dark marks dorsally and laterally; ventrally with broken dark band, with two dark parallel longitudinal marks behind gonopore and lighter brown mark medially.

Body. Habitus similar to C. sahtan sp. nov. (cf. Fig. 391). Ocular area slightly raised. Deep thoracic pit and pair of furrows diverging from pit toward posterior margin. Clypeus unmodified, only rim slightly more sclerotized than in female. Sternum wider than long (1.05/0.70), unmodified. Abdomen slightly elongated, dorso-posteriorly slightly angular.

Chelicerae. As in Figs 750-751, with three pairs of processes: lateral apophyses slightly sculptured; indistinct frontal lateral humps (arrows in Figs 750-751); and large frontal medial processes, each with one large modified hair at tip; distance between tips of modified hairs $270 \mu \mathrm{m}$; lateral stridulatory ridges fine but visible in dissecting microscope.

PALPS. As in Figs 745-747; coxa with rounded retrolateral hump; trochanter barely modified; femur short, distally strongly widened, with rounded ventral protrusion, proximally with prolateral stridulatory pick, with weak retrolateral transversal line, without retrolateral proximal process; femur-patella joints shifted toward prolateral side; tibia-tarsus joints shifted toward retrolateral side; tarsus without macrotrichia; procursus (Figs 748-749) long and slender, slightly curved towards ventral, narrowing distally, with low prolateral hump proximally set with many hairs, long dorsal hairs mostly straight, only $2-3$ hairs weakly curved, procursus tip simple, with long ventral sclerite, prolateral-dorsal sclerite, and two small retrolateral sclerotized elements; genital bulb (Figs 752-754) with simple basal sclerite connected to distal (main) sclerite, sperm duct opening not seen; distal sclerite with retrolateral ridge, with distinctive set of two large prolateral apophyses and small ridge leading to prominent ventral process.

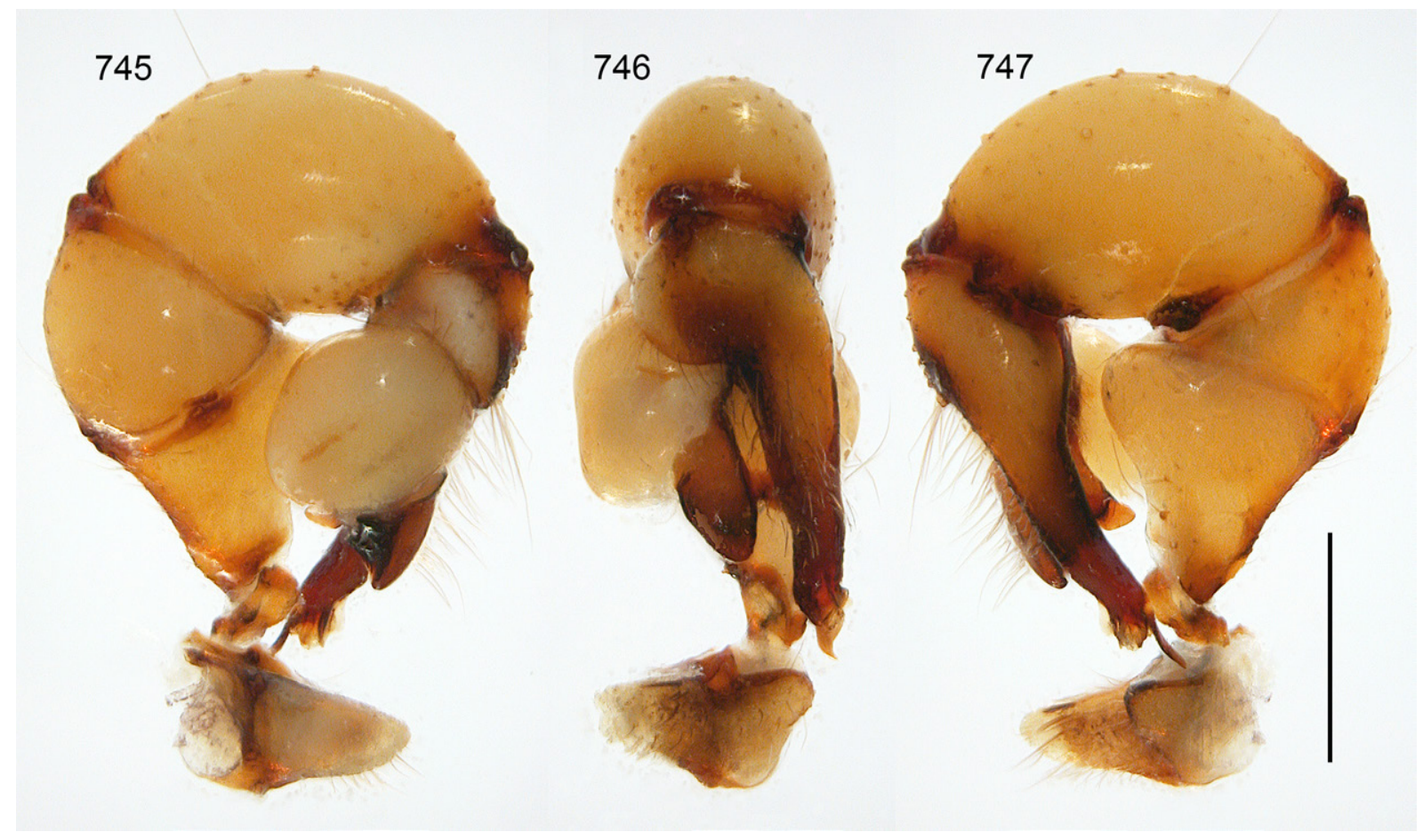

Figs 745-747. Crossopriza srinagar sp. nov.; holotype male from India, Srinagar (ZFMK Ar 22449); left palp, prolateral, dorsal, and retrolateral views. Scale bar $=0.5 \mathrm{~mm}$. 


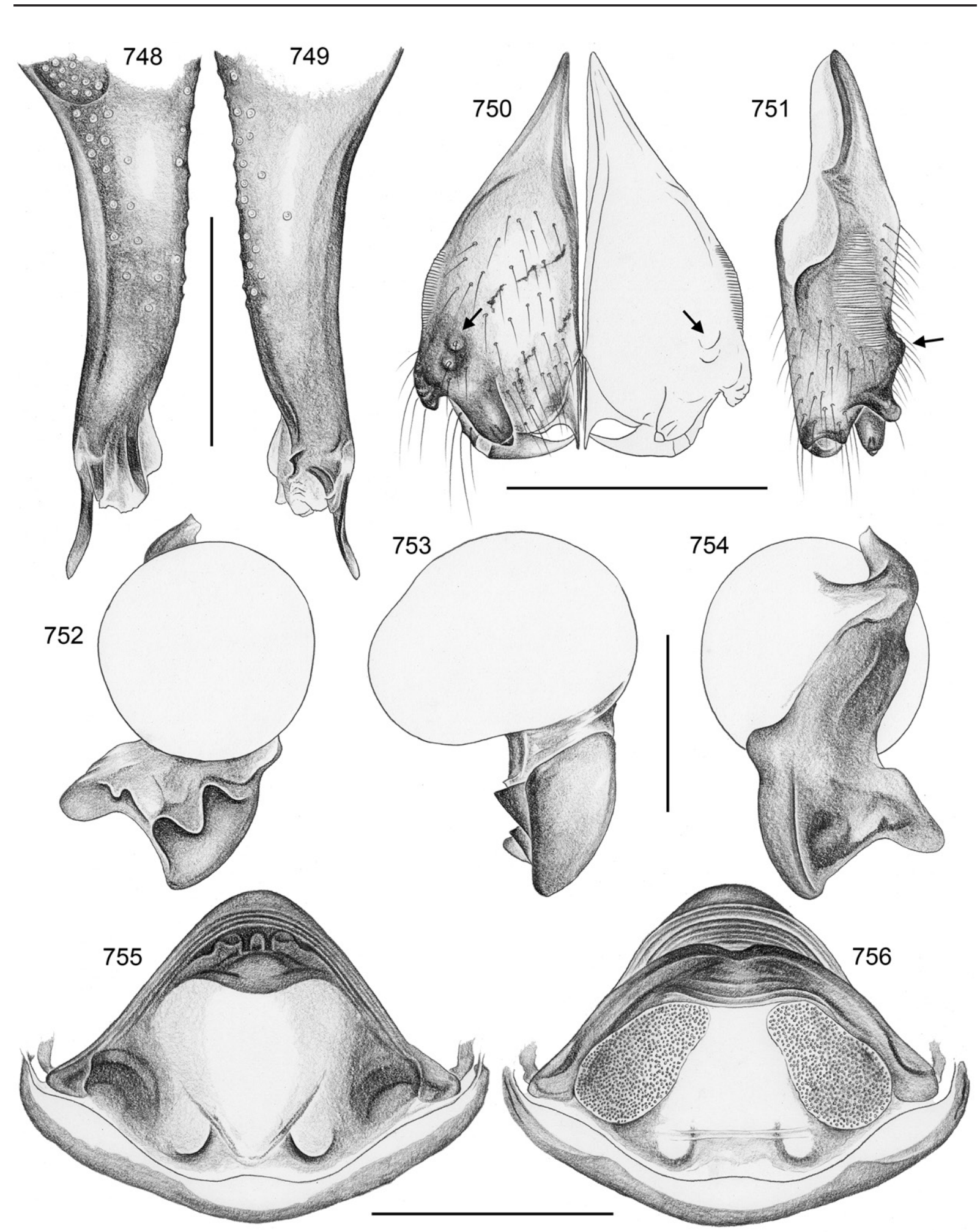

Figs 748-756. Crossopriza srinagar sp. nov.; holotype male and female from India, Srinagar (ZFMK Ar 22449, Ar 22450). 748-749. Left procursus, prolateral and retrolateral views. 750-751. Male chelicerae, frontal and lateral views; arrows: frontal humps near lateral apophyses. 752-754. Left genital bulb, prolateral, dorsal, and retrolateral views. 755-756. Cleared female genitalia, ventral and dorsal views. Scale bars: $748-749,752-754=0.3 \mathrm{~mm} ; 750-751,755-756=0.5 \mathrm{~mm}$. 

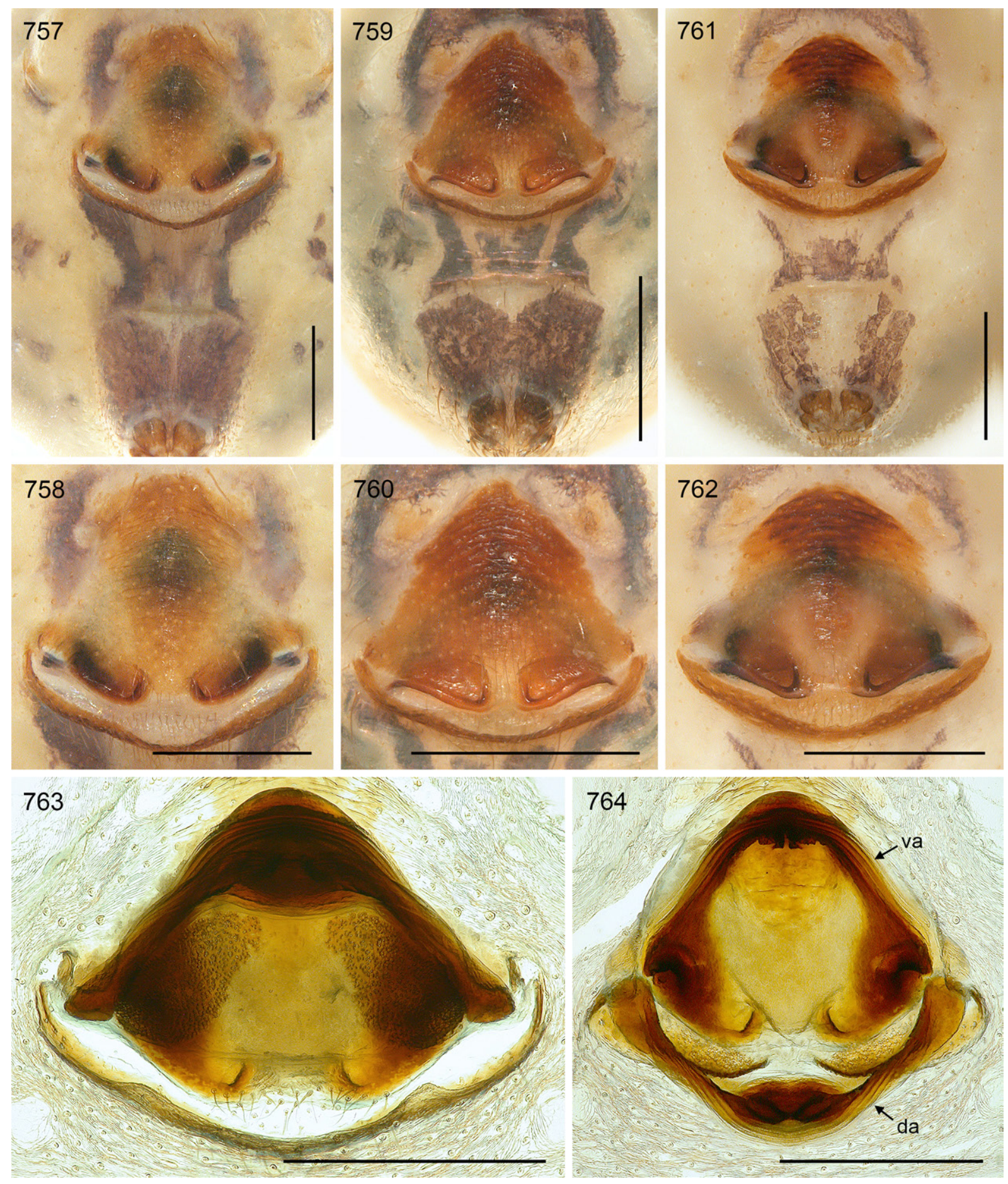

Figs 757-764. Crossopriza srinagar sp. nov. 757-758. Female from India, Srinagar (ZFMK Ar 22450), abdomen and epigynum, ventral views. 759-760. Female from Pakistan, " $N$ env. of Islamabad, National Park" (ZMMU), abdomen and epigynum, ventral views. 761-762. Female from Pakistan, $7 \mathrm{~km}$ NE Gobor-o-Bakh (ZMMU), abdomen and epigynum, ventral views. 763-764. Female from India, Srinagar (ZFMK Ar 22450), cleared female genitalia, regular dorsal view and dorsal view with dorsal arc tilted backwards. Abbreviations: da $=$ dorsal arc; va $=$ ventral arc. Scale bars $=0.5 \mathrm{~mm}$. 
LEGS. Femur 1 with single row of $\sim 18$ ventral spines; without curved hairs; few vertical hairs; retrolateral trichobothrium of tibia 1 at $4.5 \%$; prolateral trichobothrium absent on tibia 1, present on other leg tibiae; tarsal pseudosegments indistinct/irregular except $1-2$ at tip.

\section{Female}

In general similar to male but without spines on legs, apparently without stridulatory files on chelicerae, and with stridulatory organ consisting of pair of weakly sclerotized but distinct processes posteriorly on carapace and pair of small but distinct light brown plates anteriorly on abdomen. Tibia 1 in female from type locality: 7.7. Epigynum as in Figs 757-758; main epigynal plate almost as long as wide, weakly protruding, with pair of large dark pockets (distance $\sim 200 \mu \mathrm{m}$ ), anteriorly with light brown median part and ochre-whitish lateral parts, internal median structure visible in uncleared specimens; posterior plate brown, short but wide. Internal genitalia (Figs 755-756, 763-764) with large elongate pore plates slightly narrowing and converging anteriorly, dorsal arc with modified central area and widening laterally, ventral arc also with median modification, with indistinct ventral pocket (visible in lateral view only).

The two females from Pakistan have very similar but also slightly deviating epigyna; they are therefore assigned tentatively. Female from Islamabad with more evenly sclerotized anterior epigynal plate and pockets closer together (Figs 759-760; distance $\sim 95 \mu \mathrm{m}$ ); tibia 1: 4.5. Female from $7 \mathrm{~km}$ NE Gobor-oBakh with relatively (and absolutely) wider anterior epigynal plate (Figs 761-762); distance between pockets $\sim 200 \mu \mathrm{m}$; tibia 1: 5.6.

\section{Distribution}

Known from northern India (Jammu and Kashmir) and from specimens assigned tentatively from Pakistan (Islamabad Capital Territory, Khyber Pakhtunkhwa) (Fig. 353A).

$$
\begin{gathered}
\text { Crossopriza sengleti sp. nov. } \\
\text { urn:1sid:zoobank.org:act:DE567373-B1F1-499F-8F93-33B3695DDCF5 }
\end{gathered}
$$

Figs 353A, 765-782

\section{Diagnosis}

Easily distinguished from known congeners by modified male clypeus (Fig. 768; single median process); also by details of male palp (Figs 769-773; distinctive shapes of procursus tip and of ventral sclerite; distal bulbal sclerite with strong prolateral fold and small ventral pointed process), by male chelicerae (Fig. 774; distinctive position of small lateral pair of apophyses), and by epigynum (Fig. 779; wide median ridge).

\section{Etymology}

The species name honors Swiss arachnologist Antoine Senglet (1927-2015), who collected a large part of the specimens treated in this paper.

\section{Type material}

Holotype

IRAN $-{ }^{\top}$; Fars, Seridjan (Serizjan); $28.967^{\circ}$ N, 52.567 E (see Remark below); 30 Aug. 1975; A. Senglet leg.; MHNG.

\section{Other material examined}

IRAN • 1 , 2 juvs; same collection data as for holotype; MHNG $\bullet 1$, 1 ,, 4 juvs; same locality as for holotype; $28.95^{\circ}$ N, $52.55^{\circ}$ E (see Remark below); 7 Jun. 1974; A. Senglet leg.; MHNG. 


\section{Remark}

The coordinates on Senglet's label in the type vial denote a site in the hills $\sim 2 \mathrm{~km} \mathrm{NE}$ of the village of Seridjan. The coordinates in the second vial are of the village itself, which was flooded when the Tangab Dam was built ( 2003-2007) (F. Mozzaffarian, pers. com., Jun. 2021). This collecting site may thus no longer be accessible.

\section{Description}

\section{Male (holotype)}

Measurements. Total length 4.0, carapace width 1.6. Distance PME-PME $90 \mu \mathrm{m}$; diameter PME $90 \times$ $110 \mu \mathrm{m}$; distance PME-ALE $25 \mu \mathrm{m}$; diameter AME $85 \mu \mathrm{m}$; distance AME-AME $35 \mu \mathrm{m}$. Leg 1: 39.0 $(11.3+0.7+10.5+14.1+2.4)$, tibia $2: 7.2$, tibia $3: 5.3$, tibia $4: 6.1$; tibia $1 \mathrm{~L} / \mathrm{d}: 66$; femora $1-4$ diameters: $0.23,0.20,0.19,0.20$.

CoLOR (in ethanol). Carapace monochromous ochre-yellow; sternum laterally ochre-yellow, medially light brown; legs ochre-yellow, without darker rings, with distinct black lines on femora and (few) on tibiae; abdomen pale gray, with many internal whitish marks and few indistinct small dark marks posteriorly; ventrally without dark median band.

Body. Habitus similar to C. sahtan sp. nov. (cf. Fig. 391). Ocular area slightly raised. Deep thoracic pit and pair of furrows diverging from pit toward posterior margin. Clypeus with light median process (arrow in Fig. 768), 0.18 long; rim of clypeus slightly more sclerotized than in female. Sternum wider than long $(0.10 / 0.75)$, unmodified. Abdomen oval, dorso-posteriorly slightly angular.

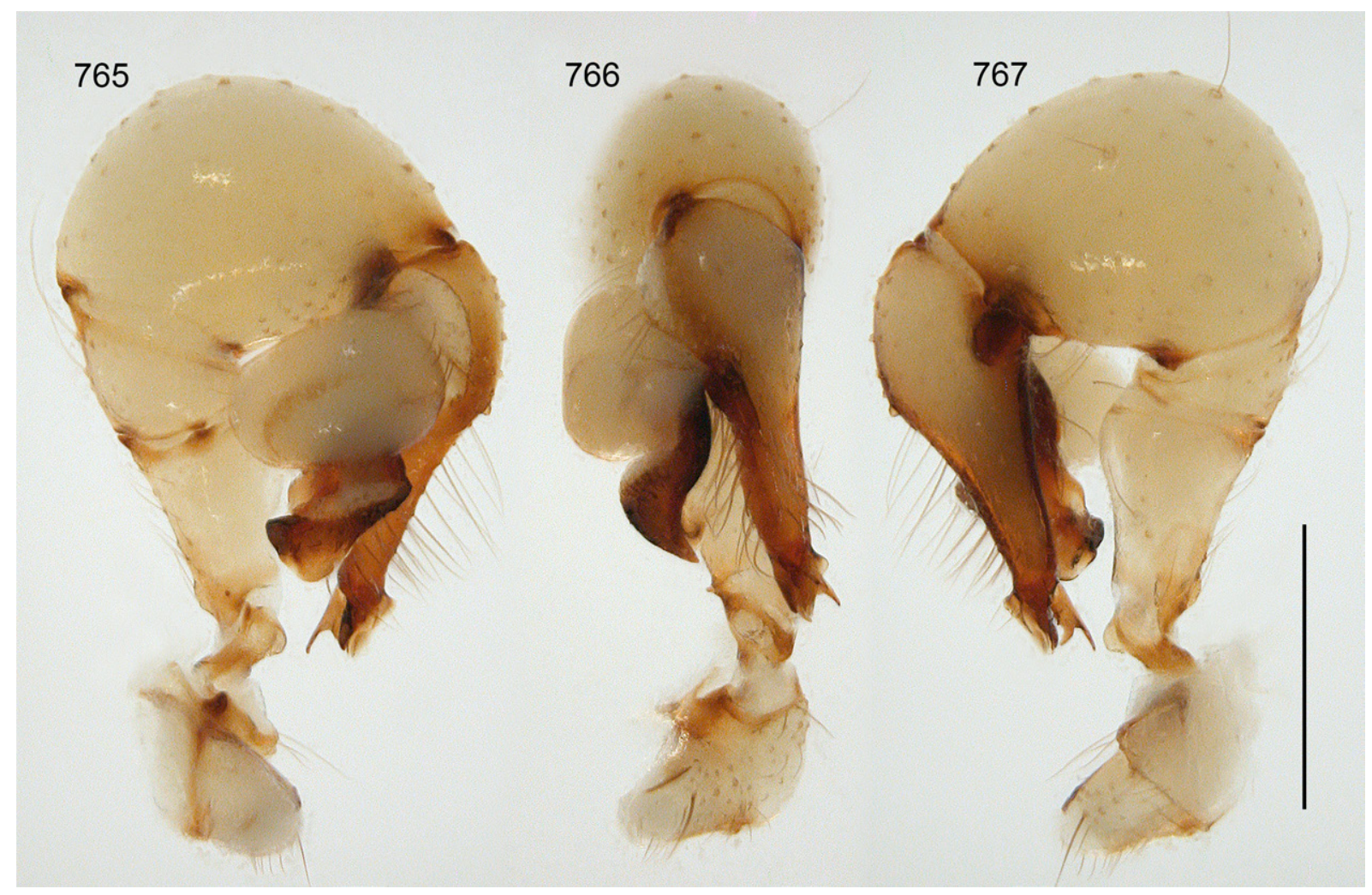

Figs 765-767. Crossopriza sengleti sp. nov.; non-type male from Iran, Fars, Seridjan (MHNG); left palp, prolateral, dorsal, and retrolateral views. Scale bar $=0.5 \mathrm{~mm}$. 


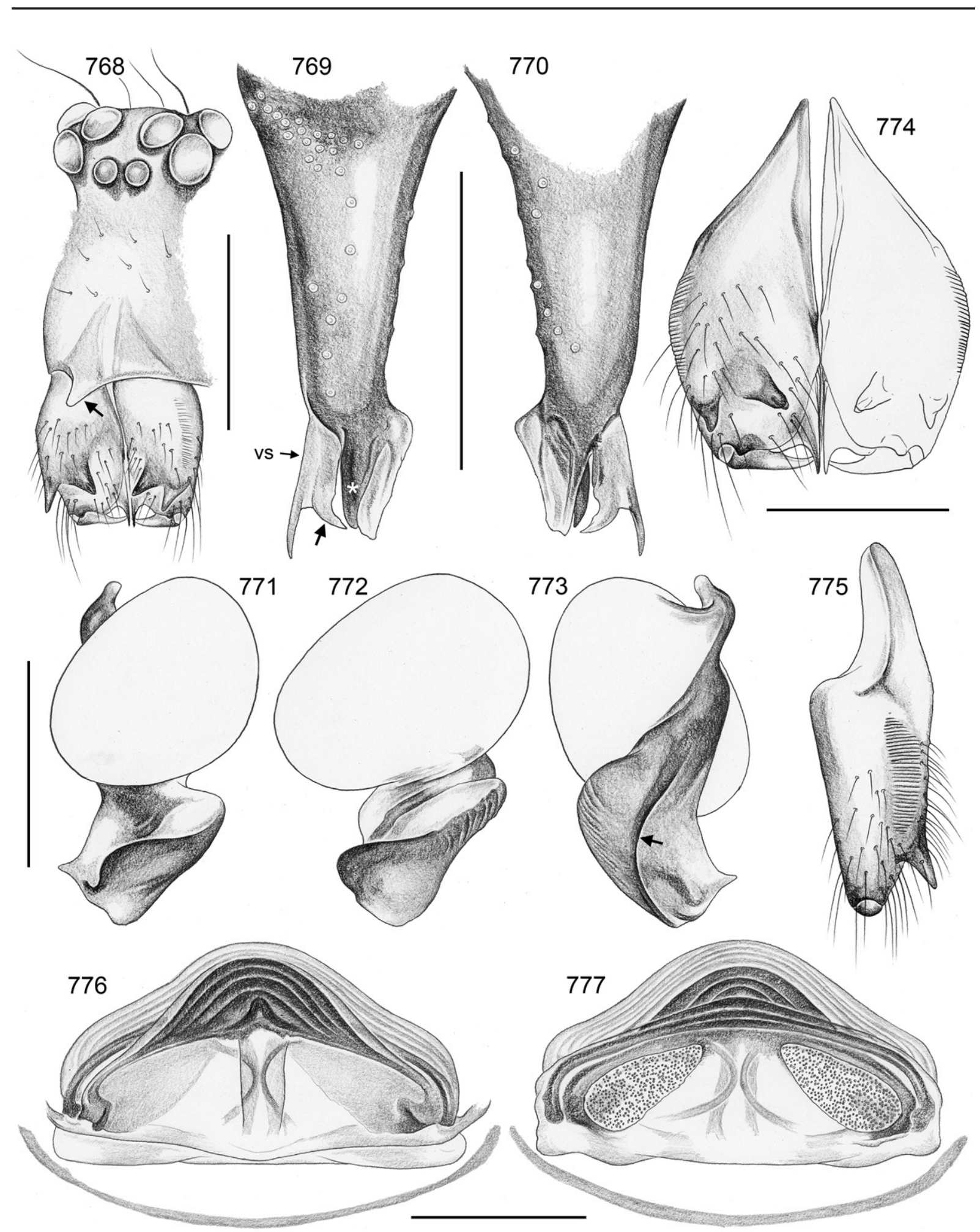

Figs 768-777. Crossopriza sengleti sp. nov.; non-type male and female from Iran, Fars, Seridjan (MHNG). 768. Male ocular area, clypeus, and chelicerae; arrow: clypeus process. 769-770. Left procursus, prolateral and retrolateral views; arrow: branch of ventral sclerite; asterisk: distinctive prolateral apophysis. 771-773. Left genital bulb, prolateral, dorsal, and retrolateral views; arrow: retrolateral ridge. 774-775. Male chelicerae, frontal and lateral views. 776-777. Cleared female genitalia, ventral and dorsal views. Scale bars: $768=0.5 \mathrm{~mm} ; 769-777=0.3 \mathrm{~mm}$. 
Chelicerae. As in Figs 774-775, with two pairs of apophyses, lateral pair simple, pointing towards distal, median pair with one large modified cone-shaped hair each; distance between tips of modified hairs $105 \mu \mathrm{m}$; lateral stridulatory ridges fine but visible in dissecting microscope.

PALPS. As in Figs 765-767; coxa with rounded retrolateral hump; trochanter barely modified; femur distally only weakly widened, proximally with prolateral stridulatory pick, without retrolateral transversal line, without retrolateral proximal process; femur-patella joints shifted toward prolateral side; tibia-tarsus joints shifted toward retrolateral side; tarsus without macrotrichia; procursus (Figs 769-770) straight and slightly tapering towards distally, proximally on prolateral side with low hump set with numerous long hairs, dorsal hairs not curved, procursus tip with distinctly bifid and weakly sclerotized ventral sclerite, distinctive prolateral apophysis (asterisk in Fig. 769), and further membranous processes; genital bulb (Figs 771-773) with simple basal sclerite connected to distal (main) sclerite, sperm duct opening not seen; distal sclerite with strong retrolateral ridge, with strong prolateral fold, and small and weakly sclerotized ventral pointed process.

LEGS. Femur 1 with single row of $\sim 25$ ventral spines; without curved hairs; few vertical hairs; retrolateral trichobothrium of tibia 1 at $3 \%$; prolateral trichobothrium absent on tibia 1, present on other leg tibiae; tarsal pseudosegments not seen.

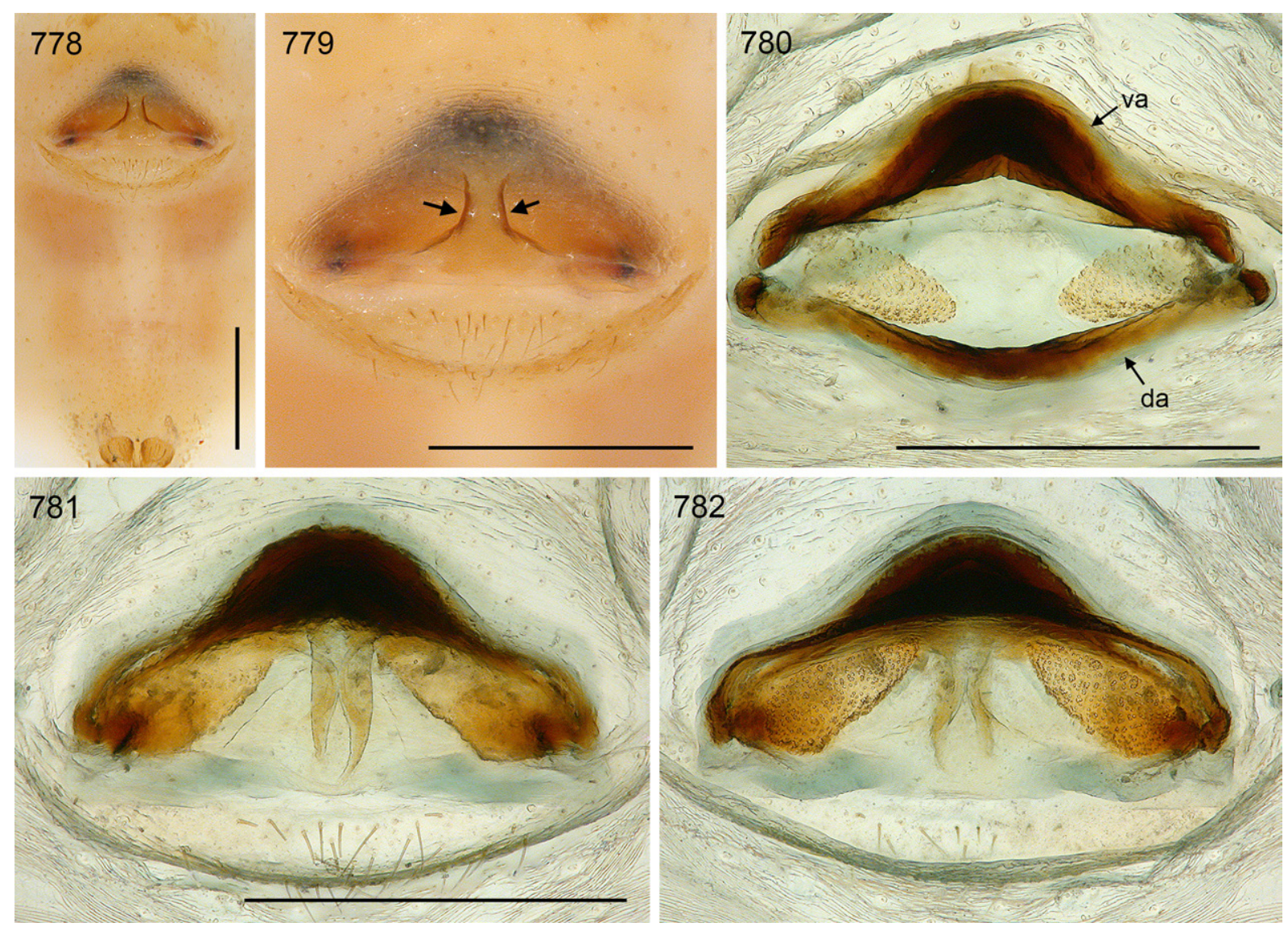

Figs 778-782. Crossopriza sengleti sp. nov.; female from Iran, Fars, Seridjan (MHNG). 778779. Abdomen and epigynum, ventral views; arrows: pockets. 780-782. Cleared female genitalia, dorsal view with dorsal arc tilted backwards, ventral view, and regular dorsal view. Abbreviations: da $=$ dorsal arc; $\mathrm{va}=$ ventral arc. Scale bars $=0.5 \mathrm{~mm}$. 
Male (variation)

Tibia 1 in second male: 9.0.

\section{Female}

In general similar to male but without spines on legs, apparently without stridulatory files on chelicerae, and with stridulatory organ consisting of pair of weakly sclerotized small processes posteriorly on carapace and pair of indistinct plates anteriorly on abdomen. Tibia 1 in two females: 8.5, 9.1. Epigynum as in Figs 778-779; main epigynal plate roughly triangular, weakly protruding; with pair of large pockets close to median line on both sides of wide median ridge (distance between pockets $30 \mu \mathrm{m}$ ); internal sclerotized arc medially visible in uncleared specimens; posterior plate indistinct, weakly sclerotized, short but wide. Internal genitalia (Figs 776-777, 780-782) with drop-shaped pore plates narrowing and converging anteriorly, dorsal arc simple, ventral arc medially strongly widened and sclerotized, apparently without median pouch or pocket.

\section{Distribution}

Known from type locality only, in Iran, Fars Province (Fig. 353A).

Crossopriza lyoni (Blackwall, 1867)

Figs 351, 702-705, 783-805

Pholcus Lyoni Blackwall, 1867: 392.

Crossopriza brasiliensis Mello-Leitão, 1935: 94, fig. 13a-c. Synonymized in Huber et al. 1999.

Crossopriza mucronata Mello-Leitão, 1942: 389, figs 1-2. Synonymized in Huber et al. 1999.

Crossopriza françoisi Millot, 1946: 154, figs 29a-b, 30b. Synonymized in Huber et al. 1999.

Crossopriza stridulans Millot, 1946: 156, fig. 31a-b. Synonymized in Huber et al. 1999.

Crossopriza nigrescens Millot, 1946: 155, fig. 30a. syn. nov.

Tibiosa caracensis González-Sponga, 2006: 11, pl. 1 figs 1-10. Synonymized in Huber 2009b.

Tibiosa casanaimensis González-Sponga, 2006: 14, pl. 2 figs 1-9. Synonymized in Huber 2009b.

Tibiosa coreana González-Sponga, 2006: 17, pl. 3 figs 1-9. Synonymized in Huber 2009b.

Tibiosa guayanesa González-Sponga, 2006: 20, pl. 4 figs 1-9. Synonymized in Huber 2009b.

Tibiosa moraensis González-Sponga, 2006: 23, pl. 5 figs 1-9. Synonymized in Huber 2009b.

Smeringopus lyonii - Thorell 1895: 70; 1898: 274.

Crossopriza lyoni - Pocock 1900: 240. — Strand 1907: 125. — Simon 1909: 80. — Sherriffs 1919: 229.

—Chamberlin 1924: 4. - Dyal 1935: 168, pl. 15 figs 90-96. - Zhu \& Wang 1963: 462, figs 4, 7-8.

— Chrysanthus 1967: 96, figs 20-24. — Tikader \& Biswas 1981: 18, pl. 1 figs 13-15. - Yaginuma 1982: 15; 1986: 31, fig. 17.5a, e, p. — Kim 1988: 35, figs 1-6. — Chikuni 1989: 29, fig. 7. - Chen \& Zhang 1991: 72, fig. 61.1-3. — Edwards 1993: 1, figs 1-2. — Majumder \& Biswas 1993: 2. — Roth 1994: 145. — Huber et al. 1999: 1, figs 1-12; 2014a: 6. — Song et al. 1999: 52, fig. 22h-o. — Huber 2000: fig. 98; 2001: 136; 2009b: 65; 2014: 140; 2019: 51. — Murphy \& Murphy 2000: 247. — Irie 2001: 7, figs 1-2(1-8); 2009: 106, figs (2-2-13) 3-5, pl. 4 fig. 2. — Van Keer \& Van Keer 2001: 82; 2004: 79. — Guarisco \& Cutler 2003: 105, fig. 1. — Van Keer 2007: 53. — Jäger 2007: 34. — Colmenares García 2008: 88, fig. 2a-c. - Beatty et al. 2008: 9, figs 17-18, 46-47. — Carvalho \& Avelino 2010: 6. — Huber \& Warui 2012: 8. — Jäger et al. 2012: 80, fig. 3. — Yin et al. 2012: 163, fig. 30. - Huber \& Kwapong 2013: 7, fig. 16. - Tong 2013: 61. — Raychaudhuri \& Saha 2015: 85, pl. 19 figs 514-518. — Bauer et al. 2016: 4, figs 1-7. — Huber \& Villarreal 2020: 57.

Crossopriza stridulans - Roth 1985: B33-1. 


\section{Justification of new synonymy}

Even though the type material of $C$. nigrescens (a single juvenile specimen) is apparently lost, the name is here synonymized, for three reasons. First, all adult Madagascan specimens of Crossopriza studied are C. lyoni (Huber et al. 1999; Irie 2001; herein); second, the type of C. nigrescens was collected in a house, suggesting a synanthropic species; third, the character that Millot (1946) considered diagnostic (the dark coloration) varies considerably among specimens, even within a locality.

\section{Diagnosis}

Distinguished from known congeners by details of male palp (Figs 786-791; procursus ventral sclerite with transparent retrolateral branch; procursus tip with wide and rounded dorsal process; distal bulbal sclerite with distinctive row of apophyses on prolateral side); from the similar C. surobi sp. nov. (identical male chelicerae) also by shorter epigynum and elongate pore plates (Figs 794-795); from the similar C. maculipes also by male chelicerae (Fig. 793; lateral apophyses in lateral view long) and by female internal genitalia (Fig. 795; pore plates elongated).

\section{Type material}

\section{Syntypes of Pholcus lyoni}

INDIA • $\overbrace{}^{\lambda} \partial^{\wedge}$,,++ (unspecified number); Delhi; $28.6^{\circ} \mathrm{N}, 77.2^{\circ} \mathrm{E}$; Meerut; $28.97^{\circ} \mathrm{N}, 77.70^{\circ} \mathrm{E}$; and Agra; $27.2^{\circ} \mathrm{N}, 78.0^{\circ} \mathrm{E}$; dates and collector(s) unknown; possibly lost.

\section{Syntypes of Crossopriza brasiliensis}

BRAZIL -1 ते, 1 q, 1 juv. (examined); Bahia, Paraguassú; $12.80^{\circ} \mathrm{S}, 38.87^{\circ} \mathrm{W}$; date unknown; O. Leonardos leg.; MNRJ 42313.

\section{Syntypes of Crossopriza mucronata}

ARGENTINA • 1 \% , 1 juv. (examined); Santiago del Estero, Beltrán; $27.83^{\circ} \mathrm{S}, 64.06^{\circ} \mathrm{W}$; date unknown; M. Birabén leg.; MLP $15800 \bullet 1$ क (not examined) (see Huber et al. 1999); same collection data as for preceding; with P. Brignoli`s (unpublished) lectotype designation; MLP.

\section{Holotype of Crossopriza francoisi}

MADAGASCAR • $\widehat{O}$ (examined); Mahajanga, Maevatanana; $16.95^{\circ} \mathrm{S}, 46.83^{\circ} \mathrm{E}$; Jul. 1945; J. Millot leg.; in bathroom; MNHN.

\section{Holotype of Crossopriza stridulans}

MADAGASCAR • $q$ (examined); Mahajanga, Majunga [= Mahajanga]; $15.71^{\circ} \mathrm{S}, 46.32^{\circ} \mathrm{E}$; date and collector unknown; MNHN.

\section{Holotype of Crossopriza nigrescens}

MADAGASCAR • 1 juv.; Antsiranana, Ankarana Sud; $12.97^{\circ}$ S, $49.14^{\circ}$ E; 1945; J. Millot leg.; apparently lost (not found in MNHN).

\section{Tibiosa spp.}

VENEZUELA. See Huber \& Villarreal (2020).

\section{Other material examined}

Arranged from West to East and (within longitudes) from North to South.

USA - California • 1 क ; Orange County, Orange; $33.80^{\circ}$ N, $117.85^{\circ}$ W; 23 Feb. 2000; J. Coleman leg.; CAS 9027397. - New Jersey • 1 o; Somerset County, Neshanic; $40.498^{\circ} \mathrm{N}, 74.720^{\circ} \mathrm{W}$; date unknown; 
E.S. Gaffney leg.; AMNH. - Texas • 1 J 3 , 3 q ; ; Brazos County, College Station; $30.63^{\circ}$ N, $96.33^{\circ}$ W; date unknown; R.G. Breene leg.; AMNH.

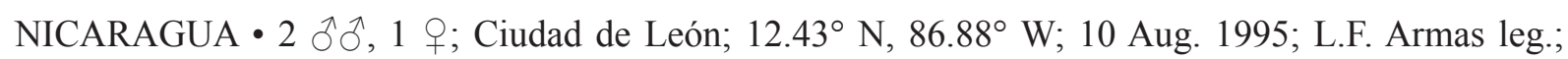
in house; MELN 1 juv.; Managua, Laguna Xiloa; $12.209^{\circ} \mathrm{N}, 86.320^{\circ} \mathrm{W} ; 13$ Jul. 1995; L.F. Armas, J.M. Maes, and J.T. Goodwin leg.; MELN.

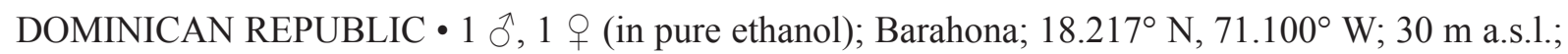
9 Dec. 2007; B.A. Huber leg.; in building; ZFMK Hai103.

COLOMBIA • 1 Oे, 1 क; Meta, Puerto Lleras, Lomalinda; $3.305^{\circ} \mathrm{N}, 73.364^{\circ} \mathrm{W} ; 300 \mathrm{~m}$ a.s.1.; Mar.

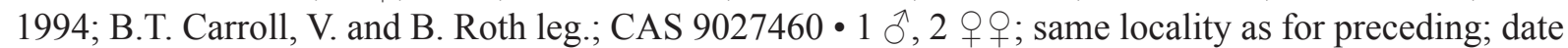
unknown; Roth leg.; CAS 9027261 • 1 q; same locality as for preceding; May 1987; B.T. Carroll leg.; CAS 9027465.

VENEZUELA • approximately $40 \hat{\jmath} \widehat{\partial}, 40$ q $\bigcirc$; see Huber \& Villarreal (2020).

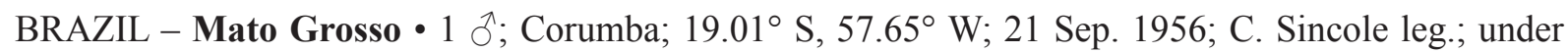
rocks; CAS 9027331. - Mato Grosso do Sul • 1 \%; Upper Paraguay River, Porto Esperança; $19.61^{\circ} \mathrm{S}$, $57.45^{\circ} \mathrm{W}$; date and collector unknown; AMNH. - Minas Gerais - 1 ô, 1 क, 1 juv.; Governador Valadares, Ibituruna; $18.845^{\circ} \mathrm{S}, 41.943^{\circ} \mathrm{W}$; 9 Sep. 1982; L. Sorkin et al. leg.; in building; AMNH 1 \%; Governador Valadares, northern part of city; $18.84^{\circ} \mathrm{S}, 41.95^{\circ} \mathrm{W}$; 1 Sep. 1982; L. Sorkin and C.E. de Assis Bandeira leg.; AMNH • 1 ỗ, 5 q $о$; Governador Valadares; $18.86^{\circ} \mathrm{S}, 41.95^{\circ} \mathrm{W}$; 26 Mar. 1981; L. Sorkin and T. Spitzman leg.; ceiling in marketplace; AMNH • 2 우; Curvelo; $18.75^{\circ} \mathrm{S}, 44.43^{\circ} \mathrm{W}$; 23 Oct. 1943; F. Pough leg.; AMNH. - Pará • 1 ô, 1 \%; Itaituba; $4.26^{\circ} \mathrm{S}, 56.00^{\circ}$ W; 7 Dec. 1991; A.A. Lise leg.; MCN • 1 Oे, 3 9 \% ; same locality as for preceding; 7 Dec. 1991; F. Guilhow leg.; MCP 4629, 4630. - São Paulo • several $q+$ and juvs (not counted); Jaboticabal; 21.25 ${ }^{\circ}$ S, $48.32^{\circ}$ W; 1979; W. and L. Miller leg.; MCZ.

PARAGUAY • 1 juv.; Boquerón, 19 km N of Filadelfia, Estancia Iparoma; $22.150^{\circ} \mathrm{S}, 60.034^{\circ} \mathrm{W}$; 5 . Oct. 1978; K.L. Anderson leg.; AMNH.

ARGENTINA - Chaco • 1 क ; Resistencia; $27.45^{\circ} \mathrm{S}, 59.00^{\circ} \mathrm{W}$; Oct. 1942; Freiberg leg.; MACN Ar 4347 - 1 क; Parque Nacional Chaco; 26.8081 ${ }^{\circ}$ S, 59.6075 ${ }^{\circ}$ W; 80 m a.s.1.; 11-13 Nov. 2007; C. Grismado et al. leg.; MACN Ar 13662. - La Rioja - 1 q; La Rioja; 29.41 ${ }^{\circ}$ S, 66.86 W; Apr. 1914; E. and P. Boman leg.; AMNH • 1 đ̃, 1 q, 1 juv.; same locality as for preceding; 15 Apr. 1914; Boman et al. leg.;

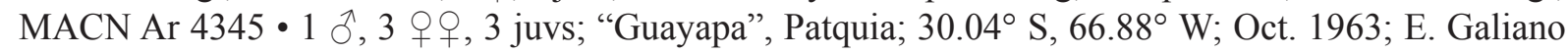
leg.; MACN Ar 20118 • 3 juvs; same locality as for preceding ("Guayapa”); Oct. 1965; Maury leg.; MACN Ar 20033. - Santiago del Estero • 2 우; Santiago del Estero; $27.786^{\circ} \mathrm{S}, 64.266^{\circ} \mathrm{W}$; Nov. 1966; E. Galiano leg.; MACN Ar 19949 • 4 우; same locality as for preceding; Aug. 1939; A. Prasen leg.; MACN Ar 4346 • 1 ; ; same locality as for preceding; 20 Sep. 1963; collector unknown; MACN Ar 20119. - Tucumán • 1 đ̃, 4 우; San Miguel de Tucumán; $26.81^{\circ}$ S, 65.22 W; 1-15 May 1950; M.L. Aczel leg.; AMNH.

GERMANY・ 1 §, 2 우우 see Bauer et al. (2016).

SENEGAL • 1 ; ; see Huber \& Kwapong (2013).

THE GAMBIA • 1 §, 4 우우 see Huber \& Kwapong (2013). 


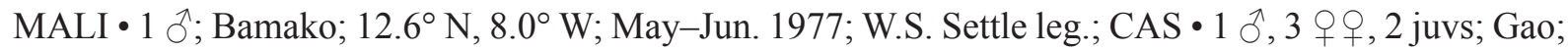
$16.275^{\circ} \mathrm{N}, 0.050^{\circ} \mathrm{W} ; 23-25$ Nov. 1948; B. Malkin leg.; AMNH $3 \partial^{\lambda} \partial^{\lambda}, 9$ q $q$; see Huber \& Kwapong (2013).

GUINEA・ 6 ふふぇ, 9 q ; s see Huber \& Kwapong (2013).

SIERRA LEONE • 1 \%; see Huber \& Kwapong (2013).

IVORY COAST • $5 \widehat{\partial} \widehat{\partial}, 14$ q $\propto$; see Huber \& Kwapong (2013).

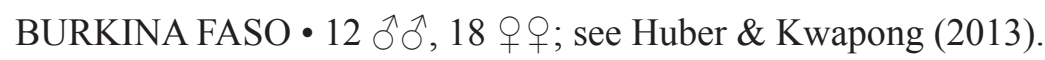

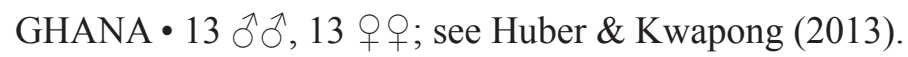

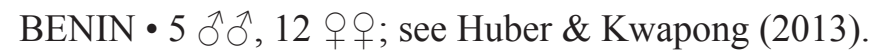

NIGER・ 8 ふึ, 28 qo+; see Huber \& Kwapong (2013).

NIGERIA • 1 đo, 1 क; Oguta Lake; $5.71^{\circ} \mathrm{N}, 6.80^{\circ} \mathrm{E}$; 10-24 Feb. 1992; H. Segers leg.; motel; MRAC 174630 • 1 क; Ile-Ife, Obafemi, Awolowo University Campus; $7.43^{\circ}$ N, 3.89 ${ }^{\circ}$ E; 1991; H. Segers leg.; MRAC 174602 part • 1 q; Lagos, Lagos University; $6.519^{\circ} \mathrm{N}, 3.392^{\circ} \mathrm{E}$; 1973; Usua leg.; BMNH.

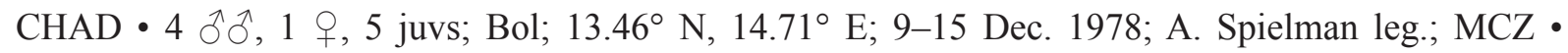
approximately 6 ऽึ, 13 우, + juvs; between Bongor ("Bougar") and N'Djamena ("Fort Lamy"), "depression inondée de la Karaska"; $11.1^{\circ} \mathrm{N}, 15.1^{\circ} \mathrm{E}$; Oct.-Dec. 1965; Y. Brandily leg.; MRAC 132894, 132896, $132897 \bullet$ approximately 9 đ̊ ô, 12 + 9 , + juvs; N'Djamena, Gardolé ("Fort Lamy”); $12.12^{\circ} \mathrm{N}$,

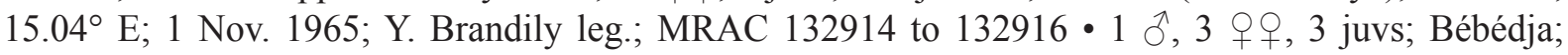
$8.68^{\circ} \mathrm{N}, 16.57^{\circ} \mathrm{E} ; 1-10$ Jul. 1977; G. Ruella leg.; MRAC 151455.

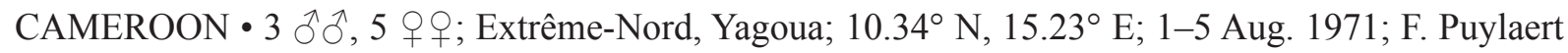
leg.; MRAC 143673・2 우; see Huber et al. (2014b).

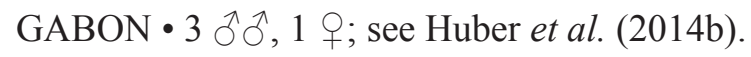

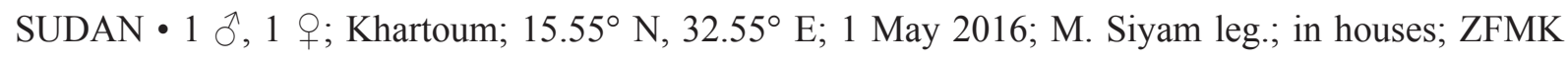
Ar 22451, Ar 22452.

ETHIOPIA $-2 \hat{\jmath}, 8$ 우, 2 juvs (in pure ethanol); Southern Nations, Nationalities and People's Region, Arba Minch, Nech Sar National Park; 6.0002 ${ }^{\circ}$ N, 37.5429 E; 1390 m a.s.1.; 19 Jun. 2011; M. Moradmand, V. Hula, and J. Niedobová leg.; in houses; SMF.

UGANDA • 3 우; see Huber \& Warui (2012).

KENYA 1 1 ; see Huber \& Warui (2012).

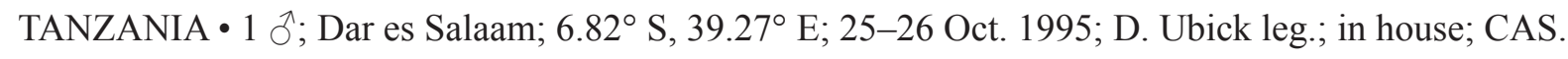

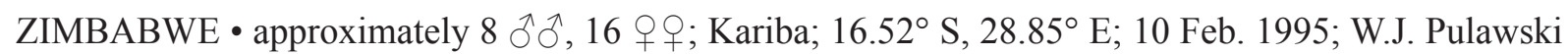
leg.; CAS • 4 ๙ิ $^{\lambda}, 8$ 우, + juvs; Victoria Falls; $17.93^{\circ} \mathrm{S}, 25.83^{\circ} \mathrm{E}$; $1-8$ Feb. 1995; W.J. Pulawski

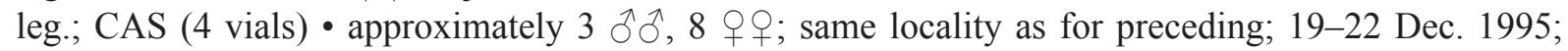

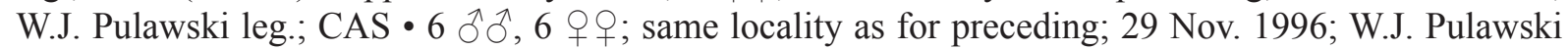
and V. Ahrens leg.; CAS. 
MADAGASCAR - Toliara • 1 đ̊; Toliara; $23.35^{\circ}$ S, $43.67^{\circ}$ E; 17 Sep. 1993; W.E. Steiner and R. Andriamasimanana leg.; USNM • 1 क ; 20 km N of Toliara "on Ferme d'Auberge rd"; $23.429^{\circ} \mathrm{S}$, $43.830^{\circ} \mathrm{E}$ (the coordinates are not $\mathrm{N}$ but SE of Toliara); $200 \mathrm{~m}$ a.s.1.; 15-16 Dec. 1999; E.I. Schlinger and M.E. Irwin leg.; CAS.

SEYCHELLES • 1 O ; Mahé, Bel Ombre; 4.6187 S, 55.3971 E; 30 m a.s.1.; 6 Mar. 2013; C. Hoareau leg.; on wall near buildings; ZFMK Ar 22453.

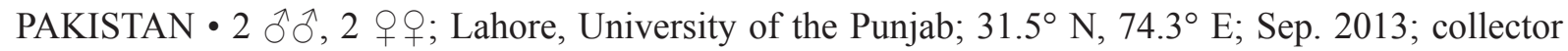
unknown; ZFMK Ar 22454.

INDIA - Andhra Pradesh • 1 9 ; Deccan highlands; $14^{\circ} \mathrm{N}, 77^{\circ} \mathrm{E}$; date and collector unknown; SMF

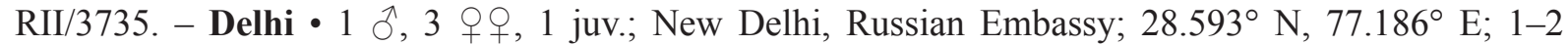
May 1999; Y.M. Marusik leg.; ZFMK Ar 5206. - Haryana • 1 đo; Kasauli; $30.1210^{\circ}$ N, $76.4075^{\circ}$ E; $240 \mathrm{~m}$ a.s.l.; 9 Mar. 2011; P. Jäger leg.; in house and surroundings, farmland; SMF. - Karnataka

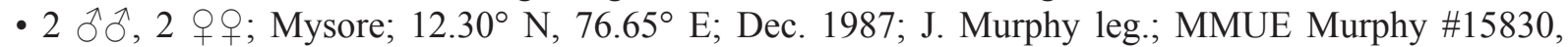
15831. - Maharashtra • 1 क; Pune, Agharkar Research Institute; $18.520^{\circ}$ N, $73.832^{\circ}$ E; 2 Sep. 1992; G.W. Kendrick leg.; WAM 99/1489 • 2 우, 2 juvs; "Kirkoskarvadt, Distr. South Sathra", probably

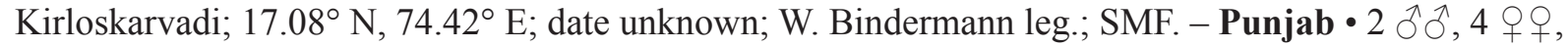
2 juvs; Patiala, University campus; $30.35^{\circ}$ N, $76.45^{\circ}$ E; $24-25$ Jun. 1999; Y.M. Marusik leg.; ZFMK Ar 5205 • 1 영 same collection data as for preceding; 3-8 May 1999; ZFMK Ar 5201 • 7 q $ᄋ$, 2 juvs; Patiala ("Patriala"); $30.35^{\circ} \mathrm{N}, 76.45^{\circ} \mathrm{E}$; date and collector unknown; SMF RII/6617 • 1 §, 1 q; Balanda; 31.0441 ${ }^{\circ}$ N, 75.4265 E; 220 m a.s.1.; 9 Mar. 2011; P. Jäger leg.; in house, courtyard, and farm; SMF. Rajastan • $1 \mathrm{O}^{\curvearrowright}, 1$ \%; Pushkar; $26.49^{\circ} \mathrm{N}, 74.55^{\circ} \mathrm{E}$; 11 Nov. 1989; V. Roth leg.; in building; CAS. - Tamil Nadu • 1 क; Chennai ("Madras"); $13.07^{\circ} \mathrm{N}, 80.25^{\circ}$ E; Aug. 1924; collector unknown; SMF Roewer \#1148 1 O; $10 \mathrm{mi} \mathrm{SW}$ of Gudiyattam; $12.86^{\circ} \mathrm{N}, 78.80^{\circ} \mathrm{E} ; 3$ Apr. 1962; Ross and Cavagnaro leg.; CAS

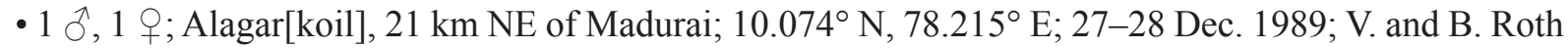

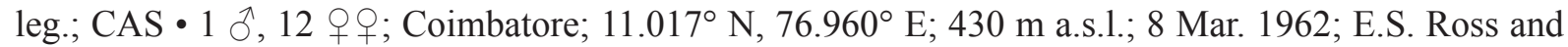
D.Q. Cavagnaro leg.; CAS • 1 \%; $80 \mathrm{~km} \mathrm{~S}$ of Madras, Mahabalipuram; $12.62^{\circ} \mathrm{N}, 80.19^{\circ} \mathrm{E} ; 5$ Mar. 1994; J.M. Waldock leg.; in house; WAM 99/1802 • approximately $6{ }^{\lambda}{ }^{\lambda}, 11$ 우; Vellore; $12.91^{\circ} \mathrm{N}, 79.13^{\circ} \mathrm{E}$;

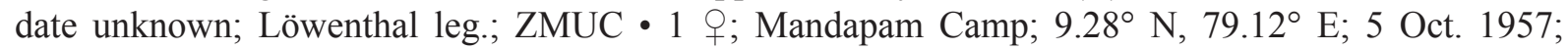
H. Lemche leg.; ZMUC. - Uttarakhand - 1 ô, 1 q; Dehradun City, Forest Research Institute, campus and nearby; $30.342^{\circ}$ N, $78.000^{\circ}$ E; 660 m a.s.1.; 6-13 May 1999; Y.M. Marusik leg.; ZFMK Ar 5204 • 2 우; Dehradun Valley; $30.34^{\circ} \mathrm{N}, 78.00^{\circ} \mathrm{E}$; $700 \mathrm{~m}$ a.s.1.; 4-13 Aug. 1978; collector unknown; ZMUC.

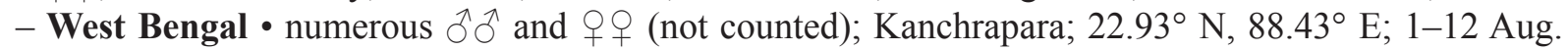
1944; M. Cazier leg.; AMNH.

NEPAL • 1 क; Kathmandu valley, Baneshwar, Ganaba-hal; $27.70^{\circ}$ N, $85.32^{\circ}$ E; 1350 m a.s.1.; 16-20 Aug. 1980; Martens and Schawaller leg.; SMF.

SRI LANKA・ $4 \hat{\jmath} \widehat{\partial}, 12$ q $\odot$; see Huber et al. (1999) and Huber (2019).

MYANMAR • approximately $10 \widehat{\partial} \widehat{\partial}, 20$ q $\odot$; Rangoon; $16.806^{\circ} \mathrm{N}, 96.150^{\circ} \mathrm{E}$; date unknown; Lövendal leg.; ZMUC.

THAILAND • 2 ๙ึ, 1 क; Saraburi Province, $8 \mathrm{mi} \mathrm{SE}$ of Saraburi; $14.45^{\circ} \mathrm{N}, 100.95^{\circ} \mathrm{E} ; 100 \mathrm{~m}$ a.s.l.; 28 Jul. 1962; Ross and Cavagnaro leg.; CAS $\bullet 2$ 우, + juvs; Bangkok (= Krung Thep), Chulalong University; $13.74^{\circ} \mathrm{N}, 100.53^{\circ} \mathrm{E}$; $18-19$ Oct. 1957; N. Meinkoth leg.; MCZ 76654 • 1 \%; Lopburi Province, Khok Samrong District, Khao Wong Phrachan; $14.9633^{\circ} \mathrm{N}, 100.7103^{\circ}$ E; $600 \mathrm{~m}$ a.s.1.; 24 Jun. 2014; P. Schwendinger leg.; dry secondary forest with bamboo; MHNG 1 ¿ ${ }^{\lambda}$; Prachuap Khiri Khan Province, Reclining Buddha Cave; $11.864^{\circ}$ N, $99.823^{\circ}$ E; 70 m a.s.1.; 13 Mar. 2015; B.A. Huber 
and B. Petcharad leg.; among rocks outside cave; ZFMK Ar 23880 • 1 $\hat{\partial}, 1$ (in pure ethanol); same collection data as for preceding; ZFMK Mal367.

LAOS - Vientiane • 1 क ; Vang Vieng, Nam Song; $18.912^{\circ}$ N, $102.456^{\circ}$ E; 230 m a.s.1.; 12 Nov. 2009; P. Jäger and S. Bayer leg.; SMF 11 ô, 2 juvs; Sisathona Distr., Sapanthong Neua; $17.957^{\circ}$ N, $102.629^{\circ}$ E; 170 m a.s.1.; 26 Mar. 2011; L. Nophasead leg.; SMF. - Oudomxai • 1 ô, 2 우; 4 km S of Oudomxai, Lipi provincial protected area; $20.653^{\circ} \mathrm{N}, 102.003^{\circ} \mathrm{E} ; 870 \mathrm{~m}$ a.s.1.; 18 Apr. 2011; L. Nophasead leg.; SMF. - Bolikhamsai • 1 क , 1 juv.; Nam Kading National Protected Area; $18.341^{\circ} \mathrm{N}, 104.144^{\circ} \mathrm{E} ; 23$ Mar. 2011; P. Jäger and L. Nophasead leg.; disturbed secondary forest; SMF • 1 juv.; same collection

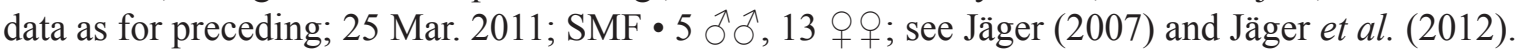

CAMBODIA • 1 juv.; Banteay Meanchey, Sisophon, La Ang Chinchian 5; $13.5905^{\circ}$ N, $102.9442^{\circ}$ E; 16 Jan. 2017; H. Steiner leg.; ZFMK Ar 22455.

VIETNAM • 2 §๊̋, 1 juv.; Kien Giang Prov., Ha Tien, Da Dung mountain; $10.427^{\circ}$ N, $104.476^{\circ}$ E; 6 Jan. 2010; H. Steiner leg.; in cave; SMF • 1 ô;; Kien Giang Prov., Kien Luong, Nui Ca Danh mountain; $10.283^{\circ}$ N, $104.565^{\circ}$ E; 9 Jan. 2010; H. Steiner leg.; SMF • 1 o; Ho Chi Minh (“Saigon”); $10.81^{\circ} \mathrm{N}$, 106.64 E; Nov. 1966-Feb. 1967; P. Fleischer leg.; MCZ.

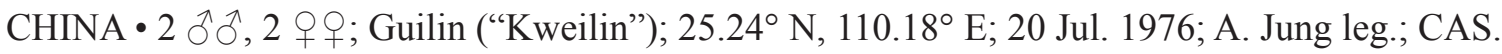

TAIWAN $-2 \widehat{\partial} \widehat{\partial}, 1$ क; Taichung, Tunghai University campus, near Tunghai Lake; $24.1815^{\circ} \mathrm{N}$, $120.6086^{\circ}$ E; 140 m a.s.1.; 17 Jun. 2013; B.A. Huber leg.; at building; ZFMK Ar 23881 • 1 (in pure ethanol); same collection data as for preceding; ZFMK Tai81.

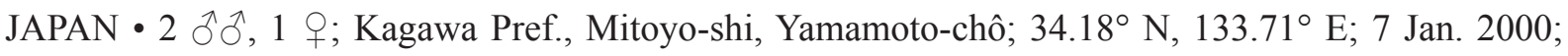
Y. Ihara leg.; ZFMK Ar 5209.

MALAYSIA -1 त, 6 우, 2 juvs; Kedah, Kedah Peak (Gunung Jerai), at foot of mountain; $5.8^{\circ} \mathrm{N}$, $100.4^{\circ} \mathrm{E} ; 6$ Jan. 1985; J.A. Beatty leg.; on buildings; BPBM.

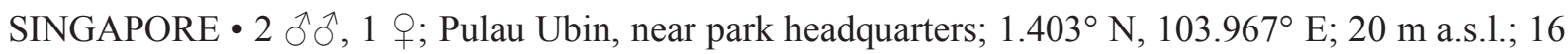
Feb. 2015; B.A. Huber, J. Koh, and D. Court leg.; in building; ZFMK Ar $23882 \bullet 1$ q (in pure ethanol); same collection data as for preceding; ZFMK Mal233.

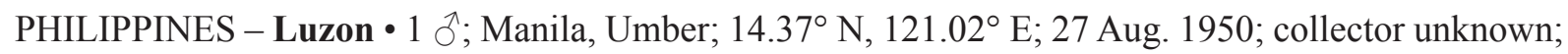
MCZ 76679 • 1 ô, 1 क; Batangas; $13.76^{\circ}$ N, 121.06 E; 4 Aug. 1986; C.K. Starr leg.; in building; AMNH • 1 q; Rizal, Alabang; $14.42^{\circ}$ N, $121.04^{\circ}$ E; Sep. 1945; B. Malkin leg.; AMNH • 1 क ; Lingayen; $16.02^{\circ} \mathrm{N}, 120.23^{\circ} \mathrm{E}$; Jun.-Jul. 1945; R.B. Burrows leg.; AMNH • 1 ``; Banaue; $16.919^{\circ} \mathrm{N}, 121.058^{\circ} \mathrm{E}$; Jan. 1980; P. Schwendinger leg.; MHNG. - Leyte • 2 ○े $\partial^{\circ}, 2$ 우; Baybay; $10.68^{\circ}$ N, $124.80^{\circ}$ E; 3-6 Sep. 1984; C.K. Starr leg.; AMNH. - Mindanao • 2 우; Bukidnon Province, Central Mindanao University; $7.859^{\circ} \mathrm{N}, 125.051^{\circ}$ E; $350 \mathrm{~m}$ a.s.1.; 10 Feb. 2014; B.A. Huber and E. Mondejar leg.; in building; ZFMK Ar 22456.

INDONESIA • 6 우 (2 vials); Papua, Merauke ("Merambe"); $8.49^{\circ}$ S, $140.40^{\circ}$ E; 1956/57; M. Monulphus leg.; P. Chrysanthus ded.; SMF.

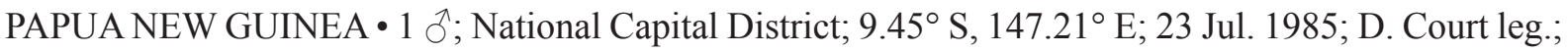
NMNL ARA 17485.

AUSTRALIA - Northern Territory • 1 q; Darwin; $12.45^{\circ}$ S, $130.84^{\circ}$ E; $20-31$ Mar. 1945; B. Malkin leg.; AMNH • 1 q; Darwin, Alawa, Eileen St.; $12.377^{\circ}$ S, 130.874 E; 23 May 1992; J.M. Waldock leg.; 
WAM 99/1672 • 1 đ̃, 2 우; 70 mi S of Darwin, Adelaide River; $13.24^{\circ} \mathrm{S}, 131.10^{\circ} \mathrm{E}$; 20-27 Mar. 1945; B. Malkin leg.; AMNH • 1 ; ; 120 mi SE of Darwin, Edge Arnhemland Res., Ben Hole Billabong; $13.8^{\circ} \mathrm{S}$, $131.8^{\circ}$ E; Jan.-Feb. 1972; J. Anderson leg.; AMNH • 1 ㅇ, 3 juvs; Alice Springs; $23.70^{\circ} \mathrm{S}, 133.88^{\circ}$ E; 29

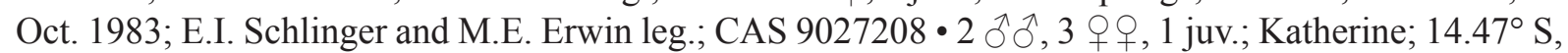

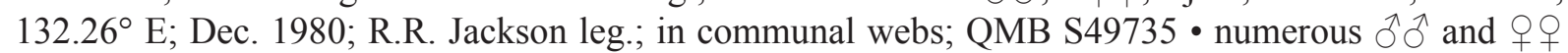
(not counted); Kakadu National Park, Narramoor; $12.696^{\circ}$ S, $132.373^{\circ}$ E; 25 m a.s.1.; 2 Jul. 1987; M.S. Harvey leg.; WAM 99/1604 to 99/1613 • 1 放; Jabiru Residency; $12.67^{\circ} \mathrm{S}, 132.84^{\circ} \mathrm{E} ; 28$ May 1992; Harvey and Waldock leg.; WAM 99/1674 • 1 क; Nhulunbuy; $12.18^{\circ}$ S, $136.68^{\circ}$ E; 8 Aug. 1991; A.F. Longbottom leg.; WAM 99/1675 • 1 \%; Berry Springs; $12.7^{\circ} \mathrm{S}, 131.0^{\circ} \mathrm{E} ; 26$ May 1992 ; Harvey and Waldock leg.; woodland, on house; WAM 99/1671 • 1 q; Humpty Doo, Marjerrison Rd; $12.57^{\circ} \mathrm{S}$, $131.1^{\circ} \mathrm{E}$; 30 Oct. 1985; J.M. Waldock leg.; WAM 99/1614 • 1 o ; Uluru National Park; $25.3^{\circ} \mathrm{S}, 131.0^{\circ} \mathrm{E}$ (label: $25.038^{\circ} \mathrm{S}, 131.070^{\circ} \mathrm{E}$ ); 15 May 1999; Gray, Milledge, and Smith leg.; in house; AMS KS 56180. - Queensland • 1 \%, 1 juv.; Widgee, NW of Gympie; $26.170^{\circ} \mathrm{S}, 152.425^{\circ} \mathrm{E}$; $16-20$ Feb. 1987; J. Gallon

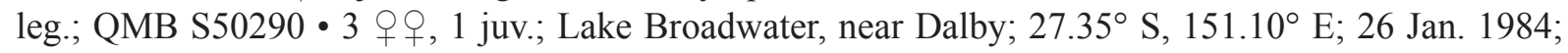
M. Bennie leg.; house; QMB S49726• 1 o ; Gatton Agricultural College; $27.554^{\circ} \mathrm{S}, 152.336^{\circ} \mathrm{E} ; 29$

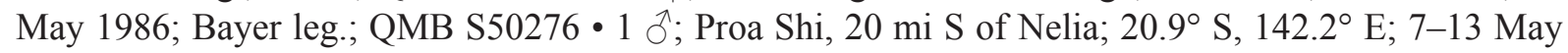
1978; J. Covacevich leg.; QMB S50262 • 1 q, 2 juvs; Mt Isa, township; $20.732^{\circ} \mathrm{S}, 139.485^{\circ} \mathrm{E}$; 16-21

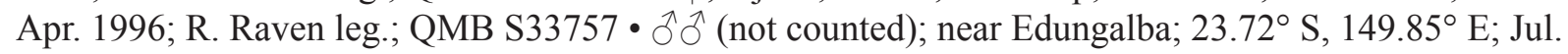
1993; D. Walker leg.; QMB S22175 • 1 o; Torres Strait, Yam Island; 9.90 S, $142.77^{\circ}$ E; 28 Nov.-2 Dec. 1986; K. Saddler and J. Gallon leg.; under bark; QMB S12418 • 1 + 1 juv.; Palm River Café; $10.96^{\circ}$ S, $143.70^{\circ}$ E; 21 Oct. 1974; V.E. Davies leg.; QMB S49730 • 1 đ; Lockerbie Scrub Wilderness Lodge; $10.78^{\circ} \mathrm{S}, 142.47^{\circ}$ E; 9 Dec. 1980; J. Gallon leg.; QMB S13021 • 1 ô, 1 q; Townsville; 19.26 ${ }^{\circ}$ S, $146.82^{\circ}$ E; Sep. 1986; collector unknown; QMB S49734 • 1 क; Boulia; $22.91^{\circ} \mathrm{S}, 139.91^{\circ} \mathrm{E}$; Jun. 1981; S. van Dyck and R. Molnar leg.; QMB S49727 • 1 क ; Doondi, 50 km SW of St. George; $28.28^{\circ}$ S, $148.56^{\circ}$ E; 10 Sep. 1979; R. Raven leg.; QMB S49732 • 1 +, 1 juv.; The Lion's Den Hotel [near Rossville?]; $15.705^{\circ} \mathrm{S}, 145.223^{\circ}$ E; 15 Apr. 1994; J. Thompson, M. Moulds, and F. Mac Killop leg.; AMS KS 45744. - Western Australia • 2 우, 1 juv.; Kalumburu; $14.295^{\circ}$ S, $126.640^{\circ}$ E; 19 Aug. 1969; A.R. Main leg.; WAM 99/1754 to 99/1758 part 2 우우; Barrow Island, WAPET camp; $20.817^{\circ}$ S, $115.433^{\circ}$ E; 1-5 Nov. 1993; M.S. Harvey and J.M. Waldock leg.; indoors; WAM 99/1739, 99/1740 •

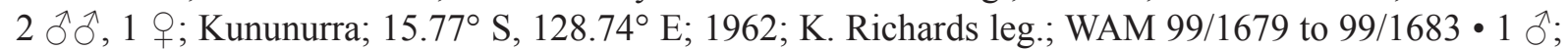
same locality as for preceding; 8 Jun. 1992; M.S. Harvey and J.M. Waldock leg.; WAM 99/1653 1 ठ, 4 juvs; same locality as for preceding; 25 Nov. 1978; D. Roberts and J. Staddart leg.; in hostel; WAM 99/1598 to 99/1602 • 1 \%; Derby; $17.317^{\circ}$ S, $123.633^{\circ}$ E; May 1963; A.R. Main leg.; WAM 99/1688 • 1 ${ }^{\text {; }}$; same locality as for preceding; 3 Nov. 1961; A.R. Main leg.; WAM 99/1702 • 1 o, 1 O ; Drysdale River Station; $15.702^{\circ}$ S, $126.380^{\circ}$ E; 15 Jan. 1994; A.F. Longbottom leg.; WAM 99/1747, 99/1748 • 1 गे, 1 क; same locality as for preceding; 21 Jun. 1993; A.F. Longbottom leg.; WAM 99/1745, 99/1746 - 1 9, 1 juv.; Geikie Gorge; $18.08^{\circ}$ S, $125.71^{\circ}$ E; 24 Jul. 1987; B.Y. Main leg.; WAM 99/1777, 99/1778 - 1 क; Geikie Gorge National Park; $18.110^{\circ}$ S, $125.697^{\circ}$ E; 7 Jun. 1999; Gray, Milledge, and Smith leg.; under bark; AMS KS 56189 • 1 क; Care Spring; $15.533^{\circ}$ S, $128.833^{\circ}$ E; 8 Jun. 1992; M.S. Harvey and J.M. Waldock leg.; WAM 99/1637 • 1 đo, 2 o o ; Fitzroy Crossing; $18.19^{\circ}$ S, $125.57^{\circ}$ E; 11 Aug. 1991; J.M. Waldock leg.; outside of house; WAM 99/1589 to 99/1591 • 1 क, 1 juv.; Meda Station; $17.4^{\circ}$ S, 124.0 E; 22 Aug. 1987; A.E. de Jong leg.; WAM 99/1616, 99/1617.

FEDERATED STATES OF MICRONESIA • 1 ^, 1 ,, 1 gynandromorphic specimen; Chuuk ("Truk") Island; $7.45^{\circ} \mathrm{N}, 151.85^{\circ}$ E; 10 Mar. 1969; Sabath leg.; MCZ 76633, 76634.

\section{Redescription}

Male (Guinea, ZFMK Ar 10456)

Measurements. Total length 6.5, carapace width 2.4. Distance PME-PME $140 \mu \mathrm{m}$; diameter PME $140 \times 170 \mu \mathrm{m}$; distance PME-ALE $50 \mu \mathrm{m}$; diameter AME $130 \mu \mathrm{m}$; distance AME-AME $45 \mu \mathrm{m}$. Leg 1: 
$63.4(18.7+0.9+17.6+22.7+3.5)$, tibia $2: 12.4$, tibia $3: 9.2$, tibia $4: 10.5$; tibia $1 \mathrm{~L} / \mathrm{d}: 70$; femora $1-4$ diameters: $0.36,0.32,0.28,0.31$.

CoLor (in ethanol). Carapace ochre-yellow; carapace pit anteriorly light brown; sternum dark brown; legs ochre-yellow, with indistinct darker rings subdistally on femora and tibiae, with black lines on femora and tibiae, patellae brown; abdomen ochre-gray, with whitish internal marks and some dark marks dorsally and laterally; ventrally with distinct black median band, partly disrupted, with three parallel longitudinal marks behind gonopore.

BoDy. Habitus as in Figs 702-703. Ocular area slightly raised. Deep thoracic pit and pair of furrows diverging from pit toward posterior margin. Clypeus unmodified, rim barely more sclerotized than in female. Sternum wider than long (1.7/1.0), unmodified. Abdomen slightly elongated, dorso-posteriorly angular.

Chelicerae. As in Figs 792-793, with two pairs of apophyses, lateral pair with 2-3 small processes (distinct in lateral view), median pair with one large modified cone-shaped hair each; distance between tips of modified hairs $80 \mu \mathrm{m}$; lateral stridulatory ridges fine but visible in dissecting microscope; distances between ridges: $\sim 7.5 \mu \mathrm{m}$ (Huber et al. 1999).

PALPS. As in Figs 783-785; coxa with rounded retrolateral hump; trochanter barely modified; femur distally widened, with rounded ventral protrusion, proximally with prolateral stridulatory pick, retrolateral-ventral rim with row of projecting and sclerotized hair-bases, with barely visible retrolateral

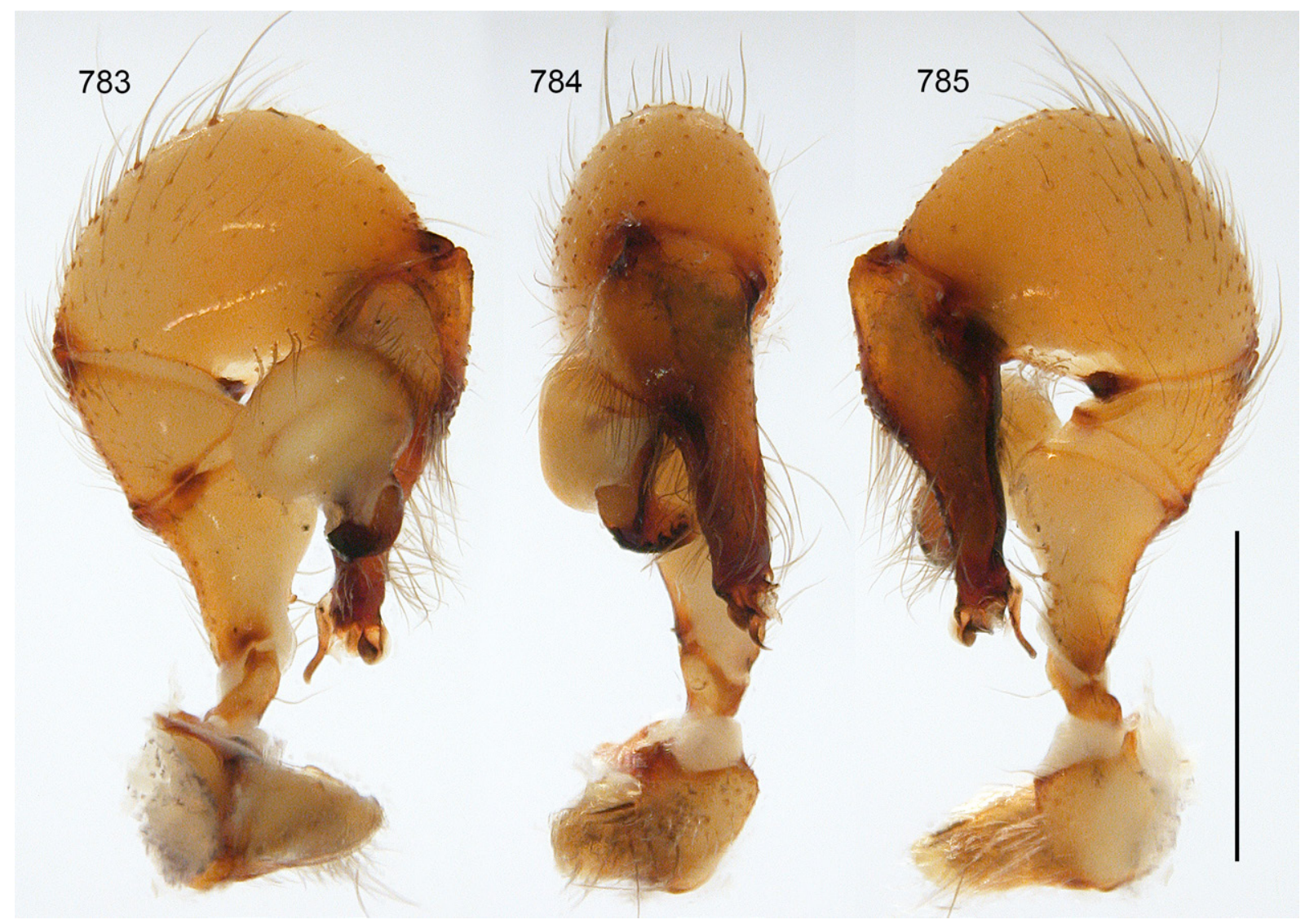

Figs 783-785. Crossopriza lyoni (Blackwall, 1867); male from Guinea, Kindia (ZFMK Ar 10456, see Huber \& Kwapong 2013); left palp, prolateral, dorsal, and retrolateral views. Scale bar $=1.0 \mathrm{~mm}$. 


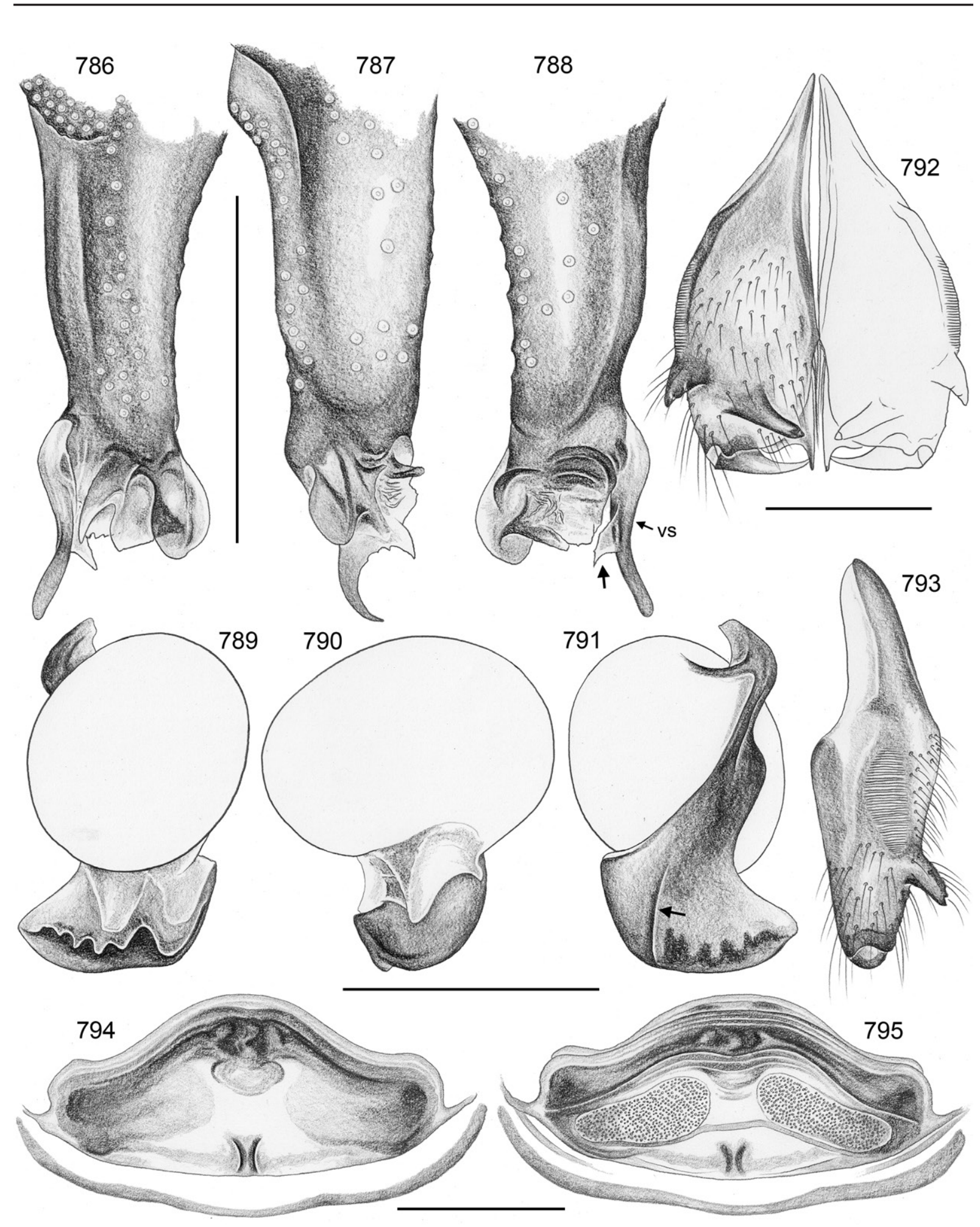

Figs 786-795. Crossopriza lyoni (Blackwall, 1867); male from Guinea, Kindia (ZFMK Ar 10456, see Huber \& Kwapong 2013), female from Japan, Yamamoto-chô (ZFMK Ar 5209). 786-788. Left procursus, prolateral, dorsal, and retrolateral views; arrow: branch of ventral sclerite. 789-791. Left genital bulb, prolateral, dorsal, and retrolateral views; arrow: retrolateral ridge. 792-793. Male chelicerae, frontal and lateral views. 794-795. Cleared female genitalia, ventral and dorsal views. Abbreviation: vs $=$ ventral sclerite. Scale bars $=0.5 \mathrm{~mm}$. 
transversal line, without retrolateral proximal process; femur-patella joints slightly shifted toward prolateral side; tibia large relative to femur, tibia-tarsus joints shifted toward retrolateral side; tarsus without macrotrichia; procursus (Figs 786-788) straight, densely set with long hairs dorsally, few hairs slightly curved upwards; proximally on prolateral side with strong hump set with numerous long hairs and followed distally by thick sclerotized ridge, procursus tip with several distinctive elements: ventral sclerite with transparent retrolateral branch (arrow in Fig. 788), rounded dorsal sclerite, semitransparent prolateral pointed process, and pair of crescent-shaped retrolateral elements; genital bulb (Figs 789-791) with simple basal sclerite connected to distal (main) sclerite, sperm duct opening
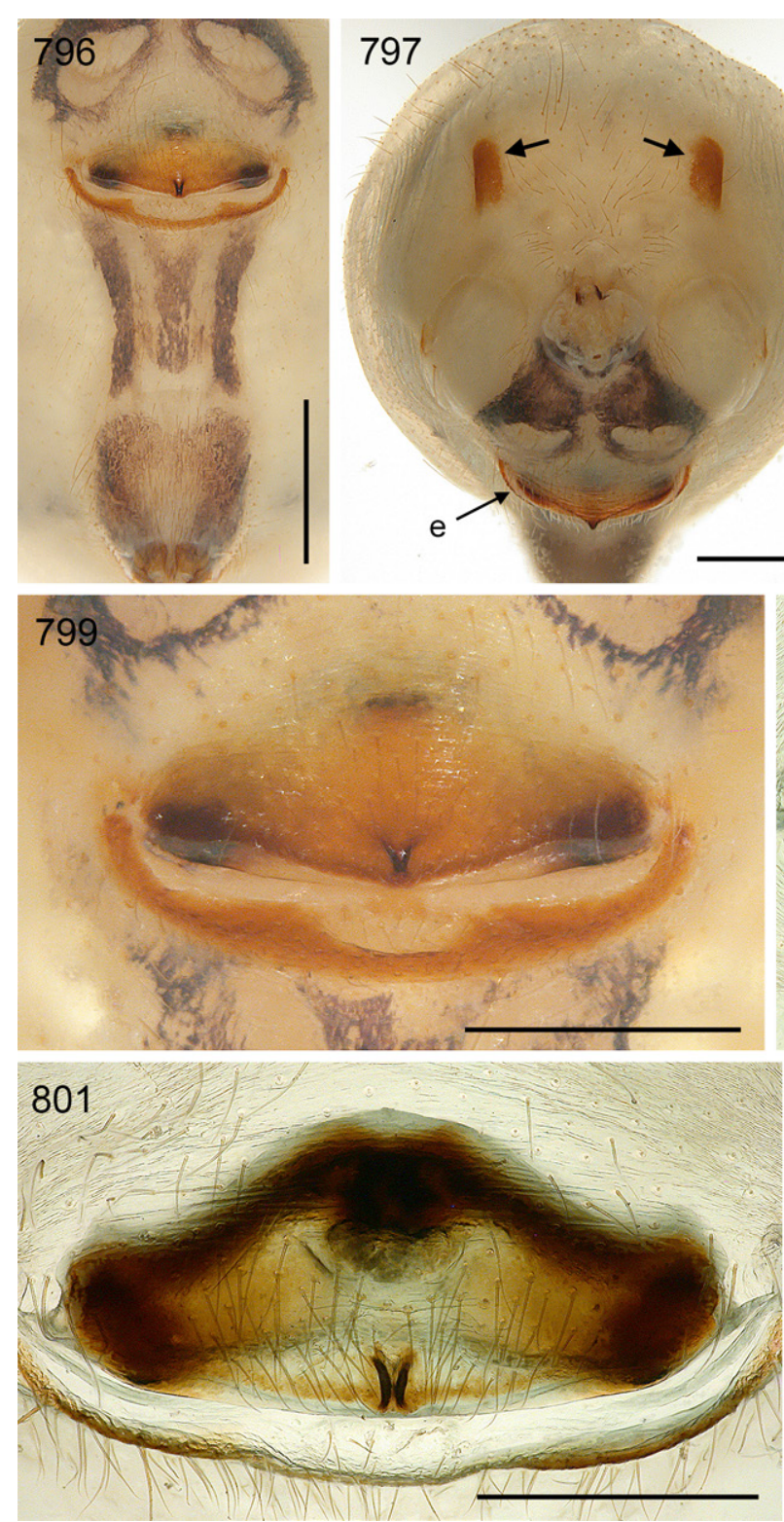
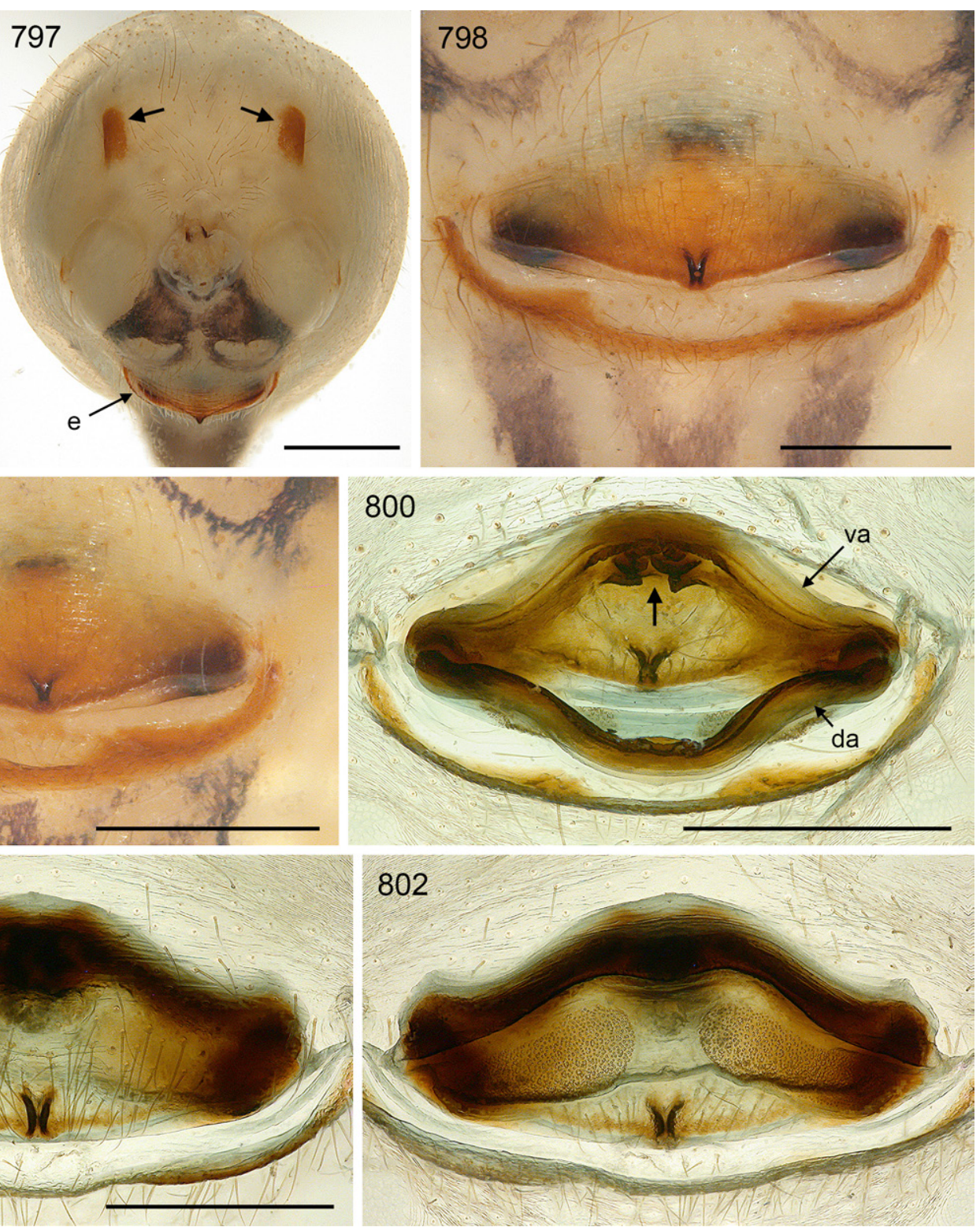

Figs 796-802. Crossopriza lyoni (Blackwall, 1867); females from Japan, Yamamoto-chô (796-798, 801-802; ZFMK Ar 5209) and from Guinea, Kindia (799-800; ZFMK Ar 10456, see Huber \& Kwapong 2013). 796-797. Abdomen, ventral and frontal views; arrows: stridulatory plates. 798-799. Epigyna, ventral views. 800-802. Cleared female genitalia, dorsal view with dorsal arc tilted backwards, ventral view, and regular dorsal view; arrow: asymmetric median sclerite. Abbreviations: da $=$ dorsal arc; $\mathrm{e}=$ epigynum; va $=$ ventral arc. Scale bars: $796-797=1.0 \mathrm{~mm} ; 798-802=0.5 \mathrm{~mm}$. 
not seen; distal sclerite with retrolateral ridge and distinctive set of one large and four smaller rounded prolateral apophyses.

LEGS. Femur 1 with single row of 23 ventral spines; without curved hairs; few vertical hairs; retrolateral trichobothrium of tibia 1 at $4 \%$; prolateral trichobothrium absent on tibia 1, present on other leg tibiae; tarsal pseudosegments indistinct except 2-3 distally.

Male (variation)

Tibia 1 in 43 males: 11.5-18.0 (mean 14.5). Dark ventral band on abdomen variably distinct, section behind gonopore with 2-4 longitudinal bands. Small apophyses prolaterally on bulbal sclerite variably distinct, usually one large plus four small apophyses, rarely one large plus three or five small apophyses. Retrolateral branch on ventral sclerite of procursus slightly variable in shape.

\section{Female}

In general similar to male (Figs 704-705) but without spines on legs, with smaller and less distinct stridulatory files on chelicerae (sometimes not visible in dissecting microscope; distances between ridges: $\sim 8.5 \mu \mathrm{m}$, Huber et al. 1999), and with stridulatory organ consisting of pair of weakly sclerotized but distinct processes posteriorly on carapace and pair of light brown plates anteriorly on abdomen (Fig. 797). Tibia 1 in 57 females: 9.2-15.5 (mean 12.5). Epigynum as in Figs 796-799, main epigynal plate short and wide, weakly protruding; posteriorly laterally strongly sclerotized, anteriorly weakly sclerotized, with variably distinct light brown median area; with pair of pockets very close to median line on both sides of median ridge (distance between pockets $30 \mu \mathrm{m}$ ), posteriorly barely protruding; internal sclerotized arc and median round structure visible in uncleared specimens; posterior plate short but wide. Internal genitalia (Figs 794-795, 800-802) with large elongate pore plates converging anteriorly, dorsal arc wide but simple, ventral arc medially slightly modified, with asymmetric sclerites (three females cleared), with simple median pouch.

\section{Gynandromorphic specimen}

Specimens that combine male and female morphological characters (sometimes in a left-right asymmetry) are extremely rare in pholcid spiders. The only previously known case was reported by Blackwall (1867), who described a specimen of $C$. lyoni from India that had a male left palp and a male left chelicera, but a female right palp, female right chelicera, and incompletely developed female genitalia (epigynum) on the right side. He noted that this was the only case of gynandromorphism among "many thousands"
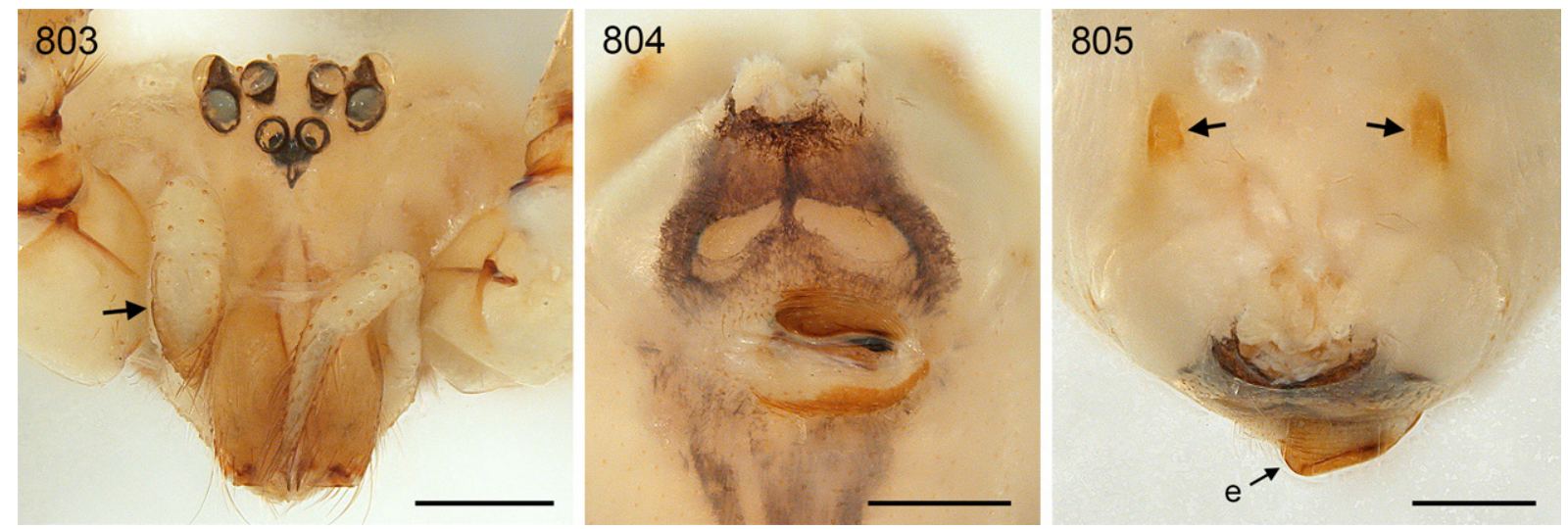

Figs 803-805. Crossopriza lyoni (Blackwall, 1867); gynandromorphic specimen from Micronesia, Chuuk Island (MCZ 76633). 803. Prosoma, frontal view; arrow: penultimate instar male palp. 804805. Abdomen, ventral and frontal views; arrows: female stridulatory plates. Abbreviation: $\mathrm{e}=$ epigynum. Scale bars $=0.5 \mathrm{~mm}$. 
of specimens of spiders studied. Surprisingly, the only gynandromorphic pholcid specimen I have ever seen (among roughtly 50,000 specimens) is also a representative of C. lyoni. This specimen (from Chuuk Island, Micronesia), has a half epigynum (Figs 804-805; relatively normally developed on the left side), but an enlarged right palp that reminds of a penultimate instar male palp (arrow in Fig. 803). The right chelicera and the right femur are not modified, as would be expected from a penultimate instar male. Surprisingly, however, the female stridulatory apparatus between prosoma and abdomen appears fully and symmetrically developed on both sides (arrows in Fig. 805). Leg length is also symmetric.

\section{Natural history}

Surprisingly little is known about the biology of this large and widely distributed synanthropic spider. Strickman et al. (1997) give basic bionomic data for spiders collected in Thailand and held at $30^{\circ} \mathrm{C}$ : eggs were laid 4-6 days after copulation; a mean of 34 spiderlings (range: 5-54) hatched form each eggsac 11-13 days after egg-laying; maturity was reached after only 74 days. Females were significantly heavier than males (mean weight $28.6 \mathrm{mg}$ vs $17.6 \mathrm{mg}$ ). As soon as spiderlings started to eat (after the first molt), they were capable of overpowering a mosquito; prey was wrapped by 'throwing' silk with the hind legs, without rotating the prey. The prey was not bitten until the moment the spider started feeding, sometimes days after wrapping. No female was observed to eat her own eggs (as reported by Downes 1987). Observations on US populations give slightly to significantly higher numbers of eggs per egg sac (or spiderlings accompanying the female): 53-58 in Edwards (1993), and 72 in Guarisco \& Cutler (2003). In Florida, Edwards (1993) observed a wide variety of insect prey in C. lyoni webs. For further observations, see Nandi \& Raut (1985) (publication only partly seen) and Irie (2001) (in Japanese).

\section{Distribution}

The closest known relatives of $C$. lyoni ( $C$. maculipes, $C$. surobi sp. nov., $C$. sengleti sp. nov.) live in an area that is mainly composed of Pakistan, Afghanistan, and Iran (Fig. 353), suggesting that C. lyoni originated from this region. It seems to have expanded from its original area at a time from which few records are available, so the early expansion is difficult to reconstruct. The earliest records from supposedly non-native regions date from the late $19^{\text {th }}$ century (Myanmar; Thorell 1895) and the early $20^{\text {th }}$ century (China; Strand 1907). Towards the east, C. lyoni may have reached the Philippines and Australia not before the $20^{\text {th }}$ century (oldest records: 1940s). Towards the west, $C$. lyoni seems to have reached South America much sooner than North America: while the oldest record from Argentina dates from 1914, and the species was "not rare" in Bahia (Brazil) by the early 1930s (Mello-Leitão 1935), it may not have reached the USA before the early 1980s (Roth 1985, Edwards 1993). In Central Europe, the species appears to be slowly spreading since approximately 2000, but it is still largely confined to buildings (Van Keer 2007, Bauer et al. 2016).

Mysteriously, this synanthropic species is largely absent from the original area of distribution of Crossopriza (Fig. 351). Such a pattern is mirrored in some other synanthropic pholcids but remains unexplained (see Discussion).

$$
\begin{aligned}
& \text { Crossopriza surobi } \text { sp. nov. } \\
& \text { urn:1sid:zoobank.org:act:6D67AB05-2114-445F-9217-35ED08B6B68F } \\
& \text { Figs 353A, 806-822, 841-853 }
\end{aligned}
$$

\section{Diagnosis}

Distinguished from known congeners by details of male palp (Figs 809-811; procursus ventral sclerite with strong retrolateral branch; procursus tip with strong dorsal process; distal bulbal sclerite with distinctive series of apophyses on prolateral side); from the similar C. lyoni (identical male chelicerae) also by longer epigynum and less elongated pore plates (Figs 816-819); from the similar C. maculipes also by male chelicerae (Fig. 815; lateral apophyses in lateral view long and pointed) and by female internal genitalia (Fig. 817; pore plates farther apart). 


\section{Etymology}

The species name refers to the type locality; noun in apposition.

\section{Type material}

\section{Holotype}

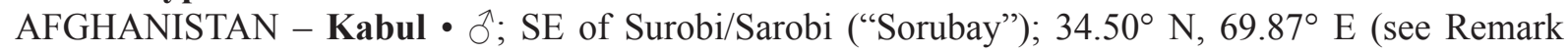
below); 800 m a.s.l.; 6 Aug. 1975; A. Senglet leg.; MHNG.

\section{Remark}

The label coordinates $\left(34^{\circ} 30^{\prime} \mathrm{N}, 69^{\circ} 52^{\prime}\right.$ E) are actually slightly outside of Kabul Province in Laghman Province. The actual collecting site might thus be slightly further west or northwest.

\section{Other material examined}

AFGHANISTAN - Kabul $\bullet 8 \hat{\partial}, 8$ q $\propto$ (partly used for SEM); same collection data as for holotype;

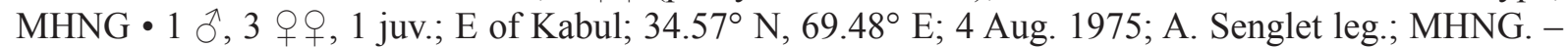
Wardak 1 1 ; [ [between] Kabul [and] Shahabuddin; $34.33^{\circ}$ N, 68.85 E; 12 Aug. 1975; A. Senglet leg.; MHNG • 1 \%; NNE of Ghazni; $33.75^{\circ}$ N, $68.57^{\circ}$ E; 12 Aug. 1975; A. Senglet leg.; MHNG.

\section{Description}

Male (holotype)

Measurements. Total length 4.2, carapace width 1.6. Distance PME-PME $100 \mu \mathrm{m}$; diameter PME $100 \times$ $130 \mu \mathrm{m}$; distance PME-ALE $25 \mu \mathrm{m}$; diameter AME $90 \mu \mathrm{m}$; distance AME-AME $20 \mu \mathrm{m}$. Leg 1: 32.8 $(9.2+0.6+8.9+11.9+2.2)$, tibia $2: 6.0$, tibia $3: 4.3$, tibia $4: 5.3$; tibia $1 \mathrm{~L} / \mathrm{d}: 64$; femora $1-4$ diameters: $0.22,0.17,0.17,0.17$.

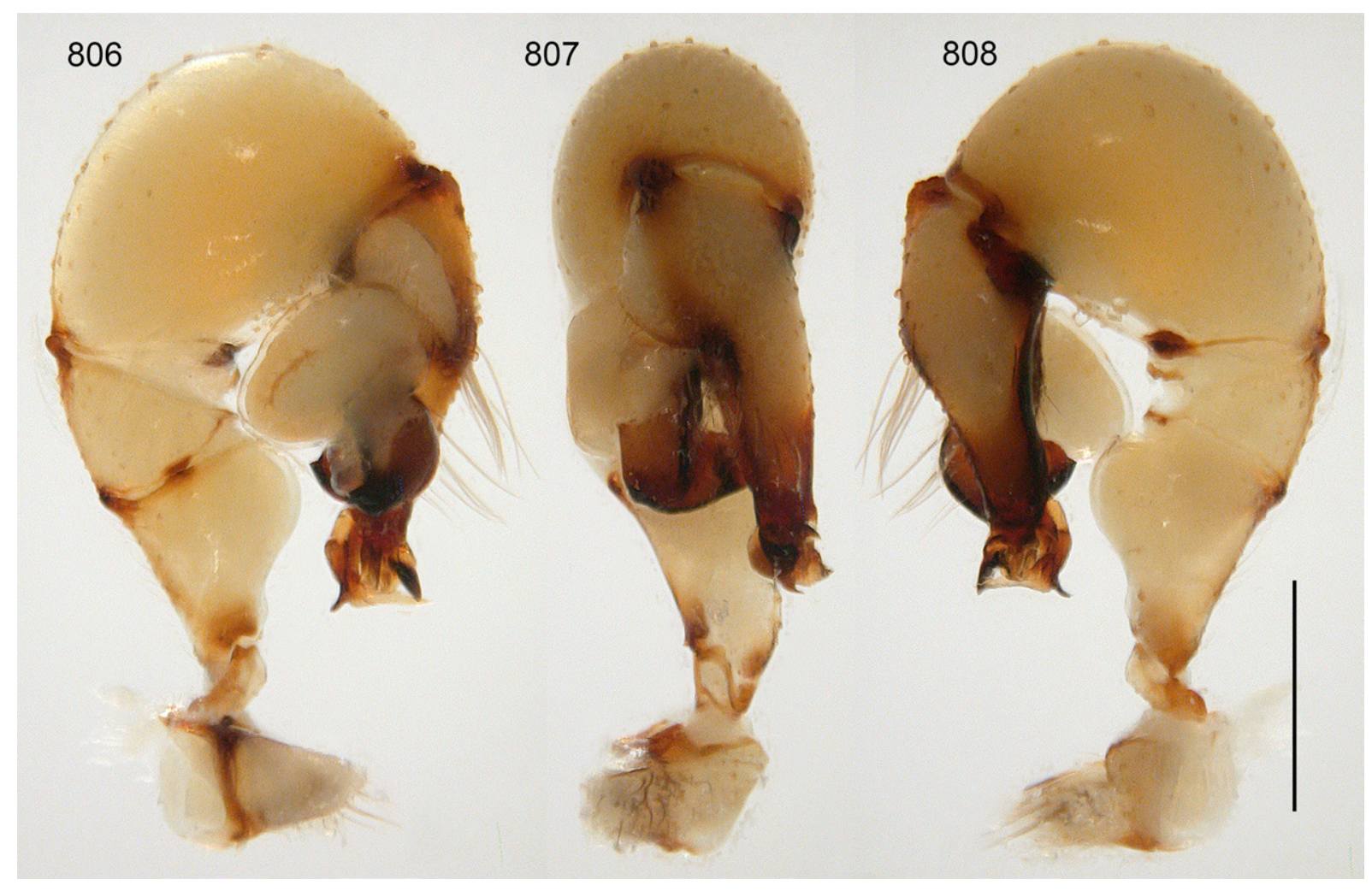

Figs 806-808. Crossopriza surobi sp. nov.; non-type male from Afghanistan, SE of Surobi (MHNG); left palp, prolateral, dorsal, and retrolateral views. Scale bar $=0.5 \mathrm{~mm}$. 

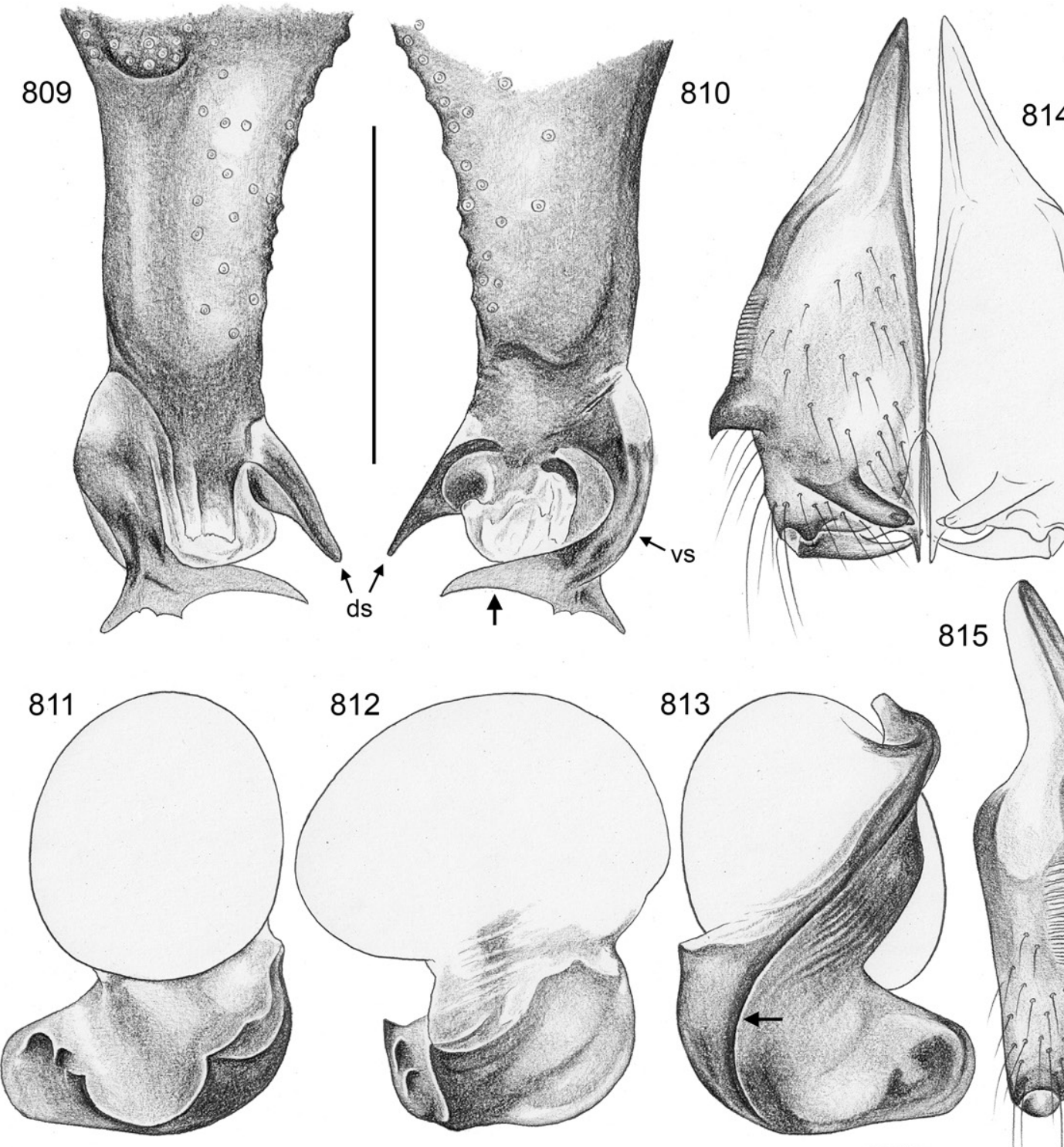

815
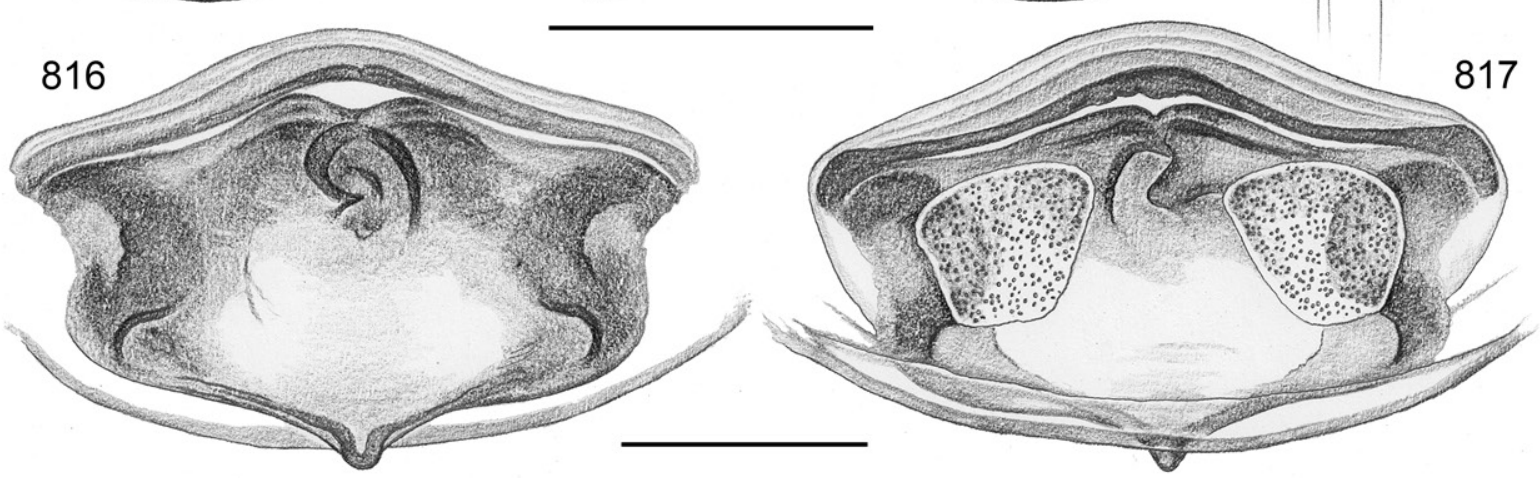

Figs 809-817. Crossopriza surobi sp. nov.; non-type male and female from Afghanistan, SE of Surobi (MHNG). 809-810. Left procursus, prolateral and retrolateral views; arrow: branch of ventral sclerite. 811-813. Left genital bulb, prolateral, dorsal, and retrolateral views; arrow: retrolateral ridge. 814815. Male chelicerae, frontal and lateral views. 816-817. Cleared female genitalia, ventral and dorsal views. Abbreviations: ds $=$ dorsal sclerite; vs $=$ ventral sclerite. Scale bars $=0.3 \mathrm{~mm}$. 
Color (in ethanol). Carapace ochre-yellow, anteriorly in median pit light brown; sternum with many small brown spots partly arranged in radial marks; legs ochre-yellow, without darker rings, with black lines on femora and (few) on tibiae; abdomen pale gray, with few indistinct small dark marks dorsally and posteriorly above spinnerets; ventrally with broken dark band, with indistinct parallel longitudinal marks behind gonopore.

Body. Habitus similar to C. tiwi sp. nov. (cf. Figs 393-394). Ocular area slightly raised. Deep thoracic pit and pair of furrows diverging from pit toward posterior margin. Clypeus unmodified, only rim slightly more sclerotized than in female. Sternum wider than long (1.05/0.65), unmodified. Abdomen slightly elongated, dorso-posteriorly angular to conical. Gonopore with four epiandrous spigots (Fig. 852).

Chelicerae. As in Figs 814-815, with two pairs of frontal apophyses; proximal pair in lateral position, pointed and directed towards lateral; distal pair directed towards median, at tip with one large modified cone-shaped hair each (Fig. 845); distance between tips of modified hairs $30 \mu \mathrm{m}$; lateral stridulatory ridges distinct (Fig. 841 ; distances between ridges $\sim 4 \mu \mathrm{m}$ ), clearly visible in dissecting microscope.

Palps. As in Figs 806-808; coxa with rounded retrolateral hump; trochanter barely modified; femur distally strongly widened, with rounded ventral protrusion, proximally with prolateral stridulatory pick (Fig. 842), without retrolateral transversal line, without retrolateral proximal process; femur-patella joints shifted toward prolateral side; tibia-tarsus joints slightly shifted toward retrolateral side; tarsus without macrotrichia; tarsal organ capsulate (Fig. 849); procursus (Figs 809-810) straight, prolateral
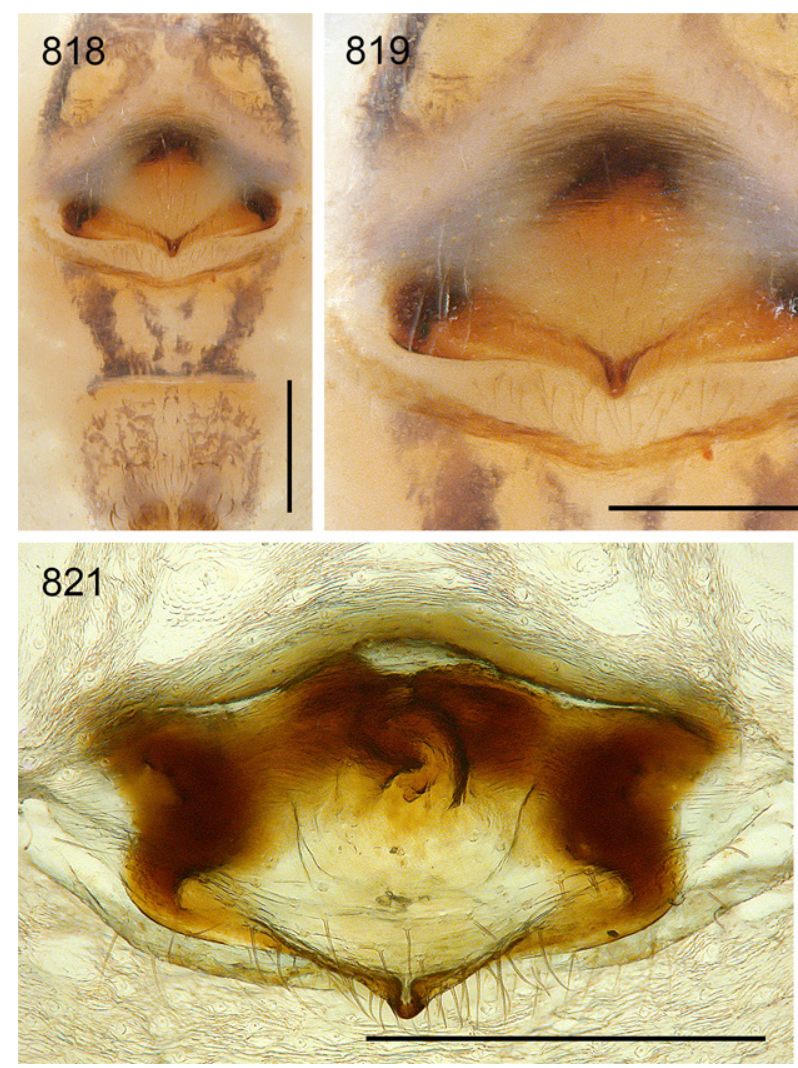
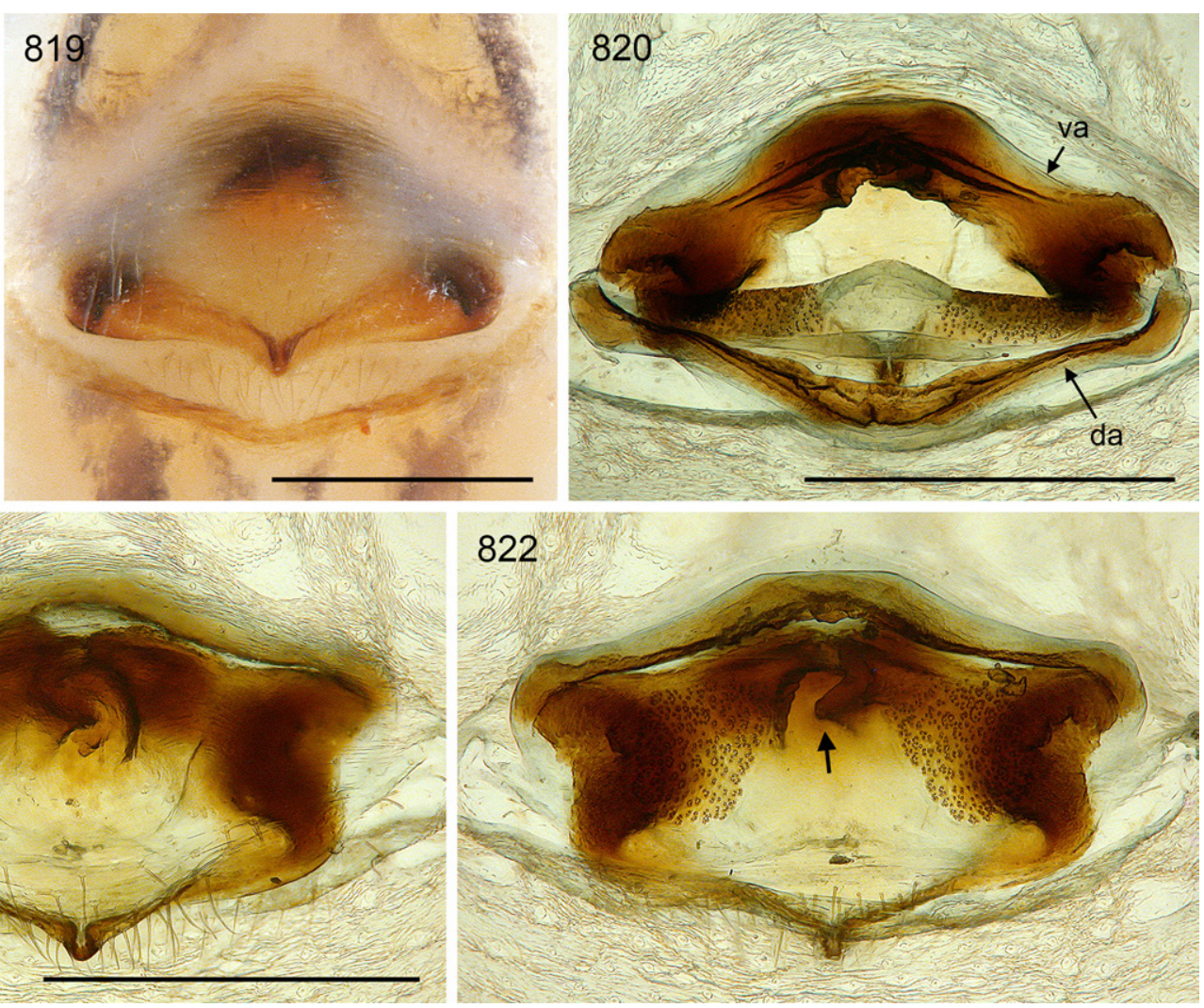

Figs 818-822. Crossopriza surobi sp. nov.; female from Afghanistan, SE of Surobi (MHNG). 818819. Abdomen and epigynum, ventral views. 820-822. Cleared female genitalia, dorsal view with dorsal arc tilted backwards, ventral view, and regular dorsal view; arrow: asymmetric median sclerite. Abbreviations: $\mathrm{da}=$ dorsal arc; $\mathrm{va}=$ ventral arc. Scale bars $=0.5 \mathrm{~mm}$. 
proximal hump set with many hairs, long dorsal hairs not curved, procursus tip with flat dorsal sclerite (wide in dorsal view), strong ventral sclerite with distinctive retrolateral branch (Fig. 846), and brush of short hair-like processes on retrolateral side (Fig. 847); genital bulb (Figs 811-813) with simple basal sclerite connected to distal (main) sclerite, sperm duct opening at basis of distal sclerite on prolateraldorsal side (arrow in Fig. 848); distal sclerite with retrolateral ridge and distinctive set of prolateral apophyses and ridge.

LEGS. Femur 1 with single row of $\sim 18$ ventral spines; without curved hairs; few vertical hairs; retrolateral trichobothrium of tibia 1 at $4.5 \%$; prolateral trichobothrium absent on tibia 1, present on other leg tibiae; tarsal pseudosegments not seen.

Male (variation)

Tibia 1 in seven males (incl. holotype): 7.8-8.9 (mean 8.4). Some males also with whitish internal marks on abdomen.

\section{Female}

In general similar to male but without spines on legs, without stridulatory files on chelicerae (Fig. 843), and with stridulatory organ consisting of pair of weakly sclerotized but distinct processes posteriorly on carapace and pair of small but distinct light brown plates anteriorly on abdomen. Tibia 1 in 12 females: 6.0-8.3 (mean 7.3). Epigynum as in Figs 819 and 853; main epigynal plate wider than long, weakly protruding, sclerotized area at posterior margin widening towards lateral; with median ridge with shallow pair of opposing pockets close together (distance $30 \mu \mathrm{m}$ ); internal sclerotized arcs and median anterior internal sclerite visible in uncleared specimens; posterior plate light brown, short but wide. Internal genitalia (Figs 816-817, 820-822) with large angular pore plates, dorsal arc simple, ventral arc stronger, with median sclerite asymmetric; apparently antisymmetric (only two females cleared; 'left' and 'right' sided).

\section{Distribution}

Known from four localities in eastern Afghanistan (Kabul and Wardak Provinces) (Fig. 353A).

Crossopriza maculipes (Spassky, 1934)

Figs 353B, 823-840, 854-865

Ceratopholcus maculipes Spassky, 1934: 361, figs 1-3.

Ceratopholcus maculipes - Senglet 2001: 49, fig. 26.

Crossopriza maculipes - Huber et al. 2014a: 420. — Ali et al. 2016: 250, fig. 3a-c. — Najim \& AlKhazali 2019: 548, figs 2-3.

\section{Diagnosis}

Distinguished from known congeners (see also Remarks below) by details of male palp (Figs 826-829; procursus ventral sclerite with distinctive retrolateral branch; procursus tip with dorsal sclerite; distal bulbal sclerite with two rows of apophyses on prolateral side); from similar species (C. lyoni, C. surobi sp. nov.) also by male chelicerae (Fig. 833; lateral apophyses in lateral view short and broadly truncated) and by female genitalia (Figs 834-835; epigynum longer than in C. lyoni; pore plates closer together than in C. surobi).

\section{Remarks}

The MHNG has a very similar species from Afghanistan (Nangarhar, NE of Djelalabad) that shares the distinctive dorsal sclerite distally on the procursus and has a possibly indistinguishable epigynum. It differs most clearly by the distal bulbal sclerite (prolateral view: only three apophyses in one row); 
also slightly by the lateral cheliceral apophyses (more slender and longer in lateral view), by the shape of the retrolateral branch on the ventral sclerite of the procursus (smaller). The available specimens are also very small compared to most $C$. maculipes specimens (male chelicerae maximum width $580 \mu \mathrm{m}$; tibia 1 in three males: 7.7, 8.2, 8.3; in three females: 6.2,6.6, 7.2). This species is not formally described because all available specimens are in very poor condition.

\section{Type material}

\section{Syntypes}

UZBEKISTAN • 2 đô, 3 우, 2 juvs, not examined; Tashkent; $41.3^{\circ} \mathrm{N}, 69.2^{\circ} \mathrm{E}$; 1928 ; Civirko leg.; presumably in Zoological Institute, Russian Academy of Sciences, St Petersburg, Russia (repeated loan requests since 2013 were unsuccessful) • 9 , , juvs, unknown number, not examined; Bukhara, Qorak'ol (“Karakul”); 39.50 N, 63.85 E; 1933; A. Alparov leg.; presumably in Zoological Institute, Russian Academy of Sciences, St Petersburg, Russia.

TAJIKISTAN - $q$, juvs, unknown number, not examined; Dushanbe ("Stalinabad"); $38.55^{\circ} \mathrm{N}$, $68.77^{\circ}$ E; 1933; A. Alparov leg.; presumably in Zoological Institute, Russian Academy of Sciences, St Petersburg, Russia.

TURKMENISTAN • 3 우, 2 juvs, not examined; Ashgabat; 37.94 N, 58.36 E; 1933; Melnikova leg.; presumably in Zoological Institute, Russian Academy of Sciences, St Petersburg, Russia.

\section{Material examined}

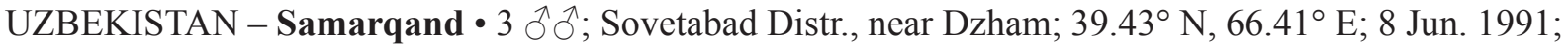
A.A. Zyuzin leg.; ZMMU. - Surxondaryo • 3 우; Uzun Distr., E slope of Babatagh Mt Range, $\sim 6.5 \mathrm{~km}$ W of Akmechet; $38.0472^{\circ} \mathrm{N}, 68.2394^{\circ}$ E; 905-1010 m a.s.1.; 28 Apr. 1995; S.V. Ovchinnikov leg.;

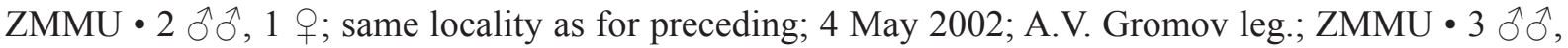
2 우; Kafirnighan river valley, Ak-Mechet; $38.047^{\circ}$ N, 68.240 ${ }^{\circ}$ E; 7 May 1994; S.V. Ovchinnikov leg.; in house; ZMMU • 1 ^, 1 ㅇ, 1 juv.; Djarkurgan [Dzharkugan] Town; $37.51^{\circ} \mathrm{N}, 67.41^{\circ} \mathrm{E} ; 25$ Apr. 1986; A.A. Zyuzin leg.; ZMMU $\bullet 1$ ô, 1 q, 3 juvs; Uzun Distr., W foot of Babatagh Mt Range, $\sim 13 \mathrm{~km}$ ESE of Denan, Argamchi canyon; $38.2128^{\circ} \mathrm{N}, 68.0531^{\circ} \mathrm{E}$; $\sim 630 \mathrm{~m}$ a.s.1.; 30 Apr. 2002; A.V. Gromov leg.; ZMMU. - Bukhara 2 O 9 ; $33 \mathrm{~km}$ SE of Bukhara; $39.57^{\circ} \mathrm{N}, 64.72^{\circ} \mathrm{E}$; $19-20$ May 1994; A.A. Zyuzin leg.; ZMMU • 2 우, 1 juv.; Bukhara; $39.7761^{\circ} \mathrm{N}, 64.4330^{\circ}$ E; 220 m a.s.1.; 20 Apr. 2002; A.V. Gromov leg.; buildings; ZMMU.

TAJIKISTAN - Dushanbe • 1 ðे, 1 क ; Dushanbe; $38.55^{\circ}$ N, $68.75^{\circ}$ E; 28 Apr. 1986; S.L. Zonstein leg.; in building; ZMMU. - Khatlon 1 万人; Vaksh river valley, Tigrovaya Balka State Res., Korolevskaia Dacha; $37.23^{\circ} \mathrm{N}, 68.38^{\circ}$ E; 3 Aug. 2006; S.V. Ovchinnikov leg.; ZMMU • 1 đ , 1 क , 1 juv.; Il'ichevsk Distr., near Gandzhino Vil., slope of Aktau Mt. Range; $37.965^{\circ}$ N, $68.560^{\circ}$ E; 850 m a.s.1.; 21 Apr. 1986;

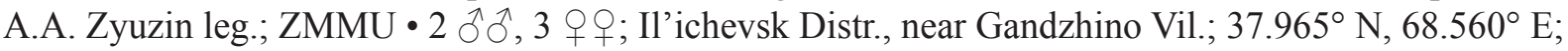
800 m a.s.l.; 19 Apr. 1986; A.A. Zyuzin leg.; ZMMU.

TURKMENISTAN - Ashgabat • 1 đ, 4 우, 2 juvs; Ashgabat; $37.94^{\circ} \mathrm{N}, 58.36^{\circ} \mathrm{E}$; date and collector

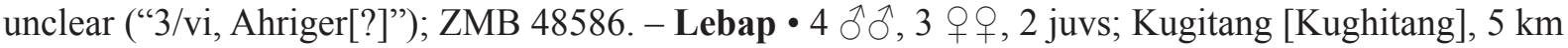
SW of Bazar-Tepe, office of Kugitang Reserve; $37.77^{\circ}$ N, $66.38^{\circ}$ E; 10-13 Jul. 1991; V.V. Dubatolov leg.; SZMN • 1 §, 1 juv.; Chardzhou Area, Amudarya Reserve, $140 \mathrm{~km} \mathrm{NW}$ of Chardzhou; $39.65^{\circ} \mathrm{N}$, $62.78^{\circ}$ E; 11 May 1988; F. Zeeleev leg.; Kabakli tugai [kind of habitat], house; ZMMU. - Balkan • 1 क ; Turkmenbashi ("Krasnovodsk"); 40.02 ${ }^{\circ}$ N, $52.97^{\circ}$ E; 4 Jul. 1929; collector unknown; house terrace, angle of window frames; ZMMU $\bullet 1 \hat{\jmath}, 1$; same locality as for preceding; in basement; date and collector unclear (“15 iv, Müller-C. Ahriger"); ZMB 48587. - Mary • 1 ○, 1 \&; Zakhmet Vil.; $37.77^{\circ} \mathrm{N}$, $62.53^{\circ} \mathrm{E}$; 6 Apr. 1991; collector unknown; ZMMU. 
AFGHANISTAN - Parwan • $2 \partial^{\wedge}$; $\mathrm{N}$ of Charikar; $35.167^{\circ} \mathrm{N}, 69.233^{\circ} \mathrm{E}$ [actual collecting site probably further west along Salang River]; 10 Aug. 1975; A. Senglet leg.; in small cave; MHNG. - Kabul • 1 q; SE of Sarobi/Surubi ("Sorubay"); $34.50^{\circ} \mathrm{N}, 69.87^{\circ} \mathrm{E}$ (label coordinates slightly outside of Kabul

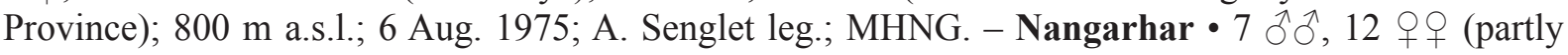
used for SEM); Djelalabad; $34.42^{\circ}$ N, $70.45^{\circ}$ E (label: 3430' N, 70 $33^{\prime}$ E); 8 Aug. 1975; A. Senglet leg.; in hotel; MHNG. - Kandahar - 1 o, 10 우; W of Kandahar; $31.617^{\circ}$ N, 65.600 ${ }^{\circ}$ E; 31 Jul. 1975; A. Senglet leg.; MHNG.

PAKISTAN - Khyber Pakhtunkhwa 1 1,1 ; Peshawar, Forestry Campus of Agricultural University;

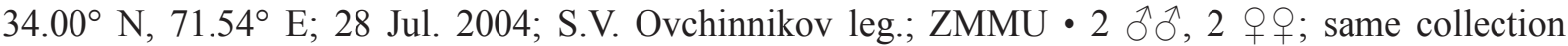

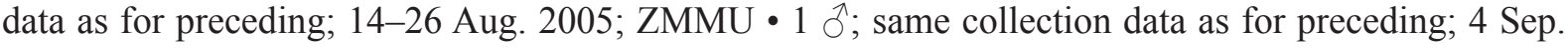
2004; ZMMU • 1 q; Chitral Distr., 7 km NE of Gobor-o-Bakh [Gobor Bakht]; $36.117^{\circ}$ N, $71.383^{\circ} \mathrm{E}$; 1 Aug. 2004; S.V. Ovchinnikov leg.; ZMMU • 1 q, 1 juv.; Kagan Valley, Shogran (= Sharan); $34.64^{\circ}$ N, 73.46 ${ }^{\circ}$ E; 2400-3000 m a.s.l.; 21 Aug. 2004; S.V. Ovchinnikov leg.; ZMMU • 1 क; Orakzai, 4 km E of "Mangu"; $33.6^{\circ}$ N, $70.7^{\circ}$ E; 29 Aug. 2005; S.V. Ovchinnikov leg.; ZMMU • 9 ỗ, 19 q ; ; Sheringal; $35.275^{\circ} \mathrm{N}, 72.005^{\circ} \mathrm{E} ; 1400 \mathrm{~m}$ a.s.1.; 6 Apr.-4 Oct. 2013; F. Perveen and N. Khan leg.; in building; ZFMK

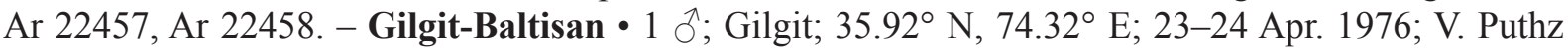

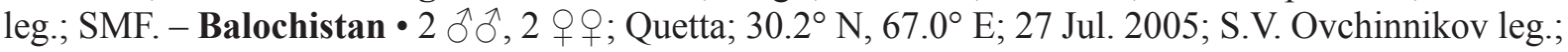
in hotel; ZMMU. - Punjab 1 q (assigned tentatively); Musa Khel, Namal gorge; $32.68^{\circ} \mathrm{N}, 71.79^{\circ} \mathrm{E}$; 300 m a.s.1.; 28 Feb. 1963; E. Kullmann leg.; ZFMK Ar 22459.

WITHOUT LOCALITY DATA • 2 우 (possibly syntypes); "Ceratopholcus maculipes", no further data; MNHN Ar 10548.

\section{Redescription}

Male (Uzbekistan, Ak-Mechet, ZMMU)

MeAsurements. Total length 5.4, carapace width 1.9. Distance PME-PME $110 \mu \mathrm{m}$; diameter PME $120 \times 140 \mu \mathrm{m}$; distance PME-ALE $30 \mu \mathrm{m}$; diameter AME $120 \mu \mathrm{m}$; distance AME-AME $20 \mu \mathrm{m}$. Leg 1 : $42.4(12.5+0.9+12.1+14.4+2.5)$, tibia $2: 8.3$, tibia $3: 6.3$, tibia $4: 7.6$; tibia $1 \mathrm{~L} / \mathrm{d}: 58$; femora $1-4$ diameters: $0.32,0.27,0.27,0.28$.

CoLOR (in ethanol). Carapace ochre-yellow; carapace pit anteriorly light brown; sternum dark brown, with radial marks; legs ochre-yellow, without darker rings, with black lines on femora and tibiae; abdomen ochre-gray, with few indistinct dark marks dorsally; ventrally with distinct black median band, partly disrupted, with two parallel longitudinal marks behind gonopore.

Body. Habitus similar to C. sahtan sp. nov. (cf. Fig. 391). Ocular area slightly raised. Deep thoracic pit and pair of furrows diverging from pit toward posterior margin. Clypeus unmodified, only rim more sclerotized than in female. Sternum wider than long (1.30/0.85), unmodified. Abdomen slightly elongated, dorso-posteriorly angular. Gonopore with five epiandrous spigots (Fig. 854); ALS with one widened spigot and one pointed spigot (Fig. 855).

Chelicerae. As in Figs 832-833, with two pairs of apophyses, lateral pair with 2-3 small processes (distinct in lateral view), median pair with one large modified cone-shaped hair each (Fig. 859); distance between tips of modified hairs $50 \mu \mathrm{m}$; lateral stridulatory ridges fine (Fig. 860; distances between ridges proximally $\sim 4 \mu \mathrm{m}$, distally $\sim 3 \mu \mathrm{m}$ ), visible in dissecting microscope.

PALPS. As in Figs 823-825; coxa with rounded retrolateral hump; trochanter barely modified; femur distally strongly widened, with rounded ventral protrusion, proximally with prolateral stridulatory pick, retrolateral-ventral rim with row of sclerotized hair-bases, with barely visible retrolateral transversal line, without retrolateral proximal process; femur-patella joints shifted toward prolateral side; tibia large relative to femur, tibia-tarsus joints slightly shifted toward retrolateral side; tarsus without macrotrichia; 
tarsal organ capsulate (Fig. 862); procursus (Figs 826-828) straight, densely set with long hairs dorsally, few hairs slightly curved upwards; proximally on prolateral side with strong hump set with numerous long hairs and followed distally by thick sclerotized ridge, procursus tip (Figs 856-857) with several distinctive elements: ventral sclerite with retrolateral branch, dorsal sclerite, prolateral partly sclerotized element, and retrolateral brush of short hair-like structures; genital bulb (Figs 829-831) with simple basal sclerite connected to distal (main) sclerite, sperm duct opening on prolateral-dorsal side (arrow in Fig. 858); distal sclerite with indistinct retrolateral ridge and distinctive set of two prolateral sclerotized ridges, each with three rounded apophyses.

Legs. Femur 1 with single row of $\sim 22$ ventral spines (Fig. 864); without curved hairs; few vertical hairs; retrolateral trichobothrium of tibia 1 at $4 \%$; prolateral trichobothrium absent on tibia 1, present on other leg tibiae; tarsal pseudosegments indistinct except 2-3 distally; tarsal organs capsulate, with round to slightly irregular rims (Fig. 863).

\section{Male (variation)}

Tibia 1 in 40 other males: 8.0-14.1 (mean 11.8). Abdomen usually also with whitish internal marks; ventral dark band on abdomen variably distinct, section behind gonopore with 2-4 longitudinal bands. Small males with fewer spines on femur 1 (smallest measured male with $\sim 12$ spines). Apophyses on prolateral bulbal ridges variably distinct, sometimes only two on each ridge; separation of ridges and angle between ridges very consistent. Retrolateral branch on ventral sclerite of procursus slightly variable in shape; in single male from Turkmenbashi slightly tilted (proximal rim bent towards retrolateral).

\section{Female}

In general similar to male but without spines on legs, with fewer and very indistinct stridulatory ridges on chelicerae (Fig. 861 ; distances between ridges $\sim 4.5 \mu \mathrm{m}$ ), and with stridulatory organ

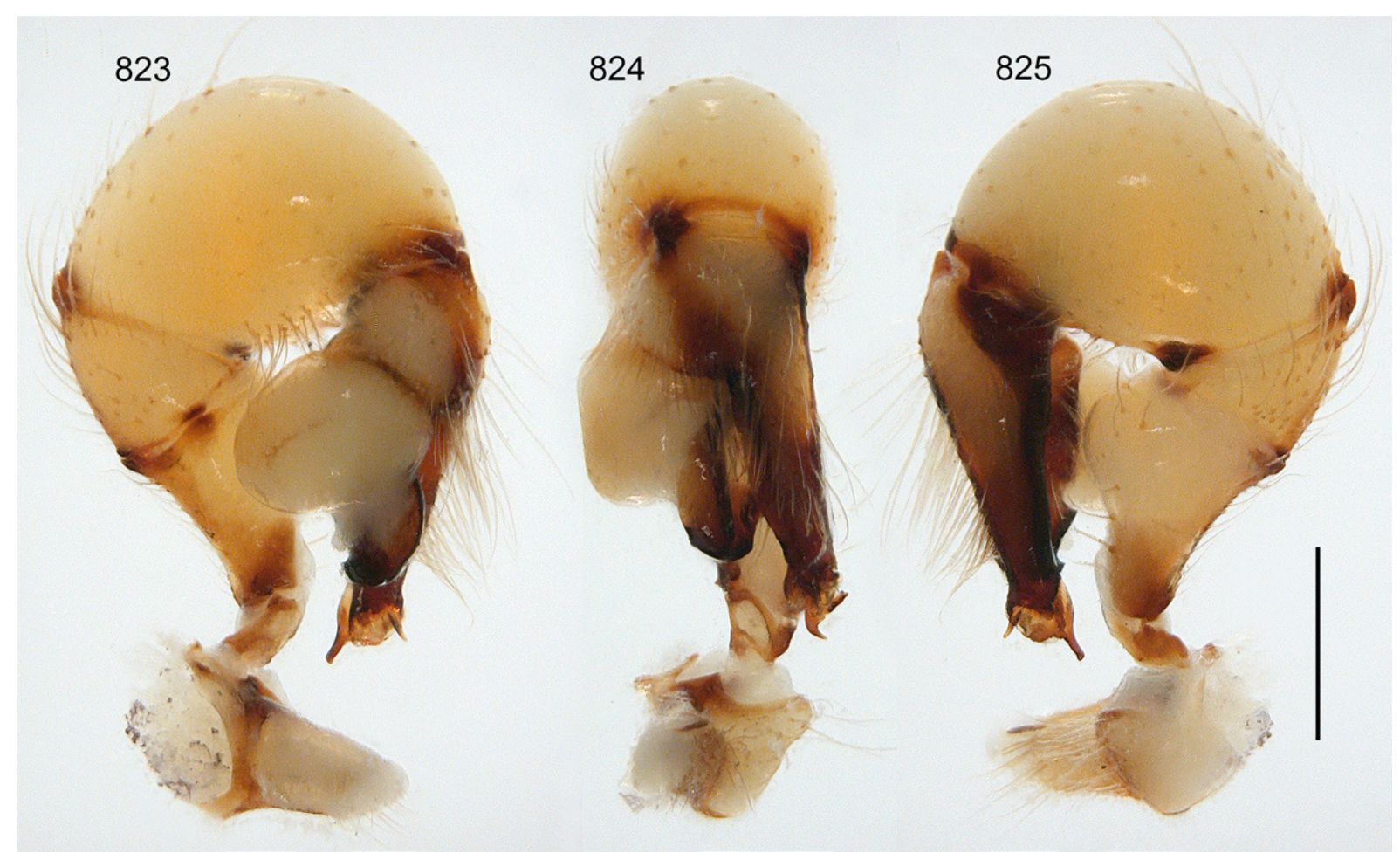

Figs 823-825. Crossopriza maculipes (Spassky, 1934); male from Turkmenistan, $5 \mathrm{~km} \mathrm{SW}$ of BazarTepe $(\mathrm{SZMN})$; left palp, prolateral, dorsal, and retrolateral views. Scale bar $=0.5 \mathrm{~mm}$. 


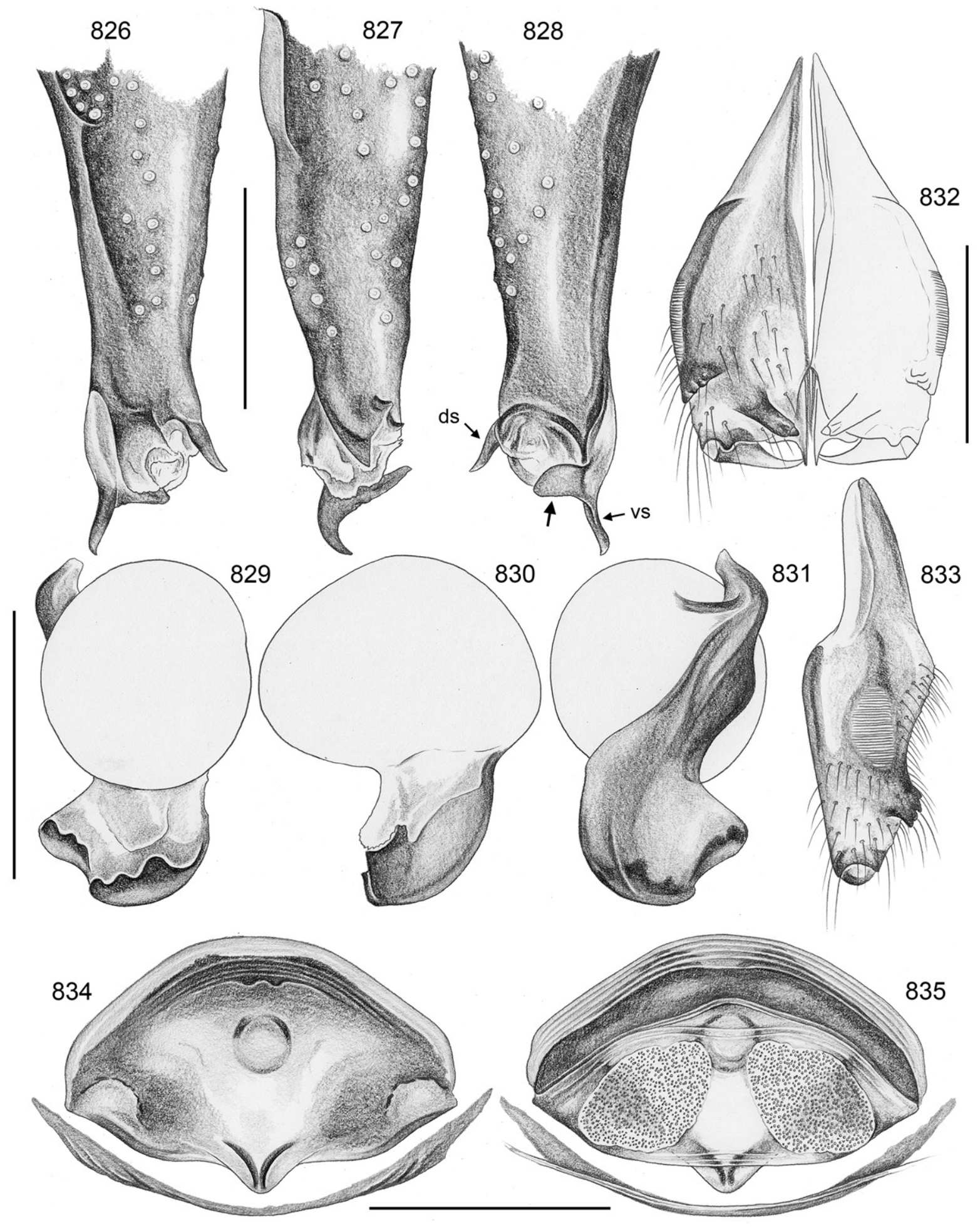

Figs 826-835. Crossopriza maculipes (Spassky, 1934); male and female from Turkmenistan, $5 \mathrm{~km}$ SW of Bazar-Tepe (SZMN). 826-828. Left procursus, prolateral, dorsal, and retrolateral views; arrow: retrolateral branch of ventral sclerite. 829-831. Left genital bulb, prolateral, dorsal, and retrolateral views. 832-833. Male chelicerae, frontal and lateral views. 834-835. Cleared female genitalia, ventral and dorsal views. Abbreviations: ds = dorsal sclerite; vs = ventral sclerite. Scale bars: $826-828=0.3 \mathrm{~mm}$; $829-835=0.5 \mathrm{~mm}$. 
consisting of pair of weakly sclerotized but distinct processes posteriorly on carapace and pair of light brown plates anteriorly on abdomen. Tibia 1 in 63 females: 6.1-12.9 (mean 10.2). Epigynum as in Figs 836-837 and 865, main epigynal plate semicircular, weakly protruding; posteriorly laterally strongly sclerotized, anteriorly weakly sclerotized, ochre-yellow with light brown median area; with pair of pockets very close to median line on both sides of median ridge (distance between pockets $25 \mu \mathrm{m}$ ), posteriorly slightly protruding; internal sclerotized arc and median round structure visible in uncleared specimens; pair of oblique black lines originating from posterior epigynal margin variably visible or not, possibly depending on degree of external sclerotization (difference not visible in cleared specimens); posterior plate short but wide. Internal genitalia (Figs 834-835, 838-840) with large oval pore plates converging anteriorly, dorsal arc strongly sclerotized but simple, ventral arc medially slightly modified, with simple median pouch. In the single female from Musa Khel (Punjab) the median epigynal area carrying the pockets is barely protruding towards posterior; this specimen is therefore assigned tentatively.

\section{Natural history}

Several records are from buildings. Beyond that, nothing is known about the biology of this widespread species.
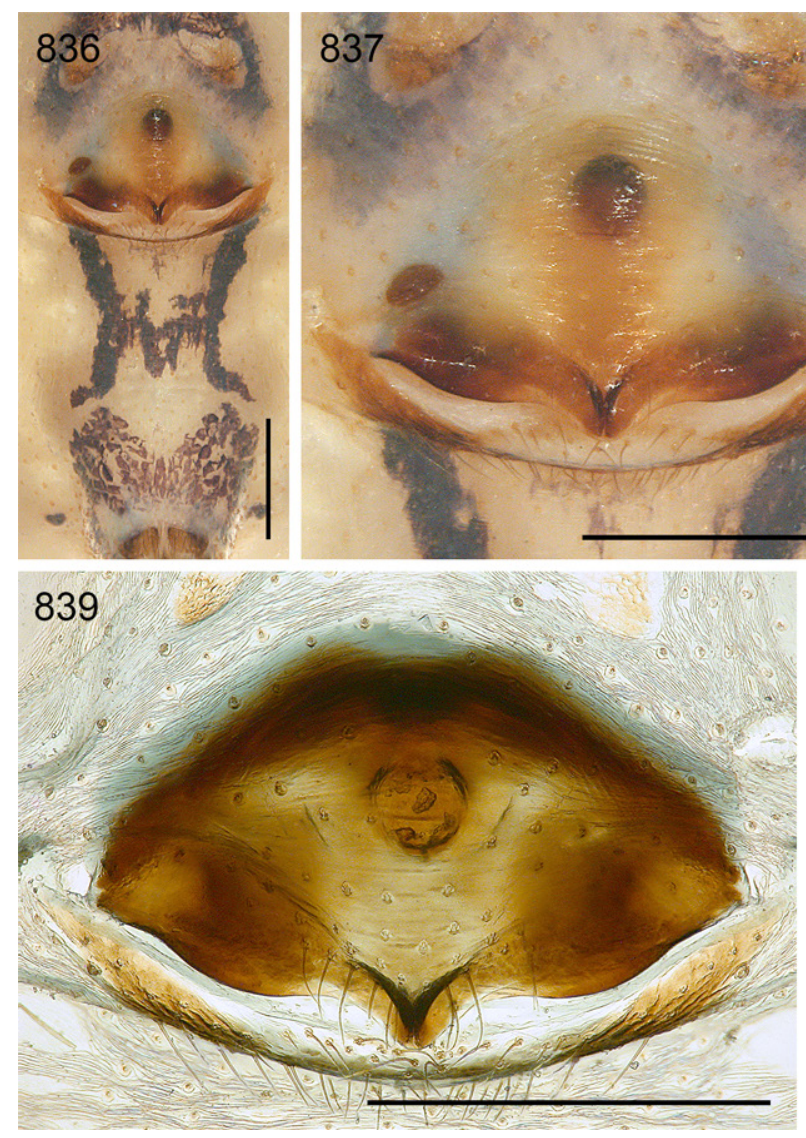
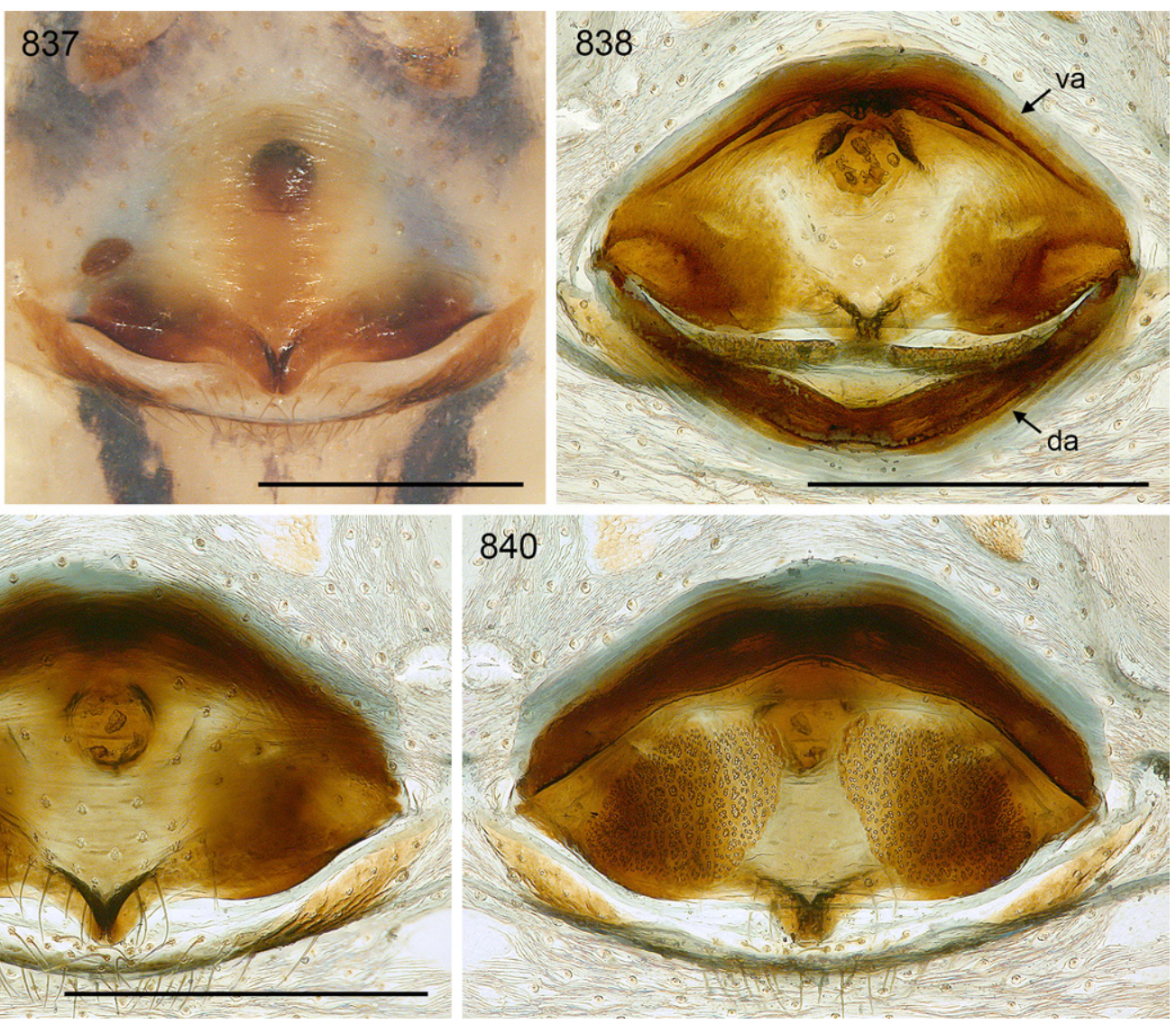

Figs 836-840. Crossopriza maculipes (Spassky, 1934); female from Uzbekistan, $6.5 \mathrm{~km} \mathrm{~W}$ of Akmechet (ZMMU). 836-837. Abdomen and epigynum, ventral views. 838-840. Cleared female genitalia, dorsal view with dorsal arc tilted backwards, ventral view, and regular dorsal view. Abbreviations: da $=$ dorsal arc; $\mathrm{va}=$ ventral arc. Scale bars $=0.5 \mathrm{~mm}$. 

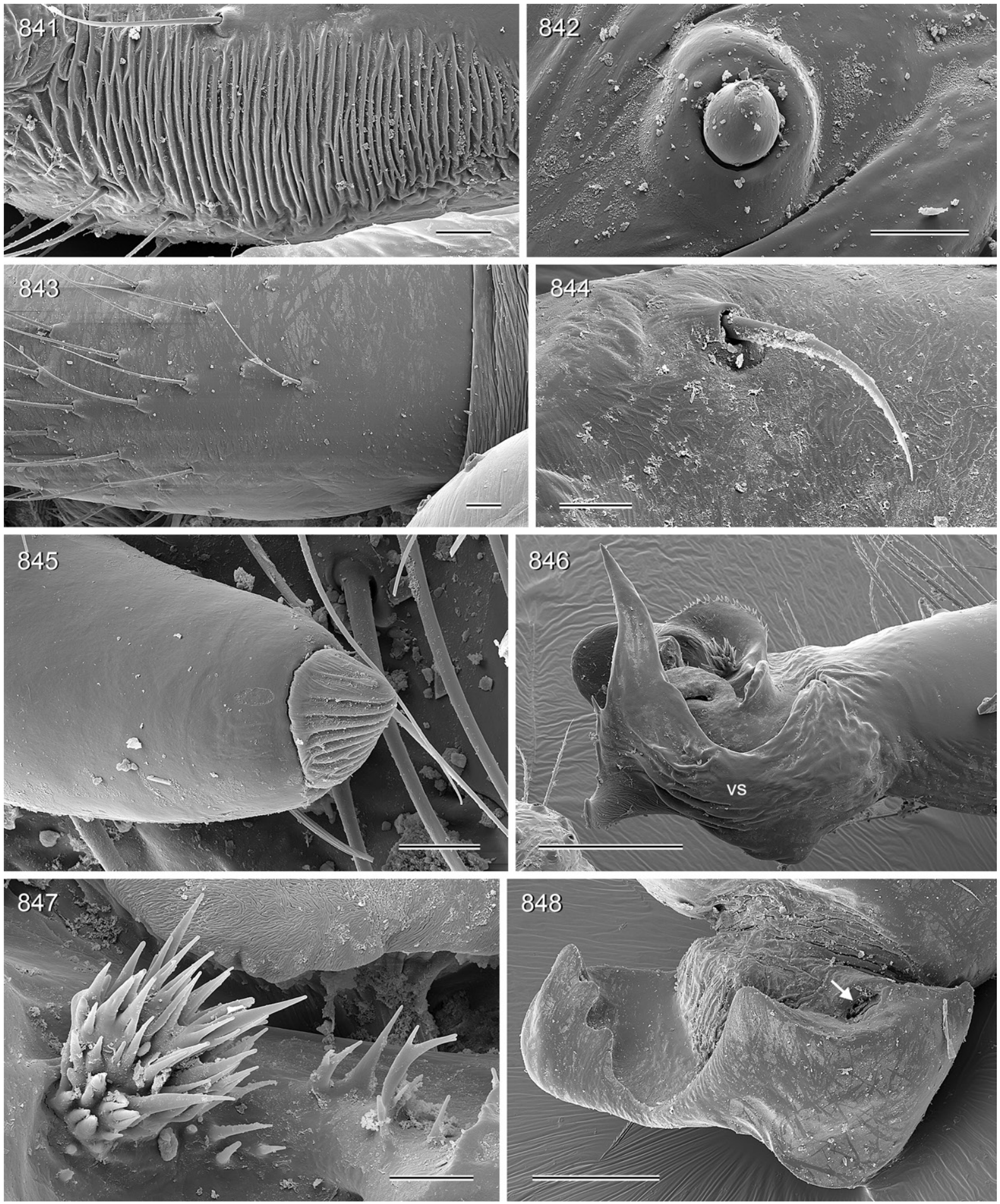

Figs 841-848. Crossopriza surobi sp. nov.; male and female from Afghanistan, SE of Surobi (MHNG). 841-842. Male stridulatory file and corresponding pick on palpal femur. 843-844. Female cheliceral side and proximal prolateral hair on female palpal femur. 845. Tip of male cheliceral apophysis. 846. Tip of left procursus, retrolateral-ventral view. 847. Detail of procursus tip. 848. Distal sclerite of left genital bulb, prolateral (slightly ventral and distal) view; arrow: sperm duct opening. Abbreviation: vs = ventral sclerite. Scale bars: $841,843=20 \mu \mathrm{m} ; 842,844-845,847=10 \mu \mathrm{m} ; 846,848=100 \mu \mathrm{m}$. 

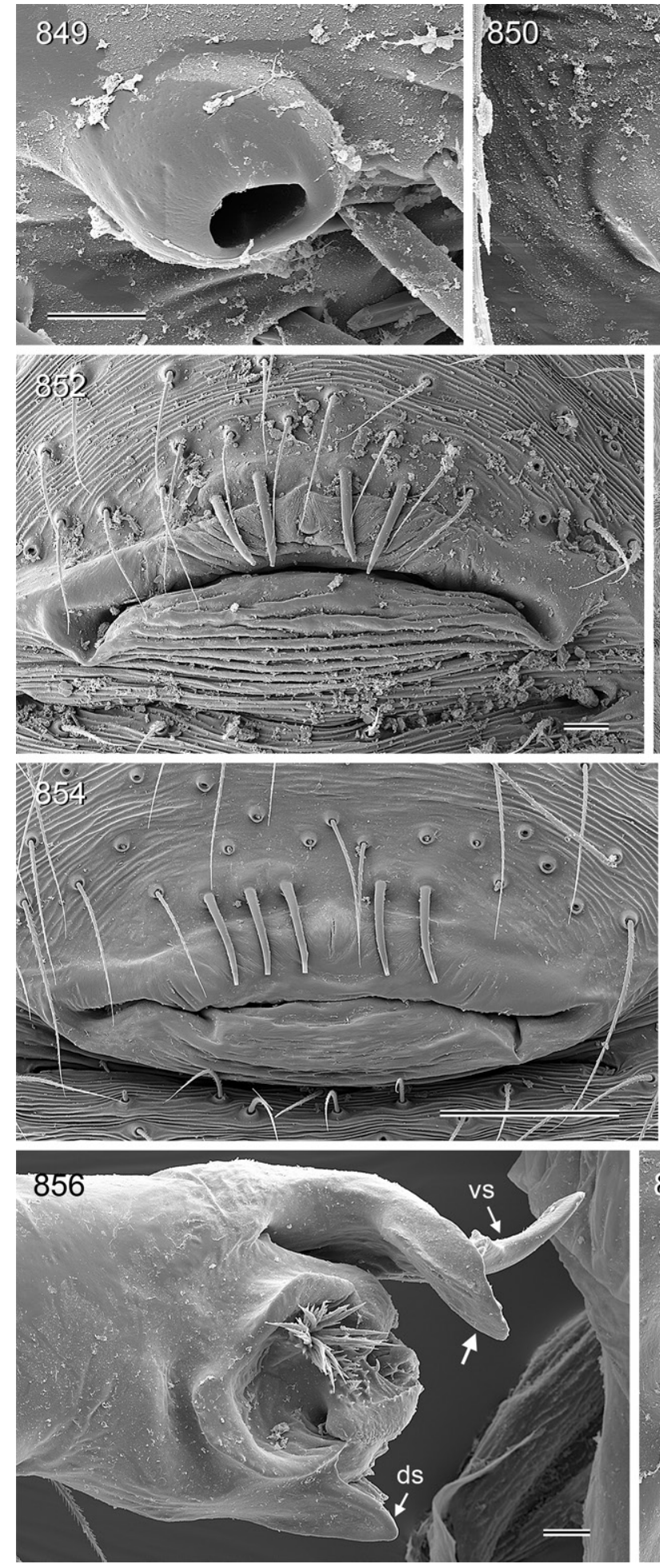
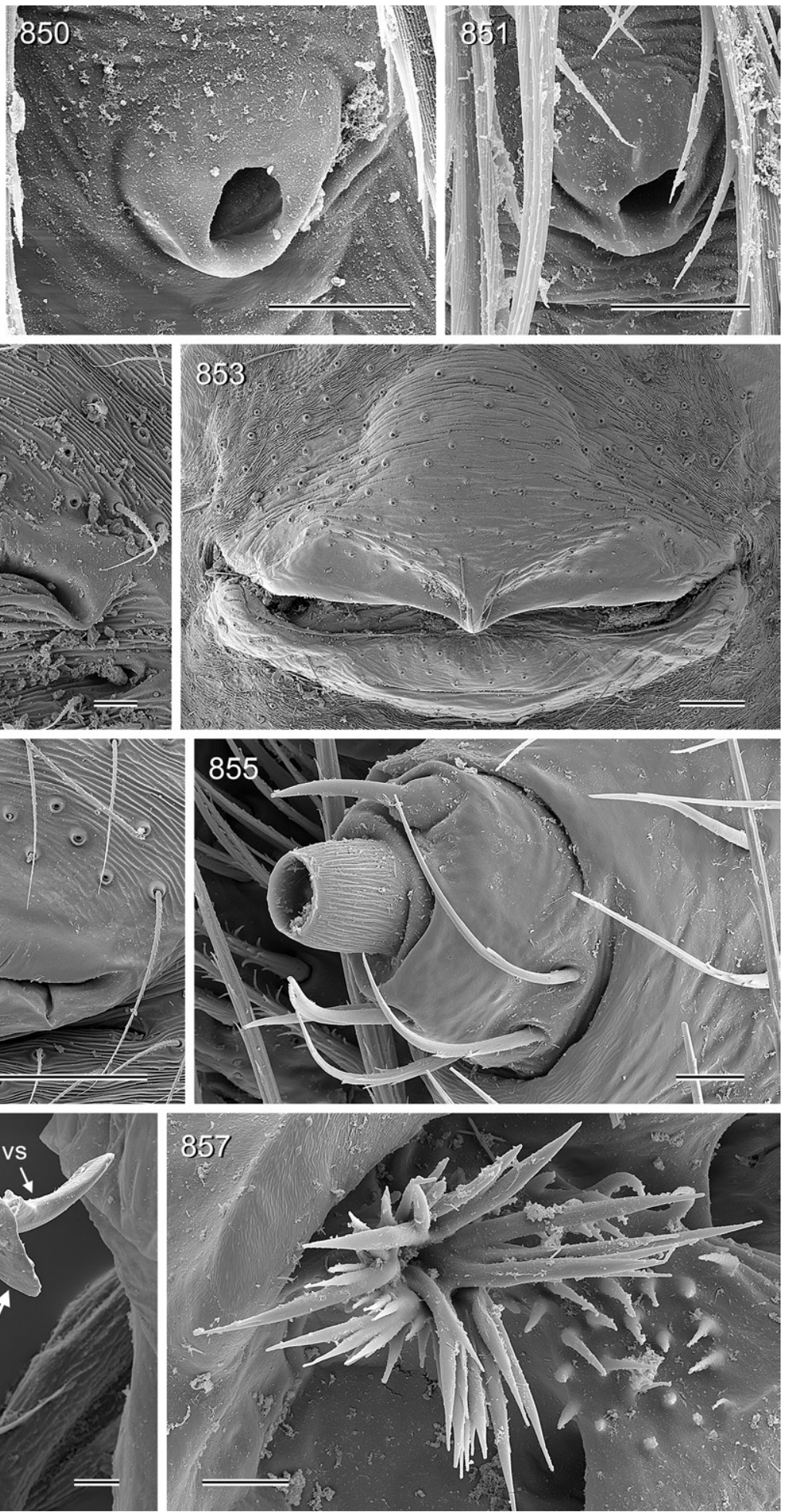

Figs 849-857. 849-853. Crossopriza surobi sp. nov.; male and female from Afghanistan, SE of Surobi (MHNG). 849-850. Male and female palpal tarsal organs. 851. Tarsal organ on female tarsus 1. 852. Male gonopore. 853. Epigynum. 854-857. Crossopriza maculipes (Spassky, 1934); male from Afghanistan, Djelalabad (MHNG). 854. Gonopore. 855. ALS. 856. Left procursus tip, retrolateral view; arrow: branch of ventral sclerite. 857. Detail of preceding figure. Abbreviations: ds $=$ dorsal sclerite; vs $=$ ventral sclerite. Scale bars: $849-851,855,857=10 \mu \mathrm{m} ; 852,856=20 \mu \mathrm{m} ; 853-854=100 \mu \mathrm{m}$. 

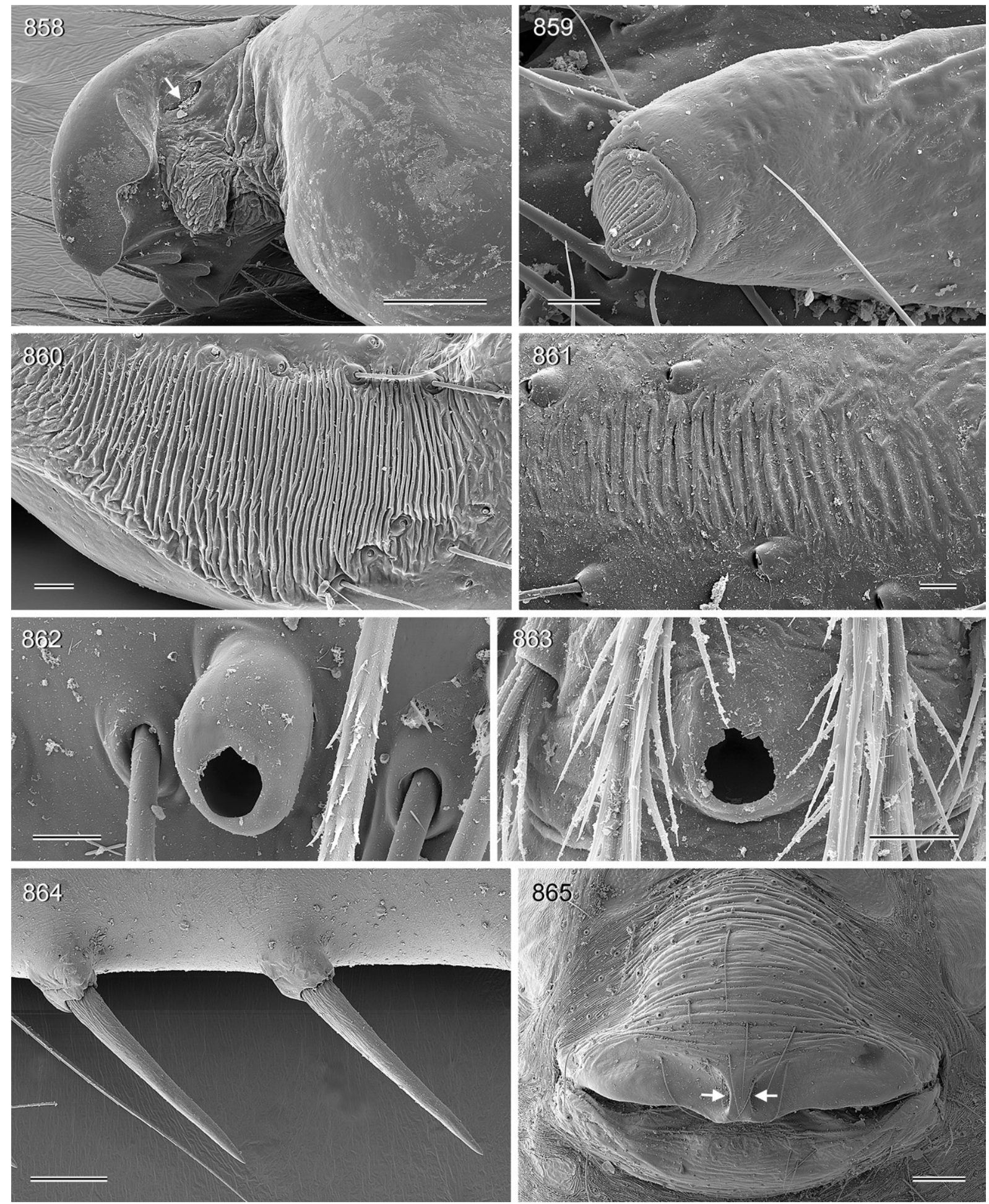

Figs 858-865. Crossopriza maculipes (Spassky, 1934); male and female from Afghanistan, Djelalabad (MHNG). 858. Right genital bulb, prolateral (slightly ventral) view; arrow: sperm duct opening. 859. Tip of male cheliceral apophysis. 860-861. Male and female cheliceral stridulatory files. 862. Male palpal tarsal organ. 863. Tarsal organ on male tarsus 1. 864. Spines on male femur 1. 865. Epigynum; arrows: pockets. Scale bars: $858,864-865=100 \mu \mathrm{m} ; 859,861-863=10 \mu \mathrm{m} ; 860=20 \mu \mathrm{m}$. 


\section{Distribution}

Widely distributed in Central Asia (Uzbekistan, Tajikistan, Turkmenistan), ranging into Afghanistan and Pakistan (Fig. 353B). I have not seen specimens from Iraq (contrary to what Najim \& Al-Khazali 2019 imply in their acknowledgements); however, judging from the illustrations in Najim \& Al-Khazali (2019), their specimens from Basrah province may be correctly identified. This suggests that the species is either also present in Iran or was somehow transported to Iraq. Sequences available in the Barcode of Life Data System (http://v3.boldsystems.org/) of two females collected in China (Hubei Province, Wuhan, in a building) are further evidence that the species has already spread far from its original distributional area.

\section{Discussion}

\section{Sexual dimorphisms}

A recent review of sexual dimorphisms in pholcid spiders (Huber 2021a) listed a wide range of characters that differ between males and females in this at first sight rather monomorphic family. Character mapping suggested more than 120 independent origins of sexual dimorphisms. The present paper adds further data to this list: a pair of sclerotized areas anteriorly on the abdomen of $H$. pluchei females, possibly acting against a strong anterior sclerite dorsally on the pedicel (Fig. 32); vertical hairs in slightly higher density on the tibiae of males than of females in some species of Maghreba gen. nov.; a second independent origin of distinct male clypeus modifications within Crossopriza (in C. sengleti sp. nov.; Fig. 768); and an indistinct difference in the degree of sclerotization of the clypeus rim in most representatives of the spotted-leg clade.

Except for this indistinct modification of the clypeus rim, and with the exception of the two species with distinct clypeus modifications (C. johncloudsleyi, C. sengleti sp. nov.), the clypeus in the spotted-leg clade is here generally described as being unmodified. This is true in the sense that males and females do not differ and that nothing 'unusual' can be seen in the light microscope. Interestingly, however, SEM images of both males and females show an area with a different cuticular texture centrally on the clypeus (e.g., Figs 44-46, 147, 416, 467, 594). Judging from the positions of similarly textured cuticle (e.g., on the carapace), these areas are here interpreted as muscle attachment sites. In Holocnemus pluchei males, this is what Calbacho-Rosa et al. (2019b) proposed to correspond functionally with the female sternum projection. An alternative that these authors did not consider is that the female sternum process might primarily function in a different context (i.e., not intersexual but intrasexual or allospecific). In this view, the contact between female projection and male clypeus might just be an occasional and relatively irrelevant by-product of the copulatory position. This interpretation is in line with the observation that male structures that obligatorily contact female structures during copulation are very unlikely to remain essentially unmodified (Eberhard 1985).

Sexual dimorphisms in the cheliceral stridulatory apparatus are common in Pholcidae and may either involve the presence vs absence of the stridulatory organ, or differences in the morphology of the files (size, distances between ridges, etc.) (Huber 2021a). Both kinds of dimorphisms occur in the spottedleg clade. While males in this group do always have stridulatory ridges, females vary from strongly developed ridges (e.g., H. pluchei; Fig. 48) to entirely smooth cheliceral sides (e.g., C. semicaudata; Fig. 600). There seems to be a tendency in females of Crossopriza, Maghreba gen. nov., and Stygopholcus to reduce the files, i.e., the files get smaller, the ridges less distinct, and the distances between the ridges larger. However, an analysis of this character requires SEM images, which are available for only a limited number of species.

\section{Male-female coevolution}

In several groups of Pholcidae, males insert the tips of their cheliceral apophyses into corresponding cavities or pockets on the female epigynal plate (Kraus 1984; Huber 1999, 2003, 2005). The exact 
significance of this interaction is unknown, but a strong grip or physical restraint appears implausible since the male chelicerae in Pholcidae are barely moveable against each other. In many or most cases, males may just use this system to achieve a correct position relative to the female before insertion of the copulatory organs (palps). Whatever the exact function, the result of this morphological interaction is a strong covariation between the distances of male and female structures. Figure 866 illustrates this covariation, but it must be noted that the distance between the female epigynal pockets is sometimes impossible to measure precisely. In general, I measured the point within the pocket that is supposedly contacted by the male cheliceral apophyses during copulation, but in the case of elongated pockets (or grooves) this is an approximate value. It must also be kept in mind that the values in Fig. 866 represent single individuals, i.e., intraspecific variation is ignored.

\section{Distribution}

The geographic distribution of Crossopriza lyoni is a further example of a rather mysterious trend in synanthropic pholcid spiders: they have spread over wide parts of the globe but are largely absent from the original areas of the respective genera. Good data for this supposed trend are difficult to obtain because for most synanthropic species the huge amount of published records has not yet been georeferenced. For C. lyoni, the map in Fig. 351 is a fairly comprehensive representation of the known distribution, and it is remarkable that there is not a single credible record from the area where the genus Crossopriza has its highest species diversity: NE Africa, the Arabian Peninsula, the Middle East, and Central Asia.

This phenomenon has been reported before for at least two other pholcid species. Artema atlanta Walckenaer, 1837 has a worldwide distribution similar to C. lyoni, but is almost entirely absent from the area where most congeneric species occur (Arabian Peninsula, Middle East, Central Asia) (Aharon et al. 2017). Psilochorus simoni (Berland, 1911) has been spreading in Europe since about a century (Fürst \& Blandenier 1993) and is now common in houses in most of Europe and beyond (Bayram et al.

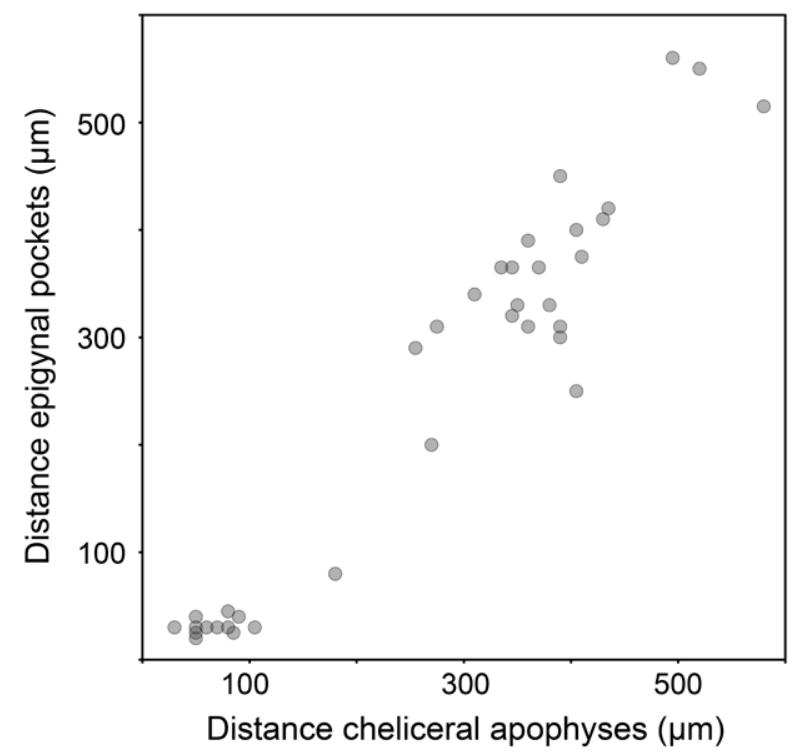

Fig. 866. Scatter diagram showing the correlation between the distances between the male cheliceral apophyses (tips) and the distances between the female epigynal pockets. Each dot represents one species (all known representatives of Holocnemus Simon, 1873, Maghreba gen. nov., and Crossopriza Simon, 1893). 
2008; Paquin et al. 2010). In North America, the native area of the genus, $P$. simoni is a rare spider, known from only four specimens (three of them females) from four localities (in California and Oregon; Slowik 2009).

This trend is mysterious in the sense that it seems hard to imagine that synanthropic habitats in the native areas of the respective genera differ in some relevant aspect from those in other parts of the world. In theory, synanthropic habitats in the native areas might be preoccupied by congeneric species, but at least for Artema and Psilochorus this is quite clearly not the case. In Crossopriza, several species invade houses (e.g., C. semicaudata, C. pristina, C. maculata), but in at least some areas, Crossopriza appears entirely absent from buildings (e.g., Oman). Finally, it must be noted that the trend is certainly not absolute. At least one synanthropic pholcid does not follow the scheme: Physocyclus globosus (Taczanowski, 1874) is common in and around buildings all over Mexico and Central America, the native area of the genus (Valdez-Mondragón 2010).

\section{Acknowledgements}

I thank the following people for sending material in loan: C. Allard, J. Altmann, J. Beccaloni, J.W. Berry, B. van der Bijl, W. van Bohemen, R. Bosmans, E.H. Buckup, C.L. Deeleman-Reinhold, C. Deltshev, J. Dunlop, A. Friederichs, M. Gray, C. Grismado, C. Griswold, J. Gruber, M. Harvey, D. Hirst, P. Jäger, R. Jocqué, C. Jonsson, M. Komnenov, L. Leibensperger, A.A. Lise, J. Lissner, L. Logunov, J.M. Maes, Y.M. Marusik, G. Milledge, E. Morano, L.A. Moscaliuc, J. Murphy, P. Oger, K. Paragamian, N. Platnick, M. Ramirez, R. Raven, C. Ribera, C. Rollard, N. Scharff, P. Schwendinger, M. Siyam, L. Sorkin, H. Steiner, M.S. Tahami, A. Topçu, J. Van Keer, and J. Waldock. Further specimens were kindly donated by R. Bosmans, A. Butt, T. Danışman, F. Gasparo, J. Hajer, S. Huber, N. Khan, B. Knoflach-Thaler, J. Lips, Y.M. Marusik, J. Moya-Laraño, P. Oger, H. Öztürk, F. Perveen, A. Russell-Smith, M. Siyam, A. van Harten, and A. Zamani. For help with coordinates I thank M. Pavlek and M.S. Harvey; J. Mederos and M. Tavano kindly prepared and sent photos of the type of Holocnemus aurouxi and of Koch's Pholcus rivulatus from Massaua, respectively; I thank P. Purcell and F. Mozaffarian for help with clarifying the dubious localities "Didi Davvs" and "Barm-e-peereGhaibi", respectively; and C. Jonsson for help with clarifying the localitites visited by K. Lindberg in the 1950s; Y.M. Marusik and D. Logunov kindly provided translations of Russian labels; H. Ono helped with translating details of a Japanese paper. R. Victor, I. Al Zakwani, and H. Belhadj helped with preparation of collecting trips, with permits, and logistics. I thank two anonymous reviewers for their valuable comments and suggestions, and the EJT editorial team for their cooperation, in particular P. Fernández for his meticulous editing.

\section{References}

Aharon S., Huber B.A. \& Gavish-Regev E. 2017. Daddy-long-leg giants: revision of the spider genus Artema Walckenaer, 1837 (Araneae, Pholcidae). European Journal of Taxonomy 376: 1-57. https://doi.org/10.5852/ejt.2017.376

Ahmed H.S.K. 2021. Biological studies on Holocnemus pluchei (Pholcidae) when fed on various prey species. Journal of Plant Protection and Pathology 12: 163-166.

https://doi.org/10.21608/jppp.2021.154422

Ali P.A., Zahid M. \& Butt A. 2016. New record of spiders fauna of Khyber Pakhtunkhwa Province, (Aranei; Sparassidae; Pholcidae) Pakistan. International Journal of Biosciences 9: 247-252. https://doi.org/10.12692/ijb/9.6.247-252

Ávila Herrera I.M., Král J., Pastuchová M., Forman M., Musilová J., Kořínková T., Št’áhlavský F., Zrzavá M., Nguyen P., Just P., Haddad C.R., Hiřman M., Koubová M., Sadílek D. \& Huber B.A. 2021. Evolutionary pattern of karyotypes and meiosis in pholcid spiders (Araneae: Pholcidae): implications 
for reconstructing chromosome evolution of araneomorph spiders. BMC Ecology and Evolution 21: 75. https://doi.org/10.1186/s12862-021-01750-8

Barrientos J.A. \& Bosco Febrer J. 2017. Arañas (Arachnida, Araneae) de Menorca (Islas Baleares, España). Nuevos datos. Revista Ibérica de Aracnología 31: 8-24.

Barrientos J.A. \& Ferrández M.A. 1982. La colección de Araneidos del Departamento de Zoología de la Universidad de Salamanca, III: arañas migalomorfas, haploginas, y cribeladas. Boletín de la Asociación española de Entomología 5: 75-86.

Barrientos J.A., Brañas N. \& Mederos J. 2019. Dos especies nuevas de Pholcidae (Araneae) de Marruecos. Revista Ibérica de Aracnología 34: 3-12.

Barriga J.C., Jiménez-Valverde A., Morano E., Morano A.G. \& Melic A. 2006. Arañas de la provincia de Ciudad Real (Arachnida: Araneae) (Castilla la Mancha, España). Revista Ibérica de Aracnología 13: $125-142$.

Bauer T., Wendt I., Holstein J. \& Gabriel G. 2016. Crossopriza lyoni new to Germany (Araneae: Pholcidae). Arachnologische Mitteilungen 52: 4-6. https://doi.org/10.5431/aramit5202

Bayram A., Allahverdi H., Danışman T., Yiğit N. \& Kunt K.B. 2008. A new genus and species record from Turkey: Psilochorus simoni (Berland, 1911) (Araneae, Pholcidae). Turkish Journal of Arachnology 1: 91-97.

Beatty J.A., Berry J.W. \& Huber B.A. 2008. The pholcid spiders of Micronesia and Polynesia (Araneae, Pholcidae). The Journal of Arachnology 36: 1-25. https://doi.org/10.1636/H05-66.1

Benhadi-Marín J., Barrientos J.A., Ferrández M.A., Henriques S. \& Morano E. 2013. Second annotated list of selected spider families (Arachnida: Araneae) held at the University of León (Spain). Revista Ibérica de Aracnología 22: 71-78.

Berland L. 1942. Polynesian spiders. Occasional Papers of the Bernice P. Bishop Museum 17: 1-24.

Blackwall J. 1858. Descriptions of six newly discovered species and characters of a new genus of Araneidea. The Annals and Magazine of Natural History (3) 1: 426-434.

https://doi.org/10.1080/00222935808696953

Blackwall J. 1867. Descriptions of several species of East-Indian spiders apparently new or little known to arachnologists. The Annals and Magazine of Natural History (3) 19: 387-394.

https://doi.org/10.1080/00222936708562695

Blanchong J.A., Summerfield M.S., Popson M.A. \& Jakob E.M. 1995. Chivalry in pholcid spiders revisited. The Journal of Arachnology 23: 165-170.

Blanke R. 1972. Untersuchungen zur Ökophysiologie und Ökethologie von Cyrtophora citricola Forskål (Araneae, Araneidae) in Andalusien. Forma et Functio 5: 125-206.

Bonnet P. 1958. Bibliographia Araneorum. Analyse méthodique de toute la littérature aranéologique jusqu'en 1939. Tome II (4me partie: $N-S$ ). Douladoure, Toulouse.

Bosmans R., Van Keer J., Russell-Smith A., Kronestedt T., Alderweireldt M., Bosselaers J. \& de Koninck H. 2013. Spiders of Crete. A catalogue of all currently known species from the Greek island of Crete. Newsletter of the Belgian arachnological Society 28 (suppl. 1): 1-147.

Breitling R. 2020. South European spiders from the Duffey collection in the Manchester Museum (Arachnida: Araneae). Arachnology 18: 333-362. https://doi.org/10.13156/arac.2020.18.4.333

Brignoli P.M. 1971a. Note sui Pholcidae d'Italia (Araneae). Fragmenta Entomologica 7: 79-101. 
Brignoli P.M. 1971b. Note sui ragni cavernicoli Italiani (Araneae). Fragmenta Entomologica 7: 121229.

Brignoli P.M. 1971c. Beitrag zur Kenntnis der mediterranen Pholcidae (Arachnida, Araneae). Mitteilungen aus dem zoologischen Museum in Berlin 47: 255-267.

https://doi.org/10.1002/mmnz.19710470203

Brignoli P.M. 1976. Ragni di Grecia IX. Specie nuove o interessanti delle famiglie Leptonetidae, Dysderidae, Pholcidae ed Agelenidae (Araneae). Revue suisse de Zoologie 83: 539-578. https://doi.org/10.5962/bhl.part.91452

Brignoli P.M. 1977. Ragni del Libano II. Un nuovo Pholcus e commenti sugli altri Pholcidae del Medio Oriente (Araneae). Fragmenta Entomologica 13: 31-39.

Brignoli P.M. 1978. Ragni di Turchia V. Specie nuove o interessanti, cavernicole ed epigee, di varie famiglie (Araneae). Revue suisse de Zoologie 85: 461-541. https://doi.org/10.5962/bhl.part.82243

Brignoli P.M. 1979. Ragni di Grecia XI. Specie nuove o interessanti, cavernicole ed epigee. Revue suisse de Zoologie 86: 181-202. https://doi.org/10.5962/bhl.part.82285

Brignoli P.M. 1984. Ragni di Grecia XII. Nuovi dati su varie famiglie (Araneae). Revue suisse de Zoologie 91: 281-321. https://doi.org/10.5962/bhl.part.81881

Brown B.V. 1993. A further chemical alternative to critical-point-drying for preparing small (or large) flies. Fly Times 11: 10.

Calbacho-Rosa L., Córdoba-Aguilar A. \& Peretti A.V. 2010. Occurrence and duration of post-copulatory mate guarding in a spider with last sperm precedence. Behaviour 147: 1267-1283.

https://doi.org/10.1163/000579510X514544

Calbacho-Rosa L., Galicia-Mendoza I., Dutto M.S., Córdoba-Aguilar A. \& Peretti A.V. 2013. Copulatory behavior in a pholcid spider: males use specialized genitalic movements for sperm removal and copulatory courtship. Naturwissenschaften 100: 407-416. https://doi.org/10.1007/s00114-013-1038-1

Calbacho-Rosa L., Córdoba-Aguilar A., Munguía-Steyer R. \& Peretti A.V. 2017. A parental care-mating dilemma? Potential risks for offspring in the pholcid spider when egg-carrying females accept mating. Journal of Insect Behavior 30: 155-169. https://doi.org/10.1007/s10905-017-9604-6

Calbacho-Rosa L., Cargnelutti F., Córdoba-Aguilar A. \& Peretti A.V. 2019a. Consistency of females' stridulatory behaviour during inter-sexual interactions in spiders. Ethology 125: 548-554.

https://doi.org/10.1111/eth.12880

Calbacho-Rosa L., Cargnelutti F., Peretti A.V. \& Costa-Schmidt L.E. 2019b. Female secondary sexual traits in spiders: adaptive interpretations of the sternum projection in the pholcid Holocnemus pluchei. Zoology 136: 125694. https://doi.org/10.1016/j.zool.2019.125694

Caporiacco L. di 1948. L'aracnofauna di Rodi. Redia 33: 27-75.

Cardoso P., Henriques S.S., Gaspar C., Crespo L.C., Carvalho R., Schmidt J.B., Sousa P. \& Szüts T. 2009. Species richness and composition assessment of spiders in a Mediterranean scrubland. Journal of Insect Conservation 13: 45-55. https://doi.org/10.1007/s10841-007-9116-3

Cargnelutti F., Calbacho-Rosa L., Córdoba-Aguilar A. \& Peretti A.V. 2018. Patterns of sperm transfer in a pholcid spider with two distinct copulatory phases. Journal of Insect Behavior 31: 616-628.

https://doi.org/10.1007/s10905-018-9702-0

Cargnelutti F., Calbacho-Rosa L., Uñates D., Costa-Schmidt L.E., Córdoba-Aguilar A. \& Peretti A.V. 2020. Copulatory behaviour increases sperm viability in female spiders. Biological Journal of the Linnean Society 131: 536-546. https://doi.org/10.1093/biolinnean/blaa130 
Carvalho L.S. \& Avelino M.T.L. 2010. Composição e diversidade da fauna de aranhas (Arachnida, Araneae) da Fazenda Nazareth, Município de José de Freitas, Piauí, Brasil. Biota Neotropica 10: 1-11. https://doi.org/10.1590/S1676-06032010000300001

Centre de Recherche en Ethnomusicologie. 2020. Archives, Collections, Tchad, Tibesti, Brandily, Monique. 1965. Available from https://archives.crem-cnrs.fr/archives/collections/CNRSMH_I_2009_001/ [accessed 30 Dec. 2021].

Chamberlin R.V. 1924. Descriptions of new American and Chinese spiders, with notes on other Chinese species. Proceedings of the United States National Museum 63: 1-38, pl. 1-7.

https://doi.org/10.5479/si.00963801.63-2481.1

Chen Z. \& Zhang Z. 1991. Fauna of Zhejiang. Araneida. Zhejiang Science and Technology Publishing House, Zhejiang.

Chikuni Y. 1989. Pictorial Encyclopedia of Spiders in Japan. Kaisei-sha Publishing Co., Tokyo.

Chrysanthus O.F.M. 1967. Spiders from South New Guinea IX. Tijdschrift voor Entomologie 110: 89105.

Colmenares García P.A. 2008. Tres nuevos registros para la araneofauna Venezolana (Arachnida, Araneae, Pholcidae). Boletín del Centro de Investigaciones Biológicas, Universidad de Zulia 42: 85-92.

Constantini G.P. 1975. I ragni della provincia di Brescia. Note di Aracnologia. Natura Bresciana 12: 81-92.

Dalmas C. de. 1920. Liste d'araignées de Boudron en Asie Mineure suive d'une étude des espèces méditerranéennes du genre Habrocestum. Annali del Museo Civico di Storia Naturale di Genova 50: $57-69$.

Damin N. 1900. Pauci Dalmajice, Hrvatske, Slavonije i Istre. Rad JAZU 143: 1-53.

Deeleman-Reinhold C.L. 1995. Redescription of Holocneminus multiguttatus Simon and description of two new species of pholcid spiders from Australia (Arachnida: Araneae: Pholcidae). Beiträge zur Araneologie 4 (1994): 31-41. [publ. in Dec. 1995]

Deeleman-Reinhold C.L. \& van Harten A. 2001. Description of some interesting, new or little known Pholcidae (Araneae) from Yemen. In: Ishwar Prakash (ed.) Ecology of Desert Environments: 193-207. Scientific Publishers, Jodhpur, India.

Deltshev C., Lazarov S. \& Petrov B.P. 2003. A contribution to the study of spiders (Araneae) from the caves of Bulgaria. Acta zoologica Bulgarica 55: 9-28.

Deltshev C., Vrenosi B., Blagoev G. \& Lazarov S. 2011. Spiders of Albania - faunistic and zoogeographical review (Arachnida: Araneae). Acta zoologica Bulgarica 63: 125-144.

Deltshev C., Komnenov M., Blagoev G., Georgiev T., Lazarov S., Stojkostka E. \& Naumova M. 2013. Faunistic diversity of spiders (Araneae) in Galichitsa mountain (FYR Macedonia). Biodiversity Data Journal 1: e977. https://doi.org/10.3897/BDJ.1.e977

Denis J. 1945. Descriptions d'araignées Nord-Africaines. Bulletin de la Société d'Histoire naturelle de Toulouse 79: 41-57.

Dimitrov D., Astrin J.J. \& Huber B.A. 2013. Pholcid spider molecular systematics revisited, with new insights into the biogeography and the evolution of the group. Cladistics 29: 132-146.

https://doi.org/10.1111/j.1096-0031.2012.00419.x

Downes M.F. 1987. Crossopriza (lyoni?) (Araneae, Pholcidae) eats her own eggs. The Journal of Arachnology 15: 276. 
Drakšić M. \& Katušić L. 2011. Preliminary results on the spider (Araneae) fauna of NP Kornati (Croatia). Entomologia Croatica 14: 163-175.

Drensky P. 1936. Katalog der echten Spinnen (Araneae) der Balkanhalbinsel. Opis na Paiatzite ot Balkanikia polouostrow. Spisanié na Beulgarskata Akademia na Naoukite 32: 1-223.

Drensky P. 1939. Faounata na Paiatzite (Araneae) w Beulgaria. III. Podrazred Arachnomorphae; II klon Trionychia; semeystwa: Urocteidae; Uloboridae; Sicaridae; Pholcidae; Eresidae. Izvestiya na Tsarskite Prirodonauchni Instituti v Sofia 12: 231-252.

Dufour L. 1820. Description de cinq arachnides nouvelles. Annales Générales des Sciences Physiques 5: 198-209.

Dutto M.S., Calbacho-Rosa L. \& Peretti A.V. 2011. Signalling and sexual conflict: female spiders use stridulation to inform males of sexual receptivity. Ethology 117: 1040-1049.

https://doi.org/10.1111/j.1439-0310.2011.01957.x

Dyal S. 1935. Fauna of Lahore. 4. Spiders of Lahore. Bulletin of the Department of Zoology of the Panjab University 1: i-ii, 119-252.

Eberhard W.G. 1985. Sexual Selection and Animal Genitalia. Harvard University Press, Cambridge, Massachusetts.

Eberle J., Dimitrov D., Valdez-Mondragón A. \& Huber B.A. 2018. Microhabitat change drives diversification in pholcid spiders. BMC Evolutionary Biology 18: 141.

https://doi.org/10.1186/s12862-018-1244-8

Edwards G.B. 1993. Crossopriza lyoni and Smeringopus pallidus: cellar spiders new to Florida (Araneae: Pholcidae). Florida Department of Agriculture \& Consumer Services, Division of Plant Industry, Entomology Circulars 361: 1-2.

Ferrández M.A., Morano E., Fernández de Céspedes H. \& Camargo M. 2006. Catálogo de las arañas (Araneae) de la Comunidad de Madrid. Graellsia 62 (n extraordinario): 53-90.

https://doi.org/10.3989/graellsia.2006.v62.iExtra.112

Foelix R.F. \& Chu-Wang I.W. 1973. The morphology of spider sensilla. II. Chemoreceptors. Tissue \& Cell 5: 461-478. https://doi.org/10.1016/S0040-8166(73)80038-2

Forsskål P. 1775. Descriptiones animalium avium, amphibiorum, piscium, insectorum, vermium; quae in itinere orientali observavit Petrus Forskål. Möller, Copenhagen [Hauniae].

https://doi.org/10.5962/bhl.title.2154

Forsskål P. 1776. Icones rerum naturalium, quas in itinere orientali depingi curavit Petrus Forskål. Möller, Copenhagen [Hauniae].

Franganillo P.B. 1925. Contribución al estudio de la geografía aracnológica de la Península Ibérica. Boletín de la Sociedad entomológica de España 8: 31-40.

Franganillo P.B. 1926a. Arácnidos nuevos o poco conocidos de la Isla de Cuba. Boletín de la Sociedad entomológica de España 9: 42-68.

Franganillo P.B. 1926b. Arácnidos de Cuba. Arácnidos nuevos o poco conocidos de la Isla de Cuba. Imprenta "El Siglo XX", Habana.

Franganillo P.B. 1926c. Arácnidos de Andalucía. Boletín de la Sociedad entomológica de España 9: 69-82.

Franganillo P.B. 1936a. Los Arácnidos de Cuba hasta 1936. Cultura, S.A., La Habana.

Franganillo P.B. 1936b. Arácnidos recogidos durante el verano de 1934. Revista 'Belen' (1936): 75-82. 
Fürst P.-A. \& Blandenier G. 1993. Psilochorus simoni (Berland, 1911) (Araneae, Pholcidae): découvertes de nouvelles stations suisses et discussion de son écologie. Bulletin de la Société neuchâteloise des sciences naturelles 116: 75-85.

Gajić I. \& Grbić G. 2016. Further notes on spiders from the Special Nature Reserve Zasavica (Serbia). Arachnologische Mitteilungen 51: 49-56.

Gasparo F. 2003. Tre campagne biospeleologiche a Creta. Progresione 48: 59-62.

Gerhardt U. 1927. Neue biologische Untersuchungen an einheimischen und ausländischen Spinnen. Zeitschrift für Morphologie und Ökologie der Tiere 8: 96-186. https://doi.org/10.1007/BF00464883

Goloboff P.A. 1993. NONA (NO NAME) ver. 2. Published by the author, Tucumán, Argentina. Available from http://www.lillo.org.ar/phylogeny/ [accessed 30 Dec. 2021].

Goloboff P.A. 1997. Pee-Wee, ver. 2.8. Published by the author. New York. Available from http://www.lillo.org.ar/phylogeny/ [accessed 30 Dec. 2021].

Goloboff P.A., Farris J.S. \& Nixon K. 2004. TNT, tree analysis using new technology, version 1.1, sponsored by the Willi Hennig Society. Available from http://www.lillo.org.ar/phylogeny/ [accessed 30 Dec. 2021].

Goloboff P.A., Farris J.S. \& Nixon K. 2008. TNT, a free program for phylogenetic analysis. Cladistics 24: 774-786. https://doi.org/10.1111/j.1096-0031.2008.00217.x

González-Sponga M.A. 2006. Arácnidos de Venezuela. Un nuevo género y cinco nuevas especies de la familia Pholcidae (Araneae). Sapiens. Revista Universitaria de Investigación 7: 9-27.

Guarisco H. \& Cutler B. 2003. Crossopriza lyoni (Araneae: Pholcidae), a synanthropic cellar spider newly discovered in Kansas. Transactions of the Kansas Academy of Sciences 106: 105-106.

https://doi.org/10.1660/0022-8443(2003)106[0105:CLAPAS]2.0.CO;2

Hajer J. 1995. Notes on spinning activity and the way of life of the spiders Cyrtophora citricola, Argyrodes gibbosus and Holocnemus pluchei. In: V. Ružicka (ed.) Proceedings of the $15^{\text {th }}$ European Colloquium of Arachnology: 84-88. Institute of Entomology, České Budějovice, Czech Republic.

Hajer J. \& Řeháková D. 2003. Spotted dome-shaped webs of the pholcids Crossopriza sp. and Holocnemus pluchei (Araneae: Pholcidae). Bulletin of the British Arachnological Society 12: 345-354.

Heimer S. \& Nentwig W. 1991. Spinnen Mitteleuropas: Ein Bestimmungsbuch. Paul Parey, Berlin.

Huber B.A. 1995. Copulatory mechanism in Holocnemus pluchei and Pholcus opilionoides, with notes on male cheliceral apophyses and stridulatory organs in Pholcidae (Araneae). Acta Zoologica, Stockholm 76: 291-300. https://doi.org/10.1111/j.1463-6395.1995.tb01001.x

Huber B.A. 1997. Redescriptions of Eugène Simon's neotropical pholcids (Araneae, Pholcidae). Zoosystema 19: 573-612. Available from https://www.biodiversitylibrary.org/page/56152852 [accessed 30 Dec. 201].

Huber B.A. 1999. Sexual selection in pholcid spiders (Araneae, Pholcidae): artful chelicerae and forceful genitalia. The Journal of Arachnology 27: 135-141.

Huber B.A. 2000. New World pholcid spiders (Araneae: Pholcidae): a revision at generic level. Bulletin of the American Museum of Natural History 254: 1-348. https://doi.org/brh26h

Huber B.A. 2001. The pholcids of Australia (Araneae; Pholcidae): taxonomy, biogeography, and relationships. Bulletin of the American Museum of Natural History 260: 1-144.

https://doi.org/10.1206/0003-0090(2001)260\%3C0001:TPOAAP\%3E2.0.CO;2 
Huber B.A. 2003. Southern African pholcid spiders: revision and cladistic analysis of Quamtana gen. nov. and Spermophora Hentz (Araneae: Pholcidae), with notes on male-female covariation. Zoological Journal of the Linnean Society 139: 477-527. https://doi.org/10.1046/j.0024-4082.2003.00082.x

Huber B.A. 2005. High species diversity, male-female coevolution, and metaphyly in Southeast Asian pholcid spiders: the case of Belisana Thorell 1898 (Araneae, Pholcidae). Zoologica 155: 1-126.

Huber B.A. 2009a. Life on leaves: leaf-dwelling pholcids of Guinea, with emphasis on Crossopriza cylindrogaster Simon, a spider with inverted resting position, pseudo-eyes, lampshade web, and tetrahedral egg-sac (Araneae: Pholcidae). Journal of Natural History 43: 2491-2523.

https://doi.org/10.1080/00222930903207876

Huber B.A. 2009b. Four new generic and 14 new specific synonymies in Pholcidae, and transfer of Pholcoides Roewer to Filistatidae (Araneae). Zootaxa 1970: 64-68; 1977: 68 (erratum).

https://doi.org/10.11646/zootaxa.1970.1.3

Huber B.A. 2011a. Revision and cladistic analysis of Pholcus and closely related taxa (Araneae, Pholcidae). Bonner zoologische Monographien 58: 1-509. Available from https://www.biodiversitylibrary.org/part/120260 [accessed 30 Dec. 2021].

Huber B.A. 2011b. Phylogeny and classification of Pholcidae (Araneae): an update. The Journal of Arachnology 39: 211-222. https://doi.org/10.1636/CA10-57.1

Huber B.A. 2012. Revision and cladistic analysis of the Afrotropical endemic genus Smeringopus Simon, 1890 (Araneae: Pholcidae). Zootaxa 3461: 1-138. https://doi.org/10.11646/zootaxa.3461.1.1

Huber B.A. 2013. Revision and cladistic analysis of the Guineo-Congolian spider genus Smeringopina Kraus (Araneae, Pholcidae). Zootaxa 3713: 1-160. https://doi.org/10.11646/zootaxa.3713.1.1

Huber B.A. 2014. Pholcidae. In: Roig-Juñent S., Claps L.E. \& Morrone J.J. (eds) Biodiversidad de Artrópodos Argentinos Vol. 3: 131-140. Sociedad Entomológica Argentina, Buenos Aires.

Huber B.A. 2019. The pholcid spiders of Sri Lanka (Araneae: Pholcidae). Zootaxa 4550: 1-57. https://doi.org/10.11646/zootaxa.4550.1.1

Huber B.A. 2020. Revision of the spider genus Hoplopholcus Kulczyński (Araneae, Pholcidae). Zootaxa 4726: 1-94. https://doi.org/10.11646/zootaxa.4726.1.1

Huber B.A. 2021a. Beyond size: sexual dimorphisms in pholcid spiders. Arachnology 18: 656-677. https://doi.org/10.13156/arac.2020.18.7.656

Huber B.A. 2021b. First Northwest African species of the spider genus Artema, from caves in Morocco, with notes on body size in pholcid spiders (Araneae: Pholcidae). Zootaxa 4984: 324-334. https://doi.org/10.11646/zootaxa.4984.1.23

Huber B.A. \& Eberle J. 2021. Mining a photo library: eggs and egg sacs in a major spider family. Invertebrate Biology 140 (4): e12349. https://doi.org/10.1111/ivb.12349

Huber B.A. \& Kwapong P. 2013. West African pholcid spiders: an overview, with descriptions of five new species (Araneae, Pholcidae). European Journal of Taxonomy 59: 1-44.

https://doi.org/10.5852/ejt.2013.59

Huber B.A. \& Villarreal O. 2020. On Venezuelan pholcid spiders (Araneae, Pholcidae). European Journal of Taxonomy 718: 1-317. https://doi.org/10.5852/ejt.2020.718.1101

Huber B.A. \& Warui C.M. 2012. East African pholcid spiders: an overview, with descriptions of eight new species (Araneae, Pholcidae). European Journal of Taxonomy 29: 1-44.

https://doi.org/10.5852/ejt.2012.29 
Huber B.A., Deeleman-Reinhold C.L. \& Pérez González A. 1999. The spider genus Crossopriza (Araneae, Pholcidae) in the New World. American Museum Novitates 3262: 1-10. Available from http://hdl.handle.net/2246/3105 [accessed 30 Dec. 2021].

Huber B.A., Colmenares P.A. \& Ramirez M.J. 2014a. Fourteen new generic and ten new specific synonymies in Pholcidae (Araneae), and transfer of Mystes Bristowe to Filistatidae. Zootaxa 3847: 413-422. https://doi.org/10.11646/zootaxa.3847.3.5

Huber B.A., Le Gall P. \& Mavoungou J.F. 2014b. Pholcid spiders from the Lower Guinean region of Central Africa: an overview, with descriptions of seven new species (Araneae, Pholcidae). European Journal of Taxonomy 81: 1-46. https://doi.org/10.5852/ejt.2014.81

Huber B.A., Pavlek M. \& Komnenov M. 2021. Revision of the spider genus Stygopholcus (Araneae, Pholcidae), endemic to the Balkan Peninsula. European Journal of Taxonomy 752: 1-60.

https://doi.org/10.5852/ejt.2021.752.1391

Irie T. 2001. Notes on the pholcid spider, Crossopriza lyoni (Blackwall 1867). Kishidaia 80: 7-20.

Irie T. 2009. Pholcidae. In: Ono H. (ed.) The Spiders of Japan, with Keys to the Families and Genera and Illustrations of the Species. Tokai University Press, Kanagawa.

Jackson R.R. 1992. Predator-prey interactions between web-invading jumping spiders and a webbuilding spider, Holocnemus pluchei (Araneae, Pholcidae). Journal of Zoology, London 228: 589-594. https://doi.org/10.1111/j.1469-7998.1992.tb04457.x

Jackson R.R., Jakob E.M., Willey M.B. \& Campbell G.E. 1993. Anti-predator defences of a webbuilding spider, Holocnemus pluchei (Araneae, Pholcidae). Journal of Zoology, London 229: 347-352. https://doi.org/10.1111/j.1469-7998.1993.tb02641.x

Jäger P. 1995. Erstnachweis von Holocnemus pluchei und zweiter Nachweis von Nesticus eremita für Deutschland in Köln (Araneae: Pholcidae, Nesticidae). Arachnologische Mitteilungen 10: 20-22. https://doi.org/10.5431/aramit1006

Jäger P. 2000. Selten nachgewiesene Spinnenarten aus Deutschland (Arachnida: Araneae). Arachnologische Mitteilungen 19: 49-57. https://doi.org/10.5431/aramit1907

Jäger P. 2007. Spiders from Laos with descriptions of new species (Arachnida: Araneae). Acta Arachnologica 56: 29-58. https://doi.org/10.2476/asjaa.56.29

Jäger P., Nophaseud L. \& Praxaysombath B. 2012. Spiders from Laos with description of a new species and new records (Arachnida: Araneae). Acta Arachnologica 61: 77-92. https://doi.org/10.2476/asjaa.61.77

Jakob E.M. 1991. Costs and benefits of group living for pholcid spiderlings: loosing food, saving silk. Animal Behaviour 41: 711-722. https://doi.org/10.1016/S0003-3472(05)80908-X

Jakob E.M. 1994. Contests over prey by group-living pholcids (Holocnemus pluchei). The Journal of Arachnology 22: 39-45. Available from https://www.americanarachnology.org/journal-joa/ [accessed 30 Dec. 2021].

Jakob E.M. 2004. Individual decisions and group dynamics: why pholcid spiders join and leave groups. Animal Behaviour 68: 9-20. https://doi.org/10.1016/j.anbehav.2003.06.026

Jakob E.M. \& Dingle H. 1990. Food level and life history characteristics in a pholcid spider (Holocnemus pluchei). Psyche 97: 95-110. https://doi.org/10.1155/1990/82891

Jakob E.M., Blanchong J.A., Popson M.A., Sedey K.A. \& Summerfield M.S. 2000. Ontogenetic shifts in the costs of living in groups: focal observations of a pholcid spider (Holocnemus pluchei). The American Midland Naturalist 143: 405-413. https://doi.org/b9dkf8 
Johnson S.A. \& Jakob E.M. 1999. Leg autotomy in a spider has minimal costs in competitive ability and development. Animal Behaviour 57: 957-965. https://doi.org/10.1006/anbe.1998.1058

Kaster J.L. \& Jakob E.M. 1997. Last-male sperm priority in a haplogyne spider (Araneae: Pholcidae): correlations between female morphology and patterns of sperm usage. Annals of the Entomological Society of America 90: 254-259. https://doi.org/10.1093/aesa/90.2.254

Kim J.P. 1988. One species of genus Crossopriza (Araneae: Pholcidae) from Southern Asia. Korean Arachnology 4: 35-38.

Koch C. 1873. Beiträge zur Kenntniss der Arachniden Nord-Afrikas, insbesondere einiger in dieser Richtung bisher noch unbekannt gebliebenen Gebiete des Atlas und der Küsten-Länder von Marocco. Berichte der Senckenbergischen Naturforschenden Gesellschaft 1873: 104-118.

Koch C.L. 1837. Die Arachniden. Vol. 4. C.H. Zeh'sche Buchhandlung, Nürnberg. https://doi.org/10.5962/bhl.title.43744

Koch C.L. 1850. Übersicht des Arachnidensystems. Vol. 5. Lotzbeck, Nürnberg. https://doi.org/10.5962/bhl.title.39561

Koch L. 1875. Aegyptische und abyssinische Arachniden gesammelt von Herrn C. Jickeli. Bauer \& Raspe, Nürnberg.

Kolosváry G. 1938. Sulla fauna aracnologica della Jugoslavia. Passegna Faunistica 5: 62-81.

Kovács G., Szinetár C. \& János E. 2006. A márváyos álkaszáspok (Holocnemus pluchei [Scopoli, 1763]) (Araneae: Pholcidae) Magyarországon. Allattani Közlemények 91: 9-18. [In Hungarian]

Kratochvíl J. 1932. Př́íspěvek k poznání araneid střední Slavonie. Sur quelques araignées de Slavonie centrale. Bulletin de l'Institut National Agronomique, Brno C 23: 1-16.

Kratochvíl J. 1940. Étude sur les araignées cavernicoles du genre Stygopholcus Krat. Acta Societatis Scientiarum Naturalium Moraviae 12: 1-26.

Kraus O. 1984. Male spider genitalia: evolutionary changes in structure and function. Verhandlungen des Naturwissenschaftlichen Vereins in Hamburg (NF) 27: 373-382.

Kritscher E. 1996. Ein Beitrag zur Kenntnis der Spinnen-Fauna der Maltesischen Inseln (Chelicerata: Araneae). Annalen des Naturhistorischen Museums in Wien 98B: 117-156. Available from http://verlag.nhm-wien.ac.at/pdfs/098B_117156_Kritscher.pdf [accessed 30 Dec. 2021].

Kumada K. 2021. New records of Holocnemus pluchei (Scopoli 1763) (Pholcidae) from Japan. Acta Arachnologica 70: 17-19. [In Japanese] https://doi.org/10.2476/asjaa.70.17

Kunt K.B., Yağmur E.A., Özkütük R.S., Durmuş H. \& Anlaş S. 2010. Checklist of the cave dwelling invertebrates (Animalia) of Turkey. Biological Diversity and Conservation 3 (2): 26-41.

Laborda A. \& Simó M. 2008. First South American records of Holocnemus pluchei (Scopoli, 1763) and Spermophora senoculata (Dugès, 1836) (Araneae: Pholcidae). Gayana 72: 261-265.

https://doi.org/10.4067/S0717-65382008000200013

Leardi in Airaghi Z. 1902. Aracnidi di Mahé e Kandy. Atti della Società italiana di scienze naturali e del Museo civico di storia naturale di Milano 40: 345-373. Available from

https://www.biodiversitylibrary.org/page/10275994 [accessed 30 Dec. 2021].

Leborgne R., Cantarella T. \& Pasquet A. 1998. Colonial life versus solitary life in Cyrtophora citricola (Araneae, Araneidae). Insectes sociaux 45: 125-134. https://doi.org/10.1007/s000400050074

Lecigne S. 2018. Sur quelques observations intéressantes d'araignées du Nord et du Pas-de-Calais (France) - $1^{\text {re }}$ note. Le Héron 51: 45-60. 
Lessert R. de. 1910. Catalogue des invertébrés de la Suisse. Fascicule 3, Araignées. Musée d'histoire naturelle de Genève, Genève.

Lucas H. 1846. Histoire naturelle des animaux articulés. In: Exploration scientifique de l'Algérie pendant les années 1840, 1841, 1842. Sciences physiques, Zoologie 1: 89-271. Paris, Imprimerie Royale. https://doi.org/10.5962/bhl.title.112444

Majumder S.C. \& Biswas B. 1993. Taxonomic studies of some pholcid spiders from India (Araneae: Pholcidae). Records of the Zoological Survey of India 92 (1992): 1-3.

Melic A. 1994. ¿Quién quiere ir a Borneo? Notas aracnológicas aragonesas, 1. Boletín de la Sociedad Entomologica Aragonesa 7: 5-19.

Mello-Leitão C. de. 1935. Three interesting new Brasilian spiders. Revista Chilena de Historia Natural 39: 94-98.

Mello-Leitão C. de. 1942. Arañas del Chaco y Santiago del Estero. Revista del Museo de La Plata (NS) 2: 381-426. Available from https://publicaciones.fcnym.unlp.edu.ar/rmlp/article/view/1617/834 [accessed 30 Dec. 2021].

Millot J. 1941. Les araignées de l'Afrique occidentale Française. Sicariides et pholcides. Mémoires de l'Académie des Sciences de l'Institut de France 64: 1-30.

Millot J. 1946. Les pholcides de Madagascar (Aranéides). Mémoires du Muséum National d'Histoire Naturelle (N.S.) 22: 127-158.

Morano E. 2001. Especies nuevas o poco conocidas de arañas (Arachnida, Araneae) de la Fauna Ibérica. Revista Ibérica de Aracnología 3: 67-68. Available from http://sea-entomologia.org/PDF/RIA_3/R03-008-067.pdf [accessed 30 Dec. 2021].

Murphy F. \& Murphy J. 2000. An Introduction to the Spiders of South East Asia. Malaysian Nature Society, Kuala Lumpur.

Najim S.A. \& Al-Khazali A.M. 2019. The first record of Crossopriza maculipes (Araneae, Pholcidae) in Iraq. Syrian Journal of Agricultural Research 6: 548-554.

Nandi N.C. \& Raut S.K. 1985. Predatory behaviour of the pholcid spider Crossopriza lyoni (Blackwall) on mosquitoes (Aedes sp.). Bulletin of the Zoological Survey of India 7: 179-183. [pp. 180-181 not seen]

Naumova M.V., Lazarov S.P., Petrov B.P. \& Deltshev C.D. 2016. New faunistic data on the cavedwelling spiders in the Balkan Peninsula (Araneae). Ecologica Montenegrina 7: 425-438.

https://doi.org/10.37828/em.2016.7.17

Naumova M.V., Lazarov S.P. \& Deltshev C.D. 2019. Faunistic diversity of the spiders in Montenegro (Arachnida: Araneae). Ecologica Montenegrina 22: 50-89. https://doi.org/10.37828/em.2019.22.5

Nicolić F. \& Polenec A. 1981. Catalogus Faunae Jugoslaviae, III/4, Aranea. Slovenska akademija znanosti in umetnosti, Ljubljana.

Nixon K.C. 2002. Winclada, version 1.00.08. Program and documentation. Available from http://www.diversityoflife.org/winclada/ [accessed 30 Dec. 2021].

Paquin P., Vink C. \& Dupérré N. 2010. Spiders of New Zealand: Annotated Family Key \& Species List. Manaaki Whenua Press, Lincoln, New Zealand.

Pérez González A. 1996. Sobre la ausencia del género Crossopriza (Araneae: Pholcidae) en Cuba, con una nueva sinonimia para Artema atlanta Walkenaer, 1837. Caribbean Journal of Science 32: 432-433. 
Pickard-Cambridge O. 1872. General list of the spiders of Palestine and Syria, with descriptions of numerous new species and characters of two new genera. Proceedings of the Zoological Society of London 40: 212-354, pl. 13-16. Available from https://www.biodiversitylibrary.org/part/67338 [accessed 1 Feb. 2022].

Pickard-Cambridge O. 1876. Catalogue of a collection of spiders made in Egypt, with descriptions of new species and characters of a new genus. Proceedings of the Zoological Society of London 44: 541-630, pl. 58-60. https://doi.org/10.1111/j.1096-3642.1876.tb02595.x

Platnick N.I. 2000. The World Spider Catalog, Version 1.0. Available from https://wsc.nmbe.ch/archive/ [accessed 30 Dec. 2021].

Pocock R.I. 1900. The Fauna of British India, including Ceylon and Burma. Arachnida. Taylor and Francis, London. https://doi.org/10.5962/bhl.title.48423

Ponomarev A.V., Snegovaya N.Y. \& Shmatko V.Y. 2019. Synaphosus shirin Ovtsharenko, Levi et Platnick, 1994 (Gnaphosidae) and Holocnemus pluchei (Scopoli, 1763) (Pholcidae) - two new species of spiders (Aranei) in the fauna of the Caucasus. Caucasian Entomological Bulletin 15: 233-235.

Porter A.H. \& Jakob E.M. 1990. Allozyme variation in the introduced spider Holocnemus pluchei (Araneae, Pholcidae) in California. The Journal of Arachnology 18: 313-319.

Raychaudhuri D. \& Saha S. 2015. Spiders (Araneae: Arachnida) of reserve forests of Dooars: Gorumara National Park, Chapramari Wildlife Sanctuary and Mahananda Wildlife Sanctuary. World Scientific News 20: 1-339.

Reiser N. \& Neumann J. 2014. Holocnemus pluchei (Araneae, Pholcidae) in Getränke- und Baumärkten in Deutschland. Arachnologische Mitteilungen 48: 24-27. https://doi.org/10.5431/ARAMIT4805

Ribera C. 2013. Las arañas (Arachnida, Araneae) de las cuevas de Jaén. In: Pérez Fernández T. \& Pérez Ruiz A. (eds) Los Invertebrados de Hábitats Subterráneos de Jaén: 58-64. Grupo de Espeleología de Villacarrillo, Jaén.

Ribera C., de Mas E. \& Barranco Vega P. 2003. Araneidos cavernícolas de la provincia de Almería (I) y descripción de cuatro especies nuevas. Revista Ibérica de Aracnología 7: 3-17.

Roewer C.F. 1928. Zoologische Streifzüge in Attika, Morea und besonders auf der Insel Kreta, II. Abhandlungen des Naturwissenschaftlichen Vereins zu Bremen 27: 92-124.

Roewer C.F. 1955. Katalog der Araneae von 1758 bis 1940, bzw. 1954. 2. Band, Abt. a (Lycosaeformia, Dionycha [excl. Salticiformia]). 2. Band, Abt. b (Salticiformia, Cribellata) (Synonyma-Verzeichnis, Gesamtindex). Institut royal des Sciences naturelles de Belgique, Bruxelles.

Roewer C.F. 1959. Die Araneae, Solifuga und Opiliones der Sammlungen des Herrn Dr. K. Lindberg aus Griechenland, Creta, Anatolien, Iran und Indien. Göteborgs Kungliga Vetenskaps och Vitterhets Samhälles Handlingar 8 (4): 1-47.

Roewer C.F. 1960. Solifugen und Opilionoiden. Araneae Orthognathae, Haplogynae und Entelegynae. Göteborgs Kungliga Vetenskaps och Vitterhets Samhälles Handlingar 8 (7): 1-53.

Rossi P. 1790. Fauna etrusca: sistens insecta quae in provinciis Florentina et Pisana praesertim collegit Petrus Rossius. Tomus secundus: 126-140. Thomae Masi \& Sociorum, Liburni.

https://doi.org/10.5962/bhl.title.15771

Roth V.D. 1985. Spider Genera of North America. American Arachnological Society, Gainesville.

Roth V.D. 1994. Spider Genera of North America, with Keys to Families and Genera, and a Guide to Literature, Third Edition. American Arachnological Society, Gainesville. 
Rozwałka R., Rutkowski T. \& Bielak-Bielecki P. 2016. New data on introduced and rare synanthropic spider species (Arachnida: Araneae) in Poland. Annales Universitatis Mariae Curie-Skłodowska 68: 127-150. https://doi.org/10.2478/v10067-012-0028-6

Savigny J.-C. \& Audouin V. 1826. Explication sommaire des planches d'arachnides de l'Égypte et de la Syrie. In: Description de l'Égypte, ou recueil des observations et des recherches qui ont été faites en Égypte pendant l'expédition de l'armée française, publié par les ordres de sa Majesté l'Empereur Napoléon le Grand. Histoire Naturelle 1 (4): 1-339 (arachnids, pp. 99-186).

Scopoli J.A. 1763. Entomologia carniolica, exhibens insecta carniolae indigena et distributa in ordines, genera, species, varietates. Methodo Linnaeana. Typis Ioannis Thomae Trattner, Vienna. https://doi.org/10.5962/bhl.title.34434

Sedey K.A. \& Jakob E.M. 1998. A description of an unusual dome web occupied by egg-laying Holocnemus pluchei (Araneae, Pholcidae). The Journal of Arachnology 26: 385-388.

Senglet A. 1971. Note sur les Pholcidae (Arachn.) de Grèce. Mitteilungen der Schweizerischen Entomologischen Gesellschaft 44: 345-359. https://doi.org/10.5169/seals-401666

Senglet A. 1972. Note sur les Spermophora (Araneae: Pholcidae) méditerranéens. Mitteilungen der Schweizerischen Entomologischen Gesellschaft 45: 307-319. https://doi.org/10.5169/seals-401694

Senglet A. 2001. Copulatory mechanisms in Hoplopholcus, Stygopholcus (revalidated), Pholcus, Spermophora and Spermophorides (Araneae, Pholcidae), with additional faunistic and taxonomic data. Mitteilungen der Schweizerischen Entomologischen Gesellschaft 74: 43-67.

https://doi.org/10.5169/seals-402796

Sherriffs W.R. 1919. A contribution to the study of South Indian arachnology. The Annals and Magazine of Natural History (9) 4: 220-253. https://doi.org/10.1080/00222931908673888

Simon E. 1866. Monographie des espèces européennes du genre Pholcus. Annales de la Société entomologique de France (4) 6: 117-124, pl. 2.

Simon E. 1873. Aranéides nouveaux ou peu connus du midi de l'Europe. Extrait des Mémoires de la Société royale des Sciences de Liège (2) 5: 187-351. [separately paginated version (pp. 1-174) seen and cited here.]

Simon E. 1874. Les Arachnides de France. Tome premier. Libraire Encyclopédique de Roret, Paris.

Simon E. 1875. Les Arachnides de France. Tome second. Libraire Encyclopédique de Roret, Paris.

Simon E. 1885. Étude sur les arachnides recueillis en Tunisie en 1883 et 1884 par MM. A. Letourneux, M. Sédillot et Valéry Mayet, membres de la mission de l'exploration scientifique de la Tunisie. In: Exploration scientifique de la Tunisie, publiée sous les auspices du Ministère de l'instruction publique. Zoologie - Arachnides. Imprimerie nationale, Paris.

Simon E. 1890. Étude sur les arachnides de l'Yemen. Annales de la Société entomologique de France 6 (10): 77-124.

Simon E. 1893. Histoire Naturelle des Araignées. $2^{\text {nd }}$ Ed. Encyclopédie Roret, Paris. https://doi.org/10.5962/bhl.title.51973

Simon E. 1907. Arachnides recueillis en Egypte et le long du Nil Blanc par la Mission zoologique suedoise, 1901. In: Results of the Swedish Zoological Expedition to Egypt and the White Nile 1901 under the Direction of L.A. Jägerskiöld Part III 21 (37): 1-10. Library of the Royal University of Uppsala, Uppsala. https://doi.org/10.5962/bhl.title.21727 
Simon E. 1908. Étude sur les Arachnides, recueillis par Mr. le Dr. Klaptocz en Tripolitaine. Zoologische Jahrbücher. Abteilung für Systematik, Geographie und Biologie der Tiere 26: 419-438. Available from https://www.biodiversitylibrary.org/page/27521606 [accessed 30 Dec. 2021].

Simon E. 1909. Étude sur les archnides du Tonkin (1 ${ }^{\text {ire }}$ partie). Bulletin scientifique de la France et de la Belgique 42: 69-147. https://doi.org/10.5962/bhl.part.24151

Simon E. 1914. Les Arachnides de France. Roret, Paris.

Siyam M., Dunlop J.A. \& El-Hennawy H.K. 2015. New spider records from the Republic of the Sudan. Arachnology 16: 264-272. https://doi.org/10.13156/arac.2015.16.7.264

Skow C.D. \& Jakob E.M. 2003 Effects of maternal body size on clutch size and egg weight in a pholcid spider (Holocnemus pluchei). The Journal of Arachnology 31: 305-308. https://doi.org/10.1636/01-85

Slowik, J. 2009. A review of the cellar spider genus Psilochorus Simon 1893 in America north of Mexico (Araneae: Pholcidae). Zootaxa 2144: 1-53. https://doi.org/10.11646/zootaxa.2144.1.1

Song D., Zhu M. \& Chen J. 1999. The Spiders of China. Hebei Science and Technology Publishing House, Shijiazhuang.

Spassky S. 1934. Araneae palaearticae novae. Fam. Pholcidae I. Bulletin du Museum d'histoire naturelle

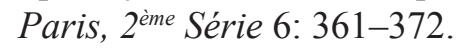

Strand E. 1907. Süd- und ostasiatische Spinnen. Abhandlungen der Naturforschenden Gesellschaft Görlitz 25: 107-215, pl. 1-21.

Strand E. 1908. Nordafrikanische Spinnen, hauptsächlich von Carlo Freiherr von Erlanger gesammelt (Dictynidae, Eresidae, Sicariidae, Dysderidae, Caponiidae, Palpimanidae, Zodariidae, Urocteidae, Pholcidae, Agelenidae, Pisauridae). Archiv für Naturgeschichte 74: 67-128.

Strickman D., Sithiprasasna R. \& Southard D. 1997. Bionomics of the spider, Crossopriza lyoni (Araneae, Pholcidae), a predator of Dengue vectors in Thailand. The Journal of Arachnology 25: 194201. Available from https://www.jstor.org/stable/3705644 [accessed 30 Dec. 2021].

Thorell T. 1895. Descriptive Catalogue of the Spiders of Burma, Based Upon the Collection Made by Eugene W. Oates and Preserved in the British Museum. British Museum, London.

https://doi.org/10.5962/bhl.title.7163

Thorell T. 1898. Secondo saggio sui ragni Birmani. II. Retitelariae et Orbitelariae. Annali del Museo civico di storia naturale di Genova 39: 271-378.

Tikader B.K. \& Biswas B. 1981. Spider fauna of Calcutta and vicinity: Part I. Records of the Zoological Survey of India, Occasional Paper 30: 1-149.

Timm H. 1976. Die Bedeutung von Genitalstrukturen für die Klärung systematischer Fragen bei Zitterspinnen (Arachnida: Araneae: Pholcidae). Entomologica Germanica 3: 69-76.

Tong Y. 2013. Haplogynae Spiders from Hainan, China. Ke xue chu ban she, Beijing.

Topçu A., Demir H., Seyyar O. \& Türkeş T. 2005. The spider fauna of the Gülek Pass (Turkey) and its environs (Araneae). Acta zoologica bulgarica, Suppl 1: 287-295.

Türkeş T. \& Karabulut H. 2013. Araneo-fauna of Kemaliye (Erzincan) from Turkey. Munis Entomology and Zoology 8: 619-633.

Valdez-Mondragón A. 2010. Revisión taxonómica de Physocyclus Simon, 1893 (Araneae: Pholcidae), con la descripción de especies nuevas de México. Revista Ibérica de Aracnología 18: 3-80.

van Helsdingen P.J. 2010. Holocnemus pluchei (Scopoli, 1763) in Nederland (Araneae, Pholcidae). Nieuwsbrief SPINED 29: 27. 
Van Keer K. 2007. Exotic spiders (Araneae): verified reports from Belgium of imported species (1976-2006) and some notes on apparent neozoan invasive species. Nieuwsbrief van den Belgische Arachnologische Vereniging 22: 45-54.

Van Keer K. \& Van Keer J. 2001. Ingeburgerde exotische trilspinnen (Araneae: Pholcidae) in Antwerpse haven en enkele algemene bedenkingen bij spinnenmigratie. Nieuwsbrief van den Belgische Arachnologische Vereniging 16: 81-86.

Van Keer K. \& Van Keer J. 2004. In België aangetroffen exoten (Araneae) uit de collectie J. Van Keer. Nieuwsbrief van den Belgische Arachnologische Vereniging 18: 78-83.

Vetter R.S., Reierson D.A. \& Rust M.K. 2011. Cobweb management and control of the spider Holocnemus pluchei (Araneae: Pholcidae) on buildings. Journal of Economic Entomology 104: 601606. https://doi.org/10.1603/ec10403

Walckenaer C.A. 1837. Histoire naturelle des Insectes. Aptères. I. Libraire Encylopédique de Roret, Paris. https://doi.org/10.5962/bhl.title.61095

Wiehle H. 1933. Holocnemus hispanicus sp. nov. und die Gattungen Holocnemus Simon und Crossoprisa Simon. Zoologischer Anzeiger 104: 241-252.

World Spider Catalog. 2021. World Spider Catalog. Version 22.0. Natural History Museum Bern. Available from http://wsc.nmbe.ch [accessed 24 Jun. 2021]. https://doi.org/10.24436/2

Wunderlich J. 1980. Zur Kenntnis der Gattung Pholcus Walckenaer 1805 (Arachnida: Araneae: Pholcidae). Senckenbergiana biologica 60: 219-227.

Yaginuma T. 1982. Occurrence of a pholcid spider, Crossopriza lyoni, in Japan. Atypus 80: 15-18.

Yaginuma T. 1986. Spiders of Japan in Color. New Edition. Hoikusha Publishing Co., Osaka.

Yin C., Peng X., Yan H., Bao Y., Xu X., Tang G., Zhou Q. \& Lui P. 2012. Fauna Hunan. Araneae in Hunan, China. Hunan Science and Technology Press, Changscha.

Zhu C. \& Wang F. 1963. Chinese Pholcidae and a new species. Journal of Jilin University 5: 461-466, pl. 1-2.

Manuscript received: 24 June 2021

Manuscript accepted: 15 November 2021

Published on: 22 February 2022

Section editor: Rudy Jocqué

Desk editor: Pepe Fernández

Printed versions of all papers are also deposited in the libraries of the institutes that are members of the EJT consortium: Muséum national d'histoire naturelle, Paris, France; Meise Botanic Garden, Belgium; Royal Museum for Central Africa, Tervuren, Belgium; Royal Belgian Institute of Natural Sciences, Brussels, Belgium; Natural History Museum of Denmark, Copenhagen, Denmark; Naturalis Biodiversity Center, Leiden, the Netherlands; Museo Nacional de Ciencias Naturales-CSIC, Madrid, Spain; Real Jardín Botánico de Madrid CSIC, Spain; Zoological Research Museum Alexander Koenig, Bonn, Germany; National Museum, Prague, Czech Republic. 
Appendix 1. Data matrix for cladistic analysis. Taxa are arranged as in the cladogram in Fig. 1. Terminals and characters are detailed in Appendices 2 and 3; "--" = non-applicable; "?" = unknown or coding ambiguous. This matrix is available online at www.pholcidae.de/matrices.html

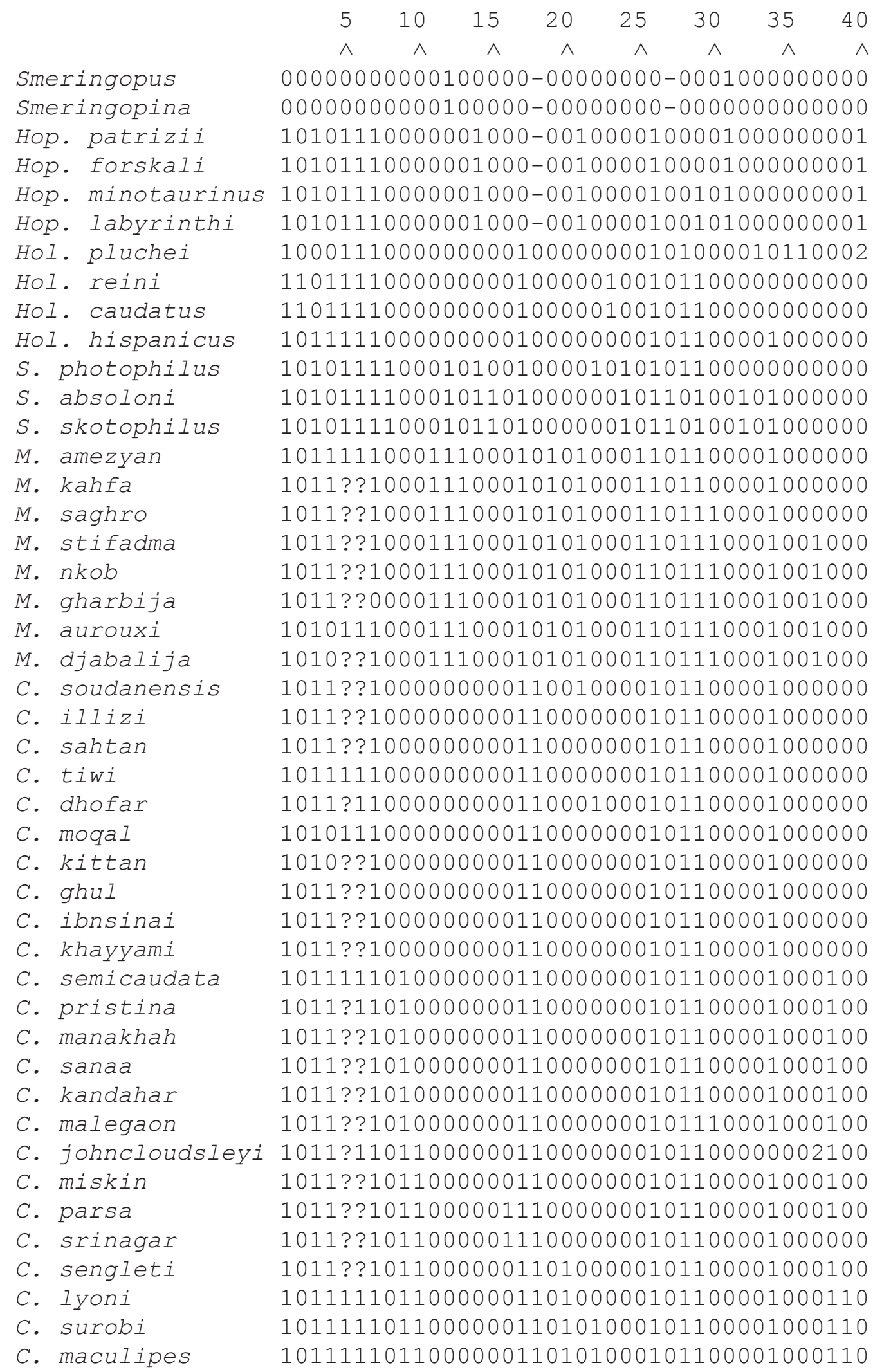


Appendix 2. Terminal taxa scored for the cladistic analysis, with origin and depository. Taxa are arranged as in the cladogram in Fig. 1.

\section{Outgroups}

Smeringopus Simon, 1890; the coding for this genus is supposed to represent the plesiomorphic states, derived from the cladistic analysis in Huber (2012).

Smeringopina Kraus, 1957; the coding for this genus is supposed to represent the plesiomorphic states, derived from the cladistic analysis in Huber (2013).

Hoplopholcus patrizii (Roewer, 1962); Turkey, Kocain Mağaras1, ZFMK Ar 20946. Turkey, Karain Mağarası, ZFMK Ar 20947.

Hoplopholcus forskali (Thorell, 1871); Hungary, Molnár János Cave, ZFMK Ar 20936. Turkey, forest below Ferzene Mağaras1, ZFMK Ar 20942.

Hoplopholcus minotaurinus Senglet, 1971; Greece, Crete, Spilaio Milatou, ZFMK Ar 20889 and CJVK 2267.

Hoplopholcus labyrinthi (Kulczyński, 1903); Greece, Crete, Spilaio Melidoniou, ZFMK Ar 20875.

\section{Spotted-leg clade}

Holocnemus pluchei (Scopoli, 1763); Israel, S of Teverya, ZFMK Ar 22306. Georgia, Sighnaghi, ZFMK Ar 21373. Greece, Daphni/Athens, MHNG.

Holocnemus reini (C. Koch, 1873) comb. nov.; Morocco, near Tilamizene, ZFMK Ar 22319. Morocco, Al Kamoun, CAS 9027140.

Holocnemus caudatus (Dufour, 1820); Spain, Valencia, Montroy, MHNG. Spain, Granada, Trevélez, MHNG.

Holocnemus hispanicus Wiehle, 1933; Spain, Conquista de la Sierra, CRB. Spain, Ronda, CRB. Spain, Huelva, “Alájar/Aracena”, MHNG.

Stygopholcus photophilus Senglet, 1971; Greece, Crete, Episkopi, ZFMK Ar 22205. Greece, Crete, Choumerion/Pérama, MHNG.

Stygopholcus absoloni (Kulczynski, 1914); Croatia, Balićeva špilja, ZFMK Ar 22195. Bosnia and Herzegovina, Zavala, MHNG. Bosnia and Herzegovina, Počitelj/Čapljina, MHNG.

Stygopholcus skotophilus Kratochvíl, 1940; Bosnia and Herzegovina, Ilijina pećina, ZFMK Ar 22198. Montenegro, Pećina u Ivici, ZFMK Ar 22196.

Maghreba amezyan gen. et sp. nov.; Morocco, between Lakhssas and Bouizakarne, ZFMK Ar 22353, Ar 22354.

Maghreba kahfa gen. et sp. nov.; Morocco, Ifri Taghrart Wankrim, CPO.

Maghreba saghro gen. et sp. nov.; Morocco, between Irherm and Tiferki, ZFMK Ar 22363, Ar 22364. 
Maghreba stifadma gen. et sp. nov.; Morocco, Setti-Fatma, ZFMK Ar 22368, Ar 22369.

Maghreba nkob gen. et sp. nov.; Morocco, E of Nkob, ZFMK Ar 22374.

Maghreba gharbija gen. et sp. nov.; Morocco, 4 km E of Ghazoua, ZFMK Ar 22379, Ar 22380.

Maghreba aurouxi (Barrientos, 2019) gen. et comb. nov.; Morocco, SE of Zebzat, ZFMK Ar 22385, Ar 22386.

Maghreba djabalija gen. et sp. nov.; Morocco, NE of Tizi n’Test, ZFMK Ar 22389, Ar 22390.

Crossopriza soudanensis Millot, 1941; Burkina Faso, Ouagadougou, MNHN Ar 10520. Mali, Bamako, MNHN Ar 10512.

Crossopriza illizi sp. nov.; Algeria, Iherir, ZFMK Ar 22392. Algeria, Aguelmane ti-n-Azzaret, CRB.

Crossopriza sahtan sp. nov.; Oman, above Wadi Sahtan, ZFMK Ar 22394. Oman, Al Ghubrat cave, ZFMK Ar 22398.

Crossopriza tiwi sp. nov.; Oman, Wadi Tiwi, ZFMK Ar 22402, Ar 22403.

Crossopriza dhofar sp. nov.; Oman, Ain Razad cave, ZFMK Ar 22412. Oman, Wadi Nahiz, ZFMK Ar 22413.

Crossopriza moqal sp. nov.; Oman, Mukal (Moqal) Cave, ZFMK Ar 22421, Ar 22422.

Crossopriza kittan sp. nov.; Oman, Ibri, Al Kittan Cave, ZFMK Ar 22424, Ar 22425.

Crossopriza ghul sp. nov.; Oman, Wadi Ghul, ZFMK Ar 22427, Ar 22428.

Crossopriza ibnsinai sp. nov.; Uzbekistan, near Dzhyra-Kuduk, SZMN.

Crossopriza khayyami sp. nov.; Iran, Izad Khast, MHNG.

Crossopriza semicaudata (O. Pickard-Cambridge, 1876); Egypt, $5 \mathrm{~km} \mathrm{~N}$ of Aswan, CAS. Egypt, Luxor, SMF. Chad, Bardaï, MRAC 132959.

Crossopriza pristina (Simon, 1890); Eritrea, Massaua, MNHN Ar 10510. Yemen, Aden, MNHN Ar 10511.

Crossopriza manakhah sp. nov.; Yemen, 12 km NW of Manakhah, ZFMK Ar 22439 to Ar 22441. Yemen, Ja'ar, ZFMK Ar 22444.

Crossopriza sanaa sp. nov.; Yemen, near Sana'a, NMNL.

Crossopriza kandahar sp. nov.; Afghanistan, W of Kandahar, MHNG.

Crossopriza malegaon sp. nov.; India, $17 \mathrm{mi}$ NE of Malegaon, CAS.

Crossopriza miskin sp. nov.; Oman, near Miskin, ZFMK Ar 22445, Ar 22446. Oman, wadi near Fasah, ZFMK Ar 22447.

Crossopriza parsa sp. nov.; Iran, Persepolis, MHNG. 
Crossopriza srinagar sp. nov.; India, Srinagar, ZFMK Ar 22449, Ar 22450.

Crossopriza sengleti sp. nov.; Iran, Fars, Seridjan, MHNG.

Crossopriza lyoni (Blackwall, 1867); Guinea, Kindia, ZFMK Ar 10456. Japan, Yamamoto-chô, ZFMK Ar 5209.

Crossopriza surobi sp. nov.; Afghanistan, SE of Surobi/Sarobi, MHNG.

Crossopriza maculipes (Spassky, 1934); Turkmenistan, 5 km SW of Bazar-Tepe, SZMN. Uzbekistan, $6.5 \mathrm{~km} \mathrm{~W}$ of Akmechet, ZMMU. Afghanistan, Djelalabad, MHNG. 
Appendix 3. Characters scored for cladistic analysis.

\title{
Prosoma
}

1. Carapace lateral marks: (0) present; (1) absent. Dark lateral marks on the carapace seem to have been lost within Smeringopinae, uniting all representatives of the northern clade. They are present in Cenemus Saaristo, 2001 (not included in the present analysis), suggesting that this mysterious Seychellois genus may be basal within the northern clade.

2. Sternum indentations: (0) absent or shallow; (1) present, deep. Deep indentations of the sternum (Figs 81, 112) are unique for the sister species Holocnemus reini and H. caudatus.

\begin{abstract}
Abdomen
3. Abdomen general shape: (0) cylindrical; (1) ventrally shortened. In the outgroup taxa Smeringopus and Smeringopina as well as in Holocnemus pluchei (and in Cenemus), the abdomen (pedicel to spinnerets, i.e., ventrally) is usually $>2$ times as long as the prosoma (pedicel to chelicerae frontal face). In Hoplopholcus and in most ingroup taxa, the abdomen is relatively shorter (usually $\sim 1.5$ times as long as prosoma, at least ventrally, i.e., discounting a dorsal elongation). The coding is problematic in Holocnemus reini and $H$. caudatus, where this value varies among specimens between 1.5 and 2.0. In the Pee-Wee analyses with conc $=1$ and 2, Hoplopholcus is nested within the spotted-leg clade, i.e., the shortening of the abdomen occurs only once.
\end{abstract}

4. Abdomen posterior dorsal shape (in lateral view): (0) rounded; (1) angular to pointed. The plesiomorphic condition in Smeringopinae (and Pholcidae) is a posteriorly rounded abdomen. A more angular or even pointed abdomen (Figs 394, 699) occurs in many representatives of the spotted-leg clade and is here interpreted to unite all species except $H$. pluchei. Several reversals within the spottedleg clade (Stygopholcus; some species of Maghreba gen. nov. and Crossopriza) and independent origins (e.g., Cantikus Huber, 2018; Ixchela Huber, 2000; Metagonia Simon, 1893; and Priscula Simon, 1893) suggest that this character is evolutionarily very flexible. In fact, one of the two most parsimonious cladograms using Pee-Wee with conc $=1$ and 2 suggests three independent origins of an angular/pointed abdomen within the spotted-leg clade.

5. Epiandrous spigots at male gonopore: (0) two; (1) four or more. The outgroup taxa Smeringopus and Smeringopina share the reduction from four to two epiandrous spigots, which is clearly the derived condition (Huber 2013). Four epiandrous spigots is the plesiomorphic condition in Pholcidae. Thus, this is not a synapomorphy of Hoplopholcus + spotted-leg clade, as suggested by the cladistic analysis. Instead, this group is characterized by a tendency to increase spigot numbers, often to five or six (Figs 161, 320, 854), rarely up to seven or eight (Fig. 93; Huber 2020: fig.72).

6. Spigots on anterior lateral spinnerets (ALS): (0) seven to eight: one widened spigot, one pointed spigot, and five or six cylindrically shaped spigots; (1) two (one widened spigot and one pointed spigot). A reduction in the number of ALS spigots has happened many times independently in Pholcidae (e.g., Huber 2000, 2011a) and distinguishes the northern clade of Smeringopinae (and Cenemus) from the Sub-Saharan clade (Smeringopus and Smeringopina).

\section{Male chelicerae}

7. Stridulatory files: (0) absent; (1) present. The cladistic analysis suggests that stridulatory files are a synapomorphy of Hoplopholcus + spotted-leg clade. However, this may be an artifact of outgroup choice (cf. char. 5). The presence of stridulatory files in most Ninetinae and Arteminae suggests that the 
alternative scenario is more plausible: that stridulatory files are plesiomorphic for Smeringopinae, and that the absence of stridulatory files is derived and unites Smeringopus and Smeringopina (cf. Huber 2012).

8. Club-shaped hairs on frontal face of chelicerae: (0) absent; (1) present. A field of $\sim 10-40$ club-shaped hairs on each frontal cheliceral face is a synapomorphy of Stygopholcus (Huber et al. 2021: figs 19, $27-$ $28,51,63-64,93-94,119,130,132)$. Such hairs occur in some distantly related groups (e.g., Blancoa Huber, 2000; Mecolaesthus Simon, 1893; Systenita Simon, 1893; Tupigea Huber, 2000) (Huber 1997, 2000; Huber \& Villarreal 2020) but not in other Smeringopinae.

9. Direction of main cheliceral apophyses: (0) towards distal; (1) towards median. The main cheliceral apophyses (i.e., those that carry one or more cone-shaped hairs at their tips) are originally directed towards distal (e.g., Figs 64, 268, 458). In a subgroup of Crossopriza, they have moved to a more frontal position and are directed towards median (e.g., Figs 585, 621,774). This character is functionally related to character 38 , which characterizes the same species group.

10. Additional pair of lateral apophyses: (0) absent; (1) present. Some of the species of Crossopriza in which the main cheliceral apophyses have moved to a more frontal position (char. 9) share an additional pair of lateral apophyses (e.g., Figs 774, 814, 832). These newly evolved apophyses never carry coneshaped hairs at their tips.

\section{Male palp}

11. Retrolateral apophysis on coxa: (0) absent; (1) present. A distinct apophysis (rather than just a rounded hump) on the retrolateral (to ventral) side of the coxa characterizes all species of Maghreba gen. nov. Such an apophysis is common in Modisiminae (e.g., Huber 2000) but otherwise rare in Pholcidae.

12. Retrolateral process proximally on femur: (0) absent; (1) present. The cladistic analysis suggests that such an apophysis was lost in the ancestor of Hoplopholcus + spotted-leg clade but regained in Stygopholcus + Maghreba gen. nov.

13. Dorsal process on femur: (0) absent; (1) present. A small dorsal process proximally on the femur characterizes all species of Maghreba gen. nov. (e.g., Figs 205, 226).

14. Palpal tarsal organ: (0) capsulate; (1) exposed. A capsulate tarsal organ is plesiomorphic for Smeringopinae (and Pholcidae). In the cladogram in Fig. 1, an exposed tarsal organ evolved independently in the ancestors of Hoplopholcus and Stygopholcus. In the analysis with Pee-Wee (with conc = 1 and 2), the exposed tarsal organ is considered a synapomorphy uniting these two genera.

15. Dorsal hairs on procursus: (0) weakly or not curved; (1) strongly curved 'upwards. The hairs dorsally on the procursus are usually straight or weakly curved. In the northern clade of Stygopholcus (represented here by S. absoloni and S. skotophilus), these hairs are strongly curved 'upwards' (Huber et al. 2021: fig. 8).

16. Procursus general shape: (0) 'regular', i.e., not particularly long and curved towards ventral; (1) unusually long and curved towards ventral. Two species of Crossopriza share a very similar procursus that is slightly longer than usual and curved towards ventral (Figs 728, 747).

17. Procursus prolateral hump: (0) absent; (1) present. All representatives of the spotted-leg clade share a more or less distinct rounded hump on the prolateral side of the procursus (e.g., Figs 137, 629). Such a hump also occurs in some species of Smeringopus but is here not considered plesiomorphic for the 
genus. In the analysis with Pee-Wee (with conc $=1$ and 2), the hump is considered secondarily lost in Hoplopholcus.

18. Hairs on procursus prolateral hump: (0) few hairs or hairless; (1) many hairs. A dense set of often longer than usual hairs on the prolateral hump of the procursus is currently the only known morphological synapomorphy of Crossopriza.

19. Procursus tip: (0) straight; (1) strongly bent towards dorsal. The main branch of the procursus is usually straight at its tip (e.g., Figs 63, 358), or slightly bent towards dorsal (e.g., Fig. 22) or ventral (e.g., Figs 138, 630). In all representatives of Maghreba gen. nov., the tip is strongly bent towards dorsal (e.g., Figs 166, 210, 229).

20. Branch on ventral sclerite of procursus tip: (0) absent; (1) present. Four species of Crossopriza (including the type species) share a retrolateral-dorsal branch on the ventral sclerite of the procursus (Figs 769, 788, 810, 828).

21. Membranous process at tip of procursus: (0) absent; (1) present. A membranous (often transparent) process at the tip of the procursus (next to the ventral sclerite; Figs 166, 210, 309, 358) is here considered to have evolved independently in Hoplopholcus, in Maghreba gen. nov., and in Crossopriza soudanensis.

22. Procursus dorsal sclerite: (0) absent; (1) present. A sclerotized process dorsally at the tip of the procursus unites Crossopriza surobi sp. nov. and C. maculipes (Figs 810, 828). Similar structures in C. dhofar sp. nov. and in Stygopholcus photophilus are considered to be convergent.

23. Retrolateral membrane on procursus tip: (0) absent; (1) present. A membranous flap that covers most of the ventral sclerite in lateral view (Fig. 63) unites Holocnemus reini and H. caudatus.

24. Slender dorsal process on genital bulb: (0) absent; (1) present. A transparent or weakly sclerotized dorsal bulbal process is a unique synapomorphy of Stygopholcus (Huber et al. 2021: figs 17, 117).

25. Dorsal pocket on distal bulbal sclerite: (0) absent; (1) present. A large and deep pocket dorsally on the distal bulbal sclerite (Figs 169, 213) is a unique synapomorphy of Maghreba gen. nov.

\section{Legs}

26. Spines on male femur 1: (0) absent; (1) present. Spines on the male femur 1 (very rarely also tibia 1 and femur 2) unite all genera of Smeringopinae except Smeringopus and Smeringopina. They are also absent in Cenemus (not included in the present analysis), which is further evidence for a basal position of this genus within the northern clade of Smeringopinae (cf. char. 1). Such spines have repeatedly evolved in distantly related taxa (e.g., Modisimus Simon, 1893; Savarna Huber, 2005; Hantu Huber, 2016; Paramicromerys Millot, 1946) (Huber 2021a).

27. Spine arrangement on femur 1: (0) in one row; (1) in two rows. In most Smeringopinae (and Pholcidae in general), spines on femur 1 (if present) are arranged in a single ventral row. In the northern clade of Stygopholcus, the spines are arranged in two rows (Huber et al. 2021).

28. Dark marks on femora and tibiae (0) absent; (1) present. Distinctive dark lines or oval marks (Figs 34, 188-189, 206-207, 558, 577, 709) are characteristic of almost all representatives of the spotted-leg clade. They have been lost in the slightly troglomorphic species of the northern clade of Stygopholcus (Huber et al. 2021). In the analysis with Pee-Wee (with conc = 1 and 2), the marks are considered secondarily 
lost also in Hoplopholcus. Such marks are rare in other pholcids. They also occur in Smeringopus cylindrogaster, a species originally assigned to Crossopriza (Huber 2009a).

29. Prolateral trichobothrium on tibia 1: (0) present; (1) absent. The plesiomorphic condition in Pholcidae is the presence of three trichobothria on each leg tibia: retrolateral, dorsal and prolateral. Only the prolateral trichobothrium of tibia 1 is sometimes lost, and this has happened repeatedly in Pholcidae (B.A. Huber, unpublished data). Within Smeringopinae, the cladogram in Fig. 1 suggests two independent losses: within Hoplopholcus, and in the ancestor of all species of the spotted-leg clade except Holocnemus pluchei. The analyses with Pee-Wee (with conc $=1$ and 2) requires a regain within Hoplopholcus (which is why the alternative topology shown in Fig. 1 is preferred).

30. Short vertical hairs on male tibiae: (0) very few, as in female; (1) in higher density than in female. Short vertical hairs (Figs 119, 120) that may function as chemoreceptors (Foelix \& Chu-Wang, 1973) occur in low densities on most leg segments of Pholcidae, mainly on the distal segments. In several pholcid taxa, males have many such hairs on the leg femora and/or tibiae (Huber 2021a). Within Smeringopinae, only some species of Maghreba gen. nov. and Crossopriza malegaon sp. nov. share this character. However, the density is not particularly high and the hairs are barely visible using a dissecting microscope.

31 Curved hairs on legs: (0) absent; (1) present. In Smeringopinae, curved hairs occur in Smeringopus and Hoplopholcus; they are absent in the spotted-leg clade. The optimization of this character is ambiguous; it is therefore not mapped on Fig. 1. In the analysis with Pee-Wee (with conc $=1$ and 2), curved hairs originate independently in Smeringopus and Hoplopholcus.

32. Rim of leg tarsal organ: (0) round or slightly irregular; (1) strongly undulating. In most Smeringopinae, the rim of the capsulate tarsal organ on the legs is either round or slightly irregular (Figs 53, 122-123, 156, 863). In the northern clade of Stygopholcus, the rim is strongly undulating (Huber et al. 2021: figs $38,75,97)$.

\section{Female body}

33. Median process on female sternum: (0) absent; 1) present. A posterior median process on the female sternum (Fig. 31) is a unique autapomorphy of Holocnemus pluchei.

34. Paired stridulatory organ between carapace and abdomen: (0) absent; (1) present. Stridulatory devices between prosoma and abdomen have evolved repeatedly in Pholcidae (Huber 2021a). Paired organs on the dorsal side occur in several distantly related genera (e.g., Trichocyclus Simon, 1908; Anopsicus Chamberlin \& Ivie, 1938; and Aymaria Huber, 2000). In Smeringopinae, this character unites a large part of the spotted-leg clade (Crossopriza, Maghreba gen. nov., Stygopholcus, and Holocnemus hispanicus). Within this group, it was secondarily lost twice (Stygopholcus photophilus and Crossopriza johncloudsleyi). The analysis with Pee-Wee (with conc $=1$ and 2) suggests two independent origins in Smeringopinae: once within Stygopholcus and once in the ancestor of Holocnemus hispanicus + Maghreba gen. nov. + Crossopriza.

35. Female palp shape: (0) 'regular', i.e., thin; (1) strongly thickened. Within Smeringopinae, only Holocnemus pluchei has female palps with strongly thickened distal segments (tibia, tarsus) (Figs 2930). A very similar thickening has independently evolved in the distantly related genus Holocneminus Berland, 1942 (Berland 1942; Deeleman-Reinhold 1995). 
36. Sclerotized ventral ridge anteriorly on female abdomen: (0) absent; (1) present. Within Smeringopinae, only Holocnemus pluchei females have such a ridge/process (Fig. 38). The only pholcid species known to have a similar structure is the distantly related Waunana modesta (Banks, 1929) (Huber 2000: fig. 1114).

\section{Female genitalia}

37. Epigynal processes: (0) absent; (1) one pair; (2) two pairs. Most Smeringopinae lack epigynal processes. Some species of Maghreba gen. nov. share a pair of more or less distinct processes (e.g., Fig. 238). The epigynum of Crossopriza johncloudsleyi with two pairs of prominent processes is unique in the spotted-leg clade.

38. Position of epigynal pockets: (0) lateral, opening towards ventral/anterior; (1) close to median line, on opposing sides of median ridge, opening towards lateral. A pair of pockets that corresponds to the male cheliceral apophyses seems to be plesiomorphic for Smeringopinae. In a subgroup of Crossopriza, these pockets have moved to a more median position and open towards lateral (e.g., Figs 587, 623, 719). This character is functionally related to character 9 , which characterizes the same species group.

39. Shape of epigynal posterior margin: (0) straight; (1) medially protruding. In three species of Crossopriza, the posterior margin of the epigynal plate is slightly (C. lyoni) to distinctly (C. surobi sp. nov., C. maculipes) protruding medially (Figs 798, 816, 834).

40. Paired pockets or pouches in female internal genitalia: (0) absent; (1) low; (2) deep and distinct. A pair of low internal pockets characterizes the genus Hoplopholcus. Within the spotted-leg clade, Holocnemus pluchei is unique in having a pair of deep conical pockets (Figs 40-42). 